Ingo Schroeter

Analyse und Bewertung der intergenerativen

Verteilungswirkungen einer Substitution des Umlage- durch das Kapitalstocksverfahren zur Rentenfinanzierung 
Ingo Schroeter

\section{Analyse und Bewertung der intergenerativen Verteilungswirkungen einer Substitution des Umlage- durch das Kapitalstocksverfahren zur Rentenfinanzierung}

Die Sicherung eines zukunftsfähigen Rentensystems ist eines der dringenden Ziele der aktuellen Sozialpolitik. Dabei spielen die Verteilungswirkungen (insbesondere zwischen verschiedenen Generationen) eine ebenso wichtige Rolle wie die allokative Effizienz. In diesem Buch wird ein Konzept entwickelt, mit dem die intergenerativen Verteilungswirkungen eines Alterssicherungssystems bewertet werden können. Im Vordergrund stehen Reformvorschläge, die auf einer (partiellen) Substitution des Finanzierungsverfahrens beruhen. Das Konzept soll es ermöglichen, konkrete Reformoptionen zu bewerten und sozialpolitische Handlungs- bzw. Entscheidungshilfen abzuleiten. Mit Hilfe des entwickelten Konzepts werden abschließend ausgewählte rentenpolitische Optionen simuliert.

Ingo Schroeter studierte von 1989 bis 1995 Wirtschaftsingenieurwesen (Fachrichtung Maschinenbau) an der Technischen Universität Darmstadt und an der Gakushuin University in Tokio. Von 1995 bis 1998 Promotion am Lehrstuhl für Finanzwissenschaft, Fachbereich Rechts- und Wirtschaftswissenschaften, der Technischen Universität Darmstadt. 


\author{
Analyse und Bewertung \\ der intergenerativen Verteilungswirkungen \\ einer Substitution des Umlage- durch \\ das Kapitalstocksverfahren zur Rentenfinanzierung
}




\section{Sozialökonomische Schriften \\ Herausgegeben von Bert Rürup}

\section{Band 18}

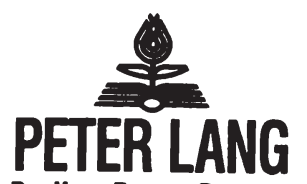

Frankturt am Main - Berlin - Bern - Bruxelles - New York - Wien 


\section{Ingo Schroeter}

\section{Analyse und Bewertung der intergenerativen Verteilungs- wirkungen einer Substitution des Umlage- durch das Kapitalstocksverfahren zur Rentenfinanzierung}

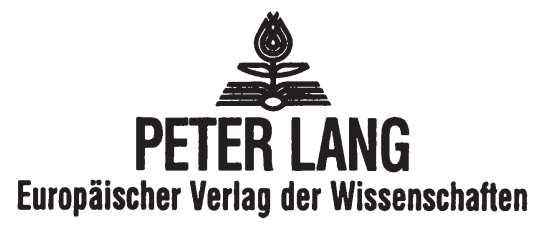


Die Deutsche Bibliothek - CIP-Einheitsaufnahme

Schroeter, Ingo:

Analyse und Bewertung der intergenerativen Verteilungswirkungen einer Substitution des Umlage- durch das Kapitalstocksverfahren zur Rentenfinanzierung / Ingo Schroeter. - Frankfurt am Main ; Berlin ; Bern ; Bruxelles ; New York ; Wien : Lang, 1999

Open Access: The online version of this publication is published on www.peterlang.com and www.econstor.eu under the international Creative Commons License CC-BY 4.0. Learn more on how you can use and share this work: http://creativecommons.org/ licenses/by/4.0.

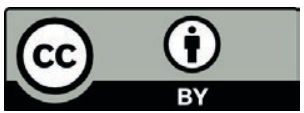

This book is available Open Access thanks to the kind support of ZBW - Leibniz-Informationszentrum Wirtschaft.

Gedruckt auf alterungsbeständigem, säurefreiem Papier.

(Sozialökonomische Schriften ; Bd. 18)

Zugl.: Darmstadt, Techn. Hochsch., Diss., 1998

ISBN 3-631-35634-X

\author{
D 17 \\ ISSN 0172-1747 \\ ISBN 3-631-35634-X \\ ISBN 978-3-631-75016-2 (eBook) \\ (C) Peter Lang GmbH \\ Europäischer Verlag der Wissenschaften \\ Frankfurt am Main 1999 \\ Alle Rechte vorbehalten.
}

Das Werk einschließlich aller seiner Teile ist urheberrechtlich geschützt. Jede Verwertung außerhalb der engen Grenzen des

Urheberrechtsgesetzes ist ohne Zustimmung des Verlages unzulässig und strafbar. Das gilt insbesondere für

Vervielfältigungen, Übersetzungen, Mikroverfilmungen und die Einspeicherung und Verarbeitung in elektronischen Systemen.

Printed in Germany $1234 \quad 67$ 


\section{VORWORT}

Die vorliegende Arbeit wurde im Sommersemester 1998 am Fachbereich für Rechts- und Wirtschaftswissenschaften der Technischen Universität Darmstadt eingereicht. Ich möchte mich bei all jenen bedanken, die mich unterstützt und zum Gelingen der Arbeit beigetragen haben. Allen voran gilt mein besonderer Dank Herrn Prof. Dr. Dr. h.c. Bert Rürup für die vielen anregenden Diskussionen und seine fachliche Unterstützung. Er ermöglichte mir, die praxisbezogene Politikberatung kennenzulernen und prägte mich als "Doktorvater" nicht nur in meiner wissenschaftlichen Tätigkeit. Dank schulde ich auch meinem Korreferenten Herrn Prof. Dr. Volker Caspari und der Studienstiftung des Deutschen Volkes, durch deren Unterstützung die Arbeit erst möglich wurde. Nicht zuletzt möchte ich mich bei Frau Dr. Dorothea Wenzel und Herrn Volker Schmitz für ihre Hilfe beim Korrekturlesen bedanken. 
Ingo Schroeter - 978-3-631-75016-2

Downloaded from PubFactory at 01/11/2019 09:14:34AM

via free access 


\subsection{INHALTSÜBERSICHT}

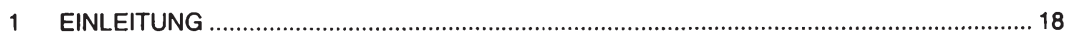

1.1 MOTIVATION UND KONKRETISIERUNG DER AUFGABENSTELLUNG ............................ 18

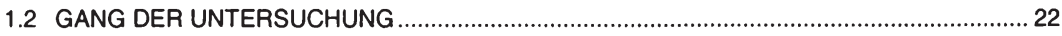

2 MODELLTHEORETISCHE ANALYSE DER FINANZIERUNGSVERFAHREN ....................... 25

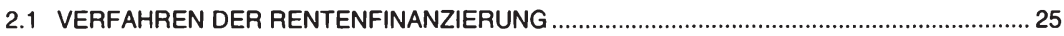

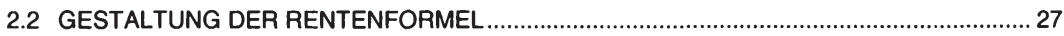

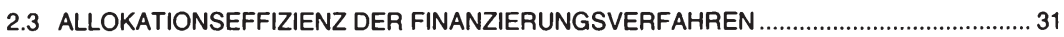

2.4 INTERGENERATIVE VERTEILUNGSWIRKUNGEN DER FINANZIERUNGSVERFAHREN . 51

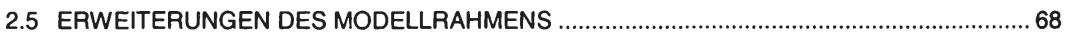

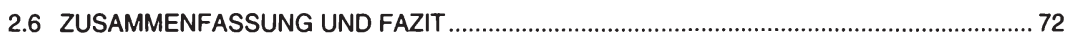

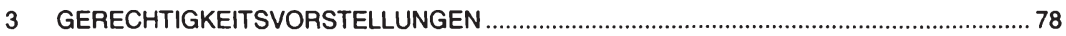

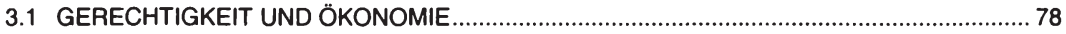

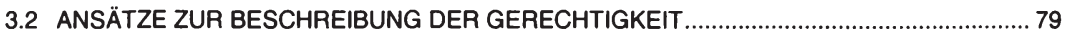

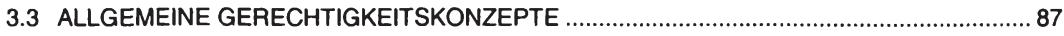

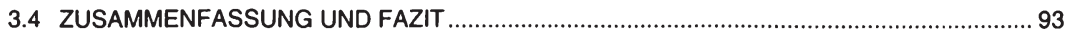

4 ANALYSERAHMEN ZUR INTERGENERATIVEN GERECHTIGKEIT DER

ALTERSSICHERUNG 98 
4.1 ABGRENZUNG DES SYSTEMS FÜR DIE BEWERTUNG DER VERTEILUNGSWIRKUNGEN

4.2 AUSWAHL DER RELEVANTEN VERTEILUNGSWIRKUNGEN FÜR DIE ANALYSE DER INTERGENERATIVEN GERECHTIGKEIT.

4.3 ÜBERWÄLZUNGS- UND ZURECHNUNGANNAHMEN ÜBER ZAHLUNGEN DRITTER..... 103

4.4 GESTALTUNGSPRINZIPIEN DER ALTERSSICHERUNG UND IHRE BEDEUTUNG FÜR DIE BEWERTUNG DER VERTEILUNGSWIRKUNGEN 105

4.5 ZUSAMMENFASSUNG UND FAZIT 124

5 OPERATIONALISIERUNG DER UNMITTELBAREN INTERGENERATIVEN UMVERTEILUNGSWIRKUNGEN

5.1 METHODEN ZUR ERFASSUNG DER INTERGENERATIVEN VERTEILUNG EINES ALTERSSICHERUNGSSYSTEMS.

5.2 WAHL EINES VERTEILUNGSNEUTRALEN REFERENZSYSTEMS 141

5.3 ABGRENZUNG DES INTERGENERATIVEN RISIKOAUSGLEICHS IN DER GERECHTIGKEITSANALYSE 153

5.4 BEWERTUNG DES VERTEILUNGSMUSTERS. 188

5.5 ZUSAMMENFASSUNG UND FAZIT 193

6 BERÜCKSICHTIGUNG DER INDIREKTEN INTERGENERATIVEN VERTEILUNGSWIRKUNGEN VERSCHIEDENER FINANZIERUNGSVERFAHREN 200

6.1 BEDEUTUNG DER THESE VOM ERSPARNIS- UND WACHSTUMSEFFEKT DES KAPITALSTOCKVERFAHRENS 200

6.2 ZUR ERFASSUNG DER ERSPARNIS- UND WACHSTUMSEFFEKTE DES KAPITALSTOCKVERFAHRENS BEI DER INTERGENERATIVEN UMVERTEILUNG. 215 


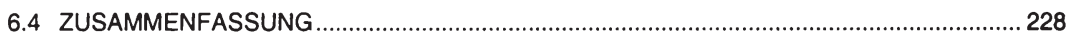

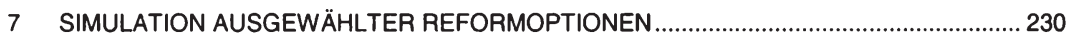

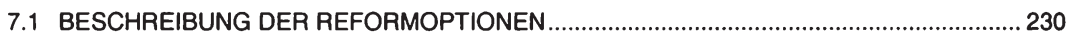

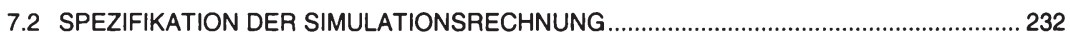

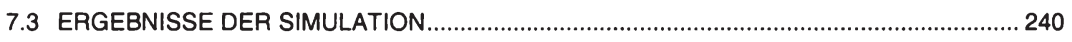

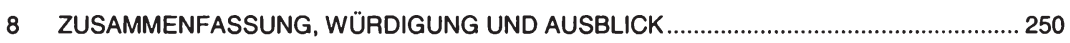

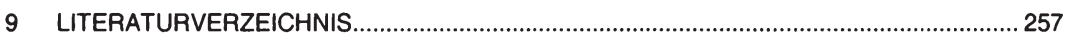

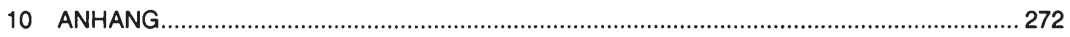




\subsection{INHALTSVERZEICHNIS}

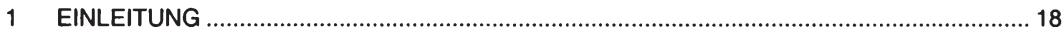

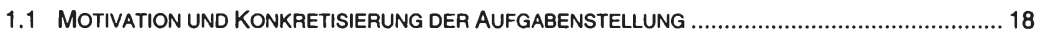

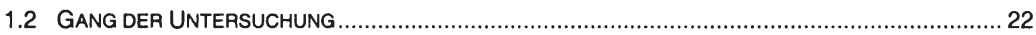

2 MODELLTHEORETISCHE ANALYSE DER FINANZIERUNGSVERFAHREN ....................... 25

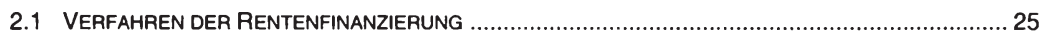

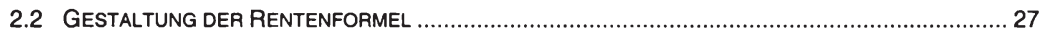

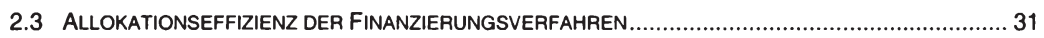

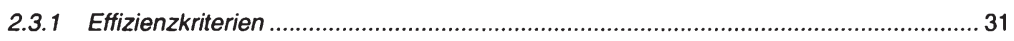

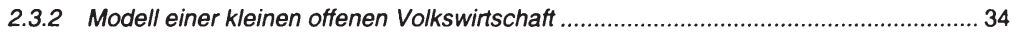

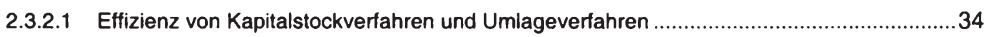

2.3.2.1.1 Interne Entragsrate der Finanzierungsverfahren ........................................................34

2.3.2.1.2 Effizienz der Allterssicherungssysteme ………………………………………..........36

2.3.2.2 Existenz einer Pareto-optimalen Umstellung des Finanzierungsverfahrens..........................38

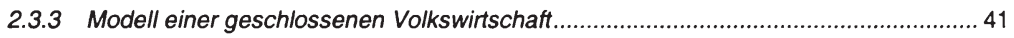

2.3.3.1 Effizienz von Kapitalstockverfahren und Umlageverfahren ................................................

2.3.3.2 Existenz einer Pareto-optimalen Umstellung des Finanzierungsverfahrens.........................47

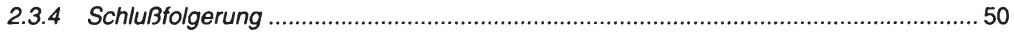

2.4 INTERGENERATIVE VERTEILUNGSWIRKUNGEN DER FINANZIERUNGSVERFAHREN..........................51

2.4.1 Identifikation relevanter Einflußfaktoren ................................................................. 51

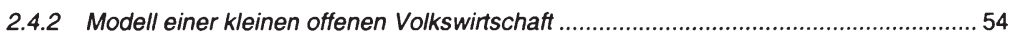

2.4.2.1 Beschreibung der Intergenerativen Verteilungswirkungen .................................................54

2.4.2.2 Modellhafte Überlegungen zur intergenerativen Verteilung................................................59

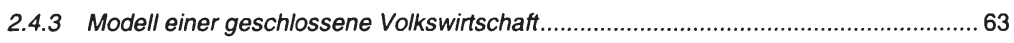

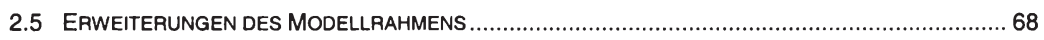

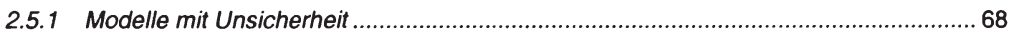

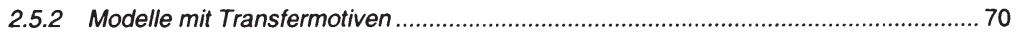

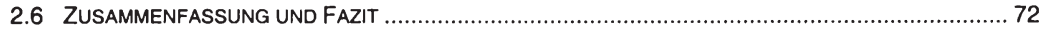

10 


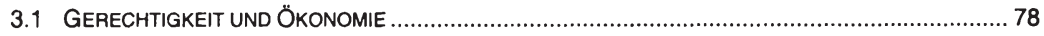

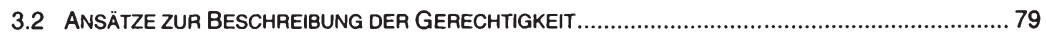

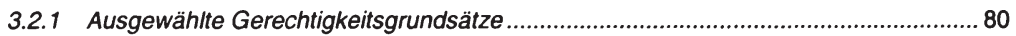

3.2.2 Beziehungen der Gerechtigkeitsgrundsätze zueinander .......................................... 84

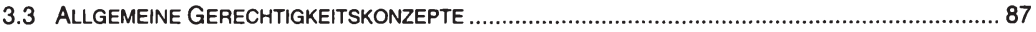

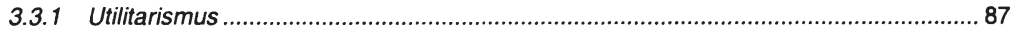

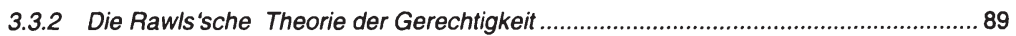

3.3.3 Nozicks Anspruchstheorie der Gerechtigkeit ........................................................... 91

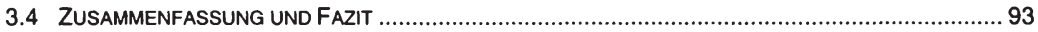

4 ANALYSERAHMEN ZUR INTERGENERATIVEN GERECHTIGKEIT DER

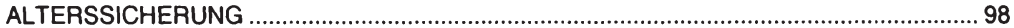

4.1 ABgRENZUNG DES SYSTEMS FüR DIE BEWERTUNG DER VERTEILUNGSWIRKUNGEN....................98

4.2 AUSWAHL DER RELEVANTEN VERTEILUNGSWIRKUNGEN FÜR DIE ANALYSE DER

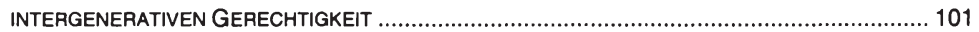

4.3 ÜBERWÄLZUNGS- UND ZURECHNUNGANNAHMEN ÜBER ZAHLUNGEN DRITTER .......................... 103

4.4 GestaltungspainZiPIEN DER ALTERSSICHERUNG UND IHRE BEDEUTUNG Für DIE

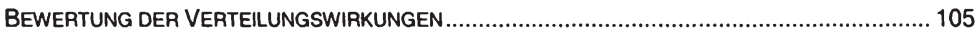

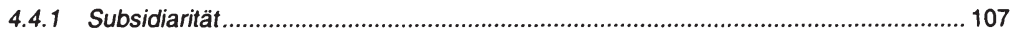

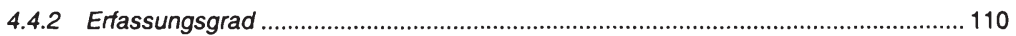

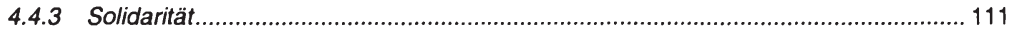

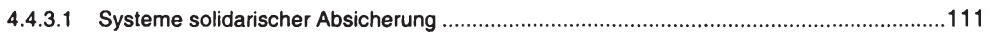

4.4.3.2 Solidarische Absicherung aus intergenerativer Perspektive...............................................118

4.4.4 Gestaltungsprinzipien der gRV und Bewertung der intergenerativen

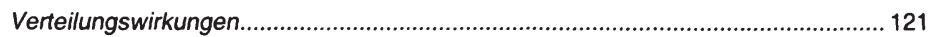

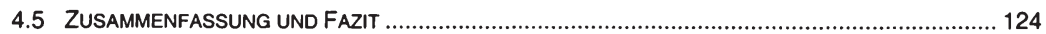

5 OPERATIONALISIERUNG DER UNMITTELBAREN INTERGENERATIVEN UMVERTEILUNGSWIRKUNGEN

5.1 Methoden ZUR ERFASSUNG DeR INTERgenerativen VerteILUNG EINES

ALTERSSICHERUNGSSYSTEMS

5.1.1 Ausgewählte Methoden zur Erfassung der intergenerationalen Verteilung. 131 


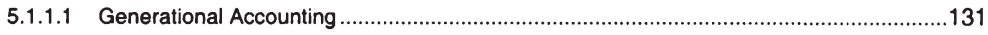

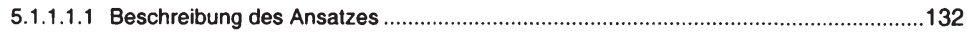

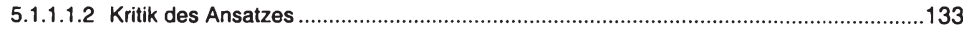

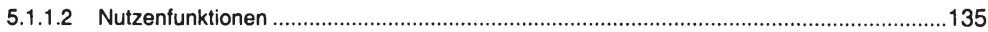

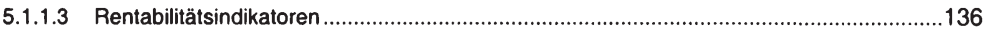

5.1.2 Auswahl der Erfassungsmethode und Formulierung des Verteilungsindikators........ 139

5.2 WAHL EINES VERTEILUNGSNEUtRALEN REFERENZSYSTEMS.......................................... 141

5.2.1 Kritik des beitragsäquivalenten Systems als Referenzmaßstab......................... 141

5.2.2 Zur Legitimation des Kapitalstockverfahrens als verteilungsneutrales Referenzsystem .................................................................................... 146

5.2.3 „Intergenerationale Durchhaltbarkeit" als Referenzsystem ...................................... 152

5.3 AbgRENZUNG DES INTERGENERATIVEN RISIKOAUSGLEICHS IN DER GERECHTIGKEITSANALYSE. 153

5.3.1 Abgrenzung der intergenerativen Risiken ......................................................... 154

5.3.1.1 Kriterien eines intergenerativen Risikoausgleichs .....................................................155

5.3.1.2 Auswahl der zu analysierenden Verteilungseffekte ................................................159

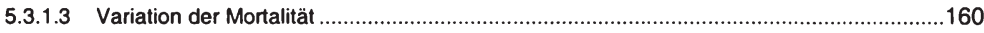

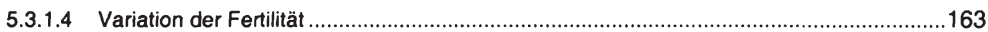

5.3.1.5 Variation der versicherungspflichtigen Enwerbstätigkeit ..............................................168

5.3.1.6 Variation des Faktorpreisverhältnisses........................................................................174

5.3.2 Ausmaß des intergenerativen Risikoausgleichs............................................. 180

5.3.3 Verteilungsregeln im Risikofall ..................................................................... 184

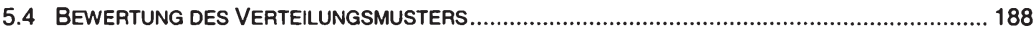

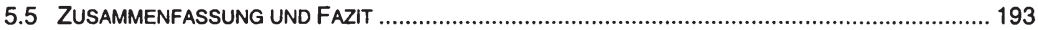

6 BERÜCKSICHTIGUNG DER INDIREKTEN INTERGENERATIVEN

VERTEILUNGSWIRKUNGEN VERSCHIEDENER FINANZIERUNGSVERFAHREN .......... 200

6.1 BEDEUTUNG DER THESE VOM ERSPARNIS- UND WACHSTUMSEFFEKT DES

KAPITALSTOCKVERFAHRENS

6.1.1 Kritik am Vermögenssubstitutionseffekt ........................................................202

6.1.2 Investitionstheoretische Kritik .................................................................... 206

6.1.3 Kritik am theoretischen Modellrahmen ...........................................................207

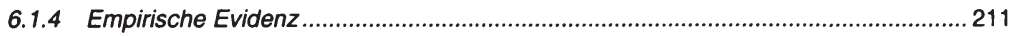

6.2 ZUR ERFASSUNG DER ERSPARNIS- UND WACHSTUMSEFFEKTE DES KAPITALSTOCKVERFAHRENS BEI DER INTERGENERATIVEN UMVERTEILUNG

12 
6.2.1 Rentenkapitalstock und gesamtwirtschaftliche Ersparnis ....................................... 216

6.2.2 Ersparnis und interne Verzinsung des Kapitalstockverfahrens............................... 218

6.2.3 Ersparnis und Wachstum der Volkswirtschaft .......................................................... 220

6.2.4 Auswirkungen auf den Verteilungsindikator der intergenerativen Gerechtigkeit........ 223

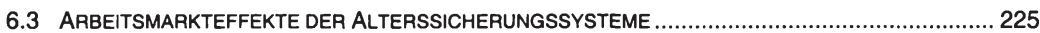

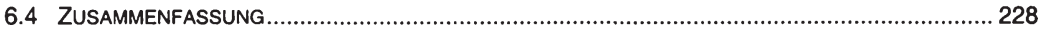

7 SIMULATION AUSGEWÄHLTER REFORMOPTIONEN ....................................................... 230

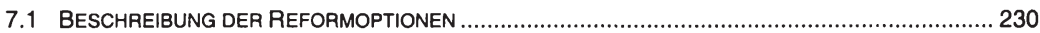

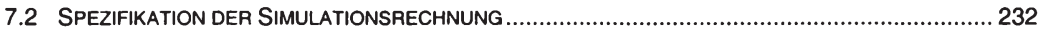

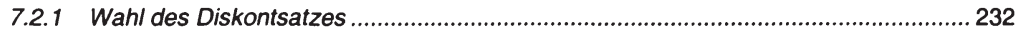

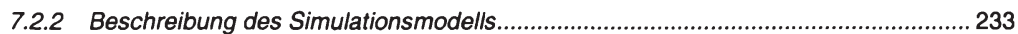

7.2.3 Modellierung der kapitalfundierten Altersvorsorge ..................................................... 236

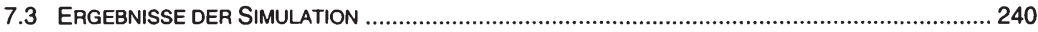

7.3.1 Wirkung des demographischen Korrekturfaktors der Reform 99............................ 240

7.3.2 Wirkung der pauschalen Senkung des Rentenniveaus ........................................... 243

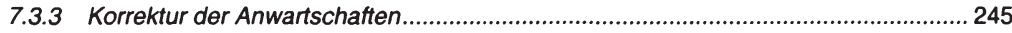

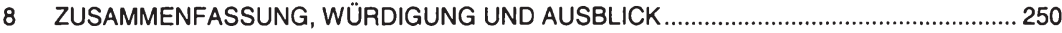

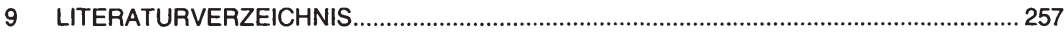

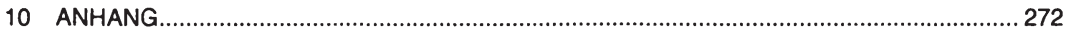




\subsection{ABBILDUNGSVERZEICHNIS}

Abbildung 1: Aufbau der Arbeit.

Abbildung 2: Veränderung der Rendite unterschiedlicher Generationen bei einer Erhöhung der Enwerbsbeteiligung der „Generation 3".

Abbildung 3: Veränderung der Rendite unterschiedlicher Generationen bei einer Erhöhung der Erwerbsbeteiligung der "Generationen 3 bis 8 ".

Abbildung 4: Übersicht Kapitel zwei 77

Abbildung 5: Übersicht Kapitel drei 96

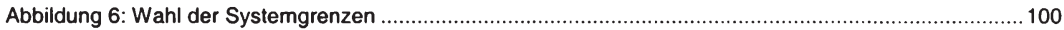

Abbildung 7: Auswahl der für die Analyse maßgeblichen Systemgrenze. 101

Abbildung 8: Gestaltungsdimensionen und -prinzipien eines Alterssicherungssystems (ohne Gestaltungsdimension „Finanzierungsverfahren")

Abbildung 9: Ausmaß an sozialen Ausgleich (Solidarität) bei verschiedenen Absicherungssystemen

Abbildung 10: Gestaltungsmöglichkeiten der Beitragsäquivalenz 115

Abbildung 11: Übersicht Kapitel vier. 128

Abbildung 12: Verlauf des generationsspezifischen Verteilungsindikators (VI) bei unterstellten vollständigen Risikoausgleich bezüglich Fertilitätsveränderungen und Akzeptanz der in der Nettoanpassung enthaltenen Lastverteilungsregel $\left(\Phi=k^{\text {netto }}\right)$.

Abbildung 13: Verlauf des generationsspezifischen Verteilungsindikators (VI) bei unterstellten unvollständigen Risikoausgleich bezüglich Fertilitätsveränderungen (bzw. einer von der Nettoanpassung abweichenden Lastverteilungsregel) $\left(\Phi>k^{\text {netto }}\right)$.....

Abbildung 14: Übersicht Kapitel fünf

Abbildung 15: Abgrenzung des intergenerativen Risikoausgleichs

Abbildung 16: Vermögen der betrieblichen Alterssicherung (Pensionsvermögen) als Prozentsatz des BIP (1990-1991) und durchschnittliche gesamtwirtschaftliche Bruttoersparnis als Prozentsatz des BSP (Sparquote) (1990-1992).

Abbildung 17: Streuungsdiagramm der Kombinationen von Vermögen der betrieblichen Alterssicherung (Pensionsvermögen) als Prozentsatz des BIP (1990-1991) und durchschnittliche gesamtwirtschaftliche Bruttoersparnis als Prozentsatz des BSP 23 ausgewählter Länder (Sparquote) (1990-1992)

Abbildung 18: Differenz der Verteilungsindikatoren der Zugangskohorten der Reform 99 zu den Verteilungsindikatoren des Status Quo......

Abbildung 19: Verlauf der Umverteilungspositionen der Zugangskohorten im Status Quo und bei der Reform 1999

Abbildung 20: Differenz der Verteilungsindikatoren der Zugangskohorten einer pauschalen Senkung des Rentennilveaus 99 zu den Verteilungsindikatoren des Status Quo.

Abbildung 21: Verlauf der Umverteilungspositionen der Zugangskohorten ibei einer pauschalen Senkung des Rentenniveaus und bei der Reform 1999.

14 
Abbildung 22: Verlauf der Umverteilungspositionen der Zugangskohorten ibei einer sukzessiven Kürzung der neu erworbenen Entgeltpunkte und im Status Quo

Abbildung 23: Verlauf der Umverteilungspositionen der Zugangskohorten bei einer pauschalen Senkung des Rentenniveaus und bei der Reform 1999.

Abbildung 24: Differenz der Verteilungsindikatoren der Zugangskohorten bei der Reform 99 und bei einer Reform ohne Halbierung der Korrekturkomponente zu den Verteilungsindikatoren des Status Quo..... 


\subsection{TABELLENVERZEICHNIS}

Tabelle 1: Varianten der Rentenformel und ihre Kompatibilität mit den Finanzierungsverfahren

Tabelle 2: Variation der Rendite verschiedener Generationen bei einer einperiodigen

Parametervariation der Generation $\mathbf{x}$.

Tabelle 3: Variation des Zinssatzes bei Veränderung der ökonomischen und demographischen

Parameter in einer geschlossenen Volkswirtschaft und bei einem Kapitalstockverfahren

mit konstantem Beitragssatz

Tabelle 4: Variation des Zins- und Beitragssatzes bei Veränderung der ökonomischen und demographischen Parameter in einer geschlossenen Volkswirtschaft und bei einem Kapitalstockverfahren mit konstantem Rentenniveau.

Tabelle 5: Ziele und Gestaltungsprinzipien der gesetzliche Rentenversicherung und Folgen für die

Bewertung intergenerativer Verteilungswirkungen

Tabelle 6: Verläufe der intergenerativen Umverteilung und ihre Bewertung durch unterschiedliche Kriterien ( $A$ > B ist zu lesen: „A wird gegenüber B als gerechter eingestuft" - fett gedruckte Bewertungen beschreiben intuitive plausible Gerechtigkeitsvorstellungen)

Tabelle 7: Abgrenzung der intergenerativen Risiken und Einfluß auf den Verteilungsindikator und das Referenzsystem

Tabelle 8: Längsschnittsanalysen zur Frage, ob das soziale Sicherungssystem die gesamtwirtschaftliche private Ersparnis mindert.

Tabelle 9: Querschnittsanalysen zur Frage, ob das soziale Sicherungssystem die gesamtwirtschaftliche private Ersparnis mindert.

Tabelle 10: Studien zur Frage, ob (kapitalfundierte) privaten Altersvorsorgemaßnahmen die private Ersparnis erhöht.

Tabelle 11: Kennziffern zum Verlauf der Umverteilungspositionen der Zugangskohorten im Lstatus Quo und bei der Reform 99

Tabelle 12: Kennziffern zum Verlauf der Umverteilungspositionen der Zugangskohorten bei einer pauschalen Senkung des Rentenniveaus und im Status Quo.

Tabelle 13: Kennziffern zum Verlauf der Umverteilungspositionen der Zugangskohorten bei einer pauschalen Senkung des Rentenniveaus und im Status Quo....

16 


\section{EINLEITUNG}

\subsection{Motivation und Konkretisierung der Aufgabenstellung}

Die Sicherung eines zukunftsfähigen Rentensystems ist eines der dringenden Ziele der aktuellen Sozialpolitik. Die verschiedenen Maßnahmen, mit denen dieses Ziel erreicht werden soll, werden in Politik, Öffentlichkeit und Wissenschaft gleichermaßen kontrovers diskutiert. Weitgehend unbestritten ist, daß aufgrund der folgenden Ursachen die Rentenversicherung reformiert werden muß:

- Kurz- und mittelfristig ergeben sich Probleme aus der Lohnzentrierung des derzeitigen Systems. In diesem Zusammenhang sind zwei Tendenzen gleichermaßen nachteilig für die Rentenversicherung: Erstens führt das anhaltende hohe Niveau der Arbeitslosigkeit einnahmeseitig zu Beitragsausfällen, während es gleichzeitig auf der Leistungsseite mit Mehrausgaben zu Buche schlägt. ${ }^{1}$ Zweitens erschwert der Wandel des Erwerbslebens und die unter dem Stichwort der „Erosion der Normarbeitsverhältnisse“ diskutierte Verschiebung der Beschäftigungsstruktur die Finanzierung der Renten.

- Mittel- und langfristig verschlechtert vor allem die prognostizierte demographische Entwicklung das Verhältnis von Leistungsempfängern zu Beitragszahlern und erschwert die Finanzierung der Alterssicherung.

- Die unter dem Stichwort der „Globalisierung“ diskutierte Zunahme und Veränderung der internationalen Verflechtung der Märkte verschärft die aus dem Arbeitsmarkt und der Demographie resultierenden Probleme: Hohe Sozialbeiträge verteuern den Faktor Arbeit und verschlechtern - neben anderen Faktoren - die internationale Wettbewerbsfähigkeit der Unternehmen. Dies akzentuiert die Arbeitsmarktprobleme und erfordert eine weitere Erhöhung der Sozialbeiträge. Diese Zusammenhänge des Rentensystems bzw. des gesamten Sozialsystems könnten in einen Circulus vitiosus ausarten.

Die Probleme der gesetzliche Rentenversicherung sind schon seit längerem bekannt und entsprechend zahlreich sind die in Wissenschaft und Politik unterbreiteten Vorschläge, wie auf diese Situation reagiert werden kann. ${ }^{2}$

Zur Bewertung eines Alterssicherungssystems, bzw. speziell dieser Reformvorschläge, werden unterschiedliche und z.T. interdependente Kriterien herangezogen. $\mathrm{Zu}$ diesen

Dieser Effekt wird noch dadurch verschärft, daß in der Vergangenheit versucht wurde, den Arbeitsmarktproblemen durch einen vorgezogenen Rentenbeginn zu begegnen.

2 Das Spektrum der systemimmanenten und systemverāndernden Reformen reicht von leichten Rentenrechtlichen Modifikationen über wertschöpfungsbezogene Arbeitgeberbeiträge und bevölkerungsdynamische Renten- und Beitragsleistungen bis zu beitrags- oder steuerfinanzierte Grundrentensystemen, kapitalstockbasierte und Universalsysteme. 
Bewertungskriterien gehören z.B. die finanzwirtschaftliche Effizienz, die politische Stabilität, die Abhängigkeit von exogenen Einflußgrößen, die Sicherheit des Lebensstandards, die Allokationseffizienz oder die (re-)distributiven Wirkungen. ${ }^{3}$

Verfolgt man die öffentliche Diskussion in den Medien zur Frage der Alterssicherung, so zeigt sich, daß diese stark von der Vorstellung eines (drohenden) Generationenkonflikts geprägt ist und somit das Kriterium der (intergenerativen) Verteilung an Bedeutung gewinnt. Politiker aller Couleur greifen diese Stimmung auf und werben für ihre Reformkonzepte mit dem Hinweis auf die damit verbundene Verbesserung der intergenerativen Gerechtigkeit. Solange jedoch nicht geklärt ist, was genau unter „intergenerativer Gerechtigkeit" zu verstehen ist und vor allem, wie sich eine Verbesserung derselben erfassen läßt, haftet solchen Bekenntnissen der Beigeschmack einer bloßen politischen Rhetorik an.

An diesem Defizit knüpft die vorliegende Arbeit an. Es soll ein Meßkonzept entwickelt werden, mit dem verschiedene Optionen zur Reform eines Alterssicherungssystems anhand ihrer intergenerativen Verteilungswirkungen bzw. nach dem Kriterium der „intergenerativen Gerechtigkeit" bewertet werden können.

Da die verschiedenen Gestaltungsmöglichkeiten der Alterssicherung und somit auch die Vielfalt an Reformoptionen auf - i.d.R. nicht explizit gemachten - unterschiedlichen Gerechtigkeitsgrundsätzen fußen, ist es nicht sinnvoll, die „Gerechtigkeit der Alterssicherung“ abstrakt zu diskutieren. Die Analyse sollte vielmehr auf die Betrachtung einer bestimmten Kategorie von Maßnahmen fokussiert werden.

Im Mittelpunkt der folgenden Arbeit stehen Reformvorschläge, die für einen (partiellen) Wechsel des Finanzierungsverfahrens plädieren.

Die Konzentration auf diese Kategorie von Reformoptionen erscheint nicht nur deshalb angebracht, weil die Diskussion um den Fundierungsgrad der Rentenfinanzierung derzeit eine Renaissance erlebt, ${ }^{4}$ sondern auch und vor allem, weil solche Maßnahmen sich aufgrund folgender Effekte unmittelbar auf die intergenerative Gerechtigkeit auswirken können:

1. Intergenerative Verteilungswirkungen entstehen in einem Alterssicherungssystem größtenteils dadurch, daß die Generationen von der demographischen Entwicklung unterschiedlich stark betroffen werden. Die Einführung eines Kapitalstockverfahrens wird regelmäßig damit begründet, daß die demographische Abhängigkeit des Systems verringert wird.

3 Vgl. z.B. Rürup/Schroeter (1997), S. 16ff..

4 Vgl. stellvertretend für viele Frankfurter Institut (1997); Symposion des Zentrums für Europäische Wirtschaftsforschunf (o.V. 13. März 1997); Jahresgutachten 1996/1997 des Sachvertăndigenrats,S.329-356; Glismann/Horn (1997); Queisser (1998); Neumann (1996) und (1986); Bahr/Kater (1997); Breyer (1996); Weltbank (1994). 
2. Sofern mit den alternativen Finanzierungsverfahren unterschiedliche Wachstumseffekte verbunden sind, hat die Wahl des Finanzierungsverfahrens einen Einfluß auf die Wohlfahrt zukünftiger Generationen.

3. Demgegenüber müssen die Übergangsgenerationen die mit einer Umstellung auf das Kapitalstockverfahren verbundenen Zusatzbelastungen einseitig tragen, was ebenfalls die intergenerative Gerechtigkeit direkt beeinflußt.

In der normativen Theorie der Alterssicherung wurde die Frage nach der intergenerativen Gerechtigkeit lange Zeit vernachlässigt, und erst seit der zweiten Hälfte der $80 \mathrm{er}$ bzw. seit Beginn der 90er Jahre steigt die Anzahl der Arbeiten, die sich mit diesem Thema auseinandersetzen. ${ }^{5}$ Angesichts der prognostizierten demographischen Herausforderungen mit denen die Alterssicherungssysteme aller Industrienationen konfrontiert sind, ist dies sicherlich erst der Beginn einer Diskussion um die intergenerative Gerechtigkeit, die in Zukunft an Bedeutung gewinnen wird. ${ }^{6}$

Die Mehrzahl der normativen Arbeiten zur intergenerativen Gerechtigkeit von Alterssicherungssystemen gehören zur Kategorie modell-theoretischer Analysen. ${ }^{7}$ Der wissenschaftliche Nutzen solcher Analysen ist unbestritten. Darüber hinaus ist eine solche Vorgehensweise für gewisse Fragestellungen auch notwendig, wenn grundlegende wirtschaftstheoretische Zusammenhänge in ihrer qualitativen Tendenz erklärt werden sollen. Andernfalls ließen sich keine eindeutigen Ergebnisse ableiten.

Oft erweisen sich die Annahmen jedoch als zu realitätsfern, um die Ergebnisse auf andere Fragestellungen zu übertragen. Insbesondere lassen sich aus diesen Arbeiten noch keine Handlungsempfehlungen für die Sozialpolitik ableiten.

Es läßt sich daher konstatieren, daß Beiträge zur intergenerativen Gerechtigkeit alternativer Finanzierungsverfahren in der Theorie einerseits stark unterrepräsentiert sind und andererseits bislang kein sozialpolitisch nutzbares Meßkonzept vorliegt, mit dessen Hilfe die intergenerative Gerechtigkeit realer Alterssicherungssysteme bewertet werden kann. Mit der vorliegenden Arbeit soll ein Beitrag zur Schließung dieser Lücke geleistet werden.

5 Vgl. Schulenburg (1989), S. 270; Speckbacher (1994); Sudhoff (1996), Breyer/Spremann (1990).

6 Ähnlich auch Sudhoff (1996), S. 3 und Schulenburg, Graf v. d. (1989), S. 289, der im Zusammenhang mit der Frage nach der intergenerativen Umverteilung und intergenerativen Gerechtigkeit ausführt: „Es ist daher nicht verwunderlich, daß man bei der Suche nach Konzepten zur Operationalisierung und Überprüfung der Konsistenz der Konsequenzen von Verteilungskriterien recht schnell an die ökonomische Forschungsfront gerät. “Neun Jahre spăter hat diese Bemerkung an ihrer Aktualităt nichts verloren.

7 Vgl. Schulenburg (1989), S. 270; Speckbacher (1994); Sudhoff (1996), Breyer/Spremann (1990); Brandts/Bartolome (1992). Einige Arbeiten, die nicht modell-theoretischer Natur sind, beschränken sich auf die Berechnung der in der gesetzliche Rentenversicherung erzielbaren Rendite (vgl. Eitenmüller (1996); Ohsmann/Stolz (1997); Preschitz (1994)) oder beziehen sich weniger aut Generationen sondern auf die Verteilungswirkungen zwischen Rentnern und Enwerbstătigen einer Periode (vgl. Abschnitt 5.3.3).

19 


\section{Das Ziel der Dissertation kann mithin wie folgt beschrieben werden:}

1. Entwurf eines Konzepts zur Erfassung und Bewertung der intergenerativen Verteilungswirkungen („intergenerative Gerechtigkeit") eines Alterssicherungssystems.

- Das Meßkonzept soll es ermöglichen, konkrete Reformoptionen zu bewerten und sozialpolitische Handlungsempfehlungen abzuleiten.

- Die Formulierung des Konzepts orientiert sich vorwiegend an Reformvorschlägen, die auf einer (partiellen) Substitution des Finanzierungsverfahrens gründen.

2. Mit Hilfe des entwickelten Konzepts werden ausgewählte Reformmaßnahmen der gesetzlichen Rentenversicherung bewertet.

Hinsichtlich der Bewertung der ausgewählten Reformmaßnahmen, muß darauf hingewiesen werden, daß im Rahmen dieser Arbeit vornehmlich das Kriterium der intergenerativen Gerechtigkeit analysiert wird, so daß von dem Ergebnis der Simulationsrechnung noch nicht auf die sozialpolitische Empfehlung einer Reformmaßnahme geschlossen werden kann. Bevor eine endgültige Entscheidung zugunsten eines Vorschlags getroffen werden kann, müssen die übrigen Kriterien (z.B. finanzwirtschaftliche Effizienz, politische Stabilität) ebenfalls ins Kalkül gezogen werden.

Ferner werden die folgenden Aspekte nicht explizit untersucht:

- In der Arbeit wird von der Existenz eines staatlichen Rentensystems ausgegangen, d.h. die fundamentale Frage nach der Legitimation einer staatlichen Zwangsversicherung wird nicht gestellt. ${ }^{8}$

- Ein weiterer Strang innerhalb der Theorie der Alterssicherung, der in den letzten zehn bis zwanzig Jahren zwar an Bedeutung gewonnen hat, aber dennoch in der vorliegenden Arbeit nicht behandelt werden soll, betrifft die Interpretation von Alterssicherungssystemen im Lichte der Public Choice Theorie. ${ }^{9}$ Dadurch wird unter anderem die Frage ausgeklammert, ob ein bestimmtes Alterssicherungssystem bzw. ein bestimmter Reformvorschlag in einer demokratischen Gesellschaft die politischen Mehrheiten erlangen kann.

- Ebensowenig fließen solche Ansätze in die Analyse ein, die darauf zielen, die Bevölkerungsentwickung zu endogenisieren, sprich bei denen die Fertilität eine Funktion des Alterssicherungssystems darstellt.

8 Vgl. Fußnote 225.

9 Eine Einführung bzw. Übersicht in den Public-Choice Ansatz der Alterssicherung bieten Homburg (1988); Breyer (1990); Breyer/Kolmar (1997), vgl. auch Fußnote 192.

20 


\subsection{Struktur der Untersuchung}

Im 2. Kapitel werden die Gestaltungsmöglichkeiten zur Finanzierung eines Alterssicherungssystems beschrieben und die grundlegenden allokativen und distributiven Wirkungen des Umlage- und des Kapitalstockverfahrens analysiert. Die Allokationseffizienz der Finanzierungsverfahren zeigt, ob das Umlageverfahren (partiell) durch das Kapitalstockverfahren substituiert werden kann, ohne daß einige Generationen schlechtergestellt werden. Die Analyse der distributiven Effekte untersucht die intergenerativen Verteilungswirkungen, die innerhalb des Alterssicherungssystems auftreten, wenn demographische und ökonomische Rahmengrößen variieren. Sowohl die allokativen als auch die distributiven Effekte werden im Modell einer kleinen offenen und einer geschlossenen Volkswirtschaft untersucht.

Das 3. Kapitel befaßt sich mit den Gerechtigkeitsvorstellungen, anhand derer die intergenerativen Verteilungswirkungen bewertet werden. Einige der üblichen Gerechtigkeitsgrundsätze stehen im Konflikt zueinander. Deshalb stellt sich das Problem, die relevanten Grundsätze auszuwählen und zu gewichten. Hierzu bieten sich zwei Vorgehensweisen an, die auf unterschiedlichen Gerechtigkeitsthesen beruhen. Diese alternativen Ansätze werden beschrieben und kritisiert. Aus der Würdigung dieser Ansätze wird die Vorgehensweise für die Bewertung der intergenerativen Verteilung in dieser Arbeit abgeleitet.

Im 4. Kapitel wird der Analyserahmen zur Messung der „intergenerativen Gerechtigkeit“ eines Alterssicherungssystems abgegrenzt. Dabei stehen drei Aspekte im Vordergrund des Interesses. Als erstes wird die Systemgrenze für die Gerechtigkeitsanalyse festgelegt. Zweitens werden aus den verschiedenen Formen von Verteilungswirkungen diejenigen herausgefiltert, die für die vorliegende Fragestellung relevant sind. Dabei zeigt sich unter anderem, daß eine umfassende Bewertung sowohl direkte als auch indirekte Verteilungswirkungen berücksichtigen muß.

Ein Alterssicherungssystem kann abhängig von Zielsetzungen nach unterschiedlichen Prinzipien gestaltet werden. In einem dritten Schritt werden daher die verschiedenen Gestaltungsdimensionen und -prinzipien der Alterssicherung beschrieben. Da diesen Zielen und Prinzipien zudem unterschiedliche Gerechtigkeitsvorstellungen zugrunde liegen, bestimmt auch die jeweilige Gestaltung des Alterssicherungssystems, ob und inwieweit auftretende Verteilungswirkungen ungerecht sind. Für die Analyse ist zu klären, welche Gestaltungsdimensionen für die Frage der intergenerativen Gerechtigkeit einer Substitution des Finanzierungsverfahrens relevant sind und welchen Einfluß die verschiedenen Gestaltungsprinzipien auf die Bewertung der Verteilungswirkungen haben.

Im 5. Kapitel wird ein Meßkonzept entwickelt, das die direkten intergenerativen Umverteilungen eines Alterssicherungssystems erfaßt. Im Gegensatz zur modelltheoretischen, qualitativen Analyse des zweiten Kapitels stellt sich nun das Problem, die Verteilungswirkungen realer Rentensysteme quantitativ zu messen. Einige der in der Literatur üblichen Ansätze werden beschrieben und bewertet, um darauf aufbauend einen geeigneten Verteilungsindikator zu formulieren. Die Verteilungseffekte können aber nur durch den Ver- 
gleich mit einem verteilungsneutralen Referenzsystem beurteilt werden. In den meisten Distributionsanalysen wird hierfür üblicherweise - explizit oder implizit - ein idealtypisches, beitragsäquivalentes Kapitalstockverfahren gewählt. Ein solches Referenzsystem vernachlässigt allerdings das Ziel der „Sicherheit des relativen Lebensstandards“ und den intergenerativen Risikoausgleich. Um das Referenzsystem und den Verteilungsindikator anzupassen, werden drei Fragen untersucht: Was gehört zu den intergenerativ versicherbaren Risiken? Wieviel an intergenerativem Risikoausgleich ist erwünscht? Wie kann die Last bei Eintritt des Risikos auf die Generationen verteilt werden? Nachdem ein Instrument zur Erfassung der direkten intergenerativen Umverteilungswirkungen hergeleitet wurde, beschreibt das Kapitel abschließend die Bewertung des resultierenden Umverteilungsmusters.

Die Analyse des zweiten Kapitels zeigt, daß die verschiedenen Finanzierungsverfahren unterschiedliche gesamtwirtschaftliche Effekte hervorrufen können. Diese dürfen bei der Bewertung der intergenerativen Gerechtigkeit nicht vernachlässigt werden. Allen voran steht die These vom Ersparnis- und Wachstumseffekt des Kapitalstockverfahrens. Kapitel 6 kritisiert zunächst diese aus einem einfachen Modell abgeleitete These. AnschlieBend wird untersucht, wie sich etwaige Ersparnis- und Wachstumseffekte bei der Bewertung der intergenerativen Umverteilung berücksichtigen lassen. Das 6. Kapitel schließt mit einem Überblick über mögliche Folgen der Finanzierungsverfahren für den Arbeitsmarkt und der Frage ob und wie diese in die Analyse einfließen sollen.

Im 7. Kapitel wird das entwickelte Meßkonzept auf ausgewählte Reformoptionen angewendet. Zunächst werden die Reformvorschläge beschrieben. Anschließend werden die Simulationsrechnungen spezifiziert und ihre wesentlichen Ergebnisse zusammenfaßt.

Mit einer zusammenfassenden Bewertung der Ergebnisse, des hergeleiteten Meßkonzepts und der politischen Optionen einer Rentenreform sowie einem Ausblick auf zukünftige Arbeiten schließt die Arbeit mit dem 8. Kapitel. 


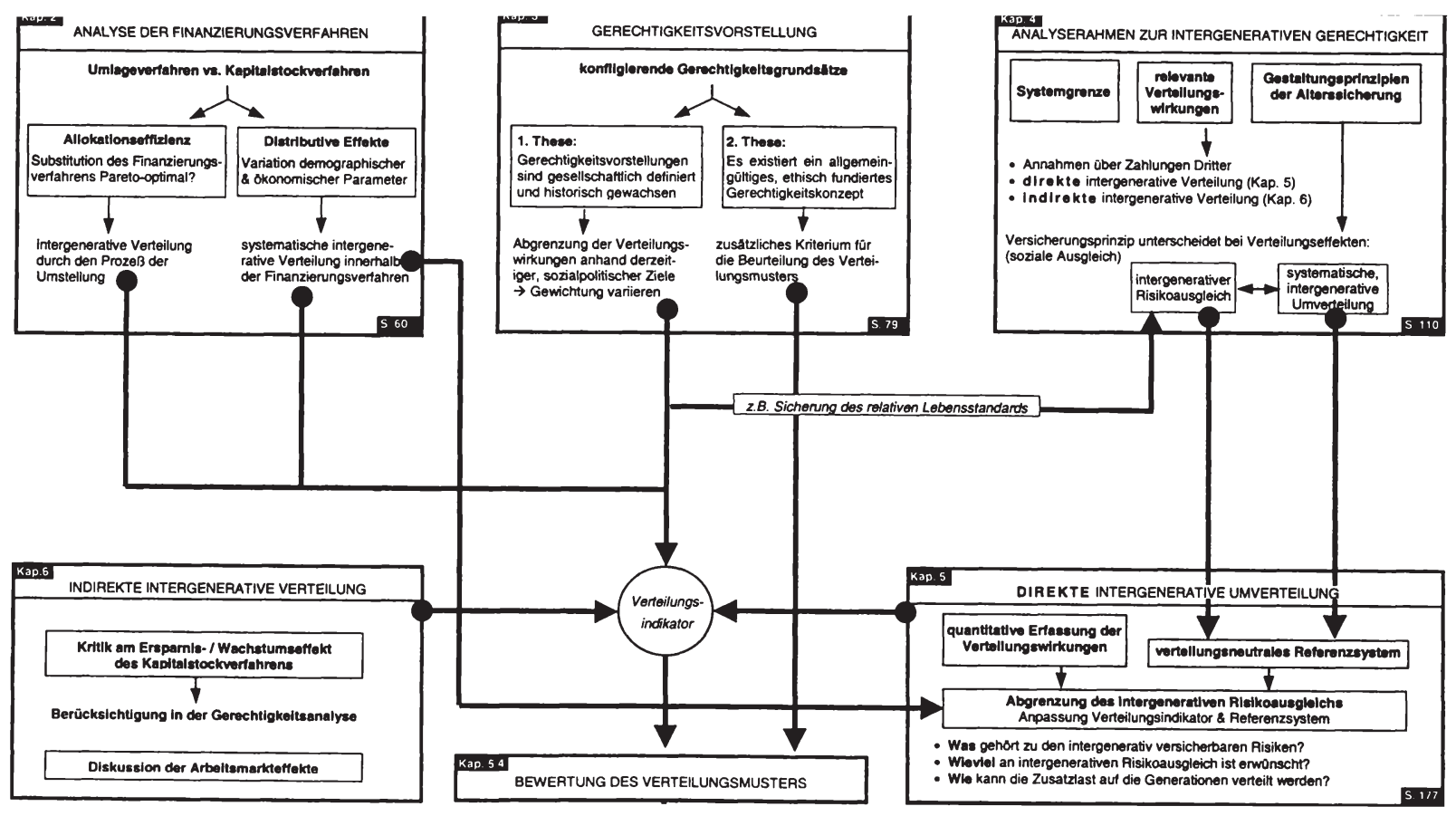




\section{MODELLTHEORETISCHE ANALYSE DER FINANZIERUNGS- VERFAHREN}

In den ersten beiden Abschnitten dieses Kapitels werden die theoretischen Gestaltungsmöglichkeiten zur Finanzierung eines Alterssicherungssystems beschrieben und für die folgende Untersuchung eingegrenzt.

Anschließend wird im Abschnitt 2.3 zunächst die Allokationseffizienz der Finanzierungsverfahren analysiert. Von ihr hängt ab, ob das Umlageverfahren (partiell) durch das Kapitalstockverfahren substituiert werden kann, ohne daß einige Generationen schlechtergestellt werden. Daraufhin werden die intergenerativen Verteilungswirkungen untersucht, die aufgrund der alternativen Finanzierungsverfahren innerhalb des Alterssicherungssystems auftreten können. Beide Effekte wirken sich unmittelbar auf die Beurteilung der intergenerativen Gerechtigkeit aus. Sowohl die allokativen als auch die distributiven Effekte werden im Modell eine kleinen offenen und einer geschlossenen Volkswirtschaft untersucht.

Im letzten Abschnitt des Kapitels werden zwei ausgewählte Erweiterungen des Modellrahmens betrachtet und untersucht, inwieweit sie die abgeleiteten Ergebnisse modifizieren.

\subsection{Verfahren der Rentenfinanzierung}

Alternative Finanzierungsarten zur Alterssicherung lassen sich grundsätzlich in beitragsfinanzierte (Vorsorge) und steuerfinanzierte Systeme (Versorgung) einteilen. Bei den Beiträgen erwirbt der Finanzier einen Anspruch auf Gegenleistung, so daß eine beitragsfinanzierte Alterssicherung regelmäßig auf einem Vorsorgesystem beruht.

Im Unterschied dazu stellen Steuern Zwangsabgaben an den Staat ohne Anspruch des Zahlers oder Trägers auf eine konkrete Gegenleistung dar. Im Fall einer Steuerfinanzierung werden die Rentner während des Ruhestands vom Staat versorgt und zwar unabhängig von den zuvor erbrachten Leistungen, gegebenenfalls jedoch nach einer vorangegangenen Bedürttigkeitsprüfung. In einem solchen Versorgungssystem wird folglich massiv und gezielt zwischen den Versicherten umverteilt. ${ }^{10}$ Der Kreis der zur Finanzierung herangezogenen Personen ist in steuerfinanzierten Systemen größer, weil alle Bürger unabhängig davon, ob sie einer Erwerbstätigkeit nachgehen, zur Finanzierung herangezogen werden. ${ }^{11}$ Sofern die Leistungen nicht an die Bedürttigkeit gekoppelt werden, ist der Kreis der Leistungsempfänger im steuerfinanzierten Systemen ebenfalls größer als in einem beitragsfinanzierten (Vorsorge-)System.

10 Dieser Aspekt der Umverteilung wird im Zusammenhang mit der Rolle der Solidarität in der Alterssicherung in Abschnitt 4.4.3 aufgegriffen. 
Innerhalb der beitragsfinanzierten Rentensysteme kann weiter unterschieden werden, ob sie auf dem Umlageverfahren, dem Anwartschaftsdeckungsverfahren oder dem Kapitalstockverfahren beruhen.

Das Umlageverfahren ist dadurch charakterisiert, daß die geleisteten Beiträge der Aktivengeneration in der gleichen Periode zur Auszahlung der Rentenleistungen herangezogen werden. Vernachlässigt man geringe Schwankungsreserven, wird entsprechend dieser Saldenmechanik aus den Beiträgen der Enwerbstätigen kein Vermögen bzw. kein Kapitalbestand angesammelt. Durch die gezahlten Beiträge erwerben die Versicherten Anwartschaften auf eine Rente, die nach Eintritt in den Ruhestand mit Hilfe der Beiträge nachkommender Generationen in Rentenzahlungen transformiert werden. Diese, das Umlageverfahren prägende und aus staatlichem Zwang $^{12}$ erwachsende Solidargemeinschaft zwischen den Generationen spiegelt den in der populären Diskussion viel verwandten, gleichwohl unpassenden Begriff des „Generationenvertrags“ wider.

Das Anwartschaftsdeckungsverfahren beruht ebenfalls auf dem Prinzip der Umlage, da auch bei dieser Variante die Einnahmen einer Periode zur Finanzierung der Renten der gleichen Periode herangezogen werden. Im Unterschied zum reinen Umlageverfahren ist allerdings die Zeitspanne, in der die Einnahmen und Ausgaben ausgeglichen werden, beim Anwartschaftsdeckungsverfahren länger als ein Fiskaljahr (in der Bundesrepublik Deutschland umfaßte diese Periode in der Vergangenheit eine Dekade). Da in diesem Verfahren folglich die Barwert-Äquivalenz von Ein- und Auszahlungsreihen über einen längeren Zeitraum erfüllt sein muß, kommt es im Anwartschaftsdeckungsverfahren zur Bildung von Rücklagen. Man könnte dieses Verfahren demzufolge auch als Mischform zwischen Umlage- und Kapitalstockverfahren charakterisieren. Das angesammelte Vermögen und die damit erzielbaren Zinseinnahmen werden aber nicht dem Individuum zugeschrieben, sondern sind „Eigentum" der Solidargemeinschaft bzw. des Trägers oder der öffentlichen Hand.

Gelegentlich wird das Anwartschaftsdeckungsverfahren auch als „Abschnittsdeckungsverfahren“ oder als „Kapitaldeckungsverfahren“ bezeichnet. Letztere Bezeichnung kann aber zu terminologische Verwirrungen führen, da der Begriff „Kapitaldeckungsverfahren“ oft auch als Synonym für das Kapitalstockverfahren verwandt wird.

Das Kapitalstockverfahren unterscheidet sich vom Umlageverfahren darin, daß die geleisteten Beiträge nicht periodengleich an die Rentenempfänger ausgeschüttet, sondern während der Erwerbszeit als Kapitalstock akkumuliert und zinsbringend angelegt werden. Im Gegensatz zum Anwartschaftsdeckungsverfahren ist der aus Beiträgen und Zinseinnahmen angesammelte Kapitalstock Eigentum der versicherten Individuen. Im Ruhestand

11 Dies gilt umso mehr, wenn das Alterssicherungssystem an die Erwerbseinkommen gekoppelt ist, aber auch ohne diese Kopplung würde beispielsweise die Ruhestandsgeneration ebenfalls über die indirekten Steuern einen Finanzierungsbeitrag leisten.

12 Die grundsätzliche Frage, wann die Teilnahme an der Versicherung freigestellt oder ein Versicherungszwang bestehen sollte, bzw. als positive Analyse, welche Folgen mit den jeweiligen Gestaltungsmöglichkeiten verbunden sind, wird im Rahmen der vorliegenden Arbeit nicht erörtert. 
wird das von dem Versicherten angesammelte Vermögen wieder abgeschmolzen und dient zusammen mit den erwirtschafteten Zinserträgen der Finanzierung der eigenen Renten. Das Kapitalstockverfahren ähnelt daher dem privatem Ansparen von Vermögen, ${ }^{13}$ welches im Idealfall bekannter Lebensdauern im Ruhestand bis zum Ableben des Individuums wieder vollkommen entspart wird. Im Gegensatz zum individuell angesparten Vermögen muß der gesamtwirtschaftliche Kapitalstock des Kapitalstockverfahrens - im Falle einer stationären Bevölkerung - jedoch nicht abgeschmolzen werden. Nach einer Aufbauphase, die in der Regel der Erwerbsphase einer Generation entspricht, würde sich das Entsparen der älteren Generationen und das Ansparen nachrückender Jahrgangskohorten gerade ausgleichen und der Kapitalstock bliebe gesamtwirtschaftlich betrachtet konstant.

Ein Alterssicherungssystem kann als Mischform auf verschiedenen Finanzierungsverfahren basieren, beispielsweise indem eine steuerfinanzierte Grundrente durch eine beitragsfinanzierte und gegebenenfalls kapitalstockbasierte Zusatzrente ergänzt wird. Auch auf Beitragszahlungen beruhende Finanzierungsverfahren können Mischformen darstellen, wenn z.B. ein Teil der Beiträge in ein Umlagesystem fließt, während mit den verbleibenden Beiträgen ein Kapitalstock für den Versicherten aufgebaut wird.

\subsection{Gestaltung der Rentenformel}

Die folgenden Ausführungen beschränken sich auf die beiden idealtypischen Formen beitragsbasierter Finanzierungsverfahren: das Umlageverfahren und das Kapitalstockverfahren. In beiden Verfahren werden die Beiträge und Rentenleistungen durch die Gestaltung der Rentenformel festgelegt. Bei der Gestaltung müssen generell zwei Entscheidungen getroffen werden:

\section{Beitragsäquivalenz vs. Teilhabeäquivalenz}

Bei der Beitragsäquivalenz berechnet sich die absolute nominelle Höhe der Leistungen unter Berücksichtigung des durchschnittlichen Risikos des Versicherungsfalls "Langlebigkeit" unmittelbar aus der absoluten nominellen Höhe aller Beiträge.

Bei der Teilhabeäquivalenz korrespondieren die Rentenansprüche zwar weiterhin mit den zuvor geleisteten Beiträgen, vorrangiges Ziel eines solchen Systems ist es jedoch, daß ein Rentner hinsichtlich seiner individuellen Rentenhöhe im Vergleich zu allen Rentnern seiner Kohorte rangmäßig die gleiche Einkommensposition einnehmen soll, die er in der Einkommenspyramide der beitragspflichtigen Erwerbstätigen

13 Zusätzlich nimmt eine im Kapitalstockverfahren finanzierte Alterssicherung gegenüber dem privaten Sparen auch die Absicherung der Unsicherheit über die individuellen Lebensdauern wahr (vgl. Abschnitt 4.4.3). 
eingenommen hat. ${ }^{14}$ Die Rentenhöhe stellt in einem solchen System eine "Teilhabe des Versicherten an der nach Eintritt seines Versicherungsfalles zur Verfügung stehenden Finanzmasse ${ }^{\text {“15 }}$ dar.

\section{Konstante Rentenleistungen vs. konstante Beiträge}

Fixierte Rentenleistungen beinhalten meist die Konstanz des Bruttorentenniveaus, ${ }^{16}$ obwohl grundsätzlich konstante absolute Rentenhöhen ebenfalls denkbar sind.

In ähnlicher Weise bezieht sich eine Beitragsfixierung oft auf konstante Beitragssätze, wenngleich es im ursprünglichen Sinne konstante absolute Beiträge beschreibt.

Zwischen den beiden extremen Varianten eines konstanten Bruttorentenniveaus oder eines konstanten Beitragssatzes sind ferner unendlich viele Variationen denkbar, bei denen beide Parameter - Beitragssatz und Bruttorentenniveau - variabel gestaltet sind. ${ }^{17}$

Mit der ersten Entscheidungsdimension wird die Struktur der Renten, mit der zweiten das Niveau der Rentenleistungen festgelegt. Es wäre beispielsweise denkbar, daß bei gleichbleibender relativer Rentenstruktur innerhalb einer Kohorte die zur Verfügung stehende Finanzmasse - auf die sich die Teilhabe bezieht - variiert. ${ }^{18}$

Wenngleich die Wahl der Rentenformel grundsätzlich eine vom Finanzierungsverfahren zu trennende Frage darstellt, ${ }^{19}$ können einige Kombinationsmöglichkeiten instabil sein. D. h., unter bestimmten Rahmenbedingungen ist das System z.B. nicht mehr zu finanzieren bzw. es werden andere Ziele der Alterssicherung nicht erfüllt. In diesen Fällen von Instabilität sind Finanzierungsverfahren und Rentenformel nicht kompatibel. Unter Vernachlässigung der Zwischenformen ergeben sich vier Gestaltungsmöglichkeiten für die beiden Finanzierungsverfahren. Tabelle 1 faßt die acht theoretischen Kombinationen sowie die Ergebnisse der nachfolgenden Analyse ihrer Stabilität zusammen.

14 Vgl. Kolb (1985), S.127f.. Wagner (1984), S. 81f. und Krupp (1981), S. 124 bezeichnen dieses Prinzip der Teilhabeäquivalenz auch als „strukturelle Beitragsäquivalenz", Meinhold (1985) nennt es das "Kohorten-Prinzip".

15 Kolb (1985), S. 126.

16 Während "Niveau“ im allgemeinen Sprachgebrauch eine absolute Größe beschreibt, wird in der Sozialpolitik mit dem Begriff "Rentenniveau“ das Verhältnis der Rentenhöhe (Standard- oder Durchschnittsrente) einer Periode zum (Brutto- oder Netto-) Einkommen (Standard- oder Durchschnittseinkommen) der selben Periode bezeichnet.

$17 \mathrm{Zu}$ den unterschiedlichen Rentenformeln mit konstanten Beitragssätzen, konstanten (Brutto-)Rentenniveau sowie den dazwischen liegenden Mischformen und ihre Auswirkungen vgl. exemplarisch Smith (1982); Berthold/Külp (1987), S. 19; Homburg (1988), S. 19-29; Dinkel (1985), S. 355ff.; Petersen (1988), S. 335ff.; Breyer (1990) und (1990a); S. 28-35; Sudhoff (1996)

19 Vgl. Dinkel (1985), S. 358. 
Tabelle 1: Varianten der Rentenformel und ihre Kompatibilität mit den Finanzierungsverfahren

\begin{tabular}{|c|c|c|c|}
\hline \multicolumn{2}{|c|}{ Rentenformel } & Umlageverfahren & Kapitalstockverfahren \\
\hline \multirow{2}{*}{ Teilhabeäquivalenz } & $\begin{array}{l}\text { Konstanter } \\
\text { Beitragssatz }\end{array}$ & kompatibel & \multirow{2}{*}{$\begin{array}{c}\text { nicht kompatibel } \\
\text { Kapitalfundierung lediglich } \\
\text { als Mischsystem (Anwart- } \\
\text { schaftsdeckungsverfahren) } \\
\text { möglich }\end{array}$} \\
\hline & $\begin{array}{l}\text { Konstantes } \\
\text { Rentenniveau }\end{array}$ & kompatibel & \\
\hline \multirow[b]{2}{*}{ Beitragsäquivalenz } & $\begin{array}{l}\text { Konstanter } \\
\text { Beitragssatz }\end{array}$ & \multirow{2}{*}{$\begin{array}{l}\text { instabiles System } \\
\text { Das Wachstum der Lohn- } \\
\text { summe darf nicht für alle Zei- } \\
\text { ten niedriger als der Zinssatz } \\
\text { sein, sonst }{ }_{n} \text { explodieren“ die } \\
\text { Beitragssätze } \\
\text { Ist das Wachstum der Lohn- } \\
\text { summe für alle Zeiten höher } \\
\text { als der Zinssatz, strebt das } \\
\text { Rentenniveau gegen Null }\end{array}$} & kompatibel \\
\hline & $\begin{array}{l}\text { Konstantes } \\
\text { Rentenniveau }\end{array}$ & & $\begin{array}{l}\text { instabiles System } \\
\text { Wenn das Lohnwachs- } \\
\text { tum den Zinssatz dauer- } \\
\text { haft übersteigt oder wenn } \\
\text { die Lohnentwicklung } \\
\text { (während der Ruhe- } \\
\text { standsphase) von der an- } \\
\text { tizipierten abweicht }\end{array}$ \\
\hline
\end{tabular}

Quelle: eigene Darstellung.

Ein auf der Teilhabeäquivalenz beruhendes Umlageverfahren kann generell sowohl mit konstanten Beitragssätzen als auch mit konstanten Rentenniveau realisiert werden. Die Wahl der Rentenformel bestimmt in diesem Fall, auf welche Weise ein (periodisches) Budgetdefizit ausgeglichen wird. Bei konstanten Beitragssätzen wird das Finanzierungsgleichgewicht hergestellt, indem die Ausgaben gesenkt und die Zusatzlast folglich den Rentenbeziehern aufgebürdet wird. Ist hingegen das Rentenniveau fixiert, werden die Einnahmen, sprich die Beiträge, solange variiert, bis das Budgetdefizit ausgeglichen ist.

Da das Deckungskapital des Kapitalstockverfahrens aus individuellen und verbrieften Vermögensansprüchen besteht, müssen die eingezahlten und verzinsten Beiträge immer - korrigiert um den Risikoausgleich der Langlebigkeit - als Rentenleistungen ausgezahlt werden. Das Kapitalstockverfahren basiert daher per definitionem auf dem Prinzip der Beitragsäquivalenz. ${ }^{20}$ Ein kapitalfundiertes und auf der Teilhabeäquivalenz beruhendes Alterssicherungssystem wäre daher lediglich als Mischsystem realisierbar, z.B. in Form eines Anwartschaftsdeckungsverfahrens, bei dem der Kapitalstock „kollektives Eigentum“ der Versichertengemeinschaft ist.

Theoretisch ist es ohne weiteres denkbar, eine Alterssicherung als umlagefinanziertes und beitragsäquivalentes System zu konzipieren. Eine eingehende Betrachtung zeigt jedoch, daß ein strikt beitragsäquivalentes Umlagesystem langfristig sehr instabil ist und daß es praktisch nur unter bestimmten Rahmenbedingungen aufrecht erhalten werden kann. Sinkt beispielsweise die Anzahl der Beitragszahler - aufgrund der demographi-

20 Die Verzinsung der Beiträge ist ein gravierendes Unsicherheitsmoment und führt in praxi zu einer Zerlegung in eine Mindestverzinsung und eine Gewinnbeteiligung. Letzteres entspricht eher dem Teilhabeals dem Beitragsäquivalenzprinzip. Die Gewinnbeteiligung bezieht sich jedoch nicht auf die Beiträge, sondern nur auf einen Teil der Verzinsung, so daß dieser Effekt bei der Frage nach der systematischen Kompatibilität des idealtypischen Kapitalstockverfahrens mit der Teilhabeäquivalenz nicht relevant ist. 
schen Entwicklung oder infolge der Arbeitsmarktsituation - , so müssen die Beitragssätze zur Finanzierung der aus früheren Beiträgen abgeleiteten Renten steigen. Die Folge sind höhere zukünftige Renten, die wiederum noch höhere Beitragssätze erfordern. Wenn die Anzahl der Beitragszahler nicht erneut steigt, setzt sich - wie sich mit einfachen Modellrechnungen unschwer zeigen läßt - schnell ein Circulus vitiosus in Gang, bei dem die erforderlichen Beitragssätze weit über 100 Prozent wachsen können. Im umgekehrtem Fall steigender Beitragszahler strebt der Beitragssatz in einem solchen System gegen Null. ${ }^{21}$ SPREMANN weist in einem einfachen zwei-Generationen-Modell nach, daß beitragsäquivalente Renten in einem Umlageverfahren langfristig nur dann gewährt werden können, wenn das Wachstum der Lohnsummen nicht für alle Zeiten niedriger als der Zinssatz ist. ${ }^{22}$

Ein mit konstanten Beitragssätzen arbeitendes, beitragsäquivalentes Alterssicherungssystem kann im Kapitalstockverfahren problemlos verwirklicht werden. Allerdings ist das im Ruhestand erzielbare Versorgungsniveau dann - ähnlich wie im Fall der Teilhabeäquivalenz - relativ unsicher.

Wird eine Sicherung des relativen und am Durchschnittslohn ausgerichteten Lebensstandards angestrebt, muß auch in einem beitragsäquivalenten System eine Politik des konstanten Rentenniveaus verfolgt werden. Um die hierfür erforderlichen Beiträge ex ante zu kalkulieren, muß die Entwicklung der Erwerbseinkommen antizipiert werden. Steigt das Pro-Kopf-Einkommen allerdings stärker als angenommen, so erweisen sich die Beiträge (bzw. das angesammelte Deckungskapital) als zu niedrig, um den Lebensstandard im Alter zu sichern. Als Folge muß das bestehende Deckungskapital durch eine zusätzliche Akkumulation (sprich durch höhere Beiträge) an das neue Lohnniveau angepaßt werden. Während auf solche Abweichungen zwischen realen und antizipierten Lohnwachstum während der Ansparphase (zumindest theoretisch) reagiert werden kann, führt eine Erhöhung des Lohnniveaus während der Ruhestandsphase im beitragsäquivalenten Kapitalstockverfahren zu einer Verringerung des Rentenniveaus. Die Kombination eines fixierten Rentenniveaus mit einem beitragsäquivalenten Kapitalstockverfahren erweist sich daher aufgrund der erforderlichen Anpassungsreaktionen in der praktischen Realisierung als äußerst schwierig und ist insofern instabil, da es nur unter bestimmten Bedingungen den relativen Lebensstandard sichern kann. Dies ist dann der Fall, wenn die tatsächliche Lohnentwicklung und der tatsächliche Zinssatz von den antizipierten Werten nicht abweicht bzw. wenn die Differenz zwischen Zinssatz und Lohnwachstum dauerhaft (im Lebenszyklus des Versicherten) höher ist als angenommen. ${ }^{23}$

21 Vgl. Dinkel (1985), S. 358/359.

22 Vgl. Spremann (1984). Zur Kompatibilität von Umlageverfahren und Beitragsăquivalenz siehe auch Bossch (1987), S. 52 sowie Anhang 1, S. 189f. ; Homburg (1988), S. 24f; Breyer (1990a).

23 Es wäre theoretisch denkbar, daß die Versicherten mit Hilfe der angenommen Lohnentwicklung und des angenommenen Zinssatzes nicht die jährlich erforderlichen Beitrăge, sondem einen konstanten Beitragssatz ermitteln. Dann wäre zumindest die Unsicherheit über die Lohnentwicklung während der Erwerbsphase verringert. Die Realităt dürtte zwischen diesen Varianten liegen, nämlich daß die Versicherten ihre Beiträge in regelmäßigen Abstānden (z.B. zehn Jahren) anpassen, wenn sich die tatsächliche Entwicklung zu sehr von der antizipierten unterscheidet.

29 
Zusammenfassend läßt sich folgern, daß die folgenden Kombinationen relevant sind:

- Ein auf der Teilhabeäquivalenz beruhendes Umlageverfahren; unabhängig davon, ob es mit konstanten Beitragssätzen; Rentenniveau oder einer Zwischenvariante arbeitet.

- Ein beitragsäquivalentes Kapitalstockverfahren mit konstanten Beitragssätzen

- Sowie ein teilhabeäquivalents Mischverfahren, das in der folgenden Analyse der idealtypischen Finanzierungsverfahren aber nicht weiter betrachtet wird.

In den nachfolgenden Abschnitten werden die Allokationseffizienz dieser Verfahren und ihre Verteilungswirkungen untersucht.

\subsection{Allokationseffizienz der Finanzierungsverfahren}

Alterssicherungssysteme ergeben sich aus einer Vielzahl von institutionellen Regelungen; es bestehen viele wechselseitige Beziehungen zwischen ihnen und den ökonomischen und demographischen Rahmenbedingungen, und sie erfordern eine zeitliche Betrachtung über mehrere Generationen hinweg. All das zeichnet sie als sehr komplexe Gebilde aus. Um ihre grundlegenden allokativen und distributiven Wirkungen dennoch eindeutig zu beschreiben, bedarf es folglich einer geeigneten Modellierung der realen Systeme. Die in der ökonomischen Theorie dominierende Vorgehensweise zur Untersuchung qualitativer Effekte der Alterssicherung gründet sich auf dem „Modell überlappender Generationen“ (englisch: Overlapping-Generation-Model, kurz OLG-Modell). ${ }^{24}$ Diese Modellierung wird auch der Analyse dieses Kapitels zugrunde gelegt, wobei die Grundannahmen des Modells im Anhang beschrieben und im folgenden vorausgesetzt werden. ${ }^{25}$

\subsubsection{Effizienzkriterien}

Während allokative Prozesse sich anhand ihrer Effizienz bewerten ließen, sei nach SCHULENBURG die Gerechtigkeit das maßgebliche Kriterium für die Untersuchung distributiver Effekte. ${ }^{26}$

Da die Auswirkungen einer Substitution des Finanzierungsverfahrens auf die intergenerative Gerechtigkeit im Mittelpunkt dieser Arbeit stehen, stellt sich die Frage, weshalb überhaupt eine Effizienzanalyse der Verteilungsanalyse vorangestellt wird?

Wenngleich die Aussage SCHULENBURGS grundsätzlich zutrifft, wäre es verfehlt daraus zu schließen, die Verteilungswirkungen eines Systems seien völlig unabhängig von seinen

24 Erstmals wurde diese Form der Modellierung von Allais (1947) angewandt. Besondere Beachtung fand das Modell durch Samuelsons Beitrag, vgl. Samuelsons (1958). Mittlerweile ist es der vorherrschende Modelltyp in den formal-analytischen Arbeiten zur Alterssicherung.

26 Vgl. Schulenburg (1989), S. 289. 
allokativen Effekten. Vielmehr bestehen Wechselwirkungen zwischen der Allokation und Effizienz auf der einen und Verteilung und Gerechtigkeit auf der anderen Seite. ${ }^{27}$

Der Begriff der Effizienz beschreibt das Verhältnis zwischen dem Zielerreichungsgrad (Effektivität) eines Verfahrens und dem dazu erforderlichen Mitteleinsatz. Werden verschiedene Verfahren hinsichtlich ihrer Effizienz verglichen, so ist bei vorgegebenem Zielerreichungsgrad dasjenige Verfahren effizienter, welches mit dem geringeren Mitteleinsatz auskommt, bzw. bei festgelegtem Mitteleinsatz gilt entsprechend das Verfahren als das effizientere, das einen höheren Zielerreichungsgrad besitzt.

Übertragen auf den Bereich der beitragsfinanzierten Alterssicherung bedeutet dies aus der Sicht eines (repräsentativen) Versicherten, daß ein Finanzierungsverfahren A dann effizienter als ein Finanzierungsverfahren $B$ ist, wenn es bei gleichen Rentenleistungen (Zielerreichungsgrad) niedrigere Beitragszahlungen zur Finanzierung (Mitteleinsatz) benötigt. ${ }^{28}$ Wenn unter allen denkbaren Optionen kein Finanzierungsverfahren existiert, welches einen höheren Zielerreichungsgrad bei gleichem oder niedrigerem Mitteleinsatz ermöglicht als das Verfahren A, bezeichnet man dieses Verfahren als Paretoeffizient.

Allgemein formuliert, ist dasjenige Verfahren Paretoeffizient, welches dem Versicherten den höchsten Nutzen stiftet. ${ }^{29}$ Dabei läßt sich der Nutzen mit Hilfe verschiedener Kriterien konkretisieren.

Die sich aus der internen Verzinsung der Rentenversicherung ergebende Rendite beschreibt das Verhältnis der zukünftigen Rentenleistung zu den geleisteten Beiträgen der aktuellen Periode. Sie kann daher als ein mögliches Kriterium zur Messung der Effizienz herangezogen werden. Ein alternatives Kriterium zur Messung der Effizienz ist das Lebenseinkommen, welches ein repräsentativer Versicherter über seinen gesamten Lebenszyklus erzielt bzw. die Höhe seines mit einem Alterssicherungssystem verbundenen Lebensnettotransfers. ${ }^{30}$ Solange keine allgemeinen Wohlfahrtseffekte beispielsweise

27 Vgl. z.B. Homann (1989), S. 54; Hackmann (1996), S.95.

Dies zeigt sich in den folgenden Ausführungen beispielsweise in den Übergangsproblemen bei einer Substitution des Umlage- durch ein Kapitalstockverfahren. Wenn letzteres gegenüber dem Umlageverfahren aus allokativer Sicht nicht Pareto-superior ist, kann eine Umstellung nicht erfolgen, ohne einige Generationen schlechter zu stellen. In gleicher Weise kann die Nichteinführung eines effizienten Systems entsprechend des Opportunitătskostenprinzips als Umverteilung gewertet werden. Andererseits ist die Effizienz immer abhängig von der Zieldefinition bzw. den gegebenen Präferenzen, diese könnten aber auch distributiver Natur sein.

Ein Altersssicherungssystem kann auch dann effizienter als ein alternatives sein, wenn es die ökonomische Rente bezüglich des Gutes "Versicherung" erhöht (vgl. Abschnitt 2.5.1). In diesem Fall kann beispielsweise mit dem Erwartungswert der Rentenleistungen argumentiert werden.

29 Solange qualitative Ergebnisse im einfachen OLG-Modell untersucht werden, ist es nicht erforderlich, die Nutzenfunktion explizit zu formulieren. Somit stellt sich auch nicht das Problem, konkrete Annahmen über die Präferenzen der Individuen treffen zu müssen.

Breyer bezeichnet dies als ${ }_{n}$ Vermőgenssaldo“, vgl. Breyer 1990, S. 20, 28. 
durch Wachstumseffekte berücksichtigt werden, sind diese Kriterien bei qualitativen Vergleichen äquivalent. ${ }^{31}$

Bei einem solchen Nutzenvergleich spiegelt sich die Wahl des Effizienzkriteriums in den Variablen der Nutzenfunktion wider. Wird beispielsweise - wie in den meisten einfachen OLG-Modellen üblich - unterstellt, daß der Nutzen eine eindeutige Funktion des Lebenskonsums ist und dieser wiederum durch das Lebenseinkommen bestimmt wird, so gilt für die Nutzenfunktion: ${ }^{32}$

$$
U_{x}=f\left(c_{x}^{1}, c_{x}^{2}\right) \text { G } 1
$$

$U_{x}=$ Nutzen der Generation $x$

$c_{x}^{1}=$ Konsum der Generation $x$ in der Periode der Erwerbstätigkeit

$c_{x}^{2}=$ Konsum der Generation $x$ in der Periode des Ruhestands

Eine solche Annahme wird auch für die folgende Effizienzanalyse getroffen, um die grundlegenden Zusammenhänge des Effizienzvergleichs zwischen einem Umlage- und einem Kapitalstockverfahren zu verdeutlichen. Gleichwohl muß darauf hingewiesen werden, daß das zugrunde gelegte Kriterium das Ergebnis des Effizienzvergleichs maßgeblich beeinflussen kann. ${ }^{33}$

Ferner kann die Effizienz eines mehrere Generationen umfassenden Alterssicherungssystems nicht beurteilt werden, indem das Effizienzkriterium nur individuell, sprich im Lebenszyklus lediglich eines (representativen) Versicherten, betrachtet wird. Das notwendige intergenerative Pareto-Kriterium läßt sich in Anlehnung an BREYER wie folgt formulie$\operatorname{ren}^{34}$ :

\section{Definition:}

Eine mit einem bestimmten Alterssicherungssystem verbundene Allokation $A^{A}$ läßt sich durch eine unendliche Folge von Konsumvektoren $\left[\left(c_{t}{ }^{1}, c_{t-1}{ }^{2}{ }^{A}\right] ; t=1,2 \ldots\right.$ beschreiben. Diese Allokation heißt pareto-superior gegenüber einer durch ein anderes Alterssicherungssystem bewirkten Allokation $A^{B}$, wenn der durch die Allokation $A^{A}$ gestiftete Nutzen $U_{t}{ }^{A}=U\left(c_{t}{ }^{1}, c_{t}{ }^{2}\right)$ für jede Generation mindestens genauso groß ist, wie im Verfahren $A^{B}$, für eine Generation jedoch strikt größer ist als in der Allokation $A^{B}$.

$$
U_{t}^{A} \geq U_{1}^{B}, \forall t \text { und } U_{1}^{A}>U_{1}^{B} \text { für mindestens ein } t
$$

Als intergenerational Paretoeffizient bezeichnet man ein Alterssicherungssystem bzw. die mit diesem System verbundene Allokation $A^{B}$, wenn keine durch ein anderes Alterssiche-

\footnotetext{
31 Vgl. Fußnote 40.

32 Der erzielbare Nutzen eines Individuums kann nur dann allein von der Höhe seines Lebenseinkommens abhängen, wenn er sein Lebenseinkommen ohne Verlust beliebig auf seine Lebenszeit verteilen kann. Es muß daher explizit oder implizit die Annahme perfekter Kapitalmärkte getroffen werden.

33 Vgl. auch Abschnitt 5.2.2.

34 Vgl. Breyer (1990), S. 19f.
} 
rungssystem verursachte Allokation existiert, die gegenüber der Allokation $A^{B}$ intergenerativ Pareto-superior ist.

\subsubsection{Modell einer kleinen offenen Volkswirtschaft}

\subsubsection{Effizienz von Kapitalstockverfahren und Umlageverfahren}

Der Modellrahmen einer kleinen offenen Volkswirtschaft zeichnet sich dadurch aus, daß die Produktionsmöglichkeiten unabhängig vom Alterssicherungssystem und die Löhne sowie Zinssätze somit exogen vorgegeben sind. ${ }^{35}$ Zur Herleitung der Effizienzbedingungen werden im folgenden zunächst nur zwei aufeinander folgende Perioden betrachtet, sprich der Effizienzvergleich aus der Perspektive eines Versicherten anhand der internen Ertragsrate geführt. Anschließend wird das oben definierte intergenerative Effizienzkriterium angewandt.

\subsection{Interne Ertragsrate der Finanzierungsverfahren}

Aus dem einfachen Finanzierungsgleichgewicht des Umlageverfahrens lassen sich durch elementare Umformungen Bestimmungsgleichungen für den Beitragssatz und die Rentenhöhe ableiten:

$$
b_{t}=\frac{p_{t}}{w_{t}} \cdot \frac{N_{t}^{R}}{N_{t}^{A}} \quad \mathbf{G} 2
$$

$$
\text { und } p_{t+1}=b_{t+1} \cdot w_{t+1} \cdot \frac{N_{t+1}^{A}}{N_{t+1}^{R}} \text {. G } 3
$$

$b_{t}=$ Beitragssatz der Periode $t$

$\mathrm{W}_{\mathrm{t}}=$ Durchschnittslohn der Periode $\mathrm{t}$

$p_{t}=$ Rentenhōhe der Periode $t$

$\mathrm{N}_{\mathrm{t}}^{\mathrm{A}}=$ Anzahl der Erwerbstätigen (Arbeiter) der Periode $\mathrm{t}$

$N^{R}$ =Anzahl der Rentenempfänger der Periode $t$

Setzt man die Rentengleichung in Relation zur Gleichung der Beiträge der Vorperiode, so erhält man die Bestimmungsgleichung der internen Ertragsrate des Umlageverfahrens (als Effizienzkriterium):

Für konstante Beitragssätze:

$$
\begin{aligned}
& I^{U}=\frac{p_{t+1}}{b \cdot w_{t}}=\frac{b \cdot w_{t+1} N_{t+1}^{A} / N_{t}^{A}}{b \cdot w_{t}}=\frac{w_{t+1}}{w_{t}} \cdot \frac{N_{t+1}^{A}}{N_{t}^{A}}=\left(1+g_{t+1}\right) \cdot\left(1+m_{t+1}\right) \quad \mathrm{G} 4 \\
& \mathrm{~g}_{\mathrm{t}}=\text { Lohnwachstum der Periode } \mathrm{t} \\
& m_{t}=\text { Bevölkerungswachstum der Periode } t \\
& p_{i}=\text { Rentenhỏhe der Periode } t \\
& \mathrm{I}_{\mathrm{t}}=\text { interne Ertragsfaktor der Generation, die in der Periode } \mathrm{t} \text { enwerbstätig ist }
\end{aligned}
$$

Für ein konstantes Rentenniveau: 


$$
I^{U}=\frac{p_{t+1}}{b_{t} \cdot w_{t}}=\frac{b_{t+1} \cdot w_{t+1} N_{t+1}^{A} / N_{t}^{A}}{b_{t} \cdot w_{t}}=\frac{w_{t+1}}{w_{t}} \cdot \frac{N_{t}^{A}}{N_{t-1}^{A}}=\left(1+g_{t+1}\right) \cdot\left(1+m_{t}\right) \quad \text { G } 5
$$

Sie entspricht der Wachstumsrate der Lohnsumme, d.h. sie setzt sich zusammen aus einer biologischen Komponente (Wachstum der Bevölkerung) ${ }^{36}$ sowie einer wirtschaftlichen Komponente (Wachstum des durchschnittlichen Lohns). Dieser Zusammenhang verdeutlicht, daß selbst im Falle einer sinkenden Bevölkerung die Rendite des Umlageverfahrens aufrecht erhalten werden kann, sofern sich das Pro-Kopf-Einkommen entsprechend erhöht.

Beim Finanzierungsgleichgewicht des Kapitalstockverfahrens müssen sich die Einnahmen und Ausgaben einer Generation in zwei aufeinander folgenden Perioden entsprechen. Die Ausgaben der Folgeperiode entsprechen definitionsgemäß den marktüblich verzinsten Einnahmen der Vorperiode. Daraus läßt sich (bei konstanten Zinssatz i) die Rendite des Kapitaldeckungsverfahrens unmittelbar ablesen als:

$$
I^{K}=1+i^{K}=1+i \quad \text { G } 6
$$

$I^{K}=$ interne Ertragsfaktor des Kapitalstockverfahrens

$i^{k}=$ interne Ertragsrate des Kapitalstockverfahrens

$\mathrm{i}=$ Marktzinssatz

Aufbauend auf diesen Ergebnissen formulierte Aaron $^{37}$ den als "SozialversicherungsParadoxon" bekannt gewordenen Effizienzvergleich für ein "repräsentatives Individuum“ des Modells:

Demnach ist eine kapitalstockbasierte Finanzierung der Alterssicherung dann effizienter bzw. rentabler als ein Umlageverfahren, wenn die Kombination aus Lohnanstieg und die Entwicklung der Bevölkerung ${ }^{38}$ (Wachstumsfaktor der Lohnsumme) niedriger ist, als der Verzinsungsfaktor des Kapitalstockverfahrens.

$$
\left(1+m_{t}\right)\left(1+g_{t}\right)<\left(1+i_{t}\right)
$$

Umgekehrt gilt, daß das Umlageverfahren immer dann rentabler ist als das Kapitalstockverfahren, wenn der Wachstumsfaktor der Lohnsumme den Zinsfaktor übersteigt.

36 Von der im einfachen Modell überlappender Generationen getroffenen Annahme, daß alle Mitglieder der Generation im erwerbstätigen Alter auch wirklich einer Erwerbstätigkeit nachgehen, kann in der Realität nicht ausgegangen werden. Maßgeblich für die interne Verzinsung des Umlageverfahrens sind mithin die Wachstumsrate des Lohns und die Wachstumsrate der Beschäftigten, so daß - wie in einigen Modellerweiterungen berücksichtigt - neben der demographischen Entwicklung auch Veränderungen beispielsweise der Enwerbsbeteiligung und der Arbeitslosigkeit einen Einfluß auf die Rendite des Umlageverfahrens haben. Schon 1958 untersuchte Samuelson (1958) die Rendite des Umlageverfahrens für den Fall konstanter Einkommen und sprach vom "biologischen Zinssatz", da das Umlageverfahren sich mit der Bevölkerungswachstumsrate verzinst. Im Jahre 1966 erweiterte Aaron (1966) diese Gleichung um die Komponente der Einkommensentwicklung. Vgl. Aaron (1966).

38 Genauer die Entwicklung der Beschäftigten (abhängig von der Entwicklung der Bevölkerung, der Erwerbsquote etc.). 


\subsection{Effizienz der Allterssicherungssysteme}

Für die Betrachtung aus Sicht des einzelnen Versicherten läßt sich die interne Ertragsrate als Effizienzkriterium formulieren. Diese individuelle Perspektive beschränkt sich jedoch auf den Zeithorizont des eigenen Lebenszyklus. Aus der gesamtwirtschaftlichen Sicht muß der Zeithorizont weiter gefaßt werden. Ein Alterssicherungssystem erweist sich dann als Paretoeffizient, wenn kein alternatives System existiert, bei dem keine Generation, z.B. hinsichtlich ihres Lebenskonsums, schlechtergestellt und mindestens eine bessergestellt wird. Um eine Aussage über die intergenerative Pareto-Effizienz eines Alterssicherungssystems zu erhalten, muß die abgeleitete Aaron-Bedingung einerseits für alle Generationen geprüft werden und andererseits müssen die Erst- und (sofern ein endlicher Betrachtungshorizont vorliegt) Letztgeneration gesondert betrachtet werden.

Sofern der Barwert des Lebenseinkommens bzw. des Lebensnettotransfers in dem Modell mit dem gleichen Marktzins diskontiert wird, mit dem sich die Beiträge in einem Kapitalstockverfahren verzinsen, ist offensichtlich, daß das Kapitalstockverfahren das Lebenseinkommen und damit den Lebensnettotransfer aller Generationen unberührt läßt. Bei der Einführung einer Umlagefinanzierung hingegen profitiert die erste Rentnergeneration von den Transferzahlungen der dann Erwerbstätigen, obwohl sie ihrerseits in der Vorperiode keine Beiträge an das System geleistet hat. ${ }^{39}$

Im Umlageverfahren gilt für den Lebensnettotransfer der Erstgeneration :

$$
\begin{gathered}
L N T_{0}^{U}=\frac{p_{1}^{U}}{1+i_{1}}-b_{0} \cdot w_{0}=\frac{b_{1} \cdot w_{0} \cdot\left(1+g_{1}\right) \cdot\left(1+m_{1}\right)}{1+i_{1}}-b_{0} \cdot w_{0}=w_{0}\left[\frac{b_{1} \cdot\left(1+g_{1}\right) \cdot\left(1+m_{1}\right)}{\left(1+i_{1}\right)}-b_{0}\right] \quad \mathbf{G} 7 \\
\mathrm{LNT}_{0}{ }^{U}=\text { Lebendnettotransfer der Erstgeneration (0) im Umlageverfahren }
\end{gathered}
$$

$\mathrm{Da} b_{0}=0$ erhält diese Generation einen positiven Lebensnettotransfer und wird somit gegenüber einem Kapitalstockverfahren besser gestellt. Für alle weiteren Generationen gilt: $^{40}$

Untersucht man jedoch (ex-post) die mit der Einführung realer Systeme verbundenen Folgen, kann diese rein modell-theoretische Betrachtung das Ergebnis verzerren. Um die Situation der Erstgeneration zu beurteilen, müßten sämtliche Formen der Alterssicherung beachtet werden, die vor der Einführung des Rentensystems bestanden haben. Auch intrafamiliäre Übertragungen müßten dann als Beitrag der Erstgeneration an die vorangegangenen Ruheständler gewertet werden. Vgl. Wagner (1984), S. 345f.; Thompson (1983), S. 1458; Bürgisser-Peters (1982), S. $297 f$.

40 Anhand dieser Gleichung läßt sich die bereits erwähnte Äquivalenz (sofern von Wohlfahrtseffekten abstrahiert wird) der Effizienzkriterien „Rendite“ und "Lebenseinkommen" bzw. „Lebensnettotransfer" verdeutlichen. Im Umlageverfahren gilt für konstante Beitragssätze:

$$
L N T_{t}^{U}=b \cdot w_{t}\left[\frac{\left(1+g_{t+1}\right) \cdot\left(1+m_{t+1}\right)}{\left(1+i_{t+1}\right)}-1\right]
$$

Daraus läßt sich erkennen, daß ein Umlageverfahren genau dann zu einem positiven (negativen) Lebensnettotransfer und damit einhergehend höheren (niedrigeren) Lebenseinkommen führt, wenn die Rendite des Umlageverfahren größer (kleiner) ist als die des Kapitalstockverfahrens:

$\left(1+g_{t+1}\right) \cdot\left(1+m_{t+1}\right)>\left(1+i_{t+1}\right) \quad$ bzw. $\left.\quad\left(1+g_{t+1}\right) \cdot\left(1+m_{t+1}\right)<\left(1+i_{t+1}\right)\right]$. Für variable Beitragssātze kann die Variation des Beitragssatzes dann als zusätzliche Komponente der Rendite interpretiert werden. 


$$
\begin{gathered}
L N T_{t}^{U}=\frac{p_{t+1}^{U}}{1+i_{t+1}}-b_{t} \cdot w_{t}=\frac{b_{t+1} \cdot w_{t} \cdot\left(1+g_{t+1}\right) \cdot\left(1+m_{t+1}\right)}{1+i_{t+1}}-b_{t} \cdot w_{t}=b_{t} \cdot w_{t}\left[\frac{b_{t+1} \cdot\left(1+g_{t+1}\right) \cdot\left(1+m_{t+1}\right)}{b_{t} \cdot\left(1+i_{t+1}\right)}-1\right] \mathbf{G ~} 8 \\
L N T_{t}^{U}>0 \Leftrightarrow \frac{b_{t+1}}{b_{t}} \cdot \frac{\left(1+g_{t+1}\right) \cdot\left(1+m_{t+1}\right)}{\left(1+i_{t+1}\right)}>1
\end{gathered}
$$

In dem zweiten Term der linken Seite in Ungleichung G9 kommt die oben hergeleitete Aaron-Ungleichung zum Ausdruck, derzufolge (bei konstanten Beiträgen) jede Generation in einem umlagefinanzierten gegenüber einem kapitalgedeckten Alterssicherungssystem besser gestellt ist, wenn für sie das Lohnsummenwachstum den Zinsfaktor übersteigt. Der erste Term zeigt, daß ähnlich wie die Erstgeneration auch Folgegenerationen in einem Umlageverfahren einen Transfergewinn erzielen könnte, sofern der Beitragssatz für nachfolgende Generationen entsprechend steigt. Bei einer solchen, oft als Ponzi-Vertrag ${ }^{41}$ bezeichneten „Kettenbriefkonstruktion“ wäre das Umlageverfahren gegenüber dem Kapitalstockverfahren immer Pareto-superior, da sich die zusätzliche Komponente der Rendite $\left(b_{t+1} / b_{t}\right)$ im Falle variabler Beitragssätze beliebig gestalten ließe. ${ }^{42}$

Sobald jedoch einem solchen Ponzi-Mechanismus Grenzen gesetzt sind, ist das Umlageverfahren in einer kleinen offenen Volkswirtschaft nicht länger unter allem Bedingungen zwangsläufig Pareto-superior bzw. Paretoeffizient. Eine solche Einschränkung ergibt sich beispielsweise, wenn bei der Analyse ein endlicher Zeithorizont zugrunde gelegt wird. Dem Nutzengewinn der Erstgeneration (und aller folgenden Generationen im PonziVertrag) steht dann immer ein Nutzenverlust der Letztgeneration gegenüber, die in ihrer Erwerbszeit zwar noch Beiträge an die Rentnergeneration zahlt, selbst aber keine Rentenleistungen aus dem System erhält und folglich einen Verlust an Lebenseinkommen erleidet. Eine weitere Einschränkung der Pareto-Superiorität des Umlageverfahrens kann sich ergeben, wenn die zulässigen Beitragssätze durch eine (beliebig hohe) obere Schranke begrenzt werden. Sobald die Beiträge in diesem Ponzi-System die Grenze erreicht haben, erleiden die betroffenen Generationen einen Nutzenverlust.

Für die Existenz einer solchen Schranke für die Beitragssätze $\left(b_{t} \leq h\right.$ für $\left.t=2,3, \ldots\right)$ zeigt Spremann, daß ein Kapitalstockverfahren bei unendlichen Zeithorizont genau dann inter-

41 Oft wird das Umlageverfahren an sich bzw. seine intergenerativen Transfers im Falle $R<M^{*} G$ als PonziVertrag bezeichnet. Vgl. v. Weizäcker (1979); Raffelhüschen (1989), S. 36f.; Breyer (1990); Famulla/Spremann (1980), S. 382ff.; Homburg (1988); Speckbacher (1994).

Hierbei handelt es sich jedoch um verschiedene Mechanismen. Besonders deutlich wird dies im Fall einer geschlossenen Volkswirtschaft, bei der sich der Zins nach Einführung des Umlageverfahrens an das Wachstum der Lohnsumme angleicht. Der Ponzi-Mechanismus einer permanenten Erhöhung des Beitragssatzes wäre auch dann noch möglich, wenn das Lohnsummenwachstum gleich oder geringer ist als der Zinsfaktor. Bösch spricht beim Ausnutzen von Effizienzgewinnen im Fall $R<M^{*} G$ daher treffender von Arbitragemöglichkeiten zur Aufhebung einer ineffizienten Situation. Vgl. Bösch (1987), S. 158; Jaeger (1990), S. 87. Im folgenden wird die Bezeichnung "Ponzi-Mechanismus" lediglich für die Möglichkeit verwandt, durch Beitragsvariation zusātzliche Gewinne zu erzielen.

42 Vgl. Spremann (1984), S. 249. Gleichung G9 verdeutlicht zudem, daß die absolute Höhe des Lebensnettotransfers durch die Beiträge der Vorperiode $\left(b_{t}{ }^{*} w_{t}\right)$ gewichtet werden. 
generational Paretoeffizient ist, wenn das Infimum ${ }^{43}$ der Folge $A^{*}=\left\{A_{2}, A_{2}{ }^{*} A_{3}, A_{2}{ }^{*} A_{3}{ }^{*} A_{4}\right.$, ... null ist. $^{44}$

Dabei gilt:

$$
\begin{gathered}
A_{t}:=\frac{G_{t} \cdot M_{t}}{R_{t}} \text { G } 10 \\
\mathrm{G}_{\mathrm{t}}=\left(1+\mathrm{g}_{t}\right)=\text { Wachstumsfaktor der Löhne } \\
\mathrm{M}_{\mathrm{t}}=\left(1+\mathrm{m}_{t}\right)=\text { Wachstumsfaktor der Bevölkerung } \\
\mathrm{R}_{\mathrm{t}}=\left(1+\mathrm{i}_{\mathrm{t}}\right)=\text { Zinsfaktor }
\end{gathered}
$$

Für eine kleinen offene Modellökonomie läßt sich der Effizienzvergleich zwischen den Finanzierungsverfahren daher wie folgt zusammenfassen:

- Bei einem endlichen Betrachtungshorizont wird die Erstgeneration im Umlageverfahren regelmäßig besser, die Letztgeneration jedoch regelmäßig schlechter gestellt als im Kapitalstockverfahren. Keines der beiden Verfahren ist gegenüber dem anderen Pareto-superior, so daß beide effizient sind.

- Bei einem unendlichen Zeithorizont ist ein in alle Zukunft reichender Vergleich der Aaron-Bedingung entscheidend. Das Umlageverfahren ist genau dann gegenüber einem Kapitalstockverfahren Pareto-superior, wenn: ${ }^{45}$

$$
\inf \prod_{t=1}^{\infty} \frac{M_{t} \cdot G_{t}}{R_{t}}>0 \text {. }
$$

In diesem Fall ist das Umlageverfahren effizient, das Kapitalstockverfahren hingegen ineffizient.

- Ist die Bedingung G 11 bei einem unendlichen Zeithorizont nicht erfüllt, ist wiederum keines der beiden Verfahren gegenüber dem anderen Pareto-superior und beide effizient.

\subsubsection{Existenz einer Pareto-optimalen Umstellung des Finanzierungsver- fahrens}

Die Betrachtung eines endlichen Zeithorizonts im vorstehenden Effizienzvergleich ergibt, daß keines der beiden Finanzierungsverfahren gegenüber dem anderen Pareto-superior ist. Ein Ergebnis, daß - wie sich nachfolgend zeigen wird - bedeutende Konsequenzen für

Das Infinum beschreibt die größte untere Schranke für die Glieder einer unendlichen Folge und ist Null, wenn eines der Glieder null ist bzw. wenn die Folge eine gegen null konvergente Teilfolge enthält. Vgl. Breyer (1990), S. 33.

44 Vgl. Spremann (1984), S. 251f. und Breyer (1990), S. 30-35.

45 Breyer führt hierzu aus: „Das Unbefriedigende an dieser „Erkenntnis" ist, daß es (...) auf die relative Hōhe der betreffenden Parameter Zinssatz, Bevölkerungs- und Lohnwachstum in den nächsten endlich vielen Perioden - selbst wenn man sie zuverlässig vorausschătzen könnte - gar nicht ankommt. Entscheidend ist vielmehr das Verhalten in unendlichen Zeiträumen. Hierfür gilt aber das bekannte - wenn auch in anderem Zusammenhang geäußerte - Zitat von John Maynard KEYNES: ,In the long run, we are all dead.' " Breyer (1990), S. 34, Hervorhebungen im Original. 
die Frage der Verteilungsgerechtigkeit eines Alterssicherungssystem (bzw. eines Reformvorschlags) und damit verbunden für die praktische Sozialpolitik hat.

Ein Übergang zur kapitalgedeckten Alterssicherung scheiterte in der praktischen Sozialpolitik bislang vor allem an dem Problem der „Doppelbelastung der Übergangsgeneration“. Im Falle einer Umstellung des Finanzierungsverfahrens obliegt es nämlich der Erwerbstätigengeneration, die angesammelten Ansprüche des Umlageverfahrens weiterhin durch Beiträge oder Steuern zu alimentieren. Trotz dieser Zahlungen hat die Übergangsgeneration allerdings keine Aussicht auf eine staatliche Rente, so daß sie gleichzeitig gezwungen ist, für ihre eigene Vorsorge einen Kapitalstock aufzubauen. Die Folge ist eine Mehrfachbelastung der Übergangsgeneration, die durch anteiligen Verzicht der Rentenbezieher zwar auf Bestandsrentner und Erwerbstätige aufgeteilt und durch Übergangsfristen verträglicher gestaltet, letztlich aber nicht vermieden werden kann. ${ }^{46} \mathrm{Da}$ es folglich immer einige, durch die Umstellung benachteiligte Generationen geben wird, ist eine Umstellung des Finanzierungsverfahrens immer auch eine Frage der Verteilungsgerechtigkeit.

Im obigen Effizienzvergleich wurde gezeigt, daß ein Kapitalstockverfahren gegenüber einem Umlageverfahren deshalb nicht Pareto-superior sein kann, weil die Erstgeneration im Umlageverfahren einen Einführungsgewinn erzielt. Das eben geschilderte Problem der Doppelbelastung bzw. die Unmöglichkeit, die mit einer Umstellung des Finanzierungsverfahrens verbundenen Lasten zu kompensieren, ist lediglich ein Reflex dieser Effizienzfrage und beruht im Grunde darauf, daß die Letztgeneration die Einführungsgewinne zahlen muß. Die Äquivalenz der Fragestellungen läßt sich im Rahmen der schuldentheoretischen Interpretation des Umlageverfahren anhand des von SPREMANN aufgestellten und bewiesenen Satzes zeigen: ${ }^{47}$

Für jedes beliebige, durch b charakterisierte Alterssicherungsverfahren gilt bei beliebiger Bevölkerungs-, Lohn- und Zinsentwicklung, daß in jeder Periode $t$ der Gesamtumfang von Transferzahlungen im Umlageverfahren dem Gegenwartswert aller früheren Wohlstandsvorteile und Wohlstandseinbußen entspricht, d.h.:

$$
\sum_{r=0}^{t}\left(E_{r}(\vec{b})-w_{r}\right) \cdot N_{r} \prod_{j=r}^{t}\left(1+r_{j+r}\right)=b_{t+1} \cdot w_{t+1} \cdot N_{t+1} \cdot \quad \mathbf{G} 12
$$

$E_{t}(\vec{b})$ beschreibt dabei das auf den Zeitpunkt $t$ diskontierte Lebenseinkommen in Abhängigkeit von dem Vektor der Beitragssätze.

46 Welche Generation (Ruheständler oder Enwerbspersonen) dabei die Hauptlast der Umstellung zu tragen hat und welches Ausmaß die intergenerativen Verteilungswirkungen haben, läßt sich a priori nicht unmittelbar feststellen. Entscheidend ist zum einen, wie die einzelnen Parameter der Umstellung konkret ausgestaltet werden (z.B. die Dauer der Umstellungsperiode, der Umfang des kapitalgedeckten Anteils der Rentenversicherung, das Ausmaß der Rentensenkung bzw. Verlangsamung der Rentendynamik, das angestrebte Versorgungsniveau und daraus resultierend die erforderlichen Aufwendungen des $\mathrm{Er}$ werbstätigen etc.). Zum anderen wird dies von der Bevölkerungsstruktur (Anzahl der Versicherten je Jahrgang) und den Regelungen des Rentensystems bestimmt. Vgl. Spremann (1984), S. $246 f$. 
Die bis zur Umstellung angesammelten und in Folge auszugleichenden Anwartschaften der geleisteten Beiträge entsprechen mithin dem Wohlfahrtsgewinn der Gründergeneration und der aller Nachfolgegenerationen. Ob die mit einer Substitution des Umlage- durch das Kapitaldeckungsverfahren verbundenen Umstellungsbelastungen kompensiert werden können, ist daher gleichbedeutend mit der Frage, ob ein Kapitalstockverfahren die Gewinne dieser „Erstgenerationen“ kompensieren kann, sprich ob es auch im unendlichen Zeithorizont Pareto-superior ist. ${ }^{48}$

Eine Reihe von Arbeiten setzte sich mit der Frage auseinander, ob sich für den Fall, daß die Bedingung $R_{t}>G_{t} * M_{t}$ für alle $t$ erfült ist, Kompensationsmöglichkeiten für diese Übergangsbelastung realisieren lassen, die einen Pareto-optimalen Übergang vom Umlage- zum Kapitalstockverfahren ermöglichen. Die wesentlichen Beiträge hierzu sollen im folgenden skizziert werden, ohne auf die formale Modellierung im Detail einzugehen.

Eine erstmals von TOWNLEY vorgeschlagene Lösung zur Kompensation der Umstellungsprobleme sieht vor, daß der Übergang vom Umlageverfahren zum Kapitalstockverfahren stichtagsbezogen erfolgt und sämtliche Anwartschaften durch staatliche Neuverschuldung ausgeglichen werden. ${ }^{49}$ Die weiteren Beiträge könnten anschließend direkt in einen sich verzinsenden Rentenfonds eingezahlt werden. Aufgrund der Voraussetzung $R_{t}>G_{t}{ }^{*} M_{t}$ wäre es möglich, in den Folgeperioden zunächst nur den Betrag an die Versicherten auszuzahlen, den sie im Umlageverfahren als Rente erhalten hätten. Diese Generationen würden folglich gegenüber dem umlagefinanzierten System gleich behandelt. Mit Hilfe des verbleibenden Teils der Erträge könnte die aufgenommene Staatsschuld beglichen werden. Mit Beginn der Periode, in der die Staatsschuld restlos beglichen ist, können die nachfolgenden Generationen in den vollen Genuß einer erhöhten Rendite kommen. Dieser zunächst plausibel erscheinende Vorschlag scheitert jedoch daran, daß ein hoher Zinssatz zwar die Rendite des Kapitalstockverfahrens erhöht, gleichzeitig aber auch zu höheren Zinszahlungen aus der Staatsverschuldung führt. Aufgrund dieser sich kompensierenden Effekte, wird keine der zukünftigen Generationen von der höheren Rendite des Kapitalstockverfahrens profitieren können. Eine Gleichstellung aller Generationen ist nur möglich, wenn die Pro-Kopf-Staatsschuld konstant bleibt. ${ }^{50}$ Ein paretooptimaler Übergang ist mithin nicht möglich.

So auch Speckbacher, der hinsichtlich der Letztgeneration in einem Umlageverfahren bei einem endlichen Zeitraum ausführt: „Damit wird auch das Problem des Übergangs von einem Umlageverfahren zum Kapitaldeckungsverfahren angeschnitten. “Speckbacher (1994), S. 75.

49 Der Vorschlag von Townley (1981) gründet sich vorwiegend auf Public Choice Überlegungen. Für die Effizienzfrage ist es aber weniger von Bedeutung, ob sich politische Mehrheiten für einen Vorschlag finden lassen, sondern ob er Pareto-optimal ist. Einen ähnlichen Vorschlag unterbreiten auch Neumann (1986), S. 67 sowie Glissmann/Horn (1996) und (1997). Diese beiden Beitrăge vernachlässigen, daß mit der Ausgabe von Staatspapieren angesammelte Rentenansprüche zwar verbrieft werden können, dies aber noch nicht hinreichend ist für einen Übergang zum Kapitalstockverfahren. Makroökonomisch läge nach wie vor ein Umlageverfahren vor. Vgl. hierzu auch Bösch (1987), S. 165 und Rürup (1997).

50 Ein analytische Nachweis für die These, daß die Staatsschuld in einem endlichen Zeitraum nicht vollkommen abgebaut werden kann, findet sich für eine Steady-Stade Ökonomie bei Breyer (1990), S. 2528. Speckbacher liefert einen Beweis, der nicht an die Annahme von Steady-State Entwicklungspfade gebunden ist. Vgl. Speckbacher (1994), S. 76f.. 
HOMBURG greift die Idee der Übergangsfinanzierung mittels Staatsschuld erneut auf, beschränkt sich aber bei den Kompensationsmöglichkeiten nicht auf die Zinseinnahmen, sondern modelliert zudem das Arbeitsangebot der Versicherten. ${ }^{51}$ Lohnabhängige Beiträge verzerren in seinem Modell eines lohnelastischen Arbeitsangebots die Arbeit(Konsum)Freizeit-Entscheidung des Versicherten. Wenn nun ein Wechsel des Finanzierungsverfahrens diese Verzerrung aufhebt, besteht die Möglichkeit, den damit verbundenen Nutzengewinn in Form von Kopfsteuern zu besteuern. Mit Hilfe dieser Zusatzeinnahmen ließe sich die Übergangsgeneration entschädigen, so daß ein Pareto-optimaler Wechsel möglich wäre. Die Allgemeingültigkeit dieses Ergebnisses ist allerdings eingeschränkt, da es zwar auf der Annahme lohnabhängiger Beiträge beruht, die Rentenzahlungen allerdings nicht beitragsabhängig, sondern in Form von lump-sum-Transfers gezahlt werden.

BRUNNER kritisiert an der Arbeit HOMBURGS, daß die Annahme lohnabhängiger Beiträge nur dann sinnvoll ist, wenn zudem unterstellt wird, daß sich die Individuen hinsichtlich ihrer Fähigkeit zur Einkommenserzielung unterscheiden. ${ }^{52}$ Unter der plausiblen Annahme, daß diese Fähigkeit nicht bekannt ist, zeigt BRUNNER in einer Erweiterung des Modells, daß eine Pareto-optimale Umstellung auch unter Einbezug etwaiger Arbeitsangebotseffekte nicht möglich ist.

Für den Fall einer kleinen offenen Volkswirtschaft bleibt das Ergebnis des obigen Effizienzvergleichs daher bestehen. Insbesondere ist nach den bisherigen Erkenntnissen keine Pareto-optimale Substitution des Umlage- durch das Kapitalstockverfahren möglich, so daß eine Umstellung immer einige Übergangsgenerationen zusätzlich belastet und somit zugleich eine Frage der intergenerativen Verteilungsgerechtigkeit ist.

\subsubsection{Modell einer geschlossenen Volkswirtschaft}

\subsubsection{Effizienz von Kapitalstockverfahren und Umlageverfahren}

In dem vorangegangenen Modellrahmen einer kleinen offenen Volkswirtschaft werden die verschiedenen ökonomischen Größen (Kapitalstock, Sozialprodukt, Lohn- und Zinsatz) als exogen vorgegebene Rahmenbedingungen der Alterssicherungssysteme behandelt. Durch die Modellierung als geschlossene Volkswirtschaft werden Wechselwirkungen zwischen diesen ökonomischen Größen und dem jeweiligen Finanzierungsverfahren zugelassen, die wiederum die Effizienz der Finanzierungsverfahren maßgeblich beeinflussen können.

Dreh- und Angelpunkt der Analyse solcher Interdependenzen ist eine mögliche, durch das Finanzierungsverfahren induzierte Veränderung der gesamtwirtschaftlichen Ersparnis. Da in einer geschlossenen Modellökonomie die Möglichkeit eines Kapitalimports bzw. -exports wegfällt, wirkt sich eine Variation der gesamtwirtschaftlichen Ersparnis unmittel-

\footnotetext{
51 Vgl. Homburg (1990).

52 Vgl. Brunner (1993).
} 
bar auf das produktive Realkapital der Volkswirtschaft aus. Gemäß der gesamtwirtschaftlichen Produktionsfunktion des jeweiligen Modells führt dies zu einem veränderten Sozialprodukt sowie zu Änderungen des Lohn- und Zinssatzes.

Ausgangspunkt der bis heute andauernden Debatte um die gesamtwirtschaftliche Ersparnisbildung bei unterschiedlichen Finanzierungsverfahren, ist der von FELDSTEIN postulierte und aus der Lebenszyklushypothese ${ }^{53}$ abgeleitete „Vermögenssubstitutionseffekt" (wealth replacement effect). ${ }^{54}$ FELDSTEIN nimmt an, daß das Wirtschaftssubjekt die durch Beitragszahlungen erworbenen Anwartschaften auf zukünftige Rentenleistungen als erspartes Vermögen wahrnimmt und entsprechend seine private Spartätigkeit reduziert. Die private Ersparnis wird mithin um den Betrag der Beitragszahlungen vermindert und durch staatliches Zwangssparen substituiert. Die Beitragszahlungen in einem umlagefinanzierten Rentensystem werden aber, im Gegensatz zu den privaten Ersparnissen, in der gleichen Periode unmittelbar ausgezahlt. Der Vermögenssubstitutionseffekt führt deshalb zu einer Verringerung der gesamtwirtschaftlichen Ersparnis der privaten Haushalte.

Eine Reihe theoretischer Arbeiten bestätigen diese (intuitiv plausible) These, wonach die Einführung eines umlagefinanzierten Rentensystems bzw. ein Anstieg des Beitragssatzes in einem bereits bestehenden Umlageverfahren einen negativen Einfluß auf die Kapitalakkumulation der jeweiligen Modellökonomie ausübt. ${ }^{55}$ Aufbauend auf dieser Annahme wird daher im folgenden innerhalb des Modells einer geschlossenen Volkswirtschaft ${ }^{56}$ die Effizienz der alternativen Finanzierungsverfahren untersucht.

Ausgangspunkt der (Steady-State) Analyse ist die volkswirtschaftliche Budgetgleichung, wonach sich die Verwendung des Sozialprodukts $\left(Y_{t}\right)$ in den gesamtwirtschaftlichen Konsum $\left(C_{t}\right)$ und die gesamtwirtschaftliche Ersparnis $\left(S_{t}\right)$ aufteilt:

$$
Y_{t}=f(k) \cdot N_{t}=C_{t}+S_{t} \quad \mathbf{G} 13
$$

$\mathrm{k}=$ Kapitalintensităt

$f(k)=$ Output (Sozialprodukt) pro Kopf

Zur vereinfachung der Darstellung beschreibt $N_{t}$ die Erwerbstātigengeneration $\left(N_{t}=N_{t}{ }^{A}=N_{t+1}{ }^{E}\right.$ )

Unter der Annahme, daß der Kapitalbestand vollständig in der Produktion aufgeht, entspricht der Kapitalbestand der Folgeperiode $\left(K_{t+1}\right)$ den Bruttoinvestitionen $\left(I_{t}\right)$ der voran-

\footnotetext{
Vgl. Modigliani/Brumberg (1954); Friedman (1957).

Vgl. Feldstein (1974).

55 Vgl. Atkinson/Stieglitz (1980), Auerbach/Kotlikoff (1987), Homburg (1988), Breyer (1990). Zur Herleitung der erspanismindernden Wirkung des Umlageverfahrens wird auf die Ausführungen von Speckbacher (1994), S. 86-93, Homburg (1988), S. 49-60 und Breyer (1990), S. 70-82 verwiesen. Jaeger (1994) kommt teilweise jedoch zu abweichenden Ergebnissen. Vgl. Abschnitt 6.1. Zur Kritik an den Annahmen der angewandten Modelle und damit verbunden zur Frage der Relevanz dieser Erkenntnisse für die praktische Sozialpolitik vgl. Abschnitt 6.1.

Die Modellannahmen werden in Anhang 1.1 beschrieben.
} 
gegangenen Periode, die sich im Gütergleichgewicht annahmegemäß mit der gesamtwirtschaftlichen Ersparnis der gleichen Periode decken: ${ }^{57}$

$$
\begin{aligned}
s_{t} \cdot N_{t} & =S_{t}=I_{t}=K_{t+1}=k \cdot N_{t+1}=k \cdot M \cdot N_{t} \quad \text { G } 14 \\
\mathrm{~S} & =\text { Ersparnis pro Kopf } \\
\mathrm{M} & =\text { Wachstumsfaktor der Bevölkerung }
\end{aligned}
$$

Für den Konsum - der sowohl den Rentnerkonsum $\left(c_{t}^{2}=\right.$ durchschnittlicher Konsum pro Rentner) als auch den der Erwerbstätigengeneration ( $c_{t}^{1}=$ durchschnittlicher Konsum pro Erwerbstätigen) umfaßt - erhält man ferner:

$$
C_{t}=c_{t}^{1} \cdot N_{t}+c_{t}^{2} \cdot N_{t-1}=\left(c_{t}^{1}+\frac{c_{t}^{2}}{M}\right) \cdot N_{t} \quad \text { G } 15
$$

Setzt man G15 und G14 in G13 ein, läßt sich die volkswirtschaftliche Budgetgleichung schreiben als:

$$
f(k)=\left(c_{t}^{1}+\frac{c_{t}^{2}}{M}\right)+k \cdot M . \quad \text { G } 16
$$

Für die Entlohnung der Produktionsfaktoren gilt ferner entsprechend der Grenzprodutivitätstheorie:

$$
\begin{aligned}
& \qquad f^{\prime}(k)=R \quad \text { G } 17 \\
& \text { und } w_{t}=f(k)-f^{\prime}(k) \cdot k=f(k)-R \cdot k . \quad \text { G } 18 \\
& \begin{array}{l}
R=\text { Zinsfaktor } \\
\mathrm{W}=\text { Lohnsatz }
\end{array}
\end{aligned}
$$

Die weiteren, für die Analyse wesentlichen Gleichungen erhält man aus dem Marginalitätskalkül des Versicherten. Er maximiert seinen Nutzen unter der intertemporalen Budgetbeschränkung genau dann, wenn er den Lebenskonsum so auf die beiden Lebensphasen aufteilt, daß die Grenzrate der Substitution zwischen dem Konsum in der Erwerbsphase und dem im Ruhestand gerade dem für die Aufteilung relevanten Zinsfaktor entspricht:

$$
\frac{\partial U}{\partial c^{1}} \cdot \frac{\partial c^{2}}{\partial U}=R . \quad \mathbf{G} 19
$$

Zusammen mit der intertemporalen Budgetbeschränkung des Versicherten

$$
c_{t}^{1}+\frac{c_{t}^{2}}{R}=w_{t} \quad \text { G } 20
$$

lassen sich somit für eine Steady-State Ökonomie die Gleichgewichtsbedingungen aufstellen, aus denen die endogenen Variablen $\mathbf{R}, \mathbf{k}, \mathbf{c}^{1}$ und $\mathbf{c}^{2}$ eines marktwirtschaftlichen

57 Eine abweichende Modellierung der Produktionsfunktion, bei der das Produktionskapital dauerhaft ist, d.h. am Ende der Periode noch zur Verfügung steht $\left(Y_{t}+K_{t}=K_{t+1}+C_{t}\right)$, hat keinen Einfluß auf das Ergebnis der Analyse. Vgl. Speckbacher (1994), S. 80-93.

42 
Systems - bei gegebener Fertilität $M$ - bestimmt werden können. ${ }^{58}$ Unter der Annahme vollkommener Märkte bildet sich dieses marktwirtschaftliche Gleichgewicht über das Zusammenspiel zweier Optimierungskalküle: Die nutzenmaximale Aufteilung des Konsums der Wirtschaftssubjekte über ihren gesamten Lebenszyklus (Gleichungen G19 und G20) sowie das gewinnmaximierende Verhalten der Unternehmungen bei gegebener Produktionstechnologie und exogener demographischer Parameter (G16, G17 und G18). Die erste Bedingung führt zur optimalen Konsumstruktur, die sich unabhängig von dem in der zweiten Bedingung ermittelten optimalen Konsumniveau bestimmen läßt. ${ }^{59}$

Neben der Bestimmung der endogenen Variablen anhand des Marktprozesses $\left(\mathbf{R}, \mathbf{k}, \mathbf{c}^{\mathbf{1}}\right.$ und $c^{2}$ ) kann aber auch danach gefragt werden, welche Werte die endogenen Variablen (bei gegebener Fertilität $M$ ) annehmen müßten, damit der vom Lebenseinkommen abhängige Nutzen des Individuums maximiert wird $\left(\underline{R}, \underline{k}, \underline{c^{1}}\right.$ und $\left.\underline{c^{2}}\right)$.

Die Maximierung der Zielfunktion

$$
\max _{c^{1}, c^{2}, k} U\left(c^{1}, c^{2}, k\right) \quad \mathbf{G} 21
$$

unter der Nebenbedingung der volkswirtschaftlichen Budgetrestriktion

$$
f(k)=\left(c_{t}^{1}+\frac{c_{t}^{2}}{M}\right)+k \cdot M . \quad \text { G } 16
$$

führt zu den Bedingungen:

$$
\begin{gathered}
\frac{\partial U}{\partial c^{1}} \cdot \frac{\partial c^{2}}{\partial U}=R=M \quad \text { G } 22 \\
\text { und } f^{\prime}(k)=R=M . \quad 60 \quad \text { G } 23
\end{gathered}
$$

In einem Steady-State-Gleichgewicht sind die endogenen Variablen folglich genau dann nutzenmaximierend für den Versicherten, wenn sie die "Goldene Regel der Kapitalakkumulation" erfüllen. ${ }^{61} \mathrm{Da}$ die aus der "Laissez-faire" Situation abgeleiteten endogenen Variablen $\left(\boldsymbol{R}, \boldsymbol{k}, \boldsymbol{c}^{\mathbf{1}}, \boldsymbol{c}^{\mathbf{2}}\right)$ bestenfalls zufällig mit den Bedingungen der „Goldenen Regel der Kapitalakkumulation“ übereinstimmen, kann sich die marktwirtschaftliche (Steady-State) Ökonomie in einem ineffizienten Zustand befinden. ${ }^{62}$ Die Ineffizienzen können darin zum

Vgl. Samuelson (1975).

Vgl. Samuelson (1968), S. 178; Bösch (1987), S. 144.

Vgl. Breyer (1990), S. 75-78. Unter Verwendung einer Produktionsfunktion mit arbeitsvermehrenden, sprich Harrod-neutralem technischen Fortschritt $\left(Y_{t}=F\left[K(t), G^{*} L(t)\right]\right.$ folgt für die obigen Bedingungen $R=$ G*M. Vgl. Bösch (1987), S. 100 und 144. Dadurch wird der Bezug zwischen der "Goldenen Regel der Kapitalakkumulation" (vgl. nachfolgend) und der "Aaron-Gleichung" offensichtlich.

Nach der Goldenen Regel der Kapitalakkumulation ist der Wachstumspfad einer Ökonomie genau dann nutzenmaximierend, wenn der Zinssatz $r$ der Wachstumsrate der Bevölkerung $m$ entspricht. Vgl. Phelps (1961) und (1965); Allais (1962), Robinson (1962), Swan (1963), von Weizäcker (1962).

62 Bösch bezeichnet diese Erkenntnis sogar als eines der "zentralsten Ergebnisse in der Literatur über überlappende Generationenmodelle ohne Vererbungsmotiv". Bösch (1987), S. 154. 
Ausdruck kommen, daß die Volkswirtschaft entweder "überkapitalisiert" ( $\boldsymbol{k}>\underline{\boldsymbol{k}}$ und $\boldsymbol{R}<\underline{\boldsymbol{R}}$ ) oder "unterkapitalisiert“ ist $(\boldsymbol{k}<\underline{k}$ und $\boldsymbol{R}>\underline{\boldsymbol{R}}){ }^{63}$

Die allokativen Wirkungen der Finanzierungsverfahren können mithin danach bewertet werden, ob und unter welchen Bedingungen ein bestimmtes Alterssicherungssystem die Volkswirtschaft auf einem Wachstumspfad hält, der der „Goldene Regel der Kapitalakkumulation" entspricht. ${ }^{64}$

Betrachtet wird hierzu ein staatliches Rentensystem, bei dem $B$ den Beitrag der Versicherten, $p$ die individuelle Rentenhöhe und $k^{s}$ dasjenige Deckungskapital je Erwerbstätigem bezeichnet, das im staatlichen Rentensystem akkumuliert wird. Im Fall $k^{s}=0$ liegt folglich ein reines Umlageverfahren vor. Aus dem Finanzierungsgleichgewicht des staatlichen Rentensystems

$$
p \cdot N_{t-1}=B \cdot N_{t}+r \cdot k^{s} \cdot N_{t}-k^{s} \cdot\left(N_{t+1}-N_{t}\right) \quad \text { G } 24
$$

kann die Bestimmungsgleichung der Rentenhöhe abgeleitet werden

$$
p=M \cdot\left[B+k^{s} \cdot(R-M)\right] \quad \text { G } 25
$$

Berücksichtigt man die staatliche Rente bei der intertemporalen Budgetbeschränkung

$$
\begin{aligned}
& \text { statt } \quad c^{1}+\frac{c^{2}}{R}=w \quad \text { nun } \\
& c^{1}+\frac{c^{2}}{R}=w+\left(\frac{p}{R}-B\right) \text { G } 26
\end{aligned}
$$

sowie bei dem Konsum der Rentner

$$
\begin{aligned}
& \text { statt } \quad \frac{c^{2}}{M}=k \cdot R \quad \text { nun } \\
& \frac{c^{2}}{M}=\left(k-k^{s}\right) \cdot R+\frac{p}{M}, \quad \mathbf{G} 27
\end{aligned}
$$

so ergibt sich ein neues Gleichungssystem für das Marktgleichgewicht einer Steady-State Ökonomie. Damit die endogenen Variablen entsprechend dieser Gleichgewichtsbedingungen der „Goldenen Regel der Kapitalakkumulation“ entsprechen, muß gelten:

$$
\frac{c^{2}}{M}=\underline{R} \cdot\left(\underline{k}-k^{s}\right)+B \quad \mathbf{G} 28
$$

wobei $\underline{R}, \underline{k}$, und $\underline{c^{2}}$ die entsprechenden "Goldene-Regel-Werte" beschreiben.

Diese Gleichung verdeutlicht,

63 Vgl. Samuelson (1958), S. 542f.; Diamond (1965), S. 1134f.; Breyer (1990), S. 77ff..

64 Vgl. nachfolgend Breyer (1990), S. 78-82.

44 
„....daß es eine unendliche Menge von Kombinationen für die Parameter des staatlichen Rentensystems, $B$ und $K^{s}$, gibt, mit denen das Marktgleichgewicht so beeinflußt werden kann, daß die ,Goldene Regel' erfüllt ist. ${ }^{\text {(65 }}$

Aus der bisherigen Analyse lassen sich für eine geschlossene Modellökonomie die folgenden Aussagen über die allokativen Wirkungen der Finanzierungsverfahren treffen:

- Nur wenn das Wachstum einer Volkswirtschaft im marktwirtschaftlichen Gleichgewicht (zufällig) dem optimalen Pfad gemäß der "Goldenen Regel der Kapitalakkumulation" folgt, ist das Kapitalstockverfahren (bei dem sich die gesamtwirtschaftliche Ersparnis annahmegemäß nicht ändert) effizient. Ansonsten ist es nicht in der Lage, die bestehende Ineffizienz zu beheben. ${ }^{66}$

- In einer durch eine überkapitalisierte Volkswirtschaft charakterisierten, ineffizienten Situation verringert eine Umlagefinanzierung der Renten die private gesamtwirtschaftliche Kapitalakkumulation. Durch geeignete Wahl der Beiträge kann die Kapitalintensität auf dem „Goldene Regel-Wert" gehalten werden. In einem solchen Fall ist der Lebensnutzen aller Generationen im Umlageverfahren größer als im Kapitalstockverfahren.

Je nach Grad der Überkapitalisierung würden die Beitragssätze und damit einhergehend das Versorgungsniveau variieren. Bei der Einführung des Umlageverfahrens nach Maßgabe eines präferierten Versorgungsniveaus würde nur zufällig der ideale Wachstumspfad erreicht werden. Wahrscheinlicher ist, daß entweder die Überkapitalisierung lediglich reduziert wird oder die Ineffizienz in eine unterkapitalisierte Volkswirtschaft umschlägt. Aus diesem Zusammenhang wird der Schluß gezogen, daß ein optimales Alterssicherungssystem als Mischsystem der Finanzierungsverfahren konzipiert sein sollte, welches zudem flexibel an veränderte Rahmengrößen angepaßt wird. ${ }^{67}$

- Ist die Ineffizienz des marktwirtschaftlichen Gleichgewichts durch eine Unterkapitalisierung der Volkswirtschaft gekennzeichnet, kann der optimale Wachstumspfad nicht durch das Deckungskapital des reinen Kapitalstockverfahren erreicht werden. Vielmehr muß das staatliche Rentensystem mehr Kapital halten, als zur Deckung der Rentenansprüche erforderlich ist. ${ }^{6 \theta}$

65 Breyer (1990), S. 79. Das Ergebnis geht zurūck auf Samuelson (1975), S. 542.

66 Bösch betont, daß sowohl für $R>M^{*} G$ als auch für $R<M^{*} G$ ein Goldener Wachstumsplad sich auch mit Hilfe des Kapitalstockverfahren erreichen ließe, sofern die entsprechenden Beiträge meritorisierend durch den Staat vorgegeben und keine vollkommenen Kapital- und Versicherungsmårkte unterstellt würden. Vgl. Bösch (1987), S. 160. Damit der Staat die Konsumstruktur meritorisch vorgeben kann, dürtte aber zusätzliches privates Sparen unter der Bedingung $R<M^{*} G$ per se nicht möglich sein. Diese Annahme ist jedoch äußerst unplausibel.

67 Vgl. stellvertretend für viele Samuelson (1975); Bösch (1987), S. 157 und 168; Männer (1974); Raffelhüschen (1989), S. 58f.

68 Vgl. Samuelson (1975), S. 542f.; Raffelhūschen (1989), S.61. Eine Pareto-Verbesserung wäre in dem Fall jedoch nicht erzielt, da die "Einführungsgeneration" auf Konsum verzichten muß, um das erforderliche Kapital anzusammeln (vgl. den nachfolgenden Abschnitt).

45 
Zuweilen wird aus diesen Erkenntnissen sogar der Vorschlag abgeleitet, das Alterssicherungssystem als Instrument der Wachstumspolitik einzusetzen. ${ }^{69}$

\subsubsection{Existenz einer Pareto-optimalen Umstellung des Finanzierungsver- fahrens}

Die vorangegangenen Ergebnisse beruhen auf einem Vergleich verschiedener SteadyState-Gleichgewichte. Die Annahme konstanter und identischer Wachstumsraten einer Volkswirtschaft ist allerdings realitätsfern und vernachlässigt insbesondere auftretende Übergangsprozesse zum neuen Gleichgewichtspfad. ${ }^{70}$

Bei einer dynamischen Betrachtungsweise würde eine (partielle) Einführung umlagefinanzierter Elemente der Alterssicherung dazu führen, daß die Ersparnisse eingeschränkt werden. $\mathrm{Zu}$ jedem Zeitpunkt des Übergangs würden die Konsummöglichkeiten die im Ausgangs- und Endzustand bestehenden Konsummöglichkeiten übersteigen. Mit anderen Worten, der Zielzustand wäre dynamisch ineffizient. ${ }^{71}$

In gleicher Weise würde die Einführung bzw. Erweiterung kapitalfundierter Elemente in einer unterkapitalisierten Volkswirtschaft allen Erwerbstätigen der Übergangsphase eine zusätzliche Ersparnisbildung, also einen Konsumverzicht abverlangen. Diese Benachteiligung einiger Versicherter entspricht der bereits erwähnten „Doppelbelastung der Übergangsgenerationen“.

Wie schon im Fall einer kleinen offenen Volkswirtschaft kann eine solche Doppelbelastung auch in einer geschlossenen Ökonomie nur dann vermieden werden, wenn ein $\mathrm{Pa}$ reto-optimaler Übergang existiert. Dies hängt wiederum von der intergenerativen Effizienz der Finanzierungsverfahren ab.

69 Vgl. z.B. Männer (1974), Janssen/Müller (1981), Spremann (1987), Jaeger (1987). Zur Kritik an solchen wirtschaftspolitischen Schlußfolgerungen aufgrund dieses Ergebnisses, das vor allem einen in alle Zukunft reichenden Vergleich der Wachstumsrate und des Zinasatzes erfordert, siehe Homburg (1988), S. 81 und die dort zitierte Literatur. Homburg führt insbesondere an, daß sich eine hohe Anpassungsflexibilität ohnehin besser mit einer Staatsverschuldung als mit einem Umlageverfahren erreichen ließe. Vgl. Homburg (1988), S. 82.

70 Bösch bestimmt daher den "nutzenmaximierenden Zeitpfad der Kapitalintensität und des Konsums der einzelnen Generationen in Abhängigkeit von gegebenen Anfangs- und Endbedingungen. " Bōsch (1987), S. 146. Dazu maximiert er eine gesamtgesellschaftliche Zielfunktion, in der die Nutzen der repräsentativen Generationsmitglieder durch einen gesellschaftlichen Diskontierungsfaktor q vergleichbar gemacht und additiv berücksichtigt werden. Das nutzenmaximale Konsumprofil ergibt sich demzufolge, wenn das Grenznutzenverhältnis der jeweils lebenden Generationen $q^{*} M$ entspricht. Die abgeleitete Optimalitātsbedingung des "Konsumniveaus" beschreibt als Differenzengleichung 2. Ordnung den Verlauf einer optimalen gesamtwirtschaftlichen Kapitalakkumulation, die der ${ }_{n}$ goldenen Nutzenregel“ $q^{*} M^{*} G=R$ folgt. Vgl. Bösch (1987), S. 147f. sowie die dort angegebene Literatur. Zu der grundsätzlichen Kritik an einer solchen utilitaristischen Vorgehensweise "des größten Glücks der größten Zahl" vgl. Abschnitt 3.3.1. Ein alternativer Ansatz wird von Speckbacher (1994) vorgeschlagen, allerdings untersucht er die Bestimmung der "optimalen im Sinne einer gerechten gesamtwirtschaftlichen Kapitalakkumulation". Ähnlich wie Bösch bestreitet auch Homburg (1988), S. 83, daß die sich aus der "Goldenen Regel der Kapitalakkumulation" abgeleitete Ineffizienz für den Effizienzvergleich zwischen Umlage- und Kapitalstockverfahren eignet.

71 Vgl. Raffelhüschen (1989), S. 59; Phelps (1965), S. 805ff.. 
Betrachtet wird zunächst eine überkapitalisierte Volkswirtschaft, bei der die Altersvorsorge durch privates Sparen verwirklicht wird, was unter Vernachlässigung von Unsicherheit einem Kapitalstockverfahren gleichkommt. Mit der Einführung eines Umlageverfahrens, dessen Umfang so bemessen ist, daß die resultierende Steady-State Kapitalintensität der der „Goldenen Regel der Kapitalakkumulation“ entspricht, konvergiert der Entwicklungspfad der Kapitalintensität der Ökonomie von oben gegen den optimalen Steady-State Wert. Da auch die Konsummöglichkeiten der Übergangsgenerationen durch die partielle Einführung eines Umlageverfahrens steigen, werden auch diese Generationen besser gestellt als im Ausgangszustand mit Kapitalstockverfahren. Folglich ist auch der Übergang von einem reinen Kapitalstockverfahren zu dem optimalen Mischsystem Pareto-optimal und das reine Kapitalstockverfahren ist somit nicht Paretoeffizient. ${ }^{72}$ Dieses Ergebnis ist unabhängig davon, daß die Übergangsgenerationen sogar besser gestellt werden, als die Generationen im Endzustand (dynamische Ineffizienz).

Es bleibt zu untersuchen, ob in einer unterkapitalisierten Volkswirtschaft ( $\boldsymbol{k}<\underline{\boldsymbol{k}}$ und $\boldsymbol{R}>$ $M$ ) mit reinem Umlageverfahren ein Pareto-optimaler Übergang zum Kapitalstockverfahren (bzw. zum idealen Mischsystem) möglich ist. ${ }^{73}$

HoMBURG vertritt die Ansicht, daß sich eine paretooptimale Umstellung auf das Kapitalstockverfahren aufgrund der mit einem Wechsel verbundenen Wachstumsimpulse realisieren läßt. ${ }^{74} \mathrm{Da}$ alle nachfolgenden Generationen aufgrund des höheren Wirtschaftswachstums über einen zusätzlichen, endlichen Güterbetrag verfügen und der erforderliche Kompensationsbetrag für die Übergangsgenerationen ebenfalls endlich ist, muß es - der Argumentation HomBURgs folgend - auch eine genügend große Periode $T$ geben, in der die Summe dieser zusätzlichen Güterbeträge den Kompensationsbetrag übersteigt. Wird der Kompensationsbetrag entsprechend aufgeteilt, so können die Übergangslasten ausgeglichen werden und alle Generationen nach der Periode $T$ können von einer höheren Rendite des Kapitaldeckungsverfahrens profitieren. ${ }^{75}$

Anders hingegen Homburg (1988), S. 84f.. Eine Verringerung der sich im Kapitalstockverfahren einstellenden Ersparnis infolge einer Einführung des Umlageverfahrens würde seiner Ansicht nach sowohl heutige Generationen, als auch alle folgende Generationen schlechterstellen. Dabei übersieht er jedoch einerseits die Einführungsgeneration und es ist nicht ersichtlich, warum sie ${ }_{n} \theta i n \theta$ als ungenügend angesehene Altersversorgung" erlangen solite. Andererseits betrachtet er lediglich die Ersparnis und blendet den Fall $R<M^{*} G$ völlig aus. Insofern ist auch seine Aussage falsch: „Der obige Satz gilt (...) bei beliebiger Wachstumsrate der Bevölkerung und beliebiger Rate des technischen Fortschritts. "Homburg (1988), S. 86.

73 Aufgrund der Äquivalenz des Problems ist es theoretisch völlig gleichwertig, die intergenerative Paretoeffizienz zweier Entwicklungspfade zu vergleichen, die von identischen Anfangswerten ausgehen und die sich einzig durch das Alterssicherungssystem bzw. konkreter dem Finanzierungsverfahren des Alterssicherungssystems unterscheiden. Vgl. Breyer (1990), S. 82-90. Das Kapitalstockverfahren wäre nur dann Paretoeffizient, wenn es gelingen kann, die Erstgeneration dafür zu entschädigen, daß sie auf die mit einem Umlageverfahren verbundenen Einführungsgewinne verzichtet.

75 Homburg kritisiert an Böschs Behauptung, ein Pareto-optimaler Wechsel sei nicht möglich: ${ }_{\text {Böschs }}$ vorausgegangene verbale Begründung ist jedoch unzureichend und im Lichte des obigen Gegenbeispiels falsch." Homburg (1988), S. 90. Homburgs Argumentation ist jedoch ebenfalls lediglich verbal und 
Dieser Mechanismus bzw. diese Argumentation ist aber nur vordergründig plausibel, denn sie blendet die intergenerative Komponente des Problems aus. Angesichts der zeitlichen Verteilung der zusätzlichen wachstumsbedingten Produktionsgewinne, kann immer nur die zur gleichen Zeit aktive Generation die Kompensationszahlungen an die jeweilige Rentnergeneration leisten. Die mit den Kompensationszahlungen belastete Generation muß in der folgenden Periode ihrerseits von der Nachfolgegeneration entschädigt werden. Ein Kapitalstockverfahren wäre mithin dann Paretoeffizient, wenn die Folge von Kompensationszahlungen zwischen den Generationen in einer beliebigen aber endlichen Zeit auf null sinkt. BREYER demonstrierte in seiner Analyse, daß die Versicherten aufgrund einer solchen „überlappenden Kompensationsstruktur" eine suboptimale Konsumaufteilung hinnehmen müssen, die durch die zusätzliche Produktion nicht kompensiert werden kann. Mit anderen Worten kann die Folge der Kompensationszahlungen niemals auf Null sinken. $^{76}$

Unter den Modellannahmen, daß die Individuen identisch, der Beitragssatz konstant und das Rentensystem intragenerational fair sind, stellen BREYER und STRAUB die Arbeit(Konsum)-Freizeit-Entscheidung des Versicherten erneut in den Mittelpunkt ihrer Untersuchung. ${ }^{77}$ Eine Aufhebung der Verzerrung des Arbeitsangebots ermöglicht eine Rentensenkung und in der Folgeperiode eine Beitragssenkung. Dieser Prozeß setzt sich nun so lange fort, bis der Beitragssatz in endlicher Zeit auf Null gesunken und der Übergang zum Kapitalstockverfahren somit vollzogen ist. Ein Pareto-optimaler Übergang, so die Schlußfolgerung der Autoren, sei demzufolge gegeben.

Analog der Kritik bei einer offenen Volkswirtschaft wird auch in diesem Fall von BRUNNER in einer neueren Analyse eingewandt, daß das Modell verschiedene Individuen berücksichtigen muß, bei denen die Fähigkeit zur Einkommenserzielung nicht bekannt ist. ${ }^{78}$ Dann zeigt sich, daß der Nutzenzuwachs durch Aufhebung der Verzerrung nicht besteuert werden kann, ohne daß sich ein Individuum verschlechtert.

Ferner ist zu bedenken, daß die Beiträge nur in dem Maß als Steuer empfunden werden, in dem die Rendite des Umlageverfahrens geringer ist als der Kapitalmarktzins. Unter dieser Voraussetzung würde, wie FENGE zeigt, ${ }^{79}$ die für die Umstellung eingegangene Staatsschuld im Verhältnis zum Volkseinkommen wachsen. Um dies zu verhindern, müßte ein Teil der Zinsen durch Steuern finanziert werden, die die gleiche Verzerrung auf das Arbeitsangebotsverhalten ausüben, wie der als Steuer empfundene Teil der Beiträge. ${ }^{80}$

\footnotetext{
- wie die weiteren Ausführungen zeigen - zudem falsch. Ferner sei darauf hingewiesen, daß Homburg inkonsistent in seiner Argumentation ist. Denn der zweite Teil seines Beweises zur Effizienz des Kapitalstockverfahrens widerspricht seiner (verbalen) Beweisführung zur Existenz einer Pareto-optimalen Umstellung des Finanzierungsverfahrens auf ein Kapitalstockverfahren. 
Das Ergebnis der bisherigen Forschung zeigt, daß in einer geschlossenen Volkswirtschaft kein Pareto-optimaler Übergang zum Kapitalstockverfahren (bzw. zum idealen Mischsystem) existiert.

\subsubsection{Schlußfolgerung}

Als Resümee der vorstehenden Effizienzanalyse läßt sich festhalten, daß sich eine Substitution des Umlage- durch das Kapitalstockverfahren bislang weder im Modell einer offenen noch einer geschlossenen Volkswirtschaft Pareto-optimal gestalten läßt. ${ }^{81}$

Einige Generationen werden während der Übergangsphase immer schlechtergestellt, so daß jede Ausweitung der Kapitalstockfinanzierung sich unmittelbar auf die intergenerative Gerechtigkeit der Reform auswirkt.

Zu diesem Ergebnis kommt auch BREYER:

„In diesem Fall läßt sich die Auswahl zwischen den verschiedenen Finanzierungsverfahren in der Rentenversicherung also nicht auf ein reines Effizienzproblem reduzieren, sondern die Problematik intergenerativer Gerechtigkeit wird relevant. ${ }^{\text {(B2 }}$

Zumindest für eine kleine offene Volkswirtschaft wird dies von SPECKBACHER bestritten. Aufgrund des Problems der Letztgeneration bzw. des Übergangs betont SPECKBACHER, daß sich der durch ein Umlageverfahren verwirklichte Zustand ebenso durch eine entsprechende Staatsverschuldung erreichen ließe. Aufgrund dieser Äquivalenz formuliert er sein Separationstheorem:

„Im Fall einer kleinen offenen Volkswirtsschaft mit vollkommenem internationalem Kapitalmarkt kann die Entscheidung für ein bestimmtes Finanzierungsverfahren (Kapitaldeckungsverfahren versus Umlageverfahren) von Gerechtigkeitsüberlegungen zur Verteilung bei konfliktären Zielen separiert werden. Ausschlaggebend sind lediglich Effizienzüberlegungen. ${ }^{83}$

Der Logik seiner Argumentation folgend würde sich jedoch nicht nur der Gerechtigkeitsvergleich sondern auch jeder Effizienzvergleich erübrigen. SUDHOFF kritisiert SPECKBACHERS Theorem daher zu recht, wenn sie ausführt:

„Zu klären bleibt jedoch selbst dann noch die Frage, ob und mit welcher Begründung diejenige intertemporale Allokation, die sich ohne jeden staatlichen Eingriff bzw. bei Anwendung eines (reinen) Kapitaldeckungsverfahrens ergibt, möglicherweise korrigiert werden soltte - sei es nun mit Hilfe eines Umlageverfahrens oder mit Hilfe einer Staatsschuld. Die Äquivalenz von Umlageverfahren und Staatsverschuldung bewirkt in diesem Zusammenhang lediglich, daß die für die Einführung eines Umlageverfahrens erforderliche Legitimation "verlagert" wird auf die Rechtfertigung für die Aufnahme einer Staatsschuld; das Legitimationserfordernis als solches bleibt aber grundsätzlich bestehen, ohne daß es auf der Grundlage von Effizienzüberlegungen generell gelöst werden kann. ${ }^{.44}$

81 Einen neuen Ansatz untersuchen derzeit Feldstein und Samwick, die davon ausgehen, daß einige Versicherte auf bereits erworbene Anwartschaften verzichten würden, wenn sie dafür aus dem umlagefinanzierten System ausscheiden können. Vgl. Feldstein (1996), S. 12.

82 Breyer (1990), S. 89. So auch Raffelhüschen (1989), S. 60: „Bei diesem intertemporalen Umverteilungsmechanismus versagt das Pareto-Kriterium“.

83 Speckbacher (1994), S. 78. Bereits Diamond (1965) wies darauf hin, daß sich die Anpassung des Zinssatzes in einer überkapitalisierten Volkswirtschaft unter bestimmten Umstānden ebenso durch eine entsprechende Staatsverschuldung realisieren läßt.

Sudhoff (1996), S. 29, Fußnote 31. 
Es bleibt also bei der obigen Schlußfolgerung, daß das Fehlen eines Pareto-optimalen Übergangs vom Umlage- zum Kapitalstockverfahren sich unmittelbar auf die intergenerative Gerechtigkeit der Reform auswirkt: Die Übergangsgenerationen werden zusätzlich belastet.

Die intergenerative Gerechtigkeit einer Reform wird aber nicht nur durch die Übergangsprozesse, sondern auch durch die Finanzierungsverfahren an sich bestimmt. Demzufolge widmen sich die nachfolgenden Abschnitte der modell-theoretischen Analyse der intergenerativen Verteilungswirkungen, die aufgrund der alternativen Finanzierungsverfahren innerhalb des Alterssicherungssystems auftreten können, um vor diesem Hintergrund die intergenerative Gerechtigkeit zu analysieren.

\subsection{Intergenerative Verteilungswirkungen der Finanzierungsver- fahren}

\subsubsection{Identifikation relevanter Einflußfaktoren}

So wie die allokativen Wirkungen der Finanzierungsverfahren nur anhand eines Effizienzkriteriums identifiziert werden können, müssen auch die distributiven Effekte anhand eines Verteilungskriteriums erfaßt werden. Hierfür eignet sich - wie bei der Allokationsanalyse die Rendite bzw. das Lebenseinkommen oder der Lebensnettotransfer einer Generation. Wenn intergenerative Verteilungswirkungen im Alterssicherungssystem auftreten, würde sich dies beispielsweise in unterschiedlichen Renditen der Generationen widerspiegein.

Die nachfolgende Untersuchung der (qualitativen) distributiven Wirkungen der Finanzierungsverfahren erfolgt in einer Partialanalyse; d.h. es wird untersucht, wie sich das Verteilungskriterium (in diesem Fall die Rendite einer Generation) verändert, wenn ceteris paribus ein bestimmter Parameter variiert wird. Bevor mit der Analyse begonnen werden kann, müssen daher zunächst diejenigen demographischen und ökonomischen Größen identifiziert werden, welche die generationenspezifische Rendite des Finanzierungsverfahrens maßgeblich beeinflussen. ${ }^{85}$

Zu den demographischen Einflußgrößen auf die Rendite zählen vor allem Veränderungen der Mortalität und der Fertilität. Hinzu kommt als dritte demographische Größe die Migra-

85 Die Frage nach den relevanten Einflußfaktoren der Rendite eines Alterssicherungssystems und vor allem danach, wie diese in die Bewertung verschiedener Reformoptionen einfließen sollen, wird in Abschnitt 5.3.1.2 für die Simulationsanalyse erneut aufgegriffen. An dieser Stelle stehen ausschließlich die Einflüsse auf das Finanzierungsverfahren in einem Modell überlappender Generationen im Vordergrund, bei denen das Verfahren bereits vollständig implementiert ist. Insbesondere werden solche Einflüssen 
tion, deren grundsätzliche Wirkung auf die Rendite, je nach Alter und Struktur der Zuwanderer sowie Zeitpunkt der Zu- bzw. Abwanderung, derjenigen der Mortalität und der Fertilität entspricht. Aus diesem Grunde werden die Migrationswirkungen in der folgenden Analyse nicht explizit modelliert.

Bei sinkender Mortalität kann weiter unterschieden werden, ob eine sinkende Sterbewahrscheinlichkeit während der Erwerbsphase oder während der Ruhephase vorliegt. Im ersten Fall steigt die Zahl der Beitragszahler und ermöglicht im Umlageverfahren niedrigere Beitragssätze, was tendenziell mit einer höheren Rendite dieser Generation verbunden ist. Andererseits erreichen mehr Versicherte das Rentenalter, so daß später auch mehr Ansprüche geltend gemacht werden können. Die Folge sind höhere Beitragssätze, und damit einhergehend geringere Renditen nachfolgender Generationen. Ein Anstieg der Lebenserwartung im Rentenalter führt zu einer Verlängerung der Rentenbezugsdauer. Sofern die längere Laufzeit in der Rentenformel über ein sinkendes Rentenniveau nicht berücksichtigt wird, ist dies für die betroffene Rentnergeneration mit Renditegewinnen verbunden. Für nachfolgende Generationen ist dies jedoch mit einem renditesenkenden Effekt verbunden, da sie steigende Beitragssätzen hinnehmen müssen.

Sinkt (steigt) die Nettoreproduktionsrate einer Generation, so verringert (vergrößert) sich die Kohortengröße nachfolgender Generationen und damit auch die Zahl potentieller Beitragszahler. Die Folge im Umlageverfahren sind steigende (sinkende) Beitragssätze, die sich wiederum negativ (positiv) auf die Rendite nachfolgender Generationen auswirken. Mit anderen Worten ausgedrückt, tragen die nachfolgenden Generation die Last (bzw. profitieren von) der Fertilitätsänderung vorangegangener Generationen.

Zu den wesentlichen ökonomischen Rahmenbedingungen zählt auf Seiten des Kapitalmarkts vor allem die Zinsentwicklung. Der Einfluß des Zinssatzes ${ }^{86}$ auf die Rendite eines Alterssicherungssystems ist beim Kapitalstockverfahren unmittelbar ersichtlich, da er die Rentabilität der Anlage direkt beeinflußt. Der Zins beeinflußt die Rendite des Umlageverfahrens vornehmlich über die Auf- bzw. Abzinsung der Zahlungsreihen, sofern dies im Modell - aufgrund der Annahme vollkommener Kapitalmärkte - zu einen homogenen Marktzinssatz erfolgt.

Da die Höhe der Rentenleistungen im Umlageverfahren mit der Höhe der Löhne steigt, ist die Lohnentwicklung eine wesentliche Komponente der Verzinsung der Rentenansprüche und somit von maßgeblicher Bedeutung für die generationenspezifische Rendite.

Die Rendite des Umlageverfahrens wird weiterhin durch die Erwerbsbeteiligung (bzw. das Ausmaß der versicherungspflichtigen Erwerbstätigkeit) auf dem Arbeitsmarkt bestimmt. Durch eine höhere Erwerbsbeteiligung können die erforderlichen Beitragssätze auf eine breite Basis umgelegt werden und die Beitragssätze im Umlageverfahren gesenkt wer-

ausgeblendet, die sich aus dem Reifegrad des Systems oder politischer Änderungen spezifischer rentenrechtlicher Regelungen ergeben.

86 Aus Grūnden der Übersicht wird zunāchst ein homogener Kapitalmarkt mit nur einem Zinssatz unterstellt. 
den. Die eigenen individuellen Rentenansprüche bleiben davon jedoch unberührt, so daß die Rendite steigt. Die Gesamtheit der Rentenansprüche steigt jedoch, so daß nachfolgende Generationen wiederum höhere Beitragssätze und somit niedrigere Renditen in Kauf nehmen müssen.

Die vorangegangenen kurzen Betrachtungen zeigten bereits, daß die Möglichkeit und Richtung einer Lastverschiebung zwischen den Generationen vor allem bei der Sterblichkeit, der Fertilität und der Erwerbsbeteiligung maßgeblich von der Entwicklung des Beitragssatzes bestimmt wird. Die intergenerative Verteilung im Umlageverfahren hängt entscheidend von dem Verfahren zum Budgetausgleich der Rentenversicherungsanstalt ab, d.h. ob die Politik eines konstanten (Brutto-)Rentenniveaus oder eines konstanten Beitragssatzes verfolgt bzw. ob eine der unendlich vielen Kombination zwischen diesen Extremen angestrebt wird und beide Parameter, Beitragssatz und Bruttorentenniveau variabel sind. ${ }^{87}$

In der gesetzlichen Rentenversicherung ist mit der Nettoanpassung der Renten eine Zwischenlösung realisiert. ${ }^{88}$ Das Ausmaß der Lastverschiebung wird hierdurch komplexer, da ein steigender Beitragssatz nun einen doppelten Effekt auf die Rendite der Erwerbsgeneration hat. Er führt nicht nur zu höheren Beitragszahlungen, sondern senkt gleichzeitig über den Rückkopplungseffekt einer sinkenden Nettoquote die zukünftigen Rentenleistungen dieser Generation. Beides führt zu einer geringeren Rendite für den Versicherten. Es muß jedoch darauf hingewiesen werden, daß sich dieser Rückkopplungseffekt der Nettoanpassung zum Teil selbst dämpft, da eine geringere Rentenanpassung einen geringeren Beitragssatz in den Folgeperioden erlaubt. ${ }^{89}$

In der folgenden Partialanalyse wird daher wie folgt vorgegangen:

1. Die zu variierenden demographischen und ökonomischen Parameter sind

- die Sterblichkeit (während der Erwerbsphase und während der Ruhephase),

- die Fertilität,

- die Erwerbsbeteiligung,

- die Lohnentwicklung und

- der Zinssatz.

2. Beim Umlageverfahren liegt der Schwerpunkt auf einem Budgetausgleich nach Maßgabe der Nettoanpassung. ${ }^{90}$

87 Vgl. Abschnitt 5.3.3.

88 D.h. die Nettorenten (nach Abzug der Sozialbeitrãge und Steuern auf den Ertragsanteil) sollen in dem Maße steigen in dem die Nettolöhne (nach Abzug der Sozialbeitrăge und Einkommensteuer) steigen.

89 Vgl. Eitenmüller (1996), S. $8 f$..

90 Die wesentlichen Unterschiede zu den alternativen Rentenformeln bestehen darin, daß bei konstanten Beitragssătzen die Lasten in Form einer schlechteren Rendite regelmäßig auf vorangegangene Generationen, in einem Alterssicherungssystem mit konstanten Bruttorentenniveau hingegen auf nachfolgende

52 
3. Die Verteilungswirkungen werden im Rahmen von zwei Modellen analysiert: dem einer kleinen offenen und dem einer geschlossenen Volkswirtschaft.

\subsubsection{Modell einer kleinen offenen Volkswirtschaft}

Die interne Ertragsrate des Kapitalstockverfahrens ist im OLG-Modell per definitionem identisch mit dem Zinssatz, ${ }^{91}$ so daß die Rendite bei einer Diskontierung mit dem Marktzins immer den Wert eins annimmt. Dieser ist in einer kleinen offenen Volkswirtschaft jedoch exogen gegeben, so daß sich eine Analyse der Verteilungswirkungen des Kapitalstockverfahren anhand des Verteilungsindikators „Rendite“ in diesem Fall erübrigt. Im Mittelpunkt dieses Abschnitts stehen daher die intergenerativen Verteilungswirkungen des Umlageverfahrens.

\subsubsection{Beschreibung der intergenerativen Verteilungswirkungen}

Um die tendenziellen Auswirkungen von Variationen der einzelnen Einflußparameter auf die Rendite der verschiedenen Generationen transparent zu machen, wird die Rentenversicherung wieder mit Hilfe eines „overlapping generations model ${ }^{492}$ abgebildet. In dem zwei-Generationen-Modell mit exogenem Zinssatz und Lohnwachstum erhält man unter Berücksichtigung der Mortalität die demographischen Beziehungen:

$$
\begin{array}{ll}
N_{x}^{R}=N_{x}^{E} \cdot l_{x} & \text { G } 29 \\
\text { und } N_{x+1}^{E}=N_{x}^{E} \cdot M_{x} & \text { G } 30
\end{array}
$$

$N_{x}^{R}=$ Kohortenstärke der Generation $x$ in der Ruhestandsphase

An dieser Stelle wird aus Gründen der Übersicht der Generationenindex x anstelle des Periodenindex angegeben, da die Veränderungen der generationsspezifischen Renditen im Mittelpunkt der Analyse stehen.

$N_{x}^{E}=$ Kohortenstärke der Generation $x$ in der Erwerbsphase

$l_{x}=$ Überlebenswahrscheinlichkeit der Generation $x$

$M_{x}=$ Reproduktionsrate der Generation $x$

Berücksichtigt man die Erwerbsbeteiligung, können die Einnahmen (E) und Ausgaben ( $A$ ) der Rentenversicherungsanstalt zum Zeitpunkt $t$ mit Hilfe der folgenden Gleichungen wiedergegeben werden:

$$
\begin{gathered}
E_{t}=b_{t} \cdot w_{t} \cdot e q_{x+1} \cdot M_{x} \cdot N_{x+1}^{E} \quad \text { G } 31 \\
A_{t}=p_{t} \cdot N_{x}^{E} \cdot l_{x} \quad \text { G } 32 \\
\mathrm{~b}_{t}=\text { Beitragssatz der Periode t } \\
\mathrm{p}_{\mathrm{t}}=\text { Durchschnittsrente der Periode } \mathrm{t}
\end{gathered}
$$

Generationen verschoben werden. Die Nettoanpassung verteilt die Last auf vorangehende und nachfolgende Generationen.

92 Vgl. zur ausführlichen Darstellung des Grundmodells Anhang 1.1. 
Dabei wird angenommen, daß die Generation $x$ zum Zeitpunkt $t$ im Ruhestand ist. Die durchschnittliche Rente $p$ ergibt sich dabei im Falle einer erwerbseinkommenszentrierten Rentengewährung und eines konstanten Bruttorentenniveaus $k \mathrm{zu}$

$$
p_{t}=w_{t} \cdot k \cdot e q_{x} \quad \mathbf{G} 33
$$

Aus den Gleichungen G31, G32 und G33 läßt sich die Bestimmungsgleichung des Beitragssatzes im Umlageverfahren mit konstantem Rentenniveau ableiten:

$$
b_{t}=\frac{e q_{x} \cdot l_{x} \cdot k}{e q_{x+1} \cdot M_{x}} \quad \text { G } 34
$$

Die Rendite der Generation x, die zum Zeitpunkt $t$ im Ruhestand ist, ergibt sich aus dem Verhältnis der empfangenen durchschnittlichen Rente zu den aufgezinsten gezahlten Beiträgen der Vorperiode:

$$
I^{x}=\frac{p_{t} \cdot l_{x}}{b_{t-1} \cdot w_{t-1} \cdot\left(1+i_{t}\right)} \quad \mathbf{G} 35
$$

Durch Einsetzen der Gleichungen G33 und G34 in G35 erhält man die Rendite einer Generation $\mathrm{x}$ in einem umlagefinanzierten Rentensystem mit erwerbseinkommenszentrierter Rentenleistung und konstantem Bruttonettoniveau:

$$
I^{x}=\frac{\left(1+g_{t}\right)}{\left(1+i_{t}\right)} \cdot M_{x-1} \cdot \frac{e q_{x}^{2}}{e q_{x-1}} \cdot \frac{l_{x}}{l_{x-1}} \quad \text { G } 36
$$

Die Rendite einer Generation $x$ hängt nicht nur vom Verhältnis der ökonomischen Parameter "Lohnwachstumsfaktor" und "Zinsfaktor" ab, sondern steigt ebenfalls mit dem Reproduktionsverhalten der vorangegangenen Generation. Darüber hinaus steigt die Rendite, wenn die Generation ihre Erwerbsbeteiligung erhöht, bzw. ihre Überlebenswahrscheinlichkeit steigt. Dies hat aber zugleich - wie Gleichung G36 ebenfalls verdeutlicht einen negativen Effekt auf die Rendite nachfolgender Generationen; vorausgesetzt die Parametervariation ist einmalig und geht für nachfolgende Generationen auf ihren Ausgangswert zurück.

Eine Verringerung der Mortalität kann allerdings - wie bereits erwähnt - während der Erwerbsphase oder während der Ruhephase vorliegen und dabei die unterschiedlichen (eingangs beschriebenen) Effekte hervorrufen. Darüber hinaus zeigt Gleichung G36, daß eine Generation durch eine Änderung ihres Reproduktionsverhaltens nicht die eigene, sondern lediglich die Rendite nachfolgender Generationen beeinflußt. Daraus wurde oft fälschlicher Weise der Schluß gezogen, daß die Fertilität in einem Umlageverfahren ein Moral Hazard Problem darstelle und der fehlende Anreiz für Kinder die Grundlagen des 
Systems selbst gefährde. ${ }^{93}$ Diese Schlußfolgerung ergibt sich jedoch nur aus der (zu) einfachen Modellierung als zwei-Perioden-Modell.

Um eine differenzierte Betrachtung der Mortalität abbilden zu können und zu zeigen, daß die Fertilität kein Morald Hazard Problem darstellt, muß das Grundmodell von SAMUELSON erweitert werden. Dies erfordert ein mindestens drei-Perioden-Modell, bei dem jede Generation sich in den ersten beiden Perioden in der Erwerbsphase und in der dritten Periode in der Ruhestandsphase befindet.

$\mathrm{Zu}$ jedem Zeitpunkt kann in der Modellökonomie folglich zwischen drei verschiedenen Generationen unterschieden werden: der jungen Erwerbstätigengeneration, der älteren Erwerbstätigengeneration und der Rentnergeneration. ${ }^{94}$

Die Generationenstärke einer Generation $x$ wird im ,jungen Erwerbsfähigenalter" also in der ersten Periode ihres Lebenszyklus durch $N_{x}^{0}$ beschrieben. Um die Generationenstärke der folgenden Periode im Lebenszyklus zu erhalten wird die Generationenstärke der Vorperiode mit der entsprechenden Überlebenswahrscheinlichkeit $I_{x}{ }^{1}$ multipliziert:

$$
\begin{array}{r}
N_{x}^{1}=N_{x}^{0} \cdot l_{x}^{1} \quad \text { G } 37 \\
\text { und } N_{x}^{2}=N_{x}^{1} \cdot l_{x}^{2}=N_{x}^{0} \cdot l_{x}^{1} \cdot l_{x}^{2}
\end{array}
$$

Die Fertilität kommt ausschließlich in der ersten Periode des Lebenszyklus zum Tragen. Die Generationenstärke der nachfolgenden Generation $x+1$ ergibt sich dann aus dem Produkt der vorangegangenen Generation mit der Reproduktionsrate dieser Generation:

$$
\begin{gathered}
N_{x+1}^{0}=N_{x}^{0} \cdot M_{x} \quad \text { G } 39 \\
M_{x}=\text { Reproduktionsrate der Generation } \mathrm{x}
\end{gathered}
$$

Für die Gesamtbevölkerung zum Zeitpunkt $t\left(B E V_{t}\right)$ - Generation $x$ befindet sich zum Zeitpunkt $t$ in der Ruhestandsphase - erhält man die Beziehung:

$$
B E V_{t}=N_{x}^{0} \cdot l_{x}^{1} \cdot l_{x}^{2}+N_{x}^{0} \cdot M_{x} \cdot l_{x+1}^{1}+N_{x}^{0} \cdot M_{x} \cdot M_{x+1} \quad \text { G } 40
$$

Die Erwerbsbeteiligung wird mit Hilfe generationenspezifischer Enwerbsquoten abgebildet:

$$
e q_{x}^{i} \quad \text { mit } \quad i \in[1,2] \text { und } 0 \leq e q_{x}^{i} \leq 1
$$

Damit läßt sich die Erwerbsbevölkerung $(E B)$ bzw. die Anzahl der Rentenempfänger $(R B)$ zum Zeitpunkt $t$ darstellen als:

$$
E B_{t}=\left(e q_{x+1}^{2} \cdot M_{x} \cdot l_{x+1}^{1}+e q_{x+2}^{1} \cdot M_{x} \cdot M_{x+1}\right) \cdot N_{x}^{0} \quad \mathbf{G} 41
$$

93 Vgl. Bösch (1987) S. 79f. Hinsichtlich des fehlenden Anreizes für das Individuum und die Folgen für die intergenerative Gerechtigkeit vgl. jedoch Breyer/Kolmar (1997).

94 Solche Modelle finden sich beispielsweise bei v. Schulenburg (1989), Dinkel (1986), Bõsch (1987) und Sudhoff (1996). 


$$
R B_{t}=N_{x}^{0} \cdot l_{x}^{1} \cdot l_{x}^{2} \quad \text { G } 42
$$

Die Einnahmen $(E)$ und Ausgaben $(A)$ der Rentenversicherungsanstalt zum Zeitpunkt t können mit Hilfe der folgenden Gleichungen wiedergegeben werden:

$$
\begin{gathered}
E_{t}=b_{t} \cdot w_{t} \cdot E B_{t} \quad \text { G } 43 \\
A_{t}=p_{t} \cdot R B_{t} \quad \text { G } 44
\end{gathered}
$$

Die durchschnittliche Rente $p$ ergibt sich dabei im Falle einer Erwerbseinkommenszentrierten Rentengewährung und eines konstanten Bruttorentenniveaus zu

$$
p_{t}=w_{t} \cdot k \cdot \frac{e q_{x}^{1}+e q_{x}^{2}}{2} \quad \mathbf{G} \mathbf{4} 5^{95}
$$

Da die Rentenleistungen in der gesetzlichen Rentenversicherung seit 1992 nach dem Prinzip des konstanten Nettorentenniveaus konzipiert werden, soll im Modell ebenfalls ein konstantes Nettorentenniveau unterstellt werden: ${ }^{96}$

$$
p_{t}=w_{t} \cdot\left(1-b_{t}\right) \cdot k \cdot \frac{e q_{x}^{1}+e q_{x}^{2}}{2} \quad \mathbf{G} 46
$$

Aus den Gleichungen G43, G44 und G46 läßt sich die bekannte Bestimmungsgleichung des Beitragssatzes im Umlageverfahren mit konstantem Rentenniveau ableiten:

$$
\begin{aligned}
b_{t} & =\frac{p_{t} \cdot R B_{t}}{w_{t} \cdot E B_{t}}=k \cdot R Q_{t} \quad \mathbf{G} 47 \\
R Q_{t} & =\text { Rentnerquotient zum Zeitpnkt } t
\end{aligned}
$$

Durch Einsetzen der Gleichungen G41, G42 und G46 erhält man die Gleichung für den Beitragssatz eines umlagefinanzierten Rentensystems mit konstantem Nettorentenniveau:

$$
b_{t, k_{-} \text {netto }}=\frac{k \cdot l_{x}^{1} \cdot l_{x}^{2} \cdot \frac{e q_{x}^{1}+e q_{x}^{2}}{2}}{e q_{x+1}^{2} \cdot M_{x} \cdot l_{x+1}^{1}+e q_{x+2}^{1} \cdot M_{x} \cdot M_{x+1}+k \cdot l_{x}^{1} \cdot l_{x}^{2} \cdot \frac{e q_{x}^{1}+e q_{x}^{2}}{2}} \quad \text { G } 48
$$

Für die weiteren Ausführungen wird zur Vereinfachung angenommen, daß sich das Erwerbsverhalten einer Generation in den beiden Erwerbsphasen nicht ändert:

$$
e q_{x}^{1}=e q_{x}^{2}=e q_{x}
$$

95 Eine höhere Erwerbsbeteiligung eq kann im Modell grundsätzlich durch eine Erhöhung der Arbeitszeit durch die bereits Enwerbstätigen oder durch eine Erhöhung der Anzahl der Erwerbstätigen erfolgen. Nach der ersten Interpretation kann der Term $\frac{e q_{x}^{1}+e q_{x}^{2}}{2}$ in Gleichung G 45 bzw. G 46 nach der zweiten in Gleichung G 42 integriert werden. Auf die hier zu untersuchenden Zusammenhänge hat dies jedoch keine Auswirkung.

Von Steuern und anderen Sozialabgaben wird in dem Modell abgesehen. 
Aus der Bestimmungsgleichung für die Rendite der Generation $x$, die zum Zeitpunkt $t$ im Ruhestand ist, ergibt sich (bei Aufzinsung der Beiträge mit dem Zinssatz l):

$$
I_{t}=\frac{p_{t}}{b_{t-1} \cdot w_{t-1} \cdot\left(1+i_{t-1}\right)+b_{t-2} \cdot w_{t-2} \cdot\left(1+i_{t-2}\right) \cdot\left(1+i_{t-1}\right)} \cdot \quad \text { G } 49
$$

Durch Einsetzen der Gleichungen G46 und G47 in G49 erhält man:

$$
I_{t}=\frac{\left(1+g_{t}\right) \cdot\left(1+g_{t-1}\right) \cdot\left[1+\frac{a_{x}}{a_{x}+c_{x}+d_{x}}\right]}{\left(1+i_{t}\right) \cdot\left(1+g_{t-1}\right) \cdot\left[1+\frac{a_{x-1}}{a_{x-1}+c_{x-1}+d_{x-1}}\right]+\left(1+i_{t}\right) \cdot\left(1+i_{t-1}\right) \cdot\left[1+\frac{a_{x-2}}{a_{x-2}+c_{x-2}+d_{x-2}}\right]}
$$

mit

$$
\begin{aligned}
& a_{x}=k \cdot l_{x}^{1} \cdot l_{x}^{2} \cdot e q_{x} \\
& c_{x}=e q_{x+1} \cdot M_{x} \cdot l_{x+1}^{1} \\
& d_{x}=e q_{x+2} \cdot M_{x} \cdot M_{x+1}
\end{aligned}
$$

Allein die Erweiterung des Modells auf drei Perioden und die Berücksichtigung der Nettoanpassung führen folglich dazu, daß die Rendite der Generation $x$ nunmehr von 20 Variablen (ohne die Vereinfachung bei der Erwerbsbeteiligung wären es sogar 24) abhängen, die insgesamt fünf Generationen betreffen. Durch Partialanalysen, bei denen für die meisten Variablen bestimmte Annahmen getroffen werden und lediglich eine Variable variiert, können einige tendenzielle Wirkungen der Parametervariationen abgeleitet werden. So ergibt sich zum Beispiel für die Annahmen:

$g_{t}=$ konstant, $i_{t}=$ konstant, $\mathrm{l}=$ konstant $=1, M=$ konstant $=1$, alle eq $=$ konstant $=1$ bis auf $e q_{x}^{1}$ die Renditegleichung:

$$
\begin{gathered}
I^{x}=\frac{(1+g)^{2} \cdot\left(k+1+e q_{x}\right) \cdot\left[1+\frac{k \cdot e q_{x}}{k \cdot e q_{x}+2}\right]}{k \cdot\left[(1+g) \cdot(1+i)+(1+i)^{2}\right]} \\
\text { mit } V=\frac{(1+g)^{2} \cdot}{k \cdot\left[(1+g) \cdot(1+i)+(1+i)^{2}\right]} \text { folgt: } \\
\frac{d I^{x}}{d e q_{x}}>0 \Leftrightarrow \frac{V \cdot k \cdot\left(2 e q_{x}+k+1\right)\left(k \cdot e q_{x}\right)-V \cdot k^{2}\left(e q_{x}+k \cdot e q_{x}+e q_{x}{ }^{2}\right)}{\left(k \cdot e q_{x}\right)^{2}}>0 \\
\Rightarrow k \cdot e q_{x}{ }^{2}+(4-k) \cdot e q_{x}+3 k+2>0
\end{gathered}
$$

Da für das Rentenniveau gilt: $k<1$ und $e q_{x}>0$, ist die obige Bedingung erfült und man kann folgern, daß die Rendite einer Generation mit ihrer Erwerbsbeteiligung steigt. Ähnlich erhält man für die Annahmen:

$g_{t}=$ konstant, $i_{t}=$ konstant, $e q=$ konstant $=1, M=$ konstant $=1$, alle $\mathrm{I}=$ konstant $=1$ bis auf $l_{x}^{1}$ die Renditegleichung: 


$$
\begin{aligned}
& \qquad I^{x}=\frac{(1+g)^{2}\left[1+\frac{k \cdot l_{x}^{1}}{k \cdot l_{x}^{1}+2}\right]}{\frac{k \cdot(1+g) \cdot(1+i)}{k+l_{x}^{1}+1}+\frac{k \cdot(1+i)^{2}}{k+2}} \\
& \text { sowie } \\
& \frac{d l^{x}}{d l_{x}^{1}}>0 \Rightarrow \frac{k\left(k \cdot l_{x}^{1}+2\right)-k^{2} \cdot l_{x}^{1}}{\left(k \cdot l_{x}^{1}+2\right)^{2}}>0 \Rightarrow 2 k>0
\end{aligned}
$$

und es läßt sich die Aussage treffen, daß die Rendite einer Generation $x$ positiv mit ihrer Überlebenswahrscheinlichkeit während der Erwerbsphase korreliert. Die eindeutigen Aussagen lassen sich jedoch nur aufgrund der Vereinfachungen ableiten. Aber selbst unter solchen Annahmen können nicht für alle Variationen eindeutige Aussagen getroffen werden. Um beispielsweise die Wirkungen einer Variation von $l_{x}^{1}$ nicht nur auf die eigene Rendite sondern auch auf die nachfolgender Generationen zu bestimmen, ist die folgende Gleichung heranzuziehen:

$$
\begin{aligned}
& I^{x}=\frac{(1+g)^{2}\left[1+\frac{k}{k+2}\right]}{\frac{k \cdot(1+g) \cdot(1+i)}{k \cdot l_{x-1}^{1}+2}+\frac{k \cdot(1+i)^{2}}{k+l_{x-1}^{1}+1}} . \\
& \frac{d I^{x}}{d l_{x-1}^{1}}>0 \Leftrightarrow-(1+g)^{2}\left[1+\frac{k}{k+2}\right] \cdot \frac{\frac{k \cdot(1+g) \cdot(1+i) \cdot\left[\left(k \cdot l_{x-1}^{1}+2\right)-k \cdot l_{x-1}^{1}\right]}{\left(k \cdot l_{x-1}^{1}+2\right)^{2}}-\frac{k \cdot(1+i)^{2}}{\left(k+l_{x-1}^{1}+1\right)^{2}}}{\left[\frac{k \cdot(1+g) \cdot(1+i)}{k \cdot l_{x-1}^{1}+2}+\frac{k \cdot(1+i)^{2}}{k+l_{x-1}^{1}+1}\right]^{2}}>0 \\
& \Rightarrow \frac{\left(k \cdot l_{x-1}^{1}+2\right)^{2}}{\left(k+l_{x-1}^{1}+1\right)^{2}}>\frac{2(1+g)}{(1+i)}
\end{aligned}
$$

Die Differentiation zeigt, daß Aussagen über die Wirkungsrichtung nur durch die Spezifikation der Werte gemacht werden können

\subsubsection{Modellhafte Überlegungen zur intergenerativen Verteilung}

Da die Wirkungen auf die generationsspezifischen Renditen sich nicht für alle Parametervariationen auf analytischen Wege bestimmen bzw. nur unter vereinfachenden Annahmen ableiten lassen, werden die tendenziellen Wirkungen im folgenden anhand von Modellrechnungen aufgezeigt. Eine Beschreibung der Modellrechnung befindet sich im Anhang 1.2 .

Für die Fertilität, die Mortalität und die Erwerbsbeteiligung werden im Referenzszenario plausible Werte angenommen, die in Sensitivitätsanalysen variiert werden. Die Werte sind innerhalb eines Szenarios für alle Generationen identisch.

Zunächst wird danach gefragt, wie sich die Rendite für die Generationen verändert, wenn einer der Parameter lediglich für die Generation $x$ variiert und für nachfolgende Generationen wieder seinen ursprünglichen Wert annimmt. 
Um die Robustheit der Ergebnisse zu prüfen, wurden die gleichen Variationen bei unterschiedlichen Konstellationen aus Zinssatz und Lohnentwicklung durchgeführt.

Tabelle 2 faßt die Ergebnisse dieser einperiodigen Parametervariation zusammen und ist wie folgt zu lesen:

Die Spalten beschreiben die Rendite der Generation x (Ix) sowie die Renditen der beiden vorangehenden und nachfolgenden Generationen. In den Zeilen sind die Parameter abgetragen, die für die Generation x variiert wurden.

Die erste Zelle gibt beispielsweise an, wie sich die Rendite $\left(I_{x-2}\right)$ der Generation, die zwei Perioden vor der Generation x geboren wurde, verändert, wenn das Reproduktionsverhalten der Generation $x$ variiert $\left(d I_{x-2} / d M_{x}\right)$.

Besteht kein Zusammenhang, so ist die Zelle unterlegt, ein positiver Zusammenhang wird durch ein + ein negativer durch ein -- angezeigt. Ein 0 gibt an, daß der Zusammenhang unbestimmt ist, d.h. von den Ausgangswerten abhängt.

Tabelle 2: Variation der Rendite verschiedener Generationen bei einer einperiodigen Parametervariation der Generation x

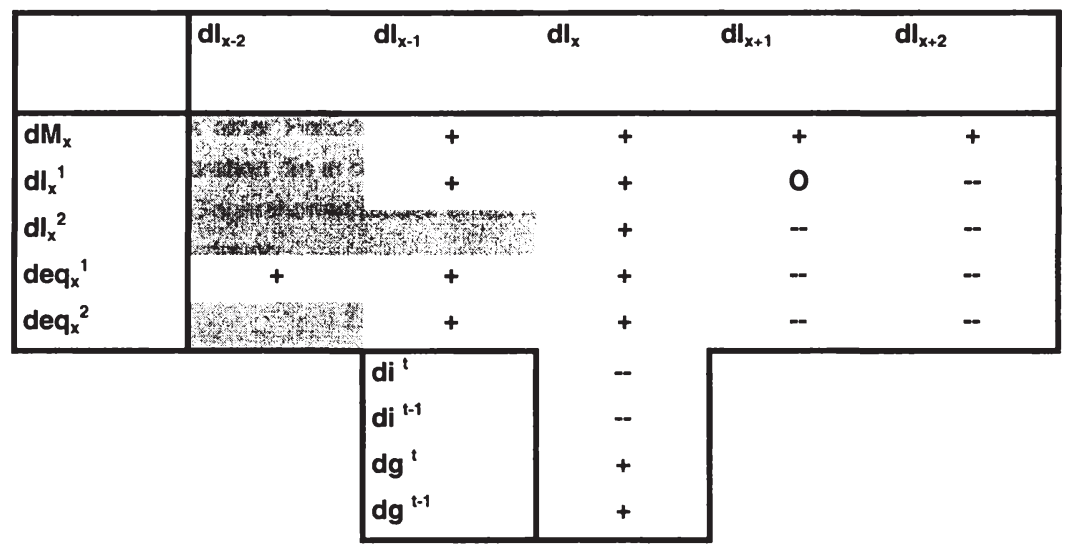

In einem zweiten Schritt wird untersucht, welche Konsequenzen sich für die intergenerative Verteilung ergeben, wenn die Parametervariationen nicht mehr nur bei einer Generation auftreten, sondern über verschiedene Generationen hinweg aufrecht erhalten werden.

Für die Einflußgrößen der Überlebenswahrscheinlichkeit und der Erwerbsbeteiligung zeigt sich, daß eine Generation die durch eine Parameteränderung ausgelöste Umverteilungslast durch Aufrechterhaltung der Variation auf andere Generationen so lange überwälzen 
kann, bis die Größe schließlich auf ihr ursprüngliches Niveau zurückfällt. ${ }^{97}$ Diese Zusammenhänge gelten im übrigen unabhängig von der konkreten Ausgestaltung der Rentenformel, sprich ob ein konstantes Netto- oder Bruttorentenniveau oder konstante Beitragssätze unterstellt werden. Die Rentenformel hat dabei lediglich einen Einfuß darauf, welche Generationen in welchem Ausmaß betroffen sind.

Die auftretenden Verteilungsmuster werden in Abbildung 2 und Abbildung 3 exemplarisch für eine Erhöhung der Erwerbsbeteiligung um zehn Prozentpunkte verdeutlicht.

Während die Variation in Abbildung 2 lediglich bei der Generation 3 auftritt, bleibt sie in Abbildung 3 für die Generationen 3 bis 8 wirksam.

Abbildung 2: Veränderung der Rendite unterschiedlicher Generationen bei einer Erhöhung der Erwerbsbeteiligung der „Generation $3^{\prime \prime}$.

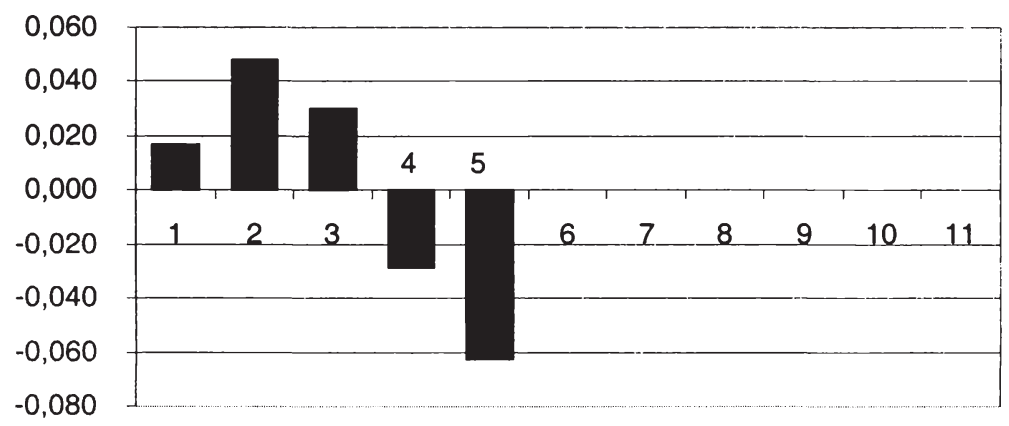

Abbildung 3: Veränderung der Rendite unterschiedlicher Generationen bei einer Erhöhung der Erwerbsbeteiligung der "Generationen 3 bis 8 “.

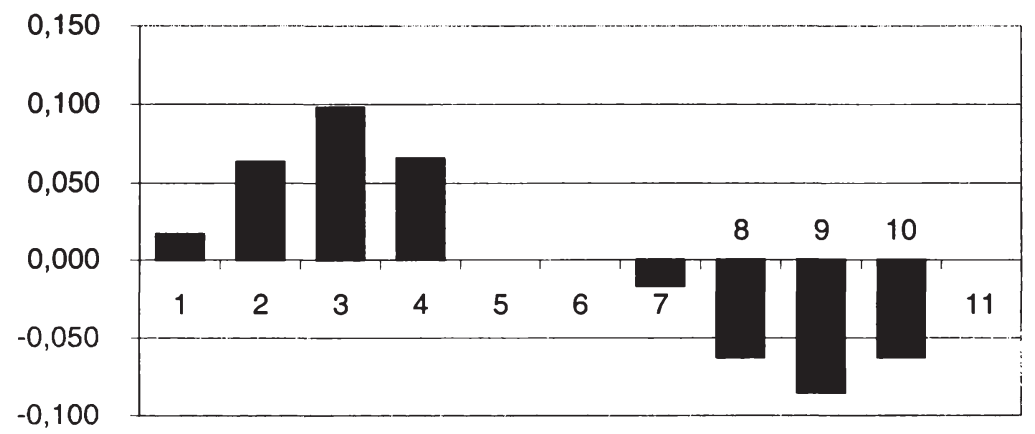

97 Ein Effekt, der in gleicher Weise auch bei der Invalidităts- und Verwitwungswahrscheinlichkeit sowie bei einer politischen Veränderung des Rentenniveaus auftritt.

60 
Diese Zusammenhänge können einfach erklärt werden. ${ }^{98}$ Ausgangspunkt ist die schuldentheoretische Interpretation der gesetzlichen Rentenversicherung, wonach es in einem Umlageverfahren immer eine Einführungsgeneration gibt, die durch empfangene Rentenleistungen profitiert, ohne jedoch zuvor entsprechende Beitragszahlungen geleistet zu haben. ${ }^{99}$ Betrachtet man einen endlichen Zeithorizont, steht dieser Einführungsgeneration immer auch eine Letztgeneration gegenüber, die zwar noch Beiträge in das System einzahlt, aber hierfür keine Rentenleistungen mehr erhält. Die Gewinne der Erstgeneration werden folglich durch die Verluste der Letztgeneration kompensiert. In einem Umlageverfahren kommt es folglich immer zu einer Umverteilung von der Letztgeneration zur Einführungsgeneration. ${ }^{100}$ Die Begriffe der Einführungs- und der Letztgenerationen sollten dabei jedoch nicht zu eng gefaßt und nicht nur auf die Einführung und Auflösung des gesamten Systems bezogen werden. Vielmehr kann jede Generation durch eine Erhöhung der Überlebenswahrscheinlichkeit, der Erwerbsbeteiligung oder des Rentenniveaus in gleicher Weise wie eine Einführungsgeneration bessergestellt werden. Ebenso wird eine Generation durch eine Verringerung dieser Faktoren zur Letztgeneration und trägt dadurch einen Teil der Umverteilungslasten. ${ }^{101}$ Bei einer einmaligen Variation dieser Parameter liegen Einführungs- und Letztgeneration folglich beieinander, während sie bei einem Anhalten der Parametervariation zeitlich auseinandergezogen werden.

Die übrigen Einflußfaktoren, die Fertilität und die Lohnentwicklung (bzw. Produktivität) sowie der Zins- bzw. der Diskontierungsfaktor, unterscheiden sich in ihren Verteilungswirkungen jedoch erheblich von den bislang untersuchten Faktoren der Überlebenswahrscheinlichkeit und der Erwerbsbeteiligung und zwar in doppelter Hinsicht.

Einerseits sind durch eine Veränderung dieser Größen die Renditen aller Generationen gleichartig betroffen. D.h. es ist nicht möglich, daß eine oder einige Generationen durch eine Variation schlechter- und andere durch dieselbe Variation bessergestellt werden. Andererseits kann die Last einer Generation nicht auf spätere Generationen verlagert werden, wenn die Parametervariation über mehrere Generationen anhält. ${ }^{102}$ Diese Zusammenhänge lassen sich recht einfach erklären. Die Fertilität und die Lohnentwicklung (Produktivität) sind die beiden Komponenten des Lohnsummenwachstums und stellen somit die Verzinsung des Umlageverfahrens dar. Verändert sich die Verzinsung eines

\footnotetext{
98 Vgl. nachfolgend auch Bösch (1987), S. 55-85.

99 In der Realität werden jedoch sowohl der Umfang der Versicherungsleistungen als auch der Versichertenkreis nur allmählich auf das angestrebte Niveau enweitert, so daß es treffender ist, nicht von einer, sondern mehreren Einführungsgenerationen zu sprechen.

100 Vgl. Bösch (1987), S. 59, Satz 4.

101 Vgl. Bösch (1987), S. 75, Satz 6; Spremann (1984). Zur schuldentheoretischen Interpretation des Umlageverfahren vgl. auch Steden (1981), S. 424f.; Spremann (1987), S.24f..

102 Vgl. auch Bösch (1987), S. 76-80.
} 
Systems, so müssen alle Mitglieder des Systems in gleicher Weise betroffen sein. Darüber hinaus ist es offensichtlich, daß das absolute Niveau der Verzinsung für die Rendite ausschlaggebend ist, während bei den übrigen Einflußfaktoren aufgrund des periodenbezogenen Budgetausgleichs das Verhältnis der jeweiligen Größe zu der vorangehenden bzw. nachfolgenden Generationen entscheidend ist.

Der allgemeine Zins- bzw. konkreter der Diskontierungssatz wirkt auf die Rendite ebenfalls wie eine Verzinsung. Vereinfacht ausgedrückt kann er als Opportunitätszinssatz bzw. als Zinssatz des Vergleichssystems (vgl. Abschnitt 5.3.1.6) aufgefaßt werden.

\subsubsection{Modell einer geschlossenen Volkswirtschaft}

In dem Modellrahmen einer kleinen offenen Volkswirtschaft ist der Zinssatz ein exogener Parameter. Wenn nun die Veränderung der generationsspezifischen Rendite das maßgebliche Verteilungskriterium ist, können intergenerative Verteilungswirkungen im Kapitalstockverfahren folglich nur aufgrund eines exogenen Effektes, nämlich der Veränderung des Zinssatzes, auftreten. Im Gegensatz dazu reagiert die Rendite des Umlageverfahrens auf Veränderungen sämtlicher exogener Parameter.

Werden die Distributionseffekte für eine geschlossene Volkswirtschaft analysiert, ist der Zinssatz eine Funktion der Kapitalintensität, die von den wesentlichen (exogenen) demographischen und ökonomischen Parametern abhängt. D.h. dieselben Größen, die die Rendite des Umlageverfahrens in einer offenen Volkswirtschaft direkt beeinflussen, bestimmen die Rendite des Kapitalstockverfahrens in einer geschlossenen Volkswirtschaft indirekt über eine Veränderung der Kapitalintensität.

Die folgende Analyse fragt danach, wie sich der Zinssatz im Modell verändert, wenn eine der exogenen Größen (Sterblichkeit während der Erwerbsphase und während der Ruhephase, Fertilität, Erwerbsbeteiligung, Lohnentwicklung bzw. technische Fortschritt) variiert. Eine grundlegende Analyse dieser Zusammenhänge findet sich bei $\mathrm{BöscH}$, auf dessen Arbeit auch die folgenden Betrachtungen beruhen. ${ }^{103}$

Ausgangspunkt der Untersuchung ist, wie im Fall einer kleinen offenen Volkswirtschaft, ein OLG-Modell mit drei Generationen. Um die Wirkung einer produktivitätsbedingten Lohnentwicklung ebenfalls zu erfassen, wird eine Produktionsfunktion mit arbeitsvermehrendem technischen Fortschritt unterstellt: ${ }^{104}$

$$
Y_{t}=F\left[K_{t}, G^{t} \cdot N_{t}\right] \quad \text { G } 50
$$

$G^{t}$ beschreibt den Faktor des technischen Fortschritts, wobei t in diesem Falle kein Index, sondern eine Potenz darstellt. Die übrigen Eigenschaften der Produktionsfunktion sind

\footnotetext{
103 Vgl. Bösch (1987). Die hergeleiteten Zusammenhänge im Umlageverfahren sind nach wie vor gültig.

104 Im Wachstumsgleichgewicht entspricht der Wachstumsfaktor des technischen Fortschritts daher dem Wachstumsfaktor der Löhne. Der Einfluß des technischen Fortschritts entspricht bei dieser Modellierung einer Erhöhung des Arbeitsangebots, so daß die Größen in der Analyse regelmäßig auf Arbeitseffizienzeinheiten bezogen werden können.
} 
analog zu dem in Anhang beschriebenen Grundmodell definiert. Insbesondere gilt wieder für die Entlohnung der Produktionsfaktoren entsprechend der Grenzproduktivitätstheorie:

$$
\begin{gathered}
\qquad\left(1+i_{t}\right)=f^{\prime}\left[k_{t}\right] \text { G } 51 \\
\hat{w}_{t}=G^{t} \cdot w_{t}=G^{t} \cdot\left[f\left[k_{t}\right]-k_{t} \cdot f^{\prime}\left[k_{t}\right]\right] \quad \text { G } 52 \\
\hat{w}_{t}=\text { Durchschnittslohn pro Kopf } \\
w_{t}=\text { Durchschnittslohn pro Arbeitseffizienzeinheit }
\end{gathered}
$$

Zu diesen drei Gleichungen des Produktionssektors kommen noch die aus der Budgetbeschränkung des Lebenszyklus abgeleiteten Bestimmungsgleichung des Konsums der jungen Erwerbstätigen $c^{1}$, der alten Erwerbstätigen $c^{2}$ und der Ruheständler $c^{3}$. In einem ersten Schritt wird ein Kapitalstockverfahren mit fixem Beitragssatz unterstellt. Weitere wesentliche Gleichungen sind die Gleichgewichtsbedingung auf dem Gütermarkt $i_{t}=s_{t}$ und die Einkommensverwendungsgleichung der Volkswirtschaft $t_{t}=c_{t}+i_{t}$.

Damit das Modell vollständig beschrieben ist und die neun endogenen Variablen des Modells $\left(c^{1}, c^{2}, c^{3}, i, s, f, r, w, k\right)^{105}$ bestimmt werden können, muß noch die Bestimmungsgleichung der Kapitalintensität $k(t)$ ermittelt werden. Sie ist der zentrale Kern der folgenden Analyse, da sämtliche endogenen Variablen $\left(c^{1}, c^{2}, c^{3}, i, s, f, r, w\right)$ von ihr abhängen. Der Kapitalstock einer Periode $t$ bildet sich im Modell ausschließlich aus der Ersparnis derjenigen Generation die in den Perioden $t-1$ und $t-2$ geboren wurden. Durch die Modellierung als drei Generationen Modell erhält man folglich eine nichtlineare Differenzengleichung zweiter Ordnung.

$$
\begin{aligned}
k_{t} & =\frac{b \cdot w\left[k_{t-1}\right]}{M \cdot G}+\frac{\left.b \cdot w\left[k_{t-2}\right] \cdot R\left[k_{t-1}\right)\right]}{M \cdot\left(M+l^{1}\right) \cdot G^{2}} \quad \text { G } 53 \\
b & =\text { Beitragssatz im Kapitalstockverfahren }
\end{aligned}
$$

Mit Hilfe dieser Differenzengleichung läßt sich auch der Steady-State Wert $\boldsymbol{k}$ der Kapitalintensität beschreiben, der eine Funktion der demographischen $\left(M, I_{1}, l_{2}\right)$, ökonomischen $(w, R, G)$ und politischen Variablen (b) darstellt. Der Einfluß dieser exogenen Parameter auf die Rendite des Kapitalstockverfahren kann nun einfach bestimmt werden, indem diese Funktion nach den jeweiligen Parametern partiell abgeleitet wird. Die Kapitalintensität läßt sich zwar nicht explizit als Funktion der exogenen Variablen formulieren, allerdings lassen sich mit Hilfe des Theorems zur impliziten Differentiation die Vorzeichen der partiellen Ableitungen bestimmen. Da eine steigende Kapitalintensität mit einem sinkenden Zinssatz einher geht und vice versa, sind somit auch die Wirkungen einer Veränderung der exogenen Parameter auf den Zinssatz, sprich auf die Rendite des Kapitalstockverfahrens festgelegt. 
Tabelle 3: Variation des Zinssatzes bei Veränderung der ökonomischen und demographischen Parameter in einer geschlossenen Volkswirtschaft und bei einem Kapitalstockverfahren mit konstantem Beitragssatz

\begin{tabular}{|l|l|l|l|l|l|l|}
\cline { 2 - 7 } \multicolumn{1}{c|}{} & $\mathrm{DM}$ & $\mathrm{DL}^{1}$ & $\mathrm{DL}^{2}$ & $\mathrm{DG}$ & $\mathrm{DB}$ & $\mathrm{DEQ}$ \\
\hline $\mathrm{DR}$ & $>0$ & $>0$ & $\prime$ & $>0$ & $<0$ & $I$ \\
\hline
\end{tabular}

Quelle: Bösch (1987), S. 107.

Dem Modell liegt die Annahme zugrunde, daß die Motivation des Sparens auf die Vorsorge für das Alter beschränkt ist. ${ }^{106}$ In der dritten Phase des Lebenszyklus wird demzufolge nicht mehr gespart, vielmehr wird das Ersparte konsumiert. Aus diesem Grunde geht $P$ auch nicht in die Differenzengleichung G53 ein und eine Veränderung der Überlebenswahrscheinlichkeit in der Ruhestandsphase hat keinen Renditeeffekt, wohl aber einen Effekt auf das Konsumniveau im Alter.

Die in Tabelle 3 angezeigte Unabhängigkeit der Erwerbsbeteiligung ist ebenfalls offensichtlich, da eine sinkende Erwerbsbeteiligung zwar die absolute gesamtwirtschaftliche Ersparnis mindert, die Anzahl der Enwerbstätigen, die mit einem Kapitalstock ausgestattet werden muß, sich aber ebenfalls im gleichen Ausmaß verringert. Die Kapitalintensität und somit auch der Zinssatz bleiben konstant. ${ }^{107}$

Die ökonomische Interpretation der positiven Korrelation zwischen der Fertilität, der Überlebenswahrscheinlichkeit im erwerbstätigen Alter sowie des technischen Fortschritts auf der einen und dem Zinssatz auf der anderen Seite beruhen alle auf den gleichen Effekt. Eine Erhöhung aller drei Parameter führt zu einer Steigerung der Arbeitseffizienzeinheiten. Das höhere Produktionsniveau führt zwar in Folge zu steigenden Beiträgen, allerdings steigt das Produktionsniveau mit abnehmenden Grenzerträgen, so daß trotz des absolut gestiegenen Kapitalstocks eine geringere Kapitalintensität in Arbeitseffizienzeinheiten vorliegt und somit der Zinssatz steigt.

Sofern das Kapitalstockverfahren ebenfalls mit einem konstanten Rentenniveau (h) arbeitet, ändert sich im obigen Modell die Gleichung des Konsums im Ruhestand zu:

$$
c^{3}{ }_{t}=h \cdot G^{t} \cdot w_{t} \quad \mathbf{G} 54
$$

Ferner muß derjenige Beitragssatz ermittelt werden, der bei vorgegebenem Rentenniveau die Beitragsäquivalenz des Kapitalstockverfahrens sichert. Aus der Identität zwischen

106 Eine Veränderung der Erspamis aufgrund anderer Motive hat zwar ebenfalls eine Wirkung auf den Zinssatz, diese ist dann jedoch nicht systemimmanent, sondern kann als „exogener Störeffekt" gewertet werden. Für das Ergebnis kann es aber dennoch von Bedeutung sein, wenn es zwischen systeminduzierten und systemautonomen Ersparnissen zu einer Substitution kommt und etwaige Effekte konterkariert werden. Vgl. Abschnitt 2.5.2 und Abschnitt 6.1.1.

107 Vgl. Bösch (1987), S. 108. An dieser Stelle offenbart sich erneut die Schwäche einer reinen SteadyState Analyse, da sich der Zinssatz auf dem Anpassungspfad einer komparativ-dynamischen SteadyState Betrachtung durchaus verändert.

64 
dem Erwartungswert der Beitragszahlungen und dem Erwartungswert der Rentenleistungen erhält man:

$$
\begin{gathered}
E(B)=E(L) \Leftrightarrow h \cdot l_{1} \cdot l_{2} \cdot w_{t}=b^{\circ} \cdot w_{t-2} \cdot(1+i)^{2}+l_{1} \cdot b^{\circ} \cdot w_{t-1} \cdot(1+i) \quad \text { G } 55 \\
\Leftrightarrow b^{\circ}=\frac{h \cdot l_{1} \cdot l_{2} \cdot G^{2}}{R^{2}+R \cdot G \cdot l_{1}} \quad \text { G } 56
\end{gathered}
$$

$b^{\circ}=$ Beitragssatz im Kapitalstockverfahren bei vorgegebenem Rentenniveau

Zwischen der Kapitalintensität G53 und dem Beitragssatz bestehen nun folgende wechselseitigen Beziehungen: Eine Erhöhung der Kapitalintensität impliziert einen sinkenden Zinssatz. Um das Rentenniveau trotz niedrigerer Verzinsung konstant zu halten, müssen die Beiträge steigen. Steigende Beiträge wiederum führen zu einem erneuten Anstieg der Ersparnis. ${ }^{108}$ Schon dieser Zusammenhang läßt vermuten, daß ein Kapitalstockverfahren mit fixiertem Rentenniveau sensibler auf Fertilitätsänderungen reagiert als eines mit fixierten Beitragssätzen.

Die Werte für den Beitragssatz und für die Kapitalintensität können folglich über das Gleichungssystem aus den Gleichungen G53 und G56 bestimmt werden. Die Veränderung des Zinssatzes bzw. des Beitragssatzes als Folge einer Variation der exogenen Parameter ließe sich wiederum durch partielle Ableitungen bestimmen. Da sich das Gleichungssystem jedoch nicht explizit als Funktion der exogenen Parameter formulieren läßt, müssen die partiellen Ableitungen mit Hilfe des Satzes über implizite Funktionen bestimmt werden. Die Ergebnisse sind in Tabelle 4 zusammengefaßt.

Tabelle 4: Variation des Zins- und Beitragssatzes bei Veränderung der ökonomischen und demographischen Parameter in einer geschlossenen Volkswirtschaft und bei einem Kapitalstockverfahren mit konstantem Rentenniveau.

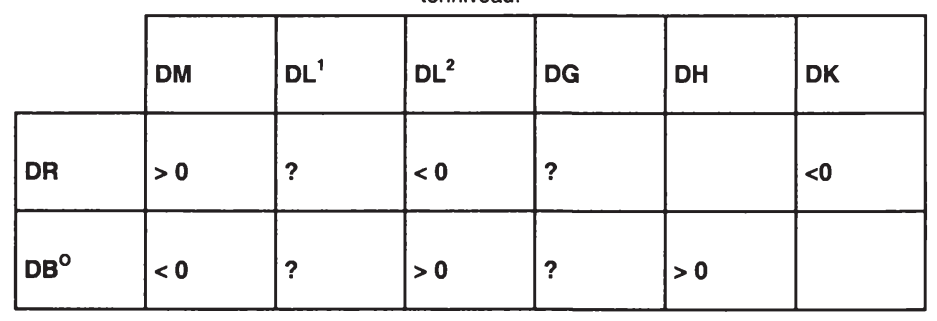

Quelle: Bösch (1987), S. 120.

Die Veränderung des Zins- und Beitragssatzes ist hinsichtlich einer Variation des technischen Fortschritts deshalb unbestimmt, weil sie zwei gegenläufige Effekte auslöst:

108 Um die Stabilität des Systems zu gewährleisten, muß diese durch den Anstieg des Beitragssatzes induzierte Erhöhung der Ersparnis geringer ausfallen, als die ursprüngliche Veränderung der Ersparnis, die für die Beitragssatzsteigerung verantwortlich war. Dies kommt im Modell darin zum Ausdruck, daß die Jakobi-Determinante des Gleichungssystems G53 und G56 kleiner Null sein muß. Vgl. Bösch (1987), S. 119.

65 
1. Eine Steigerung des technischen Fortschritts erhöht die Arbeitseffizienzeinheiten und senkt somit die Kapitalintensität. Der daraus resultierende, steigende Zinssatz ermöglicht es, das gleichgebliebene Rentenniveau mit niedrigeren Beitragssätzen zu finanzieren. Niedrigere Beitragssätze bewirken wiederum eine erneute Verringerung der Kapitalintensität etc..

2. Eine Steigerung des technischen Fortschritts führt bei einem konstanten Rentenniveau ebenfalls zu einer Erhöhung der erwarteten Rentenleistungen. Damit diese gedeckt sind, müssen die Beitragssätze steigen, was eine Steigerung der Kapitalintensität nach sich zieht.

Welcher der Effekte überwiegt, läßt sich a priori nicht bestimmen und hängt maßgeblich von der Spezifikation der Produktionstechnologie ab. Gleiches gilt für eine Variation der Überlebenswahrscheinlichkeit $l_{1}$, denn einerseits erhöhen sich bei steigendem $l$, die Arbeitseffizienzeinheiten und sinkt die Kapitalintensität, andererseits steigt die Zahl der Ruheständler und damit die erwartete Rentenleistung. Letzteres erfordert eine Erhöhung der Beitragssätze und erhöht die Kapitalintensität.

Ein zentrales Ergebnis der vorausgegangenen Analyse ist, daß die Folgen einer Fertilitätsveränderung auf die intergenerative Verteilungswirkung für das Umlageverfahren und das Kapitalstockverfahren qualitativ identisch sind. In beiden Finanzierungsverfahren mit konstantem Rentenniveau muß bei sinkenden Geburtenzahlen der Beitragssatz steigen und in beiden Finanzierungsverfahren verringert sich die Rendite unabhängig davon, ob das Rentenniveau oder der Beitragssatz fixiert wird. ${ }^{109}$

Die bisherigen Betrachtungen gründen sich alle auf Steady-State Analysen, die bestenfalls prinzipielle Wirkungen aufzeigen können. In seinem Theorem 11 zeigt HOMBURG jedoch für einen beliebigen Zustand der Wirtschaft, d.h. unabhängig von der Annahme eines Steady-State, daß der Zins gleichsinnig zur Wachstumsrate der Bevölkerung variiert. ${ }^{110} \mathrm{Ob}$ die Verteilungswirkungen sich auch in quantitativer Hinsicht entsprechen, läßt sich ohne eine genaue Spezifikation der Produktionstechnologie nicht beurteilen. ${ }^{111}$

\footnotetext{
109 Vgl. Bösch (1987), S. 120 und 123.

110 In seinem Beweis ist in Gleichung (104) jedoch ein Vorzeichen(schreib)fehler unterlaufen, vor dem rechten Term der Gleichung darf kein Minuszeichen stehen. Vgl. Homburg (1988), S. 69 f.

111 Bösch führt daher Simulationsrechnungen mit einer linear-homogenen Cobb-Douglas Produktionsfunktion durch. Dabei zeigt sich, daß die Reagibilität der Rendite des Kapitalstockverfahrens auf demographische und ökonomische Veränderungen mit steigender Produktionselastizität des Kapitals zunimmt. Für den Fall konstanter Beitragssätze entspricht die Veränderung der Wachstumsrate der Bevölkerung bereits bei einer Produktionselastizität von 0,25 der Veränderung des Zinssatzes. Für ein System mit konstantem Leistungsniveau beläuft sich der entsprechende Wert auf ungefähr 0,15 .
} 


\subsection{Erweiterungen des Modellrahmens}

Die vorangegangenen Analysen der allokativen und distributiven Wirkungen des Umlageverfahrens und des Kapitalstockverfahrens erfolgten innerhalb des Grundmodells der überlappenden Generationen.

Dieses Grundmodell ist für manche Untersuchungszwecke zu eingeschränkt und wurde daher in der Literatur je nach Fragestellung angepaßt und erweitert. Eine Systematisierung der Variationsmöglichkeiten findet sich bei BREYER. ${ }^{112}$ Es ist im Rahmen dieser Arbeit weder möglich, noch ist es das Ziel der nachfolgenden Ausführungen, einen umfassenden Überblick über die Variationsbreite dieser Modelle zu geben. Zwei Typen von Erweiterungen werden dennoch skizziert:

Dies sind zum einen Modelle, die den Faktor der Unsicherheit berücksichtigen, da sie zusätzliche Erkenntnisse über die Rolle des Risikoausgleichs von Versicherungen liefern. Dies ist notwendig, um zu unterscheiden, welche intergenerativen Verteilungswirkungen gerecht und welche als ungerecht angesehen werden (vgl. Abschnitt 4.4.3.2).

Zum anderen werden jene Ansätze umrissen, die Erbschaftsmotive in die Analyse einbeziehen. Sie spielen eine wichtige Rolle bei der Diskussion um die gesamtwirtschaftlichen Spareffekte des Kapitalstockverfahrens. Sowohl die in Abschnitt 2.4.3 abgeleiteten intergenerativen Verteilungswirkungen innerhalb des Kapitalstockverfahrens als auch die mit dem Kapitalstockverfahren verbundenen Wachstumsimpulse (vgl. Abschnitt 6.1.1) hängen entscheidend von der Rolle der Erbschaftsmotive ab.

\subsubsection{Modelle mit Unsicherheit}

Bislang wurde die Effizienz - also die Frage, ob eine Generation „besser gestellt“ wird allein anhand des Kriteriums der eigenen Konsummöglichkeiten, sprich des absoluten Lebenseinkommens beurteilt. In einer Welt mit risikoaversen Individuen und Unsicherheit über die Entwicklung der verschiedenen Einflußgrößen, können mit der Einführung eines Versicherungssystems, das eine bestimmte Sicherheit des Alterseinkommens (bzw. präziser des relativen Lebensstandards) gewährt, Effizienzgewinne verbunden sein, wenn dadurch der ex ante erwartete Nutzen steigt. Eine Reihe von Arbeiten, die sich dieser Fragestellung widmen, kommen zu den Schluß, daß ein entscheidender Vorteil des Umlageverfahrens eben in der Verbesserung einer solchen intergenerativen Streuung des Einkommensrisikos liegt:

- ENDERS/LAPAN (1982) betrachten eine Modellökonomie, in der das Lebenseinkommen der Versicherten aufgrund einer stochastischen Störung der Produktionsfunktion unsicher ist. Mit Hilfe eines umlagefinanzierten Alterssicherungssystems kann dann die Risikoteilung zwischen den Generationen verbessert werden. Unter der Bedingung einer bestehenden Risikoaversion kann dadurch der erwartete Nutzen der Individuen gesteigert werden. 
- SMITH (1982) untersucht die Einkommensrisiken, die sich bei einer Veränderung der demographischen Parameter ergeben und kommt zu dem Schluß, daß das Umlageverfahren im ungünstigsten Szenario mit einem höheren Nutzen für den Versicherten verbunden ist. Mit anderen Worten:

Ein umlagefinanziertes System mit fixierter Rentenleistung kann ein Individuum ex ante am besten gegen das Einkommensrisiko abchern, wenn das Individuum nicht weiß, in welche demographische Situation es hineingeboren wird.

- Merton (1983) bezieht die Unsicherheit des Lebenseinkommens in seiner Analyse nicht nur auf den Produktionsoutput der jeweiligen Perioden, sondern unterstellt zudem variable Anteile der Produktionsfaktoren Arbeit und Kapital an der Periodenproduktion. Die Risikostreuung in der Alterssicherung kann dann als Frage nach dem optimalen Portfolio angesehen werden, in dem Vermögen sowohl in Humankapital (Umlageverfahren) als auch in Sachkapital (Kapitalstockverfahren) investiert werden kann. Ferner modelliert MERTON den Arbeitseinsatz als Entscheidungsvariable der Versicherten. Im Falle der Nichtexistenz eines Marktes für Humankapital kann ein umlagefinanziertes Alterssicherungssystem ein geeignetes Mittel für eine Risikostreuung sein, bei dem die Einkommensrisiken von nachfolgenden Generationen auf vorhergehende übertragen werden. Die Betonung des Portfolio Aspektes verdeutlicht bereits, daß in diesem Ansatz eine Kombination der Finanzierungsverfahren als ideale Form der Alterssicherung angesehen wird.

Zur Weiterführung und Kritik dieses Ansatzes der optimalen Portfolioselektion vgl. auch RICHTER (1990).

- ABEI. (1985) betrachtet den Fall, daß ein Versicherter nur mit einer gegebenen Wahrscheinlichkeit die Ruhestandsperiode erlebt und seine Ersparnis als zufällige Erbschaft der nachfolgenden Generation zukommt. Bei einem Umlageverfahren weist der Konsum $\left(c^{1}\right.$ und $c^{2}$ ) eine geringere Varianz auf als im Kapitalstockverfahren und kann somit zu einem höheren erwarteten Lebensnutzen führen.

- GoRdon/VARIAN (1988) modellieren in ihrer Analyse zwei Einkommenskomponenten. Neben einem sicheren Pro-Kopf-Einkommen besteht im Alter ein zusätzlicher und durch exogene Einflüsse bestimmter stochastischer Ertrag. Mit Hilfe einer umlagefinanzierten Alterssicherung kann ein Teil dieses Einkommensrisikos auf nachfolgende Generationen übertragen werden, so daß der erwartete Nutzen aller Generationen ex ante erhöht werden kann.

- SPECKBACHER (1994) untersucht für eine geschlossene Volkswirtschaft und einen beliebigen, aber endlichen Zeitraum die Existenz einer gerechtigkeitsorientierten Gleichgewichtslösung bei verschiedenen Alterssicherungssystemen. Im Falle einer instationären Entwicklung exogener Größen existieren solche Lösungen sowohl im

112 Vgl. Breyer (1990), S. 4 ff.. 
Kapitalstockverfahren als auch im Umlageverfahren (aufgrund der unterstellten Ersparniswirkungen) lediglich zufällig.

„Wesentlich ist dabei die Erkenntnis, daß nur durch staatliche Eingriffe in Form einer Festlegung von Transferzahlungen, eine im Sinne dieses Kriteriums gerechte Verteilung zwischen den Generationen erreicht werden kann. (...) Nur durch staatliche Eingriffe kann ein Ausgleich der durch eine ungleichmäßige Bevölkerungsentwicklung und unterschiedliche Konsumpräferenzen der Generationen verursachten Instationaritäten erfolgen. “ ${ }^{113}$

Er warnt aber gleichzeitig vor einer unangemessenen Ausweitung des Umlageverfahrens, weil dies eine verminderte Ersparnisbildung auf Kosten nachfolgender Generationen implizieren würde. Das Umlageverfahren sollte „lediglich als Instrument zum Ausgleich von Instationaritäten aufgefaßt werden“. ${ }^{113}$

Die Bewertungen der Pareto-Effizienz eines Finanzierungsverfahrens kann folglich unterschiedlich ausfallen: je nachdem, ob bestehende Unsicherheiten und Sicherheitspräferenzen berücksichtigt werden oder ob die Rendite als einziges Effizienzkriterium herangezogen wird.

Mit der Orientierung am Erwartungswert des Effizienzkriteriums müssen auch die intergenerativen Verteilungswirkungen differenzierter betrachtet werden. Die erforderliche Trennung zwischen systematischer ex ante Verteilung und einer Verteilung, die ex post aus der Versicherung resultiert, ist von wesentlicher Bedeutung für diese Arbeit und wird in den Abschnitten 4.4.3 und 5.3 vertieft.

Vererben die Rentner allerdings ein Teil ihres Vermögens an die nachfolgende Generation, kann dies den beschriebenen „Versicherungseffekt" des Umlageverfahrens zum Teil konterkarieren. Die allgemeinen Folgen, die sich aus der Existenz solcher Transfermotive ergeben können, werden im folgenden Abschnitt beschrieben.

\subsubsection{Modelle mit Transfermotiven}

In den bisher betrachteten Modellen wurde unterstellt, daß die Wirtschaftssubjekte inre Entscheidung ausschließlich auf der Grundlage ihres eigenen Lebenszyklus fällen. Das „Ricardianische Äquivalenztheorem“, wonach die Finanzierung der Staatsausgaben mittels Steuern sich in ihrer Wirkung auf das Volkseinkommen nicht von einer öffentlichen Verschuldung unterscheidet, läßt sich auch auf die Analyse von Alterssicherungssystemen übertragen.

Sofern Vererbungsmotive in den Modellrahmen integriert werden, könnten demzufolge die hergeleiteten Wirkungen eines Alterssicherungssystems hinfällig werden: Umlageverfahren und Kapitalstockverfahren wären äquivalent.

Diese These wurde erstmals - zeitgleich, aber unabhängig voneinander - von BARRO und V. WEIZÄCKER aufgestellt. ${ }^{114}$ Die Berücksichtigung der Erbschaft verändert sowohl die

\footnotetext{
113 Speckbacher (1994), S. 5 und S. 131.

114 Vgl. Barro (1974), v. Weizäcker (1974).
} 
Zielfunktion des Modells, da das Wirtschaftssubjekt einen Nutzen aus der Vererbung zieht, als auch die Nebenbedingungen des individuellen Optimierungskalküls, da das Individuum nunmehr mit einem Erbe rechnet. In Folge wird die geringere Ersparnis der erwerbstätigen Generation durch die erbschaftsinduzierte Ersparnis der Ruhestandsgeneration kompensiert. In einer solchen „Barro-Welt" wird insbesondere automatisch der optimale Wachstumspfad gemäß der „Goldenen Regel der Kapitalakkumulation“ erreicht.

JAEGER modelliert ebenfalls ein zwei-Generationenmodell mit zweiseitigen Transfermotiven, bei dem der Nutzen der jeweils anderen Generation mit einem intergenerativen Diskontfaktor gewichtet wird. ${ }^{115}$ Seine ersten beiden Ergebnisse unterscheiden sich insofern von den oben abgeleiteten, als er Konstellationen der Transfermotive und der intergenerativen Diskontsätze beschreibt, unter denen das Kapitalstockverfahren die Rendite des Alterssicherungssystems gegen Altersstrukturveränderungen immunisieren kann. Allerdings kommt es dann zu Veränderungen des Lebenseinkommens. Im Falle des Umlageverfahrens (Mischsystems) kann durch Anpassung der Geschenke die Beitragszahlung konterkariert werden. In Folge sind die abgeleiteten allokativen Ergebnisse - ähnlich wie in der Barro-Welt - beim Kapitalstockverfahren und Umlageverfahren identisch.

Diese These war der Auslöser einer regen wissenschaftlichen Debatte. ${ }^{116}$ Als Einwand wurde u.a. auf einen kurzfristigen Planungshorizont und die Unvollkommenheit des Kapitalmarkts verwiesen.

Vor allem konzentrierte sich die Diskussion auf die verschiedenen (altruistischen oder egoistischen) Erbschaftsmotive, sowie die Möglichkeit von ungeplanten Erbschaften. ${ }^{117}$

Barro modellierte die Transfers als altruistische Erbschaften, indem er den Nutzen, den die Kinder aus dem Erbe ziehen, in die Zielfunktion der Eltern integrierte und somit einen unendlichen Regress konstruierte. Sind die Erbschaften hingegen egoistisch (bzw. ungeplant), so gehen sie als absolute Größe in die Zielfunktion der Eltern ein (bzw. finden keine Berücksichtigung). Kapitalstockverfahren und Umlageverfahren wären in dem Fall nicht länger äquivalent.

Die Diskussion um mögliche Erbschaften läßt sich dahingehend resümieren, daß die von BARRO postulierte Neutralitätstheorie in ihrer radikalen Form nicht bestätigt werden kann. Die Erweiterungen um Erbschaften steigert aber dennoch den Realitätsgehalt der Modelle und schwächt das Ausmaß der abgeleiteten allokativen und distributiven Effekte.

\footnotetext{
115 Vgl. Jaeger (1994).

116 Diese spiegelte sich nicht nur in der Barro-Feldstein-Debatte wider. Barro (1974, 1976, 1978), Feldstein (1974, 1983). Vgl. auch Becker (1974), Sheshinsky/Weiss (1981), Carmichael (1982), Aaron (1982), Burbige (1983), Kohli (1987), Bösch (1987), Berthold/Külp (1987), Homburg (1988), Blümle (1990).

117 Vgl. auch Abschnitt 6.1.1.
} 


\subsection{Zusammenfassung und Fazit}

Zu Beginn des Kapitels wurden zunächst die Verfahren beschrieben, mit denen ein Alterssicherungssystem finanziert werden kann. Die für diese Arbeit wesentlichen, beitragsfinanzierten Verfahren lassen sich einteilen in Umlageverfahren, Kapitalstockverfahren und als Mischform das Anwartschaftsdeckungsverfahren. Eine weitere Differenzierung der beitragsfinanzierten Verfahren erfolgt durch die Gestaltung der Rentenformel. Im wesentlichen müssen hier zwei Entscheidungen getroffen werden:

Die Gestaltung als beitrags- oder teilhabeäquivalentes System bestimmt die Rentenstruktur. Die Entscheidung für konstante Beiträge (bzw. Beitragssätze) oder fixierte Rentenleistungen (bzw. fixiertes Rentenniveau) legt die Rentenhöhe fest.

In einem weiteren Schritt wurde untersucht, inwieweit die verschiedenen Kombinationsmöglichkeiten der Rentenformel mit den Finanzierungsalternativen kompatibel sind:

- Während das Umlageverfahren sich problemlos mit einer teilhabeäquivalenten Rentenformel - sowohl mit konstanten Beitragssätzen als auch mit konstantem Rentenniveau - vereinbaren läßt, erweist sich eine umlagefinanzierte, streng beitragsäquivalente Alterssicherung als instabiles System.

- Das Kapitalstockverfahren läßt sich nur dann mit einer teilhabeäquivalenten Rentenformel kombinieren, wenn es als Mischform (z.B. als Anwartschaftsdeckungsverfahren) gestaltet ist. Ein beitragsäquivalentes, kapitalfundiertes System mit konstanten Beitragssätzen ist erwartungsgemäß ohne Probleme zu realisieren. Ein beitragsäquivalentes, kapitalfundiertes System mit konstanten Rentenniveau ist hingegen äußerst instabil, da Prognosen über die Entwicklung der Lohn- und Zinssätze, auf denen die Beitragsberechnung beruht, höchst unsicher sind.

Es folgte eine Analyse der allokativen Wirkungen der Finanzierungsverfahren.

Hierzu wurde in Abschnitt 2.3.1 zunächst der Effizienzbegriff definiert. Aus der Sicht des einzelnen Versicherten läßt sich die interne Ertragsrate als Effizienzkriterium formulieren, das - sofern von Wachstumseffekten abgesehen wird - zu identischen Ergebnissen führt, wie die Kriterien des Lebenseinkommens bzw. des Lebensnettotransfers. Ein Alterssicherungssystem erweist sich folglich dann als Paretoeffizient für eine Generation, wenn es kein alternatives System gibt, welches eine höhere interne Ertragsrate bietet. Diese individuelle Perspektive beschränkt sich jedoch auf den Zeithorizont eines Lebenszyklus, aus der gesamtwirtschaftlichen Sicht muß der Zeithorizont weiter gefaßt werden. Demnach ist ein Alterssicherungssystem Paretoeffizient, wenn kein alternatives System existiert, bei dem keine Generation, z.B. hinsichtlich ihres Lebenskonsums, schlechtergestellt und mindestens eine Generation bessergestellt wird. 
Der Effizienzvergleich der Finanzierungsverfahren „Kapitalstockverfahren“ und „Umlageverfahren" wurde zunächst im Modellrahmen einer kleinen offenen Volkswirtschaft geführt.

Für die individuelle Sichtweise wurde die bekannte Aaron-Bedingung hergeleitet, wonach eine kapitalstockbasierte Finanzierung der Alterssicherung immer dann effizienter bzw. rentabler als ein Umlageverfahren ist, wenn die Kombination aus Lohnanstieg und die Entwicklung der Bevölkerung ${ }^{118}$ (Wachstumsfaktor der Lohnsumme) niedriger ist, als der Verzinsungsfaktor des Kapitalstockverfahrens: $\left(1+m_{t}\right)\left(1+g_{t}\right)<\left(1+i_{t}\right)$. Ist der Wachstumsfaktor der Lohnsumme größer als der Verzinsungsfaktor des Kapitalstockverfahrens, so ist das Umlageverfahren effizienter.

Um die gesamtwirtschaftliche Effizienz eines Alterssicherungssystems beurteilen zu können, muß diese Aaron-Bedingung einerseits für sämtliche Generationen geprüft werden und andererseits müssen die Erst- und Letztgenerationen gesondert betrachtet werden. Da die Erstgeneration Transferzahlungen erhält, ohne zuvor Beiträge geleistet zu haben, erzielt sie im Umlageverfahren einen Einführungsgewinn, während die Letztgeneration bei endlichem Zeithorizont - im Umlageverfahren Beiträge zahlt, ohne selbst Rentenleistungen zu erhalten. Durch eine kontinuierliche Anhebung des Beitragssatzes ließen sich ähnliche „Einführungsgewinne" für alle nachfolgenden Generationen generieren (PonziVertrag), so daß das Umlageverfahren immer Paretoeffizient wäre. Sofern ein endlicher Zeithorizont unterstellt wird oder eine obere Schranke für den Beitragssatz existiert, ist diese Möglichkeit jedoch nicht gegeben. Für eine kleinen offene Modellökonomie läßt sich der Effizienzvergleich zwischen den Finanzierungsverfahren daher wie folgt zusammenfassen:

- Bei einem endlichen Betrachtungshorizont wird die Erstgeneration im Umlageverfahren regelmäßig besser, die Letztgeneration jedoch regelmäßig schlechter gestellt als im Kapitalstockverfahren. Keines der beiden Verfahren ist gegenüber dem anderen Pareto-superior, so daß beide effizient sind.

- Bei einem unendlichen Zeithorizont ist ein in alle Zukunft reichender Vergleich der Aaron-Bedingung entscheidend. Das Umlageverfahren ist genau dann gegenüber einem Kapitalstockverfahren Pareto-superior, wenn:

$$
\inf \prod_{t=1}^{\infty} \frac{M_{t} \cdot G_{t}}{R_{t}}>0 .
$$

In dem Fall ist das Umlageverfahren effizient, das Kapitalstockverfahren hingegen ineffizient. Ist die Bedingung nicht erfüllt, ist wiederum keines der beiden Verfahren gegenüber dem anderen Pareto-superior und beide effizient.

Durch die Modellierung als geschlossene Volkswirtschaft werden die verschiedenen ökonomischen Größen (Kapitalstock, Sozialprodukt, Lohn- und Zinsatz) zu endogenen Pa-

118 Genauer die Entwicklung der Beschäftigten (abhängig von der Entwicklung der Bevölkerung, der Erwerbsquote etc.). 
rametern. Dadurch werden Wechselwirkungen zwischen diesen ökonomischen Größen und dem jeweiligen Finanzierungsverfahren zugelassen, die wiederum die Effizienz der Finanzierungsverfahren maßgeblich beeinflussen können.

Der optimale Wachstumspfad ist im Modell dann gegeben, wenn der Zinssatz der Wachstumsrate der Bevölkerung entspricht („Goldene Regel der Kapitalakkumulation“). ${ }^{119}$ Es läßt sich zeigen, daß der gleichgewichtige Wachstumspfad einer Ökonomie bestenfalls zufällig mit dem optimalen Wachstumspfad übereinstimmt, bei dem der Nutzen bzw. die Konsummöglichkeiten aller Wirtschaftssubjekte maximiert werden.

Aus der Analyse können für eine geschlossene Modellökonomie die folgenden Aussagen über die allokativen Wirkungen der Finanzierungsverfahren abgeleitet werden:

- Nur wenn das Wachstum einer Volkswirtschaft im marktwirtschaftlichen Gleichgewicht (zufällig) dem optimalen Pfad gemäß der „Goldenen Regel der Kapitalakkumulation“ folgt, ist das Kapitalstockverfahren effizient, bei dem sich die gesamtwirtschaftliche Ersparnis annahmegemäß nicht ändert. Sonst ist es nicht in der Lage, die bestehende Ineffizienz zu beheben.

- In einer durch eine überkapitalisierte Volkswirtschaft charakterisierten, ineffizienten Situation verringert eine Umlagefinanzierung der Renten die private gesamtwirtschaftliche Kapitalakkumulation. Durch geeignete Wahl der Beiträge kann die Kapitalintensität auf den Wert der "Goldenen Regel“ gehalten werden. In einem solchen Fall ist der Lebensnutzen aller Generationen durch die Einführung der Umlagefinanzierung größer als im reinen Kapitalstockverfahren.

Die erforderlichen Beitragssätze und das Versorgungsniveau werden dabei durch den Grad der Überkapitalisierung bestimmt. Bei der Einführung eines reinen Umlageverfahrens nach Maßgabe eines höheren, präferierten Versorgungsniveaus würde die Ineffizienz umschlagen in eine unterkapitalisierte Volkswirtschaft. Wäre der Anteil der Umlagefinanzierung andererseits zu niedrig, würde die Überkapitalisierung lediglich verringert werden.

Hieraus läßt sich folgern, daß eine optimale Alterssicherung als Mischsystem der Finanzierungsverfahren konzipiert sein sollte, welches flexibel an veränderte Rahmengrößen angepaßt wird.

- Ist die Ineffizienz des marktwirtschaftlichen Gleichgewichts durch eine unterkapitalisierte Volkswirtschaft gekennzeichnet, kann der optimale Wachstumspfad nicht durch das Deckungskapital des reinen Kapitalstockverfahren erreicht werden, vielmehr muß das staatliche Rentensystem mehr Kapital halten, als zur Deckung der Rentenansprüche erforderlich ist.

119 Unter Verwendung einer Produktionsfunktion mit arbeitsvermehrenden, sprich Harrod-neutralem technischen Fortschritt $\left(Y_{t}=F\left[K(t), G^{1 *} L(t)\right]\right.$ folgt für die obigen Bedingungen $R=G * M$. Dadurch wird der Bezug zwischen der "Goldenen Regel der Kapitalakkumulation" und der "Aaron-Gleichung" deutlich. 
Die Frage nach der Effizienz der Finanzierungsverfahren ist für die intergenerative Gerechtigkeit eines Alterssicherungssystems bzw. eines Reformvorschlags von entscheidender Bedeutung. Von ihr hängt $a b$, ob sich eine Substitution des Umlageverfahrens durch das Kapitalstockverfahren Pareto-optimal realisieren läßt, also, ob eine Umstellung möglich ist, ohne daß eine Generation schlechter; mindestens eine Generation jedoch besser gestellt wird.

Ein Überblick über die bisherigen Arbeiten zu dieser Frage zeigte jedoch, daß sich eine (partielle) Substitution des Umlage- durch das Kapitalstockverfahren bislang weder im Modell einer offenen noch einer geschlossenen Volkswirtschaft Pareto-optimal gestalten läßt. Das Ergebnis der Effizienzanalyse zeigt, daß die Generationen allein durch die Umsetzung einer solchen Reform unterschiedlich belastet werden und dies zwangsläufig die intergenerative Gerechtigkeit beeinflußt.

In Abschnitt 2.4 wurden anschließend die distributiven Wirkungen der Finanzierungsverfahren untersucht.

Die Analyse der allokativen Eigenschaften der Finanzierungsverfahren sollte Erkenntnisse darüber liefern, welche Wirkungen von den Wechsel des Finanzierungsverfahrens auf die intergenerative Gerechtigkeit ausgehen. Im Gegensatz dazu wurde in der Analyse der distributiven Eigenschaften danach gefragt, ob die Finanzierungsverfahren innerhalb eines bereits eingeführten Alterssicherungssystems systematische, intergenerative Verteilungseffekte hervorrufen.

Die Untersuchung erfolgte als Partialanalyse, d.h. es wurde untersucht, wie sich das Verteilungskriterium (in diesem Fall die Rendite einer Generation) verändert, wenn ceteris paribus ein System-Parameter variiert wird. Die variierten demographischen und ökonomischen Parameter waren die Sterblichkeit (während der Erwerbsphase und während der Ruhephase), die Fertilität, die Erwerbsbeteiligung, die Lohnentwicklung und der Zinssatz. Um sämtliche relevanten Effekte abbilden zu können, wurde das Grundmodell auf ein drei-Perioden-Modell enweitert.

Da die Wirkungen auf die generationsspezifischen Renditen sich beim Umlageverfahren in einer kleinen offenen Volkswirtschaft nicht für alle Parametervariationen auf analytischen Wege bestimmen bzw. nur unter vereinfachenden Annahmen ableiten lassen, wurden tendenzielle Wirkungen anhand von Modellrechnungen aufgezeigt. Die Ergebnisse sind in Tabelle 2 auf Seite 59 zusammengefaßt.

Die Modellrechnung zeigte, daß eine Generation die Umverteilungslast, die durch eine Variation der Sterblichkeit und der Erwerbsbeteiligung ausgelöst wurde, solange auf andere Generationen überwälzen kann, bis die Größe auf ihr ursprüngliches Niveau zurückgeht. Fertilität, Lohnentwicklung und Zinsfaktor unterscheiden sich in ihren Verteilungswirkungen von den beiden anderen Parametern, da sie Zinskomponenten des Umlage- bzw. des Kapitalstockverfahrens darstellen und damit auf alle Generationen gleichermaßen wirken. 
In dem Modellrahmen einer kleinen offenen Volkswirtschaft konnten intergenerative Verteilungswirkungen beim Kapitalstockverfahren nur aufgrund eines exogenen Effektes, nämlich der Veränderung des Zinssatzes, auftreten. In einer geschlossenen Volkswirtschaft korreliert der Zinssatz positiv mit der Fertilität, der Überlebenswahrscheinlichkeit während der Erwerbsphase und dem technischen Fortschritt (der Produktivität und der Lohnentwicklung), aber negativ mit dem Beitragssatz. Die Überlebenswahrscheinlichkeit während der Ruhestandsphase und die Enwerbsbeteiligung haben keinen Einfluß auf die Verzinsung.

Die Ergebnisse beziehen sich auf ein Kapitalstockverfahren mit konstanten Beitragssätzen. Für ein Kapitalstockverfahren mit konstantem Rentenniveau ${ }^{120}$ mußte die Analyse (in einer geschlossenen Volkswirtschaft) um die Beitragseffekte erweitert werden, da der Beitrag mit der Überlebenswahrscheinlichkeit während der Ruhestandsphase und dem Rentenniveau steigt und bei höherem Zinssatz sinkt. Es zeigte sich, das die interne Ertragsrate des Kapitalstockverfahrens mit steigender Überlebenswahrscheinlichkeit während der Ruhestandsphase und steigendem Kapitalstock sowie sinkender Fertilität sinkt. Die Veränderung des Zinssatzes ist hinsichtlich einer Variation der Überlebenswahrscheinlichkeit während der Erwerbsphase und des technischen Fortschritts aufgrund gegenläufiger Effekte unbestimmt.

Die Ergebnisse der Distributionsanalyse sind für die nachfolgende Untersuchung der Gerechtigkeit von Alterssicherungssystemen deshalb von Bedeutung, weil sie zeigen, daß das Kapitalstockverfahren keineswegs demographieimmun und verteilungsneutral ist. ${ }^{121}$ Außerdem können die Erkenntnisse Grundlagen bilden, um die intergenerative Umverteilung im zu entwickelnden Meßkonzept von dem intergenerativen Risikoausgleich abzugrenzen. ${ }^{122}$

Abschließend wurde der Modellrahmen um den Aspekt der Unsicherheit und um mögliche Erbschaftsmotive erweitert. Es zeigte sich, daß das Umlageverfahren bei Unsicherheit den Erwartungswert des Nutzens steigern und somit für risikoaverse Wirtschaftssubjekte mit zusätzlichen Vorteilen verbunden sein kann.

Die Diskussion um mögliche Erbschaften läßt sich dahingehend resümieren, daß die von BARRO postulierte Neutralitätstheorie in ihrer radikalen Form zwar nicht bestätigt werden kann, die Erweiterungen um Erbschaften aber dennoch den Realitätsgehalt der Modelle steigern und das Ausmaß der abgeleiteten allokativen und distributiven Effekte schwächen können. im Vordergrund, da diese Variante sehr instabil und praktisch schwer zu realisieren ist. 
Abbildung 4: Übersicht Kapitel zwei

\section{FINANZIERUNG DER ALTERSSICHERUNG}

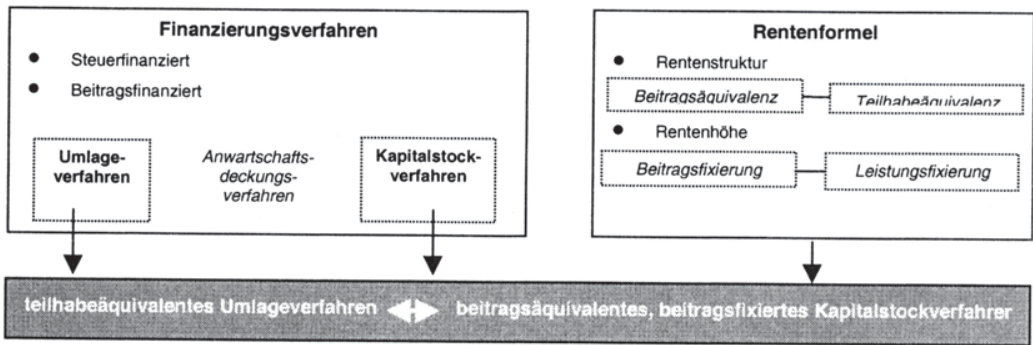

\section{Allokationseffizienz}

A) kleine offene Volkswirtschaft

Für einzelnen Versicherten gilt (Aaronbedingung)

- $\quad \mathbf{R}<\mathbf{M} * \mathbf{G} \rightarrow$ Umlageverfahren effizienter

- $\quad \mathbf{R}>\mathbf{M}^{*} \mathrm{G} \rightarrow$ Kapitalstockverfahren effizienter

Für sămtliche Generationen:
- $\quad$ Aaronbedingung für alle Generationen prüfen
Bei $\infty$-Zeithorizont:
inf $\prod_{\mathrm{t}=1}^{\infty} \frac{\mathrm{M}_{\mathrm{t}} \cdot \mathrm{G}_{\mathrm{t}}}{\mathrm{R}_{\mathrm{t}}}>0 \rightarrow$ Umlageverfahren effizienter (v.v.)
keine Pareto-optimale Substitution des
Umlage-durch das Kapitalstockverfahren

B) geschlossene Volkswirtschaft

optimaler Wachstumspfad $\rightarrow \mathbf{i}=\mathbf{m}$

(Goldene Regel der Kapitalakumulation)

- Kapitalstockverfahren nur zufällig effizient

- Ũberkapitalisierte Volkswirtschaft $\rightarrow$ durch Wah eines geeignete Umlageverfahren $\rightarrow$ effizient

- unterkapitalisierte Volkswirtschaft $\rightarrow$ mehr Kapitalbildung effizient

keine Pareto-optimale Substitution des Umlage- durch das Kapitalstockverfahren

Die Generationen werden allein durch die Umsetzung der Reform unterschiedlich belastet, es kommt zur intergenerativen Verteilung

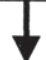

\section{Distributive Effekte}

Veränderung der generationsspezifischen Rendite bei Variation ökonomischer / demographischer Parameter

A) kleine offene Volkswirtschaft (Umlageverfahren)

\section{siehe Tabelle 2}

B) geschlossene Volkswirtschaft (Kapitalstockverfahren)

konstante Beitragssătze:

Zinssatz $=$ Funktion:

+ Fertilităt

+ Überlebenswahrscheinlichkeit Erwerbsphase

+ Lohnwachstum (Produktivităt)

- Beitragssatz

- Überlebenswahrscheinlichkeit Ruhestand

- Erwerbsbeteiligung

konstante Rentenleistungen

Zinssatz = Funktion:

+ Fertilităt

- Überlebenswahrscheinlichkeit Ruhestand

- Kapitalintensităt

- Lohnwachstum (Produktivităt)

Überlebenswahrscheinlichkeit Erwerbsphase

$+=$ positiver Einfluß

$\cdot=$ negativer Einfluß

$0=$ kein Einfluß

= unbestimmter Einfluß

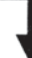

Systematische intergenerative Verteilungen bestehen innerhalb beider Finanzierungsverfahren
- Unsicherheit

- Vererbungsmotiv
Modellerweiterungen:

$\rightarrow \quad$ Bessere Absicherung durch Umlageverfahren

$\rightarrow$

gesamtwirtschaftichen Rückwirkungen des Kapitalstocks

Quelle: Eigene Darstellung 


\section{GERECHTIGKEITSVORSTELLUNGEN}

Die Analyse des vorangehenden Kapitels zeigte, daß Variationen ökonomischer und/oder demographischer Parameter sowohl beim Umlageverfahren als auch beim Kapitalstockverfahren verschiedene Generationen unterschiedlich stark belasten bzw. begünstigen. Darüber hinaus führt die Umstellung des Finanzierungsverfahrens zusätzlich zu intergenerativen Verteilungswirkungen, die bei der Beurteilung einer Reform des Alterssicherungssystems ebenfalls beachtet werden müssen.

Für die Sozialpolitik ist es wenig hilfreich zu wissen, daß jedes Alterssicherungssystem intergenerative Verteilungswirkungen erzeugt. Vielmehr müssen die intergenerativen Verteilungswirkungen der Reformmaßnahme anhand eines normativen Maßstabs bewertet werden, um sozialpolitische Empfehlungen abzuleiten. Im folgenden Kapitel werden die möglichen Wertmaßstäbe diskutiert, mit denen die Verteilungswirkungen bewertet, sprich als gerecht oder ungerecht klassifiziert werden.

\subsection{Gerechtigkeit und Ökonomie}

Zunächst ist es eine grundsätzliche Entscheidung, ob normative Fragen der Gerechtigkeit in der ökonomischen Wissenschaft überhaupt gestellt werden sollten. Der wohl bekannteste Ökonom, der diese Frage verneint, ist HAYEK:

"Der Ausdruck soziale Gerechtigkeit gehört nicht in die Kategorie des Irrtums, sondern in die des Unsinns, wie der Ausdruck 'ein moralischer Stein' ‘23

Die Begründung, mit der HAYEK seine fundamentale Kritik am Begriff der Gerechtigkeit untermauert, läßt sich wie folgt wiedergeben: Da es in der Gesellschaft keinen Akteur gibt, der eine bestimmte Einkommensverteilung absichtlich herbeiführt und folglich niemand für die Verteilung verantwortlich gemacht werden kann, verbietet es sich, die Einkommensverteilung moralisch als "gerecht" oder "ungerecht" zu kritisieren. ${ }^{124}$ BARRY weist jedoch darauf hin, daß die dieser Argumentation zugrunde liegende Prämisse keineswegs schlüssig ist, denn allein aufgrund der Tatsache, daß

"Verteilungsresultate die kumulierte Wirkung von Handlungen sind, die seitens der Akteure nicht in der Absicht unternommen wurden, diese Resultate zu erzeugen",

läßt sich nicht begründen, daß das Gesamtresultat moralisch nicht bewertet werden kann. ${ }^{125}$ Als Beispiel dafür, daß diese These nicht haltbar ist, nennt er das ökonomische Problem der "tragedy of commons", bei dem die

\footnotetext{
123 Hayek (1981), S. 112.

124 Vgl. Hayek (1981), S. 100ff, insbesondere S. 114: „Gerechtigkeitserwägungen sind im Hinblick auf die Bestimmung einer Größe, die nicht von irgendjemandes Wille oder Wunsch, sondern von Umständen abhängt, die niemand in ihrer Gesamtheit kennt, schlicht sinnlos".

Vgl. Barry (1995), S. 237/238.
} 
„unumwundene, nichtstrategische Interessenverfolgung jedes Einzelnen zu einem Ergebnis führt, das für alle schlechter ist als das, was hätte erreicht werden können, wenn man auf andere Weise gehandelt hätte. ${ }^{426}$

Das Ergebnis kann daher gerade aufgrund der mangelnden Regeln kritisiert werden, unter denen es zustande kam. BARRY führt weiter aus, daß HAYEK sich zwar kategorisch gegen jede moralische Bewertung einer Einkommensverteilung wendet, seine Argumente sich aber vornehmlich gegen ein bestimmtes Konzept sozialer Gerechtigkeit richten, demzufolge das Einkommen den individuellen Verdiensten entsprechen müsse. ${ }^{127}$ Denn HAYEK formuliert selbst eine Form von Gerechtigkeitskonzept, wenn er schreibt:

„Die gute Gesellschaft ist eine Gesellschaft, in der es wahrscheinlich ist, daß die Chancen jeder beliebigen Person so groß wie möglich sind. ${ }^{\text {“28 }}$

HAYEK stimmt zudem dem Prozeduralismus zu, wonach Institutionen der vorrangige Gegenstand moralischer Bewertung sein sollen, so daß „gerechte Institutionen“ keine ungerechten Resultate hervorbringen können. ${ }^{129}$ HAYEKS fundamentale Kritik an dem Begriff der „sozialen Gerechtigkeit" erweist sich daher bei näherer Betrachtung als Kritik an dem speziellen Konzept der Verdienstgerechtigkeit und daraus abgeleitet als Plädoyer für ein Konzept der Regelgerechtigkeit. Eine Übersicht über die verschiedenen in der Literatur diskutierten Gerechtigkeitsgrundsätze gibt der folgende Abschnitt. ${ }^{130}$

\subsection{Ansätze zur Beschreibung der Gerechtigkeit}

Wenngleich die extreme Position HAYEKS, sämtliche Gerechtigkeitsüberlegungen in der Ökonomie als unsinnig abzulehnen, angesichts der vorangegangenen Argumentation schwer nachvoliziehbar ist, bleibt es dennoch äußerst schwierig, den Begriff der „sozialen Gerechtigkeit" umfassend und präzise zu definieren. ${ }^{131}$ Die Vorstellungen darüber, was unter dem Begriff der „Gerechtigkeit“ zu verstehen ist, differieren zum Teil erheblich ${ }^{132}$ und entsprechend vielfältig sind die in der Literatur zu findenden Gerechtigkeitsgrundsätze.

\footnotetext{
126 Barry (1995), S. 238. Vgl. auch S. 241.

127 Vgl. Barry (1995), S. 235.

128 Hayek (1981), S. 178).

129 Vgl. Barry (1995), S. 240.

130 Vgl. nachfolgend Liefmann-Keil (1961); Tillmann (1991); Althammer (1991); Lampert (1990a); Zacher (1977); Döring (1995); Plant u.a. (1980); Frerich (1987); Linde (1995); Perelman (1967).

131 Vgl. auch Lampert (1990a), S. 76, der weiter ausführt, ,...daß von Seiten der Őkonomen eine allgemeinverbindliche, inhaltlich konkretisierte Festlegung der Gerechtigkeit als Beurteilungsnorm nicht vorgenommen werden kann, wie bereits 1955 Seraphim dargelegt hat." (Seraphim, H. J., "Theorie der allgemeinen Volkswirtschaftspolitik, Göttingen 1955, S. 247). 


\subsubsection{Ausgewählte Gerechtigkeitsgrundsätze}

Gemeinsam ist den meisten Grundsätzen und Konzepten lediglich die Überzeugung, daß Gerechtigkeit etwas mit Gleichheit bzw. Gleichbehandlung zu tun haben muß. ${ }^{133}$ Worauf sich diese Gleichheit allerdings beziehen soll, und welcher Grad an Gleichheit anzustreben ist, wird unterschiedlich beurteilt.

\section{Ergebnisgerechtigkeit}

Ein möglicher, an der Gleichheit anknüpfender Maßstab ist die Ergebnisgerechtigkeit. ${ }^{134}$ "Gerechtigkeit" ist entsprechend dieses Konzeptes dann gewährleistet, wenn alle Individuen den gleichen Anspruch auf die Ergebnisse des gesellschaftlichen Produktionsprozesses haben. ${ }^{135}$ Dieses Konzept entspricht weitgehend auch der Gerechtigkeitsvorstellung, die DörING als absolute Gleichheit bezeichnet und die darauf abzielt,

„daß zwischen den Mitgliedern einer Gesellschaft keine Unterschiede hinsichtlich ihrer Rechte, Pflichten, ihrer sozialen und ökonomischen Stellung bestehen sollen". ${ }^{136}$

Diese Definitionen verdeutlichen zwar die grundlegende Philosophie dieses Gerechtigkeitsgrundsatzes, nämlich die Gleichheit auf die Ergebnisse des Produktions- bzw. Verteilungsprozesses zu beziehen, zwei wesentliche Fragen bleiben aber dennoch unbeantwortet:

1. Die Definitionen lassen offen, woran der "gleiche Anspruch auf die Ergebnisse des gesellschaftlichen Produktionsprozesses" bzw. die "gleiche sozialen und ökonomischen Stellung" anknüpfen sollen. Meist wird bei der Gleichheit der Ergebnisse auf das Einkommen abgestellt ${ }^{137}$; denkbar wäre es aber auch, die Gleichheit auf die Güterzuteilung, die Lebenslagen, die absoluten Nutzen oder die Grenznutzen zu beziehen. $^{138}$

2. Döring weist ferner darauf hin,

„... daß es ein entscheidendes Argument gegen eine konsequente Realisierung" der absoluten Gleichheit "gibt, das kaum zu entkräften ist: Das Ausmaß der enforderlichen staatlichen Intervention für die Durchsetzung und Erhaltung einer Situation völliger Gleichheit ge(1958), Schlotter (1981), S. 4.

Dies entspricht in etwa dem Grundsatz, den Lampert als "Gleichheit materialer Freiheit" bezeichnet. Vgl. Lampert (1990a), S. 78. Vgl. Linde (1995), S. 859.

136 Döring (1995), S. 69. Aufgrund der Forderung nach gleichen Rechten und Pflichten bezieht das Konzept

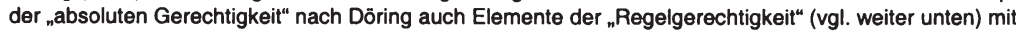
ein und ist somit im Prinzip umfassender als das Konzept der "Ergebnisgerechtigkeit". 
riete in Widerspruch zu den Voraussetzungen einer funktionierenden Marktwirtschaft ..... 139

Daraus folgt, daß hinsichtlich der absoluten Gleichheit (bzw. Ergebnisgleichheit) zwischen einer (idealen) „radikalen“ Version, die eher als theoretischer Bezugspunkt zu interpretieren ist, und einer „gemäßigten“ Version unterschieden werden muß. ${ }^{140}$ Die für die praktische Sozialpolitik relevante „gemäßigte“ Version zielt darauf $a b$, daß ein bestimmtes $\mathrm{Maß}$ an Ungleichheit nicht überschritten werden sollte, wobei sogleich die Frage aufgeworfen wird, wo dieses Maß angesetzt werden soll. ${ }^{141}$

\section{Leistungs- bzw. Verdienstgerechtigkeit}

Im Gegensatz zur Ergebnisgerechtigkeit wird nach dem Grundsatz der Leistungsgerechtigkeit der Zustand bzw. Prozeß als "gerecht" angesehen, bei dem jedes Individuum seine angeborenen und/oder erlernten Fähigkeiten für die Gesellschaft einsetzt und dafür eine äquivalente Gegenleistung erhält. ${ }^{142}$ Bei diesem, bereits bei Aristoteles anzutreffenden Gerechtigkeitskonzept wird eine bestehende Ungleichheit absoluter Art folglich dann als gerecht betrachtet, wenn sie das Ergebnis von Unterschieden in der Leistung ist. ${ }^{143}$ Charakteristisch für die Leistungsgerechtigkeit ist zudem, daß sie dem Individuum einen Anreiz gibt, wirtschaftliche Leistungen zu erbringen.

Allerdings lassen sich die verschiedenen Leistungen als Beitrag zum Sozialprodukt z.T. nur schwer miteinander vergleichen. ${ }^{144}$ Darüber hinaus resultiert eine bestimmte Leistung in der Regel aus dem Zusammenspiel verschiedener Faktoren, so daß sich der Beitrag der individuellen Leistung - wenn überhaupt - nur schwer isolieren läßt. ${ }^{145}$ Innerhalb dieses Gerechtigkeitskonzeptes stellt sich das Problem, wie die Leistung zu messen und wie sie dem Individuum zuzuordnen ist. Oftmals wird daher aus Gründen der Praktikabilität (explizit oder implizit) davon ausgegangen, daß die aus dem Marktprozeß resultierende Verteilung als leistungsgerecht anzusehen ist. ${ }^{146}$ Diese Vorgehensweise wird jedoch zurecht kritisiert, denn es ist ein Fehler anzunehmen, die Markteinkommen ließen sich allein durch die erbrachten Leistungen rechtfertigen. Vielmehr gilt, daß „der Erlös der Anstrengungen

139 Döring (1995), S. 70. Döring betont ferner, daß eine konsequente Realisierung des absoluten Gleichheitsgedankens vermutlich auch im Widerspruch gegen die Leitvorstellungen einer offenen Gesellschaft und gegen den Bestand an individuellen Rechten stehen würde. Vgl. auch Lampert (1990a), S. 77/78. Lampert (1990a), S. 78 weist daraus hin, daß dieses Problem seit einigen Jahren heftig unter dem Stichwort der "Grenzen des Sozialstaats" diskutiert wird. Vgl. hierzu auch Zimmermann (1996).

141 Ein mögliches Maß zur Begrenzung der Ungleichheit kann beispielsweise sein, daß das Einkommen keines Individuums unter ein (ebenfalls zu definierendes) Existenzminimum fallen soll.

142 Vgl. Linde (1995), S. 859.

143 Vgl. Döring (1995), S. 72. Döring bezeichnet dieses Verstăndnis des Gleichheitsgedanken im Gegensatz zur zuvor beschriebenen absoluten Gleichheit als relative Gleichheit . Neben dem Kriterium der Leistung läßt sich die relative Gleichheit auch nach Maßgabe des Bedarfs bestimmen.

144 Wie soll beispielsweise die Leistung eines Arztes im Vergleich zu der eines Politikers bewertet werden?

145 Vgl. Döring (1995), S.72.

146 So z.B. bei Lampert (1990a), S. 80. Vgl. auch Döring (1995), S. 72.

80 
der Leute nicht mit dem erkennbaren Verdienst korrespondiert ${ }^{147}$ RAWLS betont, daß der Begriff des persönlichen Verdienstes auf ökonomische Resultate nicht angewandt werden kann, da der Zufall und das Glück einen erheblichen Einfluß auf die Marktergebnisse ausübt.

Wird das Prinzip der Leistungsgerechtigkeit nicht auf die Zuteilung von „Rechten und Leistungen“ sondern von „Pflichten und Kosten“ angewandt, findet es seine Entsprechung im sogenannten Verursachungsprinzip.

\section{$\underline{\text { Regelgerechtigkeit }}$}

Regelgerechtigkeit liegt dann vor, wenn für jedes Individuum im Wirtschaftsprozeß die gleichen Regeln und Gesetze gelten. ${ }^{148}$ Wie bereits erwähnt, zieht HAYEK aus dieser Kritik an der Leistungsmessung und -zuordnung den Schluß, daß das Konzept der Regelgerechtigkeit dem der Verdienstgerechtigkeit vorzuziehen ist. Ein bestimmtes Verteilungsresultat kann demzufolge moralisch nicht kritisiert werden, die Rechtfertigung kann nur für die Ausgestaltung der Institutionen und Regeln verlangt werden, die zu diesem Ergebnis geführt haben.

Dieser Gerechtigkeitsgrundsatz zielt auf die Gleichheit von Rechten und Pflichten jedes Bürgers, weshalb er gelegentlich auch als "formale Gerechtigkeit" charakterisiert wird. Mit der Bezeichnung „formale Gerechtigkeit" soll das Prinzip von der "materiellen Gerechtigkeit“ abgrenzt werden, bei der die tatsächlichen wirtschaftlichen Verhältnisse im Mittelpunkt stehen. Zur „materiellen Gerechtigkeit" gehören die Grundsätze der Leistungs- und Ergebnisgerechtigkeit sowie der Start- und Bedarfsgerechtigkeit, die im folgenden vorgestellt werden. ${ }^{149}$

Diese Kategorisierung in formale und materielle Gerechtigkeit wird treffender als Verfahrens- und Einzelfallgerechtigkeit bezeichnet.

\section{$\underline{\text { Startgerechtigkeit }}$}

$\mathrm{Da} ß$ die aus dem Marktprozeß resultierenden Verteilungsergebnisse nicht den persönlichen Verdienst widerspiegeln, ergibt sich neben des Einflusses von „Glück und Zufall“ auch aus der unterschiedlichen Anfangsausstattung an Ressourcen, mit denen die Wirtschaftssubjekte in den Wirtschaftsprozeß treten. Aus diesem Zusammenhang läßt sich das Konzept der Startgerechtigkeit ableiten, demzufolge jedes Individuum beim Eintritt in das Wirtschaftsleben mit den gleichen bzw. mit gleichwertigen Fähigkeiten und Vermögen ausgestattet sein sollte. ${ }^{150} \mathrm{Da}$ in der Realität die Erstausstattung jedes Individuums an Ressourcen unterschiedlich ist, würde dieses Konzept auf eine Umverteilung der Res-

\footnotetext{
147 Hayek (1981), S. 104. Vgl. beispielsweise auch Rawls: „Die Gerechtigkeitsgrundsätze ... erwähnen das moralische Verdienst nicht, und die Verteilung richtet sich keineswegs nach ihm. "Rawls (1975), S. 345.

150 Vgl. Linde (1995), S. 859.
} 
sourcen hinauslaufen, die einer Enteignung gleichkäme. ${ }^{151}$ Vertreter des sogenannten „asset egalitarianism“152 halten dem entgegen, daß nicht das Individuum der Eigentümer der in seinem Besitz befindlichen Ressourcen sei, sondern die Gesellschaft, von der das Individuum seine Ressourcen (z.B. Ausbildung) erhält. ${ }^{153}$ Hinsichtlich der naturgegebenen Erstausstattung an Ressourcen argumentieren Anhänger des „asset egalitarianism“ ferner, daß sie aufgrund ihrer zufallsbedingten Verteilung nicht als gerecht angesehen werden kann. ${ }^{154}$

Die Startgerechtigkeit beschränkt sich jedoch nicht auf die materielle Ausgangssituation. Wesentlich bedeutender für die Gewährleistung der Chancengleichheit ist die Schaffung gleicher Rechte.

\section{Bedarfsgerechtigkeit}

Die Bedarfsgerechtigkeit wird von DóRING (zusammen mit der Leistungsgerechtigkeit) der Kategorie der auf einer relativen Gleichheit beruhenden Gerechtigkeitsgrundsätze zugeordnet. Sie spielt im Gesundheitswesen sowie im Pflegebereich und vor allem in der Sozialhilfe eine wichtige Rolle. ${ }^{155}$ In den anderen Bereichen der Gesellschaft ist dieser Gerechtigkeitsgrundsatz, sofern auf die individuellen Bedürfnisse abgestellt wird, nur schwer exekutierbar. Denn einerseits stellt sich das Problem, wie die individuellen Bedürfnisse erfaßt werden können und andererseits ist es angesichts begrenzter Ressourcen nicht möglich, sämtliche Bedürfnisse aller Individuen zu befriedigen. ${ }^{156}$ Als Maßstab der Bedarfsgerechtigkeit werden daher regelmäßig lediglich durchschnittliche, gesellschaftlich anerkannte Bedarfe bzw. Mindestbedarfe angewandt. In diesem Fall ähnelt das Gerechtigkeitskonzept dem absoluten Gleichheitsgedanken in gemäßigter Form. ${ }^{157}$

\section{Wirtschaftliche Effizienz}

Bei der Diskussion der Ergebnisgerechtigkeit klang bereits an, daß diese im Widerspruch zum marktwirtschaftlichen Prinzip stehen könne, während die Leistungsgerechtigkeit einen Anreiz zur wirtschaftlichen Leistung gibt. Da das Individuum durch seine Leistung zu einem wachsenden Sozialprodukt beiträgt, von dem nicht nur es, sondern alle Gesellschaftsmitglieder profitieren, wird hiermit eine weitere Dimension der Gerechtigkeitsvorstellung angesprochen. Diese manifestiert sich darin, daß eine Ungleichheit auf hohem

\footnotetext{
151 Wenn dies bei natürlichen Ressourcen (z.B. Schönheit, Begabung) nicht möglich ist, würde dies zumindest eine Umverteilung der mit diesen Ressourcen erwirtschafteten Ergebnisse erfordern.

152 Vgl. Arrow (1973), S. 248.

153 Vgl. Linde (1995), S. 864. Linde betont weiter, daß diese These nur insoweit gelten kōnne, als das Individuum für die Ressource nicht bezahlt hat (monetär aber auch in Form von Anstrengung und Verzicht). Vgl. Rawls (1975), S. 93; Linde (1995), S. 864.

155 Vgl. Lampert (1990a), S. 84/85.

156 Vgl. Lampert (1990a), S. 77.

157 Vgl. Döring (1995), S. 73, siehe auch die dritte Variante der Bedarfsgerechtigkeit bei Lampert (1990a), S. $77 / 78$.
} 
Niveau als "gerechter" empfunden wird, als eine Gleichheit auf niedrigerem Niveau. ${ }^{158}$ Eine umfassende Analyse zur "Gerechtigkeit" verschiedener Systeme oder Maßnahmen kann folglich nicht auf distributive Aspekte begrenzt sein, sondern muß allokative Gesichtspunkte ebenfalls in die Betrachtung einbeziehen. „Wirtschaftliche Effizienz" sollte folglich nicht als Gegenstück sondern als ein wesentliches Kriterium der sozialen Gerechtigkeit interpretiert werden.

\subsubsection{Beziehungen der Gerechtigkeitsgrundsätze zueinander}

Die verschiedenen Gerechtigkeitsgrundsätze können miteinander konfligieren, so daß die Wahl eines bestimmten Grundsatzes, mit dem die Wirkungen eines Systems oder einer Maßnahme analysiert werden soll, zugleich auch das Ergebnis der Untersuchung präjudiziert:

- Besonders offensichtlich ist dieser Konflikt beim Verhältnis zwischen der Leistungsund Bedarfsgerechtigkeit.

- Selbst unter der unrealistischen Annahme, daß eine vollkommene Leistungs-, Startund Regelgerechtigkeit gegeben wäre, ständen diese drei Gerechtigkeitskonzepte nur dann im Einklang mit der Ergebnisgerechtigkeit, wenn sowohl die freie Willensentscheidung des Individuums als auch der Einfluß von Glück und Zufall auf das Leistungsergebnis ausgeblendet wird. ${ }^{159}$

- Auf den Konflikt zwischen der Ergebnisgerechtigkeit und der marktwirtschaftlichen Effizienz wurde bereits hingewiesen.

Ein trade-off kann auch zwischen solchen Gerechtigkeitsgrundsätzen bestehen, die scheinbar komplementär zueinander sind. Bedenkt man, daß die Marktresultate keineswegs nur die erbrachten Leistungen widerspiegeln, so wäre entsprechend der Verteilungsgerechtigkeit eine Korrektur der marktmäßigen Einkommensverteilung gerechtfertigt. Vom Grundsatz der martwirtschaftlichen Effizienz ließe sich dagegen einwenden, daß ein Eingriff in die Marktergebnisse die marktwirtschaftliche Dynamik aushöhle. ${ }^{160}$

Auch diesbezüglich müßte entsprechend des gesellschaftlichen Konsenses ein Kompromiß gefunden werden. Ein Beispiel, bei dem der Marktmechanismus mit der Verdienstgerechtigkeit kombiniert wird, ist die progressive Einkommensbesteuerung, denn

„je höher wir die Einkommensleiter hinaufklettern, desto mehr entspringen die Einkommen entweder Erbschaften, ökonomischen Renten aufgrund seltener natürlicher Talente, der Macht, sein eigenes Einkommen festzulegen, [...], oder schierem Glück. Nichts davon hat mit

Dieser Gedanke spielt vor allem bei Fragen der intergenerativen Gerechtigkeit eine Rolle, da frühere Generationen durch ihren Einfluß auf das Wachstum der Volkswirtschaft die Möglichkeiten nachfolgender Generationen bestimmen. Ein Sachverhalt, der sich beispielsweise auch in dem von Rawls aufgestellten Spargrundsatz widerspiegelt (vgl. Abschnitt 3.3.2).

160

Vgl. Barry (1995), S. 242, der sich dabei auch auf das "umfassende Rawlsche Konzept sozialer Gerechtigkeit" bezieht. Barry weist ferner darauf hin, daß sich in der Realităt keine Option in der reinen Form finden lāßt. Als Beispiel führt er die Gestaltung von Arbeitsverträgen an, in die sowohl Verdienstaspekte einfließen als auch die Verhältnisse des Arbeitsmarktes. 
persönlichem Verdienst zu tun. Ein System progressiver Einkommensbesteuerung, das ab dem Punkt steil nach oben weist, ab dem die Einkommen weitgehend solchen Quellen entspringen, würde ein gewisses $M a ß$ an verdienstorientierter Gerechtigkeit verwirklichen und gleichzeitig jede kollektive Beurteilung individueller Einkommen vermeiden. “161

Die dieser Kombination zugrundeliegende normative Vorstellung wird auch als Leistungsfähigkeitsprinzip bezeichnet, das nicht nur in der Steuerlehre, sondern auch in verschiedenen Bereichen der Sozialversicherung (insbesondere in der gesetzlichen Krankenversicherung) zum Tragen kommt. ${ }^{162}$

Das Resümee, das eingangs bereits in Bezug auf die Ergebnisgerechtigkeit gezogen wurde, kann auf sämtliche andere Gerechtigkeitsgrundsätze übertragen werden:

Die z.T. konfligierenden Beziehungen zwischen den Gerechtigkeitskriterien (inkl. dem der wirtschaftlichen Effizienz) erfordern, Kompromisse einzugehen. Für die praktische Sozialpolitik sind daher regelmäßig die „gemäßigten Versionen“ der Gerechtigkeitsgrundsätze relevant.

In den Vordergrund tritt die Frage, welche Gerechtigkeitsgrundsätze man angesichts der z.T. konfligierenden Beziehungen zur Bewertung eines Alterssicherungssystems heranziehen soll und bis zu welchem Maße die Gleichheit bzw. Ungleichheit hinsichtlich die verschiedenen Konzepte erwünscht bzw. toleriert werden. Hierzu bieten sich grundsätzlich zwei verschiedene Vorgehensweisen an:

1. Als Referenz werden die gewachsenen gesellschaftlichen Konventionen herangezogen.

2. Es wird danach gefragt, ob sich ein allgemeingültiges und ethisch fundiertes Gerechtigkeitskonzept formulieren läßt, anhand dessen die Gerechtigkeitsgrundsätze ausgewählt werden können.

Ad. 1)

Welche Gerechtigkeitsgrundsätze angesichts der dargestellten Widersprüche auf eine konkrete Problemstellung anzuwenden sind bzw. wie die verschiedenen Grundsätze gewichtet werden sollten, läßt sich - so der Grundgedanke der ersten Vorgehensweise - auf analytischen Wege nicht entscheiden. ${ }^{163}$ Jede Gerechtigkeitsvorstellung ist vielmehr gesellschaftlich definiert bzw. historisch gewachsen. Sie ist zudem keinesfalls als konstant anzusehen, da sich der gesellschaftliche Konsens darüber, was als gerecht zu gelten hat, im Laufe der Zeit stark ändern kann. ${ }^{164}$

161 Barry (1995), S. 243.

162 Auf die methodischen Probleme, wie aufgrund dieses Prinzips eine Tarifgestaltung abgeleitet werden kann, wird an dieser Stelle nicht eingegangen.

163 Dies betont auch Liefmann-Keil (1961) wenn sie ausführt, daß Gerechtigkeit sich nicht aus einem übergeordneten, allgemeinen Maßstab ableiten läßt, sie vielmehr selbst als letzter Maßstab zu betrachten ist.

84 
Wenn die Gerechtigkeitsvorstellung sich vornehmlich im politisch-gesellschaftlichen Entscheidungsprozeß herausbildet, hat dies Konsequenzen für die Methode der Gerechtigkeitsanalyse. Es wäre daher naheliegend, diejenigen Gerechtigkeitsgrundsätze herauszuarbeiten, auf die sich die Gestaltung des derzeitigen Systems der gesetzlichen Rentenversicherung in Deutschland gründet. Anhand dieser (vorgegebenen) Gerechtigkeitsmaßstäbe könnten dann die verschiedenen Optionen zur Reform der Alterssicherung bewertet werden. Es müßten folglich zunächst die möglichen Gestaltungsprinzipien des Alterssicherungssystems beschrieben und dahingehend untersucht werden, inwieweit sie auf den verschiedenen Gerechtigkeitsgrundsätzen basieren. Ferner muß entschieden werden, in welcher Weise und in welchem Umfang sie in die Formulierung eines Meßkonzepts zur Bestimmung der intergenerativen Gerechtigkeit einfließen sollten. ${ }^{165}$

Entsprechend dieses „rechtsethischen Relativismus“ lassen sich keine zeitinvarianten und objektiven Gerechtigkeitsgrundsätze formulieren. ${ }^{166}$ Es ließe sich gegen diese Vorgehensweise einwenden, daß im Extremfall jedes existierende Alterssicherungssystem a priori gerecht wäre, solange es sich nur im demokratischen Abstimmungsprozeß bildet. Diese Interpretation entspräche einer Rechtfertigung über die Regelgerechtigkeit, bei der die demokratische Abstimmung die (sehr abstrakte) gerechte Regel darstellt. Wenn jedoch die Regelgerechtigkeit als ein Gerechtigkeitsgrundsatz darüber zu entscheiden hat, welche Gerechtigkeitsgrundsätze relevant sind, so besteht der Verdacht eines Zirkelschlusses.

Dieser Einwand gegen die erste Vorgehensweise zur Auswahl und Gewichtung der Gerechtigkeitsgrundsätze richtet sich dagegen, ein Systems, eine Institution, oder eine Vorschrift als gerecht zu bezeichnen, nur weil es auf demokratischen Wege zustande kam. Die kritisierte Vorgehensweise betont aber vor allem den evolutorische Charakter der Gerechtigkeitsvorstellung und nicht die extreme Interpretation des "rechtsethischen Relativismus" als (sehr weit gefaßte) Regelgerechtigkeit. Es wäre durchaus möglich, daß existierende Institutionen bzw. Alterssicherungssysteme zwar entsprechend gegebener Regeln geschaffen wurden, aber nicht im Einklang mit den gesellschaftlichen Gerechtigkeitsvorstellung stehen.

Allerdings verschiebt sich damit das Problem auf die Frage, ob hinsichtlich der herrschenden Gerechtigkeitsvorstellungen überhaupt ein allgemeiner gesellschaftlicher Konsens besteht und wenn ja, wie dieser und seine Veränderung identifiziert werden kann.

Ad. 2)

Mit dem eben beschriebenen, rechtspositivistischen bzw. evolutorischen Ansatz ließe sich zwar ableiten, welche Gerechtigkeitsprinzipien derzeit das Alterssicherungssystem prägen; inwieweit dieses Ergebnis jedoch die herrschende Gerechtigkeitsvorstellung aller Bevölkerungsgruppen und Generationen erfaßt ist ungewiß. Es ist nämlich wahrschein-

166 Vgl. Speckbacher (1994), S. $9 \mathrm{ff}$. 
lich, daß der identifizierte „gesellschaftliche Konsens“ nicht die Interessen aller, sondern lediglich die Interessen einflußreicher Gesellschaftsgruppen widerspiegelt. ${ }^{167}$ Ferner ist die intergenerative Fragestellungen nur unbefriedigend gelöst, denn spätere Generationen werden ebenso von dem derzeitigen System (bzw. den derzeitigen Gerechtigkeitsvorstellungen) nachhaltig betroffen, obwohl sie keine Möglichkeit haben, ihre Gerechtigkeitsvorstellung in den demokratischen Prozeß einzubringen.

Solche Defizite können nur durch eine allgemeingültige und ethisch fundierte Gerechtigkeitstheorie vermieden werden. Dabei dürtte unstittig sein, daß solche Theorien sich nicht aus übergeordneten Prinzipien ableiten lassen und zwangsweise einen axiomatischen Charakter haben. Ihre ethische Legitimation soll sich aber aus der interpersonellen Nachvollziehbarkeit und intuitiven Zustimmung ergeben.

Die folgenden Abschnitte setzen sich daher mit der Frage auseinander, inwieweit die in der Literatur beschriebenen allgemeinen Gerechtigkeitskonzepte sich auf das hier zu behandelnde Problem übertragen lassen.

\subsection{Allgemeine Gerechtigkeitskonzepte}

\subsubsection{Utilitarismus}

Ohne die utilistische Philosophie und ihre Anwendungsbereiche ${ }^{168}$ erschöpfend zu behandeln, werden im folgenden lediglich die grundlegenden Merkmale der utilitaristischen Ethik skizziert. Dadurch könnte leicht der Eindruck entstehen, es handele sich bei „dem Utilitarismus" um eine konsistente und homogene Theorie. Es muß jedoch vorab darauf hingewiesen werden, daß diese Theorie in eine Vielzahl von Forschungszweige aufgespalten ist, die zwar auf gleichen Prinzipien beruhen, diese aber unterschiedlich interpretieren. ${ }^{169}$ Im Mittelpunkt des Utilitarismus steht das utilitaristische Primärprinzip des "größten Glücks der größten Zahl“ (Prinzip der Nützlichkeit). „Gerechtigkeit“ wird in diesem Ansatz an dem kollektiven Vorteil der gesamten Gesellschaft gemessen, der wiederum aus den Nutzen der einzelnen Mitglieder der Gesellschaft aggregiert wird. Der Utilitarismus ist dadurch charakterisiert, daß er

- einen teleologischen (konsequenzialistischen) Ansatz verfolgt, da sämtliche Handlungen bzw. Handlungsweisen anhand ihrer Folgen beurteilt werden,

- diese Folgen anhand ihres (von den Individuen subjektiv wahrgenommen) Nutzens bewertet und

167 Diesem Problem des demokratischen Abstimmungsprozesses widmet sich vor allem die Public Choice Literatur zur Alterssicherung.

168 So wurde die Idee des Utilitarismus beispielsweise in der von Marschall und Pigou begrūndeten Wohlfahrtsökonomik weiterentwickelt. Vgl. Bohnen (1964), S.15.

169 Vgl. Bimbacher (1990), S. 69ff.

86 
- entsprechend des „universalistischen Prinzips“ das Wohlergehen des Kollektivs über das des Einzelnen stellt, da die einzelnen Nutzen gegeneinander aufgerechnet werden.

Die zu maximierende Zielfunktion ergibt sich aus der Aggregation der individuellen Nutzenniveaus, kann jedoch unterschiedliche Formen aufweisen. Die wichtigste Unterscheidung ist die zwischen einer Maximierung der (ggf. gewichteten) summierten Nutzen

$$
W=\sum_{i=1}^{N} u_{i}(\cdot)
$$

und einer Maximierung des durchschnittlichen Nutzens.

$$
W_{\text {Durchschnitt }}=\frac{\sum_{i=1}^{N} u_{i}(\cdot),}{N}
$$

Der Utilitarismus mag vielleicht deshalb intuitiv als gerecht aufgefaßt werden, weil er prinzipiell nicht auf Einzelinteressen gerichtet ist, sondern das Wohl aller im Auge hat. ${ }^{170}$ Wenngleich aber der utilitaristische Ansatz in der Ökonomie oft herangezogen wird, um normative Fragestellungen zu beantworten und insbesondere um intergenerative Verteilungswirkungen zu untersuchen, so ist er dennoch mit gravierenden Problemen behaftet.

Das erste Problem ergibt sich aus der Notwendigkeit, einen subjektiv empfundenen Nutzen zu messen. Zum einen müssen die den Nutzen beeinflussenden Faktoren identifiziert werden und zum anderen muß geklärt werden, durch welchen funktionalen Zusammenhang die jeweiligen Faktoren den Nutzen bestimmen. Meist hilft man sich, indem die Faktoren auf den Konsum und zuweilen die Arbeitszeit beschränkt werden und die Nutzenfunktion nicht genauer spezifiziert wird. D.h. es werden lediglich ordinale Vergleiche durchgeführt und nur qualitative Aussagen getroffen.

Eine weitere Schwierigkeit resultiert daraus, daß mikroökonomische Rationalitätserwägungen, bei denen das Individuen zwischen (marginalen) Nutzenzuwächsen und Nutzenminderungen abwägt, auf makroökonomische und intergenerative Fragestellungen übertragen werden. Zum einen ergibt sich das Problem, wie die Nutzen verschiedener Individuen bzw. Generationen verglichen und ob sie gegeneinander aufgerechnet werden können. Zum anderen erweist sich die Annahme eines repräsentativen Individuums als problematisch, da sich gesellschaftliche Präferenzordnungen nicht eindeutig aus den individuellen Präferenzordnungen ableiten lassen. ${ }^{171}$

Aus dem „universalistischen Prinzip“ und der Aggregation der Nutzen ergibt sich nicht zuletzt die Schwierigkeit, daß die Verteilungsstruktur aus der Betrachtung ausgeblendet wird.

170 Vgl. Speckbacher (1994), S. 20.

171 Vgl. Arrow (1963); Kötter (1984). 
Das Problem, ob und wie die jeweiligen Nutzen gewichtet werden sollen, beeinflußt vor allem die Beurteilung intergenerativer Fragestellungen, bei denen Ersparnis- und Wachstumseffekte eine Rolle spielen. Während einige Autoren eine Diskontierung des Nutzens zukünftiger Generationen als ethisch bedenklich einstufen und eine „soziale Zeitpräferenzrate" aus diesem Grunde ablehnen, ${ }^{172}$ betonen andere sie sei notwendig, damit früheren Generation kein übermäßig hoher Konsumverzicht abverlangt wird. ${ }^{173}$ Bei einer Diskontierung stellt sich ferner die Frage nach der adäquaten Höhe einer gesellschaftlichen Diskontrate.

Beim utilitaristischen Konzept werden die Grundsätze der Regelgerechtigkeit und zum Teil auch der Startgerechtigkeit durch die Orientierung an Resultaten nicht verfolgt. Obwohl die Handlungsweisen beim Utilitarismus anhand ihrer Folgen beurteilt werden, ist die Ergebnisgerechtigkeit in den meisten utilitaristischen Ansätzen ebenfalls von nachrangiger Bedeutung: Die Gleichheit der Ergebnisse geht bei der Aufrechnung der Nutzen unter. Entscheidend für die Beurteilung intergenerativer Fragestellungen nach dem Utilitarismus ist der Gerechtigkeitsgrundsatz der "wirtschaftlichen Effizienz".

\subsubsection{Die Rawls'sche Theorie der Gerechtigkeit}

In seinem 1971 verfaßten Werk „A theory of Justice" versucht RAWLS allgemeingültige Prinzipien aufzustellen, mit denen sich eine von allen Gesellschaftsmitgliedern als gerecht anerkannte gesellschaftliche Ordnung gestalten läßt. Seine Theorie erwuchs vorrangig aus der Kritik am Utilitarismus, insbesondere an dessen Verfahrensweise, den Nutzen verschiedener Individuen bzw. Generationen gegeneinander aufzurechnen. ${ }^{174}$ Seine Arbeit hatte das Ziel, dem Utilitarismus ein (überlegenes) Gerechtigkeitskonzept entgegenzusetzen. $^{175}$

RAWLS gründet sein Gerechtigkeitskonzept auf der klassischen Idee der Vertragstheorie, wobei er sich neben Locke und Rousseau vor allem an Kant orientierte. Ausgangspunkt seiner Überlegungen ist eine hypothetische Situation ( Urzustand“), in der die verschiedenen, rational entscheidenden und ausschließlich am eigenen Wohlergehen interessierten Individuen zusammenkommen, um die verbindlichen Regeln ihres gesellschaftlichen Zusammenlebens festzusetzen. Dabei bemüht sich jedes Individuum, seine eigene Versorgung mit Primärgütern ${ }^{176}$ zu maximieren. Die Teilnehmer dieser „Versammlung" kennen einerseits

„alle allgemeinen Tatsachen, die für die Festsetzung von Gerechtigkeitsgrundsätzen von Bedeutung sind“, d.h. „sie verstehen politische Fragen und die Grundzüge der Wirtschaftstheorie,

172 Vgl. z.B. Ramsey (1928), S. 543 und Homburg (1988), S. 98.

173 Vgl. z.B. Birnbacher (1988), S. $110 f f .$.

174 Vgl. Arrow (1973), S. 255f..

175 Vgl. Rawls (1975), S. 12.

176 Als wichtigste Primärgütern nennt Rawls Rechte und Freiheiten, Machtpositionen und Chancen sowie Einkommen und Vermögen. Vgl. Rawls 1971, S. 62/92.

88 
ebenso die Grundfragen der gesellschaftlichen Organisation und die Gesetze der Psychologie des Menschen." "17

Andererseits sind sie vom „Schleier des Nichtwissens" umgeben. Unter diesen Bedingungen kennt

"niemand seinen Platz in der Gesellschaft, seine Klasse oder seinen Status; ebensowenig seine natürlichen Gaben, seine Intelligenz, Körperkraft usw. Ferner kennt niemand seine Vorstellung vom Guten, die Einzelheiten seines vernünftigen Lebensplanes, ja nicht einmal die Besonderheiten seiner Psyche wie seine Einstellung zum Risiko oder seine Neigung zum Optimismus oder Pessimismus. " ${ }^{\text {"78 }}$

RAWLS folgert, daß eine solche Versammlung die folgenden beiden Grundsätze als Bedingung der sozialen Gerechtigkeit aufstellen würde: ${ }^{179}$

1. „Jedermann soll gleiches Recht auf das umfangreichste System gleicher Grundfreiheiten haben, das mit dem gleichen System für alle anderen verträglich ist ${ }^{180}$

2. "Soziale und wirtschaftliche Ungleichheiten sind so zu regeln, daß sie sowohl

a) den am wenigsten Begünstigsten die bestmöglichsten Aussichten bringen als auch

b) mit Ämtern und Positionen verbunden sind, die allen gemäß der fairen Chancengleichheit offenstehen. ${ }^{\prime 181}$

Gleichzeitig formuliert Rawls Vorrangregeln, nach denen dem ersten Grundsatz des absoluten Gleichheitsprinzips für einige Primärgüter die oberste Priorität zukommt. Ferner hat beim zweiten Grundsatz die im Teil b) ausgedrückte Chancengleichheit Vorrang vor der Ergebnisgleichheit des Differenzprinzips (Teil a). ${ }^{182}$

Die formulierten Grundsätze der intragenerativen Gerechtigkeit lassen sich aber nur bedingt auf die intergenerative Perspektive übertragen.

„Denn wenn das Unterschiedsprinzip auf die Frage des Sparens über Generationen hinweg angewandt wird, so führt es dazu, daß entweder überhaupt nicht oder nicht genug gespart wird, um die gesellschaftlichen Verhältnisse so weit zu verbessem, daß die gesamten gleichen Freiheiten für alle wirksam werden können. [...] Das Unterschiedsprinzip ist also auf die Frage der Gerechtigkeit zwischen den Generationen nicht anwendbar; das Problem des Sparens muß auf andere Weise gelöst werden. “183

Hinsichtlich der intergenerativen Gerechtigkeit folgert Rawls in seinem Spargrundsatz, daß jede Generation zu einer "angemessenen Kapitalakkumulation“ verpflichtet werden muß, um einen gerechten Zustand der Gesellschaft aufrecht zu erhalten. ${ }^{184}$ Wie dieser gerechter Spargrundsatz aussehen soll, vermag RAWLS allerdings nicht zu präzisieren. Es läßt sich lediglich festhalten, daß er

\footnotetext{
177 Vgl. Rawls (1975), S. $160 f .$.

178 Rawls (1975), S. 160. Ebensowenig sind die spezifischen Bedingungen der Gesellschaft (politisch, ökonomisch etc.) oder etwaige Wahrscheinlichkeiten bekannt. Vgl. Rawls (1975), S. 180.

179 Wobei der Aspekt der intergenerativen Gerechtigkeit vorerst noch ausgeklammert ist.

180 Rawls (1975), S. 81.

181 Rawls (1975), S. 104.

182 In der ökonomischen Literatur wird das Rawlssche Differenzprinzip aufgrund der Āhnlichkeit mit dem Maxmin-Regel der Entscheidungstheorie oft auch als Maxmin-Kriterium bezeichnet.

183 Rawls (1975), S. $321 f$.

184 Vgl. Rawls (1975), S. 323ff.
} 
„....die Verzichtsverpflichtung irgendwo zwischen den Sparten 1 und 0 ansiedelt und damit nicht mehr sagt, als daß jedenfalls gespart werden muß." “85

Der Spargrundsatz wird von RAWLS in die endgültige Formulierung der Gerechtigkeitsgrundsätze integriert, indem es das Differenzprinzip begrenzt:

2.a) die Ungleichheiten „....müssen unter der Einschränkung des gerechten Spargrundsatzes den am wenigsten Begünstigten den größtmöglichen Vorteil bringen,... ${ }^{186}$

Der erste, vorwiegend am Prinzip der Startgerechtigkeit ausgerichtete Grundsatz ist in seiner allgemeinen Form sicherlich konsensfähig. Offen bleibt jedoch, wie das System an Grundfreiheiten und die Verträglichkeit bei der Beurteilung der intergenerativen Gerechtigkeit realer Rentensysteme konkretisiert werden soll.

Das Differenzprinzip kann weitestgehend als "gemäßigte Ergebnisgerechtigkeit“ interpretiert werden, die für den Fall intergenerativer Fragestellungen durch den Gerechtigkeitsgrundsatz der „wirtschaftlichen Effizienz“ im Sinne des Spargrundsatzes beschränkt wird. Die fehlende Operationalisierung des gerechten Spargrundsatzes wurde bereits kritisiert. Offen bleibt darüber hinaus auch, nach welchen Kriterien festgestellt werden kann, welche Generation in einem konkreten Alterssicherungssystem die „am wenigsten Begünstigte“ ist, und wie und woran "die bestmöglichen Aussichten“ dieser Generation gemessen werden kann.

In Teil b des zweiten Grundsatzes wird vor allem die Regelgerechtigkeit und z.T. auch die Leistungsgerechtigkeit betont. Mit der Formulierung von Vorrangsregeln wird auch eine Gewichtung vorgeschlagen. Bevor sich dieser Grundsatz für Fragen der intergenerativen Gerechtigkeit in der Sozialpolitik nutzen läßt, bleibt noch zu klären, wie die „fairen Chancengleichheit" konkretisiert werden soll und wie stark sie die „gemäßigte Ergebnisgerechtigkeit" und die "wirtschaftlichen Effizienz" des Differenzenprinzips dominieren soll. Auch hierauf bietet die Theorie keine Antwort.

\subsubsection{Nozicks Anspruchstheorie der Gerechtigkeit}

Im Mittelpunkt seines 1974 erschienenen Buches „Anarchy, State and Utopia“ stand für Nozick die Frage nach der moralischen Legitimation des Staates; eine Frage, die er mit Hilfe seiner Anspruchstheorie der Gerechtigkeit so beantwortet, daß ein Minimalstaat als „Nachtwächterstaat" die einzig gerechte Staatsform darstelle.

Seine Gerechtigkeitstheorie unterscheidet sich von den beiden zuvor beschrieben im wesentlichen darin, daß nicht die Verteilungsresultate moralisch bewertet werden, sondern der Vorgang durch den sie zustande gekommen sind. Ein Vorgang gilt dabei dann als gerecht, wenn er einem der drei folgenden Grundsätze entspricht.

\footnotetext{
185 Gagern (1977), S. 273.

186 Rawls (1975), S. 336.
} 
1. Es muß sich um eine gerechte Aneignung herrenloser Gegenstände handeln, wobei eine gerechte Aneignung dann vorliegt, „wenn dadurch die Lage anderer nicht verschlechtert wird, die den Gegenstand nicht mehr frei nutzen können." ${ }^{187}$

2. Die Besitztümer werden gerecht, d.h. z.B. durch freiwilligen Tausch, Schenkung oder Erbschaft zwischen Personen übertragen, wobei wieder die „Pflicht des Eigentums" zu berücksichtigen ist. ${ }^{188}$

3. Der Vorgang korrigiert frühere Verletzungen der ersten beiden Grundsätze.

Wichtig ist dabei für NOzıcK das tatsächliche Zustandekommen einer Verteilung, nicht die Frage, ob eine Verteilung infolge der obigen drei Prinzipien zustande kommen könnte.

Kritisiert wird an NozıCKs Anspruchstheorie insbesondere die fehlende Konkretisierung seiner Grundsätze und deren ethischen Begründung, so daß zum Teil grundsätzlich in Frage gestellt wird, ob es sich um eine allgemeingültige Theorie der Verteilungsgerechtigkeit handelt. ${ }^{189}$ Darüber hinaus beschränkt NOzıCK sein Konzept einseitig auf den Gerechtigkeitsgrundsatz der Regelgerechtigkeit. Diese radikale Orientierung an der Ideologie der freien Tauschwirtschaft mag - zumindest größtenteils - noch mit der marktwirtschaftlichen Effizienz im Einklang stehen, Aspekte der Start-, Bedarfs- und Ergebnisgerechtigkeit werden aber völlig ausgeblendet, ohne dies ethisch zu begründen.

NozıCK scheint sich der Probleme einer praktischen Umsetzung seiner Grundsätze bewußt zu sein, denn er selbst führt aus, daß die von ihm abgelehnten Gesichtspunkte der „Verteilungsgerechtigkeit" und „Gleichheit" eine Rolle spielen können, wenn es angesichts des dritten Korrekturprinzips nicht möglich ist, den Zustand zu rekonstruieren, der sich ohne vorrangegangene Ungerechtigkeiten ergeben hätte. ${ }^{190}$

Insbesondere die Anwendbarkeit seiner Anspruchstheorie auf intergenerative Gerechtigkeitsaspekte - auf die NOzıCK selbst nicht eingeht - bleibt äußerst strittig. ${ }^{191}$ Denn wie läßt sich die Frage der gerechten Aneignung herrenloser Gegenstände auf verschiedene Generationen übertragen, wenn spätere Generationen einerseits keine Möglichkeit zur Aneignung haben, andererseits ihre Lage durch Aneignung früherer Generationen verschlechtert werden kann? Beispiele hierfür sind sowohl erschöpfbare Ressourcen und Umweltverschmutzung oder auch Ersparnis- und Wachstumseffekte.

Als problematisch erweist sich zudem der dritte „Berichtigungsgrundsatz", weil „zu wenig Sparen in der Vergangenheit" sich als irreversibel enweist und weil die Lebensphasen der verschiedenen Generationen auseinander fallen.

187 Sudhoff (1996), S. 132.

188 "Das schließt die Übertragung der Sache in eine Eigentumsballung aus, die die Lockesche Bedingung verletzt, sowie einen Gebrauch, (...) der die Lage anderer ungünstiger macht als die Vergleichsbasis." Nozick (1976), S. 168.

191 Vgl. Speckbacher (1994), S. 32f.; Sudhoff (1996), S.133. 
Die Ausführungen der vorangegangenen Abschnitte zeigen, daß die gängigen Ansätze für ein allgemeines und ethisch fundiertes Gerechtigkeitskonzept eine Reihe von methodischen Defiziten aufweisen und mithin ihrem Anspruch nicht genügen. Die Gerechtigkeitstheorie von RAWLS scheint am ehesten konsensfähig zu sein.

Allerdings hat auch dieser Ansatz gravierende Mängel, wenn er auf intergenerative Fragestellungen übertragen wird. Zudem ist er zu abstrakt gehalten, um inn auf die konkrete Gestaltung eines Rentensystems anzuwenden. Für die Formulierung eines sozialpolitisch nutzbaren Meßkonzepts könnte er gegebenenfalls und nur bedingt herangezogen werden, um ein zuvor ermitteltes Verteilungsmuster zu bewerten.

\subsection{Zusammenfassung und Fazit}

Das Ziel dieses Kapitels, den Gerechtigkeitsbegriff zu präzisieren, erscheint zwar zunächst trivial, dennoch erweist sich der Versuch, "Gerechtigkeit" allgemeingültig und vor allem operationalisierbar zu definieren als äußerst komplex. Es ist mithin nicht verwunderlich, daß verschiedene ökonomische Arbeiten oftmals - explizit oder implizit - von unterschiedlichen, z. T. konfligierenden Gerechtigkeitsgrundsätzen ausgehen. Somit stellt sich das grundlegende Problem, welche Gerechtigkeitsgrundsätze ausgewählt und wie diese gegebenenfalls gewichtet werden sollen. Grundsätzlich lassen sich hierzu zwei Vorgehensweisen unterscheiden, die allerdings auf alternativen Thesen zur Gerechtigkeit beruhen:

\section{These 1:}

Es läßt sich ein allgemeingültiges und ethisch fundiertes Gerechtigkeitskonzept formulieren, welches zwar einen axiomatischen Charakter hat, dessen ethische Legitimation sich aber aus der interpersonellen Nachvollziehbarkeit und Zustimmung ergibt.

\section{These 2:}

Gerechtigkeit kann weder als ein übergeordnetes, gewissermaßen naturrechtliches Prinzip interpretiert werden, noch läßt es sich auf analytischem Weg aus anderen übergeordneten Prinzipien ableiten. Jede Gerechtigkeitsvorstellung ist vielmehr gesellschaftlich definiert bzw. historisch gewachsen. Sie ist zudem keinesfalls als konstant anzusehen, da sich der gesellschaftliche Konsens darüber, was als gerecht zu gelten hat, im Laufe der Zeit ändert.

Die Ausführungen des Abschnitts 3.3 zeigen, daß sich kein allgemein akzeptiertes und ethisch fundiertes Gerechtigkeitskonzept zur Bewertung eines Alterssicherungssystems formulieren läßt bzw. daß sich bestenfalls sehr allgemeine Grundsätze aufstellen lassen, die allerdings zu abstrakt sind, um sie auf die konkrete Gestaltung eines Rentensystems anzuwenden. 
Entsprechend einer auf der zweiten Gerechtigkeitsthese beruhenden, alternativen Vorgehensweise müßten diejenigen Gerechtigkeitsgrundsätze herausgearbeitet werden, auf die sich die Gestaltung der gesetzliche Rentenversicherung in Deutschland derzeit gründet. Diese Grundsätze würden den Referenzmaßstab für die Bewertung bilden. Aber auch diese Vorgehensweise ist mit Problemen behaftet:

- Verschiede Beiträge aus dem Forschungsbereich der „Politischen Ökonomie der Sozialversicherung" weisen darauf hin, daß ein durch den demokratischen Entscheidungsprozeß legitimiertes Sozialsystem weder zwangsläufig ökonomisch effizient noch gerecht sein muß. Es ist im Gegenteil wesentlich wahrscheinlicher, daß die verschiedenen Gesellschaftsgruppen ihre eigenen Interessen durchzusetzen versuchen. Ob und in welchem Ausmaß innen dies gelingt, hängt von der politischen Macht und den damit verbundenen Einflußmöglichkeiten dieser Gruppen ab. ${ }^{192}$ Das Problem, von einer demokratischen Legitimation eines Systems nicht auf seine Gerechtigkeit schließen zu können, ist für intergenerative Fragestellungen besonders relevant. Denn es besteht die Möglichkeit, Lasten auf nachfolgende Generationen zu verschieben, die noch nicht geboren sind und ihre Interessen im demokratischen Prozeß folglich auch nicht ausdrücken können.

- Entsprechend der zweiten Gerechtigkeitsthese kann sich der politischgesellschaftliche Konsens darüber, was als gerecht anzusehen ist, im Laufe der Zeit ändern. Es wäre daher ebenfalls denkbar, daß die im derzeitigen System verankerten Gerechtigkeitsnormen die aktuelle Auffassung bzw. den gesellschaftlichen Konsens nicht mehr hinreichend widerspiegeln und somit überdacht werden müssen.

Insbesondere ist zu bedenken, daß das Alterssicherungssystem nicht nur die herrschenden Gerechtigkeitsvorstellungen widerspiegelt (bzw. widerspiegeln soll), sondern auch seinerseits die Gerechtigkeitsvorstellungen prägen, sprich verändern, kann. ${ }^{193}$

- Ferner stellt sich das Problem, den gesellschaftlichen Konsens zu definieren bzw. die grundlegende Frage, ob überhaupt von einem solchen „Konsens“ ausgegangen werden kann, angesichts differierender Präferenzen und Wertmaßstäbe der Individuen.

Durch keinen der beiden Ansätze können analytisch eindeutige, objektive und zugleich konkret anwendbare Gerechtigkeitsnormen formuliert werden. Keine der beide Gerechtigkeitsthesen ist daher optimal, um die Konsequenzen der verschiedenen Reformoptionen für die intergenerative Gerechtigkeit zu bewerten. Der vorliegenden Analyse wird daher ein pragmatischer Kompromiß zugrunde gelegt, der sich vornehmlich an der zweiten Gerechtigkeitsthese orientiert:

192 Vgl. Browning (1975), Bridges (1978), Verbon (1986), Homburg (1988), Kapitel 8, Breyer (1990) Kapitel 7 und 8. Neben dem Ansatz des Medianwähler formulierten LeGrand (1984) sowie LeGrandWinter (1987) den Mittelklassen-Ansatz. Fŭr weitere Ansătze vgl. beispielsweise Tabellini (1990). 


\section{Die Bewertung der intergenerativen Verteilungswirkungen orientiert sich an den politisch-gesellschaftlich definierten Zielen der gesetzliche Rentenversicherung.}

Sofern diese grundlegenden Ziele in Frage gestellt werden, bleibt auch die auf innen aufbauende Gerechtigkeitsanalyse angreifbar. Gerade deshalb wird bei der Analyse der intergenerativen Gerechtigkeit darauf geachtet, daß nicht die konkreten, rentenrechtlichen Bestimmungen der gesetzliche Rentenversicherung, sondern ihre ordnungspolitischen Konzeptionen und die mit ihnen korrespondierenden Gestaltungsprinzipien als Normen, sprich als Referenz für die Wahl und Gewichtung der Gerechtigkeitsprinzipien, herangezogen werden (vgl. Abschnitt 4.4). ${ }^{194}$

Dabei bleibt aber dennoch ein Interpretationsspielraum bestehen. Zudem können sich die Gerechtigkeitsvorstellungen im Laufe der Zeit wandeln und der bestehende gesellschaftliche Konsens kann von politisch machtvollen Interessengruppen zu ihren Gunsten beeinflußt sein.

Diese Probleme werden bei der Formulierung des Meßkonzepts berücksichtigt, indem die Gewichtung der Grundsätze flexibel gehalten und durch Vergleichsszenarien in der Analyse variiert wird. ${ }^{195}$

Wenngleich sich kein alternatives, normativ überlegenes Konzept - im Sinne eines „ewigen und allgemeingültigen“, objektiven Maßstabs - formulieren läßt, verdeutlicht diese Vorgehensweise, daß der Begriff der "Gerechtigkeit" mit Vorsicht zu gebrauchen ist, da er immer die Zustimmung zu den zugrunde liegenden Zielen voraussetzt.

Die Bewertung der intergenerativen Verteilungswirkungen erfolgt in zwei Schritten:

1. Quantitative Erfassung der intergenerativen Umverteilung mit Hilfe eines geeigneten Verteilungsindikators.

Dabei dienen die Gerechtigkeitsgrundsätze bzw. die mit innen korrespondierenden Gestaltungsprinzipien als Maßstab zur Abgrenzung der intergenerativen Umverteilung

2. Bewertung des Verteilungsmusters der verschiedenen Reformvorschläge.

Der Grad an Ungleichheit wird mit Hilfe unterschiedlicher Kennziffern gemessen und bewertet. Dabei kann das Differenzenkriterium nach Rawls (erste Gerechtigkeitsthese) als ein zusätzliches Kriterium herangezogen werden.

194 Es ist durchaus möglich, daß die gesetzliche Rentenversicherung aufgrund inkonsistenter Regelungen gegen ihre eigenen Gestaltungsprinzipien verstößt.

195 Interessant wäre in dem Zusammenhang die Frage, ob und welche Tendenzen sich in der Gesellschaft abzeichnen, die eine neue Definition bzw. neue Gewichtung der für die Alterssicherung relevanten Gestaltungs- bzw. Gerechtigkeitsprinzipien erforderlich machen könnten. Allerdings unterliegen solche Tendenzen - selbst wenn sie eindeutig festzustellen wären - der gleichen Kritik wie die Orientierung an den Gestaltungsprinzipien des derzeitigen Systems, so daß eine Variation nach wie vor erforderlich wäre. 
Abblidung 5: Übersicht Kapitel drei

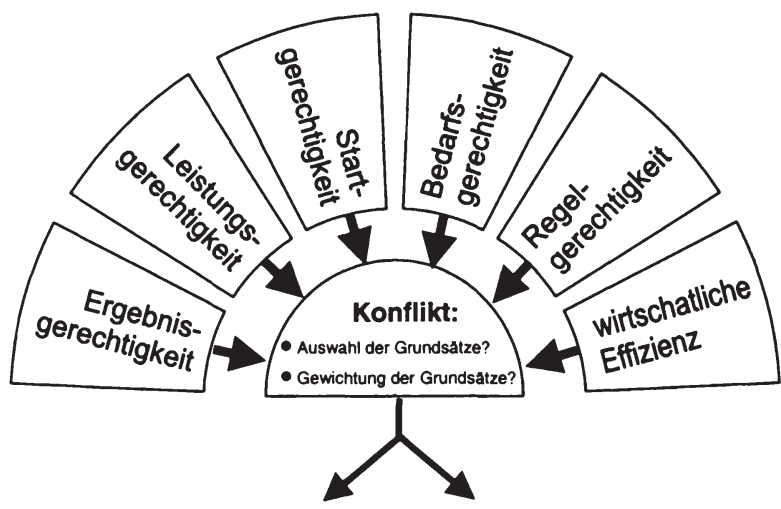

1. These:

Gerechtigkeitsvorstellungen sind gesellschaftlich definiert und historisch gewachsen

- Dominanz einzelner Interessengruppen

- Vernachlässigung nachfolgender Generationen

- veränderte Gerechtigkeitsvorstellungen nicht mehr identisch mit den Normen des derzeitigen Systems

- Transformation individueller in gesellschaftliche Vorstellungen

\section{These:}

Es lassen sich allgemeingültige und ethisch fundierte Gerechtigkeitskonzepte formulieren
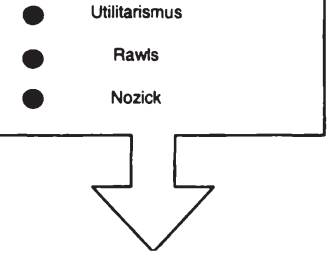

- methodische Defizite

- zu abstrakt für Bewertung sozialpolitischer Maßnahmen

- auf intergenerative Perspektive nur bedingt übertragbar

\section{PRAGMATISCHER KOMPROMIB}

- (Sozlalpolitische) Zlele des derzeitigen Systems als Referenz für dle Abgrenzung der Vertellungswirkungen

$\rightarrow$ Gewichtung durch Vergleichsszenarien variieren

- Differenzenkriterium nach Rawls nur als zusätzliches Kriterium für dle Beurteilung des Verteilungsmusters

Aufoabe der Politik:

- Auswahl der sozialpolitischen Ziele und ihrer Gewichtung

- Wahl der relevanten Kriterien für die Beurteilung des Verteilungsmusters 
Ingo Schroeter - 978-3-631-75016-2

Downloaded from PubFactory at 01/11/2019 09:14:34AM

via free access 


\section{ANALYSERAHMEN ZUR INTERGENERATIVEN GERECH- TIGKEIT DER ALTERSSICHERUNG}

Die möglichen Ansätze zur Bewertung der Verteilungswirkungen eines Alterssicherungssystems wurden im vorstehenden Kapitel beschrieben, und es zeigte sich, daß einige der formulierten Gerechtigkeitsgrundsätze im Widerspruch zueinander stehen. Abschnitt 4.1 beschreibt eine weitere Schwierigkeit der Bewertung: Ein Gerechtigkeitsgrundsatz kann die gleichen Verteilungswirkungen unterschiedlich bewerten, je nach dem, wie der Analyserahmen gesetzt wird. In diesem Abschnitt wird daher die Systemgrenze für die Analyse festgelegt.

Ein Alterssicherungssystem verursacht ferner verschiedene Verteilungswirkungen, und nicht alle beeinflussen das Untersuchungsziel dieser Arbeit: Die Folgen einer (partiellen) Substitution des Finanzierungsverfahrens für die intergenerative Verteilung bzw. die "intergenerative Gerechtigkeit". In den folgenden Abschnitten 4.2 und 4.3 werden daher die für diese Fragestellung relevanten Verteilungswirkungen abgegrenzt. Zunächst werden die möglichen Arten von Verteilungswirkungen allgemein, d.h. noch unabhängig von der Gestaltung des Alterssicherungssystems beschrieben und es wird entschieden, welche Arten von Verteilungswirkungen für die intergenerative Gerechtigkeit von Bedeutung sind. In Abschnitt 4.3 werden Annahmen darüber getroffen, wie Zahlungen Dritter (der Arbeitgeber und des Staates) in die Analyse einfließen sollen.

Das zentrale Ergebnis des dritten Kapitels besagt, daß die Gerechtigkeitsgrundsätze, mit denen die intergenerativen Verteilungswirkungen der Reformoptionen bewertet werden, entsprechend der Ziele der gesetzlichen Rentenversicherung ausgewählt und gewichtet werden sollen. Die Ziele werden durch die ordnungspolitischen Konzeptionen bzw. durch die mit innen korrespondierenden Gestaltungsprinzipien konkretisiert. Abschnitt 4.4 widmet sich daher der Analyse der Gestaltungsmöglichkeiten eines Alterssicherungssystems. Es wird untersucht, welche Ziele und Gestaltungsprinzipien für die Frage der intergenerativen Gerechtigkeit einer Substitution des Finanzierungsverfahrens wichtig sind und welchen Einfluß sie auf die Wahl der Gerechtigkeitsgrundsätze, sprich auf die Bewertung der Verteilungswirkungen haben.

\subsection{Abgrenzung des Systems für die Bewertung der Vertei- lungswirkungen}

Im folgenden werden zwei Gestaltungsmöglichkeiten eines Sozialsystems gegenübergestellt, um anhand der Leistungsgerechtigkeit zu verdeutlichen, wie gleiche Verteilungswirkungen von einem Gerechtigkeitsgrundsatz unterschiedlich bewertet werden können: 
- System A ist so konzipiert, daß seine Leistungen an das Individuum sich unmittelbar aus den vom Individuum zuvor erbrachten Beiträgen ergeben (Äquivalenzprinzip vgl. Abschnitt 4.4.3).

- Bei System B richten sich die Abgaben nicht nur nach den erwarteten Leistungen, sondern auch nach der Leistungsfähigkeit des Individuums (Leistungsfähigkeitsprinzip, alternativ können natürlich auch bei gleichen Beiträgen die Leistungen nach der Leistungsfähigkeit variieren).

Da das Individuum vom System A genau das erhält, was es zuvor eingezahlt (sprich geleistet) hat, treten keine Verteilungswirkungen auf und es läßt sich intuitiv folgern: System A ist gerecht im Sinne des Grundsatzes der "Leistungsgerechtigkeit". Je nach Ausmaß, mit dem die individuelle Leistungsfähigkeit berücksichtigt wird, werden beim System B die geleisteten Beiträge bei gleichen Leistungen differieren (bzw. bei gleichen Beiträgen differieren die erhaltenen Leistungen). System $B$ ist folglich mit Verteilungswirkungen verbunden, die den Grundsatz der Leistungsgerechtigkeit intuitiv widersprechen.

Die Leistungsfähigkeit wird allerdings an den Einkommensverhältnissen gemessen, die sich aus dem Marktprozeß ergeben. Wie in den Abschnitten 3.2.1 und 3.2.2 bereits dargelegt, korrelieren die Einkünfte umso weniger mit dem persönlichen Verdienst, je höher das Einkommen ist. Unter dieser Prämisse würden sich die vorangehenden Schlußfolgerungen gerade umkehren: Unterstellt man theoretisch, daß die Leistungsfähigkeit gerade in dem Maße berücksichtigt wird, in dem das Einkommen durch Faktoren wie Glück, Talent, ökonomische Renten etc. bestimmt wird, dann müßten die Verteilungswirkungen des Systems B nach dem Grundsatz der Leistungsgerechtigkeit als gerecht bewertet werden. System $A$ hingegen wäre trotz des Fehlens von Verteilungswirkungen ungerecht im Sinne der Leistungsgerechtigkeit. Die mit dem Leistungsfähigkeitsprinzip verbundenen Verteilungswirkungen können folglich nach dem Grundsatz der Leistungsgerechtigkeit unterschiedlich bewertet werden, und die Bewertung hängt davon ab, an welcher Stelle die „Systemgrenze“ der Analyse gezogen wird.

Betrachtet man beispielsweise die Systemgrenze 1 in Abbildung 6 und nimmt man an, daß die Beiträge für das Alterssicherungssystem aus einer Einkommensverteilung finanziert werden, die aus dem Marktprozeß resultiert und dabei nicht den Verdienst der Individuen reflektiert, dann ist eine nach dem Leistungsfähigkeitsprinzip ausgerichtete Beitragserhebung im Alterssicherungssystem mit dem Konzept der Leistungsgerechtigkeit vereinbar, obwohl bzw. gerade weil die Beiträge nicht nach dem Äquivalenzprinzip erhoben werden. Wählt man hingegen Systemgrenze 2 als relevanten Analyserahmen, so wird die primäre Einkommensverteilung als gegeben angesehen. Eine Gerechtigkeitsanalyse fragt danach, inwieweit das Alterssicherungssystem zu einer - gemessen an diesem Ausgangszustand der Primärverteilung - „ungerechteren Verteilung“ führt. In diesem Fall wäre eine an der Leistungsfähigkeit orientierte Beitragserhebung ungerecht im Sinne der Leistungsgerechtigkeit. 


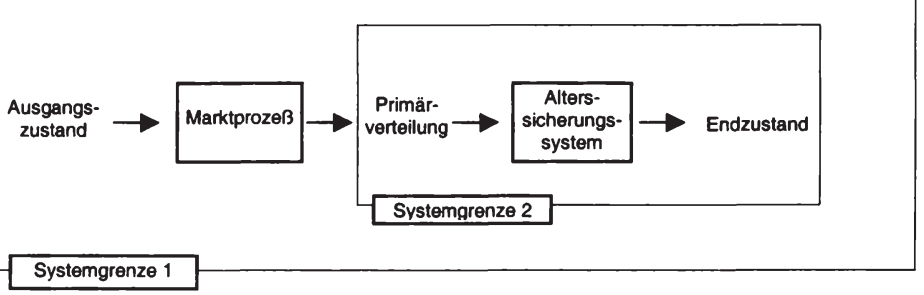

Quelle: eigene Darstellung

Um die Systemgrenze für diese Arbeit festzulegen, wird - als Vorgriff auf den folgenden Abschnitt - zwischen primärer und sekundärer Einkommensverteilung unterschieden. Die primäre Einkommensverteilung beschreibt die aus dem Marktprozeß resultierende Verteilung des Volkseinkommens. Demgegenüber bezeichnet die sekundäre Einkommensverteilung die Verteilung des verfügbaren Einkommens, die sich nach Beendigung sämtlicher staatlicher und privater Umverteilungsprozesse ergibt.

In der vorliegende Analyse wird unterstellt, daß die Zuständigkeit für die verschiedenen gesellschaftlichen Aufgaben von unterschiedlichen Institutionen wahrgenommen werden. Die erwünschte Korrektur der aus dem Marktprozeß resultierenden Primärverteilung der Einkommen soll im allgemeinen Steuer-Transfer-System (ohne Alterssicherung) erfolgen. Das Alterssicherungssystem konzentriert sich vornehmlich auf die intertemporale Verteilung von Einkommen. Die aus dem Steuer-Transfer-System (ohne Alterssicherung) resultierende Sekundärverteilung der Einkommen wird in der vorliegenden Untersuchung daher als Ausgangsverteilung akzeptiert, ohne sie auf ihre Gerechtigkeit hin zu bewerten. Es werden folglich nur solche intergenerativen Verteilungseffekte berücksichtigt, die sich aus dem Rentensystem ergeben; Verteilungseffekte aus den übrigen Zweigen der Sozialversicherung (beispielsweise dem Gesundheitssystem) werden aus der Analyse ausgeblendet. $^{196}$

Dennoch können von den sonstigen Sozialversicherungszweigen mittelbare Wirkungen auf die intergenerative Verteilung eines Alterssicherungssystems ausgehen: Beispielsweise wenn eine Veränderung der Sozialbeiträge über die Rückkopplung der Nettoanpassung den aktuellen Rentenwert und somit die Höhe der Rentenleistungen beeinflußt. Diese mittelbaren Verteilungswirkungen sind Bestandteil der intergenerativen Verteilungsanalyse (vgl. Abschnitt 4.4.3.2 und 5.3.1.5). 
Abbildung 7: Auswahl der für die Analyse maßgeblichen Systemgrenze

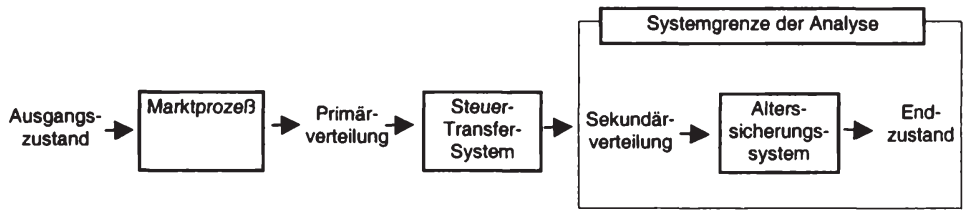

Quelle: eigene Darstellung

\subsection{Auswahl der relevanten Verteilungswirkungen für die Analy- se der intergenerativen Gerechtigkeit}

Im Mittelpunkt der Analyse ökonomischer Verteilungswirkungen steht die Frage, wie das Einkommen und das Vermögen auf bestimmte Gruppen oder Individuen aufgeteilt werden. ${ }^{197}$ Entsprechend der Kriterien, mit denen die Bezugseinheiten abgegrenzt werden, kann zwischen funktionaler und personeller Verteilung unterschieden werden. ${ }^{198}$ Die funktionale Verteilung setzt sich mit der Frage auseinander, wie sich das Einkommen auf die verschiedenen Produktionsfaktoren (Gewinn oder Einkommen aus unselbständiger Beschäftigung) aufteilt. Im Vordergrund der personellen Verteilung steht die Verteilung von Einkommen und Vermögen zwischen einzelnen Individuen oder zwischen nach bestimmten Kriterien gruppierten Individuen. Die Gruppierung kann hierbei z.B. nach sozioökonomischen Kriterien ${ }^{199}$ erfolgen und sich auf eine Periode beziehen oder aber verschiedene Generationen und mehrere Perioden umfassen. Im ersten Fall spricht man von einer interpersonellen (intragenerativen) Verteilung und im zweiten von einer intertemporalen oder intergenerativen ${ }^{200}$ Verteilung. ${ }^{201}$

In der vorliegenden Arbeit werden vornehmlich personelle Verteilungswirkungen behandelt, bei denen zudem die intergenerativen Verteilungswirkungen im Vordergrund stehen. Funktionale Verteilungswirkungen spielen nur insofern eine Rolle, als sie die relativen

Vgl. Ramser (1987), S. 1.

Vgl. nachfolgend Ramser (1987), S. 1-5.

Ein weiteres Gruppierungskriterium kann beispielsweise die Einkommensklasse sein. In dem Fall wird zwischen horizontaler und vertikaler Umverteilung differenziert. Unter die erste Kategorie fallen Umverteilungen von Einkommen zwischen Personen gleicher Einkommensklassen. Mit vertikaler Umverteilung beschreibt man eine Einkommensverteillung zwischen Gruppen hoher und niedriger Einkommensklassen.

Dabei werden die Begriffe "intergenerational", "intergenerationell" und „intergenerativ" synonym verwandt.

Die Begriffsdefinitionen sind in der Literatur keineswegs einstimmig. Während die „interpersonelle und die ${ }_{n}$ intragenerative Verteilung“ z.T. synonym verwandt werden, bezeichnet Koppelmann mit ${ }_{n}$ intragenerativer Verteilung" die Umschichtung des Lebenseinkommens eines Einkommensbeziehers von der Erwerbs- in die Ruhestandsphase. Die intergenerativen Verteilung beschreibt die Verteilung zwischen Personen bzw. Gruppen aufeinander folgender Generationen. Die intertemporale Verteilung wird als Überbegriff für intra- und intergenerationale Verteilung verstanden. Vgl. Koppelmann (1979), S. 2 und die dort angegebene Literatur. 
Faktorpreise (Lohn und Zins) verändern und auf diesem Wege je nach Finanzierungsverfahren die intergenerative Verteilung beeinflussen können.

Der Begriff der „intergenerativen Verteilung“ wird nicht einheitlich verwandt. RAMSER beschreibt sie als

„... intergenerative bzw. Verteilung auf verschiedene Generationen, d.h. auf Individuen, die (noch) im Produktionsprozeß tätig sind und Individuen, die aus Alters- und/oder Gesundheitsgründen bereits aus dem Produktionsprozeß ausgeschieden sind. ${ }^{202}$

Diese Definition kann jedoch - vor allem bei der Analyse von Alterssicherungssytemen leicht zu Mißverständnissen führen. Denn ein Vergleich von abhängig Beschäftigten bzw. Erwerbstätigen eines bestimmten Zeitpunktes (oder einer Periode) mit der Gruppe der Rentner bzw. Ruheständler des gleichen Zeitpunkts (oder der gleichen Periode) ist eher der sozioökonomischen als der intergenerativen Verteilung zuzuordnen. ${ }^{203}$ KüLP betont daher zu recht, daß es in einem System der Altersvorsorge immer dann zu einer intergenerativen Umverteilung kommt, wenn einzelne Generationen gegenüber anderen hinsichtlich der Verteilung der Einkommen bevorzugt bzw. benachteiligt werden und zwar bezogen auf die Belastungen und Leistungen der gesamten Lebensspanne einer Generation. ${ }^{204}$ Folglich müssen mindestens drei Perioden in die Betrachtung einbezogen werden: Neben der aktuellen Periode muß die vorangegangene Periode (in der die aktuelle Generation der Ruheständler noch der erwerbstätigen Generation angehörte) genauso berücksichtigt werden, wie die nachfolgende Periode (in der die erwerbstätige Generation der aktuellen Periode im Ruhestand sein wird). ${ }^{205}$

Die intergenerative Betrachtung wird im Sinne KüLPS durchgeführt, d.h. es werden die Effekte während der gesamten Lebenszeit verschiedener Generationen gegenüberstellt.

Ferner muß für jedes Verteilungskonzept festgelegt werden, wie der Einkommens- und Vermögensbegriff zu definieren bzw. abzugrenzen ist. Ein wichtiger Aspekt ist beispielsweise, ob etwaige Realtransfers in die Analyse einbezogen werden müssen oder ob es von der Zielsetzung der Untersuchung her gerechtfertigt ist, die Analyse vereinfachend auf monetäre Größen zu beschränken. Im Gegensatz zu beispielsweise der Krankenversicherung spielen Realtransfers in der gesetzlichen Rentenversicherung eine untergeordnete Rolle, ${ }^{206}$ so daß sich die Analyse der direkten (siehe unten) intergenerativen Verteilungswirkungen in dieser Arbeit auf monetäre Zahlungsströme beschränken kann.

Unter den zu analysierenden Reformoptionen befindet sich keine Variante, die für eine Ausdehnung des Versichertenkreises (beispielsweise auch auf die Gruppe der Selbständigen) bzw. einer Abkehr von der Lohnzentrierung plädiert. Aus diesem Grunde ist eine Differenzierung zwischen „zu versteuerndem Einkommen“ und „Erwerbseinkommen“ für

\footnotetext{
202 Ramser (1987), S. 2..

203 So zielt beispielsweise auch die Untersuchung von Berthold/Roppel (1994) eher auf eine statische Periodenbetrachtung und weniger auf den Aspekt der intergenerativen Gerechtigkeit.

204 Vgl. Külp (1991), S. 40+43. Vgl. auch Allekotte (1981), S.204; Dinkel (1986) und v. Schulenburg (1989).

205 Vgl. Külp (1991), S. 43f..
} 
die zu untersuchende Fragestellung von nachrangiger Bedeutung, solange die Beitragsgrundlage für alle Versicherten identisch bleibt.

Die Unterscheidung zwischen primärer und sekundärer Einkommensverteilung wurde im vorstehenden Abschnitt bereits erklärt. Es ist offensichtlich, daß ein Alterssicherungssystem sich vornehmlich auf die Sekundärverteilung auswirkt; durch verschiedene Anreizeffekte kann es aber auch die Primärverteilung beeinflussen. Für die Beurteilung der intergenerativen Gerechtigkeit eines Alterssicherungssystems müssen alle Formen intergenerativer Verteilung berücksichtigt werden: ${ }^{207}$

- Intergenerative Verteilungswirkungen können direkt durch Transferzahlungen zwischen den Generationen erfolgen bzw. treten auf, wenn die Zahlungen (Beiträge, Rentenleistungen) für verschiedene Generationen unterschiedlich erhöht/gemindert werden. Diese Effekte werden als direkte Verteilungswirkungen bezeichnet.

- Andererseits ist für die intergenerative Verteilungswirkung entscheidend, ob und in welchem Ausmaß gesamtwirtschaftliche Größen wie das BIP u.a. auch von der Art der Rentenfinanzierung bzw. der Ausgestaltung des Rentensystems bestimmt werden. Diese Zusammenhänge führen zu indirekten Verteilungswirkungen.

Beispielsweise wird das Wirtschaftswachstum durch die Entscheidung einer Generation, mehr zu konsumieren und weniger zu investieren gebremst. Nachfolgende Generationen haben dadurch einen geringeren Wohlstand und sie müssen einen relativ größeren Teil ihres Einkommens zur Finanzierung des Rentensystems ausgeben. ${ }^{208}$ Ein anderes Beispiel sind Arbeitsangebots- und Arbeitsnachfrageeffekte, die durch eine Kopplung der Finanzierung an das Erwerbseinkommen hervorgerufen werden und die gesamtwirtschaftliche Produktion bestimmen.

\section{3 Überwälzungs- und Zurechnungannahmen über Zahlungen Dritter}

In der gesetzlichen Rentenversicherung werden die Beiträge paritätisch von den Arbeitnehmern und Arbeitgebern gezahlt. Für die Analyse stellt sich damit die Frage nach der Inzidenz der Arbeitgeberbeiträge, sprich ob es den Arbeitgebern gelingt die Traglast der Beiträge ganz oder teilweise vor- bzw. rückzuwälzen. Theoretisch lassen sich die folgenden drei möglichen Hypothesen formulieren: ${ }^{209}$

206 Sachleistungen spielen lediglich bei der Rehabilitation eine Rolle, die wiederum keinen wesentlichen Einfluß auf intergenerative Verteilungswirkungen hat.

207 Vgl. Davis (1996), S. 6ff; Bösch (1987).

208 Solche gesamtwirtschaftlichen Rückkopplungseffekte werden beispielsweise in einem (finanzmathematisch ausgerichteten) Vergleich der globalen Beitragsäquivalenzziffern ausgeklammert. Vgl. Wagner (1984).

209 Vgl. Koppelmann (1979), S. 20.

102 
- Die formale Zahllast entspricht der effektiven Traglast. D.h. die Arbeitgeberbeiträge haben keinen Einfluß auf die Lohnverhandlungen und mindern ausschließlich den Gewinn der Unternehmen.

- Die Arbeitgeberbeiträge werden als Lohnbestandteil interpretiert, so daß auch die Arbeitgeberbeiträge letztendlich vom Arbeitnehmer zu zahlen sind.

- Die Arbeitgeberbeiträge werden von den Unternehmen auf die Preise vorgewälzt, so daß die Konsumenten zur Finanzierung herangezogen werden.

$\mathrm{Ob}$ und in welchem Ausmaß die Hypothesen zutreffen, hängt u.a. maßgeblich von den Preiselastizitäten der Güternachfrage und den Lohnelastizitäten des Arbeitsangebots und der Arbeitsnachfrage bzw. von der Art der Lohnverhandlung und der Macht der Tarifparteien im Verhandlungsprozeß ab.

Folgt man der dritten These, so muß zunächst die Konsumquote und -struktur der verschiedenen Gruppen (Rentner, Selbständige, unselbständig Beschäftigte etc.) bestimmt werden, um im Zusammenhang mit der effektiven Inzidenz zu klären, welcher Teil der Arbeitgeberbeiträge von den Versicherten getragen werden muß. ${ }^{210}$ WAGNER weist jedoch darauf hin, daß eine Vorwälzung auf die Preise lediglich bei Hochkonjunktur, insbesondere bei Inflation und Arbeitskräftemangel wahrscheinlich ist. ${ }^{211}$ In der Literatur wird der ersten These wenig Bedeutung zugemessen, ${ }^{212}$ und

„mit Ausnahme weniger Untersuchungen wird in allen bekannten Arbeiten der Arbeitgeberanteil als Lohnbestandteil angesehen und damit von der vollständigen Rückwälzung dieser ,Lohnsummensteuer' auf die abhängig Beschäftigten ausgegangen “213

Für die vorliegende Untersuchung werden die Arbeitgeberbeiträge mithin als Lohnbestandteil aufgefaßt, so daß die Gesamten Beiträge in die Verteilungsanalyse einfließen. ${ }^{214}$

Eine weitere zu klärende Frage ist die Behandlung des Bundeszuschusses, denn auch dieser muß finanziert werden. Abstrahiert man von der Möglichkeit der Staatsverschuldung, so wird der Bundeszuschuß aufgrund des Prinzips der Nonaffektion der Steuern durch direkte und indirekte Steuern finanziert und zwar jeweils in dem Maße in dem das gesamte Steueraufkommen sich aus direkten und indirekten Steuern zusammensetzt.

\footnotetext{
210 Vgl. Koppelmann (1979), S. 21.

211 Vgl. Wagner (1984), S. 163 und Bürgisser-Peters (1982), S. 130.

212 Vgl. Koppelmann (1979), S. 21 und die dort angegebene Literatur.

213 Wagner (1984), S. 163. Vgl. auch die dort zitierten Arbeiten (Fußnote 33 und 34).

214 Die empirischen Untersuchungen der Europäischen Union (1994) und (1995) zeigen einen eher geringen Zusammenhang zwischen der Höhe der Sozialbeiträge und den Arbeitskosten. Daraus wird der Schluß gezogen, daß die Arbeitgeberbeiträge langfristig größtenteils auf die Arbeitnehmer überwälzt werden. Auch in der Jobs Study der OECD (1994) konnte keine signifikante Korrelation zwischen dem "Steuerkeil" und den Arbeitskosten bzw. der Arbeitslosigkeit identifiziert werden. Der negative Zusammenhang zwischen den Arbeitgeberbeiträge und den Anteil der Arbeitnehmer an der Wertschöpfung scheint ebenfalls die These zu bestätigen, daß die Arbeitgeberbeiträge auf die Löhne überwälzt werden. Allerdings muß die Eindeutigkeit des Ergebnisses eingeschränkt werden, wenn nicht das Niveau sondern die Veränderung der Variablen untersucht werden. Ähnlich auch Carruth/ Schnabel (1993). Vgl. Steiner (1996) und Bean (1994) für eine Zusammenfassung der bisherigen Studien.
}

103 
Fragt man nach der effektiven Inzidenz des Bundeszuschusses, so müßte der aus direkten Steuern finanzierte Teil den Selbständigen, unselbständig Beschäftigten etc. entsprechend ihrer Lohn- und Einkommenssteuern zugeordnet werden, während der aus indirekten Steuern finanzierten Teil wieder nach Maßgabe der Konsumquote und -struktur der verschiedenen Gesellschaftsgruppen verteilt werden müßte. Im Gegensatz zu den direkten Steuern kommt es bei den aus indirekten Steuern finanzierten Teil des Bundeszuschusses auch zu einer teilweisen „Selbstfinanzierung“ der Rentner. ${ }^{215}$ Da eine solche Vorgehensweise nicht nur wenig praktikabel ist, sondern auch „ein kaum mehr durchschaubares Geflecht von Hypothesen ${ }^{216}$ erfordert, können alternativ auch die Rentenleistungen korrigiert werden, um den Bundeszuschuß zu berücksichtigen.

Von besonderer Bedeutung sind in diesem Zusammenhang die von der Rentenversicherung übernommenen gesamtgesellschaftlichen Aufgaben. Unterstellt man, daß der Bundeszuschuß zur Finanzierung der unter dem Stichwort der „versicherungsfremden Leistungen“ diskutierten Ausgaben dient, kann dieser (konstante Anteile an den Gesamtausgaben der Rentenversicherung vorausgesetzt) als "durchlaufender Posten“ angesehen werden. ${ }^{217}$ Diese Annahme scheint für die vorliegende Arbeit insbesondere deshalb vertretbar zu sein, weil kein Vergleich der Rendite mit denen privater Alterssicherungssysteme angestrebt wird, sondern verschiedene Reformoptionen hinsichtlich ihrer intergenerativen Verteilungswirkungen analysiert werden.

\subsection{Gestaltungsprinzipien der Alterssicherung und ihre Bedeu- tung für die Bewertung der Verteilungswirkungen}

Ziel eines jeden Alterssicherungssystems ist die materielle Absicherung des Alters, so $\mathrm{da} ß$ seine primäre Aufgabe darin besteht, Lebenseinkommen intertemporal auf die verschiedenen Lebensphasen zu verteilen. Daneben können mit einem Alterssicherungssystem aber auch weitere, untergeordnete Ziele verfolgt werden, die z.T. expliziert formuliert sein können, sich auf jeden Fall aber implizit in der Gestaltung des Alterssicherungssystem niederschlagen. ${ }^{218}$

Die verschiedenen Gestaltungsprinzipien lassen sich vereinfachend den drei Dimensionen der Subsidiarität, der Solidarität und des Erfassungsgrads zuordnen (Vgl. Abbildung

215 Genau genommen müßte bei den direkten Steuem auch die Besteuerung des Ertragsanteils der Renten berücksichtigt werden. Da diese Besteuerung der Renten aufgrund der hohen Freibeträge faktisch nicht relevant ist, kann dieser Effekt jedoch vernachlässigt werden.

216 Schmähl (1977), S. 555. Vgl. auch Koppelmann (1979), S. 36f..

217 Vgl. zur Diskussion um die "versicherungsfremden Leistungen" beispielsweise Berthold/Thode (1996); Vogler-Ludwig/Severin/Langmantel E. (1996); Rische (1996); Blüm (1996).

218 Werden die Ziele eines Rentensystems beispielsweise um den Schutz vor Invalidität oder die Absicherung der Langlebigkeit erweitert, ist das Alterssicherungssystem i.d.R. als Versicherung konzipiert. Es könnten dem Alterssicherungssystem auch allgemeine, sozialpolitische Aufgaben (z.B. Familienförde- 
8). ${ }^{219}$ Eine vierte Gestaltungsdimension ist die Wahl des Finanzierungsverfahrens. Diese wird hier nicht explizit diskutiert, da sie zum einen im zweiten Kapitel bereits eingehend beschrieben und analysiert wurde und weil eine Umstellung des Finanzierungsverfahrens das Untersuchungsobjekt dieser Arbeit darstellt. D.h. in diesem Abschnitt soll gerade untersucht werden, welche der übrigen Gestaltungsprinzipien für die die Bewertung einer Substitution des Finanzierungsverfahrens relevant sind.

Abbildung 8: Gestaltungsdimensionen und -prinzipien eines Alterssicherungssystems (ohne Gestaltungsdimension „Finanzierungsverfahren“)

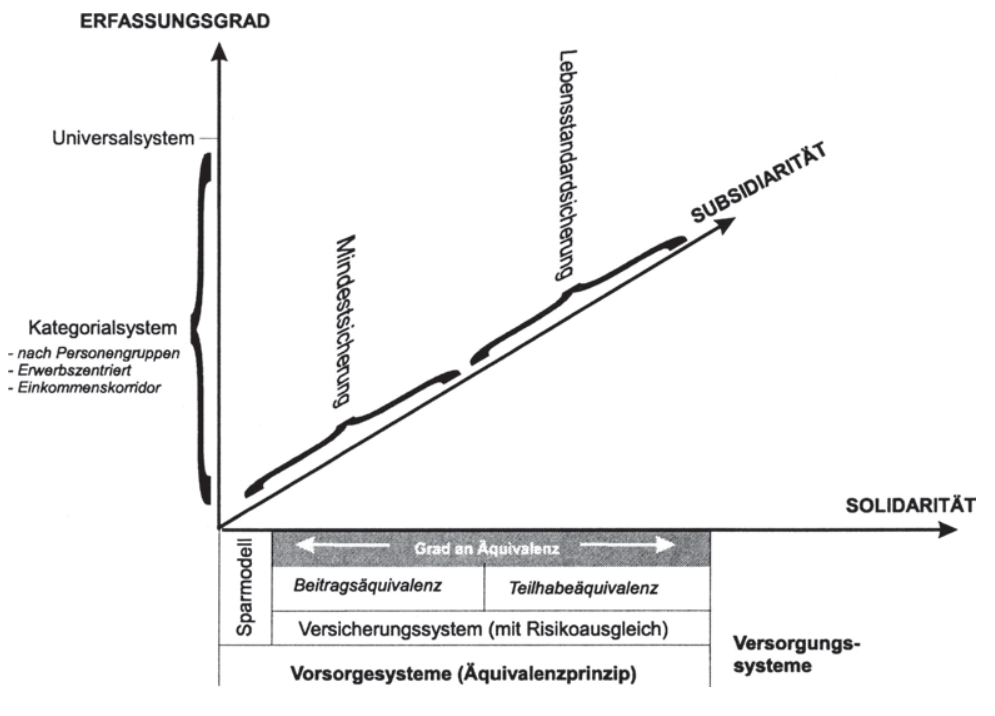

Quelle: eigene Darstellung

Ein Alterssicherungssystem kann folglich unterschiedliche Ziele verfolgen und dementsprechend auch nach unterschiedlichen Prinzipien gestaltet werden. Diese Ziele und Prinzipien sind das Ergebnis eines politisch-gesellschaftlichen Entscheidungsprozesses. ${ }^{220} \mathrm{Da}$ ihnen mithin unterschiedliche Gerechtigkeitsvorstellungen zugrunde liegen können, bestimmt auch die jeweilige Gestaltung des Alterssicherungssystems, ob und inwieweit auftretende Verteilungswirkungen ungerecht sind. Für die nachfolgende Analyse gilt es daher zu klären,

rung) dem Alterssicherungssystem übertragen werden. In dem Fall würden Elemente der Versorgung die Gestaltung des Systems mitbestimmen.

219 Die Ausführungen sollen nicht als allgemeine Typologie der Alterssicherung interpretiert werden. Vgl. hierzu Homburg (1988). Es werden vielmehr die grundlegenden Gestaltungsdimensionen betrachtet, in denen sich die verschiedenen Auffassung von Gerechtigkeit besonders widerspiegeln. Ferner werden die Gestaltungsprinzipien herausgearbeitet, auf die sich die folgende Verteilungsanalyse konzentriert bzw. die für die Analyse eine besondere Bedeutung haben. 
1. welche Gestaltungsdimensionen für die Frage der intergenerativen Gerechtigkeit einer Substitution des Finanzierungsverfahrens relevant sind und

2. welchen Einfluß die verschiedenen Gestaltungsprinzipien auf die Wahl und Gewichtung der Gerechtigkeitsgrundsätze, sprich auf die Bewertung der Verteilungswirkungen haben.

\subsubsection{Subsidiarität}

Bei der Gestaltung des Alterssicherungssystems stellt sich zunächst die Frage nach dem angemessenen Verhältnis aus Eigenverantwortung und staatlicher Vorsorge. Entsprechend dem Subsidiaritätsprinzip sollte dabei die Eigenverantwortung in einer freiheitlichen Markt- und Gesellschaftsordnung grundsätzlich Vorrang haben. ${ }^{221}$ Klammert man die Frage nach der Organisationsstruktur der Versicherungsträger aus, so bezieht sich die Frage nach der Subsidiarität in der Alterssicherung vornehmlich auf die Entscheidung "staatliche versus private Vorsorge“. ${ }^{222}$ Staatliches Eingreifen sollte nach dem Subsidiaritätsprinzip stets nachgeordnet sein, d.h. nur erfolgen, wenn der einzelne Bürger oder kleinere Gemeinschaften die Aufgabe nicht selbst erfüllen können. Die Notwendigkeit des staatlichen Eingreifens muß begründet werden.

Die Gründe, die ein staatliches Eingreifen rechtfertigen, knüpfen meist an einen meritorischen Bedarf, an Marktmängel bzw. Marktversagen oder an generell als inakzeptabel geltende Verteilungsfolgen an. ${ }^{223}$ Regelmäßig wird beispielsweise die „Kurzsichtigkeit der Versicherten" in Form einer Unterschätzung des zukünftigen Bedarfs und die daraus folgende, unzureichende Eigenvorsorge als Begründung angeführt. Einige Autoren sehen ein staatlich organisiertes, zwangsweises Sicherungssystem über Umlagefinanzierung als einziges Mittel, um gezielte intra- oder intergenerative Umverteilung sicherzustellen. ${ }^{224}$ Die unscharfe Definition „als inakzeptabel geltende Verteilungsfolgen“ deutet an, daß der optimale Umfang der staatlichen Alterssicherung sich theoretisch nicht eindeutig bestimmen läßt und stark umstritten ist. ${ }^{225}$

220 So auch Maydell (1995), S. 239: „Sicherungssysteme können sehr unterschiedlichen Sicherungszielen dienen. Die Entscheidung darüber ist politischer Natur, sie ergibt sich nicht als strikt logische Ableitung." Vgl. Spieker (1996); Glismann/Horn (1996). Knappe und Prinz bezeichnen den Zwang der Sozialpolitik in einer freiheitlichen Ordnung sogar als „Übel“. Vgl. Knappe (1995), S. 344; Prinz (1994), S. $106 f f$.

222 Bei einer Vertiefung der Fragestellung müßte weiter hinsichtlich des Zwangscharakters eines Alterssicherungssystems differenziert werden, denn eine private Zwangsversicherung ist ebenso denkbar wie eine freiwillige staatliche Vorsorge.

223 Vgl. Knappe (1995), S. 344; Berthold (1988), S. 339ff..

224 Vgl. z.B. Wagner (1985), S. 155; Breyer/Kolmar (1997). Bei dieser Argumentation steht jedoch eher der Zwangscharakter im Vordergrund. Beispielsweise arbeiten in Frankreich die Mehrzahl betrieblicher Altersvorsorgesysteme mit dem Umlageverfahren. Zu klären wäre, ob ein solcher Zwang sich auf Staatsebene nur durch staatliche Organisation realisieren ließe oder ob die Existenz von umfangreichen Rückversicherungsmöglichkeiten ausreicht.

225 Selbst wenn von Verteilungsaspekten abstrahiert und lediglich allokative Gesichtspunkte betrachtet werden, zeichnet die bisherige Theorie kein einheitliches Bild. Die klassische Begründung einer staatlichen Zwangsversicherung bei asymmetrischen Informationen gründet sich auf die von Rothschild/Stiglitz (1976) abgeleiteten Pareto-Verbesserung von staatlichen Eingriffen. Wilson (1977), Miyazaki (1977) und Spence (1977) lassen in ihrem Gleichgewicht auch kreuzsubventionierte Verträge zu und es läßt sich

106 
Wenn von dem (in der Praxis der Industriestaaten nicht anzutreffendem) Extremfall der vollständig privaten Vorsorge abgesehen wird, ${ }^{226}$ äuert sich die unterschiedliche Umsetzung des Subsidiaritätsprinzips in zwei grundsätzlichen Gestaltungsprinzipien von staatlichen Alterssicherungssystemen: Dem Lebensstandardprinzip oder dem Prinzip der Mindestsicherung.

Die Mindestsicherung strebt einen (minimalen) Ausgleich bis zu einem bestimmten Niveau an, so daß in diesem Gestaltungsprinzip die "gemäßigte Version" der Ergebnisgerechtigkeit zum Ausdruck kommt. Durch die Mindestsicherung soll Armut vermieden werden, so daß neben der gemäßigten Ergebnisgerechtigkeit auch das Konzept der Bedarfsgerechtigkeit - insbesondere im Fall einer bedürttigkeitsorientierten Mindestsicherung eine Rolle spielt. Die Abhängigkeit von der Bedürttigkeitsprüfung zeigt, daß die der Mindestsicherung zugrundeliegenden Gerechtigkeitsvorstellungen zum Teil von der konkreten Ausgestaltung (Kombination mit anderen Gestaltungsprinzipien) der Mindestsicherung abhängen. ${ }^{27}$ So trägt eine steuerfinanzierte Grundsicherung auch dem Konzept der Bedarfsgerechtigkeit und dem Leistungsfähigkeitsprinzip Rechnung.

Eine Mindestsicherung wird zuweilen mit der Voraussetzungsgerechtigkeit begründet, die sowohl die Regelgerechtigkeit als auch die Startgerechtigkeit umfassen kann. Die Begründung, weshalb eine Mindestsicherung diesen Gerechtigkeitsgrundsätzen besser entsprechen sollte als eine Lebensstandardsicherung, beschränkt sich in der Regel auf die ordnungspolitische Ideologie: Der mündige Bürger soll sich in einer freiheitlich demokratischen Grundordnung selbstverantwortlich um seine Altersvorsorge kümmern. ${ }^{228}$ Die Meinungen gehen jedoch darüber auseinander, inwieweit er dies überhaupt will und kann. ${ }^{229}$ Ferner ist nicht ohne weiteres ersichtlich, weshalb diese Forderung die Voraussetzungsgerechtigkeit der Alterssicherung verbessern sollte. ${ }^{230}$

zeigen, daß dezentrale Lösungen unter diesen Bedingungen effizient sind. (Crocker/Snow (1985)) Allerdings sind diese Modelle an eine bestimmte Form von Erwartungsbildung der Unternehmen gebunden. Andere Modellierungen (Grossman (1979), Riley (1979)) führen nicht notwendiger Weise zur ParetoEffizienz dezentraler Lösungen. Weiterhin läßt sich gegen das Ergebnis des Wilson-Miyazaki-SpenceAnsatzes einwenden, daß der Effizienz-Begriff sehr vielfältig ist und das Ergebnis stark von der Zugreihenfolge abhängt. Zu diesen Ausführungen und der zitierten Literatur vgl. Breyer/Kolmar (1997).

Die Umstellung in Chile befindet sich noch in der Umstellungsphase und läßt sich erst in einigen Dekaden umfassend beurteilen. Dennoch zeigen sich schon jetzt einige Schwierigkeiten bzw. kann noch nicht von einer originären kompletten Privatisierung gesprochen werden. Vgl. zur chilenischen Reform z.B. Schulz-Weidner (1996); Queisser, M. (1993).

Vgl. z.B. Schmähl (1993).

Vgl. z.B. Glismann/Horn (1996).

Die Erfahrungen in Großbritannien, Irland und den Vereinigten Staaten zeigten, daß ein Großteil der „mündigen Bürger" nur durch staatliche Maßnahmen zur privaten Vorsorge zu bewegen waren. Vgl. Bodie (1990); Hughes (1994); Munnel (1991).

Es ist ebenfalls möglich, daß die überdurchschnittlich verdienende Mittelschicht nicht nur einen besseren Zugang zu lukrativen privaten Anlageformen haben, sondern auch aufgrund ihrer besseren Bildung von sich aus ein stärkeres Interesse an und eine höhere Fähigkeit zur Realisierung einer eigenen Alterssicherung haben, was gerade gegen die Voraussetzungsgerechtigkeit spräche.

107 
Der einkommensbezogenen Rente eines nach dem Lebensstandardprinzip gestalteten Alterssicherungssystems kommt eine „Erwerbseinkommensersatzfunktion“ zu, d. h. sie zielt darauf ab, die Einkommensentwicklung im Lebensablauf, sprich beim Übergang in den Ruhestand zu verstetigen. ${ }^{231}$ Es ist nicht unmittelbar ersichtlich, welcher Gerechtigkeitsgrundsatz mit dem Lebensstandardprinzip verfolgt wird. Zur Begründung der lebensstandard-orientierten Rente im Regierungsentwurf zur Rentenreform 1957 hieß es:

„Wer ein erfültes Arbeitsleben hinter sich hat, der hat es verdient, daß er nach dem Ausscheiden aus dem Arbeitsleben eine Rente erhält, die seiner Lebensarbeitsleisstung entspricht. ${ }^{232}$

In dieser Formulierung kommt zwar die Vorstellung einer leistungsgerechten Gestaltung zum Ausdruck, ${ }^{233}$ allerdings stellt sich die Frage, ob die Leistungsgerechtigkeit in Form einer lebensstandard-orientierten Rente nur über das staatliche Alterssicherungssystem realisiert werden kann.

Zur Begründung kann und wird teilweise angeführt, daß sich eine beitragsfinanzierte staatliche Zwangsversicherung nur dann legitimieren und Trittbrettfahrerverhalten verhindern läßt, wenn die Höhe der Rentenleistung sich signifikant von der Höhe der ohne Vorleistung erzielbaren Sozialhilfe unterscheidet. Der Spielraum einer Senkung auf eine Mindestabsicherung sei daher begrenzt. Strittig bleibt aber weiterhin, welche Differenz als „hinreichend signifikant" angesehen werden kann.

Bleibt zu klären, welchen Einfluß die Frage der Subsidiarität auf die Bewertung der intergenerativen Gerechtigkeit einer Substitution des Finanzierungsverfahrens ausübt?

Das Ziel, den Lebensstandard im Ruhestand zu sichern, beeinflußt zwar die Bewertung der intergenerativen Gerechtigkeit, dieser Einfluß wird aber zusammen mit der Ausgestaltung des Lebensstandardsprinzips in Abschnitt 4.4.3 zur Solidarität diskutiert. Im Zusammenhang mit der Subsidiarität steht die Frage im Vordergrund, bis zu welchem Grad diese Verstetigung des Einkommens durch die staatliche Rentenversicherung erfolgen sollte.

Alle in dieser Arbeit betrachteten Reformoptionen senken das Nettorentenniveau. Unterstellt man, daß die entstehende Versorgungslücke durch eine kapitalfundierte Vorsorge ausgeglichen wird, müßte entschieden werden, ob dies staatlich oder privat erfolgen sollte. ${ }^{234}$ Wie die voranstehenden Ausführungen allerdings verdeutlichen, erweist sich eine

231 Vgl. Schmähl (1997b), S. $6 f$.

232 Prof. K. Jantz, Ministerialdirektor im Bunderarbeitsministerium, zitiert nach Ruf (1989), S. 15. Siehe auch VDR (1987), S. 12.

233 Gegebenenfalls könne auch ein Bezug zur Regelgerechtigkeit gezogen werden, dann ist es aber erforderlich, daß die Beiträge proportional zum Einkommen erhoben werden und es sich um ein universelles Sicherungssystem handelt. Vgl. Abschnitt 4.4.2.

234 Gegen eine staatliche Lösung spräche die Gefahr, daß aufgrund politischer Begehrlichkeiten ein angesammelter Kapitalstock nicht für die Alterssicherung sondern anderweitig verwendet werden könnte. Durch eine private Vorsorge erhofft man sich zudem, die Effizienzvorteile des Wettbewerbs nutzen zu können. Aus diesen Gründen plädiert auch der Autor für eine private Lōsung.

Dennoch sollte nicht verschwiegen werden, daß im Fall einer privaten Verwaltung die Gewinnerzielungsabsicht, die höheren Verwaltungskosten aber auch und vor allem die Marketing- und zusätzlichen

108 
eindeutige Zuordnung der staatlichen Mindestsicherung (eigenverantwortliche Vorsorge) und der zwangsweisen, staatlichen Lebensstandardsicherung zu bestimmten Gerechtigkeitsgrundsätzen als äußerst schwierig. Beide Formen der Alterssicherungssysteme werden häufig mit ordnungspolitischen bzw. ideologischen Argumenten begründet. Die Folgen für die intergenerative Gerechtigkeit, die sich aus der Entscheidung für eine staatliche Mindest- oder Lebensstandardsicherung ergeben, lassen sich ebenso schwer bestimmen. Aus diesem Grund wird die Diskussion um die Legitimation einer staatlichen Organisation der Lebensstandardsicherung in dieser Arbeit nicht aufgegriffen. Dies ist auch nicht erforderlich, weil in erster Linie eine Substitution des Finanzierungsverfahrens bewertet werden soll. Das Kapitalstockverfahren kann dabei sowohl privat als auch staatlich organisiert werden und die Teilnahme kann sowohl freiwillig als auch zwangsweise sein. Die Frage nach der Organisationsform der zusätzlichen Kapitaldeckung tritt für die hier durchgeführte Gerechtigkeitsanalyse in den Hintergrund.

\subsubsection{Erfassungsgrad}

Systeme der Alterssicherung lassen sich ferner danach klassifizieren, ob sie als Universalsystem oder Kategorialsystem konzipiert sind. Ein Kategorialsystem ist dadurch gekennzeichnet, daß es lediglich bestimmte Personengruppen einbezieht. Dabei sind verschiedene Selektionskriterien denkbar. Häufig wird nach dem Beruf abgegrenzt (z.B. in Arbeiter, Angestellte, Selbständige, Beamte); ein weiteres Kriterium ergibt sich beispielsweise aus dem in der Praxis relevanten Prinzip der Erwerbs- oder Lohnzentrierung der Rente. Ebenso kann sich die Begrenzung auf Personen beziehen, deren Erwerbseinkommen innerhalb eines bestimmten Korridors liegt. Ein Universalsystem fordert, daß die gesamte Wohnbevölkerung in das Sicherungssystem einbezogen wird und zwar insbesondere auch unabhängig von der jeweiligen Biographie im Erwerbsalter.

Dem Kategorialprinzip liegt implizit die Vorstellung zugrunde, daß bestimmte Personen den Schutz der (staatlichen) Alterssicherung nicht bedürfen. Die Kategorialität eines Alterssicherungssystems wird mithin regelmäßig aus der Bedarfsgerechtigkeit abgeleitet. Die Bedarfsgerechtigkeit kann jedoch ebenso herangezogen werden, um die Universalität eines Systems zu rechtfertigen. Dies gilt insbesondere dann, wenn - wie beispielsweise beim Beveridge-Plan - die Universalität mit dem Ziel der Mindestsicherung zur Armutsvermeidung verbunden wird. ${ }^{235}$ Denn solange alle Personengruppen einer Gesellschaft -

Inkassokosten einen Großteil der Rentabilitätsvorteile verzerren könnten. Darüber hinaus zeigen die Erfahrungen in Großbritannien, Irland und den Vereinigten Staaten, daß die aus Anreizmotiven (z.B. Steuervergünstigungen) herrührenden Kosten einer Privatisierung der Altersvorsorge durchaus bedeutend und nicht zu vernachlässigen sind. Vgl. Bodie, Z. (1990); Hughes, G. (1994); Munnel, A. (1991).

235 Das im Beveridge-Plan entworfene System der Alterssicherung hatte nach dem Zweiten Weltkrieg einen entscheidenden Einfluß auf die Gestaltung nicht nur des britischen, sondem auch einer Reihe weiterer europäischer Sozialsysteme. Die Gestaltung der Sozialen Sicherung sollte sich entsprechend der Empfehlung des Beveridge-Plans nach den folgenden Grundsätzen richten:

- Universalität des Sicherungssystems

- Sicherung einer Mindestversorgung (vornehmlich zur Armutsvermeidung)

- Gestaltung als Versicherungssystem, 
ob erwerbstätig oder nicht - sozial und ökonomisch heterogen zusammengesetzt sind, kann für keine dieser Gruppen ein Sicherungsbedarf a priori ausgeschlossen werden. ${ }^{236}$ Der entscheidende normative Unterschied zwischen den alternativen Gestaltungsprinzipien besteht darin, daß in einem für die gesamte Bevölkerung obligatorisches Universalsystem der Grundsatz der Regelgerechtigkeit stärker betont wird als in einem Kategorialsystem.

Die aus den unterschiedlichen Alterssicherungssystemen (=unterschiedliche Regeln) resultierenden Verteilungswirkungen und ihre Folgen für die Gerechtigkeit des Alterssicherungssystems sind allerdings vornehmlich intragenerativer Art. Wenngleich sich ein dringender Harmonisierungsbedarf des deutschen Alterssicherungssystems erkennen läßt, werden die Konsequenzen, die sich aus der Entscheidung für ein Universal- oder Kategorialsystem ergeben, in der vorliegenden Analyse der intergenerativen Gerechtigkeit folglich nicht erörtert. ${ }^{237}$

\subsubsection{Solidarität}

"Das Solidaritätsprinzip sagt, daß eine bestimmte Solidarität in einem sozialen Verband herrschen soll. Das Subsidiaritätsprinzip sagt, in welchem Verband eine bestimmte Solidarität herrschen soll. ${ }^{238}$

Während das Subsidiaritätsprinzip sich auf die Frage der Zuständigkeit bezieht, ist das konstituierende Merkmal eines solidarischen Systems bzw. einer solidarischen Gruppe, daß die Teilnehmer bzw. Mitglieder sich als Einheit verstehen und - bis zu einem bestimmten Grad - füreinander einstehen. Bei einem System der sozialen Sicherung geht dies regelmäßig mit einem gewissen $\mathrm{Ma} ß$ an Umverteilung einher.

\subsubsection{Systeme solidarischer Absicherung}

Innerhalb der in Abschnitt 4.1 festgelegten Systemgrenzen lassen sich Alterssicherungssysteme hinsichtlich des sozialen Ausgleichs in zwei Kategorien einteilen. Alterssicherungssysteme, die auf dem Äquivalenzprinzip beruhen bezeichnet man als Vorsorgesysteme. ${ }^{239}$ Demgegenüber stehen die sogenannten Versorgungssysteme (Fürsorgesysteme), bei denen der Zusammenhang zwischen Vorleistungen (Beiträge) und Rentenleistungen vollends aufgehoben und eine (massive) interpersonelle Umverteilung der Einkommen intendiert ist.

Versorgungssysteme beruhen daher vornehmlich auf dem Konzept der Ergebnisgerechtigkeit und gegebenenfalls der Bedarfsgerechtigkeit, während in den Vorsorgesystemen

Woraus sich die Forderung nach einheitlichen Beiträgen und Leistungen ableiten läßt. Vgl. Beverage (1943); Döring (1992).

236 Döring (1992), S.12/13. Beveridge (1943), Tz 118.

237 Indirekte intergenerative Wirkungen könnten sich bestenfalls ergeben, wenn sich das Verhältnis aus versicherungspflichtigen Arbeitnehmem zu unselbständig Erwerbstätigen in Zukunft, beispielsweise aufgrund der zunehmenden Bedeutung des Dienstleistungssektors, gravierend verändert.

Schlotter H.-G. (1984), S. 77 (Hervorhebungen kursiv im Original). 
die Leistungsgerechtigkeit (mehr oder weniger) zum Tragen kommt. Das Ausmaß des in einem Alterssicherungssystem verwirklichten sozialem Ausgleichs hängt mithin entscheidend davon ab, inwieweit Versorgungs- bzw. Fürsorgekomponenten im System enthalten sind.

Der Solidarausgleich beschränkt sich jedoch nicht ausschließlich auf die Existenz von Komponenten der Versorgung, sondern kann ebenfalls systematischer Bestandteil der Vorsorgesysteme sein. Lediglich die extreme Form der Vorsorge - die reinen, individuellen Sparmodelle - sind frei von Umverteilungen. Eine weit größere Bedeutung innerhalb der Kategorie der Vorsorgesysteme haben die Versicherungen (vgl. Abbildung 9).

Abbildung 9: Ausmaß an sozialem Ausgleich (Solidarität) bei verschiedenen

Absicherungssystemen

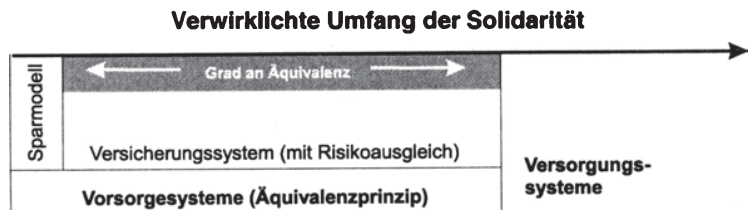

Quelle: eigene Darstellung

Die einer Versicherung zugrundeliegende Solidarität zielt weniger auf eine Nivellierung der Einkommensungleichheit im Sinne einer finalen Ergebnisgerechtigkeit, ${ }^{240}$ sondern basiert auf der Risikoaversion der einzelnen Wirtschaftssubjekte. Da "bei Versicherungen jeder Beiträge in Höhe des Geldwertes seines Risikoschutzes zahlt", ${ }^{241}$ sollte der Abschluß eines Versicherungsvertrags mithin als Erwerb des Gutes „Sicherheit“ interpretiert werden.

„Versicherungen sind Solidargemeinschaften vieler von einer gleichen Gefahr bedrohter Menschen, die das Risiko der Gefahr gemeinsam tragen. ${ }^{242}$

Im Unterschied zum reinen Sparmodell arbeitet ein Versicherungssystem folglich mit einem Risikoausgleich, um den Vorteil zu nutzen, daß die Höhe der risikoäquivalenten Versicherungsprämien geringer ist als eine individuelle Vorsorge gegen das maximale Risiko. $\mathrm{Zu}$ den verschiedenen Risiken der Altersvorsorge gehört in erste Linie die Unsicherheit über die individuelle Lebensdauer. Lebt der Versicherte - überdurchschnittlich - lange, so besteht die Gefahr einer unzureichenden Altersversorgung; im umgekehrten Fall hätte er zuviel vorgesorgt. Weitere relevante Risiken sind die Erwerbsunfähigkeit und die Versorgung der Hinterbliebenen im Todesfall. Für das Individuum sind diese Risiken sehr schwer einzuschätzen und stellen daher ein gravierendes Unsicherheitsmoment dar. In

239 Vgl. Schmähl (1997), S. 417f..

240 Vgl. Watermann, F. (1981), S. $298 \mathrm{ff.}$

241 Meinhold, H. (1976), S. 34. Vgl. zu dieser Interpretation einer Versicherung als Anbieter des Gutes Sicherheit auch Arrow (1970); Lucius (1979); Reichel (1981); Wagner (1984); Engels (1969).

242 Glismann/Horn (1995), S. 18. Zur Meinungsverschiedenheit über die Definition des Begriffs des Versicherungsprinzips vgl. Wagner (1984), S. 6f. und die dort angegebene Literatur. 
einer hinreichend großen Gemeinschaft können allerdings auch diese Risiken aufgrund des Gesetzes der großen Zahlen kalkuliert werden. ${ }^{243}$

Wenngleich die Versichertengemeinschaft im Risikofall für den Betroffenen eintritt und ein Versicherungssystem somit solidarische Gestaltungselemente aufweist, beruht ein System der Altersver sicherung nach wie vor auf dem Äquivalenzprinzip, d.h. die aus dem System zu erwartenden Leistungen ergeben sich regelmäßig aus den Vorleistungen. Das Äquivalenzprinzip verlangt lediglich, $d a ß$ ein funktionaler Zusammenhang zwischen Beiträgen und Leistungen bestehen soll.

Eine besondere Variante des Äquivalenzprinzips ist die Beitragsäquivalenz, bei der die Höhe der gesamten Rentenleistungen - unter Berücksichtigung einer Auf- bzw. Abzinsung - der Höhe der zuvor geleisteten Beitragszahlungen entspricht. Aber auch die Beitragsäquivalenz kann je nach Abgrenzung der Einheit (Individuum oder Kollektiv/Kohorte), auf die sich die Beiträge und Leistungen beziehen, bzw. je nach Abgrenzung der Risikogemeinschaft (gesamte Versichertengemeinschaft oder Teilgruppen von Versicherten) spezifiziert werden. Zur Verdeutlichung wird an dieser Stelle vornehmlich zwischen der Globaläquivalenz, der aktuarischen Beitragsäquivalenz und den risikoäquivalenten Prämien unterschieden, wobei ausdrücklich darauf hingewiesen werden muß, daß die Termini und die Abgrenzung in der Literatur keineswegs einheitlich verwendet werden.

- Die Globaläquivalenz ist dann erfüllt, wenn die Summe aller Beiträge einer Versicherungsanstalt die gesamte Summe aller Leistungen deckt. Die Globaläquivalenz darf nicht mit der periodenbezogenen Äquivalenz von Einnahmen und Ausgaben eines umlagefinanzierten Alterssicherungssystems verwechselt werden. Der für die Globaläquivalenz maßgebliche Zeitraum wird weiter gefaßt und zwar auf die Deckung der gesamten Beitrags- und Leistungssummen einer Kohorte. ${ }^{244}$

- Resultieren die Umverteilungseffekte in einem Versicherungssystem ausschließlich aus dem Eintreten des Versicherungsfalls und entsprechen die Erwartungswerte der Rentenleistungen des Einzelnen unter Berücksichtigung versicherungsmathematischer Zusammenhänge den zuvor geleisteten Beitragszahlungen, so liegt eine (individuelle) aktuarische ${ }^{245}$ Beitragsäquivalenz vor. ${ }^{246}$ Formal gesprochen bedeutet dies, daß die

243 Vgl. Eisen (1980), S. 542.

244 Vgl. Wasem (1988), S. 26; Kolb (1985), S. 123f.; Wagner (1984), S. 21/55; Thullen (1981), S. 498ff.; Innami (1966), S. 17. Kreßmann (1971) und Koppelmann (1979) hingegen bezeichnen die Globalăquivalenz als Gruppenăquivalenz, was um so verwirrender sein kann, weil Kolb (1985) beispielsweise unter dem Ausdruck Gruppenäquivalenz in etwa das bezeichnet, was in dieser Arbeit als aktuarische Beitragsäquivalenz umschrieben wird. Zuweilen wird für die Globaläquivalenz auch der Ausdruck „kollektive Äquivalenz" venwandt. Vgl. Saxer (1955).

245 Aktuarische Prämien bzw. Beitrăge bedeutet, daß sie proportional zu den Eintrittswahrscheinlichkeiten der Risiken gestaltet sind. Vgl. Eisen (1980), S. 543.

246 Kreßmann (1971), S. 235; Koppelmann (1979), S. 24; Wagner (1984), S. 21, bezeichnen diese Form der Äquivalenz als ${ }_{n}$ individuelle Äquivalenz" $z^{4}$ bzw. "Individualäquivalenz". Kolb (1985), S. 125 spricht in diesem Fall von "Gruppenäquivalenz", die sich aus den Grundsătzen der ${ }_{n}$ gerechten Prämie" und des "Nominalprinzips" zusammensetzt. "Beitragsäquiva!enz" versteht die Wissenschaftlergruppe des Sozialbeirats (BT-Drucksache 9/632, S. 31 vom 3.7.1981) als Überbegriff, zu dem sowohl die (in dieser Arbeit so

112 
auf den Zeitpunkt des Vertragsabschlusses diskontierten Barwerte der Beitragszahlungen und der erwarteten Rentenleistungen eines Versicherten sich entsprechen müssen. ${ }^{247}$ Von Bedeutung ist, daß diese Definition ex ante, d.h. bei Berücksichtigung der Wahrscheinlichkeiten des Risikoeintritts, bzw. bezogen auf das Durchschnittsrisiko gilt. Ex post können - je nach Realisation des Risikos - die Beiträge und Leistungen im Einzelfall durchaus voneinander abweichen. ${ }^{248}$

Die maßgebliche Risikogemeinschaft deckt sich bei der aktuarischen Beitragsäquivalenz zwar weiterhin mit der der Globaläquivalenz, allerdings ist die Bezugseinheit nicht mehr wie bei der Globaläquivalenz die gesamte Kohorte, sondern ein durchschnittliches Individuum der Risikogemeinschaft.

- Ob der Versicherungsfall bei einem Individuum eintritt, ist zwar grundsätzlich zufallsabhängig, dennoch können die Wahrscheinlichkeiten nach Maßgabe bestimmter Merkmale (beispielsweise Gesundheitszustand, Geschlecht, Bildungs- und Einkommensniveau etc.) differieren. ${ }^{249}$ Geht man aus diesem Grunde zur gruppenbezogenen Beitragsäquivalenz über und bestimmt die Beiträge (Prämien) gesondert nach Risikogruppen mit gleichen bzw. ähnlichen Wagnissen, spricht man von risikoäquivalenten Prämien. Risikoäquivalente Prämien können als eine Spezialform der aktuarischen Beitragsäquivalenz interpretiert werden, da auch bei innen das Individuum als Bezugseinheit zugrunde gelegt wird, die Risikogemeinschaft aber weiter differenziert wird. $^{250}$

Das Schema, mit dem die Gestaltungsmöglichkeiten der Beitragsäquivalenz klassifiziert werden können, ist in Abbildung 10 dargestellt.

bezeichnete) Beitrags- als auch Teilhabeăquivalenz zugeordnet wird. Diese Abgrenzung kann jedoch ebenfalls mißverständlich sein, da die Leistungen bei der Teilhabeáquivalenz nur indirekt über die Einkommen an die Beiträge gekoppelt sind. Vgl. z.B. Kolb (1985), S. 121f..

247 Denkbar wäre auch die Wahl des Renteneintritts als Diskontierungszeitpunkt, in dem Fall wäre jedoch zu klären, wie die Beiträge jener Versicherten behandelt werden, die vor Erreichen des Renteneintrittsalters verstorben sind. Vgl. Dinkel (1985), S. 348. Vgl. Wagner (1984), S.6.

Innerhalb einer Alterskohorte können beispielsweise die Risiken eines langen Lebens zwischen verschiedene Gruppen variieren. Die unterschiedliche Restlebenserwartung ist dabei nicht nur geschlechtsspezifisch (Dinkel 1983), vielmehr zeigen Auswertungen des Sozio-ökonomischen Panels, daß Angehörige unterer Einkommens- und Bildungsschichten eine geringere Lebenserwartung aufweisen, vgl. Klein (1995), Voges/Schmidt (1995). Während Wagner (1994), S. 44, einen solchen Risikoausgleich als gerecht einstuft (sofern man den Vertragsabschluß weit genug - ggf. sogar vor den Zeitpunkt der Geburt vordatiert), wird dies von Breyer (1997), S. 172-174, bestritten.

Vgl. Kreßmann (1971), S. 235 und Koppelmann (1979), S. 24. Sie bezeichnen diese Form der Beitragsăquivalenz als ngerechte Prämie“. 
Abblidung 10: Gestaltungsmöglichkeiten der Beitragsäquivalenz

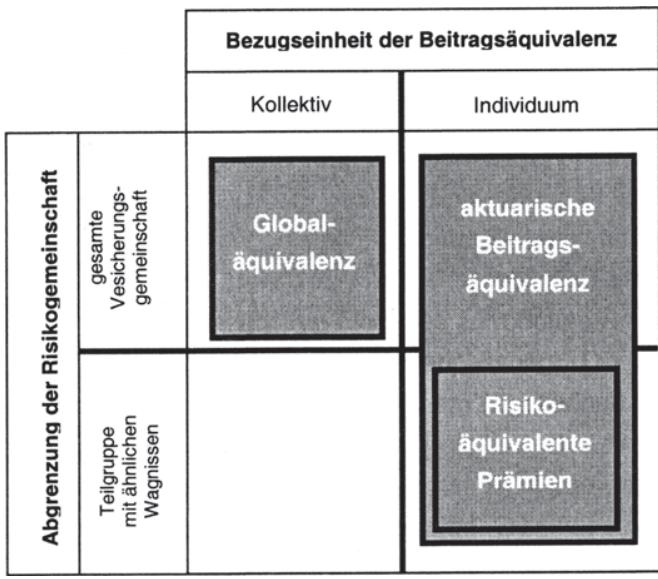

Quelle: eigene Darstellung

Anhand dieser Gestaltungsmöglichkeiten der Beitragsäquivalenz läßt sich im folgenden verdeutlichen, welchen Einfluß die Gestaltungsprinzipien eines Alterssicherungssystems auf die Bewertung der Verteilungswirkungen ausüben. Je nachdem, welches Konzept der Beitragsäquivalenz man als verteilungsneutrale Referenz einer Distributionsanalyse zugrunde legt, wird nämlich der intragenerative Risikoausgleich und somit auch die intragenerative Umverteilung eines Alterssicherungssystems unterschiedlich definiert.

A) Einfluß der unterschiedlichen Abgrenzung der Risikogemeinschaft auf die Beurteilung der Verteilungswirkungen eines aktuarisch beitragsäquivalenten Systems

Frauen und Angehörige höherer Einkommens- und Bildungsschichten erzielen in einem Alterssicherungssystem aufgrund ihrer höheren Lebenserwartung tendenziell höhere Renditen. Innerhalb eines Systems mit aktuarischer Beitragsäquivalenz, das nicht zwischen verschiedenen Risikoklassen differenziert, werden diese Verteilungswirkungen als Bestandteil des Risikoausgleichs angesehen. Sie sind versicherungsimmanent und somit gerecht. Wird hingegen ein System als Referenzmaßstab herangezogen, bei dem risikogerechte Prämien für verschiedenen Risikoklassen erhoben werden, würden die unterschiedlichen Renditen als ungerechte, interpersonelle Umverteilung gewertet werden. Je nach Abgrenzung der Risikoklassen können ähnliche Abweichungen in der Beurteilung des Solidarausgleichs z.B. bei der Bewertung der Erwerbsunfähigkeits- und Hinterbliebenenrenten auftreten. 
B) Einfluß der Bezugseinheit der Beitragsäquivalenz auf die Beurteilung der Verteilungswirkungen (aktuarische Beitragsäquivalenz vs. Globaläquivalenz)

Zu den maßgeblichen Bestimmungsfaktoren der aktuarischen Beitragsäquivalenz gehören neben der Dauer der Beitragszahlungen und der Rentenleistungen (Zeitfaktoren) auch die Höhe der Beiträge und der Rentenleistungen (Einkommensfaktoren). ${ }^{251}$ Es wurde eingangs bereits erwähnt, daß ein System sich zwar grundsätzlich an der aktuarischen Beitragsäquivalenz orientieren, die strikte Aktuarität jedoch zum Teil durch die Integration von Versorgungs- bzw. Fürsorgeelementen „aufgeweicht“ werden kann, um politisch motivierte Umverteilungen zu ermöglichen. ${ }^{252}$ Dies erfolgt durch eine Modifikation der Zeitoder der Einkommensfaktoren:

- So kann der Zeitfaktor der Leistungsseite vernachlässigt werden, indem die Dauer des Rentenbezugs bei der Bestimmung der Rentenhöhe außer Acht gelassen wird ${ }^{253}$ oder indem im Todesfall eine Hinterbliebenenrente gewährleistet wird. ${ }^{254}$

- Hinsichtlich des Zeitfaktors der Beitragszahlungen ist es möglich, daß die Versicherungsdauer bei der Bestimmung der Rentenhöhe nicht proportional bzw. nicht linear in die Berechnung einfließt, ${ }^{255}$ hinsichtlich ihres Einflusses auf die Rentenhöhe durch Grenzwerte eingeschränkt wird oder durch die Berücksichtigung von beitragsfreien Versicherungszeiten ${ }^{256}$, Mindestrenten o.ä. verzerrt wird.

- Begrenzungen der aktuarischen Beitragsäquivalenz können auch am Einkommensfaktor der Beitragsleistungen ansetzen, beispielsweise wenn nicht das gesamte beitagspflichtige Lebenserwerbseinkommen, sondern nur das Erwerbseinkommen der letzten oder der "besten“ Erwerbsjahre in die Rentenberechnung einfließen. Inwieweit dem Prinzip der aktuarischen Beitragsäquivalenz entsprochen wird, hängt ebenfalls davon $a b$, ob und in welcher Weise die Beiträge bzw. Einkommen vergangener Jahre aufgewertet werden.

251 Vgl. Kolb (1985), S. 131ff..

252 Vgl. Wasem (1988), S. $28 f$.

253 Dies gilt sowohl für die Wahl des Zeitpunkts zum Eintritt in den Ruhestand als auch für längere Rentenbezugszeiten infolge steigender Lebenserwartung. Breyer (1997), S. 169, bezieht sich auf diesen Sachverhalt, wenn er an der derzeitigen Ausgestaltung der Teilhabeăquivalenz kritisiert, daß zwei nicht vergleichbare Zahlungen einander gegenübergestellt werden: Die Summe der Beiträge während des gesamten Erwerbsleben und die Rentenansprüche pro Zeiteinheit. Vgl. auch Rürup/Schroeter (1996); Rūrup/Schroeter (1997).

Diese Sichtweise entspricht der traditionellen Auffassung (vgl. z.B. Breyer (1990a), S.129; Wasem (1988); Urteil des Verfassungsgerichts 1980 (BverfGE 53/S.290); Dinkel (1985); Berthold (1986), S. 355). Man mag allerdings auch argumentieren, daß die Möglichkeit, versorgungsbedürttige Angehörige zu hinterlassen, als ein zu versicherndes Risiko interpretiert werden könne, so daß eine Hinterbliebenensicherung nicht zwangsläufig eine Durchbrechung des Prinzips der Beitragsăquivalenz bedeutet, vgl. Kolb (1985); Wagner (1985).

Dies ist z.B. noch in dem derzeitigen österreichischen Pensionssystem der Fall, bei dem die ersten dreiBig Versicherungsjahre durch den sogenannten "Hundertsatz des Steigerungsbetrags" systematisch höher bewertet werden.

Für Erziehung, Ausbildung, Zivil- und Wehrdienst, Krankheit etc..

115 
Wählt man die aktuarische Beitragsäquivalenz als Referenzmaßstab einer Verteilungsanalyse, so werden diese Modifikationen der Zeit- bzw. Einkommensfaktoren als interpersonelle Umverteilung identifiziert und zwar unabhängig davon, ob zusätzlich nach Risikoklassen differenziert wird. Sofern diese Umverteilungen allerdings nur innerhalb einer Kohorte erfolgen, werden sie mit Hilfe der Globaläquivalenz nicht als Umverteilung ausgewiesen.

Die wesentlichen Ergebnisse dieses Abschnitts können mithin wie folgt zusammengefaßt werden:

Der soziale Ausgleich bzw. genauer die Verteilungswirkungen innerhalb eines Versicherungssystems können danach unterschieden werden, ob es sich um eine Verteilung innerhalb des versicherungsmäßigen Risikoausgleichs von den „Glücklichen“ zu den „Unglücklichen" handelt oder ob es sich um eine reine - ob politisch intendiert oder nicht, sei dahingestellt - Umverteilung handelt. Als Referenz zur Messung dieser intragenerativen Verteilungen kann die aktuarische Beitragsäquivalenz herangezogen werden. Entscheidend für das Ergebnis der Verteilungsanalyse ist allerdings, wie der versicherungsmäßige Risikoausgleich abgegrenzt wird. Dabei gilt, daß der Risikoausgleich um so enger definiert ist, je stärker bei der aktuarischen Beitragsäquivalenz nach Risikoklassen differenziert wird (risikoäquivalente Prämien). Erfolgen alle Umverteilungen innerhalb einer Kohorte, so werden bei einer Verteilungsmessung mit Hilfe der Globaläquivalenz keine Umverteilungen angezeigt. Dies legt den Schluß nahe, daß die Globaläquivalenz sich immer dann als Verteilungsmaßstab anbietet, wenn von der intragenerativen Umverteilung innerhalb einer Kohorte abstrahiert werden soll. ${ }^{257}$

Der letzte Aspekt richtet die Aufmerksamkeit auf einen bislang noch nicht angesprochenen Blickwinkel eines Versicherungssystems. Die bislang betrachtete Differenzierung von Verteilungswirkungen in „versicherungsimmanenter Verteilung“ und „systematischer Umverteilung“ bezog sich ausschließlich auf den interpersonellen Risikoausgleich. Ein Risikoausgleich ist aber ebenfalls zwischen Generationen denkbar. Dieses intergenerative, versicherungsmäßige Solidarprinzip hängt entscheidend von den Gestaltungsprinzipien eines Alterssicherungssystems ab (Gestaltung der Rentenformel) und ist Gegenstand des folgenden Abschnitts. ${ }^{258}$

257 Vgl. auch Wagner (1984), S. 55.

258 Wenn im folgenden der Begriff der "Beitragsăquivalenz" genannt wird, dann ist er - wenn nicht anders erwähnt - als allgemeiner Überbegriff oder im Sinne der Globalăquivalenz zu verstehen. 


\subsubsection{Solidarische Absicherung aus intergenerativer Perspektive}

Bislang wurden die Formen solidarischer Absicherung in einem Alterssicherungssystem (Versorgung vs. Versicherung) allgemein betrachtet. Zum einen ließen sich dadurch erste Erkenntnisse darüber ableiten, wie ein Verteilungsindikator zu gestalten ist, um die intergenerativen Verteilungswirkungen von interpersonellen Verteilungswirkungen abzugrenzen. Die allgemeine Betrachtung verdeutlichte zum anderem, wie Verteilungswirkungen aufgrund verschiedener Gestaltungsprinzipien unterschiedlich bewerten werden. Im folgenden geht es darum, diese Zusammenhänge, sprich den Solidarausgleich einer Versicherung auf die intergenerative Perspektive zu übertragen.

Im Vordergrund der Betrachtung steht dabei das Ziel, den Lebensstandard im Alter zu sichern. Dieses Ziel umfaßt im Grunde verschiedene Aspekte und wird daher durch mehrere Gestaltungsprinzipien umgesetzt:

- Die absolute Höhe der Rentenleistung bestimmt das Ausmaß der Einkommensstetigkeit beim Übergang in den Ruhestand (individuelle Einkommensersatzfunktion). Die Frage, bis zu welchem Ausmaß die gesetzliche Alterssicherung diese Verstetigung des Einkommens übernehmen soll, wird vornehmlich durch den Umfang der Subsidiarität in der gesetzlichen Alterssicherung bestimmt. ${ }^{259}$

- Es läßt sich jedoch nicht mit Sicherheit vorhersagen, ob diese absolute Höhe der Rentenleistung den Lebensstandard beim Eintritt in den Ruhestand auch tatsächlich sichern kann. Denn angesichts sich verändernder demographischer und ökonomischer Rahmenbedingungen sagt die Höhe der zu erwartenden Rente nichts über ihren zukünftigen Realwert aus. Der zweite Aspekt der Lebensstandardsicherung umfaßt daher die „Versicherung der relativen Einkommensposition“, er wird durch zwei Gestaltungsprinzipien konkretisiert:

Das Prinzip der Teilhabeäquivalenz und eine am durchschnittlichen Erwerbseinkommen orientierte Rentengewährung.

Eine auf der Teilhabeäquivalenz beruhende Rentenformel zielt darauf $a b$, daß ein Rentner hinsichtlich seiner individuellen Rentenhöhe im Vergleich zu allen Rentnern seiner Kohorte rangmäßig die gleiche Einkommensposition einnehmen soll, die er in der Einkommenspyramide der beitragspflichtigen Erwerbstätigen eingenommen hat. Als "Versicherungsobjekt" steht bei der Teilhabeäquivalenz vor allem die Sicherung der relativen Einkommensposition bzw. des "relativen Lebensstandards" im Vordergrund. Sofern ein konstantes (Netto-)Rentenniveau die Leistungshöhe zudem an die Erwerbseinkommen der aktiv Versicherten koppelt, ist der relative Lebensstandard nicht nur innerhalb der Kohorte (Teilhabeäquivalenz), sondern auch gegenüber den Er- 
werbstätigen gesichert. Eine solche Rentenformel stellt eine relative Lebensstandardsicherung im engeren Sinne dar. ${ }^{260}$

- Die Veränderungen der Rahmenbedingungen werden durch teilhabeäquivalente Renten und die Kopplung der Rentenformel an die durchschnittliche Lohnentwicklung für die Dauer der Versicherungszeit bis zum Eintritt in den Ruhestand berücksichtigt. Der Grundgedanke, den Lebensstandard gegen ungewisse Entwicklungen zu versichern, muß jedoch nicht auf die Bestimmung der Rentenhöhe beim Eintritt in den Ruhestand beschränkt werden. Erweitert man das enwerbseinkommenszentrierte Teilhabekonzept auf die gesamte Periode des Rentenbezugs, so führt dies zum dritten Aspekt des Lebensstandardprinzips: der dynamischen Rente (Rentenanpassung). Die Idee der dynamischen Rente besteht darin, auch die Ruheständler am allgemeinen Wohlstandsfortschritt teilhaben zu lassen. In der praktischen Ausgestaltung der Alterssicherung wird die Idee der dynamischen Rente meist realisiert, indem die Höhe der Renten mit den durchschnittlichen Lohnzuwächsen fortgeschrieben wird. Dies kann grundsätzlich nach Maßgabe der Netto- oder der Bruttolöhne erfolgen. ${ }^{261}$

Ein auf dem Prinzip der Teilhabeäquivalenz beruhendes Alterssicherungssystem mit konstantem Rentenniveau ist demnach weiterhin ein Versicherungssystem, legt aber im Unterschied zur (streng aktuarischen) Beitragsäquivalenz mehr Gewicht auf das Prinzip eines intergenerativen solidarischen Ausgleichs.

Die Erweiterung des solidarischen Risikoausgleichs um eine zeitliche bzw. intergenerative Komponente erscheint deshalb als sinnvoll, weil die Altersversicherung im Gegensatz zu anderen Versicherungen (wie beispielsweise der Unfall-, Kranken- oder Arbeitslosenversicherung) durch einen besonders langen Zeithorizont von rd. 60-70 Jahren geprägt ist. Infolge einer solch langen Planungsperiode ergeben sich zwangsläufig Unsicherheiten über die zukünftigen demographischen Veränderungen (Fertilität, Mortalität) aber auch über die gesamtwirtschaftliche Entwicklung (Wirtschaftswachstum, Erwerbsquoten, Invaliditätswahrscheinlichkeit, Zinsentwicklung, Inflation etc.). ${ }^{262}$ Angesichts dieser Ungewißheit über die demographische und ökonomische Entwicklung kann der Versicherte sein in der Zukunft realisiertes (reales) Versorgungsniveau im Alter nicht oder bestenfalls nur annä-

260 Bezugnehmend auf die Mackenrothsche These erachtet Dinkel eine Kopplung an ein konstantes (Netto-)Rentenniveau als einzig geeignete Rentenformel wenn er schreibt: „Da die Rentner bei jeder denkbaren Ausgestaltungsform stets aktuelles Sozialprodukt beanspruchen, ist für eine ${ }_{n}$ große" Sozialversicherung die Anknüpfung an die aktuellen Erwerbseinkommen zugleich die einzig logische und in jeder ökonomischen Ausgleichslage auch finanzierbare Lösung." Dinkel (1985), S. 357.

261 Eine in verschiedenen Alterssicherungssystemen praktizierte Alternative ist die Kopplung an einen Preisindex. In dem Fall würde die Sicherung des Lebensstandards relativ zu dem der Erwerbstätigen jedoch nach Eintritt in den Ruhestand aufgegeben werden.

262 Vgl. Dinkel (1985), 348. Wagner (1985), S. 153: „Das Prinzip der Teilhabeäquivalenz (...) hat die Funktion, dem einzelnen Versicherten in der Dynamik des Zeitverlaufs, in der Entwicklung seiner persönlicher Verhältnisse ebenso wie im Rahmen der gesellschaftlichen Entwicklung , eine gesicherte Position zuzuweisen, auf die er vertrauen und mit der er seine Alterssicherung kalkulieren kann." Kolb (1985), S. 134. Vgl. auch Wagner (1984), S. 168. Wobei der Versicherte an drei Elinkomensschicksale gebunden ist, seinem eigenen, das seiner und das der nachfolgenden Generation. Vgl. Kolb (1985), S. 128. 
hernd kalkulieren. ${ }^{263}$ Mit anderen Worten, kann ein Versicherter sich durch eine beitragsäquivalente Gestaltung zwar dagegen absichern, daß der Eintritt des Versicherungsfalls bei ihm vom durchschnittliche Risiko abweicht (interpersoneller Risikoausgleich), ob und in welchem Ausmaß sich diese durchschnittlichen Risiken in Zukunft verändern werden, läßt sich nicht antizipieren. ${ }^{264}$

Es ist daher nicht verwunderlich, daß auch in einer privat organisierten und kapitalgedeckten Alterssicherung der genaue, „beitragsäquivalente“ Anspruch immer erst im nachhinein festgestellt werden $k a n n^{265}$ und er regelmäßig nach dem Vorsichtsprinzip, sprich mit Sicherheitszuschlägen kalkuliert wird. Letztlich kann auch eine privatwirtschaftlich organisierte Lebensversicherung, die „nur" eine Mindestverzinsung bietet und ihre Gewinne erst ex post und i.d.R. proportional zu den geleisteten Beiträgen ausschüttet, keine strikte Beitragsäquivalenz, sondern - zu einem gewissen $\mathrm{Ma}$ - ebenfalls nur eine anteilsgerechte Ausschüttung garantieren. ${ }^{266}$ In einer auf der Teilhabeäquivalenz beruhenden Sozialversicherung ist ein solches Prinzip der Anteilsgerechtigkeit lediglich stärker ausgeprägt und zwar in Form der Sicherung des relativen Lebensstandards. In beiden Fällen kann die anteilsgerechte Aufteilung jedoch als intergenerativer Risikoausgleich interpretiert werden.

Je nach Eintritt des Risikos (tatsächliche Entwicklung) werden dabei die verschiedenen Generationen unterschiedlich belastet oder begünstigt. ${ }^{267}$ Von der Frage, welche dieser Risiken sich durch eine Versicherung abdecken lassen, hängt es mithin ab,

„wie die Äquivalenz von Beiträgen und Leistungen unter Berücksichtigung eines intergenerationalen Risikoausgleichs ökonomisch sinnvoll definiert werden kann. ${ }^{268}$

Die Kopplung der Rentenleistungen an die durchschnittlichen Einkommen und die Teilhabeäquivalenz sind solidarische Gestaltungsprinzipien zur Sicherung des Lebensstandards. Wie die Ergebnisse des Abschnitts 2.2 zeigen, wäre ein teilhabeäquivalentes und lebensstandardsicherndes Rentensystem im Kapitalstockverfahren instabil und müßte im Umlageverfahren organisiert sein. Das Ausmaß des angestrebten intergenerativen Solidar- bzw. Risikoausgleichs ist daher wichtig für die Bewertung der intergenerativen Gerechtigkeit einer Substitution des Finanzierungsverfahrens.

Vgl. Schulenburg (1989), S.276: „Beim Kapitaldeckungsverfahren hat eine positive Lohnsteigerungsrate einen verteuemden Effekt, da die zukünftigen Renten relativ höher im Vergleich zum jetzigen Einkommen sind, aus dem der Kapitalbetrag finanziert werden muß, der später Rentenzahlungen ermöglichen soll."

Vgl. Wagner (1984), S. 28.

Vgl. Meinhold (1985), S. 16.

Vgl. Wagner (1985), 152f. und Wagner (1984), S. 81f, Dinkel (1985), S. 350/351.

Vgl. Wagner (1984), S. $62-74$ und 81ff., exemplarisch S. 63: „Es kann daher der Fall sein, daß eine Generation stärker belastet wird, als eine andere; bei völlig unvorhersehbaren Entwicklungen könnte man darauf zurūckführende Belastungsänderungen jedoch als versicherungsmäßigen, d.h. gesamtwirtschaftlich effizienten Risikoausgleich zwischen verschiedenen Generationen ansehen." Vgl. auch die dort angegebene Literatur.

Wagner (1985), S. 147.

119 
Abschließend sei auf einen weiteren, zwar trivialen aber dennoch bedeutenden Zusammenhang des intergenerativen Solidarausgleichs hingewiesen. In Zeiten eines hohen wirtschaftlichen Wachstums sollen nicht nur die Erwerbstätigen, sondern auch die Ruheständler am steigenden Wohlstand partizipieren, umgekehrt bewirken die Kopplungsmechanismen der Erwerbseinkommenszentrierung, der Teilhabeäquivalenz und der dynamischen Rente, daß die Rentner bei ungünstiger Entwicklung der Rahmenbedingungen auch die Zusatzlast mit den Erwerbstätigen tragen. Diese Funktionsweise der Teilhabeäquivalenz und der dynamischen Rente verdeutlicht, daß Solidarität - insbesondere in der Alterssicherung - nicht ausschließlich als unidirektionales Prinzip, sprich als eine einseitige Verbesserung für die Leistungsempfänger oder als ein einseitiger Schutz der gewährten Sozialleistungen verstanden werden darf. Die Solidarität der Leistungsempfänger mit den Beitragszahlern ist ebenso Bestandteil dieses Prinzips, wie die Solidarität zur nicht enwerbstätigen Generation. ${ }^{269}$

Selbst wenn eine Alterssicherung daher grundsätzlich als teilhabeäquivalentes Rentensystem konzipiert ist und die Rentenhöhe nach Maßgabe der durchschnittlichen Löhne festsetzt, kann ihre Umsetzung in konkrete Vorschriften der Idee eines solidarischen, versicherungsmäßigen Ausgleichs zwischen den Generationen widersprechen und zu einer systematischen Umverteilung führen. ${ }^{270}$

\subsubsection{Gestaltungsprinzipien der gRV und Bewertung der intergenerati- ven Verteilungswirkungen}

Die vorangehenden Betrachtungen der verschiedenen Gestaltungsmöglichkeiten eines Alterssicherungssystems ergaben, daß die Solidarität bzw. konkreter der soziale Ausgleich in Versicherungssystemen die Bewertung einer Substitution des Finanzierungsverfahrens beeinflußt. Sofern die Ziele und Gestaltungsprinzipien der gesetzliche Rentenversicherung auf einen solchen Solidarausgleich basieren, muß dies bei der Bewertung der intergenerativen Verteilungswirkungen berücksichtigt werden (vgl. Abschnitt 3.4). Im folgenden werden daher die Ziele und Gestaltungsprinzipien der gesetzliche Rentenversicherung sowie ihre Konsequenzen für die Bewertung der intergenerativen Verteilungswirkungen herausgearbeitet

Das Zielsystem der gesetzliche Rentenversicherung in Deutschland umfaßt eine Vielzahl von Einzelzielen. ${ }^{271}$ Die grundlegenden Ziele - d.h. solche, die die ordnungspolitische Konzeption des Systems bestimmen - beschränken sich jedoch auf erstens die Vorsorge für das Alter und zweitens die Sicherung des Lebensstandards.

269 Vgl. Dinkel (1985), S. 355; Kolb (1985), S. $136 f$.

270 Aus genau diesem Grund, wurde im RRG 1992 die Bruttoanpassung der Renten zurecht durch die Nettoanpassung ersetzt.

271 Z.B. Sicherung des Unterhalts bei Berufs- und Erwerbsunfăhigkeit sowie bei Tod des „Versorgers" des Haushalts; Wiederherstellung der Enwerbsfähigkeit der Versicherten, Vorbeugende gesungheitliche Maßnahmen; Familienförderung. 
Die Betonung der Vorsorge betont die Kopplung der Leistungen an zuvor gezahlte Beiträge und steht im Gegensatz zur beispielsweise steuerfinanzierten Versorgung. Sie ist ein konstituierendes Element aller Sozialsysteme „bismarckscher Prägung“. Seit Gründung der gesetzlichen Alterssicherung in Deutschland ist es das erklärte, politische Ziel, das Rentensystem als Vorsorgesystem zu gestalten. Das mit diesem Ziel korrespondierende Gestaltungsprinzip ist das bereits beschriebene Äquivalenzprinzip. Entsprechend dieses Ziels werden die Verteilungswirkungen eines Reformvorschlags nach dem Grundsatz der Leistungsgerechtigkeit bewertet, wobei die "Leistung“ sich nach den erbrachten Beiträgen richtet.

Für die Bewertung der intergenerativen Verteilungswirkungen folgt daher, daß der Verteilungsindikator sich an der Verknüpfung zwischen Beiträgen und Rentenleistungen ansetzen muß (vgl. Tabelle 5).

Die Lebensstandardsicherung bestimmt als zweites grundlegendes Ziel erst seit der Rentenreform von 1957 die ordnungspolitische Konzeption der gesetzlichen Alterssicherung in Deutschland. Bis dahin bestand das vorrangige Ziel in der Vermeidung von Altersarmut. Die zugrunde liegenden Gerechtigkeitsgrundsätze waren demzufolge der Grundsatz der Bedarfsgerechtigkeit bzw. der gemäßigten Ergebnisgerechtigkeit.

Mit dem Paradigmenwechsel von der Armutsvermeidung zur Sicherung des Lebensstandards prägen seit 1957 nunmehr andere Gestaltungsprinzipien die gesetzliche Rentenversicherung: die Teilhabeäquivalenz, die Kopplung der Rentenleistung am durchschnittlichen Erwerbseinkommen und die Dynamisierung der Renten (vgl. Abschnitt 4.4.3.2). Mit dem Wechsel der Ziele wandelte sich auch die der gesetzliche Rentenversicherung zugrundeliegenden Gerechtigkeitsvorstellungen. Anstelle einer "gemäßigten Ergebnisgerechtigkeit" (Ausgleich der Alterseinkommen zu einem gewissen Maß zur Vermeidung von Armut) korrespondiert die Teilhabeäquivalenz mit einer auf einer relativen Gleichheit beruhenden Leistungsgerechtigkeit. Die am durchschnittlichen Erwerbseinkommen orientierte Rentenhöhe und ihre Dynamisierung, dienen (im Verbund mit der Telhabeäquivalenz) vorrangig der Absicherung gegen intergenerative Risiken. Die der Risikoabsicherung zuzurechnenden Gerechtigkeitsgrundsätze sind zum einen eine besondere Form der Regelgerechtigkeit: Denn unabhängig von den Startbedingungen, dem Bedarf oder der absoluten Gleichheit der Rentenhöhe beschreibt der Risikoausgleich die Mechanismen über die Verteilung einer Zusatzlast, die auf alle Generationen anzuwenden sind, sobald das Risikoereignis eintritt. Zum anderen spiegelt sich in der Risikoabsicherung eine Form der Leistungsgerechtigkeit wider, die sich allerdings von der an der Beitragsäquivalenz orientierenden Leistungsgerechtigkeit unterscheidet: Denn wenn der Eintritt des Risikos durch Ereignisse ausgelöst wird, die nicht dem Verdienst einer Generation zuzuordnen sind bzw. die nicht von ihr verursacht wurden, widerspricht ein (partieller) Ausgleich der durch das Ereignis verursachten Verteilungen nicht dem Prinzip der Leitungsgerechtigkeit.

Für die Bewertung der intergenerativen Verteilungswirkungen bedeutet dies, daß die Verteilungswirkungen unterschieden werden müssen in intergenerativen Risikoaus- 
rd. $70 \%$ festgeschrieben. Inwieweit dieser Wert einen gesellschaftlichen Konsens über ein „gerechtes Nettorentenniveau“ widerspiegelt ist allerdings äußerst strittig, denn einerseits wurde nur fünf Jahre später im Rentenreformgesetz 1999 eine allmähliche Senkung des Nettorentenniveaus zugelassen. Andererseits mehren sich die Beiträge, die ein Festhalten an dem 1992 beschlossenen Niveau kritisieren. ${ }^{273}$

Der Interpretationsspielraum bei der Gewichtung der Lebensstandardsicherung äußert sich beispielsweise in der Konstruktion der Teilhabeäquivalenz, wie sie der derzeit gültigen Rentenformel zugrunde liegt. Diese ist nicht konsistent und führt bei steigender Restlebenserwartung zu systematischen Verteilungseffekten: denn die Höhe der Beiträge über die gesamte Zeitperiode der Beitragspflicht wird in Beziehung zur Höhe der Rentenleistung pro Zeiteinheit gesetzt. ${ }^{274}$ Werden nun die gesamten Rentenleistungen beispielsweise nach Maßgabe der gestiegenen Restlebenserwartung über die Dauer des Rentenbezugs gestreckt, so wäre dies nicht nur nach Maßgabe der Beitragsäquivalenz, sondern auch entsprechend der Teilhabeäquivalenz als gerecht zu bewerten. Das periodische Nettorentenniveau würde in dem Fall sinken, so daß für die Bewertung ein Abwägen zwischen den Zielen bzw. den Gestaltungsprinzipien erforderlich würde. Die Sicherung des Lebensstandards könnte aber auch auf die gesamte Dauer des Rentenbezugs bezogen werden. Bei einer solchen Interpretation würde eine Korrektur der Konzeption der Teilhabeäquivalenz dem Ziel der Lebensstandardsicherung nicht widersprechen.

Dem Problem, wie die verschiedenen Bewertungsmaßstäbe "Leistungsgerechtigkeit nach Maßgabe der Beiträge" und "Solidarausgleich zur Absicherung des intergenerativen Risikos" gewichtet werden, wird durch ein flexibles Meßkonzept und Vergleichszenarien Rechnung getragen (vgl. Abschnitt 3.4).

\subsection{Zusammenfassung und Fazit}

In diesem Kapitel wurden die Verteilungswirkungen abgegrenzt und präzisiert, die für die Beurteilung der intergenerativen Gerechtigkeit der betrachteten Reformoptionen relevant sind. Zunächst wurde in Abschnitt 4.1 die Systemgrenze der Analyse festgelegt:

Die aus dem Steuer-Transfer-System (ohne Sozialversicherung) resultierende Sekundärverteilung der Einkommen wird in der vorliegenden Untersuchung als Ausgangsverteilung akzeptiert, ohne sie auf ihre Gerechtigkeit hin zu bewerten. Es werden ferner nur solche Verteilungswirkungen berücksichtigt, die sich aus dem Rentensystem ergeben, Verteilungswirkungen der übrigen Zweige der Sozialversicherung (beispielsweise dem Gesund-

273

n....aber das Prinzip der Lebensstandardsicherung kann nicht unantastbar sein, wenn es einen Rentenfrondienst der nachwachsenden Generation und eine Plünderung der Familien mit Kindern zur Folge hat.“ Spieker (1996). Vgl. auch Breyer (1997). Anders allerdings z.B. Schmähl (1997b), S.25ff..

274 Vgl. Rürup/Schroeter (1996); Rürup/Schroeter (1997); Breyer (1997), S. 169. Die Reformoptionen, die dafür plädieren, einen demographischen Korrekturfaktor in die Rentenformel zu integrieren, setzen genau an diesem Defizit an.

123 
heitssystem) werden aus der Analyse ausgeblendet, bzw. nur nach Maßgabe ihrer mittelbaren Wirkungen über die Rückkopplungen der Nettoanpassung berücksichtigt.

Anschließend wurden die verschiedenen Arten der Verteilungswirkungen abgegrenzt. Die vorliegende Arbeit beschränkt sich auf die Analyse der folgenden Verteilungswirkungen:

- Es werden vornehmlich personelle und intergenerative Verteilungswirkungen berücksichtigt.

- Intergenerative Verteilungen werden sowohl als direkte Verteilungswirkungen als auch in Form indirekter Verteilungswirkungen berücksichtigt. Letztere resultieren aus der Beeinflussung gesamtwirtschaftlicher Größen (z.B. durch ersparnisinduzierte Wachstumseffekte oder Arbeitsmarkteffekte).

- Die Analyse der direkten intergenerativen Verteilungswirkungen beschränkt sich auf monetäre Zahlungsströme. Realtransfers können vernachlässigt werden.

- Eine Differenzierung zwischen dem „zu versteuernden Einkommen“ und dem „Erwerbseinkommen" ist für die zu untersuchende Fragestellung von nachrangiger Bedeutung, solange die Beitragsgrundlage für alle Versicherten identisch bleibt.

In Abschnitt 4.3 wurden Annahmen darüber getroffen, wie die Zahlungen Dritter bei der Analyse der Verteilungswirkungen berücksichtigt werden.

- Hinsichtlich der Inzidenz der Arbeitgeberbeiträge wird unterstellt, daß sie vollständig auf die Arbeitnehmer überwälzt werden, so daß die gesamten Beiträge in der Verteilungsanalyse von den Arbeitnehmern getragen werden.

- Die Frage nach der effektiven Inzidenz des Bundeszuschusses wird vernachlässigt, da der Bundeszuschuß vereinfachend als Ausgleich für die "versicherungsfremden Leistungen“, sprich als durchlaufender Posten, interpretiert wird. Da der Bundeszuschuß für alle Reformszenarien identisch ist, hat diese Vorgehensweise keinen Einfluß auf das Ergebnis.

In einem weiteren Schritt wurde gezeigt, daß ein Alterssicherungssystem je nach Zielsetzung nach unterschiedlichen Prinzipien gestaltet werden kann. Da diesen Zielen und Prinzipien zudem unterschiedliche Gerechtigkeitsvorstellungen zugrunde liegen, bestimmt auch die jeweilige Gestaltung des Alterssicherungssystems, ob und inwieweit auftretende Verteilungswirkungen ungerecht sind. Mit anderen Worten, galt es für die nachfolgende Analyse zu klären,

1. welche Gestaltungsdimensionen für die Frage der intergenerativen Gerechtigkeit einer Substitution des Finanzierungsverfahrens relevant sind und

2. welchen Einfluß die verschiedenen Gestaltungsprinzipien auf die Bewertung der Verteilungswirkungen haben.

\section{Ad. 1)}

Abbildung 8 auf Seite 105 faßt die grundsätzlichen Gestaltungsdimensionen und -prinzipien zusammen, ohne dabei jedoch der theoretische Vielfalt und den Detailierungsmöglichkeiten vollständig gerecht zu werden. Die Systematisierung ist zudem vor- 
nehmlich als Gliederungshilfe für Alterssicherungssysteme zu verstehen, denn eine strikte Trennung der verschiedenen Prinzipien ist nicht immer möglich. ${ }^{275}$

Die Untersuchung ergab, daß nur die Dimension der Solidarität bzw. die Gestaltungsprinzipien des sozialen Ausgleichs in Versicherungssystemen die Bewertung der Verteilungswirkungen einer Substitution des Finanzierungsverfahrens maßgeblich beeinflussen.

Die Gestaltungsdimension des „Erfassungsgrads" kann in der folgenden Analyse ausgeblendet werden, da eine Umstellung des Finanzierungsverfahren die Gestaltungsform einerseits nicht verändert und andererseits der Unterschied zwischen dem derzeit herrschenden Kategorialsystem ${ }^{276}$ und einem Universalsystem für die intragenerative aber nicht für die intergenerative Gerechtigkeit von Bedeutung ist.

Die betrachteten Reformoptionen beeinflussen die „Subsidiarität" des Alterssicherungssystems, da alle Maßnahmen das Nettorentenniveau senken und - sofern der kapitalgedeckte Zweig privat organisiert wird - das Verhältnis von staatlicher (Zwangs-)Versicherung zu privater (freiwilligen) Altersvorsorge verändern. Dennoch wird dieser Effekt in der Messung der intergenerativen Gerechtigkeit nicht berücksichtigt, da sich erstens keine (intergenerativ) ethische Legitimation für ein ideales Verhältnis aus eigenverantwortlicher Vorsorge und staatlicher Zwangsversicherung formulieren läßt und zweitens die Umstellung des Finanzierungsverfahrens und nicht die Organisationsform bzw. die Zwangsversicherung bewertet werden soll. In der vorliegenden Analyse kann daher vereinfachend unterstellt werden, daß die kapitalgedeckte Altersvorsorge obligatorisch ist und von der gesetzliche Rentenversicherung ein zusätzlicher Kapitalstock verwaltet wird, der in der Höhe gebildet wird, daß das gesamte Sicherungsniveau gehalten wird, und der nach dem Prinzip der aktuarischen Beitragsäquivalenz finanziert wird. Diese theoretische Annahme der Analyse ist jedoch nicht als politische Handlungsempfehlung zu verstehen.

\section{Ad. 2)}

Hinsichtlich der Solidarität eines Alterssicherungssystems lassen sich zwei extreme Gestaltungsprinzipien unterscheiden: reine Sparmodelle, die keine Verteilungseffekte aufweisen und stark umverteilende Versorgungssysteme. Zwischen diesen beiden Polen lassen sich verschiedene Formen von Versicherungssystemen einordnen. Mit der Einfüh-

275 Wechselwirkungen bestehen untere anderem zwischen dem Lebensstandardprinzip und der Teilhabeäquivalenz, da eine Teilhabeäquivalenz sich regelmäßig an einem Lebensstandard orientiert. Andererseits ist eine Lebensstandardsicherung aber auch ohne Teilhabeäquivalenz denkbar und ein Teilhabeäquivalenz läßt sich umgekehrt auch realisieren, ohne daß eine als „angemessen empfundene“ Lebensstandardsicherung gewährleistet ist. Die Wechselwirkungen zwischen den Dimensionen der Subsidiarität und der Solidarität äußern sich beispielsweise darin, daß mit zunehmenden Maß an Solidarität auch die Notwendigkeit eines Zwangskollektivs wächst und andererseits das reine Sparmodell in der Regel privat und individualistisch gestaltet ist, also auch hinsichtlich der Subsidiarität einen Extremfall darstellt.

276 Das derzeitige Kategorialsystem ist dadurch charakterisiert, daß es sich am Erwerbseinkommen orientiert, vornehmlich unselbständig Enwerbstätige und diese zudem nur in einen durch Mindest- und Höchstbeitragsgrundlage begrenzten Einkommenskorridor erfaßt. 
rung des Versicherungsaspekts müssen die Verteilungswirkungen getrennt betrachtet werden: $:^{277}$

- Verteilungswirkungen, die als Folge von interpersonellem Risikoausgleich entstehen, lassen sich mit dem Prinzip der Beitragsäquivalenz vereinbaren.

- Wird das Prinzip der Beitragsäquivalenz z.B. durch die Anrechnung beitragsfreier Zeiten etc. durchbrochen, kommt es zu interpersonellen Umverteilungen. Diese können politisch intendiert, aber auch unbeabsichtigt sein.

Als Referenz zur Abgrenzung und zur Messung dieser interpersonellen bzw. intragenerativen Umverteilungen kann die aktuarische Beitragsäquivalenz herangezogen werden. Dabei gilt, daß der Risikoausgleich um so enger definiert ist, je stärker bei der aktuarischen Beitragsäquivalenz nach Risikoklassen differenziert wird (risikoäquivalente Prämien). Die Globaläquivalenz bietet sich immer dann als Verteilungsmaßstab an, wenn von der intragenerativen Umverteilung innerhalb einer Kohorte abstrahiert werden soll, also ausschließlich intergenerative Verteilungswirkungen beurteilt werden sollen. Sie zeigt nämlich keine Verteilungswirkungen zwischen Versicherten derselben Kohorte an.

Auch bei den intergenerativen Verteilungswirkungen ist zu unterscheiden zwischen:

- Verteilungswirkungen, die aus dem intergenerativen Risikoausgleich resultieren und die sich mit dem Prinzip der Teilhabeäquivalenz und dem Ziel der relativen Lebensstandardsicherung im Alter vereinbaren lassen, und

- Verteilungswirkungen, die auf einer systematischen, intergenerativen Umverteilung zwischen den Generationen beruhen.

Als intergenerativ ungerecht sollte lediglich die zweite Kategorie der reinen Umverteilungswirkungen bewertet werden, bei denen Lasten zwischen den Generationen verschoben werden. Mit Hilfe der Globaläquivalenz lassen sich zwar die intergenerativen Verteilungseffekte messen, allerdings kann mit diesem Indikator nicht entschieden werden, ob die Verteilungswirkungen dem intergenerativen Risikoausgleich oder der intergenerativen Umverteilung zuzuordnen sind. Im folgenden Kapitel ist folglich unter anderm zu klären, wie der intergenerative Risikoausgleich von der intergenerativen Umverteilung abgegrenzt werden kann und mit welchem Verteilungsindikator sich die intergenerative Umverteilung erfassen läßt.

277 Vgl. Stolz (1983), S. 90ff.; Schmähl (1983), S. 3ff.; Wagner (1985), S. 147.

126 
Abbildung 11: Übersicht Kapitel vier

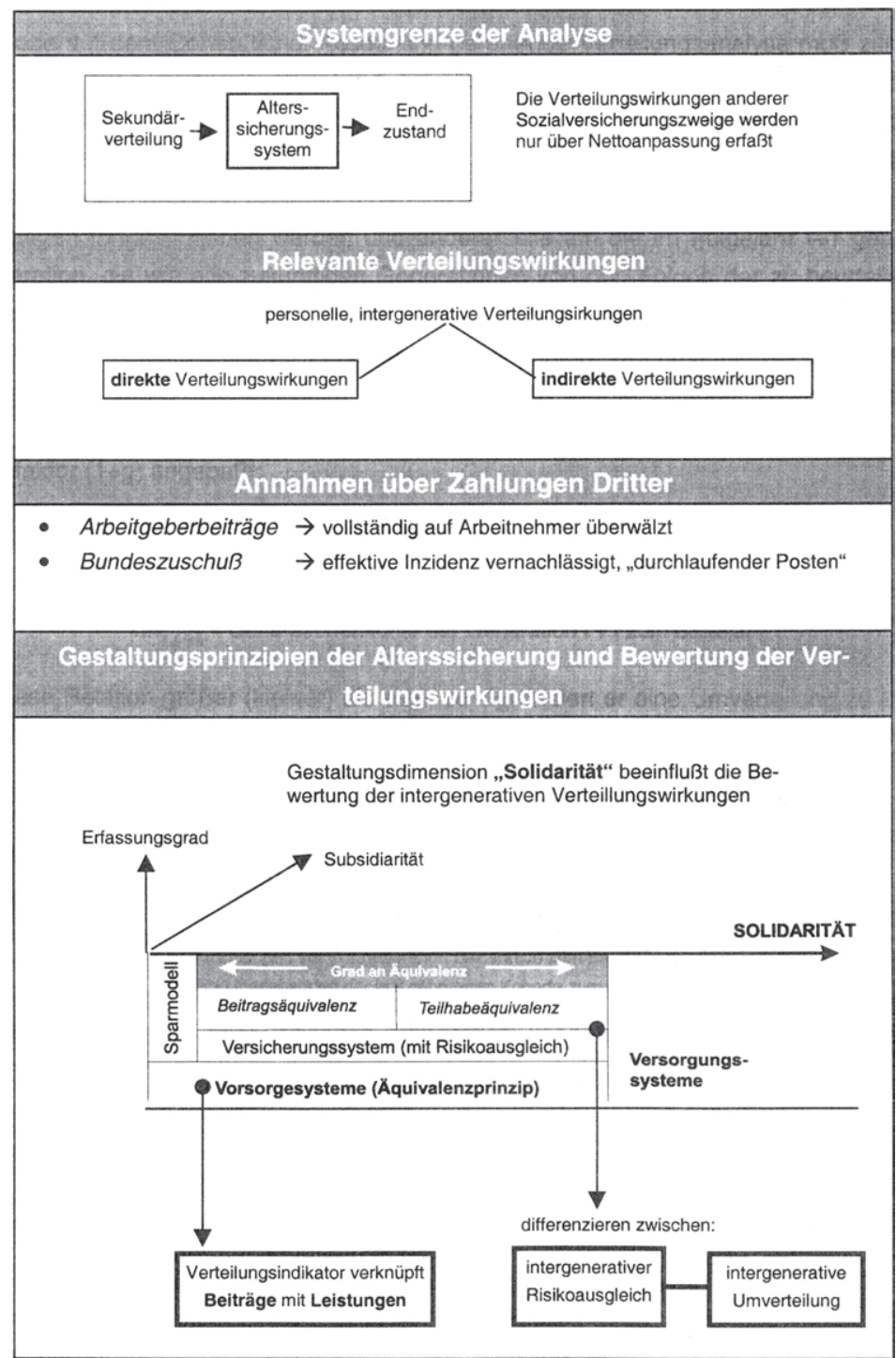

Quelle: Eigene Darstellung 
Ingo Schroeter - 978-3-631-75016-2

Downloaded from PubFactory at 01/11/2019 09:14:34AM

via free access 


\section{OPERATIONALISIERUNG DER UNMITTELBAREN INTERGE- NERATIVEN UMVERTEILUNGSWIRKUNGEN}

In den Kapiteln 5 und 6 wird ein Konzept entworfen, mit dem die intergenerative Gerechtigkeit einer (partiellen) Substitution des Finanzierungsverfahrens gemessen werden kann. Im Mittelpunkt dieses fünften Kapitels stehen ausschließlich direkte Verteilungswirkungen. Die indirekten Verteilungswirkungen, d.h. die gesamtwirtschaftlichen Rückwirkungen der Finanzierungsverfahren, werden in Kapitel 6 behandelt.

In der Literatur gibt es verschiedene Ansätze, die die intergenerativen Verteilungswirkungen eines Alterssicherungssystems quantitativ erfassen. In Abschnitt 5.1 werden diese Ansätze beschrieben und eine Methode ausgewählt, um den Verteilungsindikator für die Gerechtigkeitsanalyse zu formulieren.

Verteilungseffekte können in jeder Distributionsanalyse nur durch einen Vergleich mit einem Referenzmaßstab beurteilt werden. Die meisten Autoren wählen ein beitragsäquivalentes Kapitalstockverfahren als Referenz. Abschnitt 5.2 zeigt auf, daß diese Vorgehensweise normativ nicht begründet werden kann und sie systematische, intergenerative Umverteilungen nicht von Verteilungswirkungen unterscheidet, die aus dem intergenerativen Risikoausgleich resultieren.

Der formulierte Verteilungsindikator beruht aber implizit auf dieser Annahme, daß ein beitragsäquivalentes Kapitalstockverfahren als verteilungsneutrale Referenz dienen kann. In Abschnitt 5.3 werden daher das Referenzsystem und der Verteilungsindikator so angepaßt, daß auch der intergenerative Risikoausgleich berücksichtigt wird. Dabei stehen drei Fragen im Vordergrund:

1. Was gehört zu den intergenerativ versicherbaren Risiken? (Abschnitt 5.3.1)

2. Wieviel an intergenerativen Risikoausgleich ist enwünscht? (Abschnitt 5.3.2)

3. Wie kann die Zusatzlast bei eintritt des Risikos auf die Versichertengemeinschaft (auf die unterschiedlichen Generationen) verteilt werden? (Abschnitt 5.3.3)

Abschnitt 5.4 beschreibt, wie die Verteilungswirkungen der verschiedenen Reformoptionen bewertet werden, wenn diese (unter Berücksichtigung des intergenerativen Risikoausgleichs) erfaßt worden sind. 


\subsection{Methoden zur Erfassung der intergenerativen Verteilung ei- nes Alterssicherungssystems}

\subsubsection{Ausgewählte Methoden zur Erfassung der intergenerativen Ver- teilung}

Da die Möglichkeiten einfacher OLG-Modelle (vgl. Kapitel 2), verschiedene Reformen von Alterssicherungssystemen zu bewerten, äußerst begrenzt sind, basieren Analysen realer Systeme regelmäßig auf Simulationsrechnungen. Dabei können die verschiedenen Reformoptionen des Alterssicherungssystems hinsichtlich verschiedener Kriterien bewertet werden. Sofern die finanzwirtschaftlichen und/oder gesamtwirtschaftlichen Konsequenzen solcher Maßnahmen im Mittelpunkt der Analyse stehen, kann die Entwicklung des Beitragssatzes als zentrale Beurteilungsgröße herangezogen werden. ${ }^{278}$ Sofern die Reformoptionen jedoch anhand ihrer distributiven Wirkungen bewertet werden sollen, ist der Beitragssatz alleine wenig aussagefähig. Im folgenden werden daher zunächst die wichtigsten Ansätze skizziert, mit denen sich die Verteilungswirkungen eines Alterssicherungssystems erfassen lassen.

\subsubsection{Generational Accounting}

In der traditionellen Budgetierung des öffentlichen Sektors wird die fiskalpolitisch verursachte Lastverschiebung allein anhand des jährlichen Defizits beurteilt. Anfang der 90er Jahre kritisierten AUERBACH, GOKHALE und KOTLIKOFF, daß diese Vorgehensweise die mit der Fiskalpolitik verbundene, intergenerative Umverteilung nicht hinreichend erfaßt, ${ }^{279}$ und entwarfen als alternativen Ansatz das Konzept des Generational Accounting. ${ }^{280}$ Seitdem wächst die Anzahl an finanzwissenschaftlichen Publikationen über das Generational Accounting. Zudem wird dieser Ansatz in einigen Ländern bereits in Rahmen von Projekten erprobt und in den USA ist er bereits Teil des öffentlichen Haushaltsplans. ${ }^{281}$ Aufgrund der rasanten Verbreitung des Generational Accounting wird nachfolgend geprüft, ob sich dieser Ansatz auf die Fragestellung der vorliegenden Arbeit anwenden läßt.

278 Vgl. Jäger (1991); Prognos (1995).

279 Vgl. Auerbach/Gokhale/Kotlikoff (1991) und (1992).

280 Der eher Zeitpunktbezogene Ansatz der traditionellen Budgetierung basiert auf dem keynesianischen Paradigma, bei dem die Wirtschaftssubjekte nicht ihr gesamtes Lebenseinkommen, sondern nur ihr laufendes Einkommen in ihrem kurzfristigen Optimierungskalkül einbeziehen. Die andere extreme Sichtweise geht von der Existenz eines vollkommenen Altruismus aus, so daß die Wirtschaftssubjekte nicht nur ihr eigenes Lebenseinkommen, sondern auch das ihrer Nachkommen bei ihren Entscheidungen berūcksichtigen. Aus dieser Annahme folgt, daß sämtliche intergenerativ umverteilende Maßnahmen Anpassungen des Spar- und Erbschaftsverhaltens hervorrufen und jede intendierte Umverteilung dadurch automatisch konterkariert wird. Dieser Zusammenhang ist in der wissenschaftlichen Diskussion vor allem unter dem Stichwort des Ricardianischen Äquivalenztheorems bekannt geworden, vgl. Barro (1974). Die wirtschaftstheoretische Basis des Generational Accounting basiert auf dem neoklassischen Lebenszyklusmodell und ist daher zwischen den eben beschriebenen extremen Auffassungen angesiedelt.

281 Vgl. RaffelhüschenWalliser (1996), S.185.

130 


\subsection{Beschreibung des Ansatzes}

Für jede auf dem Generational Accounting beruhende Verteilungsanalyse muß zunächst ein Basisjahr $t$ festgelegt werden, auf das sich sämtliche Berechnungen und Ergebnisse beziehen. Um das Ausmaß an intergenerativer Umverteilung zu messen, werden die Situationen zweier repräsentativer Generationen miteinander verglichen. Dabei handelt es sich einerseits um die im Basisjahr $t$ geborene Generation, für welche die Status Quo Bedingungen fortgeschrieben werden und andererseits um die im Folgejahr $t+1$ geborene Generation, die wie alle zukünftigen Generationen von den Folgen der zu beurteilenden Maßnahmen betroffen ist. Als formales Verteilungsmaß dient die sogenannte Belastungsrelation $\Phi$, die sich als Quotient der Generationenkonten (Generational Account: GA) der beiden betrachteten Generationen definiert. Um die Vergleichbarkeit zu gewährleisten, wird das Generationenkonto der im Basisjahr geborenen Generation mit dem Wachstumsfaktor $(1+g)$ angepaßt:

$$
\begin{gathered}
\Phi=\frac{G A_{t, t+1}}{G A_{t, t} \cdot(1+g)} \quad \text { G } 57 \\
G A_{t, t+1}=\text { Generationenkonto der Generation } t+1 \text { zum Basisjahr } t
\end{gathered}
$$

Ist diese Relation größer (kleiner) als eins, so signalisiert er eine Umverteilung zu Lasten nachfolgender (gegenwärtiger) Generationen. Unter einem Generationenkonto GA versteht man dabei die pro Kopf Größe des Barwerts aller zukünftigen Nettozahlungen einer bestimmten Generation:

$$
G A_{t, k}=\frac{N_{t, k}}{P_{t, k}} \quad \mathbf{G} 58
$$

$N_{1, k}=$ Nettozahlungen der in $k$ geborenen Generation zum Basisjahr $t$.

$P_{t, k}=$ Anzahl Überlebenden der in $k$ geborenen Kohorte zum Zeitpunkt $t$

Die Nettozahlungen jeder lebenden Generation können entsprechend der folgenden Funktion bestimmt werden:

$$
\begin{aligned}
& \qquad N_{t, k}=\sum_{s=t}^{k+D} T_{s, k} \cdot P_{s, k} \cdot(1+i)^{-(s-t) \quad \text { G } 59} \\
& \mathrm{i}=\text { konstanter Zinssatz } \\
& \mathrm{D}=\text { maximale Lebensdauer } \\
& \mathrm{N}_{\mathrm{t}, \mathrm{k}}=\text { Nettozahlungen der in } \mathrm{k} \text { geborenen Generation zum Basisjahr } \mathrm{t} . \\
& \mathrm{P}_{\mathrm{o}, \mathrm{k}}=\text { Anzahl Überlebenden der in } \mathrm{k} \text { geborenen Kohorte zum Zeitpunkt s } \\
& \mathrm{T}_{\mathrm{s}, \mathrm{k}}=\text { durchschnittliche Nettozahlung im Jahr s eines in } \mathrm{k} \text { geborenen Wirtschaftssubjekts }
\end{aligned}
$$

Die durchschnittliche Nettozahlung $\left(T_{t . k}\right)$ im Jahr s eines im Jahr k geborenen Individuums setzt sich wiederum aus unterschiedlichen altersspezifischen Zahlungsarten $h_{a, i, s}$ zusammen (der Index a bezeichnet das Alter während i die Zahlungsart z.B. Einkommenssteuer, Krankenversicherungsgeld, Sozialhilfe etc. charakterisiert). Die einzelnen Komponenten lassen sich mit Hilfe von mikroökonomischen Paneldaten (z.B. Sozio-ökonomisches Panel, Einkommens- und Verbraucherstichprobe) bestimmen und so angleichen, daß die 
Summe dieser Zahlungen über alle Generationen mit den Werten der Volkswirtschaftlichen Gesamtrechnung des Basisjahrs übereinstimmen. Das auf diese Weise ermittelte, nach Alter und Zahlungsart differenzierte Belastungsprofil wird über die Zeit konstant gehalten. Die absoluten Beträge werden hingegen mit einer konstanten Wachstumsrate (meist die der Löhne) fortgeschrieben. Auf diese Weise lassen sich die Generationenkonten für jede lebende Generation, insbesondere für die in $\mathrm{t}$ geborene, bestimmen.

Zur Bestimmung der Belastungsrelation ist es ferner erforderlich, die Generationenkonten der zukünftigen Generationen (insbesondere der in $\mathrm{t}+1$ geborenen) zu bestimmen. Hierzu wird auf ein weiteres zentrales Element des Generational Accounting zurückgegriffen: die intertemporale Budgetrestriktion des Staates. Entsprechend dieser Forderung nach einem langfristige Zahlungsausgleich der Staatsausgaben $\mathrm{G}_{\mathrm{s}}$ muß die Summe der Barwerte aller gegenwärtigen und zukünftigen Staatsausgaben der Summe der Nettozahlungen aller lebenden und zukünftigen Generationen zuzüglich des im Bezugszeitpunkt verfügbaren Nettovermögens $W_{t}$ entsprechen:

$$
\sum_{s=0}^{D} N_{t, t-s}+\sum_{s=1}^{\infty} N_{t, t+s}+W_{t}=\sum_{s=1}^{\infty} G_{s}(1+i)^{t-s}
$$

Der Staatsverbrauch beschränkt sich in dieser Gleichung lediglich auf jene Ausgaben, die nicht als direkte Transfers den einzelnen Generationen zugeordnet werden können und somit bereits in den Nettozahlungen enthalten sind.

Sind das Nettovermögen im Basisjahr und der Barwert des Staatsverbrauchs (in der Regel wird der pro Kopf Staatsverbrauch ebenfalls mit dem Wachstum fortgeschrieben) bekannt, lassen sich die Nettozahlungen der zukünftigen Generationen als Residuum der obigen intertemporalen Budgetgleichung bestimmen. Diese Restgröße wird in gleichen Anteilen auf die verschiedenen Generationen aufgeteilt, d.h. sie unterscheiden sich nur durch den Wachstumsfaktor $\mathrm{g}$. In der praktischen Anwendung wird anstelle des unendlichen Zeitraums eine maximale Zeitperiode unterstellt, die so angesetzt wird, das die Berücksichtigung weiterer Generationen aufgrund des Diskontierungseffekts das Ergebnis nur noch unwesentlich beeinflußt.

Abschließend ist hinzuzufügen, daß die Generationenkonten und damit die Verteilungswirkungen sich ebenfalls nach Region, Geschlecht o.ä. getrennt berechnen lassen.

\subsection{Kritik des Ansatzes}

Das Generational Accounting versucht in erster Linie aufzuzeigen, inwieweit zukünftige Generationen durch die gegenwärtige Finanzpolitik des Staates belastet werden. Dabei sollen lediglich Erkenntnisse über die tendenzielle Wirkungen gewonnen werden, eine Prognose der genauen zukünftigen Belastungsverläufe wird nicht angestrebt. ${ }^{282}$ Wenngleich mit Hilfe dieses Ansatzes auch die intergenerative Verteilungswirkung der Alterssi- 
cherung untersucht wurde, ${ }^{283}$ ist das Generational Accounting schon allein aufgrund der fehlenden, genauen Belastungsverläufe für die Analyse solcher Fragestellungen wenig geeignet. Weitere gravierende Einwände, die gegen die Verwendung dieses Ansatzes für diese Arbeit sprechen, lassen sich wie folgt zusammenfassen:

- Der Generational Accounting Ansatz erfaßt lediglich jene Zahlungsströme, die gegenwärtig oder zukünftig geleistet werden. Mit der Vernachlässigung von Zahlungen der Vergangenheit werden die - für diese Arbeit interessierenden - Folgen der intergenerativen Umverteilung auf die gegenwärtig im System Versicherten (Beitragszahler und Rentenbezieher) ausgeblendet.

- Die Bestimmung der Generationenkonten zukünftiger Generationen werden als Restgröße der intertemporalen Budgetbeschränkung des Staates gebildet. Diese Vorgehensweise mag bei einer umfassenden Beurteilung der Finanzpolitik, die sämtliche (auch nicht monetäre) Transfers erfaßt, durchaus notwendig sein. Für eine Bewertung der Alterssicherung ist eine solche aggregierte Betrachtung nicht erforderlich und zu grob, sie erfordert eine detailliertere Abbildung der Transferzahlungen.

- Grundsätzlich stellt sich die Frage, welche gesellschaftliche Diskontrate ausgewählt werden soll. Dieses Problem ist allerdings nicht spezifisch für das Generational Accounting, sondern stellt sich bei den meisten intertemporalen Betrachtungen.

- Bei dem Staatsverbrauch wird nicht zwischen einer konsumtiven und investiven Verwendung differenziert, obwohl nachfolgende Generationen durch Investitionen bessergestellt werden können. Dieser Zusammenhang weist darauf hin, daß der Generational Accounting Ansatz sich auf Zahlungsströme beschränkt und etwaige makroökonomische Rückwirkungen - wie beispielsweise Anreizeffekte auf den Arbeitsmarkt, Preis-, Zins- oder Wachstumseffekte - ausblendet.

- Grundsätzliche Kritik ist daran zu üben, daß für lebende Generationen (dazu gehört auch die im Basisjahr t geborene Generation) die Status Quo Bedingungen fortgeschrieben wird und die Folgen der Maßnahmen ausschließlich die zukünftigen Generationen betreffen, obwohl auch die derzeit lebenden Generationen noch von den Folgen der Maßnahmen betroffen sind. ${ }^{284}$

- In gleicher Weise muß die Konstanz der Zahlungsprofile und die Fortschreibung der Zahlungen mit einer konstanten Wachstumsrate kritisiert werden.

- Wenig geeignet für eine Bewertung der intergenerativen Verteilung ist das Generational Accounting aufgrund der grundsätzlichen Beschränkung auf einen Vergleich lediglich zweier Generationenkonten. Für eine revolvierende Betrachtung (Vergleich der 
Generationen 1 und 2, dann der Generationen 2 und 3 etc.) ist der Ansatz nicht geeignet.

- Für die vorliegende Analyse interessiert vor allem die zukünftige Entwicklung der intergenerativen Verteilung über mehrere Generationen (generative Verteilungsmuster), so daß nicht zuletzt auch die gleichmäßige Aufteilung der Restgröße auf alle zukünftigen Generationen gegen die Verwendung des Generational Accounting spricht ${ }^{285}$.

- Die geäußerten Kritikpunkte ließen sich zwar durch Modifikationen des Grundmodells abmildern, grundsätzlich bleibt der eher illustrative Charakter dieses Vorgehens bestehen

„..., denn es beschreibt nicht die tatsächliche Verteilung der Lasten auf zukünttige Generationen im einzelnen, sondern liefert nur die Grundlage für die Beurteilung der Belastung all dieser Generationen insgesamt. ${ }^{\text {,286 }}$

Für den Untersuchungsgegenstand der vorliegenden Arbeit erweist sich dieser Ansatz aufgrund der obigen Kritik als ungeeignet und wird daher nicht weiter verfolgt.

\subsubsection{Nutzenfunktionen}

Die Mehrzahl der formal-analytischen Arbeiten untersuchen die allokativen und distributiven Wirkungen eines Alterssicherungssystems anhand der Nutzen, die verschiedene Generationen unter dem Regime verschiedener Alterssicherungssysteme erzielen können. Diese Vorgehensweise erlaubt es, zusätzlich zum Konsum weitere Kriterien, wie beispielsweise die Freizeit oder die Vererbung, in die Nutzenfunktion zu integrieren. Solange lediglich qualitative Zusammenhänge im Rahmen eines einfachen OLG-Modells analysiert werden, ist dieser Ansatz sehr hilfreich und bereitet wenig Probleme. Sobald er allerdings auf eine Simulationsanalyse übertragen wird, erfordert dies eine Spezifikation der Nutzenfunktion. ${ }^{287}$ Eine jährliche Simulation über einen für die Rentenversicherung notwendigen Zeitraum von rd. 70 Jahren ist dann schon aufgrund des Rechenumfangs trotz leistungsfähiger elektronischer Datenverarbeitung kaum realisierbar.

Grundsätzlich ließe sich die Rechnung zwar vereinfachen, indem die Periodenlängen auf beispielsweise 10 Jahre verlängert werden, für viele Fragestellungen - so auch für die vorliegende Analyse - wäre eine solche Vorgehensweise jedoch ungenügend. Die grundsätzlichen Probleme der Wahl des funktionalen Zusammenhangs und die Parametrisierung der Funktion blieben im übrigen weiterhin bestehen. Zudem stellt sich bei einem solchen Vorgehen das methodische Problem, wie sich die heterogenen Präferenzordnungen der Individuen zu einer repräsentativen Nutzenfunktionen komprimieren lassen .

\footnotetext{
285 Andere Verteilungsmuster sind zwar denkbar, bleiben aber ebenso spekulativ.

286 RaffelhüschenWalliser (1996), S.184, bezogen auf die gleichmäßige Aufteilung der Restgröße.

287 Die Konkretisierung der Nutzenfunktion erfordert sowohl die Festlegung des funktionalen Zusammenhangs als auch die Parametrisierung der Funktion. Ferner müßte unterstellt werden, daß der Zusam. menhang und die Parameter sich im Zeitablauf nicht ändern.
} 
Wenngleich einige Arbeiten ihr Simulationsmodell zur Analyse des Umlage- und Kapitalstockverfahrens zur Finanzierung der Renten auf solche Nutzenfunktionen gründen, ${ }^{288}$ wird dieser Ansatz aufgrund der dargelegten Kritik für die vorliegende Arbeit abgelehnt.

\subsubsection{Rentabilitätsindikatoren}

Eine Vielzahl von Arbeiten ${ }^{289}$ zur Erfassung der intergenerativen Verteilung bzw. der intergenerativen Gerechtigkeit fragen danach, ob und in welchem Ausmaß die Rentabilität des Alterssicherungssystems für unterschiedliche Kohorten variiert. Diese Vorgehensweise hat den Vorteil, daß auf bekannte mathematische Methoden zur Bestimmung der Rentabilität zurückgegriffen werden kann. Dennoch muß bei der Wahl der Rentabilität eines Alterssicherungssystems als Indikator für die intergenerativen Verteilungswirkungen beachtet werden, daß das Ergebnis der Rentabilitätsberechnung zum Teil von dem gewählten Verfahren zur Rentabilitätsrechnung abhängt.

Eine Kenngröße, mit der die Belastungen verschiedener Generationen gemessen werden kann, ist der sogenannte Lebensnettotransfer (LNT) eines Geburtsjahrgangs bzw. einer Zugangskohorte. ${ }^{290}$ Die Vorgehensweise ist der Kapitalwertmethode zur Bewertung betrieblicher Investitionsvorhaben entlehnt. Zunächst wird der Barwert aller von der Geburtsbzw. Zugangskohorte geleisteten Beiträge und der Barwert aller von der Geburtskohorte empfangenen Rentenleistungen gebildet, in dem die Zahlungen auf ein Referenzjahr (beispielsweise das Geburts- oder Rentenzugangsjahr) auf- bzw. abgezinst werden. Den LNT erhält man, indem der Barwert der Rentenleistungen von dem Barwert der Beitragsleistungen subtrahiert wird. Die verschiedenen Generationen werden von einem Alterssicherungssystem genau dann gleichbehandelt, wenn sie konstante LNT aufweisen.

Lebensnettotransfereinkommen LNT = Barwert $\Sigma$ Beiträge - Barwert $\Sigma$ Leistungen

Werden die Barwerte auf das Geburtsjahr der betrachteten Kohorte diskontiert, berechnet sich der Lebensnettotransfer nach folgender Gleichung:

$$
L N T^{J}=\sum_{a=1}^{T}(1+i)^{-a} \cdot B_{a}^{J} \cdot R L_{a}^{J} \cdot \frac{n_{a}^{J}}{n_{0}^{J}} \quad \mathbf{G} 60
$$

Vgl. Raffelhüschen (1989); Seidman (1986); Auerbach/Kotlikoff (1987).

Vgl. z.B. Prinz (1997); Ohsmann/Stolz (1997); Eitenmüller (1996); Preschitz (1994); Holzmann (1988); Weltbank (1994); Koppelmann (1979).

290 
$i=$ Diskontsatz

$B_{a}^{J}=$ Höhe der durchschnittlich in Alter a geleisteten Beiträge der Kohorte $\mathrm{J}$

$R L_{a}^{J}=$ Höhe der durchschnittlich in Alter a empfangenen Rentenleistungen der Kohorte $\mathrm{J}$

$n_{a}^{J}=$ Kohortenstärke im Alter a

$n_{0}^{J}=$ Stärke der Kohorte J (Anzahl der Neugeborenen)

Prinz schlägt als Maß für „intergenerationale Fairneß“ den sogenannten generationalen Deckungsgrad vor. ${ }^{291}$ Diese Kennzahl gibt an, welcher Teil der gesamten, während des Ruhestandes empfangenen Rentenleistungen eines Jahrgangs durch die zuvor von inm geleisteten Beitragszahlungen abgedeckt sind. Der Kehrwert dieses Indikators beschreibt dementsprechend die Leistung, die eine Geburtskohorte für eine Beitragseinheit erhält und gibt somit die Rendite der Beitragszahlungen an.

$$
\text { Generationale Deckungsgrad }=\frac{\text { Barwert } \Sigma \text { Beiträge }}{\text { Barwert } \Sigma \text { Leistungen }}
$$

Ein System wäre entsprechend dieses Maßes genau dann frei von intergenerativer Umverteilung, wenn der generationale Deckungsgrad für sämtliche Jahrgangskohorten identisch ist.

Sowohl der Lebensnettotransfer als auch der generationale Deckungsgrad bzw. die Rendite gründen sich auf die Barwerte der Leistungen und Beiträge. Während sie beim LNT subtrahiert werden, werden sie beim generationalen Deckungsgrad bzw. bei der Rendite in Relation gesetzt. Beim Ansatz des Lebensnettotransfers steht die absolute Gleichheit der Transferzahlungen im Vordergrund; der generationale Deckungsgrad hingegen beruht auf die relativ gleiche Deckung durch Vorleistung.

Das zentrale Problem beider Rentabilitätsrechnungen zur Alterssicherung besteht darin, daß die Zahlungen zu unterschiedlichen Zeiten erfolgen und gleich hohe, aber zeitlich auseinander liegende Beträge nicht gleich bewertet werden können. Um verschiedene Zahlungsreihen dennoch vergleichbar zu machen, werden die Zahlungen einer Kohorte in der Regel auf einen Zeitpunkt auf- bzw. abgezinst. Solange die Rentabilitätsberechnung auf Barwerten beruht, hängen die Ergebnisse im hohen Maße von der Wahl des Zinssatzes und der Art der Diskontierung ab. ${ }^{292}$

Einige Autoren versuchen daher dieses Problem des geeigneten Diskontsatzes zu umgehen, indem sie den internen Zinsfuß der Zahlungsreihen (Beiträge und Rentenleistungen) als Rentabilitätsmaß heranziehen. ${ }^{293}$ Der interne Zinsfuß beschreibt denjenigen Zinssatz, mit dem die Beitragszahlungen auf- und die Rentenleistungen abgezinst werden

\footnotetext{
291 Vgl. Prinz (1997), S. 4.

292 Vgl. Abschnitt 7.2.1.

293 Eine solche Vorgehensweise wählen beispielsweise Eitenmüller (1996); Dudey (1996) und Koppelmann, K.-P. (1979), S. 40ff..
} 
müßten, damit sich die Barwerte zum Bezugszeitpunkt (z.B. Geburt, Renteneintritt) genau entsprechen.

Formal erhält man den internen Zinsfuß indem die Lebensnettotransfergleichung (G 60) zu Null gesetzt und der Wert der Variable i bestimmt wird. Bedenkt man, daß für die Nullstellen eines Polynoms n-ten Grades immer $n$ komplexe Lösungen (Wurzeln) existieren, diese jedoch nur bis zum 4-ten Grad algebraisch exakt gelöst werden können, könnten angesichts des für die Alterssicherung relevanten Betrachtungszeitraum von rd. 70 Perioden numerischen Probleme entstehen. Da sich die Lösungen aber problemlos mit Hilfe von Näherungsverfahren (z.B. Näherungsverfahren nach Newton) und leistungsfähigen Rechnern bestimmen lassen, ist diese Einschränkung mittlerweile bedeutungslos.

Der interne Zinsfuß ist nicht eindeutig, wenn theoretisch rd. 70 Lösungen existieren. Imaginäre Lösungen können allerdings genauso ausgeschlossen werden, wie die ökonomisch unplausiblen Lösungen im Intervall ]- -1 [. Die für die Alterssicherung typischen Zahlungsströme weisen zudem ein bestimmtes Muster auf: Es existiert immer zunächst eine erste Periode, in der ausschließlich positive Beiträge gezahlt werden (Phase der Erwerbstätigkeit), an die sich eine zweite Periode anschließt, in der ausschließlich Leistungen (negative Beiträge) empfangen werden (Ruhestand). Mit anderen Worten, das Vorzeichen der Zahlungsströme wechselt nur einmal. Für solche Zahlungsmuster läßt sich zeigen, daß es im ökonomisch relevanten Bereich von $-1<i$ nur einen internen Zins geben kann. ${ }^{294}$

Gegen die Verwendung des internen Zinsfußes als Investitionsmaß wurde regelmäßig ebenfalls eingewandt, daß die Zinsfußmethode implizit unterstelle, jeder Einzahlungsüberschuß ließe sich zu den gleichen internen Zins investieren und Kapital könne nur zu diesem Zinsfuß beschafft werden. Dieser Einwand ist für die vorliegende Fragestellung jedoch irrelevant, da die Interpretation des internen Zinsfußes als Effektivverzinsung des gebundenen Kapitals an keinerlei Wiederanlageprämisse gebunden ist, sofern die Kapitalbildung - wie in der Alterssicherung der Fall -nicht negativ ist. ${ }^{295}$

Aus mathematisch-methodischer Sicht spricht daher nichts gegen die Anwendung des internen Zinsfußes als Indikator intergenerativer Verteilungswirkungen. ${ }^{296}$

Eine weitere Rentabilitätskennzahl ist die Beitragsrendite nach dem Barwertäquivalenzprinzip. Grob vereinfacht kann man sie als eine Mischung zwischen dem Lebensettotransferkonzept und dem interne Zinsfuß umschreiben. ${ }^{297}$ Die nach dem Barwertäquivalenzprinzip ermittelte interne Ertragsrate (Beitragsrendite) eines Alterssicherungssy-

\footnotetext{
Vgl. Koppelmann (1979), S. 42-45 und die dort angegebene Literatur. Vgl. auch Hering (1994), S. 49; Blohm/Lüder (1991). Vgl. Koppelmann (1979), S. 42-45; Hering (1994), S. 52; Blohm/Lüder (1991), S. 99ff..

296 Voraussetzung ist lediglich, daß die mit dem Alterssicherungssystem verbundenen Zahlungsströme bekannt bzw. mit Hilfe von Simulationsrechnungen prognostiziert werden. 
stems beschreibt jenen Zinssatz, mit dem sich die Beitragsleistungen verzinsen müssen, damit der Barwert der gesamten Beitragsleistungen dem vorgegebenen Barwert der Rentenleistungen entspricht. Der Barwert der Rentenleistungen wird unabhängig von der Beitragsrendite zu einem zuvor festgelegten Diskontsatz bestimmt.

Die Motivation dieser Vorgehensweise besteht vornehmlich darin, das Verfahren an die Vorgehensweise bei privaten Institutionen anzulehnen: Für einen vorhandenen Kapitalstock (aus bereits verzinsten Beiträgen) werden unter Berücksichtigung der durchschnittlichen, fernen Lebenserwartung sowie eines zuvor festgelegten Diskontsatzes die Rentenleistungen bestimmt. Da in dieser Arbeit kein Renditevergleich mit privaten Lebensversicherungen angestrebt ist, kommt dieser Ansatz für die nachfolgende Analyse nicht in betracht.

Weitere Indikatoren setzen an der relativen Einkommensposition der jeweiligen Geburts- bzw. Zugangskohorten an. ${ }^{298}$ So wird das durchschnittliche Nettoeinkommen, das ein Jahrgang während seiner gesamten Erwerbsperiode bezog mit der durchschnittlichen Rentenleistung verglichen, die dieser Jahrgang pro Rentenjahr empfing. Neben dem Vergleich dieser absoluten Größen kann auch die Veränderung der „Einkommensrelation bzw. -ersatzrate", sprich die Relation der Einkommensposition im Ruhestand zu der im erwerbstätigen Alter, wichtige Informationen über die unterschiedliche Behandlung von Generationen durch die Rentenversicherung geben. Es ist unschwer zu erkennen, daß diese Indikatoren eine gewisse Affinität zur Annuitätenmethode der Investitionsrechnung aufweisen.

\subsubsection{Auswahl der Erfassungsmethode und Formulierung des Vertei- lungsindikators}

Im vorangegangenen Abschnitt wurden verschiedene Verfahren skizziert, mit denen sich intergenerative Verteilungswirkungen erfassen lassen. Die Würdigung dieser Methoden zeigte, daß sich einige Ansätze - wie das Generational Accounting oder die Verwendung von Nutzenfunktionen - für die vorliegende Fragestellung nicht bzw. nur bedingt eignen. Angesichts der vorangegangenen Ausführungen erscheint es am zweckmäßigsten, auf generationenspezifische Rentabilitätskennziffern zurückzugreifen, um intergenerative Verteilungswirkungen zu operationalisieren. Dabei wird die Barwertmethode dem internen Zinsfuß vorgezogen, allerdings nicht, weil der interne Zinsfuß etwa methodisch unterlegen wäre, sondern weil sich die Barwertmethoden einfacher modifizieren lassen.

Die Rentabilitätsindikatoren gründen sich nämlich einseitig auf dem Prinzip der reinen Beitragsäquivalenz und orientieren sich vornehmlich am Konzept der Leistungsgerechtigkeit. Da die reine Beitragsäquivalenz weder das einzige noch das vorrangige Gestaltungsprinzip der gesetzliche Rentenversicherung darstellt, könnte man die Wahl 
der Rentabilität als adäquate Kennziffer für die intergenerative Gerechtigkeit kritisieren. ${ }^{299}$ Mit Hilfe der Rentabilitätskennziffern soll jedoch weder die Institution „gesetzliche Rentenversicherung" abschließend bewertet werden noch sollen sie das alleinige Kriterium ihrer Gerechtigkeit sein. Vielmehr dienen sie als Grundlage zur Erfassung intergenerativer Verteilungseffekte. Bei Bedarf könnten diese Kennziffern erweitert werden, um zusätzlich zum Äquivalenzprinzip weitere Gestaltungsprinzipien der Alterssicherung zu berücksichtigen. Eine solche Anpassung scheint bei Methoden der Barwertbetrachtungen einfacher zu sein als beim internen Zinsfuß, dessen Berechnung sich auf iterativen Näherungslösungen basiert.

In Anlehnung an die Renditegleichung in Abschnitt 5.1.1.3 wird daher der auf vorschüssige Zahlungen beruhende Verteilungsindikator für die weitere Arbeit formuliert:

Bei konstanten Abzinsungsfaktor:

$$
V I_{x}=\frac{\sum_{t=R E}^{T} R L^{t} \cdot(1+d)^{R E-t}}{\sum_{t=0}^{R E-1} B^{\prime} \cdot(1+d)^{R E-t}} \quad \text { G } 61
$$

\footnotetext{
$V I_{x}=$ generationsspezifischer Umverteilungsindikator der Generation $x$

$R E=$ Renteneinrtittsalter

$T$ = maximale Lebensdauer der Generation $x$

$B^{\prime}=$ gesamte Beitragshöhe der Generation $x$ in Periode $t$

$R L^{\prime}=$ gesamte Rentenleistung der Generation $\mathrm{x}$ in Periode $\mathrm{t}$

$t=0$ bezeichnet das jeweilige Geburtsjahr der Generation 300
}

und bei zeitabhängigen Auf- und Abwertungsfaktoren:

$$
V I_{x}=\frac{\sum_{t=R E}^{T}\left(R L^{t} \cdot \prod_{v=R E}^{t}\left(1+d_{v}\right)^{-1}\right)}{\sum_{t=0}^{R E-1}\left(B^{t} \cdot \prod_{v=1}^{R E-1}\left(1+d_{v}\right)\right)} \text { G } 62
$$

Der Verteilungsindikator bezieht die Leistungen einer Generation auf die von dieser Generation gezahlten Beiträge. Er erfüllt somit die Anforderung an einen Verteilungsindikator, die in Abschnitt 4.4.4 aus dem Ziel, die Rentenversicherung als Vorsorgesystem zu konzipieren, abgeleitet wurde.

Zudem erfaßt er sowohl die intergenerativen Verteilungswirkungen, die sich aus einer Umstellung des Finanzierungsverfahrens ergeben (vgl. Abschnitt 2.3) als auch die intergenerativen Verteilungswirkungen, die sich innerhalb eines mit einem bestimmten Finanzierungsverfahren arbeiten Alterssicherungssystems bei Variation der ökonomischen und loder demographischen Parameter ergeben (vgl. Abschnitt 2.4). 
Der Verteilungsindikator berücksichtigt allerdings nicht die Einkommenssituation der Versicherten, d.h. er erfaßt keine Wohlfahrtseffekte, die durch die gesamtwirtschaftlichen Wirkungen der Finanzierungsverfahren verursacht werden. In Kapitel 6 wird diesem Aspekt der Gerechtigkeitsanalyse und die eventuell notwendige Modifikation des Verteilungsindikators diskutiert.

\subsection{Wahl eines verteilungsneutralen Referenzsystems}

\subsubsection{Kritik des beitragsäquivalenten Systems als Referenzmaßstab}

Die Frage, ob und in welchem Ausmaß Verteilungseffekte auftreten, wird in jeder Distributionsanalyse durch einen Vergleich mit einem verteillungsneutralen Referenzmaßstab beurteilt. Üblicherweise wird hierzu ein (aktuarisch) beitragsäquivalentes System als Vergleichmaßstab herangezogen. ${ }^{301}$ BösCH betont weiter, daß obgleich es nicht immer explizit formuliert ist, es in der empirischen und theoretischen Literatur allgemein anerkannt sei, daß beim Referenzsysztem eine privat- bzw. marktwirtschaftlich organisierte Kapitaldeckung unterstellt wird. ${ }^{302}$ Um lediglich die systemimmanenten Umverteilungen der Alterssicherung zu bewerten und diejenigen Wirkungen auszuklammern, die sich aus dem Zwangscharakter der Sozialversicherung ergeben, wird meist kein real in der Privatwirtschaft vorzufindendes, sondern ein hypothetisches Alterssicherungssystem als Referenz zugrunde gelegt. Das fiktive Alterssicherungssystem zeichnet sich dadurch aus, daß entweder die Beiträge oder die gewährten Leistungen normiert sind und in ihrer Höhe der der gesetzliche Rentenversicherung entsprechen. ${ }^{303}$ An einem solchen Referenzsystem wird kritisiert, daß

1. es kein reales, sondern ein ideelles System ist,

2. gravierende Unsicherheiten bei den Verzinsungsannahmen bestehen und

3. der Aspekt des Sozialausgleichs im Sinne eines Risikoausgleichs vernachlässigt wird.

301 Da die intergenerative Umverteilung im Vordergrund steht, wird hier "beitragsäquivalent" im Sinne der Globaläquivalenz verstanden. Vgl. auch Wagner (1984), S. 118.

302 Vgl. Bōsch (1987), S. 34ff; Schulenburg (1990); Wagner (1984); Dinkel (1986) sowie die dort angegebene Literatur (Fußnote 7); Dinkel (1985) , S. 345. und die dort angegebene Literatur; Becker (1985), S. 152; Maydell (1983); Steden (1981); Koppelmann (1979) sowie Stolz (1983), S. 98ff.. Glismann und Horn heben diese Sichtweise in ihrem Beitrag auch semantisch hervor, wenn sie die Finanzierungsverfahren bewertend in "verteilungsneutrale Kapitalstockverfahren" einerseits und "Umverteilungsverfahren" andererseits einteilen und das Umlageverfahren zur letzten Kategorie zählen. Vgl. Glismann/Horn (1996), (1997). Raffelhüschen (1989) bezeichnet den Generationenvertrag des Umlageverfahrens pauschal als Ponzi-Mechanismus und somit als intergenerativen Umverteilungsmechanismus. Demgegenüber sei eine fundierte, staatlich oder privat organisierte Alterssicherung mit Risikopooling ohne intergenerative Umverteilung. 
Ad. 1)

Der erste Einwand gegen den hypothetischen Charakter des Vergleichssystems findet sich beispielsweise bei WAGNER. Er bezeichnet eine solche Vorgehensweise gerade deshalb als unbefriedigend, weil weltweit kein Rentenfonds existiere, der fast die gesamte Bevölkerung absichere. ${ }^{304}$ Nicht zuletzt bliebe daher auch ungeklärt, welche Zeitreihen der Beiträge und Erträge für den Vergleich heranzuziehen seien. Er bezeichnet ein solches Vorgehen daher als

„ein geradezu klassisches Beispiel dafür, daß einzelwirtschaftliche Analogien bei gesamtwirtschaftlichen Betrachtungen in die Irre führen können. ${ }^{305}$

Zudem sei aufgrund der unsicheren Verzinsung und des Sicherheitszuschlags der reale Wert des Kapitals (selbst wenn man von der Inflation absieht) unsicher, so daß auch eine kapitalgedeckte, privatwirtschaftlich organisierte Lebensversicherung nur eine Anteilsgerechtigkeit garantieren und nicht als beitragsäquivalent bezeichnet werden könne. ${ }^{306}$

Die grundlegende Kritik dieses Einwands, daß einzelwirtschaftliche Lösungen auf gesamtwirtschaftliche Probleme nicht übertragbar seien und eine solche Referenz somit fiktiv sei, ist allerdings wenig überzeugend. Denn das Referenzsystem soll lediglich ein methodisches Hilfsmittel zur Operationalisierung der Verteilungseffekte darstellen. Anhand dieses Bezugspunktes lassen sich die Verteilungswirkungen eines realen privaten Alterssicherungssystem ebenso messen, wie die der gesetzliche Rentenversicherung. Zusätzlich kann ein Vergleich beider Effekte zeigen, welches System stärker umverteilt. Es ist DINKEL daher uneingeschränkt zuzustimmen, wenn er in diesem Zusammenhang ausführt:

„Die hypothetisch äquivalente Rente, mit der die tatsächliche Rente in Umverteilungsanalysen verglichen wird, ist ein Referenzsystem für Umverteilungsaussagen und darf nicht mit einem Zielsystem verwechselt werden. ${ }^{307}$

Ad. 2)

Gravierender ist hingegen die Kritik von WAGNER, die sich darauf bezieht, für ein solches Vergleichssystem den adäquaten Zinssatz und den adäquaten Rechnungszinsfuß festzulegen. $\mathrm{Zu}$ beachten wäre beispielsweise, daß der Zinssatz in realen Ökonomien heterogen ist und zeitlich variiert. Bei größerer Kapitaldeckung stehen zudem weniger lukrative Anlagen zur Auswahl und etwaige gesamtwirtschaftliche Rückkopplungseffekte müßten beachtet werden. Der hier erhobene Einwand der starken Abhängigkeit von den Verzinsungsannahmen ist jedoch nicht grundsätzlicher, sondern methodischer Art und wird in Abschnitt 7.2.1 behandelt.

\footnotetext{
304 Vgl. Wagner (1984), S. 61; vgl. auch Dinkel (1986), S.79. Das chilenische Modell ist immer noch in der ${ }_{n}$ Einführungsphase“ und kann angesichts der zu beachtenden Besonderheiten dieser Reform aber auch aufgrund der noch bestehenden Unzulänglichkeiten kaum als Referenz herangezogen werden. Vgl. zur chilenischen Reform z.B. Schulz-Weidner (1996); Queisser, M. (1993). Wagner (1985), S. 152.

306 Vgl. Wagner (1985), S. 153 und (1984), S. 21 ..
} 
Einige Autoren kritisieren an der Wahl eines kapitalgedeckten und beitragsäquivalenten Alterssicherungssystems als Umverteilungsnullpunkt, daß es sich vornehmlich am Konzept der Leistungsgerechtigkeit orientiert und der Solidarausgleich bei einem solchen Referenzmaßstab nicht bzw. nicht hinreichend berücksichtigt wird. Da die gesetzliche Rentenversicherung aber auch auf anderen Gestaltungsprinzipien und Gerechtigkeitsgrundsätzen beruhe, könne eine auf der Beitragsäquivalenz beruhende Rendite immer nur ein ergänzendes Kriterium darstellen. ${ }^{308}$

DAVIES wendet sich beispielsweise grundsätzlich gegen die Wahl der Rendite als Umverteilungsmaß mit dem Hinweis, daß die intergenerative Verteilung einer auf solidarischen Elementen aufbauende Alterssicherung nicht anhand von Investitionskennzahlen beurteilt werden kann. Die Beiträge seien nicht als Investition, sondern als Preis für die erworbene Sicherheit zu interpretieren. ${ }^{309}$ In seinen Ausführungen kritisiert er auch die fehlende Berücksichtigung des interpersonellen Risikoausgleichs einer Versicherung. Zumindest dieser Teil der Kritik greift nur bedingt, denn in einem kapitalgedeckten äquivalenten System sind ebenfalls Elemente des interpersonellen Risikoausgleichs enthalten. Bösch formuliert sogar explizit, daß der Risikoausgleich beim hypothetischen, umverteilungsneutralem Referenzsystem nicht als redistributiver Faktor angesehen werden darf und bei einem beitragsäquivalenten System auch nicht wird. ${ }^{310}$

Dabei beschränkt er den Risikoausgleich allerdings auf den intragenerativen Aspekt, da ein intergenerativer sozialer Ausgleich im Gegensatz zum intragenerativen in den gesetzlichen Bestimmungen der gesetzlichen Rentenversicherung systematisch nicht vorgesehen sei.

308 Vgl. GVG-Papier „Alterssicherung : Finanzierung durch Umlage und Kapitaldeckung“, S.11: „Keinesfalls ist mit der Äquivalenz der unmittelbare Vergleich zur Rentierlichkeit privat angelegten Vermögens geschützt. Der Äquivalenzbegriff der gesetzlichen Rentenversicherung ist auch nicht so streng wie in der Privatversicherung“. Bomsdorf betont ebenfalls: „Im übrigen erscheint es als nicht systemadäquat, die gesetzlichen Rentenversicherung primär nach der Höhe ihrer Rendite zu beurteilen, wie dies in jüngster Zeit häufiger geschieht. Selbst wenn sie als Versicherung im klassischen Sinne angesehen werden könnte, was zweifelhaft erscheint, so ist sie doch als Bestandteil unseres Sozialversicherungssystems ein Eckpfeiler des auf dem Solidaritätsprinzip aufbauenden Sozialstaates und insoweit keinesfalls allein unter Renditegesichtspunkten zu sehen. "Bomsdorf (1997), S. 11. Ähnlich auch Eitenmüller (1996), S. 2:

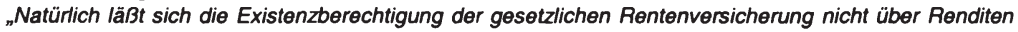
ableiten. Schließlich ist sie als Sozialversicherung und nicht als Investitionsobjekt konzipiert. “ oder Lampert (1990); Waldmann, H. (1981), S.455; Meinhold, H. (1976), S. 48. Eine andere Auffassung vertreten hingegen Glismann und Horn. Sie erheben das Prinzip der (streng aktuarische) Beitragsäquivalenz zu dem ausschlaggebenden Gerechtigkeitsmaßstab für die Alterssicherung, wenn sie ausführen: ${ }_{n} j \theta$ mehr das Verhältnis zwischen Beiträgen zur Alterssicherung und (in Form von Renten) zu erwartenden Erträgen gestört ist, desto stärker wird das Gerechtigkeitsempfinden jedes Beitragszahlers verletzt, bis schließlich das System politisch nicht mehr haltbar ist. "Glismann/Horn (1995), S. 310 und auch S. 340: "Gerecht wäre fraglos ein System, bei dem die in Tabelle 6 ausgewiesene Umverteilungsintensität (gemessen an der Differenz zu den kapitalmarktanalogen Erträgen; Anmerkung des Autors) jeweils bei Null läge. “ Zur impliziten Annahme der versicherungstechnischen Äquivalenz als unerreichbares Gerechtigkeitsideal siehe auch Dinkel (1986) und Schulenberg (1988).

309 Vgl. Davies (1996), S. 8. Siehe zu ähnlichen Äußerungen Fußnote 308.

310 Vgl. Bösch (1987), S. 34. 
„Die nachfolgend aufzuzeigenden Umverteilungsvorgänge zwischen den einzelnen Generationen ergeben sich vielmehr als Folge der Veränderung bestimmter ökonomischer, demographischer und institutioneller Faktoren, die im einzelnen nicht immer vorhersehbar sind. ${ }^{311}$

Gerade das Fehlen eines intergenerativen Ausgleichs begründe die Wahl eines Kapitalstockverfahrens als Vergleichsmaßstab: ${ }^{312}$

„Die Beiträge jeder Risikogemeinschaft und damit auch jeder Generation müssen im Kapitaldeckungsverfahren so kalkuliert sein, daß alle Risiken vollständig abgedeckt sind, ohne Beitragszahlungen anderer Generationen in Anspruch nehmen zu müssen. Aus diesem Grunde eignet sich das Kapitaldeckungsverfahren hervorragend zur Festsetzung eines Umverteilungsnullpunkts, da per definitionem keine Generation Finanzierungsverpflichtungen für andere Generationen übernehmen muß. ${ }^{\text {(313 }}$

Sofern diese These auf einer Gerechtigkeitsvorstellung basiert, bei der bereits die Existenz physischer Zahlungsströme zwischen den Generationen als intergenerativen Umverteilung gewertet wird, ist sie zwar formal zutreffend, jedoch wenig hilfreich bzw. belanglos. Ein umlagefinanziertes System, bei dem alle Generationen identisch und in gleicher Weise wie bei einem Kapitalstockverfahren behandelt werden, würde a priori und tautologisch als ungerechtes Umverteilungssystem klassifiziert werden. Insbesondere wird bei einer solchen Interpretation der intergenerativen Umverteilung ausgeblendet, daß verschiedenen Generationen auch ohne direkte Beitragszahlungen und Finanzierungsverpflichtungen durch ein System ungleich behandelt werden können bzw. sogar die Situation anderer Generationen beeinflussen können. ${ }^{314}$

Darüber hinaus wäre diese Begründung für das Kapitalstockverfahren als Umverteilungsnullpunkt nur dann unmittelbar einleuchtend, wenn eine Alterssicherung ausschließlich das individuelle Ziel einer intertemporalen Einkommensumschichtung im Lebenszyklus der Generation verfolgen würde. ${ }^{315}$ Folgt man jedoch der gängigen Auffassung, wonach eine Versicherung sich dadurch auszeichnet, das Gut „Sicherheit“ anzubieten, dann muß die obige These eingeschränkt werden. Genau hierin liegt ja die Motivation des interpersonellen Risikoausgleichs. Dieser ist aber nicht in der Lage, alle Risiken abzusichern, vielmehr bestehen auch solche Risiken, die sich nur zwischen Generationen absichern

Bösch (1987), S. 39.

Dabei bezieht sich Bösch auch auf Thullen, der den wesentlichen Unterschied zwischen dem Umlageverfahren und dem Kapitalstockverfahren darin sieht, daß das erste eine offene und das zweite Verfahren eine geschlossenen Risikogemeinschaft aufweist.

Bösch (1987), S. 39.

So führt auch Speckbacher hierzu treffend aus: „Eine weitverbreitete Auffassung stellt offensichtlich das Prinzip dar, im Idealfall solle ein Alterssicherungsverfahren so gestaltet sein, daß keine Generation (...) das Wohl der anderen Generationen vermindert (...). Die Bestimmung einer Vorschrift, die die Einhaltung dieses Prinzips gewährleistet, scheint aber gerade wegen der mit einem Alterssicherungssystem typischerweise verbundenen gegenseitigen Beeinflussung des Wohlstands der verschiedenen Generationen nicht möglich zu sein. Die Definition einer derartigen Wohlstandsänderung kann nämlich bei Beachtung dieser Interdependenzen notwendigenweise nicht absolut erfolgen, wie dies etwa mit dem Prinzip der "Beitragsāquivalenz" suggeriert zu werden scheint, sondern muß sich auf einen relativen Vergleich von Wohlstandsniveaus bei Zugrundelegung alternativer Alterssicherungsverfahren beschränken. "Speckbacher (1994), S. 66. Speckbacher betont dabei vor allem die Entwicklungspotentiale einer Volkswirtschaft (vgl. Kapitel 6) allerdings hängt die schwer zu operationalisierende Wohlfahrt auch von einer Reihe weiterer Faktoren ab.

Vgl. Wagner (1984), S.54.

143 
lassen. Akzeptiert man, daß die Sicherheit in der Altersversicherung sich auch auf den relativen Lebensstandard bzw. die Verstetigung des Einkommens im Alter bezieht, so gehören gerade die schwer vorhersehbaren demographischen oder gesamtwirtschaftlichen Entwicklungen, ${ }^{316}$ zu dieser Kategorie der Risiken.

Diese Entwicklungen haben aber nicht nur im Umlageverfahren, sondern in jedem Alterssicherungssystem einen Einfluß auf die Sicherung des relativen Lebensstandards. ${ }^{317}$ In einem kapitalgedeckten privaten System wird zwar versucht, dieser Unsicherheit durch einen Sicherheitszuschlag zu begegnen, eine Absicherung des relativen Lebensstandards gegen die Unsicherheit über die Rahmenbedingungen kann dadurch allerdings nicht gewährleistet werden. ${ }^{318}$ Bei diesen Einflußfaktoren handelt es sich folglich um Risiken, die eben nicht vollständig abgedeckt werden können, ohne andere Generationen in Anspruch zu nehmen! Dadurch, daß die Rentenzahlungen saldenmechanisch immer aus dem laufenden Sozialprodukt (ggf. ergänzt um Zahlungen aus dem Ausland) finanziert werden müssen, ${ }^{319}$ bedeutet intergenerativer Risikoausgleich immer eine Verteilung der Last auf gleichzeitig lebende Erwerbstätige und Ruheständler. ${ }^{320}$

Während diese Verteilung in einem umlagefinanzierten System im Risikofall mit Hilfe des staatlich garantierten, fiktiven „Generationenvertrags“ geregelt wird, stellen die Rentenansprüche des Kapitalstockverfahrens ein verbrieftes Recht dar, welches ebenfalls von den jeweiligen Erwerbstätigen in Form von Zinszahlungen und Liquiditätserlösen gezahlt werden muß. Im Umlageverfahren wird die Lastverteilung durch die Rentenformel bestimmt, während sie im Kapitalstockverfahren durch indirekte gesamtwirtschaftliche Anpassungsprozesse und Ausgleichsreaktionen erfolgt. ${ }^{321}$

316 Z.B. Lebenserwartung, Kinderlosigkeit, Erwerbsquoten, Invaliditätswahrscheinlichkeit etc..

317 Vgl. Abschnitt 2.4.3 und 4.4.3.2.

318 Vgl. Abschnitt 4.4.3.2. Wagner führt hierzu aus: „Das Umlageverfahren bietet ein hohes Maß an Einkommenssicherheit, und es kann flexibel auf Änderungen der demographischen und wirtschaftlichen Rahmenbedingungen reagieren. Sieht man insbesondere die Entwicklung der Lebenserwartung, der Invalidierungshäufigkeiten und der Enwerbsquoten als nur bedingt vorhersehbar an, kann man durch diese Bereiche verursachte Beitrags- und Leistungsänderungen der Altersvorsorge als intergenerationalen Risikoausgleich fassen, welcher durch eine Kapitalbildung erschwert würde." Wagner (1984) S. 111, vgl. auch S. 62-69, S.72 und S. 47. Bösch hingegen betrachtet den Sicherheitszuschlag jedoch als weiteren Risikoausgleich zwischen Überlebenden und den vorzeitig Sterbenden, der Prinzipiell jeder einzelnen Generation zugerechnet werden kann. Vgl. Bösch (1987), S, 42. Der Sichtweise Böschs kann zwar hinsichtlich der Veränderung des Enwartungswerts der Restlebenserwartung zugestimmt werden (vgl. Abschnitt 5.3.1.3) allerdings werden dabei die anderen unsicheren Faktoren außer Acht gelassen.

319 Die Kritik an der sogenannten Mackenroththese, daß diese etwaige Wohlfahrtseffekte ausblende, ist in diesem Fall ohne belang. Hier geht es ausschließlich darum, daß die durch veränderte Rahmenbedingungen verursachten gesamtwirtschaftlichen Anpassungsprozesse die verschiedenen sozioökonomischen Gruppen unterschiedlich belasten können.

320 Dies schließt jedoch nicht aus, daß die Enwerbsgeneration zum Teil ihrerseits die von ihr getragene Last durch entsprechende Verhaltensänderung an nachfolgende Generationen weiterreichen kann (vgl. Abschnitt 2.4.2.2).

321 Vgl. dazu die Ausführungen in Abschnitt 2.4.3 und Schulenburg (1989), S.276. Wagner (1985), S. 155 führt hierzu aus: „daß die Anpassung der Forderungen der Ruhestandsgeneration an geänderte wirtschaftliche Bedingungen am Kapitalmarkt automatisch erfolgt. “Ein in diesem Zusammenhang oft vorgebrachtes Argument findet sich bereits bei Mackenroth (1952), S. 267f.: Schrumpft die Bevölkerung, so kann der Kapitalstock zur Finanzierung der Renten zwar abgeschmolzen werden, die geringere Zahl von Neuzugängen, die ein Vermögen zur Altersvorsorge aufbauen senkt aber die Nachfrage nach den Ver- 
Da die Beschränkung der intergenerativen Umverteilung auf rein physische Zahlungsströme zwischen den Generationen abzulehnen ist, übernehmen auch im Kapitalstockverfahren Generationen „Finanzierungsverpflichtungen für andere Generationen“.

Das Kapitalstockverfahren ist folglich kein geeigneter Maßstab. Allerdings muß das Argument, der Risikoausgleich werde vernachlässigt, dahingehend präzisiert werden, daß bei der Wahl einer beitragsäquivalenten, kapitalgedeckten Alterssicherung als Referenzsystem der intergenerative Risikoausgleich nicht berücksichtigt wird.

Es sei nur am Rande darauf hingewiesen, daß die Einkommensstetigkeit seit 1957 zu den expliziten Zielen der Alterssicherung gehört und diese nicht zuletzt deshalb auf den Prinzipien der Teilhabeäquivalenz und der Leistungskopplung am durchschnittlichen Erwerbseinkommen beruht. ${ }^{322}$ Vor diesem Hintergrund ist auch BösCHS Behauptung, der intergenerative Risikoausgleich sei in der gesetzlichen Rentenversicherung nicht vorgesehen, äußerst strittig.

Richtig ist jedoch, daß es bislang an einer systematischen Präzisierung dessen fehlt, was zum intergenerativen Risikoausgleich gehören sollte, in welchem Umfang die Sicherung erfolgen und wie die Last bei Eintritt des Risikos verteilt werden sollte.

\subsubsection{Zur Legitimation des Kapitalstockverfahrens als verteilungsneu- trales Referenzsystem}

Bevor aufgrund der vorgenannten Kritik das idealtypische Kapitalstockverfahren als adäquates Referenzsystem verworfen werden kann, bleibt zu prüfen, ob die Wahl eines solchen verteilungsneutralen Vergleichssystems sich trotz der obigen Einwände anderweitig begründen läßt. Im Zusammenhang mit der Frage, was als ungerechte intergenerative Umverteilung eines Alterssicherungssystems zu werten sei, greift SPECKBACHER den Gedanken der Opportunitätskosten auf, wenn er ausführt:

„Solange nämlich durch die Reform eines bestimmten Alterssicherungssystems eine Umverteilung möglich wäre, welche jede Generation besser stellt, ist in der Verhinderung einer solchen Umverteilung im obigen Sinne (aus der Gerechtigkeitsperspektive) ein illegitimer Zwang zu sehen. ${ }^{323}$

Die Formulierung "welche jede Generation besser stellt" deutet schon an, daß es sich hier um einen Effizienzvergleich im pareto-optimalen Sinne handelt. D.h. entsprechend dieser Aussage könnte man ein Kapitalstockverfahren trotz der erwähnten Einwände dann als Referenzsystem heranziehen, wenn das Kapitalstockverfahren sich hinsichtlich des Lebenseinkommens als Pareto-superior erweist. Die Diskussion zur Effizienz eines Alterssicherungssystems (vgl. Kapitel 2) zeigte jedoch, daß keines der Finanzierungsver-

mögenstiteln. Die Ruheständlicher können ihr Erspartes folglich nur mit Kursverlusten liquidieren. Vgl. z.B. auch Becker (1981), S. 54ff, Meinhold (1984), S. 109. Dieser Einfluß läßt sich zwar in dem Maße vermindern, in dem das Kapital im Ausland angelegt wird, allerdings wird diese Ausweichmöglichkeit durch die Anforderungen an die Sicherheit der Auslandsanlage zum Zweck der Altersvorsorge begrenzt. Vgl. Abschnitt 4.4.4. Speckbacher (1994), S. 15. 
fahren unter beliebigen Bedingungen nach dem Pareto-Kriterium überlegen ist. Folglich läßt sich auch mit dem Effizienzargument nicht begründen, weshalb ein kapitalgedecktes System als Vergleichsmaßstab herangezogen werden soll. Dieser Argumentation zu Folge wäre es - entsprechend des Prinzip der Risikostreuung - wesentlich einsichtiger, ein Mischsystem zu befürworten und dies in der Verteilungsanalyse auch bei der Wahl des Vergleichsmaßstabs zu berücksichtigt.

BREYER verweist ebenfalls darauf, daß die Frage nach der Legitimation des Referenzsystems oft vernachlässigt wird:

"In der Literatur zur intergenerativen Gerechtigkeit von Alterssicherungssystemen wird vielfach (z.B. Dinkel 1986, oder Schulenburg, 1990) explizit oder implizit der Zustand versicherungstechnischer Äquivalenz von Beiträgen und Rentenansprüchen als ein Ideal der Gerechtigkeit hingestellt, ohne daß jedoch dieses Prinzip auf eine allgemeine ethische Norm zurückgeführt würde. ${ }^{\text {.324 }}$

Er führt weiter aus:

„In einem neueren Beitrag von Breyer/Spremann (1989) wird aufgezeigt, daß eine solche Norm aus einer modifizierten Version der Anspruchstheorie der Gerechtigkeit von Nozick (1974) gewonnen werden kann. ${ }^{324}$

Im folgenden ist daher zu untersuchen, ob die im vorangegangenen Abschnitt formulierten Einwände angesichts dieser aus der Anspruchstheorie abgeleiteten Rechtfertigung hinfällig werden. BREYER und SPREMANN modifizieren die Anspruchstheorie einerseits durch eine Erweiterung auf die intergenerative Fragestellung, ${ }^{325}$ andererseits werden hypothetische Verteilungen dann als gerecht angesehen,

„wenn sie bei gegebenen Präferenzen aus einer gerechten Verteilung durch gerechte Aneignung oder Übertragung gewonnen werden kann". ${ }^{326}$

Nach dieser Erweiterung der Anspruchstheorie sind

„insbesondere alle diejenigen Verteilungen gerecht, die zu einer gegebenen als gerecht charakterisierten Verteilung Pareto-indifferent oder Pareto-superior sind. ${ }^{327}$

Die Argumentation stützt sich folglich ebenfalls auf die Frage nach der Pareto-Effizienz eines Alterssicherungssystems, unterscheidet sich jedoch von dem vorangegangenen Effizienzargument. Ausgangspunkt ist in diesem Fall nämlich die These, daß eine auf angespartem Geldkapital oder auf Leibrenten basierende Altersversorgung bereits ein intergenerativ gerechtes System darstellt, da es auf einen vorher vereinbarten Tausch, sprich auf einer gerechten Übertragung im Sinne Nozıcks beruht. Eine solche Form der Altersvorsorge wäre - dieser Auffassung zur Folge - dann in jedem Fall intergenerativ gerecht und zwar unabhängig davon, ob sie auch Pareto-superior ist. Es wäre insbesondere unerheblich, ob Einführungsgenerationen oder andere Generationen aufgrund der Rahmen-

\footnotetext{
324 Breyer (1990a), S. 132.

„Maßgeblich für die Gerechtigkeit einer Verteilung ist dann nicht mehr, ob jedes Individuum innerhalb einer Generation Anspruch auf die von ihm gehaltenen Güter hat, sondem ob sich die Generation als ganze ihr Vermögen rechtmäßig angeeignet hat. "Breyer/Spremann (1990), S. 394.

326

327

Breyer/Spremann (1990), S.394.
} 
bedingung $G^{*} M>R$ in einem Umlageverfahren einen Wohlfahrtsgewinn haben könnten. Ein Umlageverfahren hingegen ist nur dann intergenerativ gerecht, wenn es zu einem nach Nozick zustande gekommenen Alterssicherungssystem pareto-indifferent oder pareto-superior ist.

In einem Umlageverfahren wären nach diesem Ansatz die Anforderungen der intergenerativen Gerechtigkeit für den Fall einer geschlossenen Volkswirtschaft nur dann erfüllt, wenn eine überkapitalisierte Ökonomie vorläge. ${ }^{328}$ Diese Situationen wird allerdings von den Autoren als wenig plausibel erachtet. In einer offenen Volkswirtschaft könnten die Voraussetzungen für ein intergenerativ gerechtes Umlageverfahren zwar gegeben sein, dies hängt jedoch von einem - praktisch unmöglichen - in die unendliche Zukunft reichenden Vergleich der Zins und Wachstumsraten ab. ${ }^{329}$ Konkret dürfte das Verhältnis der Lohnwachstumsrate zum Zinssatz nicht auf ewige Zeiten strikt kleiner als 1 sein. Wenngleich dies a priori nicht ausgeschlossen werden könne, wären angesichts der für die Bundesrepublik Deutschland prognostizierten Situation in den kommenden Jahrzehnten eine untragbare Abgabenlast die Folge, die wiederum entsprechende Gegenmaßnahmen erfordern würde. ${ }^{330}$ Die Bedingung seien, so wird suggeriert, so unwahrscheinlich, daß man die Fälle faktisch vernachlässigen könne.

Aber selbst wenn angenommen werden könnte, daß eine überkapitlisierte Volkswirtschaft ausgeschlossen ist und $R>M^{*} G$ für alle Zukunft gegeben sei, würde - entgegen der von BREYER und SPREMANN aufgestellten Behauptung - aufgrund der folgenden drei Kritikpunkte noch keine ethisch fundierte Legitimation der versicherungstechnischen Äquivalenz als einzig zulässigen intergenerativen Gerechtigkeitsmaßstab gegeben sein.

1. Ihr Ergebnis resultiert größtenteils aus den von ihnen implizit unterstellten Annahmen.

In ihrer Erweiterung der Anspruchstheorie betonen sie die Bedeutung der gegebenen Präferenzen. Sie unterstellen implizit, daß die Versicherten eine mindestens an der versicherungstechnischen Äquivalenz aufgerichteten Rentenleistung präferieren und daß "der maximal erreichbare Nutzen eine eindeutige Funktion des diskontierten Lebenseinkommens" darstellt. ${ }^{331}$ Angesichts dieser unterstellten Präferenzen kann es daher kaum verwundern, wenn ein umlagefinanziertes System in ihrer Analyse nur dann als intergenerativ gerecht akzeptiert wird, wenn es mindestens das gleiche diskontierte Lebenseinkommen gewährt, wie ein versicherungstechnisch äquivalentes System.

Diese Ausführungen stellen im Grunde eine Kritik an dem angewandten Kriterium zur Messung der Pareto-Effizienz dar. Bislang wurde die Effizienz - also die Frage, ob eine Generation „besser gestellt" wird - allein anhand des Kriteriums der eigenen Konsummöglichkeiten, sprich des absoluten Lebenseinkommens beurteilt. Weitere Präfe-

Breyer/Spremann (1990), S.396.
} 
renzen, die sich beispieisweise dann ergeben, wenn Unsicherheit oder unvollkommene Märkte betrachtet werden, wurden größtenteils ausgeschlossen. ${ }^{332}$ In ähnlicher Weise kritisiert auch SPECKBACHER, daß die Forderung nach barwertäquivalenten Beiträgen und Leistungen nicht nur aufgrund der erforderlichen Annahme eines vollkommenen Kapitalmarktes bedenklich ist, sondern auch,

"weil das Wohl einer Generation, auch unter der Annahme der ausschließlichen Berücksichtigung des eigenen Konsums, nicht unabhängig von Präferenzen bezüglich der zeitlichen Aufteilung des Lebenskonsums ist. Mit anderen Worten: Der Strom von Beiträgen und Leistungen kann nicht unabhängig von Präferenzen auf eine einzige Größe verdichtet werden. Entsprechend kann dann ein Gerechtigkeitskriterium, das auf das Wohl der Generationen abzielt, nicht unabhängig von Präferenzen formuliert werden. 333

Bei Unsicherheit wäre die Sicherung des relativen Lebensstandards eine durchaus plausible Präferenz, die bei einer auf angesparten Geldkapital (oder Leibrenten) basierenden Altersvorsorge von der ex ante unbekannten Entwicklung der Parameter "Lohnwachstum" und „Zinssatz" abhängt. In einer Welt mit risikoaversen Individuen und Unsicherheit über die Entwicklung der verschiedenen Einflußgrößen, können mit der Einführung eines Versicherungssystems, das eine bestimmte Sicherheit des Alterseinkommens (bzw. präziser des relativen Lebensstandards) gewährt, Effizienzgewinne verbunden sein, wenn dadurch der ex ante erwartete Nutzen steigt. Eine Reihe von Arbeiten, ${ }^{334}$ die sich dieser Fragestellung widmen, kommen zu den Schluß, daß ein entscheidender Vorteil des Umlageverfahrens eben in der Verbesserung einer solchen intergenerativen Streuung des Einkommensrisikos liegt. Wenn die Versicherten sich folglich entsprechend ihrer Präferenz für ein System mit intergenerativem Risikoausgleich entscheiden, dann muß eine Rentenleistung, die ex post aufgrund des Preises für die Sicherheit geringer ist als jene einer beitragsäquivalenten Rentenleistung, dennoch als gerechte Übertragung im Sinne Nozıcks gewertet werden. ${ }^{335}$

332 Berücksichtigl wurde lediglich der interpersonelle Risikoausgleich. So betont Breyer an anderer Stelle selbst, daß der in die Effizienzdefinition eingehende Nutzenindexwert von den angenommenen Präferenzen abhängt. Vgl. Breyer (1990), S. 19.

333 Speckbacher (1994), S. 67, der die Präferenzen vor allem auf den spezifischen Verlauf des Konsums bzw. des Sparens über den Lebenszyklus bezieht.

334 Vgl. Abschnitt 2.5.1.

335 Demgegenüber könnte eingewandt werden, daß ein solcher „Generationenvertrag" keine "gerechte Übertragung" im Sinne eines freiwilligen Tausches darstellt, da die nachfolgende Generation noch ungeboren ist und der Staat als Mittler dieses Vertrages fungieren muß. Dieser Einwand wäre jedoch grundsätzlicher Art und würde sich vor allem gegen die mangelnde Übertragbarkeit der Anspruchstheorie auf intergenerative Fragestellungen richten (vgl. Abschnitt 3.3.3). Bei einer solchen Erweiterung müßten "repräsentative Generationenvertreter" - ähnlich wie im Rawls'schen Konzept - in einer hypothetischen Versammlung zusammentreffen können.

Zudem stellt eine solch enge Interpretation der "gerechten Aneignung und gerechten Übertragung“ den Anspruch der Anspruchstheorie in Frage, eine allgemeine Gerechtigkeitstheorie zu sein. Denn wenn sie sich a priori auf Prinzipien stützt, die ausschließlich auf einer radikaten Ideologie des freien Marktes beruhen, würden Marktversagen und Marktunvollkommenheiten prinzipiell ausgeblendet werden. Jede Möglichkeit, durch zentrale Steuerung eine effizientere Situation herbeizuführen würde a priori als ungerecht angesehen werden. Diese Betrachtungsweise wäre im übrigen auch nicht konsistent mit der Grundidee der Enweiterung nach Breyer und Spremann (1990). 
2. Die mangelnde ethische Fundierung der Anspruchstheorie ${ }^{336}$ würde es ebenso ermöglichen, eine solche „dem Grundgedanken der Theorie folgende“ Modifikation der Theorie vorzunehmen, mit der sich eine „ethisch fundierte Legitimation des Umlageverfahrens als zulässigen intergenerativen Gerechtigkeitsmaßstab“ ableiten ließe.

Wie in Abschnitt 3.3.3 dargelegt, basiert die Anspruchstheorie im wesentlichen auf dem Grundsatz der Regelgerechtigkeit. NOzICKS Theorie ist im Grunde genommen lediglich ein Versuch, die relevanten Regeln aufzustellen. Die von NozıcK formulierten Regein haben jedoch ebenfalls einen ad-hoc Charakter, d.h. sie mögen zwar plausibel sein, an einer ethischen Begründung mangelt es innen aber in gleicher Weise, wie den von BREYER kritisierten Ansätzen von DINKEL und SCHULENBURG. ${ }^{337}$

Zudem wollte Nozick mit seiner Theorie ursprünglich zeigen, daß der Nachtwächterstaat die einzig gerechte Staatsform darstellt. An dieser Stelle wird nicht im einzelnen auf die Schlußfolgerung eingegangen, daß ein Minimalstaat die einzig gerechte Lösung sei. Wie seine Ausführungen allerdings zeigen, wird auch von Nozick nicht in Abrede gestellt, daß ein Staat bestimmte Aufgaben übernehmen muß, die in einer freien Tausch- bzw. Übertragungswirtschaft nicht oder nur unzureichend erfült würden. Dies wirft sogleich die Frage auf, wie sich der „gerechte Anspruch“ bei solchen Staatsaufgaben ethisch legitimieren läßt.

Folgt man der grundlegenden Logik der Anspruchstheorie, müßten die Vorgänge der Staatstätigkeit wiederum an ihrer Regelgerechtigkeit gemessen werden. Als gerechte Regel für Staatsaktivitäten wäre der demokratische Abstimmungsprozeß ebenso plausibel wie die von NOzıCK formulierten Regeln. Die Anspruchstheorie ließe sich daher wie folgt erweitern: Sofern gegebene Präferenzen nicht durch die ersten beiden Prinzipien, sondern nur durch staatliche Aktivität erfüllt werden können, gilt jene Verteilung als gerecht, bei der sich die Ansprüche aus einem demokratischen Abstimmungspro$z e ß$ ergeben. Die Parallelen zum „rechtsethischen Relativismus“338 sind evident. Denn eine solche Interpretation würde bedeuten, daß jedes existierende Alterssicherungssystem a priori gerecht ist, sofern sich bestimmte gegebene Präferenzen in einem auf klassischer Übertragungen beruhenden Altersvorsorge nicht erfüllen lassen ${ }^{339}$ und solange es sich im demokratischen Abstimmungsprozeß gebildet hat.

Es ist nicht die Absicht dieser Ausführungen, die Gerechtigkeit des Umlageverfahrens mit Hilfe der Anspruchstheorie bzw. einer Erweiterung des Ansatzes zu belegen. ${ }^{340}$ Sie sollen vielmehr verdeutlichen, daß die Anspruchstheorie (bzw. eine „plausible, der Idee der Theorie nicht widersprechende" Erweiterung des Ansatzes) sich nicht dazu eignet,

336 Vgl. Abschnitt 3.3.3.

337 Vgl. Breyer (1990a), S. 132

338 Vgl. Abschnitt 3.2.2.

339 Eine intergenerative Risikoteilung ist ohne staatliches Eingreifen beispielsweise nicht umsetzbar. Vgl. auch Sudhoff (1996), S. 46.

340 Eine solche Erweiterung wäre - wenngleich plausibel ableitbar - sicherlich nicht im Sinne Nozicks. 
ein verteilungsneutrales Referenzsystem ethisch fundiert zu begründen. Dies gilt um so mehr, weil die Anspruchstheorie sich auf intergenerative Fragestellungen wenn überhaupt dann nur sehr bedingt übertragen läßt. ${ }^{341}$

3. Das Problem der Korrektur bereits abgeschlossener Vorgänge darf nicht vernachlässigt werden.

NozıCK selbst führt aus, daß die von inm „abgelehnten Gesichtspunkte der Verteilungsgerechtigkeit und Gleichheit eine Rolle spielen ${ }^{342}$ können, wenn es angesichts des dritten Korrekturprinzips nicht möglich ist, den Zustand zu rekonstruieren, der sich ohne vorangegangene Ungerechtigkeiten ergeben hätte. Auch BREYER und SPEMANN müssen konzidieren, daß bei ihrer Legitimation des Kapitalstockverfahren als Referenzmaßstabs ein gewisses Dilemma verbleibt,

„da aus der Anspruchstheorie Nozicks nicht ableitbar ist, wie verfahren werden soll, wenn eine gerechte Lösung aufgrund von vergangenen Fehlern nicht mehr erreicht werden kann. ${ }^{343}$

In dieser Arbeit wird untersucht, welchen Einfluß Optionen zur Reform der bestehenden umlagefinanzierten gesetzliche Rentenversicherung auf die intergenerative Gerechtigkeit haben. Selbst, wenn die ersten beiden Kritikpunkte nicht greifen würden, wäre angesichts dieses dritten Einwands fraglich, ob sich ein beitragsäquivalentes Kapitalstockverfahren mit Hilfe der Anspruchstheorie als verteilungsneutrales Referenzsystem legitimieren läßt.

Als vorläufiges Ergebnis kann daher festgehalten werden, daß ein beitragsäquivalentes und kapitalgedecktes Alterssicherungssystem bestenfalls dann als Referenz einer intergenerativen Umverteilungsanalyse herangezogen bzw. begründet werden kann, wenn eine Risikoaversion hinsichtlich des relativen Lebensstandards im Alter von Null unterstellt wird und die (automatischen) gesamtwirtschaftlichen Anpassungsprozesse infolge von Variationen der ökonomischen und demographischen Rahmengrößen als gerechte Verteilung der Zusatzlast auf die Generationen angesehen wird. ${ }^{344}$

Ansonsten wäre es angemessener, ein Mischsystem als verteilungsneutralen Vergleichsmaßstab zu unterstellen. Je risikoaverser die Individuen und je unsicherer die Entwicklung des Lohn- und Zinssatzes ist, desto größeres Gewicht müßte dem Umlageverfahren zukommen. Die Frage, die sich nun stellt ist, welche Kombination aus Umlage- und Kapitalstockverfahren als optimal angesehen und als Referenzsystem zugrunde gelegt werden kann. Diese Frage läßt sich auf analytischen Wege jedoch nicht beantworten. ${ }^{345}$

\footnotetext{
$341 \mathrm{Vgl}$. Abschnitt 3.3.3.

342 Nozick (1976), S. 146.

343 Breyer/Spremann (1990), S.403.

$344 \mathrm{Vgl}$. Abschnitt 5.3.3. Eine solche Gerechtigkeitsauffassung wäre bestenfalls mit der „wirtschaftlichen Effizienz" aber mit keinen der übrigen Gerechtigkeitsgrundsätze, auch nicht mit dem der Leistungsgerechtigkeit, zu begründen.

345 Vgl. Abschnitt 5.3.2.
}

150 


\subsection{3 „Intergenerationale Durchhaltbarkeit“ als Referenzsystem}

Aufgrund der Schwierigkeit, bei einem hypothetischen Referenzsystem einen adäquaten Rechnungszinsfuß festzulegen bzw. der Unzulänglichkeit, eine Beurteilung der Sozialversicherung anhand eines einzelwirtschaftlichen Maßstabes vorzunehmen, schlägt WAGNER vor, als alternativen Vergleichsmaßstab zur Messung der direkten intergenerativen Umverteilung die sogenannte intergenerationale Durchhaltbarkeit heranzuziehen. ${ }^{346}$

In diesem Ansatz werden zwei Relationen miteinander verglichen: das Verhältnis aller Beiträge einer Versichertenkohorte zum jeweiligen Volkseinkommen während der Erwerbszeit mit dem Verhältnis aller empfangenen Rentenleistungen dieser Versichertenkohorte zum jeweiligen Volkseinkommen während der Ruhestandszeit. Entsprechen sich diese Relationen für jede Kohorte, so gilt das Alterssicherungssystem als intergenerational durchhaltbar und es liegt keine intergenerative Umverteilung vor.

Der Ansatz greift vornehmlich das Problem des adäquaten Diskontierungsfaktors auf und schreibt diese Funktion implizit der Entwicklung der Löhne (bzw. präziser des Volkseinkommens) zu. ${ }^{347}$ Eine ausschließliche Orientierung an der Einkommensentwicklung kann allerdings ebenfalls mit dem Hinweis auf das Opportunitätskostenprinzip bzw. das Prinzip der Risikostreuung im Portefeuille kritisiert werden. WAGNER formuliert dies zwar nicht explizit, führt aber dennoch alternative Diskontierungsszenarien durch, um die Abhängigkeit der Ergebnisse von der Diskontierungsannahme zu verdeutlichen. ${ }^{348}$ Durch die Kopplung an das Volkseinkommen werden die verschiedenen Faktoren der intergenerativen Umverteilung nicht bzw. lediglich zum Teil erfaßt. Profitiert eine Generation beispielsweise durch eine gestiegene Restlebenserwartung, steigen zwar bei diesem Indikator die Rentenleistungen, da jedoch das Volkseinkommen ebenfalls über eine längere Periode erfaßt wird, bleibt der Quotient aus Rentenleistung und Volkseinkommen ceteris paribus konstant. Zudem führt die intergenerative Durchhaltbarkeit nur dann zu gleichartigen Belastungen der Kohorten, wenn eine stationäre Bevölkerung unterstellt wird und es keine Ungewißheit über die relevanten durchschnittlichen Ereigniswahrscheinlichkeiten der ökonomischen Faktoren (z.B. Erwerbsquoten) gibt. ${ }^{349}$ Ansonsten

„ist zu klären, welche Beitrags- und Leistungsänderungen im Umlageverfahren zum effizienten intergenerationalen Risikoausgleich bzw. zur bewußten, im Prinzip vermeidbaren Umverteilung gezählt werden können. ${ }^{350}$

346 Vgl. Wagner (1984), S.61ff.. Wagner bezieht sich zwar bei der Beschreibung der „intergenerationalen Durchhaltbarkeit" auf Lepelmeier, dieser verwendet den Begriff jedoch in einem anderen Zusammenhang. Was Lepelmeier als „intergenerational durchhaltbar" beschreibt, entspricht weitgehend der 1992 in der gesetzliche Rentenversicherung umgesetzten Dynamisierung der Renten nach Maßgabe des Nettoprinzips. Vgl. Lepelmeier (1979), S. $160 \mathrm{f}$.

„Für diesen Maßstab zur Messung der intergenerationalen Umverteilung spricht, daß er ohne letztlich unbeweisbare Annahmen über den volkswirtschaftlich sinnvollen Rechnungszinsfuß eines fiktiven kapitalgedeckten Systems auskommt." Wagner (1984), S. 61.

Vgl. Wagner (1984), S. 249ff..

Vgl. Wagner S. $62 f$ und 168.

Wagner (1984), S. 63. 
Eine solche Differenzierung bzW. Bereinigung durch Elemente des intergenerativen Risikoausgleichs nimmt WAGNER jedoch auch nicht vor. Er umgeht das Problem, indem er die Versichertenkohorten unter Status quo Bedingungen simuliert. Dadurch klammert er automatisch die Ungewißheit über die zukünftige Entwicklung und den intergenerativen Risikoausgleich weitgehend aus. ${ }^{351} \mathrm{Da}$ für das vorliegende Untersuchungsziel aber gerade der intergenerativer Risikoausgleich wichtig ist und insbesondere keine stationäre Bevölkerung unterstellt werden kann, erweist sich auch die intergenrationale Durchhaltbarkeit als kein geeignetes intergeneratives Verteilungsmaß.

Aus der bisherigen Kritik an der Wahl eines beitragsäquivalenten kapitalgedeckten Systems bzw. der intergenerativen Durchhaltbarkeit als Referenzmaßstab haben sich zwei Aspekte herauskristalisiert, die sich bei der Wahl eines verteilungsneutralen Vergleichssystems als besonders problematisch herausstellen:

1. Die grundsätzliche Frage, welche Verteilungswirkungen als systematische intergenerative Umverteilung und welche als intergenerativer Risikoausgleich zu interpretieren sind.

2. Die eher methodische als systematische Frage nach der adäquaten Auf- bzw. Abzinsung der Zahlungsreihen.

Im folgenden Abschnitt 5.3 wird daher zunächst untersucht, wie der intergenerative Risikoausgleich bei der Formulierung des Verteilungsindikators bzw. eines verteilungsneutralen Referenzsystems berücksichtigt werden kann. Abschnitt 7.2.1 setzt sich mit der methodischen aber für das Ergebnis entscheidenden Verzinsungsfrage auseinander.

\subsection{Abgrenzung des intergenerativen Risikoausgleichs in der Gerechtigkeitsanalyse}

Die Abgrenzung eines intergenerativen Risikoausgleichs, umfaßt drei Teilbereiche:

- Als erstes muß präzisiert werden, hinsichtlich welcher Risiken überhaupt ein Bedart bzw. eine Möglichkeit nach einen intergenerativem Risikoausgleich besteht.

- Zweitens muß geklärt werden, in welchem Umfang ein Risikoausgleich präferiert wird bzw. erstrebenswert ist.

- Und schließlich stellt sich drittens die Frage, nach welchen Verteilungsregeln die bei Eintritt des Risikos entstehende Zusatzlast auf die Risikogemeinschaft verteilt werden soll.

351 Vgl. Wagner (1984), S. 168f. sowie seine Fußnote 8 zu Kapitel fünf.

152 
In den nachfolgenden Abschnitten wird versucht, Antworten auf diese Fragen nach dem "was", „wieviel“ und „wie“ eines intergenerativen Risikoausgleichs zu formulieren.

Der Begriff „versuchen“ deutet dabei bereits an, daß sich eindeutige Antworten auf analytischem Wege nur bedingt ableiten lassen. Aus diesem Grunde die Fragestellung jedoch komplett zu umgehen, kann noch weniger überzeugen. Es sollte vielmehr angestrebt werden, die verschiedenen Aspekte des intergenerativen Risikoausgleichs herauszuarbeiten, und soweit dies möglich ist, zu konkretisieren. Diejenigen Bereiche, die sich als zu unscharf bzw. als besonders strittig erweisen, sollten zumindest offen hervorgehoben und im Sinne einer positiven Analyse diskutiert werden. Mit anderen Worten sollten die je nach unterstellten Prämissen variierenden Schlußfolgerung als „wenn-dann-Aussagen“ formuliert werden.

Obwohl eine „absolut optimale (gerechte) Reformoption“ sich dann nach wie vor nicht eindeutig ableiten läßt, ist es dennoch denkbar, daß sich unter verschiedenen plausiblen Szenarien eine bestimmte Option immer gegenüber dem Status Quo als überlegen erweist. Dies wäre bereits eine hilfreiche Aussage für die Sozialpolitik. Wenn hinsichtlich mehrerer Reformoptionen keine eindeutige Rangordnung aufgestellt werden kann, sprich unter verschiedenen Prämissen unterschiedliche Reformoptionen präferiert werden, können mit einem solchen Instrument zumindest die jeweiligen Bedingungen hervorgehoben werden, unter denen eine bestimmte Option vorzuziehen wäre. Diese Ergebnisse können der Sozialpolitik ebenfalls als Entscheidungsgrundlage dienen, wobei die Wahl der adäquaten Prämissen dann aus dem politischen Entscheidungsprozeß resultieren muß.

\subsubsection{Abgrenzung der intergenerativen Risiken}

In Abschnitt 2.4.1 wurden die wesentlichen demographischen und ökonomischen Einflußfaktoren auf die generationsspezifische Rendite eines umlagefinanzierten Alterssicherungssystem identifiziert. Unter der Annahme, daß das Alterssicherungssystem auf dem Prinzip der Teilhabeäquivalenz beruht und ein konstantes Nettorentenniveau anstrebt, wurden in einem weiteren Schritt jene intergenerativen Verteilungswirkungen untersucht, die sich aus einer Variation der identifizierten Parameter ergeben. Dem schloß sich eine Untersuchung der Verteilungswirkungen im Kapitalstockverfahren an. Mit der Bestimmung der verschiedenen Verteilungswirkungen wurde jedoch noch nichts darüber gesagt, welche Verteilungen im Rahmen eines intergenerativen Risikoausgleichs erfolgen und welche von ihnen eine reine Umverteilung zwischen den Generationen darstellen. In den folgenden Abschnitten wird daher der Frage nachgegangen, hinsichtlich welcher Parameter ein Bedart nach einem intergenerativen Risikoausgleich besteht und wie dieser gegebenenfalls bei der Erfassung der intergenerativen Umverteilung beim Verteilungsindikator resp. beim Referenzsystem berücksichtigt werden kann. 


\subsubsection{Kriterien eines intergenerativen Risikoausgleichs}

Das primäre Ziel jeder Versicherung und damit jedes Risikoausgleichs besteht darin, die Abweichungen zwischen der (unvorhersehbaren) realen Entwicklung und der individuellen Lebensplanung durch einen Vermögensausgleich zu minimieren ${ }^{352}$ und dadurch ein gewisses $\mathrm{Maß}$ an Sicherheit zu gewährleisten. Beim interpersonellen Risikoausgleich wird dies i.d.R. erreicht, indem

- die Lasten beim Eintritt des Risikos auf eine möglichst große Anzahl (gleichartig gefährdeter) Versicherten verteilt werden und

- im Kollektiv der Risikogemeinschaft ein Ausgleich zwischen jenen, bei denen das Risiko nicht eintritt („den Glücklichen“), und denen, die vom Risiko betroffen sind („den Unglücklichen"), stattfindet.

- Das Eintreten des Risikos wird mit Hilfe von Methoden der Wahrscheinlichkeitsrechnung kalkuliert bzw. geschätzt (wobei die statistischen Eintrittswahrscheinlichkeiten ggf. differenziert nach Risikogruppen ermittelt werden).

In einem auf dem Prinzip der Teilhabeäquivalenz beruhenden Alterssicherungssystem bei dem die Rentenleistung durch einen bestimmten Mechanismus an das Durchschnittseinkommen gekoppelt sind, bezieht sich die Sicherheit in der individuellen Lebensplanung auch auf die folgenden Aspekte:

Zum einen können sich verschiedene ökonomische Parameter (interne Verzinsung von Kapitalanlagen, Erwerbseinkommen, Inflation) unabhängig vom Niveau anders entwikkeln, als dies von den Versicherten antizipiert wurde, und mithin den relativen lebensdurchschnittlichen Lebensstandard im Ruhestand beeinträchtigen. ${ }^{353}$ Zum anderen gibt es Risiken, wie demographische Veränderungen oder Änderungen der Beschäftigungssituation, die für jedes Alterssicherungssystem mit realen Zusatzlasten oder Gewinnen verbunden sind. Werden die Lasten nur von bestimmten Generationen getragen, besteht für einen Versicherten das Risiko, in eine Situation hinein geboren zu werden, in der diese Zusatzlast vornehmlich von seiner Generation getragen wird. Ein System, bei dem die Last bei Eintritt eines solchen Risikos von mehreren Generationen getragen wird, bietet den Versicherten mehr Sicherheit in der individuellen Lebensplanung.

Zwischen einem hierfür erforderlichen intergenerativen Risikoausgleich und den zuvor beschriebenen Mechanismen des interpersonellen Risikoausgleichs bestehen insbesondere die folgenden Unterschiede:

- Als „Verteilungseinheiten“ der Lasten treten beim intergenerativen Risikoausgleich die verschiedenen Jahrgangskohorten an die Stelle der einzelnen Versicherten.

\footnotetext{
352 Vgl. Gebler (1995) S. $38 f .$.

353 Vgl. Abschnitt 4.4.3.2.
} 
- Aufgrund der überlappenden Struktur der Risikogemeinschaft kann die Zusatzlast immer nur auf jene Kohorten aufgeteilt werden, die gleichzeitig Leben. ${ }^{354}$ Dies kann, muß jedoch nicht immer mit einem Ausgleich von "glücklichen“ zu „unglücklichen“ Generationen einhergehen, wenn die nachfolgenden Generationen in gleicher Weise von dem Risiko (z.B. schlechte Beschäftigungssituation) betroffen sind.

- Den Risiken des intergenerativen Risikoausgleichs, sprich den verschiedenen ökonomischen und zum Teil demographischen Zuständen, können im Gegensatz zum Risiko der Langlebigkeit keine bzw. nur auf subjektive Einschätzungen beruhende Eintrittswahrscheinlichkeiten zugeordnet werden.

In der traditionellen Versicherungstheorie wurde vor allem die Schätz- und Meßbarkeit von Risiken als wichtige Voraussetzung für ihre Versicherbarkeit angesehen. ${ }^{355}$ Dazu gehörte insbesondere die Kenntnis über bestimmte Wahrscheinlichkeiten und/oder die Gültigkeit des „Gesetzes der großen Zahlen“. Zwar werden diese Kriterien beim intergenerativen Risikoausgleich größtenteils nicht erfüllt, hieraus kann jedoch nicht der Schluß gezogen werden, daß ein solcher Risikoausgleich per se nicht zu realisieren wäre.

Denn erstens besteht in der neueren Literatur zur Versicherungstheorie weitgehend Einigkeit darüber, daß diese traditionellen Kriterien der Schätzbarkeit und die Gültigkeit des „Gesetzes der großen Zahlen“ keine notwendige Bedingung für die Versicherbarkeit eines Risikos mehr darstellen. ${ }^{356}$ Genauso wenig ist der Ausgleich im Kollektiv (vom "Glücklichen" zum „Unglücklichen“) ein unabdingbares Kriterium der Versicherbarkeit. ${ }^{357}$

Aber auch unabhängig von den neueren Ansätzen zur theoretischen Versicherbarkeit bleibt zweitens anzumerken, daß diese Literatur sich vornehmlich auf die privatwirtschaftliche Versicherungswirtschaft bezieht, bei der intergenerative Versicherungsaspekte wenn überhaupt - eine nur untergeordnete Rolle spielen. Ihre Übertragbarkeit auf die hier im Vordergrund stehende intergenerative Perspektive ist folglich stark eingeschränkt, zumal die Möglichkeiten eines staatlichen Sozialsystems nicht an die Bedingungen eines privaten Marktes für Versicherungen gebunden sind.

354 Solange jedoch eine Parametervariation dauerhaften Charakter hat, wird die "Last" von Generation zu Generation weitergereicht, bis der Parameter auf seinen ursprünglichen Wert zurückfält und die "letzte Generation" die Last trägt (vgl. Abschnitt 2.4.2.2).

Diese Auffassung geht vorrangig auf die Arbeiten Knights zurück, der die „Ungewißheit" nach ihrer Meßbarkeit einteilt. Beim versicherbaren Risiko ist nicht nur bekannt welche Ereignisse eintreten können sondern auch, mit weicher Wahrscheinlichkeit sie eintreten. Sind diese Voraussetzungen nicht gegeben, spricht er von Unsicherheit die nicht meßbar und folglich auch nicht versicherbar sei. Vgl. Knight (1921), vgl. auch Arrow (1951).

„In der betriebswirtschaftlichen Literatur und Praxis ist man sich inzwischen darüber einig, daß eine Versicherung auch möglich ist, wenn nicht nur für den Einzelnen, sondern insgesamt für alle Versicherten das Durchschnittsrisiko nicht berechenbar ist. "Wagner (1985), S. 153. Einen umfassenden Überblick bietet Borch et al. (1990). Vgl. auch Breyer/Kolmar (1997), S. 5; Borch/Aase/ Sandmo (1990), S.106; Härlen (1972), S. 273; Karten (1988), S. 351; Mugler (1980), S. 77. 
Im folgenden soll daher zunächst ein Kriterienkatalog formuliert werden, anhand dessen sich die reine intergenerative Umverteilung von den Verteilungswirkungen des intergenerativen Risikoausgleichs abgrenzen läßt.

Erste Anhaltspunkte hierfür lassen sich aus der in der Literatur üblichen Definition zur intergenerativen Umverteilung gewinnen. Intergenerative Umverteilung liegt entsprechend vor, wenn ein realer Einkommensgewinn (bzw. Verbesserung der Rendite) einer Generation zulasten eines dauerhaften Verlustes (bzw. Verschlechterung der Rendite) einer anderen Generation geht. ${ }^{358}$ Aus der dieser Definition zugrunde liegenden Ungleichbehandlung läßt sich das erste Kriterium für eine reine intergenerative Umverteilung ableiten:

\section{Kriterium 1:}

a) Intergenerative Umverteilung effordert, daß die verschiedenen Generation von einem Ereignis qualitativ ungleich behandelt werden, d.h. einige werden bessergestellt, während andere durch das gleiche Ereignis schlechtergestellt werden.

b) Ferner ist erforderlich, daß sich eindeutig bestimmen läßt, welche Generationen von dem umverteilenden Ereignis profitieren und welche Generationen von inm benachteiligt werden.

Das erste Kriterium gründet sich vornehmlich auf einer Gleichverteilung im Sinne der Ergebnisgerechtigkeit. Während in vorangehenden ersten Kriterium der intergenerativen Umverteilung den Generationen eine eher passive Rolle zukommt, betonen andere Autoren in ihrer Definition der intergenerativen Umverteilung explizit die aktive Rolle der Generationen. ${ }^{359}$ Diesen Aspekt berücksichtigt das folgende zweite Kriterium der intergenerativen Umverteilung:

\section{Kriterium 2:}

Bei einer intergenerativen Umverteilung läßt sich zuordnen, welche Generation das umverteilende Ereignis ausgelöst hat.

Da eine ungerechte Umverteilung entsprechend dieses Kriteriums nur dann vorläge, wenn eine bestimmte Generation dafür verantwortlich gemacht werden kann, spiegeln sie das Pendant der Leistungsgerechtigkeit - das Verursachungsprinzip - wider.

Sofern diese ersten beiden Kriterien erfüllt sind, gewinnen Moral Hazard Probleme auch im intergenerativen Zusammenhang an Bedeutung, denn dann könnte es für eine Generation möglich sein, sich durch eine gezielte Beeinflussung der Variablen zu lasten anderer Generationen besser zu stellen. ${ }^{360}$ Eine auf Moral Hazard Verhalten zurückzuführende Umverteilung kann aber nicht als Risikoausgleich gewertet werden. Jede Reformoption,

\footnotetext{
358 Vgl. beispielsweise Bösch (1987), S. 53; Dinkel (1986), S. 81; Davies (1996), S.5; Breyer/Spremann (1990); Schulenburg (1989); Speckbacher (1994), S. 66.

359 So beispielsweise Bösch (1987), S. 53; Dinkel (1986), S. 81; Speckbacher (1994), S. 66.
} 
die die Möglichkeit eines solchen Moral Hazards verhindert, vermindert mithin das Aus$\mathrm{ma}$ an intergenerativer Umverteilung. Diese Moral Hazard Probleme finden ihren Ausdruck im

\section{Kriterium 3:}

Ist eine gezielte Beeinflussung durch den Versicherten bzw. durch die jeweilige Generation möglich bzw. besteht die Gefahr einer bewußten Beeinflussung, so liegt eine intergenerative Umverteilung vor.

Sind die Kriterien 1-3 erfüllt, kann man folglich von intergenerativer Umverteilung sprechen. Allerdings gilt - wie die folgenden Ausführungen zeigen - nicht zwangsläufig auch der Umkehrschluß, daß ein Fehlen der bewußten Beeinflussung automatisch für die Existenz eines intergenerativen Risikoausgleichs spricht. Die Kriterien 2 und 3 weisen Parallelen zu HAYEKS Kritik an Gerechtigkeitserwägungen in der Ökonomie: Eine Situation die niemand bewußt herbeigeführt hat bzw. für die sich nicht ermitteln läßt, welche Generation sie verursacht hat, sollte nicht als ungerecht bewertet werden. Solche Verteilungswirkungen sollten folglich auch nicht als Umverteilung interpretiert werden. Doch ähnlich wie HAYEKS These durch BARRYS Kritik eingeschränkt werden mußte, ${ }^{361}$ kann auch an dieser Stelle eingewandt werden, daß eine solche Situation dennoch ungerecht sei (bzw. die Verteilungswirkungen als Umverteilung bewertet werden sollten), wenn die Verteilungswirkungen sich durch ein alternatives System hätten vermeiden lassen. D.h., selbst wenn bei sonst gleichen Voraussetzungen die Möglichkeit bzw. die Gefahr einer bewußten Beeinflussung im Sinne des Moral Hazard wegfällt (Kriterium 3), sind die Bedingungen eines intergenerationalen Risikoausgleichs noch nicht zwangsläufig gegeben. Zu klären bleibt, ob die auftretenden Effekte nicht durch Modifikation der Rentenformel internalisiert werden können. Eine Internalisierung könnte im übrigen auch dann in Frage kommen, wenn die auslösende Generation durch das Ereignis benachteiligt wird.

\section{Kriterium 4:}

Selbst wenn die ersten drei Kriterien nicht erfüllt sind, ist eine Verteilung dennoch als intergenerative Umverteilung zu werten, wenn sich die Verteilungseffekte mit vertretbaren Aufwand internalisieren lassen.

Die in Kriterium 4 verwandte Formulierung „vertretbarer Aufwand“ zeigt, daß es sich hierbei um ein recht unscharfes Kriterium handelt. Inwieweit eine Internalisierung möglich ist hängt unter anderem auch davon $a b$,

Zusatz 4.1 ob die auslösende Generation zugleich auch von dem umverteilenden Ereignis profitiert und

Vgl. Bösch (1987), S. 78-80. Dabei ist das Konstrukt des repräsentativen Individuums ein methodisches Hilfsmittel, um „das Verhalten“ einer Generation zu untersuchen. 
welche Rolle Externalitäten spielen, sprich ob andere Generationen ebenfalls von dem Ereignis profitieren bzw. von inm benachteiligt werden.

Die notwendige Bedingung dafür, daß ein bestimmtes Risiko zum intergenerativen Risikoausgleich gezählt werden kann, ist dann gegeben, wenn eines der ersten drei Kriterien nicht erfüllt ist. Eine hinreichende Bedingung liegt aber erst dann vor, wenn eine Internalisierung der Umverteilungseffekte ausgeschlossen (Kriterium 4) bzw. praktisch nicht umsetzbar ist (u.a. Zusatz 4.1 und 4.2).

\subsubsection{Auswahl der zu analysierenden Verteilungseffekte}

Nachfolgend werden die verschiedenen Einflußgrößen auf die generationenspezifische Rendite einer Alterssicherung im allgemeinen und auf die der gesetzliche Rentenversicherung im besonderen darauf überprüft, inwieweit sie die soeben hergeleiteten Umverteilungskriterien erfüllen. Darauf aufbauend soll dann der Risikoausgleich abgegrenzt und der Verteilungsindikator angepaßt werden.

Neben den in Abschnitt 2.4.1 bereits untersuchten ökonomischen und demographischen Parametern beeinflussen auch der Reifegrad eines Systems und die verschiedenen rentenrechtlichen Regelungen die Rendite eines Alterssicherungssystems.

In einem Umlageverfahren zeigt sich das volle Ausmaß der Leistungsverpflichtung erst, wenn es "reif“ ist, sprich wenn sich nur solche Generation im Ruhestand befinden, bei denen das System vollständig eingeführt und der angestrebte Versichertenkreis bereits voll erfaßt ist. Bevor dieser Zustand erreicht ist, spricht man von dem „Reifegrad“ des Systems. Durch die allmähliche Ausweitung der Versicherungspflicht sind die Beitragssätze zunächst niedriger als im vollständig eingeführten System. In einem „noch unreifen“ System hat der Reifegrad des Umlageverfahrens einen wesentlichen Einfluß auf die Rendite der Generationen. Von der Berücksichtigung solcher „Reife-Effekte“ bzw. Einführungsgewinne wird im folgenden allerdings abgesehen.

Von den Änderungen im Rentenrecht, wie beispielsweise die Heraufsetzung des gesetzlichen Renteneintrittsalters, die Höhe der Ab- bzw. Zuschläge bei vorgezogenem bzw. aufgeschobenen Rentenbeginn, die Anrechnung beitragsfreier Zeiten, o.ä. werden verschiedene Generationen im unterschiedlichen Ausmaß betroffen. Allein die Änderung einer Regelung kann daher die generationsspezifischen Renditen beeinflussen. Dennoch werden sie nachfolgend nicht vertieft, da der Einfluß politischer (Korrektur-)Maßnahmen nicht als systematisches Versicherungsrisiko anzusehen ist. ${ }^{362}$ Die gesetzliche Regelungen der Rentenversicherung sind lediglich dann von mittelbarer Bedeutung für die Verteilungsanalyse, wenn nicht die Tatsache ihrer Änderung, sondern die Regelung selbst zu systematischen intergenerativen Umverteilungen führt.

362 Vgl. auch Wagner: „Zu beachten ist insbesondere, daß historisch abgeschlossene Regelungen nur noch die gegenwärtige Rentnergeneration beeinflussen. Für in die Zukunft gerichtete Untersuchungen sind diese auslaufenden Regelungen weitgehend nicht mehr relevant. “Wagner (1985), S. 148. 
Im weitesten Sinne könnten die Invaliditätshäufigkeit und die Verheiratungsquote einer Generation (z.B. über Hinterbliebenenrenten) ebenfalls zu den demographischen Einflußgrößen auf die Rendite gezählt werden. Allerdings werden auch diese Effekte aus der nachfolgenden Betrachtung ausgeklammert.

Die folgende Untersuchung konzentriert sich daher im wesentlichen auf die bereits im zweiten Kapitel präzisierten ökonomischen und demographischen Einflußgrößen auf die generationsspezifischen Rendite. Aufbauend auf den in der Distributionsanalyse bereits abgeleiteten Ergebnissen (vgl. Abschnitt 2.4) werden die verschiedenen Einflußfaktoren mit Hilfe der im vorigen Abschnitt formulierten Umverteilungskriterien dahingehend untersucht, ob und inwieweit ihre Verteilungswirkungen einem intergenerativen Risikoausgleich zugeordnet werden können.

\subsubsection{Variation der Mortalität}

\section{A) Veränderung der Sterblichkeit während der Ruhestandsphase}

Verringert sich die Sterbewahrscheinlichkeit während der Ruhestandsphase einer Generation $x$, so sind - wie den Ergebnissen aus Abschnitt 2.4.2.2 zu entnehmen ist - die Kriterien 1 und 2 für eine intergenerativen Umverteilung erfülit. Denn die verschiedenen Generationen werden von der Veränderung qualitativ unterschiedlich betroffen und es läßt sich zuordnen, welche Generation benachteiligt wird bzw. von der Variation profitiert.

Rein theoretisch ist es ebenfalls möglich, daß die betreffende Generation ihre Restlebenserwartung selbst beeinflußt, indem sie beispielsweise auf einen gesunden Lebenswandel achtet (Ernährung, Sport, Vermeidung risikohafter Erwerbstätigkeit etc.). Allerdings sind einige entscheidende Faktoren, wie beispielsweise der Fortschritt in der medizinischen Forschung und in der medizinischen Technik, unabhängig von dem jeweiligen Verhalten der Generation. Der ausschlaggebende Grund, weshalb eine Veränderung der Lebenserwartung hier nicht als Moral Hazard Problem, sondern als ein exogenes Phänomen betrachtet werden sollte, ist der, daß eine längere Lebenserwartung für jede Generation ein eigenständiges erstrebenswertes Ziel darstellt, so daß etwaige Anreizeffekte durch das Alterssicherungssystem an Bedeutung verlieren und kaum das Verhalten beeinflussen dürtten. ${ }^{363}$ Das dritte Kriterium einer reinen Umverteilung ist angesichts dieser Zusammenhänge mithin nicht erfüllt.

Ob die Voraussetzungen eines intergenerativen Risikoausgleichs gegeben sind, entscheidet sich folglich entsprechend des vierten Kriteriums daran, inwieweit sich die Wirkungen der Mortalitätsänderung internalisieren, sprich in das Alterssicherungssystem integrieren lassen.

Die Mortalität spielt bereits beim interpersonellen Risikoausgleich in der Alterssicherung eine wichtige Rolle. Dieser basiert u.a. auf einer versicherungsmäßigen Einkommensum- 
verteilung von Versicherten, die vor Erreichen der durchschnittlichen fernen Lebenserwartung versterben zu jenen, deren Lebenserwartung den Erwartungswert übersteigt. Ein darüber hinausgehender Bedarf an intergenerativem Risikoausgleich könnte sich ggf. nur daraus ergeben, daß sich der statistisch zu ermittelnde Erwartungswert der Restlebenserwartung $^{364}$ über die Zeit verändert. Die durchschnittliche Restlebenserwartung, kann jedoch ohne Schwierigkeiten periodisch neu berechnet werden, sobald die aktuellen statistischen Daten zur Verfügung stehen. Die Berechnungsmethode würde sich von der im interpersonellen Risikoausgleich angewandten Vorgehensweise nicht unterscheiden, es müßte lediglich zu einer rollierenden Ermittlung der Restlebenserwartung übergegangen werden. Zusätzliche Unsicherheiten bei der Risikoabschätzung würden mithin nicht auftreten. Auch die Veränderung der durchschnittlichen, fernen Restlebenserwartung kann folglich mit Hilfe des interpersonellen Risikoausgleichs erfaßt werden, so daß ein zusätzlicher Bedarf an intergenerativen Risikoausgleich nicht konstatiert werden kann.

Wenn folglich eine längere Rentenbezugsdauer, infolge einer gesunkenen Sterblichkeit, in der Rentenformel nicht berücksichtigt und eine bestimmte Generationen somit auf Kosten nachfolgender Generationen bessergestellt wird, ist dies nicht als Risikoausgleich, sondern als intergenerative Umverteilung zu werten.

\section{B) Veränderung der Sterblichkeit während der Erwerbsphase}

Es bleibt zu klären, wie eine Veränderung der Mortalität zu beurteilen ist, wenn sie innerhalb der Erwerbsphase auftritt. Die Ausführungen in Kapitel 2 zeigten, daß die verschiedenen Generationen von einer Verminderung der Sterblichkeit der Generation x unterschiedlich betroffen sind:

a) Die Generation $x$ stellt sich durch ihre geringere Sterblichkeit besser, da die Beitragszahlungen sich auf mehr versicherte verteilen und pro Kopf sinken und mehr Personen eine Rente beziehen.

b) Alle der Generation $x$ vorangegangenen Generationen, die während der Mortalitätssenkung ebenfalls erwerbstätig sind, profitieren durch geringere Beitragssätze von dieser Variation.

c) Nachfolgende Generationen gehören, solange sie gemeinsam mit der Generation $x$ erwerbstätig sind, ebenfalls zu den Nutznießern der Variation. In der Phase, in der sie jedoch noch erwerbstätig sind, während die Generation $x$ bereits im Ruhestand ist, werden sie durch die niedrigere Mortalität allerdings benachteiligt.

d) Verlierer der Parametervariation sind all diejenigen Generationen, die in die Erwerbsphase eintreten, während Generation $x$ im Ruhestand ist.

$364 \mathrm{Zu}$ den möglichen Berechnungsverfahren mit Hilfe von Kohorten- oder Periodensterbetafeln vgl. Rürup/Schroeter (1996). 
e) Das Ausmaß der Besser- bzw. Schlechterstellung fällt dabei je nach Abstand zur Generation $x$ unterschiedlich hoch aus.

Das Kriterium 1a einer Umverteilung ist somit erfült. In Praxi ist eine eindeutige Zuordnung, welche Generation von der veränderten Sterblichkeit profitiert bzw. von ihr benachteiligt wird (Kriterium 1b), zwar äußerst schwierig, ${ }^{365}$ theoretisch ist sie jedoch möglich.

Allerdings ist auch bei der Mortalitätsänderung während der Erwerbsphase zu beachten, daß die Beeinflussung der Lebenserwartung kein typisches Moral Hazard Problem darstellt: Denn eine niedrige Sterblichkeit ist grundsätzlich als eigenständiges Ziel erstrebenswert und der Einfluß möglicher Renditewirkungen kann daher vernachlässigt werden. Das dritte Kriterium ist mithin nicht erfüllt.

Die durch eine Veränderung der Sterblichkeit während der Erwerbsphase ausgelösten intergenerativen Verteilungswirkungen sind als Umverteilung zu werten, wenn sich diese Effekte internalisieren lassen. Dies könnte mit einem Wechsel zu einer beitragsäquivalenten Leistungsgewährung erreicht werden. Eine solche Rentenformel wäre im Umlageverfahren jedoch - wie in Abschnitt 2.2 verdeutlicht - äußerst instabil, so daß diese Option einen kompletten Wechsel des Finanzierungsverfahrens erfordern würde. Ein Systemwechsel wäre allerdings nicht zwingend erforderlich, denn es ist ebenfalls denkbar, eine stärkere Beitragsorientierung in ein teilhabeäquivalentes System zu integrieren. Die Rentenhöhe würde sich weiterhin dem Grunde nach am Durchschnittseinkommen der Erwerbstätigen ausrichten und die Rentenstruktur würde nach wie vor nach dem Prinzip der Teilhabeäquivalenz gestaltet. Bei der Bestimmung der Struktur müßte lediglich zusätzlich zur Abweichung des individuellen vom durchschnittlichen beitragspflichtigen Erwerbseinkommens auch die Abweichung des jeweiligen Beitragssatzes von einem normierten Beitragssatz berücksichtigt werden. Ein Entgeltpunkt würde beispielsweise dann erworben, wenn ein Versicherter ein Jahr lang das Durchschnittseinkommen verdient und zum Referenzbeitragssatz Beiträge bezahlt hat. ${ }^{366} \mathrm{Da}$ nur derjenige Teil der Beitragssatzveränderung berücksichtigt werden soll, der auf eine Variation der Sterblichkeit während der Erwerbsphase zurückzuführen ist, würde die praktischen Umsetzung einer solchen Teilhabeäquivalenz zu einer recht komplizierten Rentenformel führen. Grundsätzlich stellt eine solche Formel aber eine Möglichkeit dar, die aus einer Veränderung der Sterblichkeit während der Erwerbsphase entstehenden Umverteilungen auch im teilhabeäquivalenten System zu kompensieren.

Ob eine Veränderung der Sterblichkeit während der Erwerbsphase als systembedingte Umverteilung oder als solidarisches Risiko interpretiert wird, hängt von der Beurteilung

365 Vgl. Punkt c) in der Aufzählung. Bei diesen Generationen sind die Wirkungen auf die Rendite schwer zu quantifizieren und hängen zudem auch in ihrer qualitativen Wirkung von weiteren Parametern ab (vgl. Abschnitt 2.4.2.1.

366

Beträgt der Referenzsatz beispielsweise 20 Prozent, der aktuelle Beitragssatz hingegen 21 Prozent, so würde der Durchschnittsverdiener 1,05 Entgeltpunkte erwerben. 
dieser Internalisierungsmöglichkeit ab. Die Erfassung und Berücksichtigung der verschiedenen Effekte in dem Verteilungsindikator würde sich als äußerst komplex erweisen, so daß man mit Hinweis auf die Kriterien (Zusatz 4.1 und Zusatz 4.2) die Ansicht vertreten kann, die Verteillungseffekte ließen sich nicht mit „vertretbaren Aufwand“ internalisieren. In der vorliegenden Arbeit wird diese Auffassung nicht vertreten. Für die Gerechtigkeitsanalyse ist diese unterschiedliche Interpretation ohnehin von nachrangiger Bedeutung Denn in Anbetracht der Tatsache, daß die Sterblichkeit während der Erwerbsphase in der Bundesrepubllik Deutschland ohnehin schon sehr niedrig ist und eine Mortalitätsänderung für Deutschland faktisch nur für die Restlebenserwartung im Rentenalter relevant ist, ist dieser Effekt bei der Erfassung der intergenerativen Umverteilung vernachlässigbar.

Alle durch Mortalitätsänderung verursachten intergenerativen Verteilungswirkungen werden daher als systematische Umverteilung und nicht als Risikoausgleich interpretiert. Der Verteilungsindikator muß nicht adaptiert werden.

\subsubsection{Variation der Fertilität}

Eine Veränderung des reproduktiven Verhaltens ließe sich theoretisch zwar einer "verursachenden Generation“ zuordnen, so daß das zweite Kriterium für eine reine Umverteilung erfüllt ist, allerdings bewirkt eine Variation der Fertilität im Gegensatz zu einer variablen Restlebenserwartung, daß sich die Rendite für alle betroffenen Generationen in die gleiche Richtung verändert. D.h. es ist nicht möglich, daß einige Generationen bessergestellt werden, während andere Generationen aufgrund der gleichen Ursache benachteiligt werden. Eine solche Verletzung des ersten Kriteriums schränkt die Möglichkeit von Moral Hazard Verhalten zwischen den Generationen ein. Die wesentlichen Motive für eine Änderung der Fertilität ergeben sich zudem weniger aus der möglichen Beeinflussung der Rendite des Alterssicherungssystems, sondern sind meistens unabhängig von der Alterssicherung. ${ }^{367}$ Auch das dritte Kriterium ist mithin nicht erfüllt.

367 Neben Faktoren wie die direkten mit der Kindererziehung verbundenen Kosten, die Einführung moderner Antikonzeptiva oder der Hōhe und Dauer der Arbeitslosigkeit sind es heutzutage vornehmlich die Opportunitätskosten von Kindern, die in den Industriestaaten für die niedrige Fertilităt verantwortlich sind (vgl. Rürup/Schroeter (1996).

Vergegenwärtigt man sich, daß die Opportunitätskosten einer Unterbrechung des beruflichen Werdegangs eine der Ursachen für sinkende Geburtenzahlen darstellen, so besteht eine negative Korrelation zwischen der Erwerbstătigkeit der Frauen und ihrem Reproduktionsverhalten. Dinkel (1986) und Sudhoff (1996) modellieren diesen Zusammenhang in ihrer Analyse sogar explizit. Ihre Arbeiten basieren allerdings auf einfachen Modellrechnungen im OLG-Modell. Eine Simulation dieser Korrelation mūßte beachten, daß sich weder das Erwerbs- noch das Reproduktionsverhalten der Frauen auf einen solch einfachen Zusammenhang reduzieren läßt. Eine Gewichtung dieses Zusammenhangs gegenüber anderen Faktoren erweist sich jedoch als ebenso schwierig, wie der Versuch, den funktionalen Zusammenhang zu spezifizieren. Selbst wenn dies gelänge, wăre weiterhin zweifelhaft, ob dieser Zusammenhang im Zeitablauf stabil ist. Ein Wandel der gesellschaftlichen Einstellung zur berufstätigen Mutter und eine Verbesserung der infrastrukturelle Möglichkeiten der Vereinbarkeit von Mutterschaft und Berufstătigkeit sind für die nächsten vierzig Jahre sehr wahrscheinlich. Die Korrelation ist aber für die Fragestellung dieses Abschnitts vor allem deshalb irrelevant, weil es keinen Einfluß auf das Meßkonzept hat, wie die exogenen Größen „Fertilität“ und „Erwerbsbeteiligung “ bestimmt werden.

162 
Erhöht (verringert) eine Generation ihr reproduktives Verhalten, so kommt es zu einer positiven (negativen) Externalität sowohl für vorangegangene ${ }^{368}$ als auch für nachfolgende Generationen. Zudem werden die verschiedenen Generationen nicht nur in gleicher Richtung, sondern auch in unterschiedlichem Ausmaß von der Variation betroffen. Beide Effekte erschweren den Versuch, die Auswirkungen zu quantifizieren, geschweige denn praktisch umsetzbar in die Rentenformel zu integrieren (Zusatz 4.2).

Eine Internalisierung wird ferner dadurch erschwert, daß die Last bzw. der Gewinn mit Hilfe der Rentenformel nicht den verschiedenen Generationen, sondern nur den einzelnen Versicherten zugeordnet werden kann. Die anhaltende Diskussion um eine bevölkerungsdynamische Rente verdeutlicht jedoch, mit welchen gravierenden Umsetzungsschwierigkeiten der Versuch verbunden ist, das individuelle Reproduktionsverhalten bei den Beitragszahlungen bzw. Rentenansprüchen des einzelnen Versicherten anzurechnen. ${ }^{369}$

Aus diesen Gründen erscheint es vertretbar, die Fertilitätsänderung als gesamtgesellschaftliches Phänomen aufzufassen, bei dem alle betroffenen Generationen qualitativ gleich behandelt werden. ${ }^{370}$ Für eine solche Interpretation spricht ebenfalls, daß die Fertilität als eine Bestimmungsgröße für das Lohnsummenwachstum im Umlageverfahren einen Zinscharakter hat. ${ }^{371}$

Wenngleich nicht im bestehenden System integrierbar, könnte gegen diese Interpretation eingewandt werden, daß mit Hilfe eines Kapitalstockverfahrens ein Alterssicherungssystem gegen Fertilitätsschwankungen immunisiert werden kann. Die Ausführungen in Abschnitt 2.4.3 zeigten jedoch, daß auch das Kapitalstockverfahren keinesfalls unabhängig von Schwankungen der Fertilität ist. Es kann lediglich erwartet werden, daß die Abhängigkeit des Alterssicherungssystems von der Fertilität vermindert wird. ${ }^{372}$

Bleibt also festzuhalten, daß bestenfalls ein nicht zu quantifizierender Anteil der fertilitätsbedingten Verteilungswirkungen als intergenerative Umverteilung gewertet

368 Vorangehende Generationen sind aufgrund des Rückkopplungseffekts der Nettoanpassung von der Fertilitätsänderung betroffen.

369 Die Diskussion des Für und Wider einer bevölkerungsdynamischen Rente soll an dieser Stelle jedoch nicht wiedergegeben werden.

370 So auch Sudhoff (1996), S. 63: „Kohortenbetrachtungen und die Einteilung in überlappende Generationen sind zwar ein hilfreiches Instrument zur Analyse von Ursache- und Wirkungszusammenhängen, jedoch sollten sie nicht dazu verleiten, kollektive Verantwortung zuzuweisen, obwohl die Ursache des Problems nicht auf kollektiven Entscheidungen beruht. “ sowie Erbe (1986), S. 201: „Die niedrige Reproduktionsrate ist sicherlich nicht das Ergebnis des kollektiven Handelns einer ganzen Generation und kann dieser daher auch nicht kollektiv angelastet werden“ Zitiert nach Sudhoff (1996), S. 63.

371 Vgl. Abschnit 2.4.2.2, vgl. Bösch (1987), S. 76f. Der diesbezüglich weiter ausführt: „Dieser zentrale Unterschied zwischen dem demographischen Faktor "Fertilität und den übrigen hier diskutierten Risikokomponenten eines Umlagesystems wird in der Literatur nicht immer deutlich gesehen."

Diese Erwartung begründet sich zum einen intuitiv darauf, daß die Einflüsse im Umlageverfahren direkter, im Kapitalstockverfahren hingegen indirekter Natur sind. Ferner bestehen (begrenzte) Möglichkeiten der Auslandsanlage. vgl. auch Fußnote 308. Darüber hinaus wäre eine Komplettumstellung auf ein Kapitalstockverfahren schon allein deshalb nicht realisierbar, weil das erforderliche Anlagevermögen GröBenordnungen annehmen würde, die das Produktivvermögen des Inlands übersteigen. Vgl. Dinkel (1984), Rürup (1995). Wenn daher lediglich eine partielle Kapitaldeckung als alternatives Alterssicherungssystem in Frage kommt, könnte das intergenerative Risiko der Fertilität - wenn überhaupt - lediglich eingeschränkt werden. 
werden kann. Die Fertilität an sich sollte als gesamtgesellschaftliches Phänomen betrachtet werden.

Nach dieser Einschätzung ist die Entwicklung der Fertilität ein versicherungsmäßiges Risiko und es stellt sich die Frage, wie dieser intergenerative Risikoausgleich bei der Gestaltung der Verteilungsindikatoren bzw. des Referenzsystems berücksichtigt werden kann. Im folgenden soll eine Korrekturkomponente in den Verteilungsindikator integriert werden. Zur Herleitung dieser Korrekturkomponente wird auf die Grundvariante des Overlapping Generation Modells zurückgegriffen. Aus der Budgetgleichung des Umlageverfahrens erhält man für ein Alterssicherungssystem mit konstantem Bruttorentenniveau $\left(p_{t}=w_{t} \cdot k^{\text {brutto }}\right)$ die bekannte Gleichung für den Beitragssatz, der sich aus dem Produkt aus Bruttorentenniveau und Altenquotienten ergibt.

$$
b_{t}=\frac{p_{t} \cdot N_{t}^{R}}{w^{t} \cdot N_{t}^{E}}=k^{\text {brutto }} \cdot A Q_{t} \quad \text { G } 63
$$

Für den Fall eines Alterssicherungssystems mit konstantem Nettoniveau $\left(p_{t}=w_{t} \cdot\left(1-b_{t}\right) \cdot k^{\text {netto }}\right)$ ergibt sich analog die Beitragsgleichung:

$$
b_{t}=k^{\text {netto }} \cdot \frac{N_{t}^{R}}{N_{t}^{E}+k^{\text {netto }} \cdot N_{t}^{R}}=k^{\text {netto }} \cdot A Q_{t, M O D}
$$

Die durch das Risiko der Fertilitätsveränderung ausgelöste Variation des Beitragssatzes (und damit auch des generationsspezifischen Verteilungsindikators) kann mit Hilfe des Altenquotienten bzw. des modifizierten Altenquotienten erfaßt werden. Für das Meßkonzept wird der modifizierte Altenquotient wie folgt definiert:

$$
A Q_{M O D}^{\prime}=\frac{B E V_{\geq 65}^{t}}{B E V_{20-64}^{\prime}+\Phi \cdot B E V_{\geq 65}^{t}}
$$

$B E V_{\geq 65}^{\prime}=$ Anzahl der über 64jährigen in der Bevökerung

$B E V_{20-64}^{\prime}=$ Anzahl der 20 bis 64jährigen in der Bevökerung

$\Phi=$ Faktor zur Bestimmung des Ausmaßes an Risikoausgleich (vgl. nachfolgende Erläuterungen)

Den Korrekturfaktor ( $F A K$ ) erhält man dann aus dem Verhältnis des (modifizierten) Altenquotienten bei konstanter Reproduktionsrate zum (modifizierten) Altenquotienten bei variabler Reproduktionsrate:

$$
F A K^{t}=\frac{A Q_{M O D}^{\prime} \text { (bei konstanter Reproduktionsrate) }}{A Q_{M O D}^{\prime} \text { (bei variabler Reproduktionsrate) }} .
$$

Sonstige demographische Einflüsse auf den Altenquotienten infolge von Migration oder einer Veränderungen der Mortalität werden durch den Faktor nicht erfaßt. Der Faktor läßt sich wie folgt in den Verteilungsindikator (VI) (vgl. Gleichung G 62) integrieren: 


$$
V I_{x}=\frac{\sum_{t=R E}^{T}\left(R L^{t} \cdot \frac{\left(1-b^{t} \cdot F A K^{t}\right)}{\left(1-b^{t}\right)} \cdot \prod_{v=R E}^{t}\left(1+d_{v}\right)^{-1}\right)}{\sum_{t=0}^{R E-1}\left(B^{t} \cdot F A K^{t} \cdot \prod_{v=t}^{R E-1}\left(1+d_{v}\right)\right)}
$$

Je nach Höhe des Faktors $\Phi$ variiert der Umfang in dem die Folgen der Fertilitätsänderung als intergenerativer Risikoausgleich interpretiert werden. Mit steigenden $\Phi$ wird ein zunehmender Teil der fertilitätsbedingten Verteilungswirkungen nicht als Risikoausgleich, sondern als systematische Umverteilung gewertet und es gilt::

$$
\lim _{\Phi \rightarrow \infty} V I^{\text {mit Risikoausgleich }}=V I^{\text {ohne Risikoausgleich }}
$$

Für den Wertebereich

$$
-\frac{B E V_{20-64}^{\prime}}{B E V_{\geq 65}^{\prime}} \leq \Phi \leq k^{\text {netto }} \quad \mathbf{G} 65
$$

signalisiert der Verteilungsindikator, daß alle von einer Senkung der Fertilität betroffenen Generationen durch den Risikoausgleich besser gestellt werden, als im System ohne Risikoausgleich, wenn keine Fertilitätsänderung auftritt. Da allerdings alle Generationen in gleicher Weise von dem veränderten reproduktiven Verhalten betroffen werden, es folglich keine Umverteilung zu gewinnenden Generationen gibt, ist dieses Ergebnis wenig plausibel und kann nicht begründet werden.

Ähnlich unplausibel sind die Ergebnisse für den Wertebereich

$$
\Phi<-\frac{B E V_{20-64}^{t}}{B E V_{\geq 65}^{\prime}} \quad \forall t \quad \mathbf{G} 66
$$

bei dem der Verlauf der Verteilungsparameter umkippt und signalisiert, daß die betroffenen Generationen durch den Risikoausgleich schlechter gestellt werden, als im Fall ohne Risikoausgleich.

Beim Wertebereich

$$
\Phi<-\frac{B E V_{20-64}^{t}}{B E V_{\geq 65}^{\prime}} \text { für mindestens ein t und }-\frac{B E V_{20-64}^{t}}{B E V_{\geq 65}^{\prime}} \leq \Phi \leq k^{\text {netto }} \text { sonst } \mathbf{G} 67
$$

zeigt der Verlauf des Verteilungsindikators für einige Generationen an, daß sie durch eine Fertilitätsänderung besser gestellt werden (sprich durch den Risikoausgleich eigentlich schlechter gestellt werden sollten, als sie es durch die Verteilungsregel werden) und für andere Generationen, daß sie durch die Variation schlechter gestellt werden, als sie es im Fall ohne Risikoausgleich werden.

Ein sinnvoll zu interpretierende Verlauf des generationsspezifischen Verteilungsindikators ergibt sich folglich nur, wenn $\Phi$ über den folgenden Wertebereich definiert ist: 


$$
\Phi \geq k^{\text {netto }} \quad \text { G } 68
$$

Wird der Faktor $\Phi$ in der Höhe des Nettorentenniveaus festgelegt, werden sämtliche durch die Veränderungen der Fertilität hervorgerufenen Verteilungswirkungen vom Verteilungsindikator als Risikoausgleich und nicht als systematische, intergenerative Umverteilung gewertet werden (vgl. Abbildung 12).

Abbildung 12: Verlauf des generationsspezifischen Verteilungsindikators (VI) bei unterstelltem vollstăndigen Risikoausgleich bezüglich Fertilitätsveränderungen und Akzeptanz der in der Nettoanpassung enthaltenen Lastverteilungsregel $\left(\Phi=k^{\text {netto }}\right)$

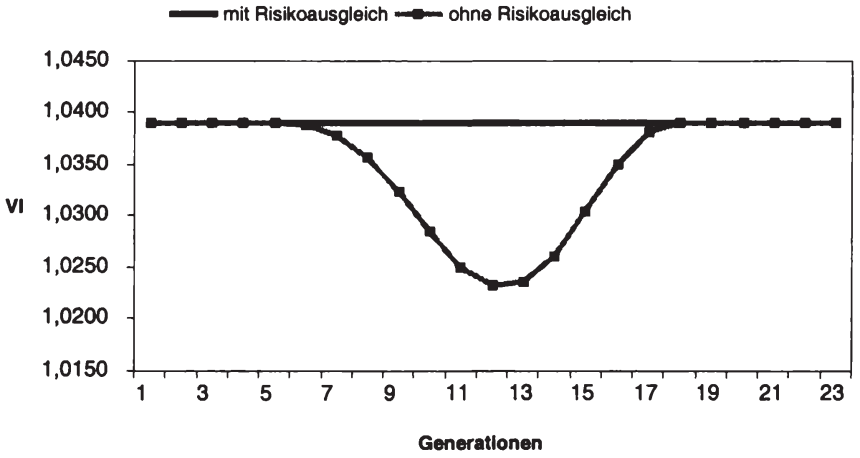

Anmerkung: Modellrechnung mit einem 6-Generationen-Modell (4 Erwerbsperioden, 2 Ruhestandsperioden). Senkung der Reproduktionsrate der Generationen 10, 11 und 12.

Quelle: eigene Darstellung

Die Gleichsetzung „ $\Phi=$ Nettorentenniveau“ ist jedoch nicht zwingend.

Zum einen kann der Modus, nach dem die Zusatzlast bei Eintritt des Risikos auf die Gefahrengemeinschaft verteilt werden soll, von der Verteilungsregel der Nettoanpassung abweichen (vgl. Abschnitt 5.3.3). Aber selbst wenn die Zusatzlast nach Maßgabe der Verteilungsregel der Nettoanpassung aufgeteilt werden soll, muß $\Phi$ nicht zwangsläufig dem Nettorentenniveau entsprechen. Wie vorangehend erläutert kann durch das Kapitalstockverfahren die Demographieabhängigkeit zumindest gemindert werden, so daß ein Teil der fertilitätsbedingten Verteilungswirkungen als intergenerative Umverteilung gewertet werden kann. Da der adäquate Anteil analytisch jedoch nicht bestimmt werden kann, könnte dieser Kritik zumindest Rechnung getragen werden, indem ein Faktor $\Phi>$ Nettorentenniveau angesetzt wird (vgl. Abbildung 13). 
Abbildung 13: Verlauf des generationsspezifischen Verteilungsindikators (VI) bei unterstelltem unvollstăndigen Risikoausgleich bezüglich Fertilitätsveränderungen (bzw. einer von der Nettoanpassung abweichenden Lastverteilungsregel) $\left(\Phi>k^{\text {netto }}\right)$

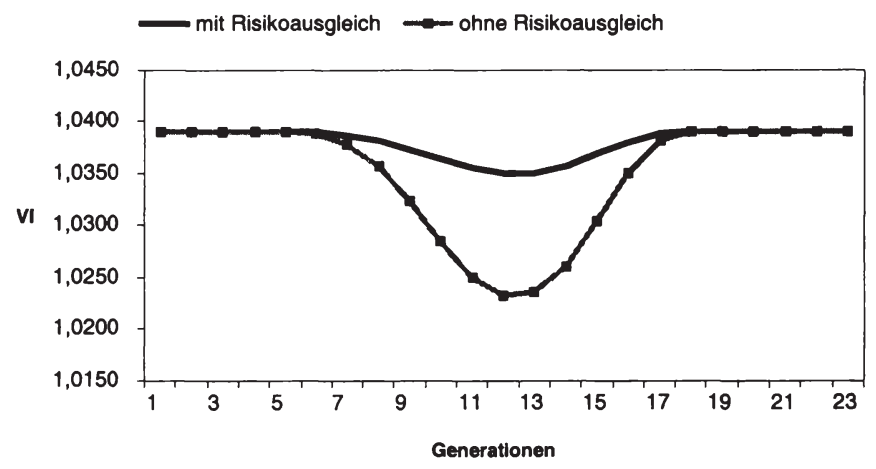

Anmerkung: Modellrechnung mit einem 6-Generationen-Modell (4 Erwerbsperioden, 2 Ruhestandsperioden). Senkung der Reproduktionsrate der Generationen 10, 11 und 12.

Quelle: eigene Darstellung

\subsubsection{Variation der versicherungspflichtigen Erwerbstätigkeit}

Im Modell des zweiten Kapitels wurde die Variation der versicherungspflichtigen Erwerbsbeteiligung als eine einzelne Einflußgröße betrachtet und nicht weiter differenziert. Sie kann jedoch unterschiedliche Ursachen haben. Schwankt die Erwerbsbeteiligung, so kann dies beispielsweise auf ein verändertes Arbeitsangebotsverhalten der privaten Haushalte zurückgeführt werden. Dabei kann sich zum einen die Lebensarbeitszeit bereits $\mathrm{Er}$ werbstätiger verändern oder zum anderen kann sich die Menge der arbeitswilligen Personen verändern. Hier ist insbesondere daran zu denken, daß Frauen verstärkt aus der Stillen Reserve und Nichterwerbstätigkeit auf den Arbeitsmarkt drängen. ${ }^{373}$ Eine Abnahme der versicherungspflichtigen Erwerbstätigkeit kann allerdings ebenso nachfragebedingt eine Folge der steigenden Arbeitslosigkeit oder der Zunahme nicht versicherungspflichtiger Arbeitsverhältnisse sein. ${ }^{374}$

Wie sich aus den Ergebnissen des Abschnitts 2.4.2.2 schließen läßt, führen sämtliche Variationen der Erwerbstätigkeit zu einer qualitativ ungleichen Behandlung der verschiedenen Generationen, die sich zudem eindeutig den Generationen zuordnen läßt (1. Krite-

373 Ein verändertes Arbeitsangebot kann sich darüber hinaus auch in der wöchentlichen oder jährlichen Arbeitszeit niederschlagen. Auf die Rentenanwartschaften würde sich dies gegebenenfalls nur über höhere Löhne auswirken. Abstrahiert man von der Zeitverzögerung der Rentenanpassung, so hätte dies bei einer lohnzentrierten Rentengewährung keine der hier diskutierten Beitragseffekte zur Folge.

374 Zu diesen unter dem Stichwort der "Erosion des Normarbeitsverhältnisses" diskutierten Arbeitsverhältnissen zählen neben der geringfügig beschäftigten vor allem die Zunahme an „Scheinselbständigen“. Vgl. Rürup/Schroeter (1996). 
rium). ${ }^{375}$ Theoretisch läßt sich ebenfalls bestimmen, bei welchen Generationen sich die Erwerbsbeteiligung verändert hat (2. Kriterium). Von einer gezielten und direkten Beeinflussung der Erwerbsbeteiligung durch die verursachende Generation (Kriterium 3) kann jedoch nur ausgegangen werden, wenn eine Veränderung des Arbeitsangebots vorliegt.

\section{A) Angebotsseitige Veränderung der Erwerbsbeteiligung}

Die durch eine angebotsseitige Veränderung der Erwerbsbeteiligung ausgelösten intergenerativen Verteilungswirkungen sind als Umverteilung zu werten, wenn die Effekte sich internalisieren lassen. Dies könnte in ähnlicher Weise wie bei der Variation der Mortalität während der Erwerbsphase durch eine stärkere Beitragsorientierung in ein teilhabeäquivalentes System erfolgen.

Auch in diesem Fall wäre nur derjenige Teil der Beitragssatzveränderung zu berücksichtigen, der auf eine Variation der Erwerbsbeteiligung zurückzuführen ist. Die die praktischen Umsetzung einer solchen Teilhabeäquivalenz würde wieder zu einer recht komplizierten Rentenformel führen. Grundsätzlich stellt eine solche Formel aber eine Möglichkeit dar, die aus einer Veränderung des Arbeitsangebots entstehenden Umverteilungen zu kompensieren.

Intergenerative Verteilungswirkungen des Alterssicherungssystems, die durch eine Veränderungen des Arbeitsangebots entstehen, sollten mithin als intergenerative Umverteilungen bewertet werden.

\section{B) Nachfragebedingte Veränderung der Enwerbsbeteiligung}

Es ist nun zu klären, inwieweit eine - von den Generationen nicht direkt beeinflußbare nachfrageseitige Variation der versicherungspflichtigen Erwerbstätigkeit als ökonomisches, durch die Versicherung zu tragendes Risiko interpretiert werden kann. Ob die Voraussetzungen eines intergenerativen Risikoausgleichs gegeben sind, entscheidet sich daran, inwieweit sich die auftretenden Verteilungswirkungen internalisieren lassen (4. Kriterium).

Sowohl ein Umlagesystem mit konstanten Beitragssätzen als auch eine Korrektur des Rentenniveaus nach Maßgabe der veränderten Erwerbstätigenquote würden lediglich zu einem anderen Verteilungsmuster führen und könnten die grundsätzlichen Verteilungswirkungen nicht vermeiden.

Die Auswirkungen einer zunehmenden „Erosion des Normarbeitsverhältnisses“ ließen sich relativ problemlos internalisieren, indem die nicht versicherungspflichtigen Arbeits-

375 Ähnlich wie bei der Variation der Mortalität in der Erwerbsphase gilt auch hier, daß eine eindeutige Zuordnung, welche Generation von der verānderten Sterblichkeit profitiert bzw. von ihr benachteiligt wird (Kriterium 1b) in Praxi äußerst schwierig aber theoretisch möglich ist.

Von indirekten Wirkungen, die sich dadurch einstellen könnten, daß eine höhere Erwerbsquoten ggf. zu einem höheren Wachstumspfad führt, wird an dieser Stelle abgesehen (vgl. Kapitel 6). Die von Wagner (1985), S. 156 angesprochene geringere "Relativbelastung ${ }^{4}$ kann bei einer an die Produktivität gekoppelten Rentenleistung vernachlässigt werden.

168 
verhältnisse (geringfügige Beschäftigungen und Scheinselbständige) durch entsprechende Gesetzesänderungen in die Versicherungspflicht mit einbezogen werden.

Die weitere Untersuchung konzentriert sich daher auf die Wirkung der zunehmenden Arbeitslosigkeit. Sie hängt ab vom Status der Arbeitslosen in der Rentenversicherung. Wenn ein Versicherter beispielsweise aus dem Alterssicherungssystem ausscheidet, sobald er arbeitslos wird, führt eine steigende Zahl von Arbeitslosen dazu, daß die verbleibenden Erwerbstätigen höhere Beiträge zahlen müssen, um die (unveränderten) Rentenleistungen zu bedienen (erster Verteilungseffekt). Für nachfolgende Generationen kann der Beitragssatz gesenkt werden, da aufgrund der vorangegangenen Arbeitslosigkeit die Rentenansprüche niedriger sind (zweiter Verteilungseffekt).

Bis zur Rentenreform 1992 wurde die Dauer der Arbeitslosigkeit als "beitragsfreie Zeit" angerechnet, so daß die Beiträge für die zeitgleich erwerbstätigen Generationen bei wachsender Arbeitslosigkeit zwar steigen, nachfolgende Generationen später jedoch nicht von fallenden Beitragssätzen profitieren. Werden die Zeiten der Arbeitslosigkeit allerdings - wie es seit der Rentenreform von 1992 in der gesetzliche Rentenversicherung praktiziert wird - als volle Beitragszeiten behandelt, so bleibt auch der erste Verteilungseffekt steigender Beitragssätze prinzipiell aus. Praktisch sind aber dennoch beide Verteilungseffekte in der gesetzliche Rentenversicherung in geringem Umfang wirksam, weil die gewährten Lohnersatzleistungen der Versicherten niedriger ausfallen als ihre vorherigen Erwerbseinkommen. ${ }^{376}$ Um beide Effekte komplett zu kompensieren, müßte die verbleibende Beitragsdifferenz durch erhöhte Zahlungen der Bundesanstalt für Arbeit oder durch einen höheren Bundeszuschuß ausgeglichen werden. ${ }^{377}$

Eine vollständige Auslagerung der Arbeitsmarktrisiken aus der Rentenversicherung wäre aus Gründen der Kostentransparenz und der Akzeptanz der Rentenversicherung grundsätzlich wünschenswert. Für die vorliegende Arbeit zeigt die Möglichkeit der Auslagerung der Arbeitsmarktrisiken aber, daß die durch die Arbeits/osigkeit induzierten Verteilungswirkungen innerhalb des Alterssicherungssystems als intergenerative Umverteilung zu werten sind.

Aber selbst wenn eine solche verursachungsadäquate Risikozuteilung realisiert würde, blieben mittelbare intergenerative Verteilungswirkungen der Arbeitslosigkeit bestehen.

376 So beziffert der VDR die durchschnittlichen jährlichen Mindereinnahmen an Beiträgen für 1997 auf 0,6 Mrd. DM je 100.000 Arbeitslose (VDR: Rentenversicherung in Zahlen 1997, S.4).

377

Denkbar wäre beispielsweise ein an die Arbeitslosigkeit gekoppelter Bundeszuschuß, dessen Erhöhung (ggf. teilweise) durch Kreditaufnahme finanziert wird (Ausbleiben des ersten Verteilungseffektes). Sobald die Arbeitsiosen in den Ruhestand gehen, sinken die Rentenansprüche und damit einhergehend die Beitragssätze. In dem $\mathrm{Maße}$ in dem die Beiträge niedriger ausfallen können Steuern erhoben werden, um die aufgenommenen Schulden (ggf. teilweise) zu tilgen (Vermeidung des zweiten Verzinsungseffekts).

Diese Zusammenhänge verdeutlichen zudem, daß der Bundeszuschuß in der gesetzliche Rentenversicherung keineswegs als "Subvention eines maroden Systems" aufgefaßt werden kann. Denn selbst, wenn die Arbeitslosenversicherung die Beiträge zahlt, können die Beitragssatzwirkungen der Arbeitslosigkeit vollends nur durch einen zusätzlichen Bundeszuschuß vermieden werden. 
Diese entstehen dadurch, daß die Rückkopplungsmechanismen der Nettoanpassung auch auf Veränderungen der Steuern und sonstigen Sozialbeiträge (beispielsweise zur Arbeitslosenversicherung) reagieren. Die Zusammenhänge lassen sich einfach in einem zwei Generationen umfassenden Modell überlappender Generationen einer kleinen offenen Volkswirtschaft zeigen.

Die Erwerbstätigengeneration teilt sich dabei auf in Erwerbstätige eq ${ }^{t} * \mathrm{~N}_{\mathrm{E}}{ }^{t}$ und in Arbeitslose $\left(1-q^{t}\right) * N_{E}{ }^{\prime}$. Für die Erwerbsquote eq der Erwerbstätigengeneration in Periode t gilt $0,5<\mathrm{eq}^{t}$ $<1$. Weiterhin wird angenommen, daß die Transfers an die Arbeitslosen durch eine Besteuerung der Erwerbstätigen mit dem Steuersatz $t$ finanziert werden, Beiträge werden nur zur Finanzierung der Alterssicherung erhoben. Die pro-Kopf Transfers entsprechen dem Durchschnittseinkommen, so daß es bei gegebenem Beitragssatz keine Beitragsausfälle gibt. ${ }^{378}$ Mit $w^{t}$ als Durchschnittslohn muß für den Steuersatz $t^{\prime}$ gelten:

$$
N_{E}^{t} \cdot e q^{t} \cdot w^{t} \cdot t^{t}=N_{E}^{t} \cdot\left(1-e q^{t}\right) \cdot w^{t}-N_{E}^{t} \cdot\left(1-e q^{t}\right) \cdot w^{t} \cdot t^{t}
$$

durch Umformung erhält man:

$$
t^{t}=1-e q^{t} \text { mit } 0<t^{t}<0,5 .
$$

Aus den Einnahmen $E^{t}=N_{E}^{t} \cdot w^{t} \cdot b^{t}$ und Ausgaben $A^{t}=p^{t} \cdot N_{R}^{t}=w^{t}\left(1-b^{t}-t^{t}\right) \cdot k \cdot N_{R}^{t}$ der Rentenversicherung erhält man die Gleichung für den Beitragssatz:

$$
\begin{aligned}
& b^{t^{*}}=\frac{N_{R}^{t} \cdot k \cdot\left(1-t^{t}\right)}{N_{E}^{t}+N_{R}^{t} \cdot k}=b^{t} \cdot\left(1-t^{\prime}\right) \\
& b^{t^{*}}=\text { Beitragsatz mit Berückschtigung der Steuer in der Nettoanpassung } \\
& b^{t}=\text { Beitragsatz ohne Berückschtigung der Steuer in der Nettoanpassung }
\end{aligned}
$$

Für die Rendite ergibt sich bei konstantem Zinssatz und konstantem Lohnwachstum die allgemeine Gleichung:

$$
R^{t}=\frac{p^{t}}{b^{t-1} \cdot w^{t-1} \cdot(1+i)}=\frac{w^{t} \cdot\left(1-b^{t}-t^{t}\right) \cdot k}{b^{t-1} \cdot w^{t-1} \cdot(1+i)}=\frac{(1+g)}{(1+i)} \cdot k \cdot \frac{1-b^{t}-t^{t}}{b^{t-1}}
$$

Unterstellt wird nun, daß es nur in Periode $t$ Arbeitslosigkeit gibt $\left(0,5<\mathrm{eq}^{\mathrm{v}}<1\right.$ für $\mathrm{v}=\mathrm{t}$ und $\mathrm{eq}^{\mathrm{v}}=1$ für alle $v \neq t$ ). Für die Generationen, die zum Zeitpunkt $\mathrm{t}$ bzw. $\mathrm{t}+1$ im Ruhestand befinden wird untersucht, wie sich die Renditen mit und ohne Berücksichtigung der Steuern bei der Nettoanpassung verhalten. Da die ersten beiden Faktoren der Rendite konstant sind, reicht es aus, jeweils die dritten Faktoren miteinander zu vergleichen.

Zunächst wird angenommen, daß die Generation, die zum Zeitpunkt $t$ im Ruhestand ist, durch die Berücksichtigung des Steuersatzes in der Nettoanpassung benachteiligt wird. Dann gilt:

$$
R^{t^{*}}<R^{\prime} \quad \Leftrightarrow \quad \frac{1-b^{\prime}-t^{t}}{b^{t-1}}<\frac{1-b^{t}}{b^{t-1}} \quad \Leftrightarrow \quad b^{t}\left(1-t^{t}\right)+t^{t}>b^{t} \quad \Leftrightarrow \quad b^{t}<1
$$

Da der Beitragssatz kleiner als $100 \%$ sein muß, wird die These bestätigt. Für die nachfolgende Generation, die zum Zeitpunkt $t+1$ im Ruhestand ist, wird angenommen, daß die von ihr erzielbare Rendite durch die Berücksichtigung des Steuersatzes in der Nettoanpassung höher ausfällt:

$$
R^{t+1^{*}}>R^{t+1} \Leftrightarrow \frac{1-b^{t+1}}{b^{t^{*}}}>\frac{1-b^{t+1}}{b^{t}} \Leftrightarrow b^{t}>b^{t}\left(1-t^{t}\right) \quad \Leftrightarrow \quad 1>1-t^{t}
$$

378 Um zu verhindern, daß die Arbeitslosen besser gestellt werden, wird auch ihr Einkommen mit dem Steuersatz $t$ besteuert und die Einnahmen gleichmäßig auf sämtliche Personen verteilt. Werden die Transferzahlungen saldiert, kann der resultierende faktische Steuersatz bestimmt werden. 
Da $0<t^{t}<0,5$ gilt, ist auch diese Annahme bestätigt.

An dieser Stelle werden nochmals die wesentlichen Unterschiede der hier diskutierten Verteilungseffekte der Arbeitslosigkeit zusammengefaßt:

- Eine zunehmende Arbeitslosigkeit kann direkte intergenerative Verteilungseffekte hervorrufen, da die erforderlichen Beitragssätze für all jene Generationen steigen, die während der Arbeitslosigkeit erwerbstätig sind und für all jene Generationen sinken, die erwerbstätig sind, während die von der Arbeitslosigkeit betroffenen Generationen sich im Ruhestand befinden.

- Die durch die Arbeitslosigkeit ausgelösten steigenden Beitragssätze werden durch das Prinzip der Nettoanpassung gedämpft, indem ein Teil der Last auf vorangegangene Generationen verschoben wird.

- Da alle diese Verteilungswirkungen sich vermeiden ließen, wenn das Risiko der Arbeitslosigkeit vollständig auf das allgemeine Steuer-Transfer-System oder die Arbeitslosenversicherung übertragen würde, wurden sie als intergenerative Umverteilung identifiziert.

- Doch selbst wenn das Arbeitsmarktrisiko vollständig aus der Alterssicherung herausgenommen wird, können noch weitere mittelbare Verteilungseffekte auftreten, sofern bei der Nettoanpassung nicht nur die Beiträge zur Rentenversicherung, sondern auch die Beiträge zur Arbeitslosenversicherung und die Steuern berücksichtigt werden, aus denen die Rentenbeiträge der Arbeitslosen finanziert werden.

Es bleibt daher zu klären, ob die durch eine Rückkopplung der veränderten Steuern und der Beiträge zur Arbeitslosenversicherung über die Nettoanpassung generierten Verteilungseffekte ebenfalls als originäre Umverteilung des Alterssicherungssystems zu bewerten sind oder ob sie als Bestandteil des allgemeinen intergenerativen Risikoausgleichs angesehen werden können. Vorangestellt ist allerdings auf folgendes hinzuweisen:

Die hier beschriebenen Effekte werden zwar in Bezug auf die Arbeitslosigkeit diskutiert, dieser Rückkopplungsmechanismus der Nettoanpassung tritt aber auch bei Variationen der übrigen Sozialbeiträge und Steuern auf und zwar unabhängig von dem ursächlichen Ereignis (höhere Morbidität oder Pflegekosten, Steuererhöhungen aufgrund einmaliger Ereignisse wie z.B. der Wiedervereinigung etc.).

Eine zunehmende Arbeitslosigkeit (resp. höhere Morbidität etc.) ist in jeder Gesellschaft und unabhängig vom Alterssicherungssystem mit einer zusätzlichen Belastung in Form höherer Steuern bzw. Beitragssätze zur Arbeitslosenversicherung verbunden. ${ }^{379}$ Dabei stellt sich die Frage, wie die Zusatzlast zu verteilen ist und ob insbesondere lediglich die Erwerbstätigen oder auch die Ruheständler zur Finanzierung herangezogen werden soll-

379 Es muß immer Einkommen zu den Arbeitslosen für deren Lebensunterhalt und deren Altersvorsorge transferiert werden. Dabei ist es vorerst unerheblich, ob der Versicherte durch diese Transfers seine 
ten. In Abschnitt 4.4.3.2 wurde betont, daß zum intergenerativen Risikoausgleich nicht nur die Solidarität zur nicht erwerbstätigen Generation gehört, sondern auch die Solidarität der Leistungsempfänger mit den Beitragszahlern. Demzufolge gehören die hier betrachteten Rückkopplungseffekte zum intergenerativen Risikoausgleich.

Diese Interpretation wird durch die folgenden Überlegungen gestützt:

Die allgemeinen Aufgaben einer Gesellschaft (inklusive der Absicherung gegen Folgen der Arbeitslosigkeit) bzw. die durch bestimmte Ereignisse hervorgerufenen Zusatzlasten werden durch den Verteilungsschlüssel des allgemeinen Steuersystems bzw. der Arbeitslosenversicherung auf die Gesellschaftsmitglieder umgelegt. Da die Renten faktisch nicht steuerpflichtig sind, wird die Gesellschaftsgruppe der Rentner nicht bzw. nur zum Teil nach Maßgabe der indirekten Steuern zur Finanzierung herangezogen. Würde die Nettoanpassung lediglich die Veränderung der Sozialbeiträge zur Rentenversicherung erfassen und die Rentenleistungen dergestalt besteuert, daß die Nettorenten denen des derzeitigen Systems entsprechen, so würden die durch erhöhte Steuern (und ggf. Beiträge zur Arbeitslosenversicherung) ausgelösten Verteilungseffekte in der Rentenversicherung formal ausbleiben, obwohl die Rentner die gleiche Leistung beziehen. Der Einfuß der Zusatzlasten auf die generationsspezifische Rendite bliebe grundsätzlich bestehen.

Das Ausmaß, in dem die Renten nach Maßgabe der höheren Steuern und Sozialbeiträge bei erhöhter Arbeitslosigkeit nicht steigen, könnte folglich - vereinfacht ausgedrückt - als einbehaltener Beitrag oder Steuer zur Beteiligung der Rentner an gesamtwirtschaftlichen Aufgaben interpretiert werden. ${ }^{380}$ Wenn folglich höhere Steuern und/oder Beiträge zur Arbeitslosenversicherung (bzw. zu den übrigen Sozialversicherungen) indirekte Verteilungseffekte über die Rückkopplung der Nettoanpassung verursachen, werden sie im folgenden als intergenerativer Risikoausgleich und nicht als originäre Umverteilung des Alterssicherungssystems interpretiert. ${ }^{381}$

Beiträge im Umlageverfahren oder im Kapitalstockverfahren zahlt (Im Fall einer beitragsfreien Anrechnung wird ein Teil der Transfers lediglich von nachfolgenden Generationen gezahlt).

380 Gleichzeitig wird die durch die Arbeitslosigkeit verursachte, höhere Belastung der erwerbstätigen Generationen durch höhere Steuern bzw. Beitragssätze zur Arbeitslosenversicherung infolge der Nettoanpassung teilweise vermindert, so daß in Folge die Renten wieder leicht steigen können.

381 Gegen eine solche Interpretation könnte wie folgt argumentiert werden:

Die im Steuersystem kodifizierten Verteilungsregeln (Progressiver Tarif, umfassender Einkommensbegriff etc.) unterscheiden sich von denen der beitragsfinanzierten Sozialsysteme. In einem Kategorialsystem wird zudem nur der Teil der Rentnergeneration in den intergenerativen Sozialausgleich einbezogen, der in der gesetzliche Rentenversicherung versichert ist. Eine modifizierte Nettoanpassung, die die Beiträge zur Arbeitslosenversicherung nicht enfaßt, aber durch eine entsprechende Steuerpflicht der Rentenleistungen flankiert wird, könnte einen intergenerativen Ausgleich (der Arbeitslosigkeitsrisiken) realisieren, der eher an die gesellschaftlichen Vorstellungen über gerechte Verteilungsregeln angepaßt werden kann.

Eine solche einseitige Ausrichtung an einer Steuerfinanzierung würde aber letztlich dazu führen, die übrigen Sozialversicherungen als Versorgungssysteme zu konzipieren. Dies widerspricht dem erklärten sozialpolitischen Ziel. Darüber hinaus beziehen sich die erwähnten Unterschiede der Verteilungsregeln im Steuersystem vornehmlich auf die interpersonelle Verteilung. Bei der Definition der Lebensstandardsicherung und daraus abgeleitet des intergenerativen Risikoausgleichs in der Alterssicherung werden die 
Zusammenfassend lassen sich die intergenerativen Verteilungswirkungen einer zunehmenden Arbeitslosigkeit daher wie folgt bewerten:

- Die direkten Beitragseffekte der Arbeitslosigkeit sowie der Teil der Rückkopplung der Nettoanpassung, der auf diese Beitragseffekte zurückzuführen ist, ließen sich durch eine Auslagerung der Arbeitsmarktrisiken auf die Arbeitslosenversicherung bzw. auf das Steuer-Transfer-System vollständig übertragen. Im Meßkonzept werden diese Verteilungseffekte mithin als originäre intergenerative Umverteilung des Alterssicherungssystems erfaßt.

Daraus folgt, daß der Verteilungsindikator der Reformoptionen nicht modifiziert werden muß, die Veränderung der Arbeitsmarkteffekte beim Referenzsystem aber keine Verteilungseffekte hervorrufen darf (vgl. Abschnitt 6.3).

- Durch die Arbeitslosigkeit steigt die Steuerbelastung bzw. steigen die Beiträge zur Arbeitslosenversicherung. Beides beeinflußt über die Rückkopplung der Nettoanpassung die generationsspezifischen Renditen. Diese Effekte beschränken sich nicht auf die Arbeitslosigkeit, sondern treten auch bei Variationen der übrigen Sozialbeiträge auf. Diese Effekte ließen sich durch eine modifizierte Nettoanpassung und Besteuerung der Renten formal aus der Rentenversicherung ausgliedern; der Effekt auf die Rendite der Generationen bliebe aber bestehen. Zudem sind diese Verteilungswirkungen mit dem intergenerativen Solidarausgleich vereinbar, denn dieser umfaßt auch die Solidarität der Leistungsempfänger mit den Beitragszahlern (vgl. Abschnitt 4.4.3.2). In dieser Arbeit werden diese Effekte daher dem intergenerativen Risikoausgleich zugeordnet. Mit anderen Worten, diese Verteilungswirkungen sind nur in dem Maße als Umverteilung zu werten, in dem die Gewichtung der Lebensstandardsicherung, sprich der verwirklichte Umfang der Risikoabsicherung das erwünschte Ausmaß übersteigt (vgl. Abschnitt 5.3.2).

Wenn weder der Verteilungsindikator der Reformoptionen noch der des Referenzsystems hinsichtlich dieser mittelbaren Verteilungswirkungen modifiziert werden, wird der Risikoausgleich mithin korrekt erfaßt.

\subsubsection{Variation des Faktorpreisverhältnisses}

In einem umlagefinanzierten System beeinflußt die Entwicklung der Erwerbseinkommen die Rendite einer Generation unmittelbar und unabhängig davon, ob beim Budgetausgleich der Beitragssatz fixiert wird oder ob konstante und am Durchschnittseinkommen orientierte Rentenleistungen gewährt werden. ${ }^{382}$ Die genauere Analyse der Verteilungswirkungen verdeutlichte zudem, daß eine Veränderung der Lohndynamik sich qualitativ

Rentenleistungen mit den Nettolöhnen verglichen, wobei es unerheblich ist, ob die Nettoquote der Löhne anders ausfallen könnte, wenn die interpersonelle Verteilung anders gelöst wird. 
auf alle betroffenen Generationen in gleicher Weise auswirkt. Somit ist bereits das erste Kriterium verletzt, welches für eine Interpretation der Verteilungseffekte als reine intergenerative Umverteilung efforderlich ist. Diese Erkenntnis überrascht wenig, wenn man bedenkt, daß die Lohnentwicklung neben der Fertilität die zweite Zinskomponente des Umlageverfahrens darstellt. ${ }^{383}$

Gemäß des zweiten und dritten Kriteriums für eine intergenerative Umverteilung ist zu klären, ob sich bei der Entwicklung der Löhne eindeutig nachvollziehen läßt, welche Generation für die auftretende Variation verantwortlich ist und ob diese Generation die Möglichkeit hat, den Parameter "Lohnsatz" bewußt zu beeinflussen. Da der Lohnsatz in einer kleinen offenen Volkswirtschaft per definitionem eine exogene Größe darstellt, ist für diesen Fall keines der beiden Kriterien ertüllt.

Aber auch in einer geschlossenen Volkswirtschaft können auftretende Lohnvariationen nicht zwangsläufig einer „verursachenden Generation“ zugeschrieben werden. Solange die Grenzproduktivitätstheorie die Entlohnung der Produktionsfaktoren bestimmt, hat die Erwerbsbeteiligung einer Generation zwar einen Einfluß auf den Lohnsatz, dennoch lassen sich weitere Einflußgrößen der Produktivität des Faktors Arbeit nicht auf eine bestimmte Generation zurückführen. Hierzu zählen beispielsweise die Kapitalausstattung und vor allem der technische Fortschritt. Diese Parameter sind größtenteils exogen vorgegeben und teilweise sogar stochastischer Natur (z.B. der Einfluß der Innovationen auf den technischen Fortschritt). Abstrahiert man von den zu restriktiven Annahmen eines vollkommenen Wettbewerbs und einer vollen Auslastung der Produktionsfaktoren, so erweist sich der Lohnfindungsprozeß als wesentlich komplexer, als es in einer einfachen neoklassischen Modellökonomie unterstellt wird. ${ }^{384}$ Angesichts dieser Zusammenhänge läßt sich die Ursache der Lohnentwicklung kaum einer bestimmten Generation zuordnen, und die Möglichkeiten einer Generation, die Lohnentwicklung bewußt zu beeinflussen, dürften angesichts der Fülle an Einflußfaktoren ebenfalls vernachlässigbar sein. ${ }^{385}$

Auch das zweite und dritte Kriterium einer intergenerativen Umverteilung, sind im Fall der Variation des Lohnwachstums mithin nicht erfüllt. Nach dem bisher gesagten wären folglich im Fall der Variation des Lohnwachstums die Voraussetzungen für einen intergenerativen Risikoausgleich gegeben, sofern sich die Verteilungseffekte nicht internalisieren lassen. Bevor dieser Frage nachgegangen wird, sollen zunächst die Wirkungen der Zinsentwicklungen betrachtet werden.

382 Vgl. Abschnitt 2.4.2.

383 Vgl. Abschnitt 5.3.1.4

384 Schwierigkeiten ergeben sich bereits beim Versuch, die relevanten Einflußgrößen auf die Lohnentwicklung abschließend zu definieren. So spielen beispielsweise die Machtpositionen der verschiedenen Tarifparteien eine wesentliche Rolle bei den Tarifverhandlungen. Diese hängen wiederum unter anderem von Faktoren wie der Arbeitslosigkeit, der Gewinnsituation der Untemehmen etc. ab. Die Entwicklung der Inflation ist eine weitere Einflußgröße, die größtenteils durch Faktoren bestimmt wird, die keiner Generation zugeordnet werden können. Für einen Überblick über die verschiedenen mikro- und makroökonomischen Arbeitsmarkttheorien siehe Sesselmeier/Blauermel (1990); Franz (1994) und (1995).

Vgl. Bösch (1987), S. 77f.. 
Vergegenwärtigt man sich die Ergebnisse der Abschnitte 2.4.2 und 2.4.3 so zeichnet sich für die Zinsentwicklung ein ähnliches Bild ab, wie für die Lohnentwicklung:

- Eine Zinsvariation hat den qualitativ gleichen Effekt auf alle betroffenen Generationen.

- Eine Zinsvariation kann nicht einer verursachenden Generation zugeordnet werden. ${ }^{386}$

- Eine Zinsvariation entzieht sich einer bewußten Beeinflussung durch eine bestimmte Generation. ${ }^{386}$

Lediglich die Richtung des Zinseffekts unterscheidet sich von der Wirkung der Lohnentwicklung, da die Rendite im Umlageverfahren mit zunehmenden Lohnsatz steigt, mit zunehmenden Zinssatz hingegen sinkt. Auch bezüglich der Zinsvariation läßt sich bislang der Schluß ziehen, daß sie als Bestandteil des intergenerativen Risikoausgleichs angesehen werden kann, sofern die damit einhergehenden Verteilungswirkungen sich nicht internalisieren lassen.

Bei der Frage, ob sich die durch eine Veränderung des Lohnsatzes bzw. des Zinssatzes hervorgerufenen Verteilungswirkungen - zumindest theoretisch - durch eine Modifikation des Rentensystems vermeiden ließen, muß gedanklich zwischen zwei Effekten unterschieden werden, über die der Zinssatz das Umlageverfahren beeinflußt: (A) Der Diskontierungseffekt und (B) der aus dem Opportunitätsprinzip abgeleitete Effekt.

\section{A) Diskontierungseffekt der Rate des Zinssatzes}

Die Gleichung G 49 läßt vermuten, daß beitragsäquivalente Rentenzahlungen die Rendite eines Alterssicherungssystems gegen schwankende Lohn- und Zinssätze immunisieren könnten. Ein variierender Zinssatz beeinflußt die generationsspezifische Rendite des Umlageverfahrens allerdings nur deshalb, weil die Beiträge und Renten nach Maßgabe des Zinssatzes diskontiert werden. Diese Diskontierungsannahme ist ebenfalls ausschlaggebend dafür, daß veränderliche Zinssätze in einem kapitalgedeckten Alterssicherungssystem keinen Einfluß auf die Rendite der Generationen haben. Würde statt dessen eine vom Zinssatz unabhängige und ggf. als konstant angenommene Zeitpräferenzrate als Rechnungszinsfuß angesetzt, wäre die Rendite - sprich die intergenerative Verteilungswirkung - im Umlageverfahren unabhängig, im Kapitalstockverfahren hingegen abhängig von etwaigen Zinsvariationen. ${ }^{387}$

Für den Fall einer kleinen offenen Volkswirtschaft, bei der der Zinssatz exogen durch die Weltwirtschaft bestimmt wird, ist dies unmittelbar einleuchtend. Aber auch in der Modellwelt einer geschlossenen Volkswirtschaft wird der Zinssatz von mehreren Variablen (Sparverhalten, Lohnsatz, technischer Fortschritt, demographischen Größen, etc. vgl. die Ausführungen des zweiten Kapitels) determiniert, die sich nicht einer bestimmten Generation zuordnen lassen. Das zweite und dritte Kriterium wird in diesem Fall ebenfalls nicht erfült. Berücksichtigt man zudem die Rolle der Bundesbank bzw. der Europäischen Zentralbank, wird diese These noch bekräftigt.

Es sei lediglich am Rande darauf hingewiesen, daß in der privaten Lebensversicherung ebenfalls ein von der (ex ante ohnehin unsicheren) real erzielbaren Verzinsung abweichender Rechnungszinssatz für kalkulatorische Zwecke angewandt wird. Die Höhe ist zwar gesetzlich vorgeschrieben, aber auch ohne gesetzliche Regelung müßte ein solcher Kalkulationszinssatz als Ausdruck des Vorsichtsprinzips angewandt werden. 
Da solche Zeitpräferenzraten sich allerdings nicht gesichert bestimmen lassen und sie zudem zwischen einzelnen Versicherten variieren würden, rekurrieren die Barwertbetrachtungen zur Bestimmung der intergenerativen Verteilungswirkungen i.d.R. auf einer Diskontierung mit Hilfe des Marktzinssatzes. Eine solche Vorgehensweise setzt allerdings implizit voraus, daß

1. der Versicherte die Möglichkeit hat, durch Sparen bzw. Verschulden sein Lebenseinkommen entsprechend seiner individuellen Präferenzen nutzenmaximal über den Lebenszyklus aufzuteilen und daß

2. diese Aktivitäten auf dem Kapitalmarkt keinen Einfluß auf die Höhe seines Lebenseinkommens haben.

Diese Bedingungen sind an die Annahme eines vollkommenen und homogenen Kapitalmarktes geknüpft, bei dem insbesondere jedem Versicherten unbegrenzte Kreditmöglichkeiten eingeräumt und bei dem identische Soll- und Habenzinssätze unterstellt werden. Ferner kann ein Einfluß auf die Höhe des Lebenseinkommens nur dann vermieden werden, wenn der Zinssatz über den gesamten Lebenszyklus konstant bleibt. ${ }^{388}$ Solche restriktiven Annahmen mögen hilfreich sein, um in einem einfachen Modell eindeutige (qualitative) Tendenzen eines Alterssicherungssystems abzuleiten, für die Beurteilung der Umverteilungswirkungen eines realen Rentensystems erweisen sie sich als zu realitätsfern.

Läßt man die Annahme eines homogenen Kapitalmarktes fallen und setzt von der (ohnehin erst ex post bestimmbare) Realverzinsung unabhängige Diskontierungsfaktoren an, wäre eine Internalisierung beider Verteilungseffekte durch die Einführung einer kapitalgedeckten Alterssicherung nicht erreichbar. Es wäre lediglich möglich, entweder eine Unabhängigkeit von der Lohnentwicklung oder eine Unabhängigkeit von dem Zinssatz zu erzielen. Folgt man dieser Auffassung, so wäre auch das vierte Umverteilungskriterium nicht erfüllt und die Parametervariationen bezüglich des Lohnwachstums bzw. des Zinssatzes zumindest teilweise als Bestandteil des intergenerativen Risikoausgleichs anzusehen.

Eine Möglichkeit, solche Verteilungswirkungen nicht als intergenerative Umverteilung zu erfassen, bestünde darin, bei der gesetzliche Rentenversicherung die Wachstumsrate des Lohnes als Diskontsatz anzusetzen. In diesem Fall würde der Verteilungsindikator im Umlageverfahren auch bei variierender Lohnentwicklung keine Verteilungseffekte anzeigen. ${ }^{389}$

\footnotetext{
388 Vgl. Speckbacher (1994), S. 67.

389 Würden in einem vergleichbaren Kapitalstockverfahren als Referenzsystem die erzielbaren Beiträge und Rentenzahlung allerdings ebenfalls mit dem Lohnwachstum diskontiert, wäre die Wirkung jedoch identisch mit der, die sich bei einer Diskontierung mit der internen Verzinsung des Kapitalstockverfahrens ergibt.
} 


\section{B) Aus dem Opportunitätskostenprinzip abgeleitete Effekt des Zinssatzes}

Die Wahl geeigneter Diskontsätze kann zwar verhindern, daß Zinsveränderungen die generationsspezifischen Renditen eines umlagefinanzierten Rentensystems beeinflussen, die Renditen eines alternativen, zinsabhängigen Systems würden aber weiterhin schwanken. Mit anderen Worten: die Opportunitätskosten der verschiedenen Generationen verändern sich.

Sobald ein zinsabhängiges Kapitalstockverfahren (oder eine Mischform) als verteilungsneutrale Referenz fungiert, wird das Opportunitätskalkül in die Umverteilungsanalyse einbezogen. Übersteigt das Lohnsummenwachstum den Zinsfaktor $\left(M^{*} G>R\right),{ }^{390}$ signalisiert dies eine positive Umverteilung im Umlageverfahren. Sofern das System nicht unendlich ist, müßte dieser Transfer bei einer Auflösung des Systems von der "Letztgeneration“ finanziert werden. Ein Lohnsummenwachstum, das niedriger ist als der Zinsfaktor ( $M^{*} G<$ R) bedeutet hingegen eine negative Umverteilung für die betroffene Generation, d.h. sie finanziert einen Teil der „Einführungsgewinne“ vorangegangener Generationen. ${ }^{391}$

Ob und gegebenenfalls in welchem Ausmaß Schwankungen der Lohn-Zins-Differenz als Umverteilung oder als intergenerativ versicherbares Risiko zu werten ist, läßt sich mithin nur aus dem Referenzsystem, sprich aus dem Umfang des präferierten Risikoausgleichs ableiten. Dieser läßt sich, wie der folgende Abschnitt verdeutlicht, analytisch nicht bestimmen. Angesichts dieses Problems verwundert es kaum, daß die Arbeiten zur intergenerativen Gerechtigkeit bislang keinen befriedigenden Ansatz zur Abgrenzung und Operationalisierung der Lohn-Zins-Effekte bieten.

Mit $R=$ Zinssatz, $M=$ Reproduktionsfaktor, $G=$ Wachstumsfaktor des Lohnes. An dieser Stelle interessieren jedoch lediglich die Lohn- und Zinsvariationen, so daß im folgenden eine konstante Fertilităt unterstellt wird.

391 Bösch bezeichnet die sich aus der Differenz zwischen Lohnsummenwachstum und Zinsfaktor ergebende Besser- bzw. Schlechterstellung der verschiedenen Generationen im Umlageverfahren als "Effizienzgewinneinkommen". Vgl. Bösch (1987), S. 55ff.. Hinsichtlich des Einflusses eines variablen Zinssatzes bzw. des technischen Fortschritts auf die Lebenseinkommenspositionen führt Bösch weiter aus: "In keinem Fall kann aber davon ausgegangen werden, daß - wie Dinkel (1984a), S. 24) argumentiert - eine Veränderung des technischen Fortschritts ein "bachward shifting of burden“ hervorruft. Selbst wenn man generationsspezifische Werte für $M$ unterstellt, gilt immer noch, daß sich keine Generation auf Kosten anderer Generationen verbessern kann, d.h. es findet kein "shifting" von "burden“ statt. "Bösch (1987), S. 78. Diese Aussage scheint sich zunächst mit der oben getroffenen Feststellung zu decken, daß das erste Umverteilungskriterium verletzt ist. Dies bezog sich allerdings auf den Einfluß auf die Rendite innerhalb eines umlagefinanzierten Systems. Bösch hingegen bezieht seine Aussage bereits auf einen Vergleich mit dem Kapitalstockverfahren. In diesem Fall verwundert seine Aussage, da sie sich nicht mit seinen vorangegangenen Ausführungen auf den Seiten $58 / 59$ deckt. So schreibt er beispielsweise auf Seite 59: „Falls das Versicherungssystem eines Tages aufgelöst wird, verbleiben allerdings der Letztgeneration Finanzierungsverpflichtungen in Höhe der verzinsten Einführungsgewinne zuzüglich oder abzüglich der anfallenden Effizienzgewinneinkommen. "Die Effiziensgewinneinkommen ergeben sich aber gerade aus der Differenz zwischen Zins und Lohnsummenwachstum, sprich andere Generationen werden von einer Variation der Parameter Zins und Lohnwachstum sehr wohl betroffen, sofern diese nicht gleich sind bzw. die Differenz durch die Fertilität ausgeglichen wird. Die Unmöglichkeit einer Lastverschiebung gilt offensichtlich nur dann, wenn ein unendlicher Betrachtungszeitraum unterstellt (und das Umlageverfahren nicht aufgelöst) wird. 
WAGNER hebt die Bedeutung des intergenerativen Risikoausgleiches zwar hervor, umgeht das Problem aber, indem er die Status-Quo-Bedingungen fortschreibt, ${ }^{392}$ was für die vorliegende Analyse jedoch unbefriedigend ist.

BöSCH, der seine Schlußfolgerung vornehmlich auf das dritten Umverteilungskriterium der gezielten Beeinflussung stützt, kommt zu einer ähnlichen Einschätzung der Lohn- und Zinsschwankungen wenn er ausführt:

„Wird allerdings - wie im Falle des Zinssatzes - wiederum argumentient, daß der technische Fortschritt zu einem bestimmten Zeitpunkt nicht als Charakteristikum einer spezifischen Generation angesehen werden darf, sondern eine exogene, generationsunabhängige Größe darstelt, macht es in diesem Zusammenhang keinen Sinn, von intergenerationaler Umverteilung oder Lastverschiebung zu sprechen. ${ }^{\text {“93 }}$

Als Referenzsystem legt er seiner Analyse aber dennoch ein beitragsäquivalentes Kapitalstockverfahren zugrunde, bei dem die gewährten Leistungen des Referenzsystems auf die des Umlageverfahrens normiert werden. Zunächst wird der für das gegebene Leistungsniveau erforderliche Beitragssatz des Referenzsystems ermittelt. In einem weiteren Schritt formuliert Bösch seinen Umverteilungsindikator, indem der Beitragssatz des Referenzsystems durch den des Umlageverfahrens dividiert und vom Ergebnis der Wert 1 subtrahiert wird. Durch diese Operationalisierung der intergenerativen Umverteilung werden etwaige Variationen des Lohn und Zinssatzes entgegen seiner eigenen Einschätzungen zur Gänze als intergenerative Umverteilung erfaßt.

Angesichts der bisherigen Ausführungen wäre es angemessener, ein Mischsystem als verteilungsneutralen Vergleichsmaßstab zu unterstellen. Je risikoaverser die Individuen und je unsicherer die Entwicklung des Lohn- und Zinssatzes sind, desto größeres Gewicht müßte dem Umlageverfahren zukommen.

Für die folgende Analyse der intergenerativen Gerechtigkeit läßt sich hinsichtlich der Bewertung der aus der Lohn- und Zinsentwicklung resultierenden Verteilungswirkungen festhalten:

Der Verteilungsindikator des Meßkonzepts muß nicht angepaßt werden, da die Abgrenzung des Anteils der Lohn-Zins-Differenz, der zum intergenerativen Risikoausgleich gehören soll, sich automatisch aus der Festlegung des erwünschten intergenerativen Risikoausgleichs, sprich aus der Bestimmung des Referenzsystems ergibt.

Die Frage, in welchem Umfang ein Ausgleich der intergenerativen Risiken erwünscht ist, wird im folgenden Abschnitt untersucht.

392 Vgl. Wagner (1984) sowie Abschnitt 5.2.3

393 Bösch (1987), S. 78. Dabei entspricht in seiner Modellierung der Wachstumsfaktor M des technischen Fortschritts dem Wachstumsfaktor der Bruttolohneinkommen pro Kopf in der Volkswirtschaft. Vgl. Bösch (1987), S. 16f.. 


\subsubsection{Ausmaß des intergenerativen Risikoausgleichs}

Unabhängig von den Verteilungsregeln der Last und der Spezifikation der relevanten Risiken ist in einem „intergenerativen Versicherungsvertrag“ festzulegen, in welchem Umfang das Risiko abgedeckt werden soll. Wird der Risikoausgleich durch ein teilhabeäquivalentes Umlageverfahren realisiert, bei dem die Leistungen sich an den aktuellen Erwerbseinkommen orientieren, spiegelt sich die Entscheidung über den Umfang des intergenerativen Risikoausgleichs in dem optimalen Verhältnis aus umlagebasierter und kapitalfundierter Finanzierung des Referenzsystems wider.

Allerdings ist der intergenerative Risikoausgleich nur ein Kriterium, an dem das optimale Verhältnis der Finanzierungsverfahren bewertet werden kann. Die Diskussion zur „Goldenen Regel der Kapitalakkumulation ${ }^{\text {‘394 }}$ und um die Portfolioansätze $e^{395}$ verdeutlichen, daß Wachstumseffekte und finanzwirtschaftliche Verzinsungsmotive ebenso eine Rolle spielen können. Es wäre Zufall, wenn das optimale Mischverhältnis aus Umlage- und Kapitalstockverfahren sich bei diesen Kriterien mit dem decken würde, der sich nach dem Bedarf an intergenerativen Risikoausgleich richtet. Dieser Trade-off müßte dementsprechend in das Optimierungskalkül integriert werden. Allen Kriterien des optimalen Mischverhältnisses ist zudem gemeinsam, daß sie sich nur schwer operationalisieren lassen. ${ }^{396}$ Für die hier interessierende Fragestellung kann zunächst von diesem Problem der verschiedenen Kriterien für ein optimales Mischverhältnis der Finanzierungsverfahren abstrahiert und lediglich die Frage verfolgt werden, welcher Risikoausgleich erwünscht wird. ${ }^{397}$ Denn das Mischverhältnis bezieht sich auf das Referenzsystem als methodisches Hilfsmittel zur Operationalisierung der Verteilungseffekte (vgl. Abschnitt 5.2.1).

Aber trotz dieser Vereinfachung der Fragestellung läßt sich der Umfang des Risikoausgleichs nicht aus übergeordneten Gerechtigkeitsprinzipien ableiten, da sie größtenteils von der Risikoaversion der Versicherten, sprich von individuellen Präferenzen, abhängt. ${ }^{398}$ Selbst wenn es eine Möglichkeit gäbe, diese genau zu ermitteln, bliebe die Schwierigkeit, daß die individuellen Präferenzen recht heterogen sind. Die Aggregation einzelner Präfe-

394

395

396

Es verwundert daher kaum, plädiert wird die Ansichten ü obschon on- aus welchen Gunnden auch inmer-fur ein Mischsystem Aussagen über eine konkrete Relation getroffen wurde. Dies wäre schon allein deshalb eine gewagte Aussage, weil das optimale Verhältnis im Zeitablauf sicherlich nicht konstant ist.

Diese methodische Vorgehensweise läßt sich damit begründen, daß an dieser Stelle keine Empfehlung für die Gestaltung des Alterssicherungssystems gegeben, sondern versucht werden soll, das Ausmaß an intergenerativen Risikoausgleich zu bestimmen. Reale Restriktionen, die sich ggf. aus der Größe des Kapitalstocks ergeben (vgl. Dinkel (1985) und (1984), S. 353; Rürup (1995)) sind für diese Fragestellung daher ebenfalls unerheblich. Als Folge solcher Einschränkungen ließe sich bspw. lediglich feststellen, daß eine minimale Umverteilung nicht vermeidbar ist.

398

So auch Wagner (1985), S. 155: „Offensichtlich liegen schwierige Probleme der gesetzliche Rentenversicherung in der Bestimmung des "Preises", den die Versicherten bereit sind für den intergenerationalen Risikoausgleich zu zahlen." 
renzen zu einer gesellschaftlichen Präferenz ist jedoch ein gleichermaßen methodisches wie ethisches Problem. ${ }^{399}$

Aus allgemeinen Gerechtigkeitskonzepten läßt sich mithin nicht herleiten, wie das Ziel des relativen Lebensstandards im Alter im Zielsystem eines Alterssicherungssystem gewichtet werden soll, sprich in welchem Umfang der intergenerative Risikoausgleich erwünscht ist. Die alternative Vorgehensweise, ${ }^{400}$ die Gegebenheiten des derzeitigen Systems als Gerechtigkeitsmaßstab zu verwenden, erscheint in diesem Fall jedoch ebenso unzulässig. Denn gerade diese Frage nach dem Grad der Kapitalfundierung der Alterssicherung prägt die derzeitig sehr kontroverse Debatte, so daß es sich verbietet, diesbezüglich von einem gesellschaftlichen Konsens auszugehen.

Das ungelöste Problem, welches Gewicht dem von den individuellen Präferenzen abhängigen Risikoausgleich zukommen soll, offenbart sich mithin als das Kardinalproblem jeder intergenerativen Verteilungs- bzw. Gerechtigkeitsanalyse. Der oft begangene Weg, die Frage vollkommen auszublenden und unter vollkommenen Marktbedingungen eine Gewichtung von Null zu unterstellen, mag die Analyse zwar erleichtern, ob und inwieweit die Ergebnisse dann aber angemessen sind, um aus innen sozialpolitische Handlungsempfehlungen abzuleiten, ist fraglich. Solange aber ein Meßkonzept einer solchen Zielsetzung dient, sollten die Prämissen über die Präferenzen transparent und veränderbar gehalten werden. Bei Aussagen im Sinne einer positiven Analyse, wie z.B.

„wenn die Risikoaversion gegen Null geht, dann werden bestimmte Optionen als intergenerativ gerecht angesehen, wenn jedoch eine Gewichtung des intergenerativen Risikoausgleichs von $\mathrm{X}$ unterstellt wird, dann erweisen sich andere Optionen, wie $\mathrm{Y}$, als vorteilhafter bzw. gerechter"

obliegt es dann dem politischen Entscheidungsprozeß, die Gewichtung vorzunehmen und eine normative Entscheidung zu treffen.

Sollen die intergenerativen Risiken z.B. zu 50\% (30\%, 80\%) abgesichert werden, so werden $50 \%(30 \%, 80 \%)$ des angestrebten Lebensstandards (Nettorentenniveau) im Referenzsystem durch das Umlageverfahren finanziert. Die Differenz zum angestrebten Zielwert des Nettorentenniveaus (beispielsweise $70 \%$ ) wird im Kapitalstockvertahren finanziert. Die erforderlichen Beiträge werden ex ante und unter Annahmen über die Lohn und Zinsentwicklung bestimmt.

Übersteigt die Zins-Lohn-Differenz die Erwartungen, würde die „Überabsicherung“ eines reinen Umlageverfahrens als (negative) intergenerative Umverteilung gewertet. Ist die

399 Angesichts dieser Schwierigkeiten mag es zunächst naheliegend erscheinen, das Problem des Risikoausgleichs in Form eines Grundrentensystems zu lösen, bei dem die Umlagefinanzierung ein Minimum an Versorgung und intergenerativen Risikoausgleich bietet und es jedem Versicherten je nach Risikoaversion und Präferenz selbst obliegt, ob er sich darüber hinaus optional weiter versichert. Auf die gravierenden Schwierigkeiten, die mit der Einführung eines Grundrentensystems verbunden sind soll an dieser Stelle nicht eingegangen werden. Eine solche Konzeption könnte jedoch aufgrund von "Adverse Selektion-Phănomenen“ auch einen effizienten intergenerativen Risikoausgleich nicht gewāhrleisten. 
Zins-Lohn-Differenz hingegen geringer als angenommen, so stellt die „Unterabsicherung“ eines reinen Kapitalstockverfahrens eine (negative) intergenerative Umverteilung dar.

Im vorherigen Abschnitt wurden die intergenerativen Umverteilungen des Umlageverfahrens vom intergenerativen Risikoausgleich abgegrenzt. Damit in der Verteilungsanalyse lediglich die Umverteilungswirkungen erfaßt werden, muß auch der Verteilungsindikator des Referenzsystems in dem Maße modifiziert werden, in dem dieses System umlagefinanziert ist. Dies gilt für die Parameter der Fertilität, der Mortalität, der versicherungspflichtigen Erwerbstätigkeit und der Lohn-Zins-Differenz.

\section{a) Fertilität}

Um die Veränderung der Fertilität als Risikoausgleich zu berücksichtigen, wird der umlagefinanzierte Anteil des Verteilungsindikators des Referenzsystems in gleicher Weise angepaßt, wie der Verteilungsindikator des zu bewertenden Systems (vgl. Abschnitt 5.3.1.4):

$$
V I_{x}^{r e f}=\frac{\sum_{t=R E}^{T}\left(R L_{U L}^{t} \cdot \frac{\left(1-b^{t} \cdot F A K^{\prime}\right)}{\left(1-b^{t}\right)} \cdot \prod_{v=R E}^{t}\left(1+d_{v}\right)^{-1}\right)+\sum_{t=R E}^{T}\left(R L_{K S}^{t} \cdot \prod_{v=R E}^{t}\left(1+d_{v}\right)^{-1}\right)}{\sum_{t=0}^{R E-1}\left(B_{U L}^{t} \cdot F A K^{t} \cdot \prod_{v=t}^{R E-1}\left(1+d_{v}\right)\right)+\sum_{t=0}^{R E-1}\left(B_{K S}^{t} \cdot \prod_{v=t}^{R E-1}\left(1+d_{v}\right)\right)}
$$

Im folgenden wird für die obige Gleichung vereinfacht geschrieben als:

$$
V I_{x}^{r e f}=\frac{\sum_{t=R E}^{T} R U+\sum_{t=R E}^{T} R K}{\sum_{t=R E}^{T} B U+\sum_{t=0}^{R E-1} B K}
$$

\section{b) Mortälität}

Um die Verteilungswirkungen, die sich aus der Variation der Mortalität ergeben, als Umverteilung zu erfassen, darf die Veränderung der Restlebenserwartung den Verteilungsindikator des Referenzsystems nicht beeinflussen. Dies wird erreicht, indem die Rentenleistungen des umlagefinanzierten Anteils mit einem kohortenspezifischen Lebenserwartungsfaktor multipliziert werden:

$$
V l_{x}^{r e f}=\frac{\sum_{t=R E}^{T}\left(R U * L E F_{x}\right)+\sum_{t=R E}^{T} R K}{\sum_{t=R E}^{T} B U+\sum_{t=0}^{R E-1} B K}
$$

Der kohortenspezifische Lebenserwartungsfaktor ist dabei so konzipiert, daß eine zunehmende Lebenserwartung die Rentenbezugsdauer zwar weiterhin erhöht, die gesamten 
Rentenleistungen über die Bezugsdauer aber gestreckt werden und die Mortalitätsentwicklung sich gegenüber der Finanzierung des Systems kostenneutral verhält und den Verteilungsindikator nicht beeinflußt. ${ }^{401}$

\section{c) Versicherungspflichtige Enwerbstätigkeit}

Durch zwei Ursachen kann die Erwerbstätigkeit variieren: Änderung der Arbeitsnachfrage und Änderung des Arbeitsangebots. In beiden Fällen kommt es zu intergenerativen Verteilungswirkungen, da die Beitragssätze bei steigender (fallender) Erwerbstätigkeit sinken (steigen) und später steigen (sinken), wenn die Generation im Ruhestand ist. Beide Verteilungseffekte wurden im Abschnitt 5.3.1.5, unabhängig von ihrer Ursache, als intergenerative Umverteilung identifiziert. Damit das Meßkonzept diese Umverteilungseffekte erfaßt, müssen die Beitragseffekte beim umlagefinanzierten Teil des Referenzsystems kompensiert werden. Dies wird erreicht, indem bei der Simulation des Referenzsystems eine Veränderung der Erwerbspersonen keine Veränderung der Beitragssätze hervorruft. In dem Finanzmodell erhält die Rentenversicherung von der Bundesanstalt für Arbeit Beitragszahlungen für Arbeitslose. Diese werden auf Grundlage einer geringeren Bemessungsgrundlage gezahlt. Um die Beitragseffekte einer steigenden Arbeitslosigkeit als intergenerative Umverteilung zu erfassen, werden diese Einnahmen der Rentenversicherung beim umlagefinanzierten Teil des Referenzmodells nicht nach Maßgabe der geringeren, sondern nach Maßgabe der normalen Bemessungsgrundlage gezahlt. Die Änderungen der Erwerbs- und Arbeitslosenquoten beeinflussen im Modell die Persönlichen Entgeltpunkte. Die im Ruhestand ausgelösten Beitragseffekte einer veränderten Erwerbstätigkeit können daher im Referenzsystem einfach kompensiert werden, indem die zeitversetzte Anpassung der Persönlichen Entgeltpunkte an die veränderten Erwerbs- und Arbeitslosenquoten weggelassen wird.

Darüber hinaus verursachen die steigenden Beiträge zur Arbeitslosenversicherung mittelbare Verteilungseffekte über den Rückkopplungsmechanismus der Nettoanpassung. Diese sind jedoch Bestandteil des intergenerativen Risikoausgleichs. Nur in dem Maße in dem das reine Umlageverfahren gegenüber dem Referenzsystem „überversichert" ist, werden diese Effekte als Umverteilung gewertet. Dies kann einfach erreicht werden, indem beim Referenzsysstem die gleichen Beitragssätze für die übrigen Sozialversicherungen angesetzt werden wie bei den zu beurteilenden Reformvorschlägen.

\section{d) Zins-Lohn-Differenz}

Übersteigt die Zins-Lohn-Differenz die Erwartungen, würde die „Überabsicherung“ eines reinen Umlageverfahrens als (negative) intergenerative Umverteilung gewertet. Ist die Zins-Lohn-Differenz hingegen geringer als angenommen, so stellt die "Unterabsicherung“ eines reinen Kapitalstockverfahrens eine (negative) intergenerative Umverteilung dar.

401 Ein solcher kohortenspezifischer Lebenserwartungsfaktor wird im Rahmen der Möglichkeiten und Konsequenzen demographischer Korrekturfaktoren in der Rentenformel in Rürup/Schroeter (1996) beschrieben.

182 
Bleibt zu klären, wie die Über- bzw. Unterabsicherung sich im Verteilungsindikator niederschlägt. Für jede Generation läßt sich für jede Periode der Quotient aus antizipierten Rentennettoniveau und tatsächlichen Rentennettoniveau bestimmen. Die Rentenleistungen können dann korrigiert werden, bevor sie im Verteilungsindikator einfließen.

\subsubsection{Verteilungsregeln im Risikofall}

Die relevante Risikogemeinschaft, auf die sich eine entstandene Zusatzlast verteilen läßt, kann sich bei einem intergenerativen Ausgleich immer nur aus gleichzeitig lebenden Generationen zusammensetzen. Dadurch, daß regelmäßig alte Generationen versterben und neue Kohorten am anderen Ende nachrücken, ergibt sich die für die intergenerative Risikogemeinschaft charakteristische Überlappungsstruktur, die in einem OLG-Modell besonders deutlich zu erkennen ist.

Änderungen der Rahmenbedingungen treffen die verschiedenen Generationen der Risikogemeinschaft je nach Alterssicherungssystem auf unterschiedliche Weise. ${ }^{402}$ In einem kapitalfundierten System erfolgt dies vornehmlich durch gesamtwirtschaftliche Anpassungsprozesse oder infolge eines sinkenden relativen Lebensstandards der Ruheständler. ${ }^{403}$ Letzteres ist beispielsweise dann gegeben, wenn die durchschnittliche Lohnentwicklung oder die Inflation höher oder die interne Verzinsung niedriger ist, als antizipiert wurde. Aber auch wenn eine Gesellschaft im Durchschnitt älter wird, sich also die demographische Struktur verändert, spiegelt sich dies in einer kapitalgedeckten Alterssicherung in sinkenden Realrenten wider. ${ }^{404}$

Sollen diese (willkürlichen) Verteilungsvorgänge auf andere Weise geregelt werden, so verhindert die überlappende Struktur der Risikogemeinschaft das Zustandekommen intergenerativer Versicherungsverträge: Die Vertragspartner (Repräsentanten der verschiedenen Generationen) können sich nicht zu einem Zeitpunkt treffen. Eine von den gesamtwirtschaftlichen Anpassungsprozessen abweichende Regelung des intergenerativen Risikoausgleichs kann folglich nur durch staatliche Intermediation zustande kommen. ${ }^{405} \mathrm{Hier}$ zu bedarf es der Vorgabe normativer Verteilungsregeln mit denen die Lasten beim Eintritt des Risikos auf die Generationen der Versichertengemeinschaft gerecht verteilt werden sollen.

In der Literatur werden verschiedene Vorschläge unterbreitet, nach welchen Regeln sich eine solche Lastverteilung zwischen den Generationen gestalten läßt. Drei dieser Vor-

\footnotetext{
402 Vgl. Abschnitt 2.4.3 und 4.4.3.2.

403 Vgl. Fußnoten 321.

404 Vgl. Fußnoten 321.

405 Vgl. z.B. Sudhoff (1996), S. 46; Wagner (1985), S. 155, der ein umlagefinanziertes Pflichtversicherungssystem für erforderlich hält.
}

183 
schläge bzw. Arbeiten zu den Verteilungsregeln zwischen (zeitgleich lebenden) Generationen werden nachfolgend exemplarisch beschrieben. ${ }^{406}$

\section{A) Petersen}

In einem umlagefinanzierten System ergeben sich die Verteilungsregel des Risikoausgleichs implizit aus denen des Budgetausgleichs der Rentenversicherungsanstalt. Solch mögliche Verteilungsregeln des Budgetdefizits werden beispielsweise bei PETERSEN beschrieben: ${ }^{407}$

- Für den Fall eines konstanten Bruttorentenniveaus würde dies bedeuten, daß die Zusatzlast allein von jenen Generationen getragen werden, die während der Wirkung der Variation zu den Beitragszahlern gehören.

- Im anderen Extremfall eines konstanten Beitragssatzes würden diejenigen Generation zur Finanzierung des jeweiligen Budgetdefizits herangezogen, die sich in der Ruhestandsphase befinden.

- Ein Alterssicherungssystem mit konstantem Nettorentenniveau nimmt eine Zwischenstellung zwischen den beiden ersten Optionen ein.

Da eine Versicherung anstrebt, die Risiken auf möglichst viele (gleichbetroffene) zu verteilen, ist es naheliegend, als Verteilungsregel eine Zwischenform zu wählen. Dann bleibt aber immer noch zu klären, welche der (theoretisch) unendlich vielen Zwischenformen gewählt werden soll. Neben einem konstanten Nettorentenniveau stellen die folgenden Vorgaben für eine Aufteilungen des Budgetsdefizits weitere Zwischenformen dar: ${ }^{408}$

- Die Zusatzlast wird zu absolut gleichen Teilen von der Gesamtheit der Beitragszahler und der Gesamtheit der Ruheständler getragen.

- Beitragszahler und Ruheständler sollen die absolut gleiche Last pro Kopf tragen.

- Beitragszahler und Ruheständler sollen die relativ (zum Nettoeinkommen bzw. zur Rente) gleiche Last pro Kopf tragen.

B) BERTHOLD und ROPPEL

Der Vorschlag von BERTHOLD und ROPPEL zielt ebenfalls auf eine solche Zwischenform beim Budgetausgleich $a b .{ }^{409}$ Sie fragen danach, wie die durch einen Anstieg des Rentnerquotienten ausgelöste Zusatzlast gleich auf die Gesellschaftsgruppen (erwerbstätige Versicherte, Arbeitgeber und Ruheständler) verteilt werden kann, damit das Ziel des Rentensystems, den Lebensstandard aufrecht zu erhalten, weiterhin erfüllt bleibt. Dies erfordert ein Nettorentenniveau von Eins, wobei in der Nettoberechnung neben den Steu-

\footnotetext{
406 Vgl. Petersen (1988), Berthold/Roppel (1984), Dinkel (1986).

407 Vgl. Petersen (1988), S. 335ff..

408 Vgl. Petersen (1988), S. 336.

409 Vgl. Berthold/Roppel (1984).
} 
ern nur die Hälfte der Beiträge (aufgrund der paritätischen Finanzierung durch Arbeitgeber und Arbeitnehmer) berücksichtigt wird. Eine Gleichverteilung der Zusatzlast erfordert ihres Erachtens, daß der gesamte Beitragssatz zur Rentenversicherung prozentual doppelt so stark ansteigen muß, wie das Bruttorentenniveau. ${ }^{410}$

C) Dinkel

Während BERTHOLD und ROPPEL inren Vorschlag vornehmlich auf die Gleichverteilung der Zusatzlast im Sinne einer Ergebnisgerechtigkeit gründen, plädiert DINKEL in seinem Aufsatz zur intergenerativen Gerechtigkeit für ein Gerechtigkeitskonzept, bei dem die Folgen demographischer Veränderungen von derjenigen Generation zu tragen seien, die die Parametervariation verursacht hat (im Sinne einer Leistungsgerechtigkeit).

Obwohl das Umlageverfahren mit konstanten Beitragssätzen sich in seiner Analyse als die resistenteste Rentenformel gegen intergenerative Lastverteilungen erwies, werden konstante Beitragssätze von DINKEL nicht favorisiert. Denn zum einen trägt die Rentnergeneration in dieser Variante sämtliche Lasten, d.h. auch die, die von ihr nicht verursacht worden sind (beispielsweise infolge allgemeiner wirtschaftlicher Entwicklungen). Zum anderen ist die zukünftig zu erwartende Rentenhöhe relativ unsicher und im Rentenalter kann es regelmäßig zu spät sein, um auf unvorhergesehene Veränderungen zu reagieren. Auch eine strikte Auslegung des Grundsatzes, daß keine Generation von der anderen beeinflußt werden sollte, kann nur schwer mit der Idee des Umlageverfahrens in Einklang gebracht werden. Eine Umstellung vom Brutto- auf das Nettoprinzip hält DINKEL hingegen als nicht ausreichend. Damit die die Veränderungen verursachende Generation zumindest den relativ größten Anteil der Last trägt, schlägt DINKEL vor, zusätzlich zum (in der 1992er Reform bereits umgesetzten) Übergang vom Brutto- zum Nettoprinzip die Veränderung des Beitragssatzes für einen bestimmten Zeitraum zu beschränken (beispielsweise nicht mehr als fünf Prozent in fünf Jahren).

BREYER/SPREMANN sowie SUDHOFF und auch SPECKBACHER kritisieren an den oben zitierten Arbeiten zur intergenerativen Gerechtigkeit, daß sie nur eine Periode betrachten und nicht den gesamten Lebenszyklus, "so daß ein Verteilungskonklikt zwischen ,den Rentnern' und ,den Enwerbstätigen' entsteht. ${ }^{\text {A11 }}$ Die Ad-hoc Regeln seien zudem auf keine ethische Norm zurückzuführen, so daß es diesen "Gerechtigkeitskonzepten“ an ethischer Fundierung und Überzeugungskraft mangele, und nicht zuletzt fehle es auch an einer eindeutigen Definition dessen, was als „zusätzliche (demographische) Last“ zu verstehen sei. ${ }^{412}$

$410 \mathrm{Vgl}$. Berthold/Roppel (1984), S. 231.

411 Breyer/Spremann (1990), S. 390.

412 Vgl. Breyer/Spremann (1990), S. 390ff.. So kritisieren Breyer und Spremann Dinkels Ansatz als: "eine Idee, die wiederum kaum aus herkömmlichen Gerechtigkeitsgrundsätzen ableitbar ist. "Breyer/Spremann (1990), S. 392. Vgl. auch Sudhoff (1996), S.62-67, exemplarisch S. 67: „A/s Beitrag zur Verwirklichung intergenrationeller Gerechtigkeit kann die Arbeit von Bertold und Roppel (1984) jedoch nicht überzeugen. [...] und zum anderen bleibt unklar, worin der normative Gehalt des politisch determinierten Verteilungsziels liegt, von dem sie ausgehen. “. Ähnlich auch Speckbacher (1994), S. 63-65, der diese Unter- 
Diese Kritik wäre - selbst wenn sich allgemeine und ethische fundierte Gerechtigkeitskonzepte formulieren ließen - allerdings nur zum Teil gerechtfertigt, nämlich dann, wenn diese Regeln allein die intergenerative Gerechtigkeit beschreiben sollten.

Wenn verschiedene Alterssicherungssysteme unterschiedliche intergenerative Umverteilungsmuster aufweisen, stellt sich die normative Frage, welches System nach dem Kriterium der intergenerativen Gerechtigkeit vorzuziehen ist. Zur Lösung dieser Problemstellung wären die obigen Ansätze in der Tat wenig hilfreich. Hierfür bedürfte es eines „allgemeinen und ethisch fundierten Gerechtigkeitskonzeptes“ - sofern ein solches sich formulieren und auf die Fragestellung übertragen läßt.

Eine davon zu trennende Frage ist, wie die intergenerative Umverteilung definiert wird. Dazu gehört beispielsweise auch, in welchem Ausmaß und in welcher Form ein intergenerativer Risikoausgleich erwünscht ist. In diese Kategorie von Fragen, wie die Zusatzlast bei Eintritt des intergenerativen Risikos auf die Erwerbstätigen und Rentner verteilt werden soll, können auch die obigen Ansätze angesiedelt werden. Wenngleich dies von DINKEL nicht explizit formuliert wurde, könnte man seinen Ansatz z.B. wie folgt interpretieren: Das Verursachungsprinzip ist der maßgebliche Gerechtigkeitsgrundsatz für die Bewertung der intergenerativen Gerechtigkeit. ${ }^{413}$ Die von inm vorgeschlagene Abschwächung dieses Prinzips soll hingegen die Frage nach dem "wie" des intergenerativen Risikoausgleichs abdecken.

Eine normative Begründung der in diesem Abschnitt zu untersuchenden Verteilungsregeln bei Eintritt des intergenerativen Versicherungsrisikos können weder die Ansätze von NOZICK und RAWLS noch die des Utilitarismus liefern. SPECKBACHER und BREYER erwähnen selbst, ${ }^{414}$ daß ein Gerechtigkeitskriterium sich nicht unabhängig von Präferenzen formulieren läßt. Sie umgehen jedoch das Problem des intergenerativen Risikoausgleichs, indem sie unter der Annahme eines vollkommenen Kapitalmarkts sowie der Abwesenheit von Unsicherheiten implizit die Präferenzen auf eine Maximierung der Lebenseinkommen beschränken. ${ }^{415}$

Die Notwendigkeit, zwischen der Bewertung des intergenerativen Umverteilungsmusters und der Definition der intergenerativen Umverteilung sowie der Aufteilung der risikobedingten Zusatzlast zu unterscheiden, ergibt sich aus dem Versicherungsgedanken und läßt sich durch eine Analogie mit dem interpersonellen Risikoausgleich verdeutlichen:

Bei einem auf risikoäquivalenten Prämien beruhenden interpersonellen Risikoausgleich stellt sich das Problem, nach welchen Kriterien und in welcher Detaillierung die Risikogruppen abgegrenzt werden sollen. Auch diesbezüglich läßt sich die Ant-

suchungen weniger als gerechtigkeitstheoretisch fundierte Überlegungen, sondern vielmehr als (praktische) Plausibilitătsüberlegungen auffaßt. Vgl. Speckbacher (1994), S. 63.

413 Inwiefern Nozicks „Regelgerechtigkeit“ ethisch fundierter sein sollte ist im übrigen nicht ersichtlich!

414 Speckbacher (1994), S. 66f., Breyer (1990), S. $19 f .$.

415 Sudhoff (1996) umgeht dieses Problem ebenfalls, indem sie den „Generationen" in ihrer Analyse nur drei zuvor definierte Optionen von Alterssicherungssystemen zur Auswahl stellt (Kapitalstockverfahren, Umlageverfahren mit festen Beitragssatz b oder mit festen Rentenniveau $\gamma$, wobei zudem $\gamma=\mathrm{b}$ unterstellt wird). 
wort nicht (!) aus einem allgemeinen und ethisch fundierten Gerechtigkeitskonzept ableiten. Vielmehr spielen versicherungstypische Aspekte wie Moral Hazard und Adverse Selektion aber auch Aspekte wie Praktikabilität und Kosten-Nutzen Abschätzungen eine Rolle. Eine normative Begründung der Kriterien und der Detaillierung kann sich dabei lediglich auf gesellschaftlich gebildete Gerechtigkeitsvorstellungen gründen.

In gleicher Weise bleibt auch im umlagefinanzierten, teilhabeäquivalenten System die Verteilung der durch den Risikofall auftretenden Zusatzlasten (durch die Rentenformel) immer ein offenes Forschungsfeld. Denn der intergenerative Risikoausgleich muß nicht zwangsläufig zwischen Generationen erfolgen die einerseits von Risiko betroffen andererseits verschont geblieben sind. Die Frage nach der richtigen, also "gerechten“ Aufteilung einer ökonomisch und/oder demographisch bedingten Zusatzlast in der Alterssicherung kann sich ebenfalls nur auf gesellschaftlich gebildete Gerechtigkeitsvorstellungen und Aspekte wie „Praktikabilität“ gründen. ${ }^{416}$

Entsprechend der zweiten Gerechtigkeitsthese, ${ }^{417}$ wonach die Gerechtigkeitsvorstellung gesellschaftlich definiert bzw. historisch gewachsen ist, orientieren sich die Verteilungsregeln für die im Risikofall auftretende Zusatzlast in dieser Arbeit an der in der gesetzlichen Rentenversicherung verwirklichten Nettoanpassung. Dies entspricht einem Grundsatz der relativen Ergebnisgerechtigkeit.

Diese Vorgehensweise präjudiziert nicht das Ergebnis der Analyse zugunsten des derzeitigen Systems, denn die Frage, welche Lasten in welchem Ausmaß auf die Risikogemeinschaft verteilt werden sollen, wird hiervon nicht betroffen.

\subsection{Bewertung des Verteilungsmusters}

Bislang wurde beschrieben, wie der Verteilungsindikator für die zu bewertende Option und für das Referenzsystem ermittelt wird, um dem Ziel der intergenerativen Risikoabsicherung Rechnung zu tragen. Die Differenz dieser Indikatoren zeigt an, ob eine Generation durch ein bestimmtes Alterssicherungssystem bzw. durch eine Reformoption benachteiligt oder begünstigt wird.

416 "Die Vorstellungen zur intergenerationalen Leistungsgerechtigkeit sind bislang nur wenig präzisiert. Insbesondere ist nicht geklärt, ob und in welcher Weise Schwankungen in der wirtschaftlichen Entwicklung, welche als die ,Rendite' der Beitragszahlungen verändern, auf Rentner oder Erwerbstätige umgelegt werden sollen. Weiterhin ist nicht geklärt, inwieweit durch veränderte Kinderhäufigkeiten verursachte Belastungen des Rentenversicherungssystems auf Erwerbstätige oder Rentner umgelegt werden sollen. D.h., daß letztlich nicht klar ist, inwieweit die Forderung nach Stetigkeit des Einkommens im Alter und dessen Bedarfsgerechtigkeit mit dem Ziel der intergenerationalen Leistungsgerechtigkeit zu vereinbaren sind. "Wagner (1984), S. 46f.. Vgl. zu diesen Zusammenhängen auch S. 72 und S. 31, wo Wagner hinsichtlich der Diskrepanz zwischen der intergenerationalen Leistungsgerechtigkeit und der Forderung nach Einkommensstetigkeit fordert, zunächst zu prüfen sei, ob diese nicht einen ökonomisch sinnvollen Risikoausgleich darstelle.

417 Vgl. Abschnitt 3.4. 


$$
P O S_{x}=V I_{x}^{o p t}-V I_{x}^{r e f} \quad \text { G } 69
$$

$$
\begin{aligned}
& P O S_{x}=\text { Umverteilungsposition der Genration } x \\
& V I_{x}^{\text {opt }}=\text { Verteilungsindikator der Genration } x \text { bei der betrachteten Reformoption } \\
& V I_{x}^{\text {ref }}=\text { Verteilungsindikator der Genration } x \text { beim Referezsystem }
\end{aligned}
$$

Eine negative (positive) Position signalisiert, daß eine Generation im Vergleich zum Referenzsystem benachteiligt (begünstigt) wird. Wenn diese Positionen für alle betrachteten Generationen berechnet werden, beschreibt ihr Verlauf das Umverteilungsmuster jeder Reformoption. Bevor beurteilt werden kann, ob eine bestimmte Reform gerechter ist als eine andere, müssen diese Verteilungsmuster bewertet, d.h. in eine ordinale Reihenfolge gebracht werden. Die Bewertung der Verläufe kann anhand verschiedener Kriterien erfolgen, beispielsweise durch

- Konzentrationsmaße (bzw. Disparitätsmaße),

- Streuungsmaße,

- das Pareto-Kriterium,

- Lagemaße oder

- das Differenzenkriterium nach Rawls.

Intuitiv wäre es plausibel, daß ein vollkommen gleicher Verlauf der Positionen ein intergenerativ gerechtes Verteilungsmuster beschreibt und mit zunehmenden Grad an Ungleichheit ein Verteilungsmuster zunehmend ungerechter wird. Entsprechend dieser Vorstellung scheinen Streuungs- und Konzentrationsmaße besonders geeignete Kriterien zu sein Konzentrations- bzw. Disparitätsmaße (z.B. Variationskoeffizient, Gini-Koeffizient) werden vornehmlich als Methode zur Messung der Einkommensverteilung angewandt. ${ }^{418}$ Die verschiedenen Maße sind allerdings nicht äquivalent und unterscheiden sich vor allem hinsichtlich ihrer Sensitivität. ${ }^{419}$ Für jede Analyse muß daher zunächst das adäquate Maß ausgewählt werden. Zwei weitere Einwände sprechen ebenfalls dagegen, die intergenerative Gerechtigkeit mit Hilfe von Disparitätsmaßen zu bewerten: Zum einen steht bei der Einkommensverteilung die Frage im Vordergrund, wie sich eine vorgegebene Gesamtheit auf die verschiedenen Untersuchungseinheiten verteilt. Die Konzentrationsmaße sind daher skaleninvariant, d.h. sie bleiben gleich, wenn die Verteilungsfunktion mit einem Parameter multipliziert wird. Für die Bewertung der intergenerativen Umverteilung ist diese Eigenschaft weniger geeignet. Beispielsweise würden die Verteilungsfunktionen $A$ und $B$ des 2. Falls in Tabelle 6 als "gleich gerecht“ eingestuft werden, obwohl die Verteilung B sicherlich vorzuziehen wäre. Zum anderen sind die Positionen einiger Generationen ne-

418 Ginikoeffizient, logarithmische Varianz, Variationskoeffizient, Atkinsonsches Maß, Theilsches Maß etc. Die Terminologische Abgrenzung zwischen "Konzentrationsmaßen“ und "Disparitătsmaßen“ bzw. "Variationsmaßen“ ist nicht immer einheitlich. Vgl. z.B. Pflug (1979); Schaich (1990); Piesch (1975).

419 Eine Beschreibung der verschiedenen Maße bzw. ihrer Sensitivität vgl. z.B. Schmid (1991); Pflug (1979); Schaich (1990); Piesch (1975); Atkinson (1970); Champernowne (1974).

188 
gativ (Benachteiligung gegenüber das Referenzsystem) und liegen nicht mehr im Definitionsbereich der Konzentrationsmaße.

Streuungsmaße, wie beispielsweise die Varianz oder die Standardabweichung, haben die Eigenschaft, invariant gegenüber einer Translation der Verteilungsfunktion zu sein. Die Verteilungsmuster A und B aus Fall 1 in Tabelle 6 würden durch diese Kennziffer als "gleich gerecht" gewertet, obwohl die Reform mit dem Verteilungsmuster B alle Generationen besserstellen würde. Die Bewertung anhand von Streuungsmaßen führt beispielsweise bei Verläufen wie in Fall 5 Tabelle 6 (gegebenenfalls auch Fall 4) zu plausiblen Ergebnissen, wenn das Pareto-Kriterium einerseits nicht anwendbar ist und andererseits die Ergebnisse der Konzentrationsmaße sowie des Mittelwerts nicht befriedigen.

Die Kritik an den Streuungs- und Konzentrationsmaßen legt es nahe, das ParetoKriterium zur Bewertung der Verteilungsmuster heranzuziehen, und in der Tat liefert dieses Kriterium in den Fällen 1 bis 3 (vgl. Tabelle 6) plausible Ergebnisse. Das ParetoKriterium versagt aber in den Fällen, in denen sich die Funktionen schneiden. ${ }^{420}$ Gerade solche Verteilungsmuster sind allerdings bei einer partiellen Substitution des Finanzierungsverfahrens zu erwarten, wenn der Zinssatz zwar höher ist als die Wachstumsrate der Lohnsumme, die Doppelbelastung der Übergangsphase die zusätzlichen Zinseinkünfte jedoch übersteigt. Im zweiten Kapitel wurde gezeigt, daß eine Substitution des Umlage- durch das Kapitalstockverfahren nicht Pareto-optimal gestaltet werden kann. Zumindest theoretisch wäre es allerdings möglich, daß gerade diejenigen Generationen durch die Umstellung relativ zum Status quo benachteiligt werden, die im Vergleich zum Referenzsystem begünstigt werden. Eine solche Senkung einer starken Begünstigung einiger Generationen bei Minderung der Ungleichverteilung (Verlauf B in Fall 7, Tabelle 6) könnte entsprechend der folgenden Vorstellung von Gerechtigkeit als gerecht betrachtet werden: Wenn alle Generationen gegenüber dem Referenzsystem in beiden Systemen qualitativ gleich behandelt werden, gilt jenes Alterssicherungssystem als intergenerativ gerechter, bei dem die Ungleichheit der Verteilung gemindert wird (Streuungsmaß als Kriterium).

Dabei wurde das Pareto-Kriterium auf das Status quo bezogen. Die Verteilungsmuster ergeben sich aus einem Vergleich mit einem Umverteilungsneutralen Referenzsystem, so daß bei dem Verteilungsmuster die Pareto-Bedingung theoretisch dennoch erfült sein kann.

Ähnlich wie das Pareto-Kriterium würde auch ein Lagemaß, wie beispielsweise der Mittelwert, in den Fällen 1 bis 3 (vgl. Tabelle 6) plausible Ergebnisse liefern. Für Verläufe von Typ 4 in Tabelle 6 würde der Mittelwert die Verteilung B favorisieren. Diesem Ergebnis läge die Vorstellung zugrunde, daß wenigen Generationen ein geringer Verlust zuzumu-

420 Der Übersicht halber werden hier stetige Verteilungsfunktionen beschrieben, obwohl der Verlauf der Umverteilungspositionen der verschiedenen Generationen diskret ist. 
ten sei, wenn viele Generationen dadurch einen großen Gewinn haben. ${ }^{421}$ Eine solche Auffassung mag in einigen Fällen wirtschafts- und sozialpolitisch erforderlich sein, dies ist dann aber eine rein politische Entscheidung, bei der offen dargelegt werden sollte, daß „ein gewisses Maß an Ungerechtigkeit in Kauf genommen wird“. Ethisch läßt sich dieses Vorgehen nicht begründen. Der Mittelwert kann mithin lediglich als Zusatzinformation, nicht als allgemeines Kriterium für die Bewertung der intergenerativen Umverteilung dienen.

Die verschiedenen Streuungs-, Konzentrations- und Lagemaßen werden bestimmt, indem man die Positionen der Generationen zu einer Kenngröße aggregiert. Auf diese Weise gehen viele Informationen über den Verlauf des Verteliungsmusters verloren. So ist es denkbar, daß die gesamte Streuung des Umverteilungsmusters durch eine Reformmaßnahme minimiert wird, obwohl gleichzeitig extreme Ausreißer auftreten. (Fall 6 in Tabelle 6) Ein solches Verteilungsmuster, bei dem die meisten Generationen zwar annähernd gleich behandelt, einige wenige jedoch extrem belastet werden, dürfte sozialpolitisch kaum als erwünscht bzw. gerecht angesehen werden. In einem solchen Fall kann auf das Differenzenkriterium nach Rawls zurückgegriffen werden (vgl. Abschnitt 3.3.2) ${ }^{422}$ Von zwei intergenerativen Verteillungsmustern ist dann dasjenige vorzuziehen, bei dem das Ausmaß der Ungleichbehandlung der am stärksten belasteten Generation minimiert wird. ${ }^{423}$ Bei Verläufen der Umverteilung, wie sie beispielsweise in den Fällen 2 und 3 in Tabelle 6 dargestellt sind, ist das Differenzenkriterium allerdings wenig hilfreich. In diesen Fällen müßte daß Differenzenkriterium mit dem Pareto-kriterium kombiniert werden.

421 Vgl. Raffelhüschen (1989), S. 238; Neumann (1996). Jäger (1991), S. 275 betrachtet eine solche Verfahrensweise zwar nicht als optimalzustand, spricht aber dennoch von einer Verbesserung der intergenerativen Gerechtigkeit.

422 Vgl. Rawls (1975), S. 31f.. Auf diesem Kriterium gründen beispielsweise Schulenburg (1989) und Sudhoff (1996) ihre Analysen der intergenerativen Gerechtigkeit eines Alterssicherungssystems.

423 Rawls betont zwar, daß sich dieses Differenzenkriterium nicht auf die intergenerative Perspektive übertragen läßt (vgl. Rawls (1975), S. 322), dieses Problem stellt sich jedoch nur bei einem Optimierungsproblem. Bei der Beurteilung der Folgen vorgegebener Reformoptionen, ist dieser Einwand nicht relevant. Vgl. Sudhoff (1996), S. 111-114. 
Tabelle 6: Verläufe der intergenerativen Umverteilung und ihre Bewertung durch unterschiedliche Kriterien

( $A$ > B ist zu lesen: „A wird gegenüber $B$ als gerechter eingestuft“ - fett gedruckte Bewertungen beschreiben intuitive plausible Gerechtigkeitsvorstellungen)

\begin{tabular}{|c|c|c|c|c|c|c|}
\hline Fall & Verteilungsfunktionen *) & $\begin{array}{c}\text { Streuungs- } \\
\mathrm{ma} \beta\end{array}$ & $\begin{array}{l}\text { Variations- } \\
\text { koeffizient }\end{array}$ & Mittelwert & Pareto & Rawls \\
\hline 1 & & $A=B$ & $B>A$ & B $>$ A & B $>$ A & B $>$ A \\
\hline 2 & & $A>B$ & $A=B$ & $B>A$ & B $>$ A & $A=B$ \\
\hline 3 & & $A>B$ & $A>B$ & B $>$ A & B $>$ A & $A=B$ \\
\hline 4 & & $A>B$ & $A>B$ & $B>A$ & $\begin{array}{c}\text { keine } \\
\text { Aussage }\end{array}$ & $A>B$ \\
\hline 5 & & $A>B$ & $A=B$ & $A=B$ & $\begin{array}{c}\text { keine } \\
\text { Aussage }\end{array}$ & $A>B$ \\
\hline 6 & A & $B>A$ & $B>A$ & $B>A$ & $\begin{array}{l}\text { keine } \\
\text { Aussage }\end{array}$ & $A>B$ \\
\hline 7 & & $B>A$ & B $>$ A & $A><B$ & $\begin{array}{l}\text { keine } \\
\text { Aussage }\end{array}$ & B $>$ A \\
\hline
\end{tabular}

•) Aus Gründen der Übersicht werden stetige Verläufe dargestellt.

Quelle: eigene Darstellung

Die meisten Informationen enthält die Darstellung des Verlaufs der Umverteilung. In d folgenden Analyse werden die graphischen Verteilungsmuster daher als „Hauptkriteriur herangezogen. Ergänzend werden die verschiedenen, hier diskutierten Kriterien als Z satzinformation ausgewiesen. Dieses „Menü an Bewertungskriterien“ soll der Sozialpolit 
als Entscheidungshilfe dienen, wenn Reformen der Rentenversicherung nach ihrer Auswirkung auf die intergenerative Gerechtigkeit bewertet werden.

\subsection{Zusammenfassung und Fazit}

Im Abschnitt 5.1 wurden verschiedene Ansätze skizziert, mit denen sich die intergenerativen Verteilungswirkungen eines Alterssicherungssystems quantitativ erfassen lassen. Die Kritik dieser Ansätze zeigte, daß sich generationenspezifische Rentabilitätskennziffern für die vorliegende Fragestellung besser eignen, als das Generational Accounting oder die Verwendung von Nutzenfunktionen. Von den verschiedenen Rentabilitätskennziffern wurde die auf der Barwertmethode beruhende Rendite ausgewählt. Allerdings nicht, weil der interne Zinsfuß methodisch unterlegen wäre, sondern weil sich die Barwertmethoden einfacher modifizieren lassen.

Die Möglichkeit einer Modifikation ist notwendig, da die Rentabilitätsindikatoren sich zum einen an dem Prinzip der reinen Beitragsäquivalenz bzw. der Leistungsgerechtigkeit orientieren, obwohl weitere Gestaltungsprinzipien der Alterssicherung (intergenerative Risikoausgleich) bei der Beurteilung der Gerechtigkeit berücksichtigt werden müssen. Zum anderen vernachlässigt der formulierte Verteilungsindikator die gesamtwirtschaftlichen Wirkungen der Finanzierungsverfahren und muß in Kapitel 6 ggf. angepaßt werden, um auch diesen Aspekt der Gerechtigkeitsanalyse zu berücksichtigen.

Der formulierte Verteilungsindikator erfaßt sowohl die intergenerativen Verteilungswirkungen, die durch eine Substitution des Finanzierungsverfahrens ausgelöst werden als auch jene, die bei Variationen ökonomischer und demographischer Parameter innerhalb des Rentensystems auftreten. Insbesondere bezieht der Indikator die Leistungen einer Generation auf die von dieser Generation gezahlten Beiträge und erfült die Anforderung, die aus dem Ziel abgeleitet wurde, die gesetzliche Alterssicherung als Vorsorgesystem zu gestalten (vgl. Abschnitt 3.4).

Die Verteilungswirkungen eines Alterssicherungssystems müssen mit einem Referenzmaßstab vergleichen werden, um sie zu beurteilen. Meist dient ein beitragsäquivalentes Kapitalstockverfahren als Referenz. Abschnitt 5.2 zeigte allerdings, daß diese Vorgehensweise normativ nicht begründet werden kann, da sie die Präferenz der Versicherten nach Sicherheit eines relativen Lebensstandards im Alter vernachlässigt. Mit anderen Worten ausgedrückt: Ein beitragsäquivalentes Kapitalstockverfahren als Referenz unterscheidet nicht zwischen „systematischer, intergenerativer Umverteilung“ auf der einen und „intergenerativen Risikoausgleich“ auf der anderen Seite. Der Versuch, die Vorgehensweise mit Hilfe der Anspruchstheorie von NozıcK zu begründen, kann ebenso wenig überzeugen, wie der alternative Ansatz der „intergenerationalen Durchhaltbarkeit“.

Für die Analyse der intergenerativen Gerechtigkeit müssen daher sowohl das Referenzsystem als auch der Verteilungsindikator so angepaßt werden, daß dem intergenerativen 
Risikoausgleich Rechnung getragen wird. Dies wurde in Abschnitt 5.3 versucht, indem drei Fragen analysiert wurden:

1. Was gehört zu den intergenerativ versicherbaren Risiken? (Abschnitt 5.3.1)

2. Wieviel an intergenerativen Risikoausgleich ist erwünscht? (Abschnitt 5.3.2)

3. Wie kann die Zusatzlast bei Eintritt des Risikos auf die Versichertengemeinschaft (auf die unterschiedlichen Generationen) verteilt werden? (Abschnitt 5.3.3)

Ad 1)

In Kapitel 2 wurden bereits die Einflußfaktoren auf die Rendite eines Alterssicherungssystems identifiziert und ihre tendenziellen, intergenerativen Verteilungswirkungen analysiert. Offen blieb, welche Wirkungen der Umverteilung und welche dem Risikoausgleich zuzuordnen sind. In Abschnitt 5.3.1.1 wurden daher vier Kriterien für eine intergenerative Umverteilung aufgestellt und auf die verschiedenen demographischen und ökonomischen Einflußfaktoren angewandt:

Die durch eine Variation der Mortalität verursachten Verteilungswirkungen erwiesen sich als systematische Umverteilung, sofern die Veränderung der Sterblichkeit während der Ruhestandsphase auftritt. Eine Veränderung der Sterblichkeit während der Erwerbsphase könnte, mit Hinweis auf die Zusatzkriterien 4.1 und 4.2, als solidarisches Risiko interpretiert werden, da die Internalisierung der Verteilungseffekte zu einer recht komplizierten Rentenformel führen würde. Diese Auffassung wird in der vorliegenden Arbeit jedoch nicht gefolgt, was auch deshalb vertretbar ist, weil die Sterblichkeitsänderung während der Erwerbsphase in der Bundesrepublik Deutschland relativ gering und vernachlässigbar ist.

Die Veränderung der Fertilität erwies sich als gesamtgesellschaftliches Phänomen, und die von ihr induzierten Verteilungswirkungen wurden dem intergenerativen Risikoausgleich zugeordnet. Der Verteilungsindikator wurde so modifiziert, daß er die durch Fertilitätsänderungen hervorgerufenen Verteilungswirkungen nicht ausweist. Da ein Kapitalstockverfahren die Abhängigkeit eines Alterssicherungssystems von der Fertilität zwar nicht aufheben, ggf. aber mindern kann, könnte bestenfalls ein nicht zu quantifizierender Anteil der fertilitätsbedingten Verteilungswirkungen als intergenerative Umverteilung gewertet werden. Auch dies läßt sich einfach durch die Anpassung des Faktors $\Phi$ im modifizierten Verteilungsindikator berücksichtigen.

Bei der Variation der versicherungspflichtigen Erwerbstätigkeit wurde zwischen zwei Kategorien von Ursachen differenziert: Veränderungen beim Arbeitsangebot und Veränderungen bei der Arbeitsnachfrage. Verteilungswirkungen, die auf eine Veränderung des Arbeitsangebots zurückzuführen sind, wurden als intergenerative Umverteilung bewertet. Bei den Veränderungen der Nachfrage an versicherungspflichtiger Erwerbsarbeit wurde weiter unterschieden, ob es sich um eine Verschiebung der Beschäftigungsstruktur hin zu nichtversicherungspflichtigen Arbeitsverhältnissen oder um eine Zunahme der Arbeitslosigkeit handelt. Die Folgen einer veränderten Beschäftigungsstruktur ließen sich durch entsprechende Gesetzesänderungen vermeiden, so daß die hierdurch entstehenden Verteilungswirkungen ebenfalls intergenerative Umverteilungen darstellen. Die Zunahme 
der Arbeitslosigkeit ist in der gesetzliche Rentenversicherung mit mehreren Verteilungswirkungen verbunden, die vom Status der Arbeitslosen in der Rentenversicherung abhängen. Scheidet der Arbeitslose aus der Rentenversicherung aus, werden erwerbstätige Genrationen durch steigende Beiträge stärker, nachfolgende durch sinkende Beiträge geringer belastet. In der gesetzliche Rentenversicherung werden die Zeiten der Arbeitslosigkeit als Beitragszeiten angerechnet, so daß die Beiträge zur Rentenversicherung in einem geringeren Umfang steigen. Diese direkten Beitragseffekte der Arbeitslosigkeit, sowie der Teil der Rückkopplung der Nettoanpassung, der auf diese Beitragseffekte zurückzuführen ist, ließen sich durch eine Auslagerung der Arbeitsmarktrisiken auf die Arbeitslosenversicherung bzw. auf das Steuer-Transfer-System vollständig übertragen. Sie werden daher als originäre intergenerative Umverteilung des Alterssicherungssystems erfaßt. Rückkopplungseffekte der Nettoanpassung, die sich aus der steigenden Steuerbelastung oder dem Anstieg der Beiträge zur Arbeitslosenversicherung ergeben, werden hingegen als intergenerativer Solidarausgleich gewertet.

Ob und gegebenenfalls in welchem Ausmaß eine Variation der Lohn- und Zinsentwicklung als Umverteilung oder als intergenerativ versicherbares Risiko zu werten ist, läßt sich nur aus dem Referenzsystem, sprich aus dem Umfang des präferierten Risikoausgleichs ableiten (Punkt 2)).

Ad 2)

Das Referenzsystem muß beiden ordnungspolitisch relevanten Zielen bzw. den mit innen verbundenen Gestaltungsprinzipien der gesetzliche Rentenversicherung (Vorsorgesystem und Lebensstandardsicherung vgl. Tabelle 5) Rechnung tragen. Die Berücksichtigung beider Ziele kann im Referenzsystem durch eine Kombination aus Umlageverfahren und Kapitalstockverfahren erreicht werden. Dabei stellt sich das Problem, wie die beiden Anteile gewichtet werden sollen.

Die Risikoaversion der Versicherten ist das maßgebliche Kriterium für den erwünschten Umfang an intergenerativen Risikoausgleich. Die individuellen Präferenzen lassen sich aber nur schwer ermitteln, sind heterogen und ihre Aggregation zu einer gesellschaftlichen Präferenz ist ein gleichermaßen methodisches wie ethisches Problem. Deshalb allerdings das Ziel "Sicherung des relativen Lebensstandards" vollkommen auszublenden und unter vollkommenen Marktbedingungen eine Gewichtung von Null zu unterstellen, erleichtert zwar die Analyse, aus den Ergebnisse können dann aber keine sozialpolitische Handlungsempfehlungen abgeleitet werden. Solange dies aber das ausdrückliche Ziel des Meßkonzepts ist, sollten die Prämissen über die Präferenzen transparent und veränderbar gehalten werden. Die Gewichtung des Status quo kann ebenso wenig als Referenz dienen, da gerade der Grad der Kapitalfundierung derzeit kontrovers diskutiert wird.

Die Analyse basiert daher auf konditionalen Aussagen, wie z.B. „wenn die Risikoaversion gegen Null geht, dann werden bestimmte Optionen als intergenerativ gerecht angesehen". Die Gewichtung und die normative Entscheidung sind dann Aufgaben der Politik. 
Um die Abgrenzung der intergenerativen Umverteilung vom Risikoausgleich (vorangegangener Punkt 1) im Meßkonzept zu erfassen, mußte das Referenzsystem bzw. der Verteilungsindikator des Referenzsystems entsprechend angepaßt werden.

Ad 3)

Bei der Beschreibung der intergenerativen Verteilungswirkungen in Abschnitt 4.2 wurde betont, daß die „intergenerative Gerechtigkeit" nicht anhand der Verteilung zwischen „den Rentnern" und "den Erwerbstätigen" in einer Periode bewertet werden kann. Eine andere Frage ist die nach der Aufteilung der risikobedingten Zusatzlast. Sie kann nur zwischen Rentnern und Erwerbstätigen einer Periode erfolgen (überlappende Risikogemeinschaft). Die Regeln, nach denen die Zusatzlast bei Eintritt des Risikos auf die unterschiedlichen Generationen der Versichertengemeinschaft verteilt werden, lassen sich nicht aus einem allgemeinen, ethisch fundierten Gerechtigkeitskonzept ableiten. Sie bilden sich im politischen Entscheidungsprozeß und lassen sich nur auf gesellschaftlich gebildete Gerechtigkeitsvorstellungen gründen. Die Verteilungsregeln für die im Risikofall auftretende Zusatzlast orientieren sich daher in dieser Arbeit an der in der gesetzlichen Rentenversicherung verwirklichten Nettoanpassung. Diese Vorgehensweise präjudiziert nicht das Ergebnis der Analyse zugunsten des derzeitigen Systems, denn die Frage, welche Lasten in welchem Ausmaß auf die Risikogemeinschaft verteilt werden sollen, wird hiervon nicht betroffen.

Die Umverteilungsposition jeder Generation erhält man, indem man den Verteilungsindikator der zu beurteilenden Reformoption von dem des Referenzsystems subrahiert. Auf diese Weise entsteht ein Umverteilungsmuster über alle betrachteten Generationen. In Abschnitt 5.4 wurde untersucht, wie die verschiedenen Umverteilungsmuster der Reformoptionen bewertet werden können. Dabei zeigte sich, daß keines der möglichen Kriterien (weder Konzentrations-, Streuungs- und Lagemaße noch das Pareto- oder das Differenzenkriterium nach Rawls) für sich allein genommen eine ausreichende Bewertung garantiert. In der folgenden Analyse werden daher die verschiedenen Verläufe graphisch dargestellt und die diskutierten Kriterien als Ergänzung ausgewiesen. 
Abbildung 14: Übersicht Kapitel fünf

\section{DIREKTE INTERGENERATIVE VERTEILUNGSWIRKUNGEN}
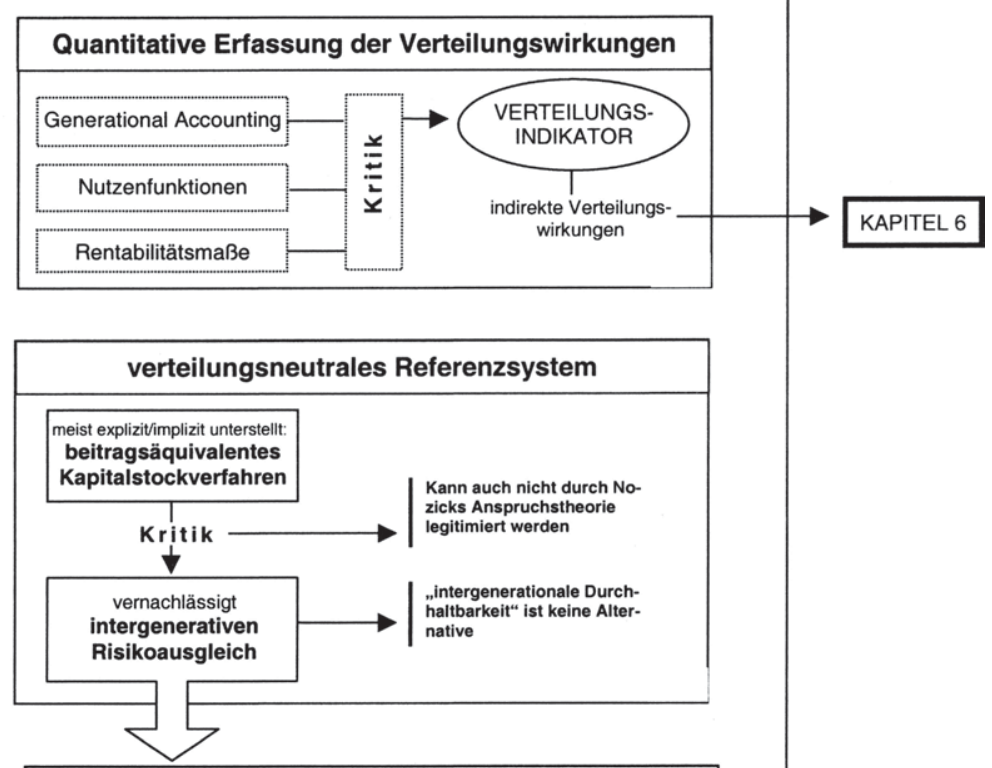

\section{Abgrenzung des intergenerativen Risikoausgleichs}
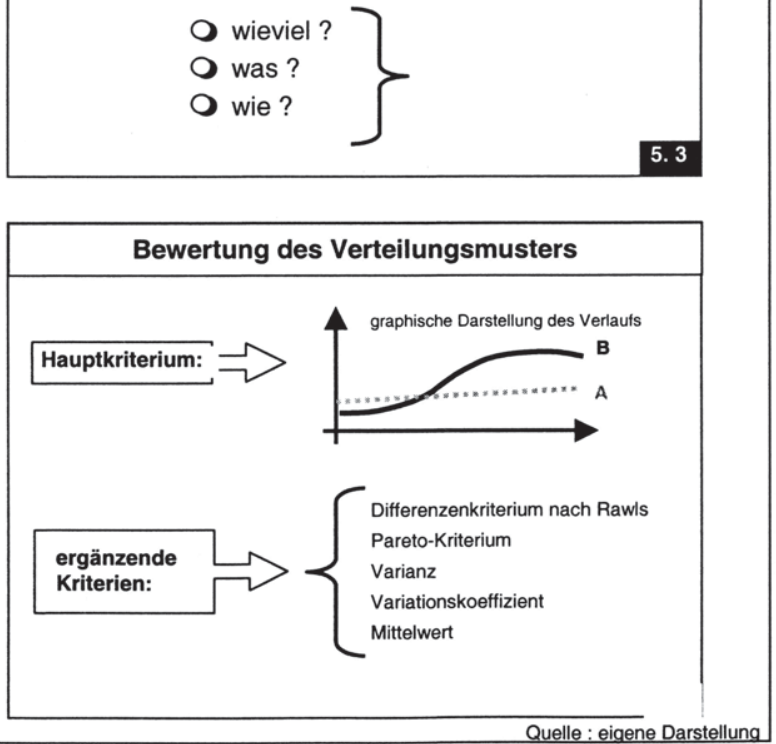
Abbildung 15: Abgrenzung des intergenerativen Risikoausgleichs

\section{WIEVIEL ?}
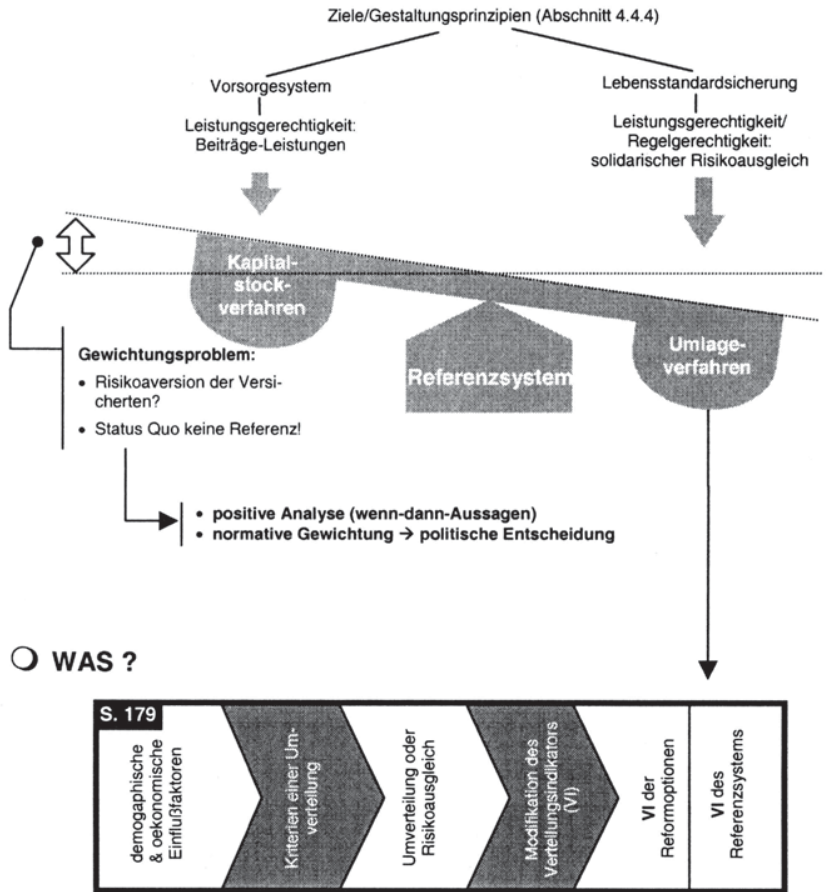

\section{WIE ?}

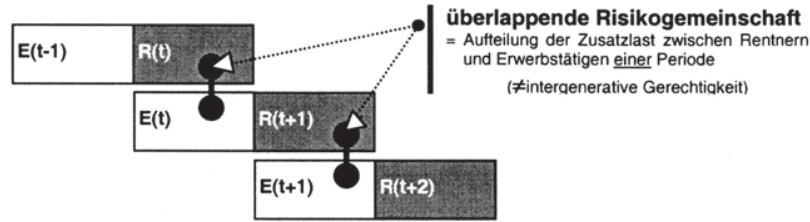

Aufteilungsregeln

- können nicht durch allgemeines Gerechtigkeitskonzept begründet werden

- werden vorwiegend durch gesellschaftliche Vorstellungen und Praktikabilitätserwägungen bestimmt 
Tabelle 7: Abgrenzung der intergenerativen Risiken und Einfluß auf den Verteilungsindikator und das Referenzsystem

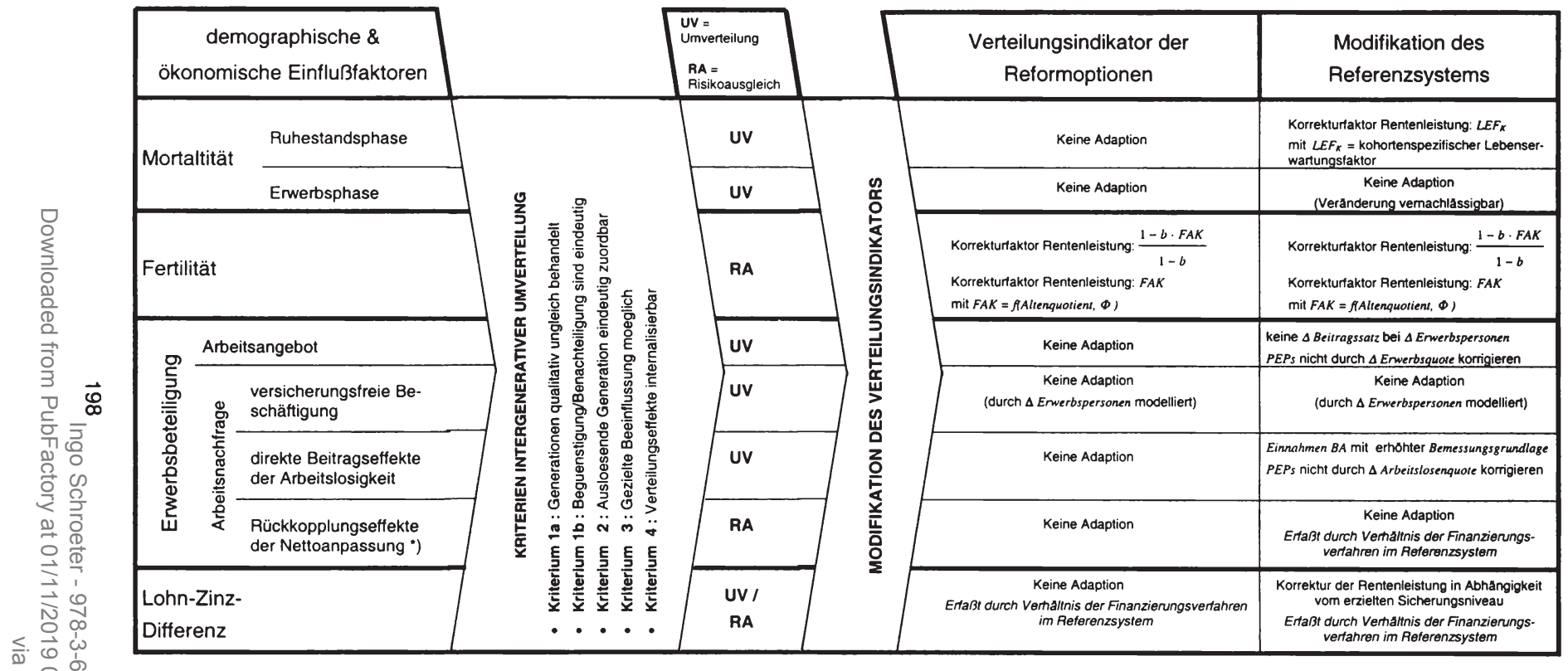

*) Gilt ebenfalls für die Rückkopplungseffekte der Nettoanpassung durch Beiträge zu den übrigen

Sozialversicherungszweigen sowie durch Veränderung der durchschnittlichen direkten Steuersätze 


\section{BERÜCKSICHTIGUNG DER INDIREKTEN INTERGENERATIVEN VERTEILUNGSWIRKUNGEN VERSCHIEDENER FINANZIE- RUNGSVERFAHREN}

Die modelltheoretische Analyse des zweiten Kapitels zeigte, daß die Finanzierungsverfahren zur Altersvorsorge auch einen Einfluß auf gesamtwirtschaftliche Parametern ausüben. In Abschnitt 6.1 wird daher zunächst geprüft, inwieweit die These vom Ersparnis- und Wachstumseffekt des Kapitalstockverfahrens valide bleibt, wenn von den dort unterstellten restriktiven Annahmen abstrahiert wird. Sofern von gesamtwirtschaftlichen Rückwirkungen ausgegangen wird, muß der formulierte Verteilungsindikator (vgl. Abschnitt 5.1.2) angepaßt werden, um auch die aus diese indirekten intergenerativen Verteilungseffekte zu berücksichtigen. Dieser Fragestellung widmet sich Abschnitt 6.2. Abschließend werden in Abschnitt 6.3 die mit einem Umlageverfahren verbundenen Arbeitsmarkteffekte diskutiert.

\subsection{Bedeutung der These vom Ersparnis- und Wachstumseffekt des Kapitalstockverfahrens}

Im Zentrum der Diskussion zur Frage „Umlageverfahren versus Kapitalstockverfahren“ stehen vor allem die gesamtwirtschaftlichen Rückwirkungen der Finanzierungsverfahren auf die private Ersparnisbildung und damit einhergehend auf den Wachstumspfad einer Volkswirtschaft. Ein Übergang zum Kapitalstockverfahren, so eine weit verbreitete Meinung, sei mit massiven Wachstumseffekten verbunden. Wie die folgenden Beispiele zeigen, prägt diese „Kapitalakkumulations- bzw. Wachstumsthese“ die rein modelltheoretischen Analysen in gleicher Weise wie die sozialpolitische Debatte:

- Der Wachstumseffekt ist der zentrale Kern aller Arbeiten zur Allokationseffizienz eines Finanzierungsverfahrens, die auf die Goldenen Regel der Kapitalakkumulation rekurrieren.

- Die distributiven Wirkungen innerhalb einer kapitalfundierten Alterssicherung beruhen größtenteils auf der steigenden gesamtwirtschaftlichen Ersparnisbildung, die einen sinkenden Zinssatz nach sich zieht.

- Einen besonderen Einfluß hat die unterstellte Kapitalakkumulation erwartungsgemäß auf sämtliche Simulationsrechnungen zur Umstellung des Finanzierungsverfahrens. ${ }^{424}$ Im Zusammenhang mit ihrer Simulation einer (kompletten) Systemsubstitution behaupten GLISMANN und HORN, daß es sich bei der viel zitierten Doppelbelastung im 
Übergang um bloße Vermutungen handele. ${ }^{425}$ Eine genaue Analyse ihres Vorschlages zeigt jedoch, daß auch sie keinen Königsweg für die Bewältigung des Übergangsproblems gefunden haben: Ein Teil der Belastungen wird auf die Bestandsrenten verschoben und die Anwartschaften der Übergangsgeneration werden, entgegen ihren Angaben, gekürzt. Ein Großteil der Lastminderung resultiert zudem aus (zweifelhaften) Wachstumsannahmen. ${ }^{426}$ Gerade bei Simulationsrechnungen besteht die Gefahr, die Ergebnisse durch die Stellgröße „Wachstumseffekte“ massiv zu beeinflussen.

- Die These, daß von einem kapitalfundierten System Wachstumsimpulse auf die gesamte Volkswirtschaft ausgehen, ist auch für die Untersuchung der intergenerativen Gerechtigkeit von zentraler Bedeutung. Da die Konsummöglichkeiten künftiger Generationen unmittelbar von der Wachstumsrate des Sozialprodukts und somit von dem Konsumverzicht vorangegangener Generationen abhängen, stellt sich für jede Generation die normative Frage nach dem intergenerativ gerechten Verhältnis aus Konsum und Sparen während der Erwerbsphase. Insbesondere die utilitaristischen Ansätze fokussieren ihre Analyse auf diese Frage nach dem optimalen Entwicklungspfad des Sparens. ${ }^{427}$ Wenngleich RAWLS die utilitaristische Methodologie vehement kritisiert, konzidiert er aber, daß die sich im gerechten Spargrundsatz ${ }^{428}$ manifestierende Frage nach der intergenerative Gerechtigkeit für jede ethische Theorie eine ernste, wenn nicht unertüllbare Bewährungsprobe darstellt. ${ }^{429}$ SPECKBACHER widmet seine modelltheoretische Arbeit (2-Generationenmodell) zur intergenerativen Gerechtigkeit von Alterssicherungssystemen ebenfalls dem Problem, gerechte Sparraten zu bestimmen. Auch er stützt sich auf eine Maximierung der gewichteten generativen Summe der Konsumanteile. Im Gegensatz zu den utilitaristischen Ansätzen wird die Gewichtung nicht explizit durch die Zielfunktion vorgegeben, sondern sie ergibt sich endogen nach Maßgabe des Beitrags der jeweiligen Generation zur gesamten Leistungsfähigkeit der betrachteten Ökonomie.

JAEGER vertritt jedoch die Meinung, daß die These, ein umlagefinanziertes System vermindere die Kapitalakkumulation einer Volkswirtschaft, theoretisch zumindest als umstritten angesehen werden muß..$^{430}$ In einem OLG-Modell stellt die Gleichung der Kapitalak-

Vgl. Jäger (1991); Raffelhüschen (1989) sowie die dort zitierte Literatur.

Vgl. Glismann/Hom (1996), S. 19.

Es wird angenommen, daß die Höhe der gesamtwirtschaftlichen Zusatzerspamis $40 \%$ der gesamten Rentenzahlungen entspricht, die sich bei einer Fortführung des derzeitigen Systems mit 2,5prozentiger Wachstumsrate ergeben würde und daß das BIP aufgrund dieser Zusatzersparnis jăhrlich mit einer Rate von $2,94 \%$ anstatt von $2,5 \%$ wachsen würde. Die umstellungsbedingten Wachstumsimpulse werden folglich mit 0,44 (l) Prozentpunkte per anno angesetzt.

Vgl. Abschnitt 3.3.1. Dabei werden die mit der gesellschaftlichen Zeitpräferenzrate gewichteten Generationennutzen in einer (unendlichen) Gesellschaftlichen Nutzensumme zunächst aggregiert und anschlieBend maximiert.

Rawls (1975), S. 320: „Jede Generation muß [...] stets auch eine angemessene Kapitalakkumulation betreiben."

Vgl. Rawls (1975), S. 319.

Vgl. Jaeger 1994, S. 358. 
kumulation eine Differenzengleichung erster Ordnung dar, für die es zwei Arten von lokaler Stabilität gibt. Die schwingungsfreie Stabilität, der das Walras'sche Gleichgewichtskonzept zugrunde liegt, und die Stabilität mit gedämpfter Schwingung die auf dem Gleichgewichtskonzept nach Marshall beruht. Für den Fall schwingungsfrei stabiler steady-stade Pfade bestätigt er das Standardergebnis der Literatur, daß ein Umlageverfahren verglichen mit einem Kapitalstockverfahren mit einer niedrigeren gesamtwirtschaftlichen $\mathrm{Er}$ sparnisbildung einhergeht. Für den Fall stabiler Steady-State Pfade mit gedämpfter Schwingung ist das Umlageverfahren mit einer höheren gesamtwirtschaftlichen Ersparnisbildung verbunden. Letzteres erfordert, daß der Substitutionseffekt geringer ist als der Einkommenseffekt und daß die private Ersparnis mit steigenden Zinssatz sinkt. Angesichts dieser notwendigen Bedingung für JAEGERS Ergebnis in einem zyklischen Gleichgewicht, kann zwar die Frage nach der empirischen Relevanz dieses Falles gestellt werden, ${ }^{431}$ seine Analyse verdeutlicht aber dennoch, wie sehr die "Ersparnisthese“ von dem unterstellten Modell und den getroffenen Annahmen beeinflußt wird.

Angesichts der oben skizzierten Bedeutung der „Wachstumsthese“ für weiterführende Fragestellungen insbesondere auch für die Frage der intergenerativen Gerechtigkeit, wird im folgenden daher zunächst die Validität dieser These geprüft, die aus einem einfachen Modell überlappender Generationen neoklassischer Provenienz abgeleitet wurde. Anschließend muß entschieden werden, ob und in welcher Weise etwaige Wachstumseffekte in dem Meßkonzept zur intergenerativen Gerechtigkeit berücksichtigt werden sollen.

\subsubsection{Kritik am Vermögenssubstitutionseffekt}

FELDSTEIN formulierte 1974 den sogenannten Vermögenssubstitutionseffekt, demzufolge die Versicherten ihre Beitragszahlungen an ein Alterssicherungssystem als erspartes Vermögen wahrnehmen, das ihre private Spartätigkeit ersetzt. Da die Beitragszahlungen in einem umlagefinanzierten Rentensystem nicht gespart werden, führt dieses Alterssicherungssystem zu einer Verringerung der gesamtwirtschaftlichen Ersparnis der privaten Haushalte. Feldsteins These von der ersparnismindernden Wirkung des Umlageverfahrens wurde von verschiedenen Seiten kritisiert, und er selbst revidierte seine ursprüngliche Schlußfolgerung einer vollständigen Substitution, da sich beide Vermögensformen (private Ersparnis und Rentenanwartschaften) hinsichtlich der Liquidität wesentlich unterscheiden. $^{432}$

Ein Ersparnisverlauf, wie er der Lebenszyklushypothese zugrunde liegt, impliziert ferner einen vollkommenen Kapitalmarkt. Diese Modellannahme ist in der Realität nicht anzu-

\footnotetext{
431 Allerdings weist auch Raffelhüschen darauf hin, daß die Wirkung der Zinsvariation unbestimmt ist, da neben den Substitutions- und Einkommenseffekten auch Humanvermögenseffekte berücksichtigt werden müssen. Raffelhüschen (1989), S. 79ff. Mit steigender Periodenzahl steigt die Anzahl der zu berücksichtigenden Effekte (bei 55 Perioden müßten bereits 12.319 verschiedene Effekte berücksichtigt werden), so daß „eine positiv vom Zinssatz abhängige Erspamis nicht zwingend“ ist. Raffelhüschen (1989), S. 84. Vgl. auch Boadway/ Wildasin (1984), S. 304.

Vgl. Feldstein (1983), S.3f..
} 
treffen. So zeigt es sich z.B., daß junge Menschen mit hohem Ausbildungsniveau trotz der zu erwartenden, hohen zukünftigen Einkommen keine potentiellen Kreditgeber finden, solange sie nicht über ausreichende Sicherheiten vertügen. ${ }^{433} \mathrm{Im}$ Falle eines unvollkommenen Kapitalmarktes kann auch der Zwangscharakter der umlagefinanzierten Rentenversicherung das Ausmaß des Vermögenssubstitutionseffekts weiter einschränken und zwar dann, wenn die Beiträge zur Rentenversicherung höher ausfallen, als die ursprünglich geplanten privaten Ersparnisse des Individuums. Einige private Haushalte, die aufgrund ihres niedrigen Einkommens kein privates Vermögen während der Erwerbsphase auflösen können und die keinen oder nur einen beschränkten Zugang zum Kapitalmarkt haben, sparen zwangsweise mehr als geplant. ${ }^{434}$

Ferner wurde Kritik daran geübt, daß die alleinige Motivation des Sparens entsprechend der Lebenszyklushypothese darin besteht, für Konsummöglichkeiten im Alter vorzusorgen, in dem kein oder nur ein geringes Einkommen bezogen werden kann. ${ }^{435}$ Treten weitere Sparziele zu dem der Altersvorsorge hinzu, so fällt die Verringerung der privaten Spartätigkeit geringer aus, als in der Lebenszyklushypothese angenommen. ${ }^{436}$ Das Modell vernachlässigt zudem den Aspekt der Vererbung. Geht man davon aus, daß Vererbung altruistisch motiviert ist, sie also ein eigenständiges Sparziel darstellt, so wird - entsprechend einer Argumentation BARROS - ein durch das Finanzierungsverfahren verändertes Sparverhalten durch intrafamiliäre Transfers ausgeglichen und der Vermögenssubstitutionseffekt kompensiert. ${ }^{437}$ Gegen BARROS Beweisführung läßt sich einwenden, daß von einem mikroökonomischen Verhalten in einer Familie nicht auf das Verhalten von Generationen geschlossen werden kann und zum anderen sehr hohe Anforderungen an die Planungsfähigkeit und Voraussicht gestellt werden. ${ }^{438}$ Problematisch ist aber vor allem, daß BARRO (und auch BECKER) ${ }^{439}$ den Altruismus modellieren, indem das Nutzenniveau der Kinder und nicht der Erbschaftsbetrag direkt in die Nutzenfunktion der Eltern eingeht. Die gegenwärtige Sparentscheidung wird dadurch vom erwarteten Nutzen aller zukünftigen Generationen beeinflußt. Wenngleich die Kritik an der extremen „Barro-

433 Vgl. Meier (1995), S.89.

434 Vgl. Berthod/Külp (1987).

435 Der Terminus der "Lebenszyklustheorie" wird in der Literatur nicht einheitlich verwandt. Während einige inn streng im Sinne Modiglianis verstehen, sprechen andere vom Lebenszyklusmodell, wenn vorausschauende Agenten mit intertemporalen Konsumproblemen konfrontiert sind; Erbschaften und andere Sparmotive sind dann mit der Lebenszyklustheorie durchaus vereinbar.

436 Browning / Lusardi (1996) S. 1797 verweisen in diesem Zusammenhang auf die 1936 von Keynes aufgelisteten acht Sparmotive, die sie um ein weiteres (Sparen für Luxus- und langlebige Güter) ergänzen.

437 Vgl. Barro (1974), Barro (1976) und Kohli (1987), S.397. Vererbung muß aber nicht zwangsläufig altruistisch motiviert sein. Ungewolltes Vererben kann schon allein deshalb unvermeidbar sein, weil das Individuum den Zeitpunkt seines Ablebens nicht genau vorhersagen kann (vgl. Abel (1985)). Denkbar ist auch, daß Eltern deshalb ein Erbe in Aussicht stellen, um dadurch die Zuwendung der Kinder "zu erkaufen" (vgl. Bernheim et al. (1985)). Solche Motive des Vererbens lassen sich mit der Lebenszyklushypothese in Einklang bringen.

Vgl. Blümle (1990), S.40; Homburg (1988) S. 112. Allerdings sind die Anforderungen an das Individuum im Lebenszyklusmodell Feldsteins nicht minder hoch. Vgl. Jobelius (1991) S. 18.

439

Vgl. Becker (1974). 
Welt ${ }^{440}$ berechtigt ist, so wird ein durch Erbschaft motiviertes Sparen der Rentnergeneration den Vermögenssubstitutionseffekt dennoch einschränken.

Sparen zum Zwecke der Absicherung muß aber nicht auf die Altersvorsorge beschränkt sein, sondern umfaßt auch altersunabhängige, allgemeine Risiken und Freuden des Lebens. Das altersunabhängige Vorsichtsmotiv des Sparens ist um so bedeutsamer, je eingeschränkter der Zugang zum Kapitalmarkt ist. ${ }^{441}$ Sowohl das Vorsichtsmotiv als auch das Sparen, um Luxusgüter, Immobilien oder andere langlebige Güter erwerben zu können, bewirken, daß ein Übergang zum Kapitaldeckungsverfahren weniger eine zusätzliche Vermögensansammlung induziert, sondern eher durch Vermögensumschichtungen realisiert wird. ${ }^{442}$

Neben diesen Einschränkungen wurden in der Diskussion weitere Effekte angeführt, die dem Vermögenssubstitutionseffekt entgegenwirken und inn zumindest teilweise kompensieren können. ${ }^{443}$ So weist DARBY darauf hin, daß das Ausmaß des Vermögenssubstitutionseffekts von den Erwartungen der Versicherten teilweise abgeschwächt wird. ${ }^{444}$ Wird erwartet, daß die interne Verzinsung des Umlageverfahrens niedriger ausfällt als die voraussichtliche Rendite privater Ersparnisse, kommt es, aufgrund dieses Zinseffektes, nicht zu dem Rückgang privater Ersparnisbildung, den der Vermögenssubstitutionseffekt erwarten ließe $e^{445}$.

Ferner muß ein Individuum, das früher aus dem Erwerbsleben ausscheidet, mehr sparen, um den einmal erreichten Lebensstandard in der Ruhestandsphase aufrecht zu erhalten. BERTHOLD und KüLP bezeichnen dieses Phänomen als „Substitutionseffekt des Rentenversicherungssystems" auf die private Ersparnisbildung, der dem Vermögenssubstitutionseffekt (als "Einkommenseffekt") entgegenwirkt. Aufgrund besserer Risikoabsicherung bei unsicheren Lebenserwartungen, unvollkommener Märkte und faktischer Umverteilung, löse die umlagefinanzierte Rentenversicherung solche Ruhestandseffekte aus und erhöhe dadurch den Anreiz zur privaten Ersparnisbildung. ${ }^{446}$

Unter der Annahme, daß die Versicherten die Funktion der Rentenversicherung als intertemporale Verteilung von Einkommen zwischen den Lebensphasen nicht erkennen bzw.

440 Vgl. auch Berthold/Külp (1987), S. 137ff. anders jedoch Jobelius (1991) S. 19ff.. Bestätigt wird die Kritik auch zum Teil durch Studien, die das Ricardianische Äquivalenztheorem als empirisch irrelevant ausweisen. Vgl. z.B. Altonji et al. (1992); Bernheim (1989). Vgl. Meier (1995), S. 90, Siehe auch Abel (1985).

Auch Ribhegge weist darauf hin, daß Teile der Vermögensansammlung mit Konsum- oder Vermögensumschichtungen innerhalb der Enwerbsbevölkerung einhergehen, sofern man hinreichend zwischen finanztechnischer und realwirtschaftlicher Betrachtungsweise differenziert. Vgl. Ribhegge (1990), S. 362. Homburg (1988), S.32 argumentiert hingegen, daß andere Sparmotive als die der Altersvorsorge quantitativ vernachlässigbar seien.

Cagan (1965) und Katona (1965) verweisen fermer auf den ${ }_{n}$ goal-gradient-effect" und den ${ }_{n}$ recognition effect" hin, die dem Vermögenssubstitutionseffekt entgegen wirken können. Beide Effekte gründen sich auf psychologische Verhaltensstrukturen. Zudem dürtte ihnen nur ein geringes Gewicht zukommen, so daß sie hier nicht weiter verfolgt werden.

Vgl. Darby (1979), S.2. 
der Funktionsweise nicht trauen, formulierte SCHNIEWIND ${ }^{447}$ in Anlehnung an die Steuerlasttheorie die Mißtrauenshypothese. Die Wirkung der Beitragszahlungen betrifft dann lediglich die laufenden, absolut verfügbaren Einkommen. Während Schniewind daraus folgert, daß alle Einkommensverwendungsarten (Konsum, Sparen und Transferzahlungen) eingeschränkt werden ${ }^{448}$, halten MüLLER/ROPPEL ${ }^{449}$ und MEIER ${ }^{450}$ sogar eine Erhöhung der Ersparnis für möglich.

Ausschlaggebend für das Wachstumspotential einer Volkswirtschaft ist darüber hinaus nicht die individuelle Spartätigkeit sondern die von weiteren Faktoren abhängige gesamtwirtschaftliche Nettoersparnis. ${ }^{451}$ So gelangen BERTHOLD und KüLP in einer einfachen saldenmechanische Betrachtung im Zwei-Generationen-Modell zu dem Ergebnis

"[...], daß das Ausmaß der Nettoersparnisse in einer Volkswirtschaft bei einem kapitalfundierten Alterssicherungssystem nicht nur von der individuellen Sparneigung, sondern auch von der Entscheidung der Erwerbstätigen über den Ruhestandsbeginn, sowie von makroökonomischen Größen, wie demographischen Schwankungen, der Entwicklung der Produktivitäten der Produktionsfaktoren und der Lohn- und Zinsentwicklung beeinflußt wird. ${ }^{452}$

Hinsichtlich des demographischen Einflusses vermindert sich im Fall einer schrumpfenden Bevölkerung die Anzahl der ersparnisbildenden Erwerbstätigen relativ zu den entsparenden Alten, wodurch es auch bei einer kapitalstockfundierten Alterssicherung zu einer Verringerung der gesamtwirtschaftlichen Ersparnisbildung kommt. ${ }^{453}$ Selbst bei einer stationären Bevölkerung führt das Kapitaldeckungsverfahren lediglich in der Übergangsphase zu einer höheren Nettoersparnis. Im Beharrungszustand ist kein Zuwachs mehr zu erwarten. Vergegenwärtigt man sich, daß die aktive Generation in der Übergangsphase ohnehin aufgrund der Umstellung einer zusätzlichen Belastung ausgesetzt und daher zu Sparumschichtungen gezwungen ist, so ist bei einer umfassenden Betrachtungsweise selbst in der Übergangsphase eine Zunahme der Nettoersparnis sehr ungewiß. Ein spürbares Anwachsen der gesamtwirtschaftlichen Ersparnis wäre daher auch beim Kapitaldeckungsverfahren nur bei einer wachsenden Bevölkerung zu erwarten. ${ }^{454}$

So plausibel der hergeleitete Zusammenhang zwischen dem Kapitaldeckungsverfahren und der Ersparnis im einfachen Modell erscheinen mag, so zeigte die durch die Feldstein-

447

448

449

450

451

452

454

Vgl. Schniewind (1989) S. 58f..

Vgl. Schniewind (1989) S. $60 f .$.

Vgl. Müller/Roppel (1990) S. 120.

Vgl. Jobelius (1991), S. 21.

Vgl. Jobelius (1991), S. 22.

Berthold / Külp (1987), S.148. Vgl. auch Meier (1995). Ferner weisen Berthold und Külp auf die Existenz sogenannter ${ }_{n}$ second-order-Effekte" hin, die sich aus Rückwirkungen der Veränderung der Nettoersparnis auf die relativen Faktorpreise (Zins- und Lohnsatz) ergeben. Eine geringere Nettoerspamis senkt die Kapitalintensităt und damit auch das Lohn-Zins-Verhăltnis, wodurch die volkswirtschaftliche Gewinnquote erhőht wird. Unter der erfahrungsgestützten Annahme, daß die Sparneigung der Empfänger von Gewinneinkūnften die der Lohnempfänger übersteigt, ergibt sich, daß eine (durch das Umlageverfahren induzierte) Verringerung der gesamtwirtschaftlichen Nettoersparnis sich über " second-order-Effekte ${ }^{\mu}$ abschwächt.

Vgl. Berthold/Külp (1987) S.149ff. ; Meier (1995) S. 91f..

Zur ausführlicheren Darstellung zum Einfluß der Demographie auf die Ersparnis siehe Meier (1995).

204 
Kontroverse ausgelöste Debatte, daß es eine Reihe mikro- und makroökonomischer Einwände gibt, die das Ausmaß des Vermögenssubstitutionseffekts auf die private Ersparnisbildung in relevanten Maße einschränken.

\subsubsection{Investitionstheoretische Kritik}

Die These, eine (partielle) Umstellung auf ein kapitalgedecktes System sei zwingend mit Wachstumsimpulsen verbunden, basiert auf den (beiden) Prämissen, daß die Substitution erstens eine erhöhte Ersparnisbildung induziert und zweitens diese in produktives Realkapital investiert wird. Der Vermögenssubstitutionseffekt wird sich aufgrund der soeben diskutierten Effekte nicht in vollem Maße auf die Sparquote niederschlagen. Zugleich muß man davon ausgehen, daß auch die zweite Voraussetzung einer entsprechenden Transformation der Vermögensansammlung in Realkapitalbildung sich nur bedingt erfüllen läßt.

Abstrahiert man nämlich von den Modellannahmen und betrachtet den realen Kapitalmarkt, so sind die sich bietenden Anlagemöglichkeiten recht heterogen. Von einer Umwandlung in Realkapital kann nur dann ausgegangen werden, wenn die institutionellen Versicherer ihr Vermögen in Risikokapital, sprich in Aktien, Schuldverschreibungen von Unternehmen o.ä investieren. Daß dies nicht zwangsläufig der Fall ist, zeigen die amerikanischen Pensionfonds, die einen nicht unwesentlichen Teil des Vermögens in staatliche Schuldtiteln investieren und somit das Staatsdefizit finanzieren. ${ }^{455}$ Davon abgesehen, daß dies gesamtwirtschaftlich lediglich eine Lastverlagerung darstellt, da auch die Zinserträge aus Staatsschuldtiteln durch späteres Steueraufkommen finanziert werden müssen, haben solche Investitionen keinen unmittelbaren Einfluß auf das Produktivvermögen der Volkswirtschaft. $^{456}$

Der auch empirisch belegte Trade-off zwischen Realinvestition eines Landes und seiner Kapitalbildung ist schon allein deshalb plausibel, weil die Determinanten des Investitionsverhaltens andere sind als die des Sparverhaltens. ${ }^{457}$

Ferner wird diese Isolierung von Kapitalbildung und Sparen durch die zunehmende internationale Verflechtung verstärkt. Denn zu recht verweisen die Verfechter des Kapitalstockverfahrens in Zusammenhang mit der Demographieabhängigkeit und der durch den zusätzlichen Kapitalstock induzierten Zinssenkung auf die Globalisierung des Kapitalmarktes hin. In gleicher Weise richten sich aber auch die Investitionen in einer Volkswirtschaft bei globalen Finanz- und Kapitalmärkten nicht mehr nach der inländischen Spartätigkeit. Sofern die Investitionen in einem Land attraktiv erscheinen, stellen internationale

Vgl. Schmähl, W. (1995). Auch die chilenischen Pensionsfonds investierten 1994 40\% ihres Vermögens in staatliche Schuldtitel. Weiterhin ist augenfällig, daß in Chile faßt alle der Aktienanleihen Anfang der 90 er Jahre ehemals staatliche Unternehmen betrafen, die im Laufe der Umstellung privatisiert wurden.

Eine andere Meinung vertritt Feldstein (1996), S. 6. Er Argumentiert, daß auf diese Weise ein Teil der "crowding-out" Effekte verhindert werden. Die privaten Haushalte, die ansonsten die staatlichen Schuldtitel gekauft hätten, würden nun auf andere Anlageformen ausweichen müssen und es kommt zu einer hŏheren Realinvestition.

Vgl. Krupp (1997a) und (1995). 
Finanzströme das nötige Kapital bereit. Selbst wenn man die eingangs zitierten Einwände beiseite schiebt und unterstellt, daß das Kapitalstockverfahren eine massive Zunahme der inländischen Ersparnis hervorruft, werden ceteris paribus keine bzw. keine relevanten Wachstumseffekte auftreten, da die Investitionen nicht von der volkswirtschaftlichen $\mathrm{Er}$ sparnis, sondern von der Attraktivität der Anlage abhängen. ${ }^{458}$

\subsubsection{Kritik am theoretischen Modellrahmen}

Die Periode von 1945 bis zu den siebziger Jahren wird zuweilen auch als "wirtschaftstheoretische Zeitalter der Neoklassischen Synthese" beschrieben. Auch in den theoretischen Erklärungsansätzen des wirtschaftlichen Wachstums dominierte das Paradigma der neoklassischen Wachstumstheorie, das im Grunde eine Kombination aus neoklassischer Produktionsfunktion und keynesianischer Sparhypothese $S(Y)$ darstellt. Es verwundert daher kaum, daß auch die Rückwirkungen der Alterssicherungssysteme auf das wirtschaftliche Wachstum vorwiegend im Rahmen dieser theoretischen Modellierung erforscht wurden. Angesichts neuerer Erklärungsansätze des Wachstums ist zu prüfen, inwieweit die abgeleiteten Ergebnisse durch die Wahl des Untersuchungsrahmens präjudiziert werden.

Mit Hilfe der neoklassischen Wachstumstheorie werden die Bedingungen für langfristig gleichgewichtige Wachstumspfade einer Ökonomie abgeleitet. Die fundamentale Differentialgleichung der Kapitalakkumulation ist ein zentrales Element dieser Theorie und beschreibt das kurzfristige Gleichgewicht von Anpassungsprozessen:

$$
\begin{aligned}
& \qquad \dot{k}=s \cdot f(k)-n \cdot k \quad \text { G } 70 \\
& \dot{k}=\text { Wachstumsrate der Pro }- \text { Kopf }- \text { Kapitalintensität } \\
& f(k)=\text { Pro }- \text { Kopf -Output der gesamwirtschaftlichen Produktionsfunktion } \\
& s=\text { marginale Sparquote } \\
& n=\text { Wachstumsrate der Bevölkerung } \\
& k=\text { Pro-Kopf-Kapitalintensität }
\end{aligned}
$$

Das heißt, die Pro-Kopf-Kapitalintensität wächst mit den Pro-Kopf-Investitionen korrigiert um das Kapital, welches notwendig ist, um bei wachsender Bevölkerung die zusätzlichen Wirtschaftssubjekte mit Kapital auszustatten. Aufgrund abnehmender Grenzerträge des Kapitals wird mit jeder zusätzlichen Pro-Kopf-Investitionen immer weniger zusätzlich produziert. Langfristig nähert sich das Wirtschaftssystem daher einem Steady state, bei dem alle Niveauparameter mit gleicher Rate wachsen und die Quotienten, allen voran die Kapitalintensität, konstant bleiben. ${ }^{459}$ Im traditionellen Untersuchungsrahmen der Neoklassik beeinflussen die individuellen Konsum- bzw. Sparpräferenzen daher lediglich in der kurz-

458 Eine andere Meinung vertritt Feldstein (1996), S. 8, Fußnote 24, der diese Zusammenhänge zumindest einschränkt. 
fristigen Übergangsphase zum Steady state die Wachstumsrate der Volkswirtschaft. Um diese Theorie mit dem empirisch zu beobachteten Wachstum des Pro-Kopf-Einkommens in Einklang zu bringen, wurde die Wachstumsrate des technischen Fortschritts - meist als Verbesserung der Arbeitsproduktivität - modelliert. ${ }^{460}$ Die Modellgleichungen beziehen sich dann nicht mehr auf Pro-Kopf Größen, sondern auf Arbeitseffizienzeinheiten.

Als grundlegende Prämissen unterstellt der traditionelle neoklassische Modellrahmen, daß die Entwicklung der originären Wachstumsursachen „technischer Fortschritt" und „Bevölkerungswachstum“ exogene Parameter darstellen und die intertemporalen Präferenzen - sprich die Höhe der Sparquote - nicht die Wachstumsrate, sondern nur das $\mathrm{Ni}$ veau des gleichgewichtigen Wachstumspfads bestimmen.

Die aus der Logik der neoklassischen Wachstumstheorie resultierende „Konvergenzthese" war empirisch jedoch ebenso wenig valide wie die langfristige Konstanz der Kapitalintensität. ${ }^{461}$ Aus den Bestrebungen diese Defizite der Theorie mittels geeigneter Modellverfeinerungen zu beheben, erwuchs Ende der achtziger Jahre der Forschungszweig der sogenannten "Neuen Wachstumstheorien“ bzw. der „Endogenen Wachstumstheorien“. Das innovative Element der bahnbrechenden Arbeiten von ROMER und LUCAS ${ }^{462}$ besteht in der Integration von positiven Externalitäten in das Wachstumsmodell. Während ROMER sein Modell auf externe Effekte des technischen Wissens ${ }^{463}$ gründete, betrachtete LUCAS vorrangig die externen Effekte des Humankapitals. ${ }^{464}$ Durch die Berücksichtigung externer Effekte war es möglich, gleichgewichtige Wachstumspfade mit konstanten oder gar steigenden Skalenerträgen zu vereinbaren.

Diese Erweiterungen bleiben insofern nicht ohne Folgen für die Analyse der allokativen Wirkungen von Alterssicherungssystemen, als die individuellen Präferenzen nunmehr auch die langfristige Wachstumsrate beeinflussen. Die fundamentale Gleichung der Ka-

459 Das heißt, im langfristigen Wachstumspfad muß die obige Bewegungsgleichung der Kapitalakkumulation G 70 folglich den Wert Null annehmen.

Vgl. Solow (1956).

Vgl. Barro (1991).

Vgl. Romer (1986), Lucas (1988). Grundzüge dieser Ansätze finden sich jedoch schon Anfang der sechziger Jahre ins Arrows „Learning-by-Doing"-Ansatz, vgl. Arrow (1962) und bei Uzawa (1965).

Modelliert wird dieser Ansatz indem das technische Wissen des einzelnen Unternehmens $\left(a_{i}\right)$ als auch das über alle Unternehmen aggregierte technische Wissen (A) als zusätzliche Argumente der Produktionsfunktion berücksichtigt wird. Eine Steigerung des eigenen technischen Wissens $\left(a_{i}\right)$ ist folglich mit einem technischen externen Effekt verbunden, da dies über die Veränderung von $A$ den Output aller übrigen Unternehmen ebenfalls beeinflußt. Das Gleichgewicht bleibt trotz steigender Skalenerträge dennoch bewahrt, weil das einzelne Unternehmen den Faktor A bei seinem Kalkül als konstant unterstellt.

Das von Romer modellierte dokumentierte und frei zugängliche technischen Wissen würde in letzter Konsequenz zu einer räumlich und zeitlich unbegrenzten Externalität führen, insbesondere auch unabhängig davon, inwieweit (in unterschiedlichen Regionen oder Ökonomien) die Fähigkeiten vorhanden sind, dieses Wissen umzusetzen. Diese Schwierigkeit wird beim personengebundenen Humankapital vermieden. Als Produktionsfaktoren werden demnach Sach- und Humankapital betrachtet, wobei letzteres sich aus dem Humankapital pro Kopf und Anzahl der Erwerbstätigen ergibt. Das gesamtwirtschaftliche Vermögen kann demzufolge in Human- oder Sachkapital investiert werden. Vgl. zum Humalkapitalansatz auch Homburg (1995), der ebenfalls eine Kritik an der traditionellen neoklassischen Wachstumstheorie anführt. 
pitalakkumulation muß langfristig mithin nicht mehr den Wert Null annehmen. Ein Ergebnis, das beispielsweise die aus der „Goldene Regel der Kapitalakkumulation“ abgeleiteten Schlußfolgerung zum optimalen Mischverhältnis der Finanzierungsverfahren ${ }^{465}$ in Frage stellt!

Einige Ansätze der endogenen Wachstumstheorie sind so modelliert, daß die externen Effekte von dem aggregierten Kapitalstock ausgehen und dessen Niveau mithin die Gesamtproduktivität der Produktionsfunktion erhöht. Auf den ersten Blick scheinen diese Ansätze die ursprüngliche Wachstumsthese des Kapitalstockverfahrens noch zu verstärken. Vergegenwärtigt man sich jedoch, daß unter dem Terminus „Externalitäten des aggregierten Kapitalstocks“ auch "spill-over-Effekte“ von technischen Wissen und Humankapitaleffekte subsumiert werden, relativiert sich der Eindruck, da diese Effekte nicht zwangsläufig mit der gesamtwirtschaftlichen Ersparnis korrelieren müssen. Ob und welche Wachstumseffekte zu erwarten sind, hängt je nach Modell unter anderem auch davon ab, welche Anreize von den alternativen Finanzierungsverfahren auf die Investition in Humankapital ausgehen.

Wenngleich die Neue Wachstumstheorie zweifelsohne einige empirische Phänomene erklären kann, die im in der traditionellen neoklassischen Wachstumstheorie Schwierigkeiten bereiten, gründet sie sich dennoch weiterhin auf dem Paradigma der neoklassichen Wachstumstheorie. Gleichgewichtsbetrachtungen stehen nach wie vor im Mittelpunkt der Analysen. Eine fundamentalere Kritik ${ }^{466}$ wendet sich gerade gegen diesen Gleichgewichtsansatz der „alten“ und „neuen“ neoklassischen Wachstumstheorie. Andere theoretische Erklärungsansätze des wirtschaftlichen Wachstums erachten gerade die - beispielsweise aus Wettbewerbs- und Anpassungsprozessen resultierenden - ungleichgewichtigen Situationen als maßgeblichen Wachstumsmotor. Solche Ansätze betonen vor allem die Rolle und den evolutorischen Charakter von Produkt- und Prozeßinnovationen bzw. den schumpeterischen Prozeß der „schöpferischen Zerstörung““ ${ }^{467}$ Im Lichte dieser Theorien, bei denen den Innovationsprozessen eine weit größere Bedeutung zukommt, als einer durch eine Veränderung der intertemporalen Präferenzen induzierten Kapitalakkumulation, verliert auch die Argumentation an Überzeugungskraft, wonach eine kapitalfundierte Alterssicherung aufgrund steigender gesamtwirtschaftlicher Ersparnis und zunehmender Kapitalakkumulation zu einem höheren Sozialprodukt führt. ${ }^{468}$

Auf Ablehnung stößt die These von den dem Kapitalstockverfahren zugeschriebenen Wachstumsimpulsen unter anderem auch deshalb, weil die neoklassische Wachstum-

465 Vgl. Abschnitt 2.3.3.1.

466 Vgl. z.B. NelsonWinter (1982): „The neoclassic approach to growth theory has taken us down a smooth road to a dead end" Vgl. auch Boltho und Holtham (1982).

467 Vgl. Schumpeter (1950).

468 Angesichts der Akzentverschiebung bei den Wachstumsursachen auf die Rolle von Innovationen, wird die klassische Begrūndung für die Wachstumseffekte zwar entkräftet, völlig ausschließen lassen sich etwaige Wachstumsimpulse als folge einer verstärkten Kapitalfundierung der Altersvorsorge a priori jedoch nicht. Allerdings gründen diese sich dann auf einen völlig anderen Argumentationsstrang. Vgl. Abschnitt 6.2.3.

208 
stheorie vollkommene Märkte, insbesondere eine Vollbeschäftigung der Produktionsfaktoren unterstellt.

So betont KRUPP, daß die Knappheit der Produktionsfaktoren und Nachfrageeffekte die gesamtwirtschaftlichen Rückwirkungen eines bestimmten Finanzierungsverfahrens wesentlich beeinflussen. ${ }^{469}$ Ausgehend von der Frage, welcher Produktionsfaktor, Kapital oder Arbeit, die limitierende Größe bei der Erstellung des Sozialprodukts darstellt, können zwei Zustände der Wirtschaft unterschieden werden. Der eine zeichnet sich durch hohe Arbeitslosigkeit, sprich durch ein Überangebot an Arbeit aus. Die zweite ist durch eine tendenzielle Arbeitsknappheit charakterisiert.

Um das Wachstumspotential der Volkswirtschaft möglichst gut auszuschöpfen, erfordern Phasen der Kapitalknappheit und Phasen der Arbeitsknappheit unterschiedliche Strategien. Eine in der Phase der Arbeitsknappheit zu verfolgende Strategie müßte zum Ziel haben, möglichst viele Arbeitslose wieder in den Arbeitsmarkt zu integrieren. Das Persistenz- und Hysteresephänomen läßt darauf schließen, daß ein relevanter Teil der gegenwärtigen Arbeitslosigkeit struktureller Natur ist. Daraus aber (vorschnell) den Schluß zu ziehen, Nachfrageeffekte seinen gänzlich irrelevant, wäre verfehlt. Denn solange ein, wenn auch nur lockerer, Zusammenhang besteht, werden die Arbeitsmarktprobleme durch eine Schwächung der Nachfrage akzentuiert. ${ }^{470}$ In einer Phase der Kapitalknappheit müßte die Strategie darauf abzielen, die Erwerbsquoten zu erhöhen und den Arbeitseinsatz möglichst effizient zu gestalten, indem die Arbeit mit mehr Kapital ausgestattet wird.

Überträgt man diese Überlegungen auf die Gegebenheiten der Bundesrepublik Deutschland auf die Zeit bis zum Jahre 2030, so können zwei Perioden unterschieden werden: Die erste zeichnet sich durch hohe Arbeitslosigkeit, sprich durch ein Überangebot an Arbeit aus. Wenngleich die Demographie nicht die alleinige Einflußgröße für die Beschäftigung und Arbeitslosigkeit ist, spricht einiges dafür, daß aufgrund der zu erwartenden demographischen Veränderung ab etwa 2010/15 das Arbeitsangebot zurückgeht. Es erfolgt der Übergang zur zweiten Periode, die durch eine tendenzielle Arbeitsknappheit charakterisiert ist.

Auffallend an der derzeitigen Arbeitsmarktsituation ist, daß trotz hoher gesamtwirtschaftlicher Sparquote arbeitsplatzschaffende Investitionen weitgehend ausbleiben. Dies scheint im Widerspruch zu der Annahme zu stehen, daß die Investitionen hauptsächlich eine Funktion der Ersparnis sind. Ferner ist der derzeitige Langfristzins vergleichsweise niedrig. Beide Phänome können als Indiz dafür gewertet werden, daß die gegenwärtige Wachstumsschwäche nicht durch Kapitalmangel und eher durch eine Nachfrageschwäche zu erklären ist. Wenn nun in einer solchen Situation eine zunehmende Kapitalbildung - infolge einer Umstellung auf eine kapitalgedeckte Rentenfinanzierung die Nachfrageprobleme verstärkt, so wäre, nach der Argumentation KRUPPS, weniger mit handelt. Das heißt, daß eine Nachfrageausweitung nicht zwangsläufig eine Senkung der Arbeitslosigkeit und Ausweitung des Wachstums induziert. Krupp (1997) S. 206.
}

209 
einem Wachstumsimpuls und vielmehr mit einer Schwächung des Wachstums zu rechnen. ${ }^{471}$

\subsubsection{Empirische Evidenz}

Da aus der theoretischen Analyse (bislang) keine eindeutigen Schlüsse über die Wirkung des Umlageverfahrens auf die Sparquote bzw. auf das Wachstum abgeleitet werden können, ist diese These nur empirisch zu falsifizieren. ${ }^{472}$ Unterstellt man, daß die These vom wachstumshemmenden Umlageverfahren zutrifft, so ist zu vermuten, daß die gesamtwirtschaftliche Sparquote der Länder mit weitgehend kapitalfundierter Alterssicherung die Sparquote der Länder mit umlagefinanzierten Systemen übersteigt. Ein erster Querschnitts-Vergleich der Vermögen aus (kapitalfundierter) betrieblicher Alterssicherung als Prozentsatz des BIP (1990-1991) [PV/BIP] mit der durchschnittlichen gesamtwirtschaftlichen Bruttoersparnis als Prozentsatz des BSP (1990-1992) [ $\mathrm{s} /$ BSP $]$ von 23 Staaten ergab, daß kein signifikanter Unterschied zwischen Staaten mit hohem ( $\mathrm{PV} / \mathrm{BIP}>30 \%$ ) und solchen mitniedrigem ( $\mathrm{PV} / \mathrm{BI}<10 \%$ ) betrieblichen Alterssicherungsvermögen festzustellen ist ( $\mathrm{vgl}$. Abbildung 16 und Abbildung 17). ${ }^{473}$

471 Die Untertunnelungsstrategie beruht darauf, in der demographisch noch "guten Phase" einen Kapitalstock zu bilden, den man spăter in einer Phase eines besonders hohen Altenquotienten abschmelzen kann, um den Beitragssatz zu stabilisieren. Eine solche Strategie wäre angesichts der beschriebenen gesamtwirtschaftlichen Rahmensituation insofern mit kontraproduktiven Effekten verbunden, als die gesamtwirtschaftlichen Entzugsprozesse des Kapitalaufbaus die inlåndische Nachfrage in der ersten Periode mindern und mithin das Wachstum schwächen würden (Vgl. auch Meinhold (1980) S.18ff.). Eine vollstăndige Kompensation dieses Effektes durch Exportsteigerungen wăre, so Krupp weiter, unwahrscheinlich. Zusätzlich zu den Folgen eines Nachfrageausfalls muß damit gerechnet werden, daß ein Wechsel des Finanzierungssystems zu einer Verunsicherung in der Bevőlkerung führt, was sich ebenfalls nachteilig auf die Investitionsbereitschaft der Unternehmen auswirke (vgl. Ribhegge (1990), S. 375; Berthold/Külp (1987) S.164).

Ein späteres Abschmelzen des Vermögensbestands ab etwa 2020 dem Produktionsprozeß Kapital in einer Periode entziehen, in der der Faktor Arbeit eigentlich mit mehr Kapital ausgestattet werden müßte. Wenn zudem in der Phase einer möglichen Arbeitskrăfteknappheit nachfragebedingt an der Kapazitätsgrenze produziert würde, würden die auflösungsbedingten Expansionseffekte zu einem Anstieg der Preise und damit de facto zu einer Senkung der realen Renten führen. Ein Ausgleich der Expansionseffekte ist zwar durch eine Steigerung des Imports denkbar, die damit einhergehende Passivierung der Leistungsbilanz ist jedoch ebenfalls mit - wenn auch geringeren - Preiseffekten verbunden, da sie zu einer Abwertung der Währung führt. Unterstellt man, daß die Wechselkurse weniger von den tatsăchlichen Handelsströmen bestimmt werden und statt dessen die Einschătzung der deutschen Situation durch die Finanz- und Kapitalmärkte der maßgebliche Faktor ist, so dürtte eine massive Kapitalauflösung eher Mißtrauen erwecken und ebenfalls zu einer Währungsabwertung führen.

Für eine privatwirtschaftliche Kapitaldeckung (im Gegensatz zur Untertunnelungsstrategie) mūssen die mit der Auflōsung verbundenen Effekte allerdings relativiert werden, da das Vermögen nicht vollstăndig, sondern nur nach Maßgabe des Bevölkerungsrückganges abgeschmolzen wird. Die obige Argumentation hinsichtlich der Phase des Kapitalaufbaus gilt jedoch analog.

472 Vgl. auch Jaeger (1994), S. 366; Hughes (1996), S.9; Feldstein (1974), S. 910 und (1996), S. 7; Jobelius (1991), S. 22.

473 Ein ähnliches Ergebnis ergibt sich bei einem Vergleich zwischen 23 Ländem, allerdings unter der Fragestellung, ob die sozialen Transfers als Prozentsatz des BSP einen Einfluß auf die Wachstumsraten der Volkswirtschaften haben. (Lindert 1996a), S. 19/20. Auch die verschiedenen ökonometrischen Untersuchungen zu dieser Frage konnten keinen positiven Zusammenhang ausmachen. Vgl. Lindert (1996); Crandall (1993); Kusmin (1994) und Helms (1985). 
Abbildung 16: Vermögen der betrieblichen Alterssicherung (Pensionsvermögen) als Prozentsatz des BIP (1990-1991) und durchschnittliche gesamtwirtschaftliche Bruttoersparnis als Prozentsatz des BSP (Sparquote) (1990-1992)

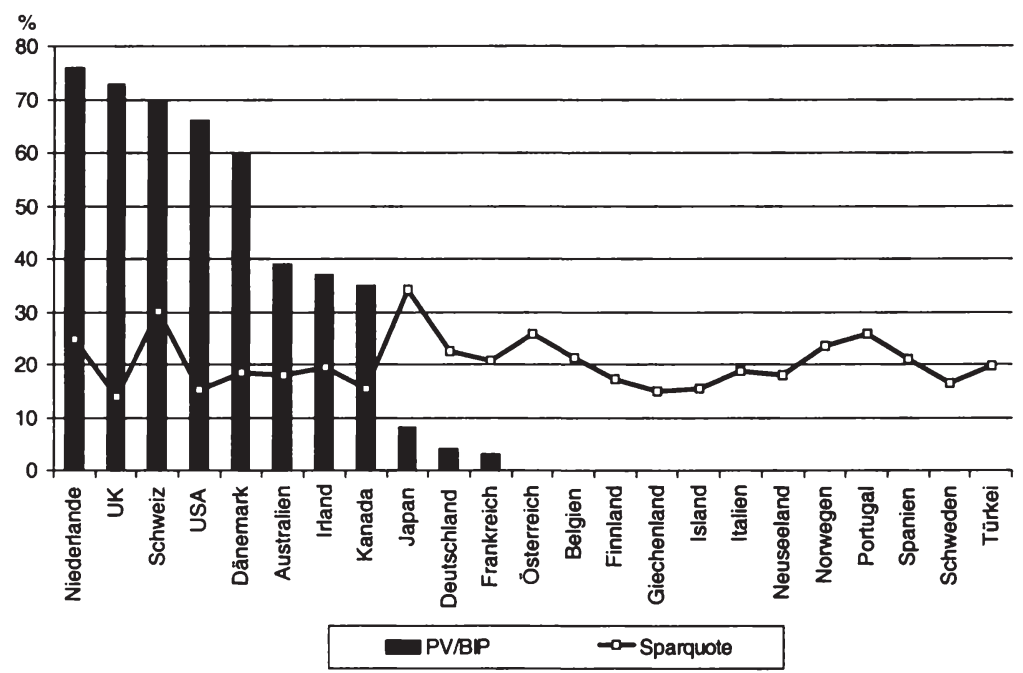

Quelle: Hughes (1996) Table 2, S. 7.

Abbildung 17: Streuungsdiagramm der Kombinationen von Vermögen der betrieblichen Alterssicherung (Pensionsvermőgen) als Prozentsatz des BIP (1990-1991) und durchschnittliche gesamtwirtschaftliche Bruttoersparnis als Prozentsatz des BSP 23 ausgewählter Länder (Sparquote)

(1990-1992)

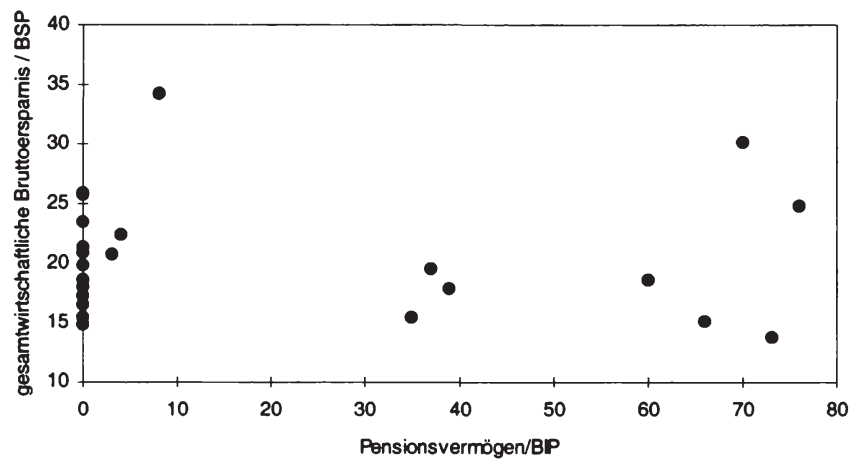

Quelle: Hughes (1996) Table 2, S. 8. 
Die durchschnittliche Sparquote der ersten Ländergruppe lag bei 19,4\%, während die der zweiten $20,1 \%$ betrug. $^{474}$

Die voranstehende Gegenüberstellung vergleicht lediglich aggregierte Größen und differenziert nicht zwischen unterschiedlichen Einflußvariablen und kann daher auch nicht die verschiedenen mit innen verbundenen Kausalzusammenhänge analysieren. Obschon die These eines ersparnissenkenden Effektes nicht widerlegt ist, spricht der Vergleich zumindest als vorläufiger Indiz gegen deren pauschale Gültigkeit.

Dennoch wäre es möglich, daß ein bestehender Zusammenhang nicht unmittelbar aus den obigen Daten abzulesen ist, weil weitere Einflußfaktoren auf die gesamtwirtschaftliche Ersparnis den Effekt des Finanzierungsverfahrens überdecken können. ${ }^{475}$ Eine Reihe ökonometrischer Analysen widmete sich daher dem Problem, aus der komplexen Verflechtung verschiedener Einflußfaktoren, die Wirkung des Finanzierungsverfahrens zu isolieren.

In verschiedenen ökonometrischen Längsschnittanalysen wurde beispielsweise danach gefragt, ob die Existenz sozialer Sicherungssysteme die private Ersparnis mindert. Die Ergebnisse sind sehr kontrovers, wobei sich die positiven und negativen Antworten auf die Frage in etwa die Waage halten (vgl. Tabelle 8).

474 Vgl. Hughes (1996) S. 6, basierend auf Daten der Weltbank und der OECD.

475 Die Frage, welches die maßgeblichen Einflußgrößen der privaten Ersparnis sind, war Gegenstand zweier umfassende Studien des IWF, die sowohl Industrienationen als auch Entwicklungslănder in die Untersuchung einschlossen. Zu den die Ersparnis fördernden Faktoren gehörten neben höheren Wachstumsraten und höheren realen Zinssătzen auch verbesserte terms of trade. Die Zunahme von Überschüssen im Staatsbudget oder des Pro-Kopf-Einkommens relativ zu dem der Vereinigten Staaten führten hingegen tendenziell zu einer Verringerung der privaten Sparquote. Vgl. Masson, Bayoumi, Samiei (1995, 1996).

212 
Tabelle 8: Längsschnittsanalysen zur Frage, ob das soziale Sicherungssystem die gesamtwirtschaftliche private Ersparnis mindert.

\begin{tabular}{|l|l|l|l|}
\hline & \multicolumn{1}{|c|}{ Schätzperlode } & \multicolumn{1}{|c|}{ Land } & \multicolumn{1}{|c|}{ Ergebnis } \\
\hline Feldstein (1974) & $1930-71$ & USA & Ja** \\
\hline Munnell (1974) & $1929-69$ & USA & Ja \\
\hline Barro (1978) & $1929-74$ & USA & Nein \\
\hline Markowski / Palmer (1979) & $1954-75$ & Schweden & Ja \\
\hline Boyle / Murray (1979) & $1946-69$ & Kanada & Nein \\
\hline Denny / Rea (1979) & $1946-75$ & Kanada & Nein \\
\hline Pfaff, Hurler, Dennerlein (1979) & $1965-78$ & Deutschland & Ja* \\
\hline Carmichael / Hawtrey (1981) & $1961-79$ & Australien & Nein \\
\hline Leimer / Lesnoy (1982) & $1930-74$ & USA & Nein \\
\hline Feldstein (1982) & $1929-76$ & USA & Ja \\
\hline Browning (1982) & $1966-76$ & U.K. & Ja \\
\hline Bentzel / Berg (1983) & $1955-79$ & Schweden & Nein \\
\hline Lee / Chao (1988) & $1930-74$ & USA & Nein \\
\hline Rossi / Visco (1993) & $1954-90$ & Italien & Unklar* \\
\hline Magnussen (1994) & $1966-90$ & Norwegen & Nein \\
\hline
\end{tabular}

Quelle: Atkinson (1987), Magnussen (1994) nach Hughes (1996)

- Die Schlußfolgerungen dieser Studien wurden um entsprechende Fehler korrigiert und entsprechen daher nicht jenen der Autoren (vgl. Hughes 1996, S. 11).

** Leimer und Lesnoy (1978) entdeckten einen entscheidenden Fehler in Feldsteins Berechnungen und kamen bei einer erneuten Schätzung seines Modells zu dem Ergebnis, daß die private Ersparnis nicht reduziert wurde.

Ein ähnlich diffuser Befund resultiert aus der Gegenüberstellung der einschlägigen Querschnittsanalysen. Während die Hälfte der Untersuchungen eine Reduktion der privaten Ersparnis infolge des sozialen Sicherungssystems bejaht, kommt die andere Hälfte zu dem gegenteiligen Ergebnis (vgl. Tabelle 9). ${ }^{476}$

476 Vgl. auch Cagan (1965); Kantona (1965); Munnel (1976). 
Tabelle 9: Querschnittsanalysen zur Frage, ob das soziale Sicherungssystem die gesamtwirtschaftliche private Ersparnis mindert.

\begin{tabular}{|l|l|l|l|l|}
\hline \multicolumn{1}{|c|}{ Autoren } & \multicolumn{1}{|c|}{ Daten } & Periode & Beobachtungen & Ergebnis \\
\hline Diamond und Hausmann (1980) & LDSMM & & & Nein \\
\hline King und Dicks-Mireaux (1982) & CSCF & 1977 & 12.734 & Ja \\
\hline Dicks-Mireaux und King (1984) & CSCF & 1977 & 8.279 & Ja \\
\hline Kurz (1984) & PCPP & 1979 & 4.293 & Nein \\
\hline David und Menchik (1985) & WS & $1947-64$ & 720 & Nein \\
\hline Avery, Elliehausen und Gustafson (1986) & SCF & 1983 & 3.800 & Nein \\
\hline Mariger (1986) & SFC & 1962 & 623 & Ja \\
\hline Leimer und Richardson (1992) & CES & $1982-83$ & 2.715 & Ja \\
\hline
\end{tabular}

Quelle: Magussen (1994) und Munnel und Yohn (1992)

Anmerkung: CES = Consumer Expenditure Survey; CPRT = Central Personal Registration Number Data; LDSMM = Labor Department Survey of Mature Men; PCPP = President's Commission on Pension Policy; SCF = Survey of Consumer Finances; SFC = Survey of Financial Characteristics.

Tabelle 10: Studien zur Frage, ob (kapitalfundierte) privaten Altersvorsorgemaßnahmen die private Ersparnis erhöht.

\begin{tabular}{|l|c|c|c|r|c|}
\hline \multicolumn{1}{|c|}{ Autoren } & Daten & Land & Periode & Beobachtungen & Ergebnis \\
\hline Venti und Wise (1990) & CES & USA & $1983-85$ & 3.994 & Ja \\
\hline Gale und Scholz (1994) & CES & USA & $1983-86$ & 2.822 & Nein \\
\hline Florentsen (1996) & CPRN & Dänemark & $1984,86,88$ & 100.000 & Nein \\
\hline
\end{tabular}

Quelle: Venti und Wise (1990), Gale und Scholz (1994), Florentsen (1996)

Anmerkung: CES = Consumer Expenditure Survey; $C P R N=$ Central Personal Registration Number.

Die empirisch fundierten Antworten auf die Frage, ob das soziale Sicherungssystem die gesamtwirtschaftliche Ersparnis beeinflußt, führten ebenfalls - wie die theoretische Analyse - zu keiner validen Aussage, so daß das Resümee zulässig erscheint, daß es bislang nicht möglich ist, einen negativen Effekt des Umlageverfahrens auf die gesamtwirtschaftliche Ersparnis aufzuzeigen.

\subsection{Erfassung der Ersparnis- und Wachstumseffekte des Kapi- talstockverfahrens bei der intergenerativen Umverteilung}

Angesichts der vorgenannten Kritik sowohl an der Vermögenssubstitutionshypothese als auch an den wachstumsfördernden Effekten des Kapitalstockverfahrens müssen die folgenden Fragen für die Analyse der intergenerativen Gerechtigkeit geklärt werden:

1. Welcher funktionale Zusammenhang soll zwischen dem Kapitalstock zur Rentenfinanzierung und der privaten gesamtwirtschaftlichen Ersparnis unterstellt werden? 
2. Wie beeinflußt eine Veränderung der Ersparnis die interne Verzinsung des kapitalfundierten Anteils der Altersvorsorge?

3. Sollen eventuelle Wachstumseffekte modelliert werden und wenn ja, in welchem Ausmaß sollten sie wirken?

Die folgenden drei Abschnitte setzen sich in dieser Reihenfolge mit den vorangehenden Fragestellungen auseinander.

\subsubsection{Rentenkapitalstock und gesamtwirtschaftliche Ersparnis}

RAFFELHÜSCHEN gründet seine Analyse auf die Anreizeffekte, die von den alternativen Finanzierungsverfahren auf das individuelle Sparverhalten (und Arbeitsangebotsverhalten) ausgehen. Anstatt eine gesamtwirtschaftlichen Spar- bzw. Konsumfunktion in Abhängigkeit vom Alterssicherungsverfahren zu schätzen, ${ }^{477}$ versucht er, seine Simulationsanalyse mikroökonomisch zu fundieren, indem er das Kalkül der privaten Haushalte in sein Modell integriert. Aus einer über sieben Perioden zu maximierenden, spezifizierten CESNutzenfunktion leitet er die Verhaltensfunktionen eines repräsentativen Wirtschaftssubjekts einer Generation t hinsichtlich seines Konsums, seines Arbeitsangebots, seiner Ersparnis und seiner (strategischen) Erbschaft ab. Diese Vorgehensweise zur Ermittlung der privaten Ersparnisbildung kann jedoch aufgrund der nachfolgenden Einwände wenig befriedigen.

- Da die Lebensnutzenfunktionen nicht zur Ableitung qualitativer Tendenzen sondern zur quantitativen Ermittlung der Wirkungen herangezogen werden, müssen die Nutzenfunktionen genauer spezifiziert werden. Die Entscheidung für eine bestimmte Form des funktionalen Zusammenhangs läßt sich theoretisch nicht herleiten, und eine empirische Ermittlung stellt ein schwieriges, wenn nicht gar unmögliches Unterfangen dar. Es besteht die Gefahr, daß die mathematische Bequemlichkeit einen großen Einfluß auf die Wahl des funktionalen Zusammenhangs hat.

- Als ähnlich problematisch muß die Wahl der Parameter für die Nutzenfunktion angesehen werden, deren Komplexität mit zunehmender Zahl an Parametern steigt. ${ }^{478}$

- Die Formulierung einer nicht nur konsistenten sondern zudem auch im Zeitablauf stabilen Nutzenfunktion dürfte in praxi mit unüberwindlichen Schwierigkeiten verbunden sein.

477 Vgl. zur Schätzung solcher Funktionen und den widersprüchlichen Ergebnissen Abschnitt 6.1.4 sowie Kune (1983), S. 377.

478 Allein in Raffelhüschens 2-Generationen-Modell müssen neben der Zeitprăferenzrate, altersspezifischer Intensitätsparameter und der intratemporalen Substitutionselastizităt auch die intertemporale Substitutionselastizităt berūcksichtigt werden. In einem 7-Generationenmodell steigen die zu schätzenden Parameter entsprechend, bzw. müssen eine Reihe von vereinfachenden Stationäritătsannahmen getroffen werden. 
- Auch methodisch wird diese Vorgehensweise ihrem Anspruch einer mikoökonomischen Fundierung wenig gerecht, solange die Annahme eines repräsentativen Individuums aufrecht gehalten wird. Insbesondere stellt sich das methodische Problem, wie und ob sich Präferenzordnungen in Nutzenfunktionen ausdrücken lassen bzw. wie und ob sich einzelne Nutzenfunktionen aggregieren lassen. ${ }^{479}$

- Das Modell basiert trotz der Erweiterung um strategische Erbschaften vornehmlich auf der Lebenszyklushypothese. Zusätzliche Sparmotive und etwaige Vermögensumschichtungen werden nicht ausreichend erfaßt, geschweige denn hinsichtlich der Art und des Ausmaßes ihres Einflusses spezifiziert.

- Ferner ist anzumerken, daß andere Variablen des Sparverhaltens in diesem wahlhandlungstheoretischen Nutzenmaximierungskalkül nicht berücksichtigt werden. ${ }^{480}$

- Bei einem auf Jahresbasis beruhenden Wahlhandlungsmodell wird das private Optimierungskalkül zu komplex, um eine Lösung zu erhalten. ${ }^{481}$ Der zusätzliche Erkenntnisgehalt, der mit einem Übergang von einem 2 zu einem 7-Generationenmodell (,goldener Mittelweg") verbunden sein soll, ist allerdings äußert fragwürdig.

Allgemein werden die erforderlichen Annahmen durch die „mikroökonomische Fundierung" des privaten Sparverhaltens lediglich auf die Konkretisierung der Nutzenfunktion vorgelagert. Eine solche Vorgehensweise ist nur dann den „ad-hoc-Annahmen“ überlegen und mit einem Erkenntnisgewinn verbunden, wenn die erklärenden Größen des Modells sich besser vorhersagen bzw. schätzen lassen als eine makroökonomische Schätzung der Ersparniseffekte selbst. Dies ist jedoch äußerst fragwürdig. Zudem ist es für einen solchen Ansatz erforderlich, daß die unterstellten Zusammenhänge zwischen den erklärenden und der zu erklärenden Größe (gesamtwirtschaftliche Nettoersparnis) über den Zeitraum der Voraussage hinweg konstant sind.

Abstrahiert man von rein qualitativen modell-theoretischen Analysen, läßt sich vom derzeitigen theoretischen und empirischen Kenntnisstand der Ersparniseffekt des Kapitalstockverfahrens ex ante weder vorhersagen noch hinreichend modellieren. Es kann lediglich angenommen werden, daß sowohl ein vollständiges ausbleibenden des Spareffektes als auch ein 1:1 Anstieg der gesamtwirtschaftlichen Ersparnis nach Maßgabe des Rentenkapitalstocks unplausibel erscheinen. Angesichts dieser Überlegungen und der obigen Einwände ist der von JÄGER gewählte Ansatz seiner Simulationsanalyse vorzuziehen. ${ }^{482}$ Demzufolge werden pauschale Annahmen über die gesamtwirtschaftlichen Spareffekte getroffen und die verbleibende Unsicherheit durch verschiedene Szenarien gemindert.

\footnotetext{
479 Beispielsweise zeigt das Allais'sche Paradoxon, daß bei bestimmten plausiblen Präferenzordnungen eine Nutzenfunktion nicht existieren kann. Vgl. hierzu die Ausführungen bei Thießen (1993), S. 158f..

480 Vgl. Masson, Bayoumi, Samiei $(1995,1996)$.

481 Vgl. Raffelhüschen (1989), S. 84f..

482 Vgl. Jăger (1991), S. 90ff..
} 
Eine Integration der wahlhandlungstheoretischen Ansätze zur Bestimmung der gesamtwirtschaftlichen Ersparniseffekte würde den Realitätsgehalt der Simulationsanalyse nicht steigern. Es würde lediglich eine Scheingenauigkeit der Simulation vorgetäuscht, die vor allem die kontroversen Ergebnisse der empirischen und theoretischen Analysen zur "Ersparnisthese" des Kapitalstockverfahrens sowie die Abhängigkeit von den jeweiligen Annahmen verschleiert. Auch diesbezüglich hätte die vorgeschlagene Vorgehensweise den Vorteil, daß die Abhängigkeit der simulierten Wirkungen von den Annahmen transparenter wird.

\subsubsection{Ersparnis und interne Verzinsung des Kapitalstockverfahrens}

Eine zunehmende private Ersparnisbildung war in dem geschlossenen Modellrahmen des zweiten Kapitels gleichbedeutend mit einem steigenden gesamtwirtschaftlichen Kapitalstock. Als Folge der abnehmenden Grenzproduktivität des Kapitals sinkt der Zinssatz mit der Größe des gesamtwirtschaftlichen Kapitalstocks. In welchem Ausmaß sich der Zins verändert, hängt in der klassischen Theorie von den Zinselastizitäten des Kapitalangebots und der Kapitalnachfrage ab. Diese wiederum werden unter anderem von dem Offenheitsgrad der Ökonomie bestimmt. Im Extremfall einer unbeschränkt offenen und kleinen Volkswirtschaft würden sich zu einem gegebenen Zinssatz dem Anleger weltweit eine schier unbegrenzte Menge an lukrativen Investitionen bieten. Dies entspräche einer vollkommen zinselastischen gesamtwirtschaftliche Kapitalnachfrage. Etwaige Zinseffekte würden in dem Fall - in Übereinstimmung mit den Ergebnissen des zweiten Kapitels trotz Variationen des Kapitalangebots ausbleiben.

Die Mängel der neoklassischen Wachstumstheorie wurde bereits in Abschnitt 6.1.3 beschrieben. Im Hinblick auf die vorangegangene investitionstheoretische Kritik ${ }^{483}$ gilt es daher zunächst zu klären, welche Rolle die internationale Verflechtung der Kapitalmärkte für Deutschland spielt.

In seiner Studie zum Entlastungspotential eines Teilkapitalstocks untersucht das IfoInstitut, wie der Nominal- und Realzinssatz auf Änderungen des Kapitalangebots und der Kapitalnachfrage reagiert. ${ }^{484}$ In ihrer nach verschiedenen Sektoren untergliederten Analyse gelangen die Autoren für die Bundesrepublik Deutschland zu dem Ergebnis: „Die Hypothese, daß mit zusätzlichem Kapitalangebot der Kapitalmarktzins sinkt, muß somit abgelehnt werden. ${ }^{A 85}$ Dennoch weisen ihre Modellrechnungen für die Einführung einer Teilkapitalstockfinanzierung einen leichten Rückgang des Zinssatzes aus. ${ }^{486}$ KAEHLER und KORN ermitteln in ihrer empirischen Analyse zur Kapitalnachfrage in Deutschland eine Zinselastizität der gesamtwirtschaftlichen Kapitalnachfrage von $-0,17 .{ }^{487}$ Auch aufgrund dieses

\footnotetext{
483 vgl. Abschnitt 6.1.2.

484 Vgl. Langmantel/Vogler-Ludwig (1997).

485 Vgl. Langmantel/Nogler-Ludwig (1997), S. 37.

486 Vgl. Langmantel/Vogler-Ludwig (1997), S. 121.

487 Vgl. Kaehler/Korn (1995).
} 
Ergebnisses wären, entsprechend eines klassischen Kapitalmarktmodells, leichte Zinseffekte zu erwarten.

Die Annahme eines zumindest leicht variierenden Zinssatzes erscheint auch deshalb als zweckmäßig, weil das steigende Kapitalangebot aus einem spezifischen Motiv, nämlich dem der Altersvorsorge, resultiert. Solche zweckgebundenen Anlageformen müssen bestimmte Sicherheitsanforderungen genügen, so daß tendenziell (aufgrund von Wechselkursschwankungen u.ä.) eher inländische Anleihen favorisiert werden. Trotz eines generell eher offenen Kapitalmarktes wäre daher zu erwarten, daß ein erheblicher Teil der zusätzlichen Ersparnis im Inland investiert würde. Zwar könnte man argumentieren, daß bestehende inländische Sparanlagen, die nicht zur Altersvorsorge dienen, zumindest theoretisch in Auslandsanlagen verdrängt werden könnten, eine vollständige Investition ins Ausland wäre jedoch unwahrscheinlich bzw. nur bei einem geringen Fundierungsgrad denkbar. Mit dieser Argumentation wird das Augenmerk auf zwei wesentliche, für die Simulationsanalyse relevante Aspekte gelenkt:

- In der zitierten Untersuchung des ifo-Instituts wurde die Fiktion eines homogenen Zinssatzes weiterhin aufrechterhalten, obwohl ein realer Kapitalmarkt eine Vielzahl heterogener Anlagemöglichkeiten bietet.

- Ferner wurde bislang die Korrelation zwischen Kapitalangebot und allgemeinen Marktzins betrachtet. Für die nachfolgende Analyse ist jedoch vorrangig die Frage interessant, wie eine Veränderung des Kapitalangebots die interne Verzinsung des kapitalfundierten Anteils der Altersvorsorge beeinflußt.

Selbst wenn man daher konzidiert, daß das Zinsniveau der verschiedenen Anlagemöglichkeiten unabhängig von der Höhe des inländischen Kapitalangebots ist, dürfen etwaige Rationierungen beim Volumen der verschiedenen Anlageformen nicht außer Acht gelassen werden.

Innerhalb der Anlagemöglichkeiten, die sich angesichts der erwähnten Sicherheitsanforderung für Anlagen zum Zwecke der Altersvorsorge eignen, sind die verfügbaren Mengen besonders rentabler Anlageformen sehr begrenzt. Faktisch dürften sie durch die institutionellen Anleger größtenteils bereits ausgeschöpft sein. Eine zusätzliche Kapitalfundierung in der Altersvorsorge müßte folglich auf weniger rentable Anlagemöglichkeiten ausweichen, so daß die durchschnittlich erzielbare interne Verzinsung des kapitalfundierten Anteils der Altersvorsorge sinkt. ${ }^{488}$ Schon allein deshalb kann die interne Verzinsung, die sich in der Vergangenheit mit privaten kapitalgedeckten Alterssicherungssystemen erzielen lies, nicht ohne weiteres auf die Zukunft übertragen werden. Dieser Einwand resultiert aus der selbstinduzierten Wirkung der partiellen Substitution des Finanzierungsverfahrens und gilt unabhängig von bzw. zusätzlich zu dem grundsätzlichen Problem, daß bei jeder 
Projektion vergangener Werte in die Zukunft der Einfluß des für die damalige Zeit spezifischen, ökonomischen Kontextes berücksichtigt werden muß.

Für die Simulation der intergenerativen Verteilungswirkungen ist nun entscheidend, in welchem Ausmaß die interne Verzinsung des Rentenfonds durch die beschriebenen Wechselwirkungen verringert wird. Versucht man, diese Effekte auch nur halbwegs schlüssig zu quantifizieren, stößt man schnell an die Grenzen einer systematischen Deduktion. Denn eine solche Vorgehensweise würde erfordern, daß die Konsequenzen einer zusätzlichen Kapitalfundierung der Altersvorsorge auf den Kapitalmarkt der Bundesrepublik Deutschland hinreichend erforscht sind. Insbesondere müßten umfassende theoretische und empirische Analysen zu den folgenden Fragestellungen zugänglich sein:

- Welche Auswirkungen hat eine zusätzliche Kapitalfundierung der Altersvorsorge auf die Kapitalmarktstruktur? ${ }^{489}$

- Welche Konsequenzen hat dies auf mikroökonomischer Ebene auf das Anlageverhalten der Finanzinstitutionen und auf die Förderung neuer innovativer Finanzprodukte?

- Wie werden die zu erwartenden Anpassungsprozesse auf dem Kapitalmarkt durch exogene Effekte, allen voran die Einführung der Europäischen Währungsunion beeinflußt?

Umfassende Untersuchungen zu diesen Fragestellungen sind bislang nicht verfügbar. ${ }^{490}$ Solange keine gesicherten Erkenntnisse über diese Wechselwirkungen vorliegen, die sich in ein makroökonometrisches Modell integrieren ließen, muß auch hinsichtlich des Einflusses einer Teilfundierung auf die interne Verzinsung des Kapitalstockverfahrens der (unbefriedigende) Weg beschritten werden, lediglich pauschale Annahmen vorzugeben. Die Ergebnisse können dann bestenfalls mittels Szenarientechnik auf ihre Robustheit geprüft werden.

\subsubsection{Ersparnis und Wachstum der Volkswirtschaft}

Die These, ein Übergang zur Kapitaldeckung würde mit Wachstumsimpulsen verbunden sein, gründet sich vornehmlich auf neoklassisches Gedankengut, demzufolge die induzierte erhöhte Ersparnisbildung das produktive Realkapital der Volkswirtschaft anwachsen läßt. In Anbetracht der Kritik die gegen diese Argumentationskette geäußert wurde, erweist sich die gängige Wachstumsthese als äußerst zweifelhaft. Sozialpolitisch relevante

489 Darunter fallen beispielsweise Veränderungen des Anteils der Aktien bzw. der Staatstitel an den gesamten Finanzaktiva, die Relation von gehaltenen inlāndischen zu ausländischen Finanztitel, die Liquidität des Options- und Aktienhandels, das Verhältnis der Finanzaktiva in privater Hand zu den Finanzaktiva institutioneller Anleger etc. Einige Ansätze hierzu finden sich bei Langmante/Nogler-Ludwig (1997), S.39ff.. Dabei werden allerdings die Erhöhung der Liquidität des Kapitalmarkts, die vermehrte Steuerung von Finanzierungssmitteln über Finanzinstitutionen und eine vermehrte Anlage in Eigenkapital "quasi automatisch" unterstellt. 
Entscheidungen, wie eine partielle Substitution des Verfahrens zur Finanzierung der Renten, sollten sich nicht auf solch ungesicherte bzw. unwahrscheinliche Wachstumseffekte stützen.

Wenn jedoch an der neoklassischen Makroökonomie die Prämisse vollkommener Märkte bemängelt wurde, so muß diese Kritik auch gegen die Annahme reibungslos funktionierender Finanzmärkte vorgebracht werden. Sofern nicht die extreme Sichtweise geteilt wird, daß der Finanzsektor lediglich ein „Schleier“ sei, der keinerlei Auswirkungen auf die realwirtschaftlichen Prozesse habe, ${ }^{491}$ können Finanzinstitutionen einen wesentlichen Beitrag zum Abbau von effizienzmindernden Friktionen ${ }^{492}$ leisten und gleichermaßen wachstums- und wohlfahrtssteigernd wirken.

Etwaige wohlfahrtssteigernde Wirkungen infolge einer effizienteren Finanzintermediation, könnten in der neoklassischen Wachstumstheorie allenfalls pauschal durch die Annahme einer veränderten Produktionstechnologie (steigende Produktivität des Faktors Kapital) oder ebenso pauschal durch die Annahme einer vermehrten Kapitalakkumulation abgebildet werden. Beides würde lediglich das Niveau des Wachstumspfads nicht jedoch die langfristige Wachstumsrate der Volkswirtschaft beeinflussen. Demgegenüber bieten die endogenen Wachstumstheorien einen theoretischen Rahmen, der es erlaubt, die durch eine effizientere Kapitalmarktallokation hervorgerufenen Wohlfahrtseffekte mit langfristigen Wachstumseffekten zu verknüpfen. ${ }^{493}$ Solche theoretischen Ansätze werden zunehmend auch durch empirische Arbeiten flankiert, in denen die Korrelation zwischen Wirtschaftswachstum und dem Entwicklungsgrad der Finanzintermediation ermittelt wird. ${ }^{494}$

Wenn sich folglich bei einer partiellen Substitution des Umlageverfahren durch eine kapitalfundierte Finanzierung positive Effekte auf die Gestaltung von Finanzinstitutionen eruieren lassen, so kann die Wachstumsthese des Kapitalstockverfahrens mithin durch das Zustandekommen einer effizienteren Kapitalallokation (zumindest teilweise) "gerettet" werden.

Ausgangspunkt einer solch modifizierten Wachstumsthese ist nach wie vor ein Spareffekt des Kapitalstockverfahrens. Infolge einer verstärkten gesamtwirtschaftlichen Ersparnis werden mehr Finanzierungsmittel über den Kapitalmarkt gelenkt, was mit positiven externen Effekten für den Entwicklungsgrad der Finanzintermediation verbunden ist. Die Zu-

490 Eine mikroökonomische Analyse möglicher Auswirkungen einer partiell kapitalfundierter Finanzierung der Renten auf die Entwicklung innovativer Finanzprodukte befindet sich jedoch bei Davies. Vgl. Davies (1993), S. 36-40.

491 Vgl. Lucas (1988).

492 Eine wesentliche Rolle spielen beispielsweise Informationsasymmetrien bei der Kreditvergabe. Durch die Übernahme der Monitoring-Funktion durch den Finanzintermediär können u.a. die Kontroll- bzw. allgemeiner die Transaktionskosten verringert werden. Vgl. zu entsprechenden Modellen Diamond (1984) und (1991); Williamson (1986); Allen (1993).

493 Solche Ansătze finden sich beispielsweise bei Greenwood/Jovanovic (1990); Boyd/Smith (1992); Bencivenga/Smith (1991); Ireland (1994). Einen Überblick über die Literatur bietet Pagano (1993).

494 Vgl. King/Levine (1992) und (1993); Atje/Jovanovic (1993); Neusser/Kugler (1994). Zu den Problemen solcher empirischen Untersuchungen treten neben der Frage nach adăquaten Indikatoren auch die grundsätzliche Frage nach dem hinter der Korrelation stehenden kausalen Zusammenhang.

220 
sammenhänge, die zu einer verbesserten Allokationsfunktion des Kapitalmarktes führen, gründen sich vornehmlich darauf, daß der Kapitalmarkt erstens durch die zusätzlichen Finanzierungsmittel aus der Altersvorsorge liquider wird und zweitens die Rolle des Eigenkapitals gestärkt wird. ${ }^{495}$

Naheliegend ist, daß eine höhere Liquidität die Gründung zusätzlicher Institutionen wie Rating-Agenturen, Informationsbroker etc. fördert, und somit die Transparenz und den Organisationsgrad des Kapitalmarktes erhöht. Auch positive Impulse auf die Entwicklung neuer Finanzinstrumente dürtten zu erwarten sein. Ferner wird davon ausgegangen, daß mehr Finanzströme über Finanzinstitute geleitet werden. Dies gewährleistet nicht nur eine professionellere und somit effizientere Kapitalallokation, sondern führt auch dazu, daß der in Eigenkapital investierte Anteil der Finanzmittel im Vergleich zum Portfolio privater Haushalte steigt. Dies wiederum erleichtert vor allem (innovativen) kleinen und mittleren Betriebe den für risikoreiche Expansion notwendigen Zugang zur Eigenkapitalfinanzierung. Nicht zuletzt wird auch auf den Anreizeffekt auf das Management verwiesen, der mit einer höheren Eigenkapitalquote verbunden sei.

Insbesondere die makroökonomische Bedeutung des letzten Aspekts dürtte strittig sein. Auch die Verfügbarkeit von Risikokapital für kleinere und mittlere Unternehmen hängt teilweise wieder von dem Liberalisierungsgrad und der internationalen Verflechtung der Kapitalmärkte ab. Welches Gewicht den einzelnen Argumenten daher zukommt, dürfte sich letztlich nur empirisch beantworten lassen.

Der Versuch einer solchen empirischen Überprüfung der Auswirkungen eines Teilkapitalstocks zur Finanzierung der Renten findet sich beispielsweise in einer Studie des lfoInstituts. Die Autoren schätzen die Auswirkungen verschiedener Finanzindikatoren ${ }^{496}$ auf die totale Faktorproduktivität und kommen zu dem Ergebnis, daß durch einen Teilkapitalstock von 100 Mrd. DM sich eine Steigerung des Wachstums um 0,2 -0,25\% erreichen läßt.

Bei diesen Ergebnissen muß allerdings auf einige Schwächen der Schätzgleichung hingewiesen werden. ${ }^{497}$ Die Autoren weisen ferner zu recht darauf hin, daß die Ergebnisse aufgrund des kräftigen Wachstumsschubs der Wiedervereinigung verzerrt werden. Dieser Effekt spiegelt sich nicht nur in den niedrigen Bestimmtheitsmaßen wider, sondern verdeutlicht das grundsätzliche Problem des kausalen Zusammenhangs, sprich welche Parameter als zu erklärende und erklärende Variablen angesetzt werden sollten. Auf den

495 Vgl. für die nachfolgenden Wirkmechanismen Langmantel/Nogler-Ludwig (1997), S. 38.

496 Zu den Finanzindikatoren gehören 1) der finanzielle Überbau gemessen am Quotienten aus finanziellen Passiva einer Volkswirtschaft zum Kapitalstock 2) Verhältnis der Finanzpassiva der Finanzinstitute zum Gesamtfinanzpassiva und 3) der Aktienanteil an den Gesamtfinanzpassiva.

497 So konzidieren die Autoren, daß die einbezogenen Variablen die totale Faktorproduktivität in Deutschland nicht allzu gut erklären. Da die derzeitige Arbeitslosigkeit vorrangig nicht konjunkturell bedingt ist, dürtte inre Venwendung als Proxivariable für die Konjunktur die Ergebnisse der Schătzung massiv beeinflussen. Grundlegend stellt sich weiter die Frage nach der geeigneten wirtschaftstheoretischen Fundie- 
Zusammenhang zwischen Teilkapitalstock und Finanzmarktindikatoren wird nur unzureichend eingegangen. Die Erhöhung der Liquidität des Kapitalmarkts, die vermehrte Steuerung von Finanzierungssmitteln über Finanzinstitutionen und eine vermehrte Anlage in Eigenkapital werden „quasi automatisch“ unterstellt. ${ }^{498}$ Wenngleich ihre Studie sicherlich als weiterer Indiz dafür gewertet werden kann, daß ein Zusammenhang zwischen dem Entwicklungsgrad der Finanzintermediation und dem Wirtschaftswachstum besteht, erscheinen die ermittelten Elastizitäten als eine zu grobe Schätzung, um sie als eindeutige, quantitative Richtwerte heranzuziehen.

Am Rande sei noch darauf hingewiesen, daß auch bei der "modifizierten Wachstumsthese" die internationale Verflechtung der Kapitalmärkte nicht unerheblich ist. Denn sofern Finanzdienstleistungen international gehandelt werden können, kann es zu einer zumindest teilweisen Entkopplung des Entwicklungsgrad der Finanzintermediation einer Volkswirtschaft von den Auswirkungen auf den Wachstumsprozeß dieser Volkswirtschaft kommen.

Die Versuche, diejenigen Wachstumseffekte des Kapitalstockverfahrens zu quantifizieren, die sich aus den positiven externen Effekten auf die Finanzintermediation einer Ökonomie ergeben, sind folglich mit dem selben Problem konfrontiert, wie der Versuch des vorangehenden Abschnitts, etwaige Verzinsungseffekte zu operationalisieren. Bislang sind die Konsequenzen einer zusätzlichen Kapitalfundierung der Altersvorsorge auf die Kapitalmarktstrukturen und -institutionen der Bundesrepublik Deutschland mikro- und makroökonomisch noch nicht hinreichend erforscht.

Möchte man etwaige Wachstumseffekte daher simulieren, ließen sich als Ausgangsbasis zwar die vom Ifo-Institut ermittelten Ergebnisse verwenden, diese dürtten jedoch nur als ein Szenario berücksichtigt werden und müßten ebenfalls durch Variationen ergänzt werden.

\subsubsection{Auswirkungen auf den Verteilungsindikator der intergenerativen Gerechtigkeit}

In der modelltheoretischen Analyse der Finanzierungsverfahren wurde darauf hingewiesen, daß eine Generation im Umlageverfahren dann einen positiven (negativen) Lebensnettotransfer erhält, wenn das Lohnsummenwachstum dauerhaft über (unter) dem Zinsfaktor liegt. Aufgrund dieser Zusammenhänge wurden der Lebensnettotransfer und die Rendite als äquivalente Umverteilungskriterien eines Alterssicherungssystem angesehen.

rung, sprich inwieweit Ansätze aus der endogenen Wachstumstheorie in der Schätzgleichung berücksichtigt werden sollten.

498

Hinsichtlich des relativ höheren Anteils an Anlagen in Eigenkapital der Lebensversicherung und Fonds müßte ebenfalls die Ursache des „Knicks“ ab 1989 geklärt werden (vgl. Langmante/Vogler-Ludwig (1997) Abbildung 2.4.8, S. 42). Die Frage nach eventuellen Verhaltensänderungen der Anleger wird nicht gestellt, insbesondere bleibt ungeklärt, warum der Aktienanteil auf $50 \%$ der Fondsmittel anwachsen wird. 
Wenn nun die durch ein spezifisches Finanzierungsverfahren induzierten indirekten Wachstumseffekte ebenfalls in die Gerechtigkeitsanalyse einfließen sollen, muß das allgemeine Wohlfahrtsniveau ebenfalls ins Kalkül gezogen werden. Die Gerechtigkeitsindikatoren "Lebenseinkommen“ (bzw. Lebensnutzen) und "Rendite“ sind dann nicht mehr identisch. Solange sowohl Beitragszahlungen als auch Rentenleistungen an die aktuellen Erwerbseinkommen und somit zumindest tendenziell an die allgemeine Wirtschaftsentwicklung gekoppelt sind, vermag der an die Rendite angelehnte Verteilungsindikator (vgl. Gleichung G 62) daher nicht (bzw. nur bedingt und über die veränderte Verzinsung), diese gesamtwirtschaftlichen (Wachstums-)Effekte zu erfassen. Eine alternative Normierung des Rentenniveaus oder des Beitragssatzes verschafft auch keine Abhilfe, da in dem Fall Zähler und Nenner des Indikators sich gleich verändern.

Da das Ausmaß der intergenerativen Umverteilung aus dem Vergleich mit einem Referenzsystem ermittelt wird und entweder die Beitrags- oder die Leistungsseite des Referenzsystems, sowie aller Reformoptionen entsprechend normiert sind, ließen sich etwaige Wachstumseffekte in die Umverteilungsanalyse problemlos integrieren, wenn der Verlauf der Beitragssätze als Verteilungsindikator herangezogen wird. In einem OLG-Modell mit zwei oder drei Generationen bereitet dies wenig Probleme. ${ }^{499}$ Bei einer Simulationsrechnung wie sie hier angestrebt wird, muß der Verlauf des Beitragssatzes jedoch auf eine Kenngröße verdichtet werden. Zudem müßte der Ansatz weiterentwickelt werden, um die Wohlfahrtsgewinne, von denen ein Versicherter in einem Mischsystem auch im Ruhestand noch profitiert, ebenfalls zu erfassen.

Aus diesem Grund wird hier ein anderer Ansatz gewählt. Der Verteilungsindikator, der das Verhältnis aus empfangenen Leistungen zu gezahlten Beiträgen beschreibt, wird zusätzliche um einen Niveauparameter ergänzt.

Wenn die Versicherten infolge höherer Einkommen auch indirekt von der Reform profitieren, ist die Beitragsbelastung bei gleichen absoluten Beiträgen relativ geringer. Im Verteilungsindikator wird dies durch fiktive, niedrigere Beiträge berücksichtigt, die durch Multiplikation mit einem Niveauparameter $(\psi)$ ermittelt werden. Der Niveauparameter dient als Indikator des Wohlfahrtseffekts und bestimmt sich aus dem Verhältnis der je nach Reformoption erzielbaren durchschnittlichen Nettoeinkommen. ${ }^{500}$

$$
\begin{gathered}
B_{t}^{\mathrm{mod}}=\psi_{t} \cdot B_{t}, \text { mit } \\
\psi_{t}=\frac{w_{t}^{S Q}}{w_{t}^{V G L}}
\end{gathered}
$$

$w_{1}^{\text {so }}=$ Nettolohn im Status Quo Szenario in der Periode t

$w_{L}{ }^{\text {VGL }}=$ Nettolohn im Vergleichsszenario in der Periode $t$

499 Vgl. Bösch (1987).

500 Alternativ dazu könnte auch der Quotient der Beitragssätze als Niveauparameter gewählt werden. Das Ergebnis bliebe zwar gleich, allerdings müßte zunächst die Veränderung des Beitragssatzes, die sich aus dem Wachstumseffekt ergibt von der Veränderung separiert werden, die eine Folge einer Differenz bei der Verzinsung ist $\left(I \neq G^{*} M\right)$. 


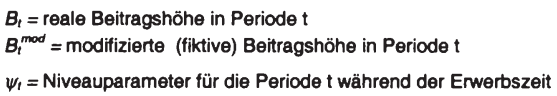

Bei einem reinen Umlageverfahren nimmt dieser Niveauparameter den Wert 1 an. Bei einem reinen Kapitalstockverfahren sind die Rentenleistung unabhängig vom Einkommen, so daß lediglich der Nenner korrigiert werden müßte. Sobald jedoch ein Mischsystem betrachtet wird, profitiert der Versicherte im Ruhestand weiterhin nach Maßgabe des umlagefinanzierten Anteils von den steigenden Einkommen. Dieser Effekt ließe sich im Zähler des Verteilungsindikators durch fiktive, höhere Rentenleistungen berücksichtigen.

$$
R L_{t}^{\bmod }=\xi_{t} \cdot R L_{t}
$$

$$
\begin{aligned}
& R L_{t}=\text { reale Höhe der Rentenleistung in Periode t } \\
& R L^{\text {mod }}{ }_{t}=\text { modifizierte (fiktive) Höhe der Rentenleistung in Periode t } \\
& \xi_{t}=\text { Niveauparameter für die Periode t während des Ruhestands }
\end{aligned}
$$

Die Veränderung des Nettoeinkommens darf dabei nur nach Maßgabe des umlagefinanzierten Anteils der Altersvorsorge in den Niveauparameter einfließen.

$$
\xi_{t}=1+\theta_{t} \cdot\left(\frac{w_{t}^{V G L}}{w_{t}^{S Q}}-1\right)
$$

$\theta_{t}=$ Anteil der umlagefinanzierten Rentenleistung an der gesamten Rentenleistung

Mit dieser Erweiterung nimmt der Verteilungsindikator (Gleichung G 62) die folgende Form an:

$$
V I_{x}=\frac{\sum_{t=R E}^{T}\left(R L_{t} \cdot \xi_{t} \cdot \frac{\left(1-b_{t} \cdot F A K_{t}\right)}{\left(1-b_{t}\right)} \cdot \prod_{v=R E}^{t}\left(1+d_{v}\right)^{-1}\right)}{\sum_{t=0}^{R E-1}\left(B_{t} \cdot \psi_{t} \cdot F A K_{t} \cdot \prod_{v=t}^{R E-1}\left(1+d_{v}\right)\right)} .
$$

\subsection{Arbeitsmarkteffekte der Alterssicherungssysteme}

Arbeitsmarkteffekte können danach unterschieden werden, ob sie an der Nachfrageseite oder der Angebotsseite des Arbeitsmarktes ansetzen. Beide Formen prägen die Diskussion um die gesetzliche Alterssicherung.

Nachfrageeffekte entstehen in erster Linie, weil die Sozialversicherungsbeiträge eines Umlageverfahrens Teil der Lohnnebenkosten sind: Steigen die Beiträge, verteuert sich der Faktor Arbeit. Angesichts des zunehmenden globalen Wettbewerbs sind die Unternehmen zu Kosteneinsparungen gezwungen, so daß hohe Lohnnebenkosten zwangsläufig zur Lohnreduzierung oder zum Arbeitsplatzabbau führen. ${ }^{501}$ Dabei muß allerdings auch erwähnt werden, daß die Wettbewerbsfähigkeit zum einen nicht ausschließlich von den 
Lohnkosten, sondern auch von Produktivitätsgewinnen, Produktinnovationen und anderen Faktoren abhängt und zum anderen die Lohnnebenkosten nicht nur durch Abgaben zur Sozialversicherung verursacht werden. Eine wichtige Rolle bei der Beurteilung der Nachfrageeffekte auf dem Arbeitsmarkt spielen die Überwälzungsmöglichkeiten der Unternehmen. ${ }^{502}$ Wälzen die Unternehmen die höheren Kosten durch Preiserhöhungen oder Lohnsenkungen (bzw. ausbleibenden Lohnerhöhungen) auf die Konsumenten respektive die Arbeitnehmer über, so werden die anteilsmäßig vom Arbeitgeber finanzierten Sozialleistungen faktisch von den Konsumenten und Arbeitnehmern getragen. Die effektiven Lohnkosten blieben konstant. In welcher Größenordnung sich die Vor- oder auch Rückwälzungsprozesse abspielen, hängt von den Angebots- und Nachfrageelastizitäten auf dem Arbeitsmarkt und den Produktmärkten ab. In dieser Arbeit wird unterstellt (vgl. Abschnitt 4.3), daß die Arbeitgeberbeiträge „rückgewälzt“ werden, d.h. die Arbeitnehmer müssen bei steigenden (Arbeitgeber-)Beiträgen niedrigere Reallöhne akzeptieren. Da die effektiven Arbeitskosten der Unternehmen sich nicht verändern, werden die Nachfrageeffekte in dieser Arbeit nicht modelliert.

Die weiteren Ausführungen konzentrieren sich daher auf die von einem Finanzierungsverfahren ausgehenden Angebotseffekte. Auswirkungen auf das Arbeitsangebot ergeben sich zum einen dadurch, daß für die Arbeitnehmer mit der Höhe der Sozialabgaben auch der Anreiz steigt, sich dem Zugriff zu entziehen und verstärkt in die Schattenwirtschaft abzuwandern. ${ }^{503}$ Ein weiterer Effekt ist weniger offenkundig, wird aber vor allem in jüngster Zeit in die Debatte um das Umlageverfahren eingebracht ${ }^{504}$ : der „excess burden“Effekt.

Der Begriff „Excess burden“ stammt aus der Finanzwissenschaft und bezeichnet die Zusatzlast, die durch eine Einführung oder Erhöhung eines Steuersatzes entsteht. Betrachtet man in einem einfachen Marktmodell die aggregierte fallende Nachfragekurve und die aggregierte steigende Angebotskurve, so wird deutlich, daß einige Konsumenten ihre Güter zu niedrigeren Preise erhalten, als sie zu zahlen bereit wären. Einige Anbieter erzielen für ihre Güter höhere Preise, als sie fordern würden. Diese Anbieter und Nachfrager erzielen bei einer Transaktion zusätzliche (allerdings nicht materielle) „Gewinne“: die ökonomischen Renten. Wenn in diesem Modell auf das betrachtete Gut eine Steuer in Form eines Steuersatzes erhoben wird (die beispielsweise von Nachfragern und Anbietern getragen wird), so verändern sich die effektiven Preise. D.h. der Verlauf der Nachfrage- und Angebotsfunktionen ändert sich. Die durch die Steuern erzielten Einnahmen sind allerdings geringer, als die Verringerung der ökonomischen Renten der Konsumenten und der Produzenten. Diese Differenz wird als „excess burden“ bezeichnet. Sie stellt einer Verringerung der Wohlfahrt einer Volkswirtschaft dar. Die Zusammenhänge werden wie folgt auf die Alterssicherung übertragen: Die Beiträge zur gesetzlichen Alterssicherung entsprechen den Steuern, die die effektiven Preise auf dem Arbeitsmarkt (Löhne) beein-

\footnotetext{
502 Vgl. Abschnitt 4.3.

503 Vgl. Schmähl (1986).

504 Vgl. z.B. Dudey (1996), S. 122ff.; Feldstein (1996); Homburg/Richter (1990).
} 
flussen: Für die Versicherten vergrößert sich der Abstand zwischen Brutto- und Nettolohn, für den Arbeitgeber steigt der Effektivlohn. ${ }^{505}$ Selbst wenn ein vollkommen unelastisches Arbeitsangebot vorläge, käme es zu excess-burden-Effekten, da das entscheidende Kriterium die Veränderung der relativen Preise ist (so würde das Gut „Freizeit“" teuerer bzw. billiger werden). Es ist ein Standardergebnis der Finanzwissenschaft, daß excess-burdenEffekte der Besteuerung nur durch eine Kopfsteuer vermieden werden.

Inwieweit der Beitragssatz zur Rentenversicherung das Arbeitsangebot der Versicherten beeinflußt, hängt davon ab, wie der Versicherte die Beiträge wahrnimmt. Betrachtet er sie als Zahlung für eine spätere Gegenleistung, ändert er sein Verhalten nicht bzw. nur gering. Empfindet er die Beiträge als Steuern, beeinflussen sie sein Angebot an Arbeit. Oft wird argumentiert, daß die Beiträge zumindest in dem Maße als Steuern aufgefaßt werden, in dem die zukünftigen Leistungen von einer marktüblichen Verzinsung abweichen. ${ }^{506}$ Zuweilen wird angeführt, die Regeln der Rentenversicherung seien so komplex und intransparent, daß einige Versicherte die gesamten Beiträge als Steuern wahrnähmen. ${ }^{507}$

HOMBURG und RichteR schätzten das Ausmaß solcher durch den „Steuersatz der Alterssicherung" entstehenden excess-burden-Effekte für Deutschland auf 36 Milliarden DM per anno. ${ }^{508}$ FELDSTEIN betont, daß excess-burden-Effekte ${ }^{509}$ i.d.R. höher ausfallen, als man auf den ersten Blick vermuten mag, da die Beiträge zur Sozialversicherung zum einen zusätzlich zu den übrigen Steuern erhoben werden und die Effekte nicht linear, sondern quadratisch von dem Steuersatz abhängen. Zum anderen äußert sich die Zusatzlast nicht nur in der Verzerrung des zeitlichen Arbeitsangebots. Es ändert sich auch das „Konsumverhalten" der Versicherten, da die Arbeitnehmer eine monetäre Entlohnung zunehmend durch steuerfreie Leistungen, gute Arbeitsbedingungen o.ä. zu ersetzen suchen. $\mathrm{Er}$ schätzt daher den Umfang der gesamten Zusatzlast für die USA im Jahre 1995 auf 68 Mrd. US\$, wobei sich diese Größe nicht allein auf die Alterssicherung, sondern auf das gesamte umlagefinanzierte Sozialsystem bezieht. ${ }^{510}$

Solche Schätzungen sind allerdings mit einer Reihe von Unsicherheiten verbunden. Denn die Annahmen über die Elastizität des Arbeitsangebots und insbesondere über den Verlauf der Indifferenzkurven der Versicherten und die Substitutions- und Einkommenseffekte bestimmen den Umfang der "Last" im relevanten Maße. Vor allem handelt es sich um "nichtmaterielle Gewinne“, die aus den Opportunitätskosten abgeleitet wurden. Es stellt sich somit das Problem, wie man diese Gewinne materialisieren sollte. Eine Operationalisierung im Sinne einer kardinalen Nutzentheorie ist äußerst strittig und birgt die Gefahr,

\footnotetext{
505 Werden die in dieser Arbeit getroffenen Annahmen über Überwälzungsprozesse berücksichtigt, würden die Beitragssātze lediglich die Versicherten, d.h. das Arbeitsangebot, beeinflussen. 
einen allzu willkürlichen „Jocker“ in die Analyse einzubauen, mit dem die Ergebnisse der Simulationen (faßt beliebig) gesteuert werden können.

In der nachfolgenden Analyse werden die hier diskutierten Verzerrungen des Arbeitsangebots daher nicht modelliert.

\subsection{Zusammenfassung}

Die modelltheoretische Analyse des zweiten Kapitels zeigte, daß die Finanzierungsverfahren zur Altersvorsorge und verschiedene, gesamtwirtschaftliche Parameter sich gegenseitig beeinflussen. In den vorstehenden Ausführungen wurde zunächst geprüft, inwieweit die abgeleitete These vom Ersparnis- und Wachstumseffekt des Kapitalstockverfahrens valide bleibt, wenn von den dort unterstellten restriktiven Annahmen abstrahiert wird.

Die Kritik am Vermögenssubstitutionseffekt zeigte, daß es eine Reihe mikro- und makroökonomischer Einwände gibt, die das Ausmaß des Vermögenssubstitutionseffekts auf die private Ersparnisbildung in relevanten Maße einschränken. Gegen die Wachstumsthese bestehen ebenfalls investitions- und modelltheoretische Einwände. Beide Prämissen der Wachstumseffekte, die erhöhte Ersparnisbildung als auch ihre Transformation in produktives Realkapital, sind mithin umstritten. Da aus der theoretischen Analyse (bislang) keine eindeutigen Schlüsse über die Wirkung des Umlageverfahrens auf die Sparquote bzw. auf das Wachstum abgeleitet werden können, ist diese These nur empirisch zu falsifizieren. Die Auswertung verschiedener Untersuchungen ergab: die empirischen Befunde sind ebenfalls diffus und erlauben keine eindeutige Schlußfolgerung.

In einem weiteren Schritt wurde untersucht, wie die indirekten Effekte berücksichtigt werden können, wenn die intergenerative Gerechtigkeit verschiedener Optionen zur Reform der Alterssicherung bewertet wird. Die Ergebnisse lassen sich wie folgt resümieren:

- Eine Integration wahlhandlungstheoretischer Ansätze zur Bestimmung der gesamtwirtschaftlichen Ersparniseffekte wurde ebenso abgelehnt, wie der Rekurs auf eine einfache neoklassische Produktionsfunktion zur Ermittlung von Zins- und Wachstumseffekten.

- Die Auswirkung einer partiellen Kapitaldeckung auf die gesamtwirtschaftliche private Ersparnis wird in der Simulation durch pauschal getroffene Annahmen berücksichtigt. Dabei wird der Vermögenssubstitutionseffekt jedoch nicht im Verhältnis 1:1 wirksam. Die Ergebnisse werden mittels alternativer Szenarien auf ihre Robustheit geprüft.

- Die Folgen einer höheren Ersparnis auf die interne Verzinsung des Kapitalstockverfahrens werden in gleicher Weise durch gesetzte Annahmen und Sensitivitätsanalysen erfaßt.

- Wachstumseffekte resultieren ausschließlich deshalb, weil das Kapital infolge einer verbesserten Finanzintermediation effizienter alloziiert werden kann. Da einschlägige 
mikro- und makroökonomische Untersuchungen zu den Auswirkungen einer zusätzlichen Kapitalfundierung der Altersvorsorge auf den Entwicklungsgrad der Finanzintermediation für Deutschland bislang nicht verfügbar sind, muß auch diesbezüglich auf die Szenarientechnik ausgewichen werden. Die Ergebnisse der makroökonometrischen Schätzung des Ifo-Instituts dienen dabei als Ausgangsbasis.

Diese Vorgehensweise mag unbefriedigend erscheinen, da die indirekten Wirkungen ausschließlich durch exogene Annahmen erfaßt werden und die nachfolgende Simulationsanalyse somit anfechtbar bleibt.

Solange der Wissenschaftsstreit um das Ausmaß der Ersparniswirkung des Kapitalstockverfahrens jedoch nicht geklärt ist und sich die Zusammenhänge zwischen Anpassungsvorgängen auf dem Kapitalmarkt und dem gesamtwirtschaftlichen Wachstum nicht endogen in Rahmen einer konsistent formulierten Theorie erklären lassen, bietet sich aber keine überlegene alternative Vorgehensweise. Häufig werden etwaige Wachstumsimpulse in Modellen neoklassischer Provienienz mittels einer einfachen Cobb Douglas Produktionsfunktion simuliert. Im Lichte der dargelegten Kritik dürfte jedoch unstrittig sein, daß dieser Ansatz ebenso willkürbehaftet ist, wie jede ad-hoc Annahme. In diesem Zusammenhang ist die Äußerung Grohmanns nach wie vor aktuell:

Es sollte "letztlich verlockender sein, mit unvollkommenen Mitteln die wirkliche Welt zu analysieren als mit vollkommenen eine nur hyphothetische. ${ }^{\text {511 }}$

Abschließend wurden die mit einem Umlageverfahren verbundenen Arbeitsmarkteffekte diskutiert. Angesichts der in Abschnitt 4.3 getroffenen Annahme, daß die Arbeitgeberbeiträge von den Arbeitnehmern zu tragen sind, werden etwaige Nachfrageeffekte nicht modelliert. Eine Operationalisierung von angebotsseitigen excess-burden-Effekten im Sinne einer kardinalen Nutzentheorie wurde aus methodischen Gründen abgelehnt, sie fließen ebenfalls nicht in die Analyse ein.

511 Grohmann (1977), S. 367 zitiert nach Wagner (1984). 


\section{SIMULATION AUSGEWÄHLTER REFORMOPTIONEN}

\subsection{Beschreibung der Reformoptionen}

Das in dieser Arbeit entwickelte Konzept wird im folgenden exemplarisch angewendet, um die intergenerativen Verteilungswirkungen verschiedener Gestaltungsmöglichkeiten des Alterssicherungssystems in Deutschland zu bewerten. Neben der Bewertung des derzeitigen Systems der gesetzlichen Rentenversicherung werden Reformoptionen betrachtet, die sich einerseits auf eine pauschale Substitution des Finanzierungsverfahrens gründen und andererseits auf das Rentenreformgesetz 1999, das eine demographische Korrekturkomponente in die Rentenformel integriert.

\section{Pauschale Substitution des Finanzierungsverfahrens:}

Reform 1: "Korrektur der Anwartschaften“"

Sukzessive Substitution des Umlage- durch das Kapitalstockverfahren beginnend ab dem Jahr 2000. Bereits erworbene Anwartschaften werden nicht gekürzt. Die neu erworbenen Anwartschaften werden allmählich über einen Zeitraum von 20 Jahren derart korrigiert, daß ein Versicherter im Jahre 2020 einen halben Entgeltpunkt erwirbt, wenn er ein Jahr lang zum Durchschnittseinkommen Beiträge bezahlt hat. Ein Durchschnittsverdiener würde dann im Jahr 2000 0,975 Entgeltpunkte, im Jahr 2001 0,95 Entgeltpunkte etc. erwerben. Der Eckrentner des Rentenzugangs 2010 würde dann 43,625 Entgeltpunkte angesammelt haben. Erst beim Rentenzugang 2065 wäre die Umstellung vollständig vollzogen, d.h. diese Zugangskohorte würde dann lediglich $50 \%$ der Leistungen des derzeitigen Systems aus der umlagefinanzierten Rentenversicherung beziehen. Die Differenz wäre durch eine (private) kapitalgedeckte Rentenvorsorge zu decken.

Reform 2: "Senkung des Rentenniveaus“

Sukzessive substitutiion des Umlage- durch das Kapitalstockverfahren beginnend ab dem Jahr 2000, indem das Nettorentenniveau über einen Zeitraum von 20 Jahren pro Jahr um ein Prozentpunkt gesenkt wird. Die Differenz wird durch ein Kapitalstockverfahren abgesichert.

Integration einer demographischen Korrekturkomponente in die Rentenformel

Reform 3: „Reform 99“

Berücksichtigung einer demographischen Komponente in der Rentenformel entsprechend des Rentenreformgesetzes 1999. Auch hierbei wird unterstellt, daß die erwerbstätigen Generationen die entstehende „Versorgungslücke“ durch verstärkte kapitalfundierte Vorsorge ausgleichen.

229 
Die modifizierte Rentenanpassung des Rentenreformgesetzes 1999 basiert auf der folgenden Fortschreibungsformel:

$$
\begin{aligned}
a R W_{t}=a R W_{t-1} \cdot \frac{B L G S_{t-1}}{B L G S_{t-2}} \cdot \frac{N Q_{t-1}}{N Q_{t-2}} \cdot \frac{R N Q_{t-2}}{R N Q_{t-1}} \cdot L E F_{t} & \text { G } 71 \\
& \text { mit } L E F_{t}=\frac{\left(\frac{R L E_{t-9}^{65}}{R L E_{t-8}^{65}}-1\right)}{2}+1 \\
a R W & =\text { aktueller Rentenwert } \\
B L G S & =\text { Bruttolohn- und -gehaltssumme je durchschnittlich beschäftigten Arbeitnehmer } \\
N Q & =\text { Nettoentquote des Arbeitsentgelts nach VGR } \\
R N Q & =\text { Rentennettoquote } \\
L E F & =\text { Lebenserwartungsfaktor } \\
R L E^{65} & =\text { Restlebenserwartung der 65jährigen (gewichtetes, arithmetisches Mittel aus Frauen } \\
& \text { und Männer, Ost und West) }
\end{aligned}
$$$$
\text { RNQ = Rentennettoquote }
$$

Eine systematische Berücksichtigung der steigenden Lebenserwartung in der Rentenversicherung hält die Summe der gewährten Rentenleistungen konstant und verteilt sie lediglich über die längere Bezugsdauer. Folglich reduzieren sich die monatlichen Bezüge, so daß sich Möglichkeiten für einen weiteren Ausbau der kapitalgedeckten Vorsorge ergeben. Da durch die Umsetzung der Reformmaßnahmen der Anstieg der Beitragssätze ebenfalls gedämpft werden kann, ergibt sich für den Versicherten auch ein finanzieller Handlungsspielraum zur privaten Vorsorge.

Der entscheidende Unterschied und Vorteil zu einer pauschalen Umstellung des Finanzierungsverfahrens der gesetzlichen Rentenversicherung besteht darin, daß der durch die gestiegene Lebenserwartung neu entstehende zusätzliche Sicherungsbedarf kapitalgedeckt finanziert wird. Auf diese Weise ließe sich ein Großteil der umstellungsbedingten Doppelbelastung der Übergangsgeneration vermeiden. Systematisch wäre dies vergleichbar mit der Finanzierung eines neu einzuführenden „Versicherungssystems“ mit dem zusätzlichen Vorteil, daß auch die Leistungen in diesem "System" erst sukzessive fällig werden. Es ist also durchaus möglich, mittels systemimmanenter Reformen die mit einer Umstellung des Finanzierungsverfahrens verbundenen Nachteile zu vermeiden und dennoch die Vorteile eines größeren Kapitalstocks zu nutzen.

Allerdings gilt dieser Vorteil für die Reform 3 nur bedingt. Systematisch korrekt wäre nämlich eine Korrektur der persönlichen Entgeltpunkte (PEP) nach Maßgabe kohortenspezifischer Lebenserwartungsfaktoren. Die Reform 3 modifiziert jedoch - aus pragmatischen Gründen und aufgrund der Akzeptanz bei den Versicherten - die Rentenanpassung mit Hilfe von periodischen Lebenserwartungsfaktoren. ${ }^{512}$

\footnotetext{
512 Vgl. Rürup/Schroeter (1996).
} 


\subsection{Spezifikation der Simulationsrechnung}

\subsubsection{Wahl des Diskontsatzes}

Der Diskontsatz kann als Zeitpräferenzrate ${ }^{513}$ interpretiert werden oder man betrachtet die Diskontierung als ein Vergleich der Altersvorsorge mit einer Verzinsung der Beiträge, die sich auf dem Kapitalmarkt erzielen ließe (Opportunitätskostenprinzip). Folgt man der zweiten Interpretation, entspricht der Diskontsatz in jeder Periode dem Kapitalmarktzins. Dieses Vorgehen wirt folgende Fragen auf:

- Welche Zinssätze werden angesichts des heterogenen und unvollkommenen Kapitalmarkts als Vergleichsgröße herangezogen?

- Wie wird bei den Vergleichsrenditen des Kapitalmarkts zwischen einem reinen Sparprozeß und einem Versicherungssystem mit Risikoausgleich abgegrenzt?

- Die in der Vergangenheit erzielten Renditen ergaben sich aus den damaligen Rahmenbedingungen und können bei ex ante Betrachtungen nicht auf die Zukunft übertragen werden. Welche Annahmen können daher für die Zukunft unterstellt werden?

- Welche Rückwirkungen des Alterssicherungssystems auf die Kapitalmarktrenditen müssen beachtet werden?

In dieser Arbeit werden die intergenerativen Verteilungswirkungen des Alterssicherungssystems bewertet, so daß zusätzlich zu diesen methodischen Unsicherheiten auch systematische Einwände dagegen sprechen, den Kapitalmarktzins als Diskontsatz anzusetzen:

1. Ein wesentliches Ziel der Rentenversicherung ist die Sicherung des Lebensstandards im Alter. Die Variation der Zins-Lohnwachstums-Differenz erwies sich als Risiko bei der Umsetzung dieses Ziels, von dem die Generationen im unterschiedlichen Maße betroffen sind. Werden die Beiträge und Leistungen mit dem Kapitalmarktzins diskontiert, kompensiert dies den durch die Zinsänderung induzierten Teil des Risikos und die unterschiedliche Behandlung der Generationen wird vom Verteilungsindikator nicht erfaßt.

2. HOMBURG plädiert in seiner Kritik an der Diskontierung zukünftiger Bedürfnisse für eine stärkere Orientierung an der Lebenszyklushypothese, bei der „das persönliche Lebensalter bestimmt, welcher Nutzen einem Zukunftsgut zugemessen wird. ${ }^{414}$ Eine Diskontierung nach Maßgabe der schwankenden Verzinsung des Kapitalstocks würde das Gegenteil bewirken: Für einige Generationen könnte in der Mitte ihres Lebens ein hoher, gegen Ende ihres Lebens ein niedriger Diskontsatz wirken, während für andere Gene- 
rationen in der Mitte ihres Lebens ein niedriger und in ihrem letzten Lebensabschnitt ein hoher Diskontsatz angewandt wird.

Aus den hier dargelegten Gründen werden die Beiträge und Leistungen der Generationen im Verteilungsindikator nicht mit den Kapitalmarktzinsen, sondern mit einer vorgegebenen Zeitpräferenzrate diskontiert. Grundsätzlich ist der Diskontsatz über die betrachtete Periode konstant, periodische Variationen werden nicht zugelassen. ${ }^{515}$ Die Barwerte werden aber auf den Zeitpunkt des Renteneintritts bezogen und es ist festzulegen, ob die Zinssätze zum Aufzinsen der Beiträge bzw. zum Abzinsen der Leistungen identisch sein sollen. In der vorliegenden Arbeit werden identische Diskontsätze unterstellt; die Diskontierung kann allerdings ohne weiteres auf differierende Beitrags- und Leistungssätze erweitert werden. Die Entscheidung über die Diskontierungsfunktion beeinflußt ebenfalls das Ergebnis. Die vorliegende Arbeit gründet sich - wie allgemein üblich - auf das lineare Standarddiskontmodell, ${ }^{516}$ obwohl eine nichtlineare Diskontierung theoretisch ebenfalls denkbar wäre. ${ }^{517}$

Nachdem der Diskontierungsmodus festgelegt ist, stellt sich die Frage welcher Wert der Diskontsatz annehmen soll. Jede (in die Zukunft gerichtete) Rentabilitätsrechnung muß hinsichtlich der Zins- und Diskontsätze sowie des Diskontierungsmodi mit Annahmen und Vereinfachungen arbeiten. Diese Annahmen können gewiß immer kritisiert und angefochten werden. Diese Schwäche ist jedoch unumgänglich und kennzeichnend für jede Art von Simulationsrechnung. Es kann lediglich versucht werden, die Annahmen soweit wie möglich plausibel zu begründen und die Robustheit der Ergebnisse durch Sensitivitätsanalysen zu überprüfen. Im Basisszenario wird daher ein Diskontsatz von 4 Prozent unterstellt, der durch Vergleichsrechnungen mit 5 Prozent ergänzt wird. ${ }^{518}$

\subsubsection{Beschreibung des Simulationsmodells}

Das Simulationsmodell ${ }^{519}$ gliedert sich in zwei Teile: die Prognose der Bevölkerungsentwicklung bis zum Jahr 2070 und die Prognose der gesetzliche Rentenversicherung. Der demographische Teil basiert auf der mittleren Variante der 8. koordinierten Bevölkerungsprognose des Statistischen Bundesamtes.

515 Möglich wäre, dem Vorschlag Homburgs zu folgen und keine periodischen Diskontsätze, sondern für jede Generation die gleichen, altersspezifischen Diskontsätze anzuwenden. Die vorliegende Arbeit basiert jedoch auf Zugangs- und nicht auf Geburtskohorten, so daß ein solches Vorgehen ausscheidet. Durch die Verwendung von einheitlichen Diskontsätzen kann allerdings der unerwünschte Effekt der Zinsschwankungen vermieden werden.

516 Vgl. Strotz (1955), S. 56, der zeigt, daß die Diskontierung mit dem Faktor $(1+d)^{t}(d=$ Diskontsatz, $t=$ Periode mit $t=0$ als Periode für die die Barwerte berechnet werden) im Gegensatz zu anderen Faktoren plankonsistent ist.

517 Vgl. Ahlbrecht/Weber (1995).

518 Ohsmann/Stolz (1997) arbeiten mit den Disskontsätzen 4 Prozent, 5,5 Prozent und 7 Prozent auf der Beitragsseite und 5,5 Prozent auf der Leistungsseite. Die 5,5 Prozent entsprächen der durchschnittlichen, langfristigen Kapitalverzinsung auf den deutschem Kapitalmarkt.

519 Für eine detaillierte Beschreibung des Modells siehe Bosduganov (1998).

232 
Im Modell der gesetzliche Rentenversicherung werden in einem ersten Schritt die Bestandsgrößen (Beitragszahler-Rentenbezieher) und in einem zweiten Schritt die Finanzpositionen bestimmt. Sowohl die Bestands- als auch die Finanzpositionen werden für die folgenden Kriterien getrennt ausgewiesen: Neue/Alte Bundesländer, Männer/Frauen, Arbeiter-/Angestelltenversicherung (ArV/AnV) sowie Knappschaftsversicherung (KnV). ${ }^{520}$ Sämtliche Daten werden pro Jahr und pro Altersgruppe berechnet.

Die Entwicklung der Erwerbs- und Arbeitslosenquoten werden exogen vorgegeben. Die Fortschreibung der Erwerbsquoten orientiert sich an den Annahmen des Prognosgutachtens von $1995 .{ }^{521}$ Die Versicherten werden für jedes Jahr in folgender Aufteilung ermittelt:

- pflichtversicherte Beitragszahler

- freiwillig versicherte Beitragszahler

- Arbeitslose, deren Beiträge von der Bundesarbeit für Arbeit gezahlt werden

- Bezieher von Altersrenten

- Bezieher von Hinterbliebenenrenten

- Bezieher von Berufs- und Erwerbsunfähigkeitsrenten.

Um die intergenerativen Verteilungswirkungen analysieren zu können, werden die Bezieher von Rentenleistungen als Zugangskohorten erfaßt und durch die Bestimmung der jährlichen Zu- und Abgänge getrennt fortgeschrieben. Der Rentenbestand bis 1995 wird „en bloc“ fortgeschrieben. Der gesamte Rentenbestand ergibt sich für jedes Jahr als Summe der Kohortenbestände und des Rentenbestands bis 1995. Die intergenerativen Wirkungen einer Reform werden daher nicht auf Basis von Geburtskohorten, sondern auf Basis von Zugangskohorten analysiert.

Sieben Positionen bilden die Einnahmeseite des Finanzmoduls:

- Beiträge der Pflichtversicherten

- Beiträge der freiwillig Versicherten

- Beiträge der Bundesanstalt für Arbeit

- Beiträge der gesetzlichen Krankenversicherung (GKV)

- Beiträge der gesetzlichen Pflegeversicherung (GPV)

- Sonstige Einnahmen

- Bundeszuschuß

Die Gesamtausgaben setzen sich aus fünf Positionen zusammen:

- Rentenzahlungen für Altersrenten

- Rentenzahlungen für Witwen( $r$ renten

- Rentenzahlungen für Waisenrenten

- Rentenzahlungen für Berufs- und Erwerbsunfähigkeitsrenten (EU/BU-Renten)

- Sonstige Ausgaben gestelltenversicherung. 
Der Beitragssatz der Arbeiter- und Angestelltenversicherten wird in einem iterativen Prozeß bestimmt, bei dem die gesamten Einnahmen und Ausgaben abgeglichen werden. Der Beitragssatz zur Knappschaftsversicherung verändert sich mit der gleichen rate wie der Beitragssatz zur Arbeiter- und Angestelltenversicherung.

Zu den Finanzpositionen im einzelnen:

Die Beiträge ergeben sich aus der Multiplikation der Faktoren Bestand, Beitragssatz und Bezugsgröße. Die Bezugsgrößen werden für das Ausgangsjahr 1995 aus dem finanziellen und statistischen Bericht des VDR für die Pflichtversicherten, freiwillig Versicherten und die Beiträge der Bundesanstalt für Arbeit ermittelt und mit der Bruttolohn- und -gehaltssumme fortgeschrieben. Der Bundeszuschuß wird ausgehend vom Startwert im Jahre 1995 entsprechend der gesetzlichen Bestimmungen fortgeschrieben. ${ }^{522}$ Die sonstigen Einnahmen sowie Beiträge der GKV und PKV werden so fortgeschrieben, daß das Verhältnis zu den Beitragseinnahmen konstant ist.

Die Rentenleistungen sind das Produkt aus den Rentenbeziehern und der Rentenhöhe, wobei die Rentenhöhe nach der folgenden Formel ermittelt wird:

$$
\begin{aligned}
& \quad R_{M B}=R A F \cdot P E P \cdot a R W \quad G 72 \\
& R_{M B}=\text { Monatsbetrag der Rente } \\
& \text { RAF = Rentenartfaktor } \\
& \text { PEP = Persönliche Entgeltpunkte } \\
& \text { aRW = aktuelle Rentenwert }
\end{aligned}
$$

\section{a) Rentenartfaktor}

Die Rentenartfaktoren der Altersrenten ergeben sich aus $\S 67$ SGB VI und $\S 82$ SGB VI (RAF = 1 für ArV/AnV, RAF = 1,3333 für KnV). Für die EU/BU-Renten wurden die gewichteten, gemittelten Werte nach Maßgabe der $§ 67$ SGB VI und § 82 SGB VI für das Ausgangsjahr 1995 bestimmt und konstant gehalten. ${ }^{523}$ Witwen- bzw. Witwerrenten haben in der ArV/AnV (KnV) einen Rentenartfaktor von $0,6(0,8)$, Waisenrenten von $0,1(0,1333)$

\section{b) Persönlichen Entgeltpunkte}

Ausgehend von den Rentenzahlbeträgen und dem durchschnittlichen Monatsbetrag des Jahres 1995 aus der Statistik des Verbands Deutscher Rentenversicherungsträger (VDR) sowie der Sonderauswertung des VDR, werden die PEP für 1995 bestimmt. Die PeP werden pro Altersklasse und Zugangskohorte mit der Veränderungen der Erwerbsquoten während der Erwerbszeit angepaßt. ${ }^{524}$ Analog wird die Veränderung der Arbeitslosigkeit berücksichtigt. Durch eine Abschätzung ${ }^{525}$ wird berücksichtigt, daß die

521 Vgl. Prognos (1995).

Vgl. § 213 SGB VI (alten Bundeslände); § 287 SGB VI (neue Bundeslănder). Für die Knappschaft besteht eine volle Defizitdeckung des Bundes (§ 215 SGB VI).

523 Durch die arithmetische Mittelung differieren die Rentenartfaktoren nach Altersklassen.

524 Vgl. Bosduganov (1998), Abbildung 6.

525 Vgl. Bosduganov (1998), S. 35. 
PEP der wegfallenden Renten des „Rentenbestands bis 1995“ niedriger sind als ihr Durchschnittswert.

c) aktuelle Rentenwert

Die Fortschreibung des aktuellen Rentenwerts erfolgt entsprechend der Formel des $\S$ 69 SGV VI. Für die Neuen Bundesländer wird er bis zur Herstellung einheitlicher Einkommensverhältnisse getrennt fortgeschrieben (§ 228b SGV VI).

Die Entwicklung der Bruttolohn- und -gehaltssumme wird exogen vorgegeben. Im Basisszenario wird sie in den Alten Ländern mit 3 Prozent und in den Neuen Ländern mit 4 Prozent per anno fortgeschrieben, bis sich die Werte entsprechen. Anschließend wird eine einheitliche jährliche Wachstumsrate von 3 Prozent unterstellt.

Der Lohnsteuersatz (exogener Parameter) bleibt für die Alten Länder auf dem Niveau von 1995 und verändert sich für die Neuen Länder, bis er im Jahre 2010 dem Satz der Alten Länder entspricht. Die exogene Entwicklung der Beitragssätze zur GKV und GPV werden vom Prognosgutachten übernommen. Die Beitragssätze zur Arbeitslosenversicherung entwickeln sich entsprechend der beiden Szenarien proportional zur Entwicklung der Arbeitslosenquote.

Der Anteil der Ausgaben für die KVdR und PVdR an den Rentenzahlungen wird für 1995 bestimmt und entsprechend der Entwicklung der Beitragssätze fortgeschrieben.

Die Entwicklung der sonstigen Ausgaben orientiert sich bei der Entwicklung der Verwaltungs- und Verfahrenskosten sowie den Rehabilitationsausgaben an der Prognose des ifo-Instituts ${ }^{526}$ und berücksichtigt zudem, daß einige der als „versicherungsfremd“ bezeichneten Leistungen in Zukunft wegfallen werden. Für die Alten Länder werden die Ausgaben so fortgeschrieben, daß der Zuschlagssatz zunächst bis 2020 und dann nochmals bis 2039 halbiert wird. In den Neuen Länder gleicht sich der Zuschlagssatz bis zum Jahre 2020 dem Wert der Alten Länder an.

\subsubsection{Modellierung der kapitalfundierten Altersvorsorge}

\section{A) Bestimmung des Kapitalgedeckten Anteils der Alterssicherungssystems}

Ein Vergleich der Verteilungswirkungen verschiedener Alterssicherungssysteme erfordert die Normierung entweder der Leistungs- oder der Beitragsseite. In der Simulationsanalyse wird die Leistungsseite normiert. Denn einerseits ist die Sicherung eines - wie auch immer definierten - Lebensstandards im Alter die Motivation für jede Altersvorsorge. Andererseits hat das Ziel der Lebensstandardsicherung im hier entwickelten Konzept zur Bewertung der intergenerativen Verteilungswirkungen eine besondere Bedeutung für den intergenerativen Risikoausgleich.

526

Vgl. Langmantel/Vogler-Ludwig (1997).

235 
Alle betrachteten Reformoptionen erfordern eine zusätzliche kapitalfundierte Vorsorge des Versicherten, um das ursprüngliche Versorgungsniveau im Alter aufrecht zu erhalten. Dabei stellt sich die Frage, mit welchen jährlichen Beiträgen der erforderliche Kapitalstock angesammelt wird. Um die Unsicherheiten der Lebensstandardsicherung zu modellieren, wird wie folgt vorgegangen:

1. Die Kohorten bestimmen zunächst den Barwert der erforderlichen Rentenleistungen für den Zeitpunkt des Renteneintritts. Die Rentenleistungen ergeben sich, indem zunächst die zukünftigen Nettolöhne mit dem (je nach Reformoption variierenden) korrigierten Rentennettoniveau (<70 Prozent) multipliziert werden. Die Differenz zu den Nettolöhnen multipliziert mit einem Rentennettoniveau von 70 Prozent ergibt die erforderliche jährliche Summe, die durch das Kapitalstockverfahren aufgebracht werden muß. Für die Projektion der Nettolöhne antizipieren die Versicherten, daß das Lohnwachstum dem entspricht, das zum Zeitpunkt des Sparbeginns vorliegt. Die Diskontierung der Rentenleistungen erfolgt ebenfalls mit der antizipierten Verzinsung des Kapitalstocks, d.h. mit dem Zinssatz der dem zum Zeitpunkt des Sparbeginns entspricht. Bei der Abzinsung werden vorschüssige Zahlungen unterstellt:

$$
B W_{x}^{R L}=\sum_{t=R E}^{T} n_{t, x} \cdot\left(R L_{t}^{0,7}-R L_{t, x}^{k, r}\right) \cdot\left(1+i_{a, x}\right)^{R E-t} \quad \text { G } 73
$$

$B W_{x}^{R L}=$ Barwert der erforderlichen Rentenleistungen der Generation $x$

$R L_{t}^{0.7}=$ Rentenleistungen bei einem Rentennettoniveau von $70 \%$ im Jahr $t$

$R L_{t, x}^{k o r}=$ Rentenleistungen des korrigierten Rentennettoniveaus der Generation $x$ im Jahr $t$

$n_{t, x}=$ Größe der Generation $x$ im Jahr $t$

$i_{a . x}=$ antizipierter Zinssatz der Generation $x$

$R E=J a h r$ des Renteneintritts der Generation $x$

$T=$ Jahr in dem der Letzte Angehörige der Generation x stirbt

2. Im nächsten Schritt wird der Sparplan während der Erwerbsphase bestimmt. Die jährlichen Beiträge werden so festgelegt, daß der Endwert der aufgezinsten, vorschüssigen Zahlungen dem Barwert der erforderlichen Rentenleistungen entspricht. Dabei werden keine Annuitätenzahlungen in dem Sinne unterstellt, daß die Beiträge über die gesamte Erwerbsphase in absolut gleicher Höhe gezahlt werden. Vielmehr soll die Relation zum Bruttoeinkommen konstant sein. Dies ermöglicht einen Vergleich der Entwicklung des effektiven Beitragssatzes der verschiedenen Reformen. Das antizipierte Lohnwachstum und die antizipierte Verzinsung entsprechen wieder den Werten zum Zeitpunkt des Sparbeginns. Formal bestimmen sich die Beiträge wie folgt:

$$
\begin{gathered}
B_{l, x}=b_{\text {basis }, x} \cdot B L_{\text {basis }, x} \cdot\left(1+w_{a, x}\right)^{t-\text { basis }} \\
\text { mit }
\end{gathered}
$$




$$
b_{\text {basis }, x}=\frac{B W_{x}^{R L}}{B L_{\text {basis }, x} \cdot n_{\text {basis }, x}} \cdot \frac{\frac{\left(1+w_{a, x}\right)}{\left(1+i_{a, x}\right)}-1}{\left(1+w_{a, x}\right)^{(T-R E)}-\left(1+i_{a, x}\right)^{(T-R E)}} \quad \text { G } 75
$$

$B W_{x}^{R L}=$ Barwert der erforderlichen Rentenleistungen der Generation $x$

$B L_{\text {basis } x}=$ Bruttolohn bei Sparbeginn der Generation $x$

$b_{\text {basis }, x}=$ rechnerischer Beitagssatz der Generation $x$ bei Sparbeginn

$B_{t, x}=$ Beiträge der Generation $x$ zur kapitalfundierten Vorsorge im Jahr $t$

$n_{\text {basis }, x}=$ Größe der Generation $x$ zum Sparbeginn

$i_{a, x}=$ antizipierter Zinssatz der Generation $x$

$w_{a, x}=$ antizipierter Lohnwachstum der Generation $x$

$R E=J a h r$ des Renteneintritts der Generation $x$

$T=$ Jahr in dem der Letzte Angehörige der Generation $x$ stirbt

3. Die Rentenleistungen ergeben sich, indem die gezahlten Beiträge mit dem tatsächlichen Zinssätzen verzinst werden.

B) Berücksichtigung der Abweichung vom Ziel der Lebensstandardsicherung

Die tatsächlichen Zinssätze werden i.d.R. von denen abweichen, die die Versicherten ex ante angenommen hatten. In dem Fall unterscheiden sich die Rentenleistungen, die sich tatsächlich aus dem kapitalfundierten Zweig der Alterssicherung ergeben, von den Rentenleistungen, die ursprünglich angestrebt wurden, um ein Nettorentenniveau von 70 Prozent aufrecht zu erhalten. Auch wenn sich das Lohnwachstum anders entwickelt als angenommen, weicht das realisierte Nettorentenniveau von dem angestrebten Nettorentenniveau ab. Mit anderen Worten, in dem Maße, in dem die Zins-Lohnwachstum-Differenz von der antizipierten abweicht, weicht der kapitalfundierte Teil der Rentenleistungen von dem angestrebten Ziel der Lebensstandardsicherung ab (vgl. Abschnitt 4.4.4). Um die positiven oder negativen Umverteilungen zu erfassen, die sich aus einer „Unter- bzw. Überversicherung“ ergeben, werden die Rentenleistungen im Verteilungsindikator wie folgt erfaßt:

$$
R N_{-} F A K T O R_{t}^{x}=\frac{R L_{a, t}}{B L_{\text {basis }, x} \cdot\left(1+w_{a, x}\right)^{t-\text { basis }} \cdot N Q_{t}} \cdot \frac{B L_{t} \cdot N Q_{t}}{R L_{t, . x}^{\text {tat }}} \quad \text { G } 76
$$

mit

$$
R L_{t, x}^{V G L}=R N_{-} F A K T O R_{t}^{x} \cdot R L_{t, x}^{t a t} \quad \mathbf{G} 77
$$


$R N_{-}$FAKTOR ${ }_{t}^{x}=$ Korrekturfaktor nach Maßgabe des erzielten Renteniveaus der Generation $x$ im Jahr $t$

$R L_{a, t}=$ antizipierte Rentenleistungen im Jahr $t$

$R L_{t, x}^{t a t}=$ tatsächliche Rentenleistungen der Generation $\times$ im Jahr $t$

$R L_{t, x}^{V G L}=$ Vergleichs - Rentenleistung der Generation $x$ im Jahr $t$

$N Q_{t}=$ Nettoquote im Jahr $t$

$w_{a, x}=$ antizipierte Lohnwachstumsrate der Generation $x$

$B L_{\text {basis }, x}=$ durchschnittlicher Bruttolohn beim Sparbeginn der Generation $x$

$B L_{t}=$ durchschnittlicher Bruttolohn im Jahr $t$

Die Lebensstandardsicherung wird in gleicher Weise auch im Referenzsystem berücksichtigt. Entscheidend für die Bewertung der Umverteilungen ist dadurch nicht die Abweichung des Nettorentenniveaus von dem Wert von 70 Prozent, sondern von dem Nettorentenniveau, das sich im Referenzsystzem einstellt. Denn das Referenzsystem gibt an, in welchem Ausmaß eine Absicherung des intergenerativen Risikos „Einfluß des ZinsLohnwachstum-Differenz auf den relativen Lebensstandard" erwünscht wird.

Zukünftige Kohorten haben eine längere Ansparphase als jene, die nur kurz nach Bekanntgabe der Reformmaßnahme in den Ruhestand treten. Die Kohorte, die bei Bekanntgabe gerade ins Erwerbsleben eintritt und alle nachfolgenden können während der gesamten Erwerbsphase ihren Kapitalstock ansammeln. Es ist wahrscheinlich, daß sie in einer solchen Zeitspanne ihre Beiträge immer wieder anpassen, wenn ihre Annahmen über Lohnwachstum und Zinssatz von der tatsächlichen Entwicklung abweichen. Zumindest während der Erwerbsphase würde das Unsicherheitsmoment der „ZinsLohnwachstum-Differenz" eingeschränkt werden. Diese Verhaltensannahmen ließen sich einfach in das Modell integrieren, indem die antizipierten Zinsätze und das antizipierte Lohnwachstum in bestimmten Abständen an die aktuellen Werte angepaßt werden.

\section{C) Bestimmung des gesamtwirtschaftlichen Kapitalstocks und seine gesamtwirtschaftli- chen Rückwirkungen}

Für jedes Jahr existieren Kohorten, die Kapital ansammeln und Kohorten, die ihren Kapitalstock auflösen. Der gesamtwirtschaftliche Kapitalstock der Vorperiode korrigiert um diese Zahlungsströme ergibt den Kapitalstock der aktuellen Periode, der wiederum mit dem tatsächlichen Zinssatz der aktuellen Periode verzinst wird. Aus der Höhe des gesamtwirtschaftlichen Kapitalstocks wird durch Multiplikation mit einem „Vermögenssubstitutionsfaktor" die Zunahme der gesamtwirtschaftlichen Ersparnis berechnet (vgl. Abschnitt 6.2.1).

$$
\Delta S_{t}=\left(K S_{t}-K S_{t-1}\right) \cdot V S_{-} F A K T O R \quad \text { G } 78
$$

$V S_{-}$FAKTOR $R_{t}^{x}=$ Vermögessubstitutionsfaktor

$\Delta S_{t}=$ Veränderung der gesamtwirtschaftlichen Ersparnis im Jahr $t$

$K S_{t}=$ gesamte Kapitalstock der zusätzlichen, kapitalfundierten Vorsorge im Jahr $t$ 
Das Verhältnis aus der Zunahme der gesamtwirtschaftlichen Ersparnis zur Bruttolohnund -gehaltssumme dient als Indikator für etwaige gesamtwirtschaftlichen Rückwirkungen. Die Wirkungen auf die mit dem Kapitalstockverfahren erzielbare Ertragsrate (vgl. Abschnitt 6.2.2) werden wie folgt erfaßt:

$$
i_{t}^{V G L}=i_{t}^{S Q}-\frac{\Delta S_{t}}{B L G S_{t}} \cdot \frac{1}{i_{-} F A K T O R_{t}^{K S}} \cdot \quad \mathbf{G} 79
$$

$i_{-} F A K T O R_{t}^{K S}=$ Faktor zur Erfassung der Verzinsungsändenung durch den Veränderten Kapitalstock $\Delta S_{t}=$ Veränderung der gesamtwirtschaftlichen Erspamis im Jahr $t$

$i_{i}^{V G L}=$ Vergleichszinssatz bei Berücksichtigung des Einflusses einer emöhten gesamtwirtschaftlichen Ersparnis

$i_{t}^{\text {SQ }}=$ Zinssatz Status quo (ohne Berücksichtigung des Einflusses einer erhöhten gesamtwirtschaftlichen Ersparnis)

$B L G S_{1}=$ Bruttolohn - und - gehaltssumme im Jahr $t$

Die Wirkungen auf das gesamtwirtschaftliche Wachstum (vgl. Abschnitt 6.2.3) werden durch die Gleichung berücksichtigt:

$$
w_{t}^{V G L}=w_{t}^{S Q}+\frac{\Delta S_{t}}{B L G S_{t}} \cdot w_{-} \text {FAKTOR } R_{t}^{K S} \cdot \mathbf{G} \mathbf{8 0}
$$

$w_{-}$FAKTOR $_{t}^{K S}=$ Faktor zur Erfassung der durch die Veränderung der gesamtwrtschaftlicn Ersparnis verursachten Wachstumseffekte

$\Delta S_{1}=$ Veränderung der gesamtwirtschaftlichen Ersparnis im Jahr $t$

$w_{t}^{V G L}=$ Vergleichswachstumsrate der Bruttolohn - und - gehaltssumme im Jahr $t$

bei Berücksichtigung des Einflusses einer erhöhten gesamtwirtschaftlichen Ersparnis

$w_{t}^{V C L}=$ Wachstumsrate der Bruttolohn - und - gehaltssumme im Jahr $t$ beim Status quo

(ohne Berücksichtigung des Einflusses einer erhöhten gesamtwirtschaftlichen Ersparnis)

BLGS, = Bruttolohn - und - gehaltssumme im Jahr $t$

\subsection{Ergebnisse der Simulation}

\subsubsection{Wirkung des demographischen Korrekturfaktors der Reform 99}

Der direkte Vergleich der Verteilungsindikatoren einer demographischen Korrektur der Rentenformel mit denen des Status Quo zeigt, daß die Zugangskohorten in drei Gruppen eingeteilt werden können (vgl. Abbildung 18):

\section{Bestandsrenten}

Ihre Situation verschlechtert sich relativ zum derzeitigen System, da sie in beiden Systemen zwar die gleichen Beiträge zahlen, die Leistungen bei einer demographischen Korrektur aber geringer steigen und sie damit absolut geringer ausfallen. 


\section{Kohorten, die bis zum Jahr 2013 in Rente gehen}

All jene Kohorten, die nach dem Jahr 1998 (Bekanntgabe der Reform) in Rente gehen, können die entstehende Versorgungslücke durch eine kapitalgedeckte Vorsorge ausgleichen. Für diese Kohorten unterscheiden sich die Rentenleistungen der beiden Systeme nicht. Allerdings zahlen sie im Fall der korrigierten Rentenformel zusätzliche Beiträge für die eigene Vorsorge. Für die Zugangskohorten bis zum Jahr 2013 ist diese Ansparphase jedoch relativ kurz, so daß der Zinseffekt nicht ausreicht, die höheren Beitragszahlungen auszugleichen. Die Positionen dieser Kohorten verschlechtern sich ebenfalls im Vergleich zum Status Quo.

Abbildung 18: Differenz der Verteilungsindikatoren der Zugangskohorten der Reform 99 zu den Verteilungsindikatoren des Status Quo

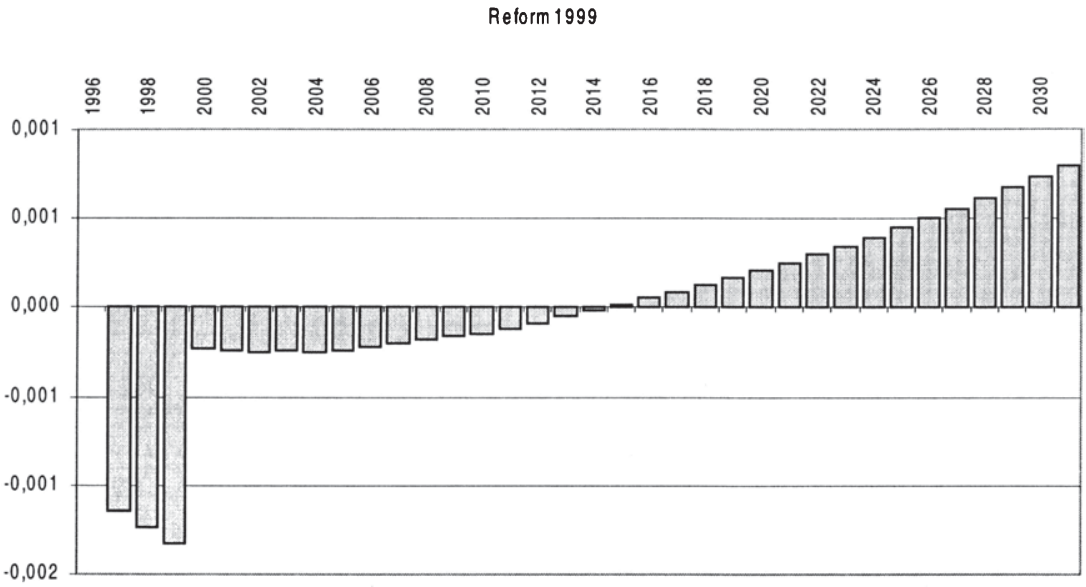

Reform 1999

Quelle: eigene Berechnungen

3. Kohorten, die nach dem Jahr 2013 in den Ruhestand wechseln

Kohorten, die nach dem Jahr 2013 in den Ruhestand wechseln, profitieren zum einen von einem höheren Zinseffekt, zum andern profitieren sie stärker von dem geringeren Anstieg der Beitragssätze durch die Reform. Diese Kohorten werden folglich besser gestellt als im derzeitigen System.

Da einige Generationen im Vergleich zum derzeitigen System benachteiligt werden, ist die Reform 99 nicht optimal im Sinne Paretos. Entscheidend für die Beurteilung der „Gerechtigkeit" der Reform ist jedoch der Verlauf der Umverteilungspositionen der Zugangskohorten relativ zu dem Verlauf der Umverteilungspositionen des Referenzsystems (vgl. Abbildung 19). 
Abbildung 19: Verlauf der Umverteilungspositionen der Zugangskohorten im Status Quo und bei der Reform 1999

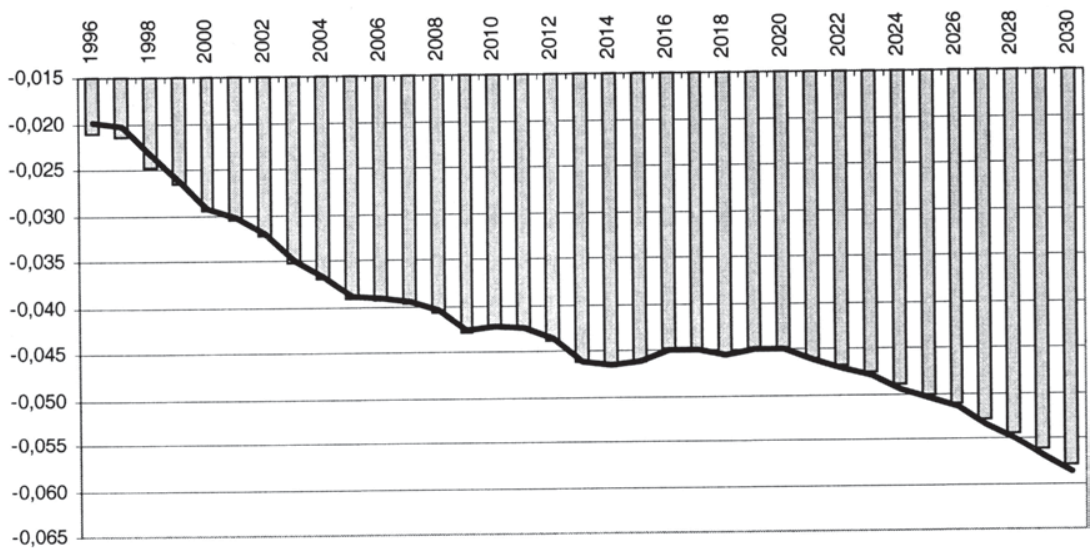

Reform1999 - Status Quo

Quelle: eigene Berechnungen

In diesem Vergleich zeigt sich, daß die ersten beiden Gruppen der Zugangskohorten (jene, die ihre Position relativ zum Status Quo verschlechtern) bereits im derzeitigen System besser gestellt sind. Die Lasten der Alterssicherung werden durch die Reform folglich gleichmäßiger verteilt. Auch die ergänzenden Kennzahlen der Verteilung zeigen, daß das Rentensystem durch die Reform 99 "gerechter" wird (vgl. Tabelle 11).

Tabelle 11: Kennziffern zum Verlauf der Umverteilungspositionen der Zugangskohorten im Lstatus Quo und bei der Reform 99

\begin{tabular}{|l|c|c|}
\cline { 2 - 3 } \multicolumn{1}{c|}{} & Status Quo & Reform 99 \\
\hline Standardabweichung & 0,009645 & 0,009190 \\
\cline { 1 - 1 } Variationskoeffizient & 0,230580 & 0,219695 \\
\cline { 1 - 1 } Rawls Differenzenkriterium & $-0,058704$ & $-0,057912$ \\
\hline
\end{tabular}

Quelle: eigene Berechnungen 


\subsubsection{Wirkung der pauschalen Senkung des Rentenniveaus}

Die pauschale Senkung des Rentenniveaus setzt an den gleichen „Stellschrauben“ an, wie der demographische Korrekturfaktor. Der Unterschied besteht lediglich in dem Ausmaß und der Begründung der Korrektur. Die Wirkungen der Reform entsprechen daher in der Tendenz denen der Reform 99 (vgl. Abbildung 20). Allerdings ist das Ausmaß der Effekte höher. Bei dieser Reformoption werden zudem erst die Zugangskohorten ab dem Jahr 2019 gegenüber dem derzeitigen System begünstigt.

Abbildung 20: Differenz der Verteilungsindikatoren der Zugangskohorten einer pauschalen Senkung des Rentennilveaus zu den Verteilungsindikatoren des Status Quo

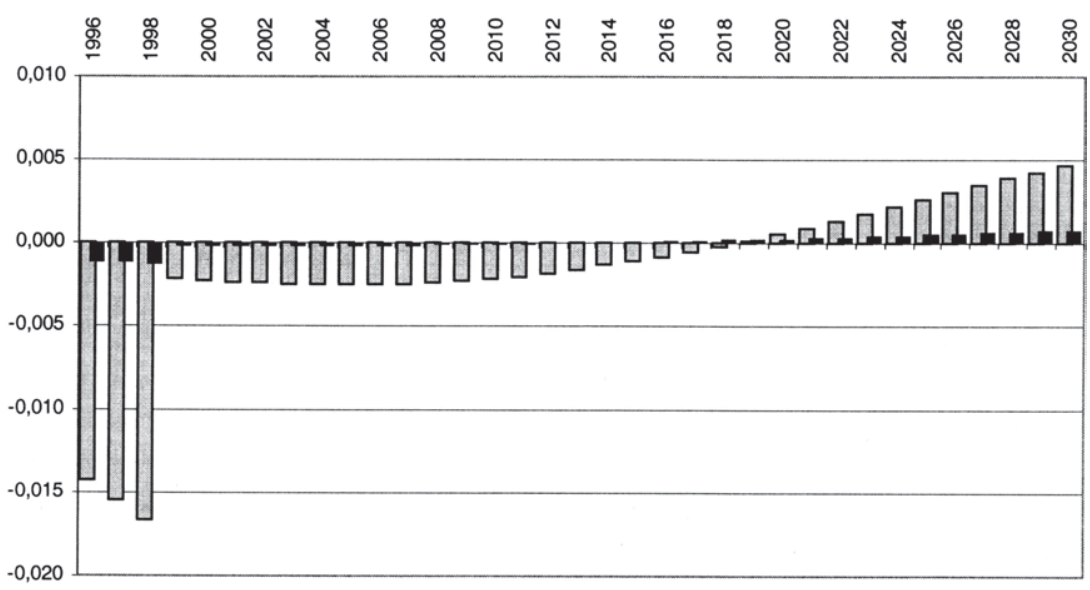

Senkung Rentenniveau $\mathbf{\square}$ Reform1999

Quelle: eigene Berechnungen

Der Vergleich des Verlaufs der Verteilungsindikatoren dieser Reformoption mit dem des Referenzsystems signalisiert ebenfalls eine Verbesserung der intergenerativen Gerechtigkeit durch eine pauschale Senkung des Rentenniveaus ( vgl. Abbildung 21 und Tabelle 12). 
Abbildung 21: Verlauf der Umverteilungspositionen der Zugangskohorten bei einer pauschalen Senkung des Rentenniveaus und bei der Reform 1999

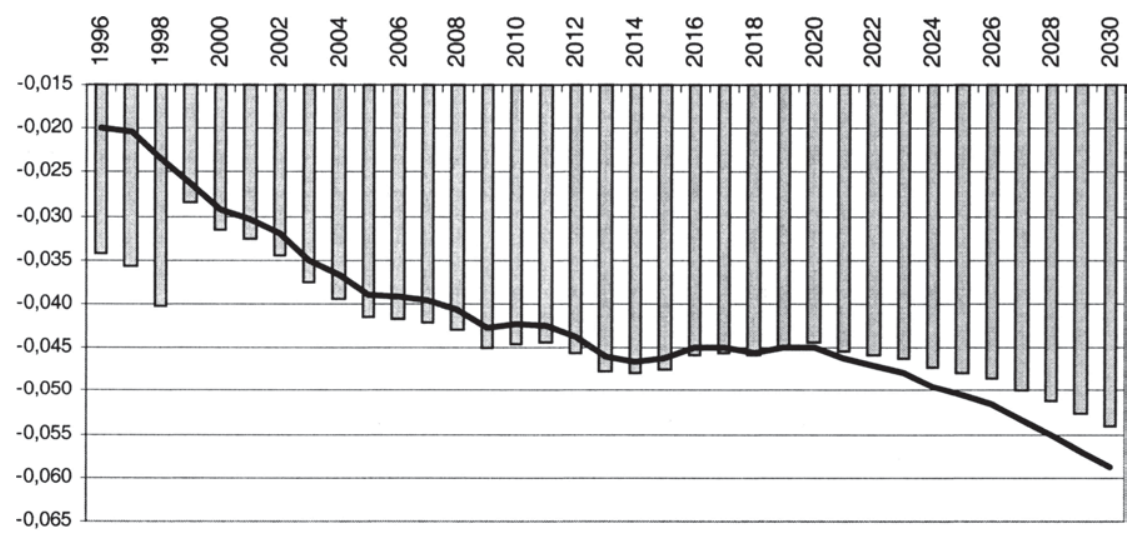

Senkung Rentenniveau $\longrightarrow$ Status Quo

Quelle: eigene Berechnungen

Tabelle 12: Kennziffern zum Verlauf der Umverteilungspositionen der Zugangskohorten bei einer pauschalen Senkung des Rentenniveaus und im Status Quo

\begin{tabular}{|l|c|c|}
\cline { 2 - 3 } \multicolumn{1}{c|}{} & Status Quo & Absenkung Rentenniveau \\
\hline Standardabweichung & 0,009645 & 0,005944 \\
\cline { 1 - 1 } Variationskoeffizient & 0,230580 & 0,136908 \\
\cline { 1 - 1 } Rawls Differenzenkriterium & $-0,058704$ & $-0,054009$ \\
\hline
\end{tabular}

Quelle: eigene Berechnungen

Im dritten Kapitel wurde gezeigt, daß die Gerechtigkeit einer Reform der gesetzlichen Rentenversicherung durch die beiden Gestaltungsziele der Vorsorge und der Lebensstandardsicherung maßgeblich beeinflußt wird. Der Sicherung des Lebensstandards wurde im Modell Rechnung getragen, indem die Leistungsseite bei den Reformmaßnahmen konstant gehalten wurde, sprich die entstehende Differenz durch kapitalfundierter Vorsorge ausgeglichen wurde. Abweichungen vom angestrebten Sicherungsniveau, die durch Variationen der Faktorpreise verursacht werden, werden durch den Vergleich mit dem Referenzsystem sowie durch den RN_Faktor erfaßt. Für die Bestandsrenten besteht allerdings keine Möglichkeit mehr vorzusorgen. Sowohl bei der Reform 99 als auch bei der pauschalen Senkung des Rentenniveaus müssen die Bestandsrenten daher gesondert bewertet werden. Mit andern Worten, für eine Umstellung des Finanzierungsverfahrens 
muß, sofern sie an dem aktuellen Rentenwert ansetzt, eine Sicherungsgrenze für die Bestandsrenten angegeben werden.

\subsubsection{Korrektur der Anwartschaften}

Durch die dritte Reformoption wird das Sicherungsniveau aller Zugangskohorten, d.h. insbesondere auch der Bestandsrenten nicht gravierend verändert. Das Ziel der Lebensstandardsicherung wird nicht verletzt und die „intergenerativ gerechte“ Lösung wäre gegebenenfalls ohne das Hilfsmittel einer Sicherungsuntergrenze für die Bestandsrenten zu verwirklichen.

Beim Vergleich der Umverteilungspositionen der Zugangskohorten bei dieser Reform mit denen des Status Quo können wieder unterschiedliche Gruppen von Zugangskohorten unterschieden werden (vgl. Abbildung 22):

Abbildung 22: Verlauf der Umverteilungspositionen der Zugangskohorten bei einer sukzessiven Kürzung der neu erworbenen Entgeltpunkte und im Status Quo
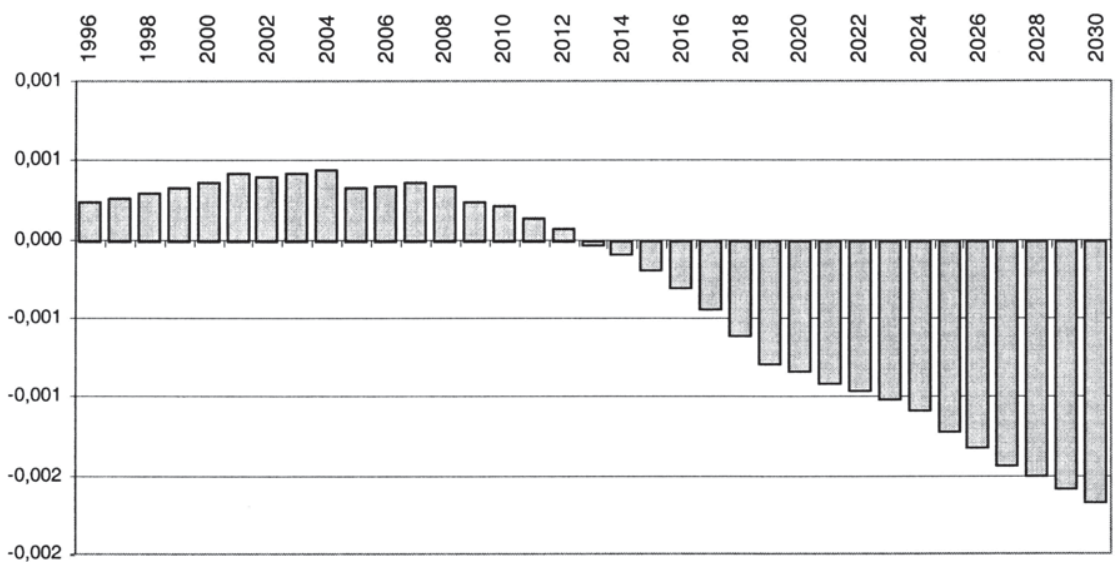

Korrektur der Anw artschaften

Quelle: eigene Berechnungen

Durch das sukzessive Senken der enworbenen Entgeltpunkte steigt der Beitragssatz geringer als im Status Quo. Aufgrund der Rückkopplung über die Nettoanpassung steigt dadurch auch der aktuelle Rentenwert stärker als im derzeitigen Rentensystem. Als Folge dieses Effektes erhalten Bestandsrentner bei einer Korrektur der Anwartschaften höhere Renten, obwohl sie die gleichen Beiträge gezahlt haben, wie im Status Quo. Die Bestandsrenter werden daher vergleichsweise bessergestellt. 
Bei den Zugangskohorten, die nach dem Jahr 1998 (Bekanntgabe der Reform) in den Ruhestand wechseln, treten drei unterschiedliche Effekte auf: Erstens profitieren sie ebenfalls von dem Anstieg des aktuellen Rentenwertes. Da sie die entstehende Versorgungslücke durch eine kapitalgedeckte Vorsorge ausgleichen, kommt innen zweitens der Zinseffekt zugute. Allerdings finanzieren sie die bestehenden Anwartschaften, enwerben mit ihren Beiträgen aber vergleichsweise geringere Anwartschaften (Entgeltpunkte). Dieser dritte Effekt spiegelt die im zweiten Kapitel beschriebene Doppelbelastung der Übergangsgeneration wider und wirkt den ersten beiden entgegen.

Bei den Zugangskohorten, die bis zum Jahre 2012 in den Ruhestand wechseln, überwiegt der erste Effekt, sie werden daher wie die Bestandsrenten im Vergleich zum Status Quo besser gestellt. Für Zugangskohorten ab dem Jahr 2013 überwiegt der dritte Effekt, d.h. sie werden relativ zum derzeitigen System benachteiligt. Für Zugangskohorten, die noch später in Rente gehen - wenn die Umstellung bereits zum Großteil erfolgt ist - wird der Zinseffekt überwiegen, so daß diese wieder im Vergleich zum Status Quo von der Reform profitieren. Von welchem Jahr an dies zutrifft, kann nicht genau gesagt werden, da die Zugangskohorten nur bis zum Jahr 2030 untersucht wurden.

Diese unterschiedliche Behandlung sagt noch nichts darüber aus, wie "gerecht" die Reform ist. Ein Blick auf den Verlauf der Umverteilungspositionen relativ zu dem Verlauf der Umverteilungspositionen des Referenzsystems (vgl. Abbildung 23) zeigt allerdings, daß die Reform die ungleiche Behandlung noch verstärkt. Alle zusätzlichen Kennzahlen der Verteilung weisen ebenfalls aus, daß das Rentensystem durch eine solche Reform intergenerativ ungerechter würde (vgl. Tabelle 13).

Abbildung 23: Verlauf der Umverteilungspositionen der Zugangskohorten bei einer pauschalen Senkuna des Rentenniveaus und bei der Reform 1999

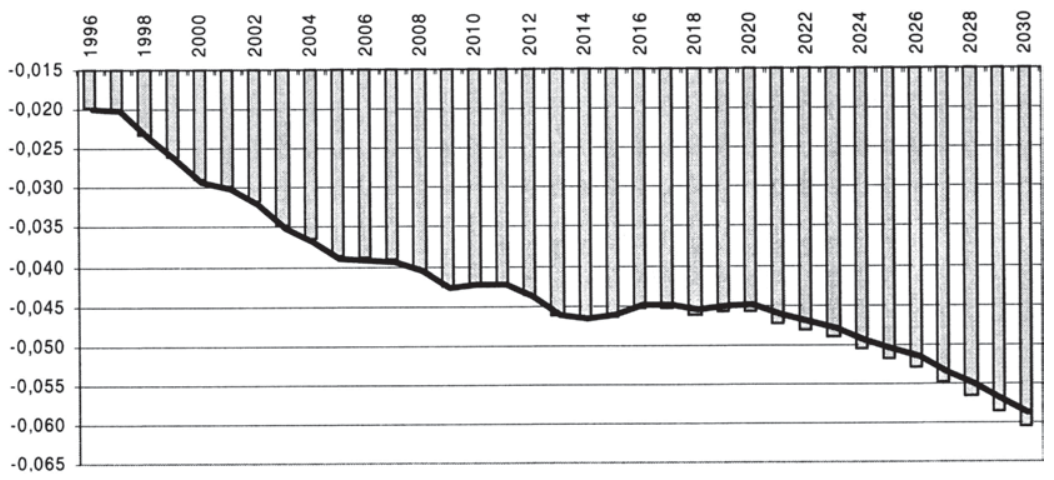

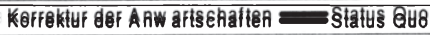

Quelle: eigene Berechnungen 
Tabelle 13: Kennziffern zum Verlauf der Umverteilungspositionen der Zugangskohorten bei einer pauschalen Senkung des Rentenniveaus und im Status Quo

\begin{tabular}{|l|c|c|}
\cline { 2 - 3 } \multicolumn{1}{c|}{} & Status Quo & Korrektur der Anwartschaften \\
\hline Standardabweichung & 0,009645 & 0,010213 \\
Variationskoeffizient & 0,230580 & 0,242411 \\
\cline { 1 - 1 } Rawls Differenzenkriterium & $-0,058704$ & $-0,060364$ \\
\hline
\end{tabular}

Quelle: eigene Berechnungen

Die hier beschriebene Modellrechnung wurde durch weitere exemplarische Szenarien ergänzt (vgl. Anhang 1.3). Die beschriebenen grundsätzlichen Ergebnisse erwiesen sich dabei als robust.

Zusammenfassend lassen sich aus den exemplarischen Berechnungen mit dem vorliegenden Meßkonzept die folgenden Ergebnisse formulieren:

- Der in dem Rentenreformgesetz 1999 vorgesehene demographische Korrekturfaktor trägt dazu bei, die Alterssicherung nach Maßgabe des in dieser Arbeit beschriebenen Meßkonzepts intergenerativ gerechter zu gestalten. Trotz dieser Verbesserung bleiben aber gravierende intergenerative Umverteilungen zwischen den Zugangskohorten bestehen.

- Mit einer weitergehenden Senkung des aktuellen Rentenwerts könnte erreicht werden, daß die Umverteilungspositionen der Zugangskohorten weiter angeglichen werden. Dieser Gewinn an Gerechtigkeit muß allerdings mit einer Verletzung des Ziels der Lebensstandardsicherung bei den Bestandsrenten abgewogen werden.

- Eine alternative Maßnahme, um das Umlageverfahren partiell durch das Kapitalstockverfahren zu substituieren, besteht darin, die neu erworbenen Anwartschaften (Entgeltpunkte) sukzessive zu senken. In dem Fall muß nicht mehr abgewogen werden, ob das Ziel der Lebensstandardsicherung auch bei den Bestandsrenten hinreichend gewährleistet ist. Diese Reformoption führt bei den in der Modellrechnung erfaßten Zugangskohorten (Zugangsjahr 1996 bis 2030) allerdings zu einer Verschlechterung der intergenerativen Gerechtigkeit.

Eine Begrenzung der Korrektur des aktuellen Rentenwerts, wie sie auch im Rentenreformgesetz vorgesehen ist, mag zunächst als willkürlich erscheinen. Vor dem Hintergrund der obigen Ergebnisse ist sie jedoch auch aus Überlegungen zur intergenerativen Gerechtigkeit erforderlich. Angesichts der noch bestehenden massiven Ungleich- 
behandlung der Zugangskohorten stellt sich allerdings die Frage, ob die nur halbe Berücksichtigung des Lebenserwartungsfaktors eine angemessene Abwägung darstellt:

Bei einer vollen Berücksichtigung der Lebenserwartung würden die Umverteilungspositionen der Zugangskohorten noch stärker angeglichen werden (vgl. Abbildung 24). Das Rentenniveau würde in den Modellrechnungen statt auf $66,6 \%$ auf $63,2 \%$ sinken. Zudem wird dieses Niveau erst im Jahr 2030 erreicht sein, so daß dieses Niveau für den Großteil der Bestandsrenten ohnehin nicht relevant ist.

Abbildung 24: Differenz der Verteilungsindikatoren der Zugangskohorten bei der Reform 99 und bei einer Reform ohne Halbierung der Korrekturkomponente zu den Verteilungsindikatoren des Status Quo

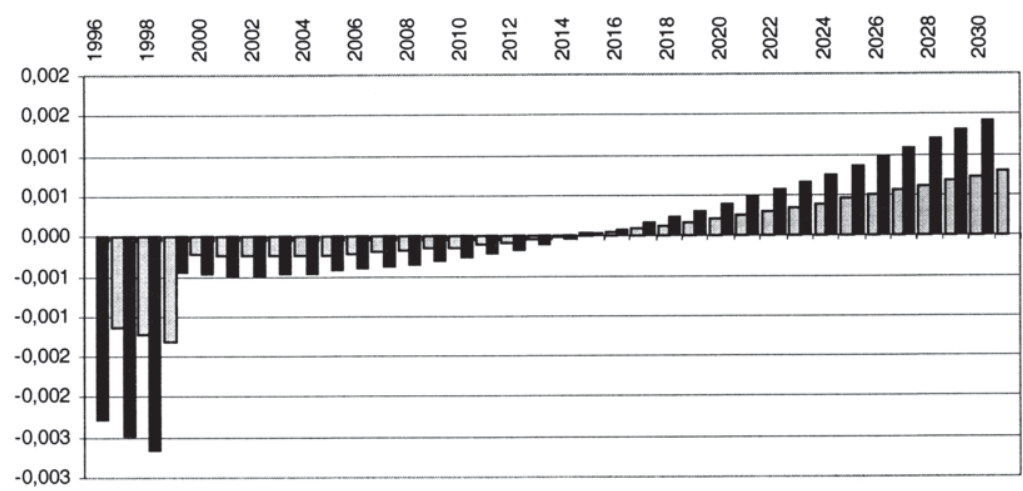

Reform1999 [ LE Faktor ohne Halbierung 
Ingo Schroeter - 978-3-631-75016-2

Downloaded from PubFactory at 01/11/2019 09:14:34AM

via free access 


\section{ZUSAMMENFASSUNG, WÜRDIGUNG UND AUSBLICK}

Ziel der vorliegenden Arbeit ist die Entwicklung eines Konzepts, mit dem die intergenerativen Verteilungswirkungen eines Alterssicherungssystems bzw. einer Reformoption erfaßt und bewertet werden, um Aussagen über die intergenerative Gerechtigkeit der Systeme bzw. Maßnahmen zu treffen.

Im Vordergrund stehen Reformvorschläge, die auf einer (partiellen) Substitution des Finanzierungsverfahrens beruhen. Im 2. Kapitel werden daher zunächst die allokativen (a) und distributiven (b) Wirkungen der Finanzierungsverfahren analysiert:

a) Selbst wenn angenommen wird, daß der Zinssatz das Lohnsummenwachstum dauerhaft übersteigt, erweist sich das Kapitalstockverfahren nicht als Paretoeffizient, da die Einführungsgenerationen in einem Umlageverfahren bessergestellt würden. Diese Zusammenhänge beschränken sich nicht auf die Einführung eines Alterssicherungssystems, sondern sind ebenfalls von Bedeutung, wenn ein bestehendes Umlageverfahren durch ein Kapitalstockverfahren substituiert wird. In dem Fall finden die Probleme der Einführungsgenerationen ihre Entsprechung in der Doppelbelastung der Übergangsgenerationen. Sowohl in einem Modell einer kleinen offenen als auch einer geschlossenen Volkswirtschaft ist es nicht möglich, diese Generationen für die Zusatzbelastung zu entschädigen, ohne andere Generationen schlechter zu stellen. Eine Umstellung auf ein Kapitalstockverfahren ist Pareto-optimal nicht realisierbar und wirkt mithin unterschiedlich auf die Generationen, mit anderen Worten: sie beeinflußt zwangsläufig die intergenerative Gerechtigkeit.

b) Bei der Analyse der distributiven Wirkungen stand die Frage im Mittelpunkt, ob und wie verschiedene Generationen innerhalb eines Finanzierungsverfahrens unterschiedlich behandelt werden, wenn einige ökonomische und demographische Parameter ceteris paribus variieren. Sowohl im Umlageverfahren als auch im Kapitalstockverfahren beeinflussen solche Variationen die Generationen im unterschiedlichen Maße; sie verursachen folglich in jedem Finanzierungsverfahren eine intergenerative Verteilung. Insbesondere erwies sich das Kapitalstockverfahren ebenfalls als abhängig von der demographischen Entwicklung. Die Ergebnisse beruhen auf einer qualitativen Analyse. Quantitative Aussagen, vor allem ob das Ausmaß der Wirkungen zwischen den Finanzierungsverfahren variiert, konnten nicht getroffen werden.

Im 3. Kapitel wird danach gefragt, wie die intergenerativen Verteilungswirkungen bewertet werden, d.h. wonach entschieden wird, ob eine Verteilung zwischen den Generationen gerecht oder ungerecht ist. In Verteilungsanalysen können (und werden) verschiedene Gerechtigkeitsgrundsätze als Norm herangezogen, die sich allerdings zum Teil widersprechen. Die für die Fragestellung relevanten Gerechtigkeitsgrundsätze müssen daher ausgewählt und gegebenenfalls gewichtet werden. Grundsätzlich lassen sich hierzu zwei Vorgehensweisen unterscheiden, die allerdings auf alternativen Thesen zur Gerechtigkeit 
beruhen. Die erste These unterstellt, daß ein allgemeingültiges und ethisch fundiertes Gerechtigkeitskonzept existiert. Entsprechend der zweiten These läßt sich die Wahl der Gerechtigkeitsnormen nicht analytisch aus übergeordneten Prinzipien ableiten. Jede Gerechtigkeitsvorstellung ist gesellschaftlich definiert bzw. historisch gewachsen. Sie ist zudem nicht konstant, sondern kann sich im Laufe der Zeit ändern. Ein allgemein akzeptiertes und ethisch fundiertes Gerechtigkeitskonzept zur Bewertung eines Alterssicherungssystems kann nicht formuliert werden. Es lassen sich bestenfalls sehr allgemeine Grundsätze aufstellen, die allerdings zu abstrakt sind, um sie auf die konkrete Gestaltung eines Rentensystems anzuwenden. Wenngleich die zweite Vorgehensweise ebenfalls mit methodischen Mängeln verbunden ist, kann sie eher für die Bewertung konkreter Reformmaßnahmen verwendet werden. Der für diese Arbeit gewählte Kompromiß orientiert sich daher vornehmlich an der zweiten Gerechtigkeitsthese:

Die Bewertung der intergenerativen Verteilungswirkungen orientiert sich an den politischgesellschaftlich definierten Zielen und den darauf beruhenden ordnungspolitischen Konzeptionen der gesetzliche Rentenversicherung. Um den Interpretationsspielräumen Rechnung zu tragen, werden die Gewichtung der Grundsätze in Vergleichsszenarien variiert. Das Differenzenkriterium nach Rawls wird lediglich als zusätzliches Kriterium bei der Bewertung des Verteilungsmusters herangezogen.

Um das Meßkonzept zu entwerfen, muß zuvor der Analyserahmen im 4. Kapitel weiter abgegrenzt werden. Die Gerechtigkeit der Verteilungswirkungen wird auf die als gerecht unterstellte Sekundärverteilung ohne Alterssicherungssystem bezogen. Die Verteilungswirkungen anderer Sozialversicherungszweige werden nur in dem Maße erfaßt, in dem sie über die Rückkopplung der Nettoanpassung auf die Rentenleistung wirken. Für die vorliegende Fragestellung interessieren lediglich die personellen, intergenerativen Verteilungswirkungen, wobei letztere sich auf den Vergleich unterschiedlicher Generationen über ihren gesamten Lebenszyklus beziehen. Intergenerative Verteilungswirkungen können sowohl direkt als auch indirekt durch Wechselwirkungen mit anderen gesamtwirtschaftlichen Größen entstehen. Beide Kategorien müssen bei der Bewertung der Alterssicherungssysteme berücksichtigt werden. Ferner wird angenommen, daß die Arbeitgeberbeiträge vollständig auf die Arbeitnehmer überwälzt werden. Die effektive Inzidenz des Bundeszuschusses wird vernachlässigt. Er wird als durchlaufender Posten für die als versicherungsfremd bezeichneten Leistungen interpretiert und für sämtliche Reformoptionen unverändert beibehalten.

Die Ziele und ordnungspolitischen Konzeptionen eines Alterssicherungssystems spiegeln sich in unterschiedlichen Gestaltungsprinzipien wider, die folglich die Bewertung der Verteilungswirkungen beeinflussen. Es wird untersucht, welche Gestaltungsprinzipien für die Frage der intergenerativen Gerechtigkeit einer Substitution des Finanzierungsverfahrens wichtig sind und welchen Einfluß sie auf die Bewertung der Verteilungswirkungen haben. Dabei erweist sich die Gestaltungsdimension der Solidarität, bzw. konkreter der Sozialausgleich eines Versicherungssystems als wichtiges Kriterium bei der Bewertung der Verteilungswirkungen. Hinsichtlich der Solidarität können zwei dominante Ziele der gesetzliche Rentenversicherung identifiziert werden: Das erste Ziel fordert, das Alterssiche- 
rungssystem als Vorsorgesystem zu gestalten und findet seine gestalterische Umsetzung im Äquivalenzprinzip. Das zweite Ziel strebt die Sicherung des Lebensstandards an und korrespondiert mit den Gestaltungsprinzipien der Teilhabeäquivalenz, der Kopplung der Rentenleistungen an das durchschnittliche Erwerbseinkommen und der Dynamisierung der Renten. Ausgehend von diesen Zielen und ordnungspolitischen Konzeptionen können folgende Schlußfolgerungen für das zu entwickelnde Konzept gezogen werden:

- Grundlage des Verteilungsindikators ist die Verknüpfung von Beiträgen und Leistungen.

- Bei den Verteilungswirkungen muß zwischen einem intergenerativen Risikoausgleich und der intergenerativen Umverteilung unterschieden werden.

In den Kapiteln 5 und 6 wird ein Konzept entwickelt, mit dem die intergenerative Umverteilung erfaßt und bewertet wird. Dabei ist es das erklärte Ziel der Arbeit, daß mit dem Konzept konkrete Reformoptionen bewertet und sozialpolitische Handlungs- bzw. Entscheidungshilfen abgeleitet werden können. Im Gegensatz zum modell-theoretischen Ansatz des zweiten Kapitels ist es daher nicht mehr ausreichend, sich auf qualitative Wirkungen in einem OLG-Modell zu beschränken. Kapitel 5 beschreibt das Vorgehen zur Erfassung und Bewertung der direkten Umverteilungswirkungen. Die indirekten Wirkungen werden im 6 . Kapitel berücksichtigt.

Zunächst werden verschiedene Ansätze skizziert, mit denen sich die intergenerativen Verteilungswirkungen eines Alterssicherungssystems quantitativ erfassen lassen. Es wird ein Verteilungsindikator formuliert, der auf der Barwertmethode, bzw. genauer auf der generationsspezifischen Rendite basiert. Gegenüber dem interne Zinsfuß hat diese Rentabilitätskennziffer den Vorteil, daß sich der Verteilungsindikator einfacher modifizieren läßt, um neben der reinen Beitragsäquivalenz weitere Gestaltungsprinzipien der Alterssicherung (intergenerative Risikoausgleich) sowie etwaige gesamtwirtschaftliche Wirkungen der Finanzierungsverfahren bei der Beurteilung der Gerechtigkeit zu berücksichtigen. Die Verteilungswirkungen eines Alterssicherungssystems müssen mit einem Referenzmaßstab vergleichen werden, um sie zu beurteilen. Die Analyse zeigte, daß die übliche Vorgehensweise, ein beitragsäquivalentes Kapitalstockverfahren als Referenz zugrunde zu legen, normativ nicht begründet werden kann. Sie vernachlässigt die Präferenz der Versicherten nach Sicherheit eines relativen Lebensstandards im Alter. Der Versuch, die Vorgehensweise mit Hilfe der Anspruchstheorie von NOZICK zu begründen, kann ebenso wenig überzeugen, wie der alternative Ansatz der „intergenerationalen Durchhaltbarkeit“. Für die Analyse der intergenerativen Gerechtigkeit wird der Verteilungsindikator daher angepaßt, um dem intergenerativen Risikoausgleich Rechnung zu tragen. Im Mittelpunkt stehen drei Fragen:

1. Wieviel an intergenerativen Risikoausgleich ist erwünscht?

2. Was gehört zu den intergenerativ versicherbaren Risiken?

3. Wie kann die Zusatzlast bei Eintritt des Risikos auf die Versichertengemeinschaft (auf die unterschiedlichen Generationen) verteilt werden? 
Beide Ziele der gesetzliche Rentenversicherung (Vorsorgesystem und Lebensstandardsicherung vgl. Tabelle 5) bestimmen das Referenzsystem. Die Gewichtung des Ziels der Lebensstandardsicherung entscheidet über das erwünschte $A u s m a ß$ des intergenerativen Risikoausgleichs. Beide Ziele werden im Referenzsystem durch eine Kombination aus Umlageverfahren und Kapitalstockverfahren berücksichtigt, wobei durch den Anteil der Umlagefinanzierung festgelegt wird, in welchem Umfang der intergenerative Risikoausgleich berücksichtigt wird. Die Risikoaversionen der Versicherten, d.h. die individuellen Präferenzen, sind das maßgebliche Kriterium für die Gewichtung des intergenerativen Risikoausgleichs. Diese lassen sich aber nur schwer und unpräzise ermitteln. Die Gewichtung des Status quo kann ebenso wenig als Referenz dienen, da gerade der Grad der Kapitalfundierung derzeit kontrovers diskutiert wird. Die Analyse basiert daher auf konditionalen Aussagen, wie z.B. "wenn die Risikoaversion gegen Null geht, dann werden bestimmte Optionen als intergenerativ gerecht angesehen". Die Gewichtung als normative Entscheidung ist dann Aufgabe der Politik.

Ad 2)

Um zu beurteilen, was zu den intergenerativ versicherbaren Risiken gezählt werden kann, werden vier Kriterien für eine intergenerative Umverteilung aufgestellt. Diese Kriterien werden für die im 2. Kapitel identifizierten, demographischen und ökonomischen Einflußfaktoren geprüft. Anschließend werden die Verteilungsindikatoren der Reformoptionen und des Referenzsystem so modifiziert, daß sie ausschließlich die intergenerative Umverteilung erfassen. Die Ergebnisse der Bewertung und die erforderlichen Modifikationen der Verteilungsindikatoren sind in Tabelle 7 auf Seite 198 wiedergegeben.

Ad 3)

Das "wie" eines intergenerativen Risikoausgleichs betrifft die Frage nach der Aufteilung der risikobedingten Zusatzlast. Die Aufteilung kann nur zwischen Rentnern und Erwerbstätigen einer Periode erfolgen (überlappende Risikogemeinschaft).

Die Regeln, nach denen die Zusatzlast bei Eintritt des Risikos auf die unterschiedlichen Generationen der Versichertengemeinschaft verteilt werden, lassen sich nicht aus einem allgemeinen, ethisch fundierten Gerechtigkeitskonzept ableiten. Sie bilden sich im politischen Entscheidungsprozeß und lassen sich nur auf gesellschaftlich gebildete Gerechtigkeitsvorstellungen gründen. Die Verteilungsregeln für die im Risikofall auftretende Zusatzlast orientieren sich daher in dieser Arbeit an der in der gesetzlichen Rentenversicherung verwirklichten Nettoanpassung.

Subtrahiert man den Verteilungsindikator des Referenzsystems von dem der zu beurteilenden Reformoption, so erhält man die Umverteilungsposition jeder Generation. Die verschiedenen Ansätze zur Bewertung der entstehenden Umverteilungsmuster wurden dis- 
kutiert und es zeigte sich, daß keines der möglichen Kriterien (weder Konzentrations-, Streuungs- und Lagemaße noch das Pareto- oder das Differenzenkriterium nach Rawls) für sich allein genommen eine ausreichende Bewertung garantiert. In der Analyse werden daher die verschiedenen Verläufe graphisch dargestellt und die diskutierten Kriterien als Ergänzung ausgewiesen.

Im 6. Kapitel wird untersucht, wie etwaige indirekte Verteilungswirkungen erfaßt werden können. Zunächst wurde die These vom Ersparnis- und Wachstumseffekt des Kapitalstockverfahrens eingehender untersucht. Die Kritik am Vermögenssubstitutionseffekt sowie die investitions- und modelltheoretische Einwände verdeutlichen, daß beide Prämissen der Wachstumseffekte, die erhöhte Ersparnisbildung als auch ihre Transformation in produktives Realkapital, theoretisch umstritten sind. Die empirischen Befunde erlauben ebenfalls keine eindeutige Schlußfolgerung.

Für die Simulationsanalyse wird daher unterstellt, daß die gesamtzwirtschaftliche Ersparnis durch den Kapitalstock der Alterssicherung nicht im Verhältnis 1:1 wächst. Die Auswirkungen werden durch pauschale Annahmen getroffen, wahlhandlungstheoretische Ansätze werden abgelehnt. Ebensowenig werden die Zins- und Wachstumseffekte durch einen Rekurs auf eine einfache neoklassische Produktionsfunktion ermittelt. Auch für diese Effekte werden exogene Annahmen getroffen und durch Szenarien variiert.

Etwaige Nachfrageeffekte des Umlageverfahrens auf dem Arbeitsmarkt werden nicht modelliert, da angenommen wird, daß die Arbeitgeberbeiträge von den Arbeitnehmern in Form von niedrigeren Reallöhnen zu tragen sind. Eine Operationalisierung von angebotsseitigen excess-burden-Effekten im Sinne einer kardinalen Nutzentheorie wird aus methodischen Gründen ebenfalls abgelehnt.

Abschließend wurde das entworfene Konzept angewandt, um ausgewählte Reformvorschläge hinsichtlich ihrer Konsequenzen für die intergenerative Gerechtigkeit zu untersuchen. Die exemplarischen Berechnungen zeigten die folgenden Ergebnisse:

- Der in dem Rentenreformgesetz 1999 vorgesehene demographische Korrekturfaktor trägt dazu bei, die Alterssicherung nach Maßgabe des in dieser Arbeit beschriebenen Meßkonzepts intergenerativ gerechter zu gestalten. Trotz dieser Verbesserung bleiben aber gravierende intergenerative Umverteilungen zwischen den Zugangskohorten bestehen.

- Mit einer weitergehenden Senkung des aktuellen Rentenwerts könnte erreicht werden, daß die Umverteilungspositionen der Zugangskohorten weiter angeglichen werden. Dieser Gewinn an Gerechtigkeit muß allerdings mit einer Verletzung des Ziels der Lebensstandardsicherung bei den Bestandsrenten abgewogen werden.

- Eine alternative Maßnahme, um das Umlageverfahren partiell durch das Kapitalstockverfahren zu substituieren, besteht darin, die neu erworbenen Anwartschaften (Entgeltpunkte) sukzessive zu senken. In dem Fall muß nicht mehr abgewogen werden, ob das Ziel der Lebensstandardsicherung auch bei den Bestandsrenten hinreichend gewährleistet ist. Diese Reformoption führt bei den in der Modellrechnung erfaßten 
Zugangskohorten (Zugangsjahr 1996 bis 2030) allerdings zu einer Verschlechterung der intergenerativen Gerechtigkeit.

Eine Begrenzung der Korrektur des aktuellen Rentenwerts, wie sie auch im Rentenreformgesetz vorgesehen ist, mag zunächst als willkürlich erscheinen. Vor dem Hintergrund der obigen Ergebnisse ist sie jedoch auch aus Überlegungen zur intergenerativen Gerechtigkeit erforderlich. Angesichts der noch bestehenden massiven Ungleichbehandlung der Zugangskohorten stellt sich allerdings die Frage, ob die nur halbe Berücksichtigung des Lebenserwartungsfaktors eine angemessene Abwägung darstellt: Bei einer vollen Berücksichtigung der Lebenserwartung würden die Umverteilungspositionen der Zugangskohorten noch stärker angeglichen werden. Das Rentenniveau würde in den Modellrechnungen statt auf $66,6 \%$ auf $63,2 \%$ sinken. Dieses Niveau ist auch deshalb aus Überlegungen zur intergenerativen Gerechtigkeit vertretbar, weil es erst im Jahr 2030 erreicht wird, so daß dieses Niveau für den Großteil der Bestandsrenten ohnehin nicht relevant ist.

Die verschiedenen Vorschläge zur Reform des Alterssicherungssystems werden anhand unterschiedlicher, z.T. interdependenter Kriterien bewertet. Wenngleich dabei das Kriterium der intergenerativen Gerechtigkeit aufgrund der demographischen Entwicklung an Bedeutung gewinnt, fehlt es bislang an einem Instrument, wie dieses Kriterium operationalisiert werden kann. Das in dieser Arbeit entwickelte Konzept bewertet die intergenerativen Verteilungswirkungen eines Alterssicherungssystems und leistet somit einen Beitrag zur Schließung dieser Lücke. Dennoch besteht nach wie vor ein Forschungsbedarf auf diesem Gebiet. Beispielsweise wurde bei der Berücksichtigung der indirekten Verteilungswirkungen auf die Defizite bisheriger Ansätze hingewiesen. Die Modellierung der indirekten Verteilungen kann jedoch auch in dem hier entwickelten Konzept verbessert werden, indem das Simulationsmodell durch ein makroönonometrisches Modul ergänzt wird. Dabei sollten vor allem die folgenden Forschungsschwerpunkte im Mittelpunkt stehen:

1. Wachstumseffekte resultieren vornehmlich deshalb, weil das Kapital infolge einer verbesserten Finanzintermediation effizienter alloziiert werden kann. Forschungsbedarf besteht daher vor allem hinsichtlich der Auswirkungen einer zusätzlichen Kapitalfundierung der Altersvorsorge auf die Kapitalmarktstrukturen ${ }^{527}$ und den Entwicklungsgrad der Finanzintermediation in Deutschland.

2. Ferner müßten die Auswirkungen externer Effekte, allen voran die der Europäischen Währungsunion, untersucht und modelliert werden.

527 Vgl. Davies (1993), S. 36-40 
An einigen Stellen werden normative Vorgaben benötigt ${ }^{528}$ oder es müssen Annahmen getroffen werden. ${ }^{529}$ Ein Vorteil des Konzepts ist, daß diese Annahmen und Vorgaben offen gelegt werden und sich im Konzept einfach variieren lassen. Auf diese Weise wird ihr Einfluß auf das Ergebnis transparent und erleichtert die Bewertung verschiedener Alterssicherungssysteme. Trotz der noch offenen Aufgaben, kann das vorliegende Konzept der Sozialpolitik daher als wertvolle Entscheidungshilfe bei der Bewertung verschiedener Reformoptionen dienen. Wenn die Arbeit darüber hinaus die wissenschaftliche Diskussion um die Bewertung der intergenerativen Verteilung eines Alterssicherungssystems anstößt und sich aus der Kritik an dem hier erarbeiteten Konzept weiterführende Ansätze ergeben, so wäre schon viel erreicht.

528 Z. B. bei der Gewichtung der beiden Ziele im Referenzsystzem, bei dem Ausmaß, in dem die Fertilität als intergeneratives Risiko erfaßt wird, bei dem Verteilungsschlüssel der Zusatzlast im Risikofall oder bei der Bewertung der resultierenden Verteilungsmuster.

529 Z. B. bei der Erfassung der Ersparnis- und Wachstumseffekte des Kapitalstocks sowie bei seinem Einfluß auf die erzielbare Verzinsung der Anlagen oder bei den Annahmen über die Inzidenz der Arbeitgeberbeiträge. 


\section{LITERATURVERZEICHNIS}

Aaron, J. J. (1966); "The Social Insurance Paradox“, Canadian Journal of Economics and Political Science, Bd. 33, S. 371-374.

Aaron, J. J. (1982); „Economic Effects of Social Security“, Washington, 1982.

Abel, A. (1985); „Precautionary Savings and Accidental Bequests“, in: American Economic Review, 75, S.777-791.

Achinger, H. (1958); „Sozialpolitik als Gesellschaftspolitik“, Hamburg.

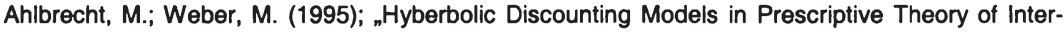
temporal Choice", in: Zeitschrift für Wirtschafts- und Sozialwissenschaften, 115, S. 535-568.

Allais, M. (1947); „Economie et intérét“, Paris, Imprimerie Nationale.

Allais, M. (1962); „The Influence of Capital-Output Ratio on Real National Income“, in: Econometrica, 30, S. 700-728.

Allekotte, H. A. (1981); „Möglichkeiten eines intertemporalen Belastungsausgleichs im Rahmen des Generationenvertrags der gesetzliche Rentenversicherung ${ }^{4}$, in: Herder Dorneich (Hrsg.), Dynamische Theorie der Sozialpolitik“, (1981), S. 203-223.

Allen, F. (1993); „Stock Markets and Resource Allocation“, in: Mayer, C.; Vives, X. (Hrsg), "Capital Markets and Financial Intermediation“, Cambridge.

Althammer, W. (1991); ,Steuern und Gerechtigkeit. Einige Bemerkungen zum Problem der ökonomischen Theorie der Gerechtigkeit“, in: Wahl, J. (Hrsg.), Steuerpolitik vor neuen Aufgaben. - Regensburg, 1991, S. $105-112$.

Althammer, W. (1991); „Steuern und Gerechtigkeit. Einige Bemerkungen zum Problem einer ökonomischen Theorie der Gerechtigkeit", in: Wahl, J. (Hrsg.), ${ }_{n}$ Steuerpolitik vor neuen Aufgaben“. Regensburg (1991), S. 105-112.

Altonjii, J.; Hayashi, F.; Kotlikoff, L. (1992); „IS the Extended Family Altruistically Linked? Direct Tests Using Micro Data“, in: American Economic Review, 82, 5, S. 1177-1198.

Arrow, K. J. (1951); „Alternative Approaches to the Theory of Choice in Risk-Taking Situations“", in: Econometrica, 19, 1951, S. 404-437.

Arrow, K. J. (1962); ${ }_{n}$ The Economic Implications of Learning by Doing“, in: Review by Economic Studies, 29, 1962, S. 155-173.

Arrow, K. J. (1963); „Social choice and Individual Values“, New York, 1963 “.

Arrow, K. J. (1970); „Essays in the Theory of Risk-Bearing“, Amsterdam u.a. 1970.

Arrow, K. J. (1973); ${ }_{n}$ A Theory of Justice by John Rawls: Two Review Articles“, in: Journal of Philosophy, 70 Jg. (1973), S. 245-263.

Atje, R. ; Jovanovic, B. (1993); „Stock Markets and Development", in: European Economic Review, Bd. 37, S. $632-640$.

Atkinson, A. B.; Stieglitz, J. E. (1980); „Lectures on Public Economics“, New York et al. McGrawHill. 
Auerbach, A. ; Gokhale, J.; Kotlikoff, L. J. (1991); „Generational Accounting: a meaningful alternative to deficit accounting", in: Bradford, D. (Hrsg), Tax policy and the economy, Bd. 5, Cambridge, S. 55 - 110.

Auerbach, A. ; Gokhale, J.; Kotlikoff, L. J. (1992); „Generational Accounting: a new approach to understanding the effects of fiscal policy on saving“, in: Scandinavian Journal of Economics, 94, S. 303-318.

Auerbach, A. J.; Kotlikoff, L. J. (1987); „Dynamik Fiscal Policy“, Cambridge: Cambridge University Press.

Bahr, H.; Kater, U. (1997); „Umlageverfahren versus Kapitaldeckungsverfahren - quo vadis Rentenversicherung?", in: Wirtschaftsdienst 4/97.

Barro, R. J. (1974); „Are Government Bonds Net Wealth?“, in: Journal of Political Economy, Bd. 82, S.1095-1117.

Barro, R. J. (1976); „Reply to Feldstein and Buchanan“, in : Journal of Political Economy, Bd. 84, 1976 , S. 343 - 349.

Barro, R. J. (1978); „The Impact of Social Security on Private Saving:Evidence from the U.S. Time Series", American Enterprise Institute for Public Policy Research, Washington D.C..

Barry, B. M. (1995); „Ist soziale Gerechtigkeit eine Illusion?“, Prokla, Heft 99, 25.Jg. 1995, Nr. 2, S. 235-243.

Bean, C. R. (1994); „European Unemployment: A Survey“, in: Journal of Economic Literature, 32, S. 573-619.

Beattie, R.; McGillivray, W. (1995); „A Risky Strategy: Reflections on the World Bank Report, 'Averting the Old Age Crisis', in: International Social Security Review, Volume 48, 3-4, 1995, S. 12.

Becker, B. (1981); „Probleme der Kapitaldeckung durch die Altersvorsorge“, Frankfurt/M.

Becker, G. S. (1974); „A Theory of Social Interaktions“, in: Journal of Political Economy, 82, S.1063-1093.

Becker, I. (1985); „Umverteilung durch positive und negative Transfers im Jahr 1981“, in: Hauser, R./Engel, B. (Hrsg.), ,Soziale Sicherung und Einkommensverteilung ${ }^{4}$, Frankfurt, Now York, 1985.

Bencivenga, V. ; Smith, B.; (1991); „Financial Intermediation and Endogenous Growth“, in: Review of Economic Studies, Bd. 58, S. 195-209.

Bernheim, D. (1989); "A Neoclassical Perspective on Budget Deficits.", in: Journal of Economic Perspectives, 3, Nr. 2, S. 55-72.

Bernheim, D. ; Schleifer, A.; Summers, L. (1985), „The Strategic Bequest Motive“, in: Journal of Political Economy, 93, S.1045-1076.

Berthold, N. (1986); ${ }_{n}$ Versicherungsprinzip und soziale Sicherung“, in: Finanzarchiv, 44, 1986, S. 350-358.

Berthold, N. (1988); "Marktversagen, staatliche Intervention und Organisationsformen Sozialer Sicherung“, in: Rolf, G.; Spahn, P.B.; Wagner G. (Hrsg.): „Sozialvertrag und Sicherung", Frankfurt/ New York 1988.

Berthold, N.; Külp, B. (1987); „Rückwirkungen ausgewählter Systeme der Sozialen Sicherung auf die Funktionsfähigkeit der Marktwirtschaft", in: Sozialpolitische Schriften Heft 47, Berlin 1987. 
Berthold, N.; Roppel, U. (1984); „Demographic Change and Old-Age Security“, in: G. Steinmann (Hrsg.), Economic Consequences of Population Change in Industrialized Countries, Berlin u.a., S. 218-237.

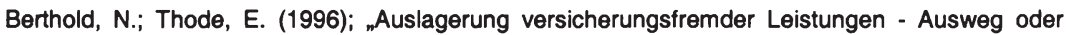
Kreisverkehr?", in: Wirtschaftsdienst 7/1996.

Beverage, W. H. (1943); „Der Beverage Plan. Sozialversicherung und verwandte Leistungen“, Zürich, New York.

Birnbacher, D. (1988); „Verantwortung für zukünftige Generationen“, Stuttgart, 1988.

Birnbacher, D. (1990); „Der Utilitarismus und die Ökonomie“, in: Biervert, B. u.a. (Hrsg.), „Sozialphilosophische Grundlagen ökonomischen Handelns, Frankfurt am Main, S. 65-85.

Blohm, ; Lüder (1991); „Investition“, 7. Auflage, München.

Blüm, N. (1996); „Zur Diskussion über die Fremdleistungen“, in: Bundesarbeitsblatt 6/1996, S. 5-9.

Blümle, G. (1990); „Der Einfluß verschiedener Alterssicherungssysteme auf das private Sparverhalten“, in Gahlen B. et. al. (Hrsg.): „Theorie und Politik der Sozialversicherung“, Tübingen 1990, S. $65-81$.

Boadway R. W.; Wildasin D. E. (1984); „Public Sector Economics“, Toronto, 1984.

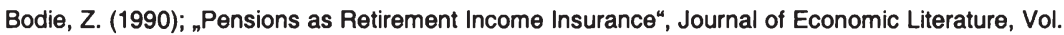
XXVIII.

Böhm-Bawerk, E. v. (1921); „Kapital und Kapitalzins, Zweite Abteilung:Positive Theorie des Kapitals ${ }^{u}, 4$. Auflage, Jena.

Bohnen, A. (1964); „Die utilitaristische Ethik als Grundlage der modernen Wohlfahrtsökonomik“, Monographien zur Politik, Heft 6, Göttingen.

Boll, S. (1996); „Intergenerative Verteilungseffekte offentlicher Haushalte“, Diskussionspapier 6/96 Volkswirtschaftliche Forschungsgruppe der Deutschen Bank.

Boll, S.; Raffelhüschen, B.; Walliser, J. (1992); „Social Security and Intergenerational Redistribution: A Generational Accounting Perspective“, Diskussionspapier, Institut für Finanzwissenschaft und Sozialpolitik, Universität Kiel, Nr. 41.

Boltho, A.; Holtham, G. (1982); ${ }_{n}$ The Assassment: New Approaches to Economic Growth“, in: Oxford Review of Economic Policy, 8, 1982, S. 1-14.

Bomsdorf, E. (1997); Die Renten sichern - Ein Weg aus der Rentenkrise“. in: Farny, D. (Hrsg.),Reihe: Versicherungswirtschaft Band 21,1997.

Borch, K. H.; Aase, K. K. ; Sandmo A. (1990); „Economics of Insurance“, North-Holland, Amsterdam u.a.

Bösch, M. (1987), „Umverteilung, Effizienz und demographische Abhängigkeit von Rentenversicherungssystemen“, Berlin / Heidelberg.

Bosduganov, N. (1998); ${ }_{n}$ Entwurf und Implementierung eines mikroökonomischen Modells der deutschen Rentenversicherung“, Diplomarbeit TU-Darmstadt, Fachgebiet Finanzwissenschaft.

Boyd, J.; Smith, B. (1992); „Intermediation and the Equilibrium Allocation of Capital. Implications of Economic Development", Journal of Monetary Economics, Bd.30, S. 409-432.

Brandts, J.; Bartolome, C.A.M. de (1992); ${ }_{n}$ Population Uncertainty, Social Insurance, and Actuarial Bias“, in: Journal of Public Economics, 47, S. 361- 380. 
Breyer, F. (1997); „Sind äquivalente" Renten fair?“, in: Hauser, R. (Hrsg.),Reform des Sozialstaats I, 1997.

Breyer, F. (1990); „Ökonomische Theorie der Alterssicherung“, München 1990.

Breyer, F. (1990a); „Das Aequivalenzprinzip in der Rentenversicherung aus wohlfahrtsoekonomischer Sicht", in: ,Fanzarchiv. - N.F.,48, S. 127 - 142; 1990.

Breyer, F. (1996); „Leitlinien für eine Systermkorrektur in der Rentenversicherung“, (BertelsmannStiftung), Siebert H. (Hrsg), Markt und Staat, 1996.

Breyer, F.; Spremann, K. (1990); „Übergangsprobleme der Rentenversicherung bei abnehmender Bevölkerung und Nozicks Theorie der Gerechtigkeit", in: Felderer, B. „Bevökerung und Wirtschaft" (1990), Berlin.

Breyer, F.; Kolmar, M. (1997); „Sozialpolitik und Verteilung: Ist Äquivalenz effizient, effektiv und stabil?".

Breyer, F; Straub M. (1993); WWelfare Effects of Unfunded Pension Systems When Labor Supply is Endogenous" Journal of Public Economics, 50, S.77-91.

Bridges, B. (1978); "Why the social Insurance Budget is too Large in a Democracy", Economic Inquiry 16, S. 133-138.

Browning, E.K. (1975); „Why the social Insurance Budget is too Large in a Democracy“, Economic Inquiry 13, S. 373-388.

Browning, M.,Lusardi, A. (1996); „Household Saving: Micro Theories and Micro Facts“, in: Journal of Economic Literature, 34, December 1996, S. 1797-1855.

Brunner, J. K. (1993); „Redistribution and the Efficiency of the Pay-as-You-Go Pension System“, Diskussionsbeitrăge Universität Konstanz Serie I - Nr. 265.

Brunner, J. K. (1996); Transition from a Pay-as-you-go to a Fully Funded Pension System: The Case of Differing Individuals and Intragenerational Fairness", in: Journal of Public Economics, 60, S. 131-146.

Burbige, J. (1983); „Social Securities and Saving Plans in Overlapping Generation Modells“, in: Journal of Public Economics, 21, S. 79-92.

Bürgisser-Peters, P. (1982); „Verteilungswirkungen der staatlichen Altrsvorsorge in der Schweiz“, Zürich.

Cagan, P. (1965); The Effect of Pension Plans on Aggregate Saving: Evidence from a Sample Survey“, National Bureau of Economic Research, Occasional Paper No. 95 New York 1965.

Carmichael, J. (1982); „On Barro's Theorem of Debt Neutrality: The Irrelevance of Net Wealth“ in: American Economic Review, 72, S. 202-213.

Carruth, A.; Schnabel, C. (1993); " The Determination of Contract Wages in West Germany“, in: Scandinavian Journal of Economics, 95, S. 297-310.

Champernowne, D. G. (1974); „A Comparison of Inequality of Income Distribution“, in: Economic Journal, 84, S. $787-816$.

Commission of the European Union (1994); ${ }_{n}$ Employment in Europe“, Brüssel.

Commission of the European Union (1995); „Employment in Europe“, Brüssel.

Crandall, R. W. (1993); „Manufacturing on the Move“, Whashington: Brookings Institution, 1993.

Crawford V. P.; Lilien D. M. (1981); " Social Security and the Retirement Decision“, in: Quarterly Journal of Economics, Vol. 96, 1981, S. 505 - 529. 
Darby, M. R. (1979) in The Effects of Social Security on Income and the Capital Stock“, Washington D.C.

Davies, B. (1996); „Equity Within and Between Generations“, Konferenz der GVG 13.-16. Juni 1996.

Davies, E. P. (1993); ${ }_{n}$ The Structure, Regulation, and Performance of Pension Funds in Nine Industrial Countries“, Policy Research Working Paper No. 1229, World Bank.

Diamond, D. (1984); „Financial Intermediation and Delegated Monitoring“, in: Review of Economic Studies, 51, 393-414.

Diamond, D. (1991); Monitoring and Reputation: The Choice Between Bank Loans and Directly Placed Debt", in: Journal of Political Economy, 99, S. 689-721.

Diamond, P. (1965); ${ }_{n}$ National Debt in a Neoclassical Growth Model“, American Economic Review, Bd. 55, S. 1126-1150.

Dinkel, R. (1983); „Werden Frauen durch die gesetzliche Rentenversicherung benachteiligt?“, in: Finanzarchiv, 41, Heft 1, S. 60-72.

Dinkel, R. (1984); „Umlage- und Kapitaldeckungsverfahren als Organisationsprinzipien einer allgemeinen Sozialversicherung“, WiSt Heft 4/84, S. 165-169.

Dinkel, R. (1985); „,Das Äquivalenzprinzip in Privat- und Sozialversicherung eine kritische Auseinandersetzung mit der herrschenden Oethodoxie", in: Zeitschrift für die gesamte Versicherungswissenschaft 74.Jg., S.345-369.

Dinkel, R. (1986), „Sozial Security and intergenerational Equity“, in: J.-M. Graf v.d. Schulenburg (Hrsg.), Essasy in Social Security Economics, Berlin u. a.O., S. 77-107.

Döring, D. (1992); „Alterssicherung in der EG“, Diskussionspapier Nr. 2, Akademie der Arbeit, Universität Frankfurt.

Döring, D. (1995); „Anmerkungen zum Gerechtigkeitsbegriff der Sozialpolitik mit besonderer Berücksichtigung liberaler Vorstellungen zur Sozialversicherungspolitik“, in: Döring, D. u.a., Gerechtigkeit und Wohlfahrtsstaat, Marburg, S.67-113.

Dudey, S. (1996); "Verteilungswirkungen des Sozialversichrungssystems der Bundesrepublik Deutschland und Modellierung seiner zukünftigen Entwicklung“.

Eisen R. (1980); „Das Äquivalenzprinzip in der Versicherung - unterschiedliche Folgerungen aus verschiedenen Interpretationen,,Zeitschrift für die gesamte Versicherungswissenschaft, 69 Jg., S. 529-556.

Eisen R. (1980); ,Das Äquivalenzprinzip in der Versicherung - unterschiedliche Folgerungen aus verschiedenen Interpretationen, Zeitschrift für die gesamte Versicherungswissenschaft, 69 Jg., S. 529-556.

Eitenmüller, S. (1996); „Die Rentabilität der gesetzlichen Rentenversicherung - Kapitalmarktanaloge Renditeberechnungen für die ferne Zukunft“, VDR 12/1996.

Enders, W.; Lapan, H. E. (1982); ${ }_{\text {}}$ Social Security Taxation and Intergenerational Risk Sharing“, in: International Economic Review, 23, S. 647-658.

Engels, W. (1969); „Rentabilität, Risiko und Reichtum“, Tübingen, 1969.

Famulla, R.; Spremann, K. (1980); "Generationsvertrăge und Rentenversicherung als Ponzi $\mathrm{GmbH}^{4}$, in: Zeitschrift für öffentliche und Gemeinwirtschaftliche Unternehmen, 3, Heft 3, S. 379-403.

Feldstein, M. (1974); ${ }_{n}$ Social Security, Induced Retirement and Aggregate Capital Accumulation, in: Journal of Political Economy, 82, S. 905-926. 
Feldstein, M. (1983); „Social Security Benefits and the Accumulation of Pre-retirement Wealth“, in: F. Modigliani und R, Hemming (Hrsg.), „The Determinants of National Saving and Wealth“, S.3 -23.

Feldstein, M. (1996); „The Missing Piece in Policy Analysis: social Security Reform“, in: AEA Papers and Proceedings, May 1996, 86, Nr. 2, S.1-14.

Fenge, R. (1996); „Pareto-efficiency of the Pay-as-you-go Pension System with Intragenerational Fairness", S. 357-363.

Frankfurter Institut (1997); „Rentenkrise. Und wie wir sie meistern können“, Band 21.

Franz, W. (1994); „Arbeitsmarktökonomik“, Berlin.

Franz, W. (1995); ${ }_{n}$ Theoretische Ansätze zur Erklärung der Arbeitslosigkeit: Wo stehen wir 1995“, Diskussionspapier 27, Centre for International Labor Economics, Universität Kon$\operatorname{stanz.}$

Frerich, J. (1987); „Sozialpolitik“, München.

Friedman, Milton (1957);A theory of the consumptrion function. Princeton: Princeton U. Press, 1957.

Gagern, M. (1977); „Das Sparprinzip. Politisch-ökonomische Betrachtungen zur Rawlsschen Gerechtigkeitstheorie", in: Höffe, O. (Hrsg.), Über John Rawls' Theorie der Gerechtigkeit, Frankfurt am Main, 1977, S. 259-282.

Gebler, O. (1995); „Das Versicherungsprinzip in der gesetzlichen Rentenversicherung“, Hamburg .

Gijsel P. de (1984); „Individuum und Gerechtigkeit in ökonomischen Verteilungstheorien“, in: Gijsel, P. et al. (Hrsg.), "Ökonomie und Gesellschaft", Jahrbuch 2: Wohlfahrt und Gerechtigkeit, Frankfurt am Main, 1984.

Glismann, Hans H./ Horn, Ernst-Jürgen 1997, ,Hat das umlagefinanzierte staatliche Rentensystem noch eine Chance?", Konjunkturpolitik 35. Jahrg. H. 46.

Glismann, Hans H./ Horn, Ernst-Jürgen, 1995, ,Die Krise des deutschen Systems sozialer Sicherung. I. Die staatliche Alterssicherung,ORDO, Jahrbuch für Ordnung, Wirtschaft und Gesellschaft Bd. 461995

Glissmann, H. H.; Horn, E. J. (1996) iz Zur Reform des deutschen Systems der Alterssicherung“, Kieler Arbeitspapiere Nr. 767.

Gordon, R. H..; Varian H. R. (1988); „Intergenerational Risk Sharing“, in: Journal of Public Economics, 37, S. 185-202.

Gordon, S. (1976); „The New Contractarians“ in: Journal of Political Economy, 84, S. 573-590.

Greenwood, J.; Jovanovic, B. (1990); „Financial Development, Growth and the Distribution of Income“, Journal of Political Economy, Bd. 98, S. 1076-1107.

Grohmann H. (1981), "Wege zur Bewahrung der langfristigen Stabilität der Rentenversicherung im demographischen, ökonomischen und sozialen Wandel", in: Deutsche Rentenversicherung, 1981, S. 265-290.

Grohmann, H. (1977); „Bevölkerungsmodelle und sozialpolitische Entscheidung“, in: Allgemeines Statistisches Archiv, Heft 4, 61, S. 349-370.

Grohmann, H. (1987); „Probleme der Abschätzung des für ein Kapitaldeckungsverfahrenin der gesetzlichen Rentenversicherung notwendigen Deckungskapitals - Theoretische Überlegungen und empirische Ergebnisse“; in: Felderer, Bernhard (Hrsg), „Kapitaldeckungsverfahren versus Umlageverfahren“, Schriften des Vereins für Socialpolitik, Band 163, Berlin 1987, S. 67 - 89.

261 
GVG-Papier „Alterssicherung : Finanzierung durch Umlage und Kapitaldeckung“,

Hackmann, J. (1996); „Verteilungsgerechtigkeit aus volkswirtschaftlicher Sicht: Vorüberlegungen zu einer Steuerverteilungslehre“, in: Nutzinger, H. G. (Hrsg.), Wirtschaftsethische Perspektiven III: Unternehmensethik, Verteilungsprobleme, methodische Ansätze, Schriften des Vereins für Socialpolitik N.F., 228/III, Berlin, S. 95-136.

Härlen, H. (1972); „Die Grenzen der Versicherbarkeit, zum Beispiel in der Lebensversicherung stark erhöhter Risiken “, in: Zeitschrift für die gesamte Versicherungswissenschaft, S. 269-282.

Haveman, R. (1994); „Should Generational Accounts Replace Public Budget and Deficit", in: Journal of Economic Perspectives, 8, Nr.2, S. 95-112.

Hayek, F. A. (1981); „Recht, Gesetzgebung und Freiheit“, Bd. 2: Die Illusion der sozialen Gerechtigkeit, Landsberg am Lech 1981.

Helms, L. J. (1985); „The Effects of States and Local Taxes on Economic Growth: A Time Series Cross Section Approach“, in: Review of Economics and Statistics, 67, 4 (November 1985): S. 574-82.

Hering, T. (1994); „Investitionstheorie aus der Sicht des Zinses“, Wiesbaden.

Hoffmann, J. (1989) ${ }_{n}$ Sparen im Dienste der Altersvorsorge als volkswirtschaftliches Problem“, Untersuchungen zur Wirtschaftspolitik, Band 78, Köln.

Holzmann, R. (1988); „Zu ökonomischen Effekten der österreichischen Pensionsversicherung: Einkommensersatz, Ruhestandsentscheidung und interne Ertragsraten“, in: Holzmann, R. (Hrsg.), Ökonomische Analyse der Sozialversicherung, Wien, S. 153-205.

Homann, K. (1989); „Vertragstheorie und Property-Rights-Ansatz - Stand der Diskussion und Möglichkeiten der Weiterentwicklung“, in: Biervert, B.; Held, M. (Hrsg.), Ethische Grundlagen der ökonomischen Theorie. Eigentum, Verträge, Institutionen, Frankfurt, New York, S. $37-69$.

Homburg, S. (1988); „Theorie der Alterssicherung“, Berllin et. al. Springer-Verlag.

Homburg, S. (1990); ${ }_{n}$ The Efficiency of Unfunded Pension Systems“, in: Journal of Institutional and Theoretical Economics, 146, S. 640-647.

Homburg, S. (1995); „Humankapital und endogenes Wachstum“, Zeitschrift für Wirtschafts- und Sozialwissenschaften, Bd. 115, S. 339-366.

Homburg, S.; Richter, W. F. (1990); „Eine effizienzorientierte Reform der GRV“, in: Felderer, B. (Hrsg.), Bevölkerung und Wirtschaft, Berlin, S. 183- 191.

Hughes, G. (1996); „Pension Financing, the Substitution Effect and National Savings“, Paper for the international conference on Pensions in the European Union, Munster 14-15 June 1996.

Hughes, G. (1994); „Private Pensions in OECD Countries: Ireland“, Paris OECD.

Innami, H. (1966); „Das Äquivalenzprinzip in der Versicherungswirtschaft", in: Zeitschrift für die gesamte Versicherungswissenschaft, 55, S. 17-30.

Ireland, P. (1994); „Money and Growth“, American Economic Review, Bd. 84, S. 47-65.

Jaeger, K. (1994); On the Influence of Pay-as-you-go Financed Pension Systems on Saving: The Role of Stability", in: Scotisch Journal of Political Economy, Vol. 41, No. 4, November 1994. 
Jaeger, K. (1987); " “Optimale Anpassung der Rentenfinanzierung bei abnehmenden Wachstumsraten der Bevölkerung", in: Felderer, B. (Hrsg.),Kapitaldeckungsverfahren versus Umlageverfahren, Berlin, 1987.

Jaeger, Klaus, 1990, Allokations- und Distributionseffecte schrumpfender Bevölkerungen bei alternativen Alterssicherungssystemen in Modellen überlappender Generationen, in Theorie und Politik der Sozialversicherung - Schriftenreihe des Wirtschaftswissenschaftlichen Seminars Ottobeuren, Tübingen S. 67-95

Jäger, N. (1991); „Die Umstellung der gesetzlichen Rentenversicherung auf ein partiell kapitalgedecktes Finanzierungsverfahren: Eine Simulationsanalyse“, Europäische Hochschulschriften Reihe 5 Bd.1991.

Janssen, M.; Müller, H. (1981); „Der Einfluß der Demographie auf die Aktivitäten des Staates: die Finanzierung der 1. und 2. Säule der Altersvorsorge", in: Schweizerische Zeitschrift für Volkswirtschaft und Statistik, 117, S. 297-314.

Jobelius, H.-J. (1991);,Kapitaldeckungs- und Umlageverfahren und ihr Einfluß auf die private Ersparnisbildung“, ZeS-Arbeitspapier Nr. 5/91.

Kaehler, J.; Korn, O. (1995); „Zusammenhänge zwischen Zinsen und makroökonomischer Aktivität“", Baden-Baden.

Karten, W. (1988); „Existenzrisiken der Gesellschaft”, in: Zeitschrift für die gesamte Versicherungswirtschaft, S. 343-362.

Katona, G. (1965);,Private Pensions and Individual Saving“, Monograph No. 40, Survey Research Center, Institute for Social Research, Ann Arbor 1965.

King, R.; Levine, R. (1992), „Financial Indicators and Economic Growth in a Cross Section of Countries“, PRE Working Paper, Nr. 819, The World Bank.

King, R.; Levine, R. (1993), „Financial Intermediation and Economic Development", in: Mayer, C.; Vives, X. (Hrsg.), Capital Markets and Financial Intermediation, Cambridge.

Klein, T. (1995); „Mortalităt in Deutschland - Aktuelle Entwicklungen und soziale Unterschiede“, in: Zarpf, W.; Schupp, J.; Habich, R. (Hrsg.), Lebenslagen im Wandel: Sozialberichtserstattung im Längsschnitt, Frankfurt, New York.

Knappe, E. (1995); „Umbau des Sozialstaates - Kranken-, Renten- und Arbeitslosenversicherung“, List Forum Band 21, 1995 Heft 4. S.342-371.

Knight, F. H. (1921); „Risk, Uncertainty and Profit“, Chicago, 1971 (Original 1921).

Kohli, M. (1987) inRuhestand und Moralökonomie“, in: Heinemann K. „Soziologie des wirtschaftlichen Handelns", Opladen 1987, S.393-416.

Kolb, R. (1985); „Die Bedeutung des Versicherungsprinzips für die gesetzliche Rentenversicherung“, in: Schmăhl, W. (Hrsg.), Versicherungsprinzip und soziale Sicherung, Tübingen, 1985.

Koppelmann, K.-P. (1979), "Intertemporale Einkommensumverteilung in der gesetzlichen Rentenversicherung der Bundesrepublik Deutschland“, Göttingen 1979.

Kotlikoff, L. J. (1979); , Testing the Theory of Social Security and Life Cycle Accumulation“, in: American Economic Review, Vol. 69, 1979, S. 396 ff.

Kötter, R. (1984); „Distributive Gerechtigkeit und Wohhlfahrt“, in: Gijsel, P. et al. (Hrsg.), „Ökonomie und Gesellschaft", Jahrbuch 2: Wohlfahrt und Gerechtigkeit, Frankfurt am Main, 1984.

Kreßmann, K. (1971); „Das versicherungstechnische Äquivalenzprinzip in der gesetzlichen Altersversicherung der BRD, Dissertation, Frankfurt a.M., 1971.

263 
Krupp, H.-J. (1981); "Grundlagen einer zielorientierten und integrierten Alterssicherungspolitik“, Einzelgutachten für die Wissenschaftlergruppe des Sozialbeirats, in: Sozialbeirat, BD. 2, S. 95-142.

Krupp, H.-J. (1995); „Bestimmungsfaktoren volkswirtschaftlicher Kapitalbildung“, in: arbeitgeber Sonderausgabe Juni 1995, Kapitalbildung und Investitionstätigkeit im Zeichen der Globalisierung.

Krupp, H.-J. (1997); Makroökonomische Perspektiven einer Teilkapitaldeckung der Rentenversicherung“, in: Wirtschaftsdienst 1997/IV, S. 203-211.

Krupp, H.-J. (1997a); „Ist das Kapitaldeckungsverfahren in der Alterssicherung dem Umlageverfahren überlegen?", in: WSI Mitteilungen 7/1997.

Külp, B. (1991); „Unterschiedliche Finanzierungssysteme der gesetzlichen Rentenversicherung und ihr Einfluß auf die Verteilung zwischen den Generationen“, Hamburger Jahrbuch 1991, S. 35-54.

Kune, J. B. (1983); "Studies on the Relationships between Social Security and Personal Saving“, in: Kredit und Kapital, 16, 1983, S. 371-380.

Küng, E. (1967), „Wirtschaft und Gerechtigkeit“, Tübingen, 1967.

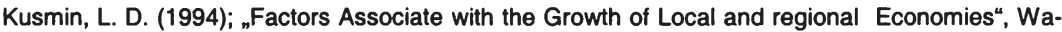
shington: USDA, Economic Research Service, 1994.

Lampert, Heinz (1990), „Notwendigkeit, Aufgaben und Grundzüge einer Theorie der Sozialpolitik“, in: ${ }_{n}$ Theoretischen Grundlagen der Sozialpolitik", Schriften des Vereins für Socialpolitik Band 193, 1990.

Lampert, Heinz (1990a), „Die Bedeutung der Gerechtigkeit im Konzept der Sozialen Marktwirtschaft." in: Hamburger Jahrbuch für Wirtschafts- und Gesellschaftspolitik 35 (90),S. 75-91.

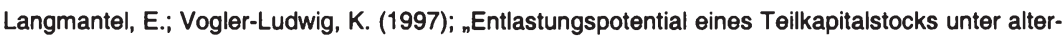
nativen Bevölkerungsannahmen“, Studie im Auftrag der Enquete-Kommission Demographischer Wandel Deutscher Bundestag.

Le Grand, J. (1984); ${ }_{n}$ The Future of the Welfare State ${ }^{4}$, in: New Society, 68, S. 385-386.

Le Grand, J.; Winter, D. (1987); ${ }_{n}$ The Middle Classes and the Defence of the Britisch Welfare State ${ }^{4}$, in: Goodin, R.E.; Le Grand, J. (Hrsg.), Not only the Poor. The Middle Classes and the Welfare State, London, ${ }_{n}$. 147-168.

Lepelmeier, D. (1979); „Soziale Sicherung und Parafiskalität“, Frankfurt u.a.

Liefmann-Keil, E. (1961); „Ökonomische Theorie der Sozialpolitik“, Berlin u.a.

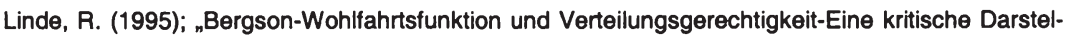
lung", in: WISU Heft 10, 1995, S.852-867.

Lindert, P, H. (1996a) inDoes Social Spending Deter Economic Growth?“, in: Challenge, 5/6 1996, S. 17-23.

Lindert, P. H. (1996); "What Limits Social Spending?“ Explorations in Economic History 33, 1 (January 1996), S.1-34.

Lübbe, G. (1978); ${ }_{n}$ Robert Nozicks Naturrechtsidealismus. Zu einer Karrikatur des klassischen Liberalismus“, Rechtstheorie 9, S. 217-228.

Lucas, R. E. Jr. (1988); „On the Mechanics of Economic Development", Journal of Monetary Economics, Bd. 22, S. 3-42. 
Lucius, R.-R. (1979); „Die Grenzen der Versicherbarkeit“, Frankfurt a. M..

Mackenroth, G. (1952); „Die Reform der Sozialpolitik durch einen deutschen Sozialplan“, in: Külp, B; Schreiber, W. (Hrsg.), Soziale Sicherung, S. 265-275, Köln, Berlin.

Männer, L. (1974), „Überlegungen zum optimalen Finanzierungsverfahren in der gesetzlichen Rentenversicherung“ "Fanzarchiv N.F. (32), 1974, S. 244ff..

Masson P.R.; Bayoumi T. und Samiei, H. (1995);Saving behavior in Industrial and Developing Countries, Staff Studies for the World Economic Outlook. Washington DC: International Monetary Fund.

Masson P.R.; Bayoumi T. und Samiei, H. (1996);International Evidence on the Determinants of Private Saving. London: Centre for Economic Policy Research, Discussion Paper No. 1368.

Maydell, B. v. (1983); "Lebensversicherung“, Sachverständigenkommission Alterssicherungssysteme, Darstellung der Alterssicherungssysteme in der Besteuerung von Alterseinkommen, Gutachten der Sachverständigenkommission 2, S. 405-423, Bonn.

Maydell, B. v. (1995); „Alterssicherungssysteme im Transformationsproze $\beta^{4}$, Die Angestelltenversicherung Heft 7-8/95.

Meier, C.-P. (1995) in Demographie und Ersparnis“, in: Zeitschrift für Wirtschaftspolitik Jg. 44 (1995), Heft 1, S. 85-110.

Meinhold, H. (1976), „Sozialpolitik durch sozialpolitische Parafisci“, Tübingen, 1976.

Meinhold, H. (1980); „Der Generationenvertrag und die verschiedenen Formen unserer Alterssicherung“, in: „Bevölkerungsentwicklung und Generationenvertrag“, Besters H. (Hrsg.), Baden-Baden, S.18ff.

Meinhold, H. (1984); „die finanziellen und wirtschaftlichen Rahmenbedingungen der 80er Jahre“, in: Nachrichtendienst des deutschen Vereins öffentliche und private Fürsorge, Heft 3, 1984, S. 107-112.

Meinhold, H. (1985); „Die ordnungspolitische Bedeutung des Versicherungsprinzips in der deutschen Sozialpolitik“, in: Schmähl, W. (Hrsg.), Versicherungsprinzip und soziale Sicherung, Tübingen, 1985.

Merton, R.C. (1983); "On the Role of Social Security as a Means for Efficient Risk Sharing in an Economy where Human Capital is not Tradable“, in: Bodie, Z.; Shoven, J.B. (Hrsg.), Financial Aspects of the United States Pension System, Chicago, S. 325-358.

Modigliani, F.; Brumberg, R. (1954); „Utility Analysis and the Consumption Function: An Interpretation of the cross-section Data“, in: Kurihara K. (Hrsg.), Postkeynesian economics, New Brunswick, NJ: Rutgers U. Press, 1954, pp. 388 - 436.

Mugler, J. (1980); „Risikopolitische Strategien im Grenzbereich des Versicherbaren“, in: Zeitschrift für die gesamte Versicherungswissenschaft, 69, S. 71-87.

Müller, H.-W.; Roppel, U. (1990); Lösung der demographisch bedingten Probleme der Alterssicherung durch Kapitaldeckung? - Zur Abschätzung des Deckungskapitals der Anwartschaften in der gesetzlichen Rentenversicherung der Bundesrepublik Deutschland", in: Acta Demographica Band 1, Heidelberg 1990, S. 107-130.

Munnel, A. (1976); „Private Pensions and Savings: New Evidence“, Journal of Political Economy, Bd. 84, S. 1012-1032.

Munnel, A. (1991); „Are Pensions Worth the Cost?", National Tax Journal, September. 
Nell-Breuning, O. V. (1985), Gerechtigkeit und Freiheit, Grundzüge katholischer Soziallehre, 2. Auflage, München 1985.

Nelson, R. R.; Winter, S. G. (1982); „An Evolutionary Theory of Economic Change“, Cambridge, 1982.

Neumann, M. (1986), „Möglichkeiten zur Entlastung der gesetzlichen Rentenversicherung durch kapitalbildende Vorsorgemaßnahmen“, Tübingen.

Neumann, M. (1996); „Wie kann ein Übergang vom bestehenden Rentenversicherungssystem zum Kapitaldeckungsverfahren gestaltet werden?"

Neusser, K.; Kugler, M. (1994), "Manufactoring Growth and Financial Sector Development in OECD Countries“, mimeo.

Nozick, R. (1976); „Anarchie, Staat, Utopia“, Übersetzung von Vetter, H., München.

Nullmeier, F.; Vobruba, G. (1995); „Gerechtigkeit im sozialpolitischen Diskurs“, in: Döring, D.; Nullmeier, F.; Pioch, R.; Vobruba, G. (1995), Gerechtigkeit im Wohlfahrtsstaat, Marburg, S. 11-66.

o.V. (1997); „Bei der Rente ist ein sanfter Übergang zur Kapitaldeckung möglich“, in: Frankfurter Allgemeine Zeitung vom 13 März 1997.

OECD (1994); The OECD Jobs Study, Evidence and Explanations - Part II, The Adjustment Potential of the Labour Market, Paris.

Ohsmann, S.; Stolz, U. (1997); „Beitragszahlungen haben sich gelohnt“, in: Die Angestelltenversicherung Heft $3 / 97$.

Pagano, M. (1993); "Financial Markets and Growth: An Overview", in: European Economic Review, 37, S. 613-622.

Perelman, C. (1967); „Über die Gerechtigkeit", München.

Petersen, J. H., (1988), "A Note on Old-Age Pensions, Demography and the Distribution Between Generations“, Journal of Institutional and Theoretical Economics 144, S. 333-342.

Pflug, G. (1979); ${ }_{n}$ Statistische Konzentrationsmaße - ein mathematischer Überblick“, in: Allgemeines Statistisches Archiv, 63, S. $240-259$.

Phelps, E. S. (1961); „The Golden Rule of Accumulation: A Fable for Grothmen“, in: American Economic Review, 51, 1961, S. 628-643.

Phelps, E. S. (1965); ${ }_{n}$ Second Essay on the Golden Rule of Accumulation: A Fable for Grothmen“, in: American Economic Review, 55, 1965, S. 792-814.

Piesch, W. (1975); „Statistische Konzentrationsmaße“, Tübingen.

Plant, R.; Lesser, H.; Taylor-Gooby, P. (1980); „Political Philosophy and Social Welfare. Essays on the Normative Basis of Welfare Provision, London u.a.

Preschitz, G. (1994); „Offentliche und private Pensionsversicherung“, Diplomarbeit TU Wien.

Prinz, A (1994), ${ }_{n}$ Der Zwang ist aber ein Übel, wenngleich ein notwendiges ${ }^{u}$ : Von der ${ }_{n}$ Armenassekuranz" zum "Voll eigenständigen System"?, in: Jahrbücher für Nationalökonomie und Statistik 213 (1994), S. 106ff. .

Prinz, C. (1997); "Verlierer und Gewinner des Generationenvertrages“, Finanznachrichten Heft 20/21 15. Mai 1997.

Prognos (1995); Eckerle, K.; Schlesinger, M.; ${ }_{n}$ Perspektiven der gesetzliche Rentenversicherung für Gesamtdeutschland vor dem Hintergrund veränderter politischer und ökonomischer Rahmenbedingungen“, in: DRV-Schriften, Bd. 4, Frankfurt a.M.. 
Queisser, M. (1993); „Vom Umlage- zum Kapitaldeckungsverfahren: Die chilenische Rentenreform als Modell für Entwicklungsländer?", ifo Institut für Wirtschaftsforschung, München / Köln / London.

Queisser, M. (1998); „Die Rente mit Kapital unterlegen“, in: Frankfurter Allgemeine Zeitung vom 24. Januar 1998.

Raffelhüschen, B. (1989); „Anreizwirkungen des Systems der sozialen Alterssicherung - Eine dynamische Analyse“, Frankfurt.

Raffelhüschen, B.; Walliser, J. (1996); „Generational Accounting: Eine Alternative zur Messung intergenerativer Verteilungswirkungen“, Wirtschaftswissenschaftliches Studium, 4/1996, S. 181-188.

Ramser H. J. (1987), "Verteilungstheorie“, Heidelberg.

Ramsey, F. P. (1928); „A Mathematical Theory of Saving“, in: Economic Journal, 38, S. 543-559.

Rawls, J. (1971), „A Theory of Justice“, Cambridge.

Rawls, J. (1975), „Eine Theorie der Gerechtigkeit“, Frankfurt a. M..

Reichel, G. (1981); „Operations Research im Versicherungswesen“, in: Zeitschrift für Operations Research, 25, S. B1-B8.

Ribhegge, H. (1990); „Denkfehler zum Thema Alterssicherung. Kapitaldeckungs- versus Umlageverfahren“, in: Jahrbuch für Sozialwissenschaft Heft 41 1990, S. 359-376.

Richter, W.F. (1990); "Social Security as a Means of Overcoming the Nonmarketability of Human Capital", in: Finanzarchiv, 48, S. 451-466.

Rische, H. (1996); „Bundeszuschuß und versicherungsfremde Leistungen in der Rentenversicherung“, Vortrag zur Sitzung der Kommission „Fortentwicklung der gesetzliche Rentenversicherung“ am 30.9.1996.

Robinson, J. (1962); „A Neoclassical Theorem“, in: Review of Economic Studies, 29, S. 219-226.

Romer, P. M. (1986); „Increasing Returns and Long-Run Growth“, Journal of Political Economy, Bd. 94, S. 1002-1037.

Ruf, T. (1989); „Rentenreform: Abschied von Illusionen?“, in: Arbeit und Sozialpolitik 2, $1989 .$.

Rürup, B. (1995); „Rentenfinanzierung nach dem Kapitalstockverfahren - Möglichkeiten und Probleme“, in: Forschungsinstitut der Friedrich-Ebert-Stiftung (Hrsg.), Harmonisierung der Rentenversicherungssysteme - Sprengen Pensionslasten die Staatskasse?, Gesprächskreis Arbeit und Soziales, 53, 1995, Bonn..

Rürup, B. (1997); „Hat das umlagefinanzierte Rentensystem noch eine Chance?“, in: Beihefte der Konjunkturpolitik, 46, 1997, S. 75-88.

Rürup, B.; Schroeter, I. (1996); „Perspektiven der gesetzliche Rentenversicherung“, Gutachten im Auftrag des Bundesministeriums für Arbeit und Sozialordnung, Darmstadt.

Rürup, B.; Schroeter, I. (1997); „Perspektiven der Pensionsversicherung in Osterreich“, Gutachten im Auftrag des Bundesministeriums für Arbeit, Gesundheit und Soziales, Darmstadt.

Sachverständigenrat (1996); ${ }_{n}$ Sachverständigenrat zur Begutachtung der gesamtwirtschaftlichen Entwicklung - Jahresgutachten 1996/97“, Fünftes Kapitel: Reform der sozialen Sicherung, S.321-409.

Samuelson, P. A. (1958); „An Exact Consumption-Loan Model of Interest with or without the Social Contrivance of Money", Journal of Political Economy, Bd. 66, S. 467-482 
Samuelson, P. A. (1968); " "The Two-Part Golden Rule Deduced as the Asymtotic Turnpike of Catenary Motions", in: Western Economic Journal, S. 85-89.

Samuelson, P. A. (1975); „Optimum Social Security in a Life-Cycle Growth Model“, International Economic Review, Bd. 16, S. 539-544.

Saxer, W. (1955); „Versicherungsmathematik“, Berlin..

Schaich, E. (1990); „Sensitivitätsanalyse von Disparitätsmaßen“, in: Allgemeines Statistisches Archiv, 74, S. 457-488.

Schlotter, H. G. (1981); „Intertemporale (intergenerationale Gerechtigkeit und Chancengleichneit“, in: Schriften des Vereins für Socialpolitik, 123.

Schlotter, H.-G. (1984); "Die Grenzen des Sozialstaats als normatives Problem“, in: HerderDornreich, Ph.; Klages, H.: Schlotter, H.-G., „Überwindung der Sozialstaatskrise“, Baden-Baden 1984.

Schmähl (1997b); „Die Zukunft des Sozialstaats im Spannungsfeld zwischen Solidarität und Subsidiarität“" Vortrag anläßlich des 50jährigen Bestehens des GVG am 28. Oktober 1997. Informationsdiesnt Nr. 262, S. 11-35.

Schmähl W. (Hrsg.) (1993); ;Mindestsicherung im Alter. Erfahrungen, Herausforderungen, Strategien."

Schmähl, W. (1977); „ Alterssicherung und Einkommensverteilung“, Tübingen.

Schmähl, W. (1983); „Lebenseinkommensanalysen - Einige methodische und empirische Fragen im Überblick“, in: Schmähl W. (Hrsg.): Ansätze der Lebenseinkommensanalyse, Tübingen 1983, S. 1-55.

Schmähl, W. (1986); „Soziale Sicherung und Schattenwirtschaft - Ein Problem überblick“, in: Winterstein, H. (Hrsg.), Sozialpolitik in der Beschäftigungskrise I., Schriften des Vereins für Socialpolitik, Gesellschaft für Wirtschafts- und Sozialwissenschaften, N.F., Bd. 152/l., Berlin, S. 137-206.

Schmähl, W. (1997); „Altersversicherung Quo vadis?“, in: Jahrbücher für Nationalökonomie und Statistik, 216,S. 413-435.

Schmähl, W. (1995); „Perspektiven der Alterssicherung - Anmerkung zur deutschen und internationalen Diskussion“, Arbeitsunterlage 13/17 der Enquete-Kommission Demographischer Wandel.

Schmid, F. (1991); „Zur Sensitivität von Disparitătsmaßen“, in: Allgemeines Statistisches Archiv, 75 , S. $155-167$.

Schniewind, H.-J. (1989); Gesetzliche Rentenversicherung und Konsum“, Freiburg 1989.

Schulenburg J.-M. Graf v.d. (1990); ${ }_{\text {N }}$ on der Marktwirtschaft zur ,sozialen Marktwirtschaft' - eine Operationalisierung des Begriffs ,sozial' “, in: W. Fischer (Hrsg.), Währungsreform und soziale Marktwirtschaft; Berlin, S. 197-213.

Schulenburg von der, J.-M. (1989); Demographischer Wandel, intergenerationale Gerechtigkeit und Stabilität des Generationenvertrages“, S.269-300.

Schulz-Weidner, W. (1996); "Das "chilenische Modell“ einer Privatisierung der Rentenversicherungmehr Leistung für weniger Beiträge?“, in: Deutsche Renten Versicherung 3/96, S. 158175.

Schumpeter, J.A. (1950); „Kapitalismus, Sozialismus und Demokratie“, Tübingen, 1993 (Original: München, 1950). 
Seidman, L. S. (1986); „A Phase Down of Social Security: The Transition in a Life-Cycle Growth Model“, in: National Tax Journal, 39, S. 97- 107.

Seng, P. (1989); „Informationen und Versicherung“, Wiesbaden.

Sesselmeier, W.; Blauermel, G. (1990); „Arbeitsmarkttheorien“, Heidelberg.

Sheshinsky, E.; Weiss, Y. (1981); "Uncertainty and Optimal Social Security Systems“, in: The Quarterly Journal of Economics, 96, Nr.2, S. 189-206.

Smith, A. (1982); „Intergenerational Transfers as Social Insurance“, in: Journal of Public Economics, 19, S. 97-106.

Solow, R. M. (1956); „A Contribution to the Theory of Economic Growth“,in: Quarterly Journal of Economics, 81, S. 65-94.

Speckbacher, G., (1994); „Alterssicherung und intergenerationale Gerechtigkeit: Eine modellorientierte Analyse“, Heidelberg 1994.

Spieker, M. (1996), „Solidarität oder Rentenfrondienst“, FAZ vom 31. Mai 1996.

Spremann, K. (1984), „Intergenerational Contracts and Their Decomposition“, Zeitschrift für Nationalökonomie, Bd. 44, S.237-253.

Spremann, K. (1987); "Generationenverträge bei instationärer Wirtschaftsentwicklung“, in: Felderer, B. (Hrsg.), Kapitaldeckungsverfahren versus Umlageverfahren, Schriften des Vereins für Socialpolitik, 163, Berlin, S. 15-25.

Steden, W. (1981); „Die Finanzierung der gesetzliche Rentenversicherung in der Bundesrepublik Vermögenstheoretische Implikationen“, Finanzarchiv, N.F., 39, S. 409-461.

Steiner, V. (1996); „Employment and Wage Effects of Social Security Financing“, Discussion Paper No. 96-14, ZEW, Mannheim.

Stolz, E. (1983); „Einkommensumverteilung in der Bundesrepublik Deutschland“, Frankfurt, New York.

Strotz, R. H. (1955); „Myopia and Inconsistency in Dynamic Utility Maximization“, in: Review of Economic Studies, 23, S. 165-180.

Sudhoff, B. (1996); „Alterssicherung, demographischer Wandel und intergenerationelle Gerechtigkeit : eine theoretische Untersuchung", Hamburg.

Swan, T. W. (1963); „Growth Models of Golden Ages and Production Functions“, in: Berrill, K. E. (Hrsg.), Economic Development with Special Reference to East Asia.

Tabellini, G. (1990); „A Positive Theory of Social Security, National Bureau of Economic Research, Working Paper No. 3272.

Thießen F. (1993); „Das Paradoxon von Maurice Allais: zeigt es die Notwendigkeit zu erneuernden Verhaltenstheorien?", in: Schmalenbachs Zeitschrift für betriebwirtschaftliche Forschung, Bd. 45, 1993, S. $157-174$.

Thompson, L. H. (1983); „The Social Security Reform Debate“, in: Journal of Economic Literature, 21, S. 1425-1467.

Thullen, P. (1981); „Der Begriff der Beitragsäquivalenz aus der Sicht der Versicherungsmathematik", in: Deutsche Rentenversicherung, 1981, S. $497-513$.

Tillmann, G. (1991); „Steuern und Gerechtigkeit“ ,in: Wahl, J. (Hrsg.), Steuerpolitik vor neuen Aufgaben. - Regensburg, 1991, S. 83 - 104.

Townley, T.G.C.(1981); „Public Choice and the Social Insurance Paradox: A Note“, Canadian Journal of Economics 14, S.712-717.

269 
Uzawa, H. (1965); nOptimum Technical Change in an Aggregative Model of Economic Growth“, in: International Economic Review, 6, S. 18-31.

VDR (1987), Verband Deutscher Rentenversicherungsträger: Kommissionsgutachten, „Zur langfristigen Entwicklung der gesetzlichen Rentenversicherung“, Kurzfassung, Franfurt a.M. 1987, S. $12 f$.

VDR (1997), Rentenversicherung in Zahlen 1997

Verbon, H. A. A. (1986); „Altruism, Political Power and Public Pensions“, in: Kyklos, 39, S. 343-358.

Voges, W.; Schmidt, C. (1995); „Lebenslagen, die Lebenszeit kosten - Zum Zusammenhang von sozialer Lage, chronischer Erkrankung und Mortalität im zeitlichen Verlauf", in: Zapf, W.; Schupp, J.; Habich, R. (Hrsg.), Lebenslagen im Wandel: Sozialberichterstattung im Längsschnitt, Frankfurt, New York..

Vogler-Ludwig, K.; Langmantel E. (1997); „Entlastungspotential eines Teilkapitalstocks unter alternativen Bevölkerungsannahmen“, Studie des ifo Instituts im Auftrag der Enquete Kommission Demographischer Wandel, Deutscher Bundestag.

Vogler-Ludwig, K.; Severin, C.; Langmantel E. (1996); „Versicherungsfremde Leistungen in der Sozialversicherung", Studie des ifo Instituts.

Wagner, G. (1984); „Umverteilung in der gesetzlichen Rentenversicherung: Eine theoretische und empirische Analyse zum Versicherungsprinzip in der gesetzlichen Rentenversicherung", Frankfurt/New York.

Wagner, G. (1985); „Zur Meßbarkeit eines versicherungsgemäßen Risisikoausgleichs und der Umverteilung in der gesetzliche Rentenversicherung“, in: Schmähl, W. (Hrsg.), Versicherungsprinzip und soziale Sicherung, Tübingen, 1985, S. 142-203.

Wagner, G. (1994); „Möglichkeiten des Sozialversicherungsstaates - Prinzipielles und Reformvorschläge an den Beispielen der Gesundheits- und Umweltvorsorge“, in: Riedmüller, B; Olk, T. (Hrsg.), Grenzen des Sozialversicherungsstaates, Opladen.

Waldmann, H. (1981); „Zum Problem der Beitragsäquivalenz in der gesetzliche Rentenversicherung“, in: Deutsche Rentenversicherung, Heft 7, 1981, S. 453-465.

Wasem, J. (1988); „Äquivalenzprinzip und Leistungsfaehigkeit in der Sozialversicherung“, in: Groser et. al. Beiträge zur Sozialen Ordnungspolitik, Baden-Baden; S. 15-41.

Watermann, F. (1981), „Die Sozialversicherung im Spannungsfeld von Kausalität und Finalität", in: Zeitschrift für Sozialreform, Jg. 981, S. 298ff.

Weizäcker, C. C. v. (1962); „Wachstum, Zins und optimale Investitionsquote“, Tübingen.

Weizäcker, C. C. v. (1974); „Intergenerationale Einkommensverteilung: Einfache Beispiele für Wirkungen steuerlicher Maßnahmen und für die optimale Steuerstruktur", in: Bombach, G.; Frey, B.S.; Gahlen, B. (Hrsg.), Neue Aspekte der Verteilungstheorie, Tübingen.

Weizäcker, C. C. v. (1979); „Das Eherne Zinsgesetz“, in; Kyklos, 32, S. 270-282.

Weltbank (1994);,Averting the Old Age Crisis“, Washington (Oxsford University Press).

Williamson, S. (1986); „Costly Monitoring, Financial Intermediation, and Equilibrium Credit Rationing“, in: Journal of Monetary Economics, 18, S. 159-179.

Zacher, H. F. (1977); „Sozialstaatsprinzip“, in: Handbuch der Wwirtschaftswissenschaften, Stuttgart u.a., S. 152-160.

Zimmermann, H. (1996); „Wohlfahrtsstaat zwischen Wachstum und Verteilung“, München. 


\section{Anhang}

Ingo Schroeter - 978-3-631-75016-2

Downloaded from PubFactory at 01/11/2019 09:14:34AM

via free access 


\section{Inhalt}

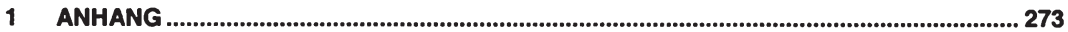

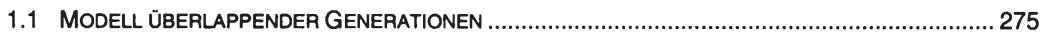

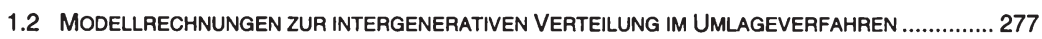

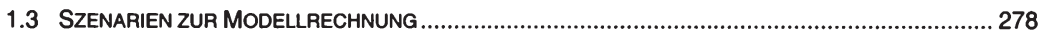

\section{Abbildungen}

Abbildung 1: Differenz der Verteilungsindikatoren der Reformen zum Referenzsystem (Szenario 1)......

Abbildung 2: Verlauf der Umverteilungspositionen der Zugangskohorten

(Szenario 1: Status Quo - Reform 99).

Abbildung 3: Verlauf der Umverteilungspositionen der Zugangskohorten

(Szenario 1: Status Quo - Senkung des Rentenniveaus)

Abbildung 4: Verlauf der Umverteilungspositionen der Zugangskohorten

(Szenario 1: Status Quo - Korrektur der anwartschaften)

Abbildung 5: Differenz der Verteilungsindikatoren der Reformen zum Referenzsystem (Szenario 2).

Abbildung 6: Verlauf der Umverteilungspositionen der Zugangskohorten

(Szenario 2: Status Quo - Reform 99).

Abbildung 7: Verlauf der Umverteilungspositionen der Zugangskohorten

(Szenario 2: Status Quo - Senkung des Renitenniveaus)

Abbildung 8: Verlauf der Umverteilungspositionen der Zugangskohorten

(Szenario 2: Status Quo - Korrektur der Anwartschaften) .286

Abbildung 9: Differenz der Verteilungsindikatoren der Reformen zum Referenzsystem (Szenario 3) 288

Abbildung 10: Verlauf der Umverteilungspositionen der Zugangskohorten (Szenario 3: Status

Quo - Reform 99)

Abbildung 11: Verlauf der Umverteilungspositionen der Zugangskohorten (Szenario 3: Status

Quo - Senkung des Rentenniveaus) . 289

Abbildung 12: Verlauf der Umverteilungspositionen der Zugangskohorten (Szenario 3: Status Quo - Korrektur der Anwartschaften). 290 


\section{Tabellen}

Tabelle 1: Verteilungsindikatoren im Basisszenario

Tabelle 2: Verteilungsindikatoren im Szenario 1

Tabelle 3: Kennziffern zum Verlauf der Umverteilungspositionen der Zugangskohorten 1996 bis

Tabelle 4: Verteilungsindikatoren Szenario 2

Tabelle 5: Kennziffern zum Verlauf der Umverteilungspositionen der Zugangskohorten 1996 bis 2030 (Szenario 2)

Tabelle 6: Verteilungsindikatoren im Szenario 3

Tabelle 7: Kennziffern zum Verlauf der Umverteilungspositionen der Zugangskohorten 1996 bis 2030 (Szenario 3) 
Ingo Schroeter - 978-3-631-75016-2

Downloaded from PubFactory at 01/11/2019 09:14:34AM

via free access 


\section{ANHANG}

\subsection{Modell überlappender Generationen}

Die Mehrzahl der theoretischen Abhandlungen zur Alterssicherung beruhen auf dem auf Samuelson zurückgehende "Modell überlappender Generationen“530. Definiert man eine Generationendauer als den Altersabstand zwischen Eltern und Kindern (ca. 25 Jahre), so kann man zu jeder Zeit zwischen drei Generationen unterscheiden und jedes Individuum durchlebt in der Regel drei Generationenperioden. Im Modell überlappender Generationen wird vereinfachend nur zwischen den Generationen der Erwerbstätigen und der Rentner unterschieden. Diese Beschränkung erscheint zulässig, da der Konsum der Kinder mit dem der Eltern zusammengefaßt werden kann und es weiterhin plausibel ist, daß auch die wichtigen Entscheidungen für die Kinder von den Eltern getroffen werden. Da die Analyse keine quantitativen Prognosen liefert, sondern die qualitativen Wirkungsweisen herausarbeiten soll, ist es nicht von Belang, daß das Individuum im Modell nur zwei Generationsdauern durchlebt.

Im Modell wird die Zeit in diskrete Perioden aufgeteilt, wobei die Länge einer Periode ( $t$ ) genau einer Generationsdauer entspricht, die für Erwerbstätige und Rentner gleich lang ist. Weiterhin geht man vereinfachend zunächst davon aus, daß alle Mitglieder der Bevölkerung im enwerbsfähigen Alter auch einer Erwerbstätigkeit nachgehen. Wenn $\mathrm{N}^{A}$ die $A n-$ zahl der Erwerbstätigen (Aktiven) und $\mathrm{N}^{A}$ die Anzahl der Rentner darstellt, so ergibt sich im Fall sicherer und einheitlicher Lebensdauern der Zusammenhang:

$$
N_{t-1}^{A}=N_{t}^{R}
$$

Es mag verwundern, daß das Modell eine einheitliche Lebensdauer unterstellt, diese Annahme läßt sich aber rechtfertigen, wenn man Finanzierungssysteme betrachtet, die mit einem Risikoausgleich für das Risiko der ungewissen individuellen Lebensdauer arbeiten. Das Modell arbeitet ausschließlich mit Durchschnittsgrößen, so daß sich die Ergebnisse, die sich für ein repräsentatives Individuum ergeben, ohne weiteres auch auf die gesamte Generation übertragen lassen. Entsprechend der zu untersuchenden Fragestellung, kann das Modell hinsichtlich verschiedener Parameter variiert werden. ${ }^{531}$ Zur Analyse der zunächst interessierenden, grundlegenden Zusammenhänge der Alterssicherung, ist das Basismodell ausreichend, das von den weiteren Annahmen einer sicheren Lebensdauer, der Abwesenheit von Altruismus und Vererbung, eines gesetzlich fixierten Rentenzugangsalters und rationalen Verhalten ausgeht. Desweiteren sind der Zins, der Lohnsatz

Vgl. Samuelson 1958. Homburg 1988, S. 15 und Malinvaud 1987 weisen jedoch darauf hin, daß zuvor schon Allais 1947 von diesem Modell Gebrauch machte. 
und die Bevölkerungsentwicklung im einfachen Modell exogen und i.d.R. werden konstante Wachstumsraten von Zins, Lohn und Bevölkerung (sogenannter Steady-State) unterstellt. Definitionsgemäß erfordert das Finanzierungsgleichgewicht des Umlageverfahrens, daß sich in jeder Periode die Einnahmen (E) und die Ausgaben ( $A$ ) entsprechen müssen.

$$
A_{t}=E_{t} \quad(G 2)
$$

Die Einnahmen einer Periode $t\left(E_{t}\right)$ setzen sich hierbei zusammen aus dem Beitragssatz $\left(b_{t}\right)$, dem Durchschnittslohn $\left(w_{t}\right)$ und der Anzahl der Erwerbstätigen der Periode $\left(N_{t}^{A}\right)$. Die Ausgaben erhält man durch Multiplikation der Durchschnittspension $\left(p_{t}\right)$ mit der Anzahl der Pensionsbezieher der Periode $\left(N_{t}^{R}\right)$.

$$
E_{t}=b_{t} \cdot w_{t} \cdot N_{t}^{A} \text { und } A_{t}=p_{t} \cdot N_{t}^{R} \quad(\mathrm{G} 3)
$$

Für den Lohnwachstumsfaktor $\left(1+g_{t+1}\right)$, als Verhältnis des Durchschnittslohnes einer Periode $t+1$ zum Durchschnittslohn der Vorperiode $t$, und für den Bevölkerungswachstumsfaktor $\left(1+m_{t+1}\right)$ gelten ferner die Beziehungen:

$$
1+g_{t+1}=\frac{w_{t+1}}{w_{t}} \quad \text { sowie } \quad 1+m_{t+1}=\frac{N_{t+1}}{N_{t}}
$$

Weitere Kenngrößen der Alterssicherung sind der Rentnerquotient (RQ) und das Rentenniveau (RN). Den Rentnerquotient erhält man, wenn man die Anzahl der Rentenempfänger zur Anzahl der Beitragszahler ins Verhältnis setzt. Das Rentenniveau ist definiert als Quotient aus Durchschnittspension und Durchschnittslohn.

$$
\begin{aligned}
& R Q=\frac{N_{t}^{R}}{N_{t}^{A}} \\
& R N=\frac{p_{t}}{w_{t}}
\end{aligned}
$$

Setzt man die Beziehungen G3 in das Finanzierungsgleichgewicht G2 ein, so ergibt sich unter Verwendung von G5 und G6, daß der erforderliche Beitragssatz des Umlageverfahrens durch das Produkt aus Rentnerquotient und Rentenniveau bestimmt wird.

$$
b_{t} \cdot w_{t} \cdot N_{t}^{A}=p_{t} \cdot N_{t}^{R} \Leftrightarrow b_{t}=\frac{p_{t}}{w_{t}} \cdot \frac{N_{t}^{R}}{N_{t}^{A}}=R N \cdot R Q
$$

Das Finanzierungsgleichgewicht des Kapitaldeckungsverfahrens ergibt sich daraus, daß sich die Einnahmen und Ausgaben einer Generation in zwei aufeinander folgenden Peri- 
oden entsprechen müssen. Die Ausgaben der Folgeperiode entsprechen definitionsgemäß den marktüblich verzinsten Einnahmen.

$$
A_{t+1}=E_{t} \cdot\left(1+r_{t}\right) \quad(\mathrm{G} 8)
$$

\subsection{Modellrechnungen zur intergenerativen Verteilung im Umla- geverfahren}

Im Referenzszenario haben die verschiedenen Parameter für alle Generation die folgenden als plausibel angenommenen Werte:

- Fertilität $M=1$;

- Überlebenswahrscheinlichkeit während der Erwerbsphase $I^{1}=0,9$;

- Überlebenswahrscheinlichkeit während der Erwerbsphase $\mathrm{I}^{2}=0,8$;

- Erwerbsbeteiligung während der ersten Periode $\mathrm{eq}^{1}=0,6$;

- Erwerbsbeteiligung während der zweiten Periode $\mathrm{eq}^{2}=0,6$;

- Wachstumsrate der Löhne $w=$ konstant $=0,03$;

- $\quad$ Zinssatz $\mathrm{i}=$ konstant $=0,03$;

Die Startwerte für die Kohortengröße und für die Lohnhöhe sind für die Generation $x-4$ auf eins normiert $\left(N_{x-4}{ }^{0}=1 ; w^{1-4}=1\right)$, wobei gilt, daß die Generation $x$ zum Zeitpunkt $t$ im Ruhestand ist. Es werden zunächst einmalige Parametervariationen durchgeführt, bei denen lediglich die entsprechenden Werte für die Generation x variieren und die Werte der anderen Generationen konstant bleiben. Um die Robustheit der Ergebnisse zu prüfen, wurden die gleichen Variationen bei den folgenden Konstellationen aus Zinssatz und Lohnentwicklung durchgeführt:

\begin{tabular}{|l|c|c|c|c|}
\hline Konstellation & 1 & 2 & 5 & 6 \\
\hline Zinssatz & $2 \%$ & $4 \%$ & $3 \%$ plus 0,25 \%-punkte pro Periode & $3 \%$ \\
\hline Lohnwachstum & $4 \%$ & $2 \%$ & $3 \%$ & $3 \%$ plus $0,25 \%$-punkte pro Periode \\
\hline
\end{tabular}

Weitere Sensitivitätsanalysen wurden für die folgenden Werte durchgeführt:

\begin{tabular}{|c|c|c|c|c|c|c|c|}
\hline \multicolumn{2}{|c|}{$\mathbf{M}$} & \multicolumn{2}{|c|}{$\mathbf{I}^{\mathbf{1}}$} & \multicolumn{2}{c|}{$\mathbf{I}^{2}$} & \multicolumn{2}{c|}{ eq } \\
$\mathbf{1 , 2}$ & 0,8 & 1 & 1 & 0,8 & 0,8 & 0,5 & 0,7 \\
\hline
\end{tabular}




\subsection{Szenarien zur Modellrechnung}

\section{Basisszenario}

- Zinssatz ab 1996: 5\%

- Lohnwachstum ab 1996: $3 \%$

- Diskontsatz: $4 \%$

- Vermölgenssubstitutionsfaktor: 0,5

- Wachlstumsfaktor (w_Faktor) =0,07 (Steigerung des Wachstums um 0,2 Prozentpunkte bei 100 Mrd. zusătzlicher gesamtwirtschaftlicher Ersparnis bezogen auf 3,5 bio. DM BIP)

- Verzinsungsänderung (i_Faktor) $=1 /$ w_Faktor

- $\Phi$ (Faktor für das Ausmaß des Risikoausgleichs bei Fertilitätsänderungen) = 3,5

Tabelle 14: Verteilungsindikatoren im Basisszenario

Werte der Verteilungsindikatoren

\begin{tabular}{|c|c|c|c|c|c|}
\hline Kohorte & Reforenz & Status Quo & Reform1999 & $\begin{array}{c}\text { Senkung des Renten- } \\
\text { niveaus }\end{array}$ & $\begin{array}{c}\text { Korrektur der } \\
\text { Anwartschaften }\end{array}$ \\
\hline 1996 & 0,10083 & 0,080878 & 0,079736 & 0,066669 & 0,081117 \\
\hline 1997 & 0,09986 & 0,079575 & 0,07834 & 0,064168 & 0,079845 \\
\hline 1998 & 0,10181 & 0,078321 & 0,076992 & 0,061688 & 0,078625 \\
\hline 1999 & 0,10279 & 0,076464 & 0,076236 & 0,074306 & 0,076798 \\
\hline 2000 & 0,10425 & 0,074937 & 0,074693 & 0,072642 & 0,075304 \\
\hline 2001 & 0,10337 & 0,073165 & 0,072919 & 0,070768 & 0,073587 \\
\hline 2002 & 0,10293 & 0,070886 & 0,070643 & 0,068445 & 0,071285 \\
\hline 2003 & 0,10444 & 0,069402 & 0,069157 & 0,066899 & 0,069832 \\
\hline 2004 & 0,10493 & 0,068151 & 0,067912 & 0,065606 & 0,068603 \\
\hline 2005 & 0,1048 & 0,06588 & 0,065663 & 0,063399 & 0,066217 \\
\hline 2006 & 0,1044 & 0,065225 & 0,065029 & 0,06273 & 0,065574 \\
\hline 2007 & 0,10433 & 0,064827 & 0,064646 & 0,062335 & 0,065196 \\
\hline 2008 & 0,10434 & 0,063771 & 0,063609 & 0,061354 & 0,064116 \\
\hline 2009 & 0,10573 & 0,063015 & 0,06287 & 0,060688 & 0,063263 \\
\hline 2010 & 0,10461 & 0,062282 & 0,062167 & 0,060075 & 0,062508 \\
\hline 2011 & 0,10341 & 0,061033 & 0,06095 & 0,059012 & 0,06118 \\
\hline 2012 & 0,10379 & 0,060068 & 0,060018 & 0,058248 & 0,060144 \\
\hline 2013 & 0,10489 & 0,05881 & 0,058792 & 0,057232 & 0,058788 \\
\hline 2014 & 0,10467 & 0,058109 & 0,058128 & 0,056771 & 0,05803 \\
\hline 2015 & 0,10372 & 0,057483 & 0,057535 & 0,056385 & 0,057302 \\
\hline 2016 & 0,10184 & 0,056901 & 0,056985 & 0,056079 & 0,0566 \\
\hline 2017 & 0,10141 & 0,056447 & 0,056569 & 0,055928 & 0,056014 \\
\hline 2018 & 0,1014 & 0,055824 & 0,055989 & 0,055616 & 0,055227 \\
\hline 2019 & 0,10039 & 0,055399 & 0,055605 & 0,055545 & 0,054622 \\
\hline 2020 & 0,09973 & 0,054765 & 0,055017 & 0,055302 & 0,053939 \\
\hline 2021 & 0,10039 & 0,054127 & 0,054422 & 0,05505 & 0,05322 \\
\hline 2022 & 0,10068 & 0,053601 & 0,05394 & 0,054947 & 0,052655 \\
\hline 2023 & 0,10102 & 0,053121 & 0,053511 & 0,054899 & 0,052108 \\
\hline 2024 & 0,10207 & 0,052628 & 0,053075 & 0,054849 & 0,051548 \\
\hline 2025 & 0,10258 & 0,052021 & 0,052518 & 0,054633 & 0,05081 \\
\hline 2026 & 0,10306 & 0,0515 & 0,052055 & 0,054525 & 0,050188 \\
\hline 2027 & 0,10442 & 0,050994 & 0,051606 & 0,054433 & 0,049572 \\
\hline 2028 & 0,10539 & 0,050309 & 0,050982 & 0,054171 & 0,048818 \\
\hline 2029 & 0,10657 & 0,049614 & 0,050345 & 0,053902 & 0,048038 \\
\hline 2030 & 0,10752 & 0,048816 & 0,049608 & 0,053511 & 0,047156 \\
\hline
\end{tabular}




\section{Szenario 1}

- Zinssatz ab 1996: $5 \%$

- Lohnwachstum ab 1996: $3 \%$

- Diskontsatz: $5 \%$

- Vermölgenssubstitutionsfaktor: 0,5

- Wachlstumsfaktor (w_Faktor) $=0,07$ (Steigerung des Wachstums um 0,2 Prozentpunkte bei 100 Mrd. zusätzlicher gesamtwirtschaftlicher Ersparnis bezogen auf 3,5 bio. DM BIP)

- Verzinsungsänderung (i_Faktor) $=1 / w_{-}$Faktor

- $\Phi$ (Faktor für das Ausmaß des Risikoausgleichs bei Fertilitätsänderungen) $=3,5$

Tabelle 15: Verteilungsindikatoren im Szenario 1

\begin{tabular}{|c|c|c|c|c|c|}
\hline \multicolumn{6}{|c|}{ Werte der Verteilungsindikatoren } \\
\hline Kohorte & Referenz & Status Quo & Reform1999 & $\begin{array}{c}\text { Senkung des Renten- } \\
\text { niveaus }\end{array}$ & $\begin{array}{c}\text { Korrektur der Anwart- } \\
\text { schaften }\end{array}$ \\
\hline 1996 & 0,10083 & 0,080878 & 0,079736 & 0,066669 & 0,081117 \\
\hline 1997 & 0,09986 & 0,079575 & 0,07834 & 0,064168 & 0,079845 \\
\hline 1998 & 0,10181 & 0,078321 & 0,076992 & 0,061688 & 0,078625 \\
\hline 1999 & 0,10279 & 0,076464 & 0,076236 & 0,074306 & 0,076798 \\
\hline 2000 & 0,10425 & 0,074937 & 0,074693 & 0,072642 & 0,075304 \\
\hline 2001 & 0,10337 & 0,073165 & 0,072919 & 0,070768 & 0,073587 \\
\hline 2002 & 0,10293 & 0,070886 & 0,070643 & 0,068445 & 0,071285 \\
\hline 2003 & 0,10444 & 0,069402 & 0,069157 & 0,066899 & 0,069832 \\
\hline 2004 & 0,10493 & 0,068151 & 0,067912 & 0,065606 & 0,068603 \\
\hline 2005 & 0,1048 & 0,06588 & 0,065663 & 0,063399 & 0,066217 \\
\hline 2006 & 0,1044 & 0,065225 & 0,065029 & 0,06273 & 0,065574 \\
\hline 2007 & 0,10433 & 0,064827 & 0,064646 & 0,062335 & 0,065196 \\
\hline 2008 & 0,10434 & 0,063771 & 0,063609 & 0,061354 & 0,064116 \\
\hline 2009 & 0,10573 & 0,063015 & 0,06287 & 0,060688 & 0,063263 \\
\hline 2010 & 0,10461 & 0,062282 & 0,062167 & 0,060075 & 0,062508 \\
\hline 2011 & 0,10341 & 0,061033 & 0,06095 & 0,059012 & 0,06118 \\
\hline 2012 & 0,10379 & 0,060068 & 0,060018 & 0,058248 & 0,060144 \\
\hline 2013 & 0,10489 & 0,05881 & 0,058792 & 0,057232 & 0,058788 \\
\hline 2014 & 0,10467 & 0,058109 & 0,058128 & 0,056771 & 0,05803 \\
\hline 2015 & 0,10372 & 0,057483 & 0,057535 & 0,056385 & 0,057302 \\
\hline 2016 & 0,10184 & 0,056901 & 0,056985 & 0,056079 & 0,0566 \\
\hline 2017 & 0,10141 & 0,056447 & 0,056569 & 0,055928 & 0,056014 \\
\hline 2018 & 0,1014 & 0,055824 & 0,055989 & 0,055616 & 0,055227 \\
\hline 2019 & 0,10039 & 0,055399 & 0,055605 & 0,055545 & 0,054622 \\
\hline 2020 & 0,09973 & 0,054765 & 0,055017 & 0,055302 & 0,053939 \\
\hline 2021 & 0,10039 & 0,054127 & 0,054422 & 0,05505 & 0,05322 \\
\hline 2022 & 0,10068 & 0,053601 & 0,05394 & 0,054947 & 0,052655 \\
\hline 2023 & 0,10102 & 0,053121 & 0,053511 & 0,054899 & 0,052108 \\
\hline 2024 & 0,10207 & 0,052628 & 0,053075 & 0,054849 & 0,051548 \\
\hline 2025 & 0,10258 & 0,052021 & 0,052518 & 0,054633 & 0,05081 \\
\hline 2026 & 0,10306 & 0,0515 & 0,052055 & 0,054525 & 0,050188 \\
\hline 2027 & 0,10442 & 0,050994 & 0,051606 & 0,054433 & 0,049572 \\
\hline 2028 & 0,10539 & 0,050309 & 0,050982 & 0,054171 & 0,048818 \\
\hline 2029 & 0,10657 & 0,049614 & 0,050345 & 0,053902 & 0,048038 \\
\hline 2030 & 0,10752 & 0,048816 & 0,049608 & 0,053511 & 0,047156 \\
\hline
\end{tabular}


Tabelle 16: Kennziffern zum Verlauf der Umverteilungspositionen der Zugangskohorten 1996 bis 2030 (Szenario 1)

\begin{tabular}{|l|r|r|r|r|}
\hline & Status Quo & Reform1999 & $\begin{array}{c}\text { Senkung } \\
\text { Rentenniveau }\end{array}$ & \multicolumn{1}{|c|}{$\begin{array}{c}\text { Korrektur der } \\
\text { Anwartschaften }\end{array}$} \\
\hline Sandardabweichung: & 0,0044 & 0,0041 & 0,0026 & 0,0049 \\
\hline Variationskoeffizient & 0,1543 & 0,1412 & 0,0835 & 0,1683 \\
\hline Rawls Differenzenkriterium & $-0,0344$ & $-0,0338$ & $-0,0357$ & $-0,0365$ \\
\hline
\end{tabular}

Quelle: eigene Berechnungen

Abbildung 25: Differenz der Verteilungsindikatoren der Reformen zum Referenzsystem (Szenario 1)

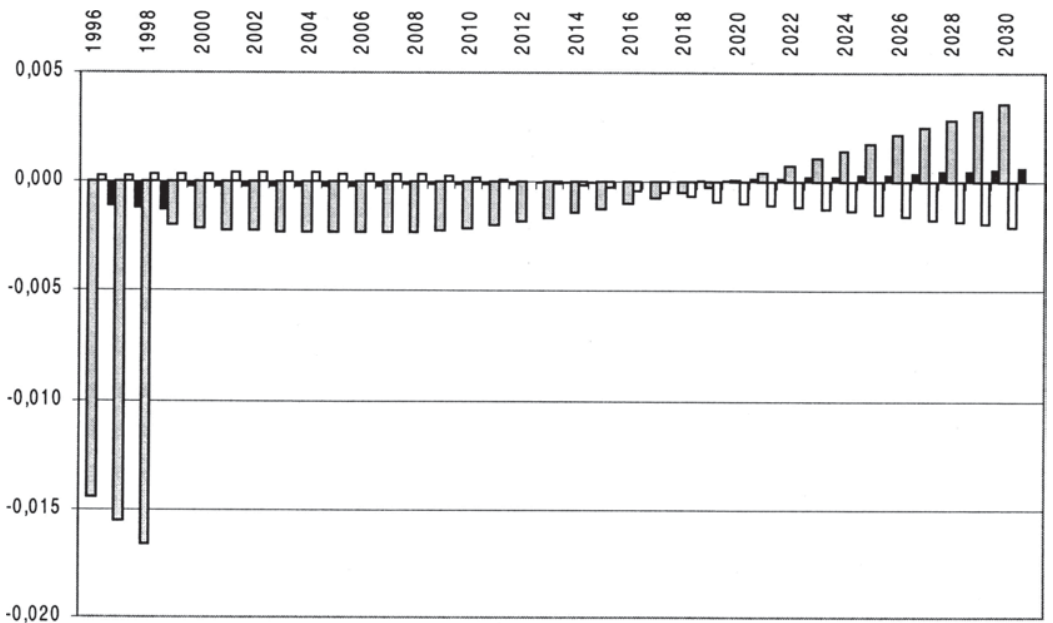

Reform 1999 Senkung Rentenniveau $\square$ Korrektur der Anwarts chaften 
Abbildung 26: Verlauf der Umverteilungspositionen der Zugangskohorten

(Szenario 1: Status Quo - Reform 99)

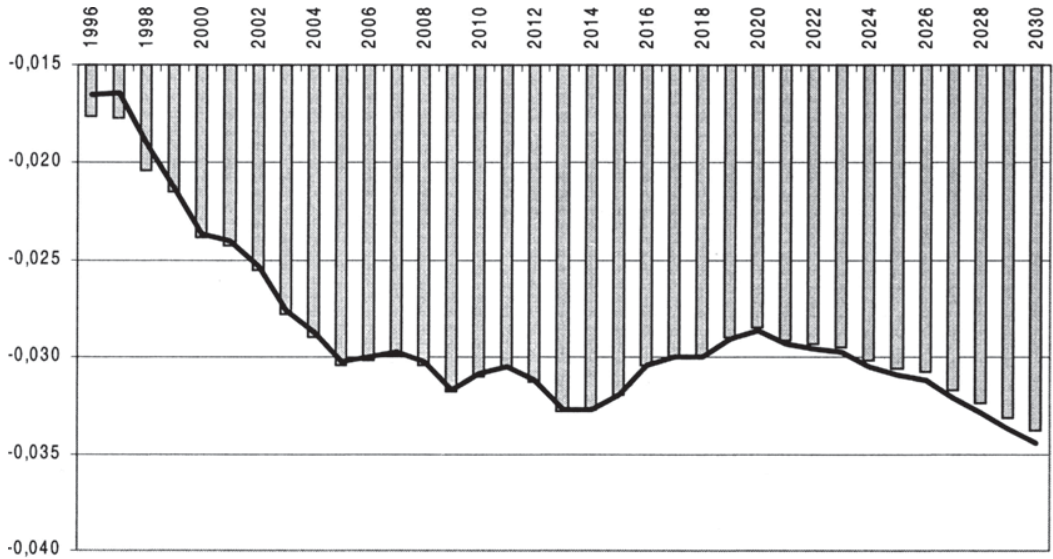

$\square$ Reform $1999 \longrightarrow$ Status Quo

Quelle: eigene Berechnungen

Abbildung 27: Verlauf der Umverteilungspositionen der Zugangskohorten

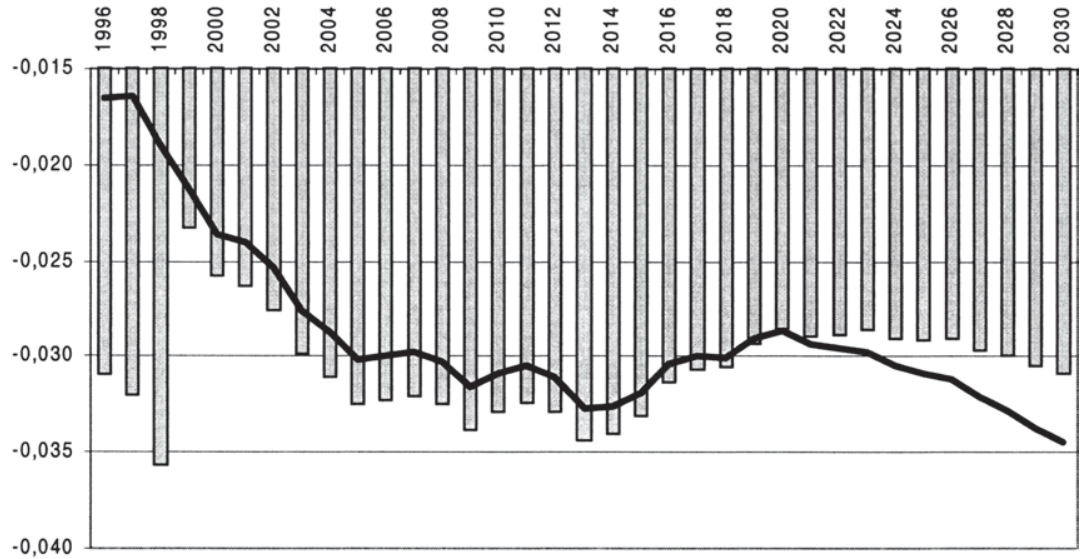

Senkung Rentenniveau $\longrightarrow$ Status Quo

Quelle: eigene Berechnungen 
Abblldung 28: Verlauf der Umverteilungspositionen der Zugangskohorten

(Szenario 1: Status Quo - Korrektur der Anwartschaften)

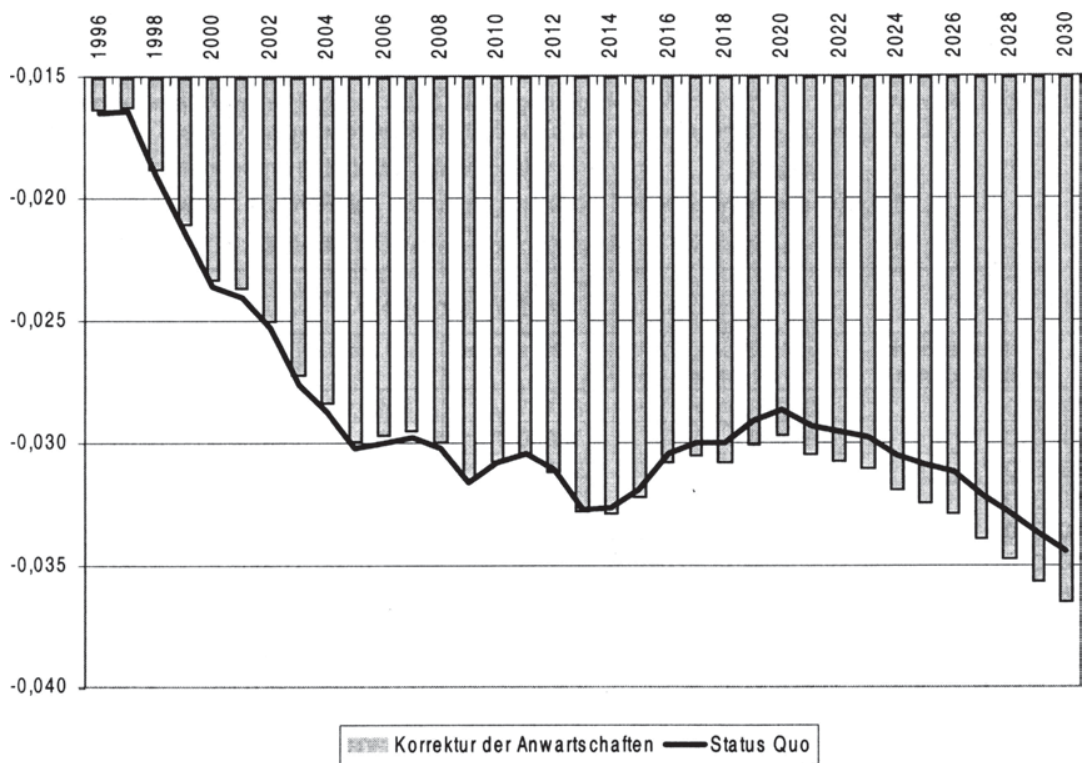

Quelle: eigene Berechnungen 


\section{Szenario 2}

- Zinssatz ab 1996: 5\%

- Lohnwachstum ab 1996: $2 \%$

- Diskontsatz: $4 \%$

- Vermölgenssubstitutionsfaktor: 0,5

- Wachlstumsfaktor (w_Faktor) =0,07 (Steigerung des Wachstums um 0,2 Prozentpunkte bei 100 Mrd. zusätzlicher gesamtwirtschaftlicher Ersparnis bezogen auf 3,5 bio. DM BIP)

- Verzinsungsänderung (i_Faktor) $=1 /$ w_Faktor

- $\Phi$ (Faktor für das Ausmaß des Risikoausgleichs bei Fertilitätsänderungen) = 3,5

Tabelle 17: Verteilungsindikatoren Szenario 2

\begin{tabular}{|c|c|c|c|c|c|}
\hline \multicolumn{6}{|c|}{ Werte der Verteilungsindikatoren } \\
\hline Kohorte & Referenz & Status Quo & Reform1999 & $\begin{array}{c}\text { Senkung des Renten- } \\
\text { niveaus }\end{array}$ & $\begin{array}{c}\text { Korrektur der Anwart- } \\
\text { schaften }\end{array}$ \\
\hline 1996 & 0,096758 & 0,072571 & 0,071757 & 0,065947 & 0,072725 \\
\hline 1997 & 0,09546 & 0,070698 & 0,069821 & 0,063542 & 0,070873 \\
\hline 1998 & 0,097172 & 0,068936 & 0,067995 & 0,061234 & 0,069135 \\
\hline 1999 & 0,09793 & 0,066701 & 0,066477 & 0,065636 & 0,066922 \\
\hline 2000 & 0,099107 & 0,064815 & 0,064582 & 0,063688 & 0,065059 \\
\hline 2001 & 0,097873 & 0,062778 & 0,062548 & 0,06161 & 0,063054 \\
\hline 2002 & 0,097234 & 0,060381 & 0,060165 & 0,059201 & 0,060645 \\
\hline 2003 & 0,098492 & 0,058678 & 0,058479 & 0,057474 & 0,058969 \\
\hline 2004 & 0,098642 & 0,057194 & 0,057014 & 0,055973 & 0,057508 \\
\hline 2005 & 0,098345 & 0,054963 & 0,054808 & 0,053775 & 0,055187 \\
\hline 2006 & 0,097469 & 0,054028 & 0,053891 & 0,052833 & 0,054268 \\
\hline 2007 & 0,096907 & 0,053311 & 0,053193 & 0,052118 & 0,053583 \\
\hline 2008 & 0,096603 & 0,052109 & 0,052018 & 0,050956 & 0,052374 \\
\hline 2009 & 0,097658 & 0,051163 & 0,051102 & 0,050058 & 0,051389 \\
\hline 2010 & 0,096048 & 0,050261 & 0,050229 & 0,049212 & 0,0505 \\
\hline 2011 & 0,094576 & 0,049009 & 0,049013 & 0,048047 & 0,049212 \\
\hline 2012 & 0,094695 & 0,048011 & 0,048058 & 0,047154 & 0,048205 \\
\hline 2013 & 0,09566 & 0,046804 & 0,046896 & 0,046074 & 0,046962 \\
\hline 2014 & 0,095041 & 0,046053 & 0,046193 & 0,045451 & 0,046215 \\
\hline 2015 & 0,093669 & 0,045369 & 0,045556 & 0,044899 & 0,045515 \\
\hline 2016 & 0,091362 & 0,044727 & 0,044962 & 0,04441 & 0,044853 \\
\hline 2017 & 0,090554 & 0,044206 & 0,044493 & 0,044062 & 0,044306 \\
\hline 2018 & 0,090267 & 0,043573 & 0,043912 & 0,043609 & 0,04363 \\
\hline 2019 & 0,088754 & 0,0431 & 0,043494 & 0,043341 & 0,043114 \\
\hline 2020 & 0,087713 & 0,042484 & 0,042934 & 0,042952 & 0,04241400 \\
\hline 2021 & 0,088113 & 0,041869 & 0,04237 & 0,04255 & 0,04176200 \\
\hline 2022 & 0,088017 & 0,041365 & 0,041929 & 0,042279 & 0,04117700 \\
\hline 2023 & 0,087982 & 0,040905 & 0,041539 & 0,042066 & 0,04064600 \\
\hline 2024 & 0,088724 & 0,040454 & 0,041161 & 0,041879 & 0,04011500 \\
\hline 2025 & 0,088927 & 0,039928 & 0,040716 & 0,041604 & 0,03955300 \\
\hline 2026 & 0,089025 & 0,039484 & 0,040357 & 0,041429 & 0,03902900 \\
\hline 2027 & 0,090094 & 0,039059 & 0,040021 & 0,041284 & 0,03852700 \\
\hline 2028 & 0,090843 & 0,038518 & 0,039579 & 0,041042 & 0,03786700 \\
\hline 2029 & 0,091853 & 0,037985 & 0,039145 & 0,040814 & 0,03722100 \\
\hline 2030 & 0,092687 & 0,037381 & 0,038643 & 0,040512 & 0,03649900 \\
\hline
\end{tabular}


Tabelle 18: Kennziffern zum Verlauf der Umverteilungspositionen der Zugangskohorten 1996 bis 2030 (Szenario 2)

\begin{tabular}{|r|r|r|r|r|}
\hline & Status Quo & Reform1999 & $\begin{array}{r}\text { Senkung } \\
\text { Rentenniveau }\end{array}$ & $\begin{array}{r}\text { Korrektur der } \\
\text { Anwartschaften }\end{array}$ \\
\hline Sandardabweichung: & 0,0075 & 0,0070 & 0,0055 & 0,0077 \\
\hline Variationskoeffizient & 0,1715 & 0,1616 & 0,1245 & 0,1761 \\
\hline Rawls Differenzenkriterium & $-0,0553$ & $-0,0540$ & $-0,0522$ & $-0,0562$ \\
\hline
\end{tabular}

Quelle: eigene Berechnungen

Abbildung 29: Differenz der Verteilungsindikatoren der Reformen zum Referenzsystem (Szenario 2)

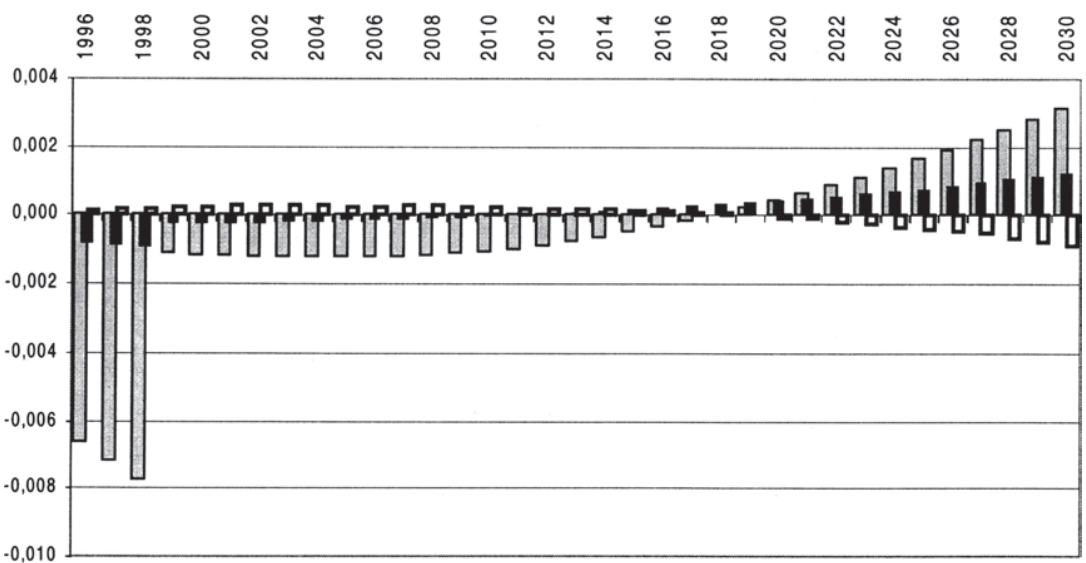

Senkung Rentenniveau $\mathbf{a}$ Reform1999 $\mathbf{\square}$ Korrektur der Anw artschaften

Quelle: eigene Berechnungen 
Abbildung 30: Verlauf der Umverteilungspositionen der Zugangskohorten

(Szenario 2: Status Quo - Reform 99)

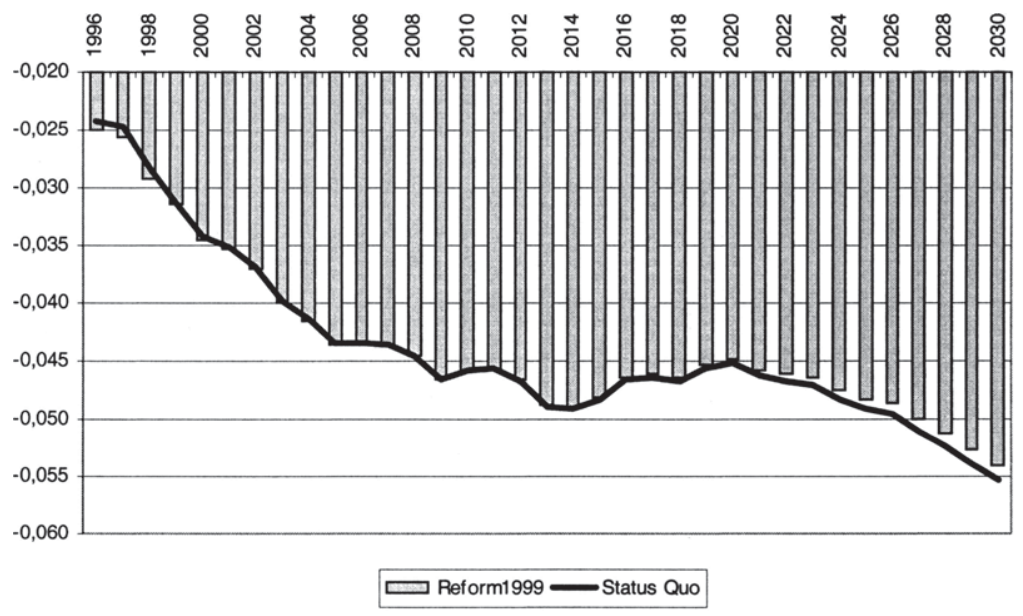

Quelle: eigene Berechnungen

Abbildung 31: Verlauf der Umverteilungspositionen der Zugangskohorten (Szenario 2: Status Quo - Senkung des Renltenniveaus)

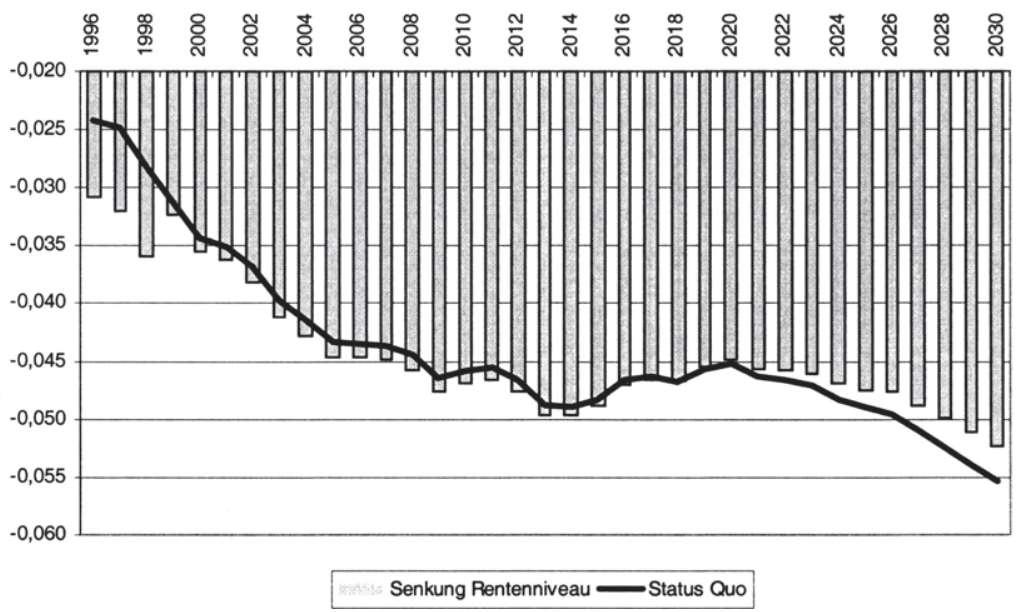

Quelle: eigene Berechnungen

285 
Abblldung 32: Verlauf der Umverteilungspositionen der Zugangskohorten

(Szenario 2: Status Quo - Korrektur der Anwartschaften)

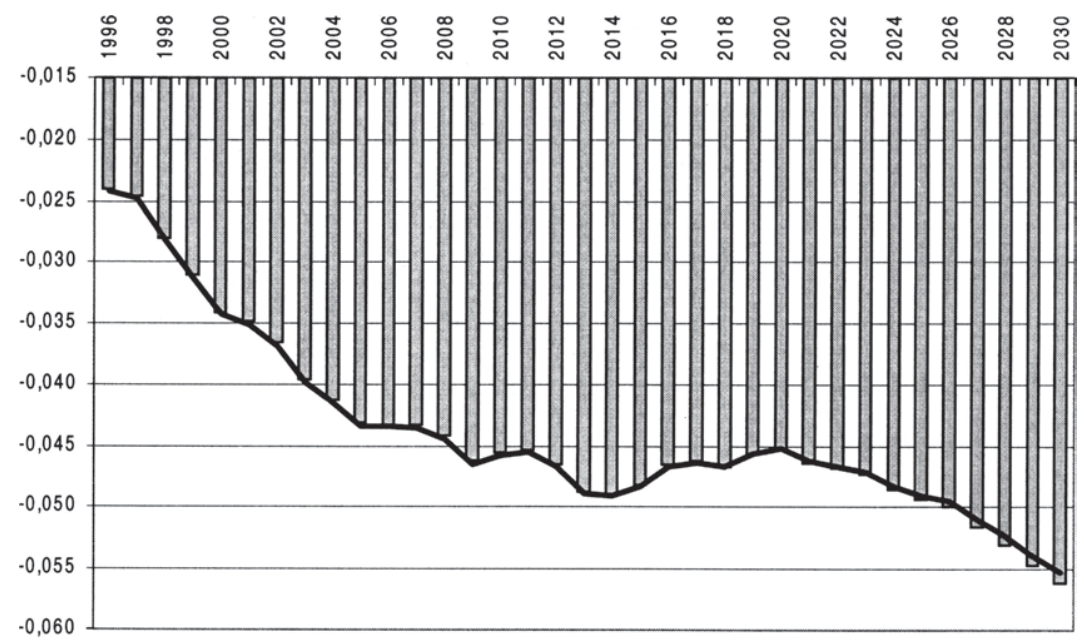

Korrektur der Anwartschaften $\longrightarrow$ Status Quo

Quelle: eigene Berechnungen 


\section{Szenario 3}

- Zinssatz ab 1996: $4 \%$

- Lohnwachstum ab 1996: $3 \%$

- Diskontsatz: $4 \%$

- Vermölgenssubstitutionsfaktor: 0,5

- Wachlstumsfaktor (w_Faktor) $=0,07$ (Steigerung des Wachstums um 0,2 Prozentpunkte bei 100 Mrd. zusätzlicher gesamtwirtschaftlicher Ersparnis bezogen auf 3,5 bio. DM BIP)

- Verzinsungsänderung (i_Faktor) $=1 /$ w_Faktor

- $\Phi$ (Faktor für das Ausmaß des Risikoausgleichs bei Fertilitätsänderungen) =3,5

Tabelle 19: Verteilungsindikatoren im Szenario 3

\begin{tabular}{|c|c|c|c|c|c|}
\hline \multicolumn{6}{|c|}{ Werte der Verteilungsindikatoren } \\
\hline Kohorte & Referenz & Status Quo & Reform1999 & $\begin{array}{c}\text { Senkung des Renten- } \\
\text { niveaus }\end{array}$ & $\begin{array}{c}\text { Korrektur der Anwart- } \\
\text { schaften }\end{array}$ \\
\hline 1996 & 0,10145 & 0,080878 & 0,079736 & 0,066669 & 0,081117 \\
\hline 1997 & 0,10041 & 0,079575 & 0,07834 & 0,064168 & 0,079845 \\
\hline 1998 & 0,10234 & 0,078321 & 0,076991 & 0,061688 & 0,078625 \\
\hline 1999 & 0,10326 & 0,076464 & 0,076235 & 0,074296 & 0,076799 \\
\hline 2000 & 0,10461 & 0,074937 & 0,074691 & 0,072626 & 0,075304 \\
\hline 2001 & 0,10356 & 0,073165 & 0,072917 & 0,070745 & 0,073587 \\
\hline 2002 & 0,10301 & 0,070886 & 0,07064 & 0,068415 & 0,071284 \\
\hline 2003 & 0,10438 & 0,069402 & 0,069153 & 0,066862 & 0,069831 \\
\hline 2004 & 0,10469 & 0,068151 & 0,067908 & 0,065561 & 0,068603 \\
\hline 2005 & 0,10444 & 0,06588 & 0,065659 & 0,063347 & 0,066212 \\
\hline 2006 & 0,10381 & 0,065225 & 0,065024 & 0,062669 & 0,065567 \\
\hline 2007 & 0,10352 & 0,064827 & 0,06464 & 0,062264 & 0,065188 \\
\hline 2008 & 0,10338 & 0,063771 & 0,063602 & 0,061275 & 0,064104 \\
\hline 2009 & 0,10458 & 0,063015 & 0,062863 & 0,0606 & 0,063246 \\
\hline 2010 & 0,10317 & 0,062282 & 0,06216 & 0,059978 & 0,062487 \\
\hline 2011 & 0,10178 & 0,061033 & 0,060942 & 0,058906 & 0,061153 \\
\hline 2012 & 0,10198 & 0,060068 & 0,060009 & 0,058135 & 0,06011 \\
\hline 2013 & 0,10287 & 0,05881 & 0,058783 & 0,057114 & 0,058746 \\
\hline 2014 & 0,10236 & 0,058109 & 0,058119 & 0,056651 & 0,057984 \\
\hline 2015 & 0,10117 & 0,057483 & 0,057528 & 0,056275 & 0,057255 \\
\hline 2016 & 0,0991 & 0,056901 & 0,056978 & 0,055975 & 0,056551 \\
\hline 2017 & 0,0985 & 0,056447 & 0,056562 & 0,055832 & 0,055965 \\
\hline 2018 & 0,0983 & 0,055824 & 0,055984 & 0,055545 & 0,055186 \\
\hline 2019 & 0,09706 & 0,055399 & 0,055601 & 0,055486 & 0,054586 \\
\hline 2020 & 0,0962 & 0,054765 & 0,055014 & 0,055257 & 0,053912 \\
\hline 2021 & 0,09662 & 0,054127 & 0,05442 & 0,055018 & 0,053201 \\
\hline 2022 & 0,09659 & 0,053601 & 0,053939 & 0,054927 & 0,052645 \\
\hline 2023 & 0,09661 & 0,053121 & 0,053511 & 0,054888 & 0,052105 \\
\hline 2024 & 0,09729 & 0,052628 & 0,053075 & 0,054852 & 0,051557 \\
\hline 2025 & 0,0974 & 0,052021 & 0,052521 & 0,054679 & 0,050853 \\
\hline 2026 & 0,09746 & 0,0515 & 0,05206 & 0,054608 & 0,050263 \\
\hline 2027| & 0,098337 & 0,050994 & 0,051612 & 0,054548 & 0,049674 \\
\hline 2028 & 0,098811 & 0,050309 & 0,050989 & 0,054319 & 0,048951 \\
\hline 2029 & 0,099436 & 0,049614 & 0,050353 & 0,05408 & 0,048198 \\
\hline 2030 & 0,099816 & 0,048816 & 0,049616 & 0,053716 & 0,047341 \\
\hline
\end{tabular}


Tabelle 20: Kennziffern zum Verlauf der Umverteilungspositionen der Zugangskohorten 1996 bis 2030 (Szenario 3)

\begin{tabular}{|r|r|r|r|r|}
\hline & Status Quo & Reform1999 & $\begin{array}{c}\text { Senkung des } \\
\text { Rentenniveaus }\end{array}$ & $\begin{array}{c}\text { Korrektur der Anwart- } \\
\text { schaften }\end{array}$ \\
\hline Sandardabweichung: & 0,0076 & 0,0071 & 0,0041 & 0,0081 \\
\hline Variationskoeffizient & $-0,1917$ & $-0,1804$ & $-0,0995$ & $-0,2027$ \\
\hline Rawls & $-0,0510$ & $-0,0502$ & $-0,0461$ & $-0,0525$ \\
\hline
\end{tabular}

Quelle: eigene Berechnungen

Abbildung 33: Differenz der Verteilungsindikatoren der Reformen zum Referenzsystem (Szenario 3)

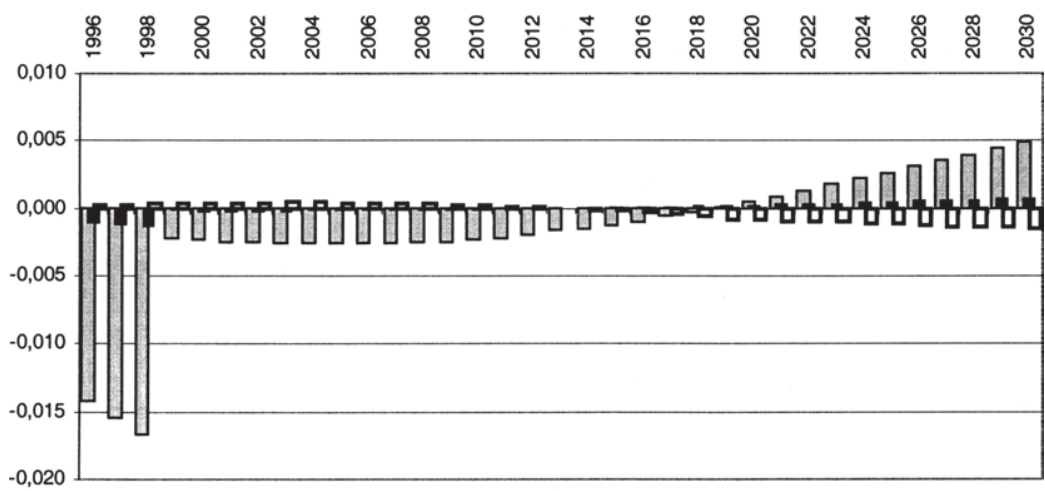

Senkung Rentenniveau $\mathbf{a}$ Reform1999 $\mathbf{0}$ Korrektur der Anw artschaften

Quelle: eigene Berechnungen 
Abbildung 34: Verlauf der Umverteilungspositionen der Zugangskohorten

(Szenario 3: Status Quo - Reform 99)

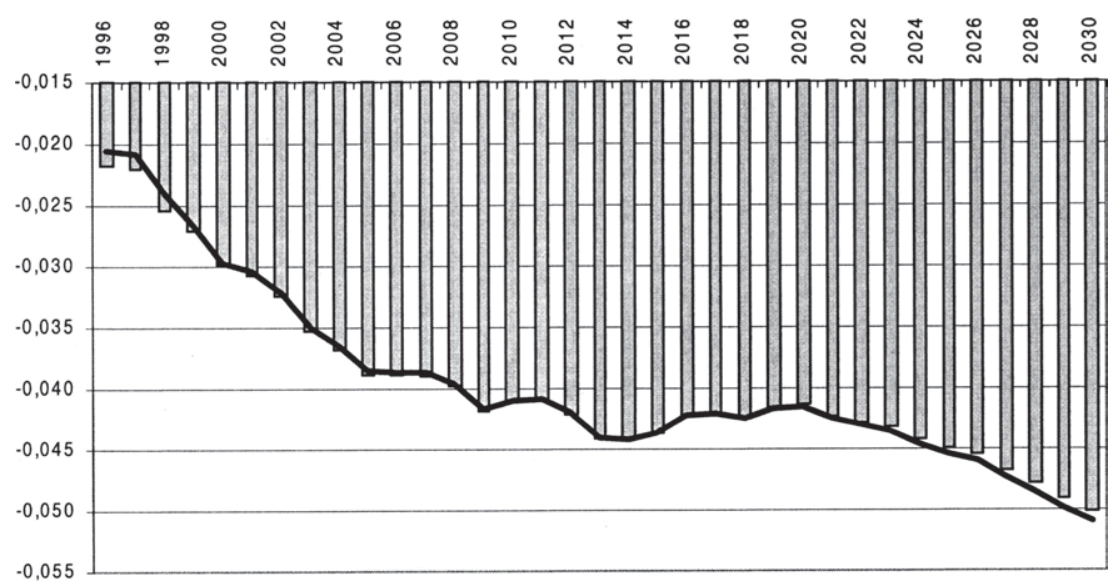

Reform $1999 \longrightarrow$ Status Quo

Quelle: eigene Berechnungen

Abbildung 35: Verlauf der Umverteilungspositionen der Zugangskohorten

(Szenario 3: Status Quo - Senkung des Rentenniveaus)

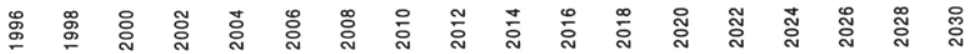

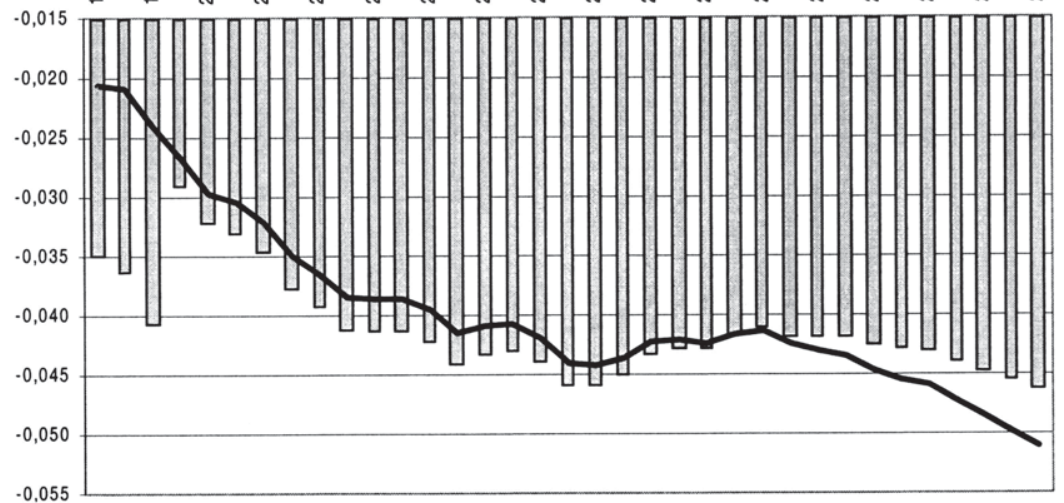

Senkung Rentenniveau $\longrightarrow$ Status Quo 
Abblldung 36: Verlauf der Umverteilungspositionen der Zugangskohorten

(Szenario 3: Status Quo - Korrektur der Anwartschaften)

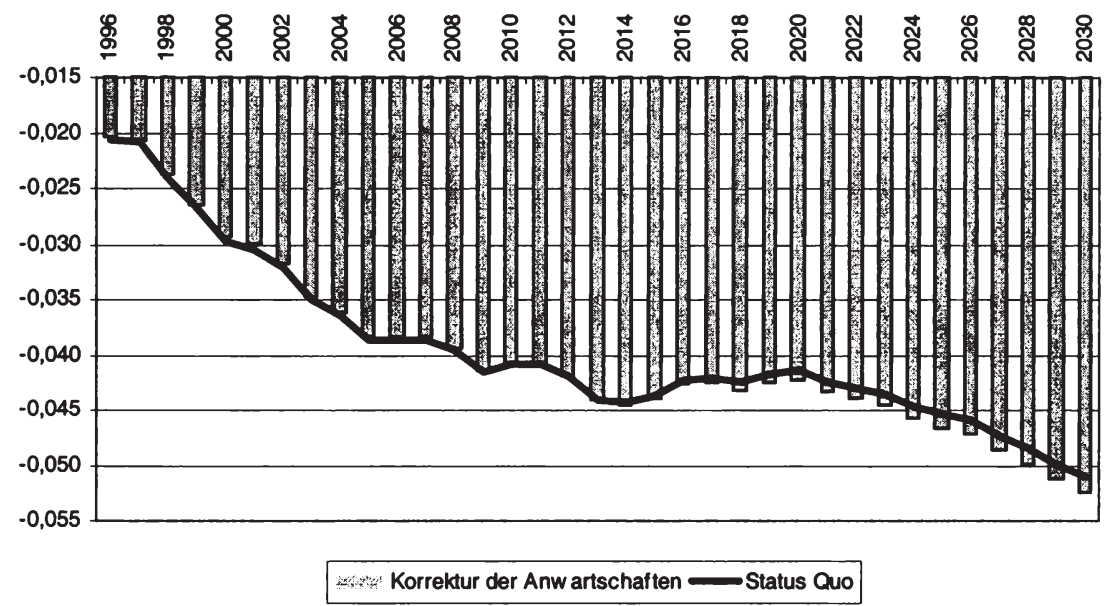

Quelle: eigene Berechnungen 


\section{SOZIALÖKONOMISCHE SCHRIFTEN}

Herausgegeben von Professor Dr. Dr. h.c. Bert Rürup

Band 1 Marietta Jass: Erfolgskontrolle des Abwasserabgabengesetzes. Ein Konzept zur Erfassung der Gesetzeswirkungen verbunden mit einer empirischen Untersuchung in der $\mathrm{Pa}$ pierindustrie. 1990.

Band 2 Frank Schulz-Nieswandt: Stationäre Altenpflege und "Pflegenotstand" in der Bundesrepublik Deutschland. 1990.

Band 3 Helmut Böhme, Alois Peressin (Hrsg.): Sozialraum Europa. Die soziale Dimension des Europäischen Binnenmarktes. 1990.

Band 4 Stephan Ruß: Telekommunikation als Standortfaktor für Klein- und Mittelbetriebe. Telekommunikative Entwicklungstendenzen und regionale Wirtschaftspolitik am Beispiel Hessen. 1991.

Band 5 Reinhard Grünewald: Tertiärisierungsdefizite im Industrieland Bundesrepublik Deutschland. Nachweis und politische Konsequenzen. 1992.

Band 6 Bert Rürup, Uwe H. Schneider (Hrsg.): Umwelt und Technik in den Europäischen Gemeinschaften. Teil I: Die grenzüberschreitende Entsorgung von Abfällen. Bearbeitet von: Thomas Kemmler, Thomas Steinbacher. 1993.

Band 7 Mihai Nedelea: Erfordernisse und Möglichkeiten einer wachstumsorientierten Steuerpolitik in Rumänien. Dargestellt am Beispiel der Textil- und Bekleidungsindustrie. 1995.

Band 8 Andreas Schade: Ganzjährige Beschäftigung in der Bauwirtschaft - Eine Wirkungsanalyse. Analyse und Ansätze für eine Reform der Winterbauförderung. 1995.

Band 9 Frank Schulz-Nieswandt: Ökonomik der Transformation als wirtschafts- und gesellschaftspolitisches Problem. Eine Einführung aus wirtschaftsanthropologischer Sicht. 1996.

Band 10 Werner Sesselmeier / Roland Klopfleisch / Martin Setzer: Mehr Beschäftigung durch eine Negative Einkommensteuer. Zur beschäftigungspolitischen Effektivität und Effizienz eines integrierten Steuer- und Transfersystems. 1996.

Band 11 Sylvia Liebler: Der Einfluß der Unabhängigkeit von Notenbanken auf die Stabilität des Geldwertes. 1996.

Band 12 Werner Sesselmeier: Einkommenstransfers als Instrumente der Beschäftigungspolitik. Negative Einkommensteuer und Lohnsubventionen im Lichte moderner Arbeitsmarkttheorien und der Neuen Institutionenökonomik. 1997.

Band 13 Stefan Lorenz: Der Zusammenhang von Arbeitsgestaltung und Erwerbsleben unter besonderer Berücksichtigung der Erwerbstätigkeiten von Frauen und Älteren. 1997.

Band 14 Volker Ehrlich: Arbeitslosigkeit und zweiter Arbeitsmarkt. Theoretische Grundlagen, Probleme und Erfahrungen. 1997.

Band 15 Philipp Hartmann: Grenzen der Versicherbarkeit. Private Arbeitslosenversicherung. 1998.

Band 16 Martin Setzer / Roland Klopfleisch / Werner Sesselmeier: Langzeitarbeitslose und Erster Arbeitsmarkt. Eine kombinierte Strategie zur Erhöhung der Wiederbeschäftigungschancen. 1999.

Band 17 Dorothea Wenzel: Finanzierung des Gesundheitswesens und Interpersonelle Umverteilung. Mikrosimulationsuntersuchung der Einkommenswirkung von Reformvorschlägen zur GKV-Finanzierung. 1999.

Band 18 Ingo Schroeter: Analyse und Bewertung der intergenerativen Verteilungswirkungen einer Substitution des Umlage- durch das Kapitalstocksverfahren zur Rentenfinanzierung. 1999. 


\section{Bremer Gesellschaft für Wirtschaftsforschung e.V. (Hrsg.)}

\section{Der Euro und die Folgen}

\section{Probleme und Perspektiven}

\section{Konzeption und Koordination: Alfons Lemper}

Frankfurt/M., Berlin, Bern, New York, Paris, Wien, 1998. 215 S.

ISBN 3-631-34021-4 · br. DM 38.- *

Die Einführung des Euro zum 1.1.1999 bringt nicht nur eine Fülle schwieriger und kostspieliger technischer Probleme mit sich, an deren Bewältigung allseits gearbeitet wird, sondern auch eine Reihe von Konsequenzen, die auf den ersten Blick nicht in Erscheinung treten, die gleichwohl für die Beurteilung des Gesamtprojektes „Europäische Währungsunion" (EWU) äußerst wichtig sind. Sie betreffen neben der prospektiven Stabilität der künftigen Währung und den Konsequenzen für die Kapitalanleger etwa Fragen nach den verbleibenden wirtschaftlichen Gestaltungsspielräumen für nationale Regierungen oder Tarifpartner, die Wahrscheinlichkeit eines innergemeinschaftlichen Finanzausgleichs oder Folgen für die Sozialstandards. Tiefgreifende Probleme ergeben sich auch für künftige Unternehmensmärkte. Die Lösung dieser - großenteils politischen - Probleme auf längere Sicht dürften für den Erfolg des Projektes EWU entscheidender werden als die bank- und bilanztechnischen Probleme, die derzeit meist im Vordergrund stehen.

Aus dem Inhalt: Prospektive Stabilität des Euro und seine internationale Position - Eingeschränkte Spielräume nationaler Wirtschaftspolitik · Finanzausgleich und Sozialstandards - Konsequenzen für Unternehmensmärkte

Frankfurt/M - Berlin - Bern - New York - Paris - Wien

Auslieferung: Verlag Peter Lang AG

Jupiterstr. 15, CH-3000 Bern 15

Telefax (004131) 9402131

*inklusive Mehrwertsteuer

Preisänderungen vorbehalten 